for

\title{
Synthesis of Fluorenes and Dibenzo[g,p]chrysenes Through an Oxidative Cascade
}

\author{
Cody F. Dickinson"a , Glenn P. A. Yap ${ }^{\mathrm{b}}$, and Marcus A. Tius ${ }^{* a}$ \\ ${ }^{a}$ Chemistry Department, University of Hawaii at Manoa, Honolulu, Hawaii 96822, United States \\ bDepartment of Chemistry and Biochemistry, University of Delaware, Newark, Delaware 19716, United \\ States \\ Email: tius@hawaii.edu
}

\section{Table of Contents}

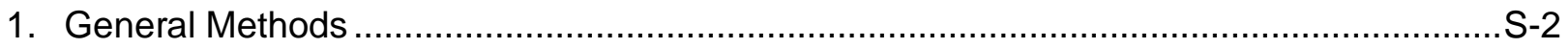

2. Experimental Section for Compounds 1-17 …………................................................

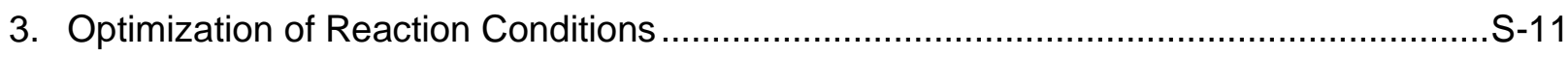

4. Synthesis of Aldol Adducts .......................................................................................

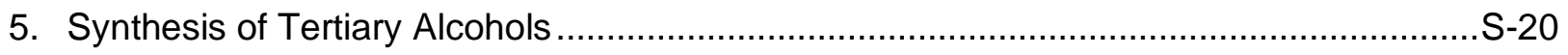

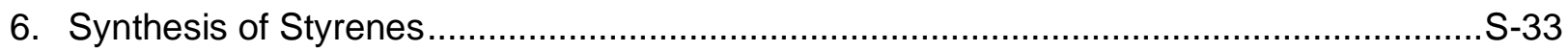

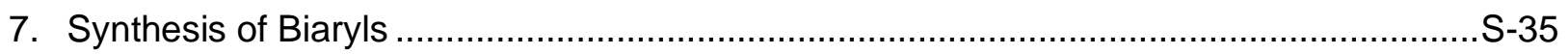

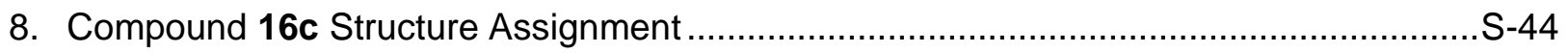

9. Postulated Reaction Mechanism for the Formation of 17a .......................................... -45

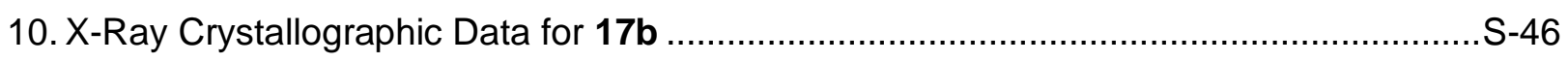

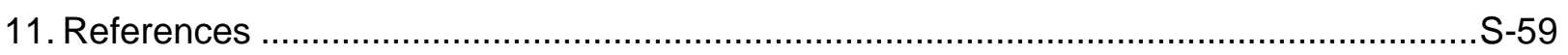

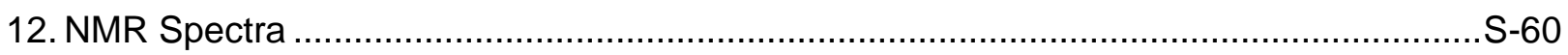




\section{General Methods}

All moisture and air sensitive reactions were performed under an argon atmosphere in oven-dried or flame-dried glassware. Reactions that required heating where carried with a stir-hot plate using a heated external oil bath. Tetrahydrofuran (THF) and diethyl ether $\left(\mathrm{Et}_{2} \mathrm{O}\right)$ were dried using a Glass Contour solvent purification system. Acetonitrile $(\mathrm{MeCN})$ was purchased from Alfa Aesar $(>99.5 \%)$ and used without further purification. ${ }^{1} \mathrm{H}$ NMR and ${ }^{13} \mathrm{C}\left\{{ }^{1} \mathrm{H}\right\}$ NMR spectra were measured on a Varian Mecury-300 (300 MHz/75 MHz), Agilent 400 DD2 (400 MHz/100 MHz), Varian INOVA$500(500 \mathrm{MHz} / 125 \mathrm{MHz})$, or Agilent $600 \mathrm{DD} 2(600 \mathrm{MHz} / 150 \mathrm{MHz})$ spectrometer at ambient temperature. Chemical shifts are reported in parts per million ( $\mathrm{ppm})$ and are referenced to the solvent (e.g., $\delta 7.26$ for $\mathrm{CHCl}_{3} ; \delta 77.0$ for $\left.\mathrm{CDCl}_{3}\right) .{ }^{19} \mathrm{~F} \mathrm{NMR}$ spectra were measured on a Varian Mercury-300 (282 MHz), Varian INOVA-500 (470 MHz), or an Agilent 600 DD2 (565 MHz) spectrometer and reported in ppm relative to TFA $(-76.5 \mathrm{ppm})$. Multiplicities are indicated as follows: br (broadened), s (singlet), d (doublet), t (triplet), q (quartet), pent (pentet), sext (sextet), sept (septet), etc. or m (multiplet). Coupling constants $(J)$ are reported in Hertz (Hz). Infrared (IR) spectra were recorded on a Shimadzu IRAffinity-1 Fourier transform or a ThermoFisher Nicolet Summit FTIR spectrophotometer. High-resolution mass spectra (HRMS) were obtained with an Agilent 1100 quaternary LC system. High-resolution mass spectra that were measured using $\mathrm{El}^{+}$ were obtained at the University of Illinois Mass Spectrometry Laboratory (Dr. Furong Sun and Dr. Xiuli Mao) or at the University of Delaware Mass Spectrometry Facility. Melting points were recorded on a DigiMelt MPA160 instrument and are uncorrected. Thin layer chromatography (TLC) was performed on glass plates, $250 \mu \mathrm{m}$, particle size 5-17 $\mu \mathrm{m}$, pore size $60 \AA$. All reactions were monitored by TLC and analyzed under UV (254 and/or $365 \mathrm{~nm})$ light and visualized using either PAA, $\mathrm{KMnO}_{4}$, PMA, CAM, or DNP stains. Flash column chromatography was performed on silica gel, $200-400$ mesh or premium silica gel, $60 \AA, 40-75 \mu \mathrm{m}$. Purity and homogeneity of all materials was determined by TLC, ${ }^{1} \mathrm{H} N \mathrm{NMR},{ }^{13} \mathrm{C}\left\{{ }^{1} \mathrm{H}\right\} \mathrm{NMR}$, and LCMS. 


\section{Experimental Section for Compounds 1-17}

Synthesis of Fluorenes. Representative Procedure. To a stirred solution of (E)-7-benzylidene8-(4-methoxyphenyl)-1,4-dioxaspiro[4.5]decan-8-ol 4a (348 mg, $1.0 \mathrm{mmol}, 1.0 \mathrm{eq}$ ) in MeCN (70 $\mathrm{mL}, 5 \mathrm{mg} / \mathrm{mL}$ ) under air at room temperature was added $\mathrm{Sc}(\mathrm{OTf})_{3}(48 \mathrm{mg}, 0.1 \mathrm{mmol}, 10 \mathrm{~mol} \%)$ and stirred for 30 minutes. The colorless reaction mixture immediately became a pinkish color upon the addition of $\mathrm{Sc}(\mathrm{OTf})_{3}$ which slowly reverted back to colorless. At this point, $\mathrm{CuBr}_{2}$ (446 $\mathrm{mg}, 2.0 \mathrm{mmol}, 2.0 \mathrm{eq}$ ) was added at once. After stirring for $30 \mathrm{~min}$ the reaction mixture was quenched with a sat. aq. solution of $\mathrm{NH}_{4} \mathrm{Cl}$ and stirred for $20 \mathrm{~min}$. The aqueous phase was separated and extracted with diethyl ether (x3). The combined organic extracts were washed with a saturated aqueous solution of $\mathrm{NaHCO}_{3}(x 2)$ and brine. The combined organic extracts were dried over anhydrous sodium sulfate. After filtration, the solvent was removed in vacuo and the crude oil was purified by flash column chromatography on silica gel using a gradient of ethyl acetate in hexane as eluent (load as PhMe solution; 0, 5, 7, 10, 15, 20\%) to afford 5 a as a white solid (222 $\mathrm{mg}, 78 \%$ yield).

7-Methoxy-9-phenyl-9H-fluoren-2-ol (5a): white solid (222 mg, 78\% yield); mp $163-164{ }^{\circ} \mathrm{C} ;{ }^{1} \mathrm{H}$ NMR (500 MHz, $\left.\mathrm{C}_{6} \mathrm{D}_{6}\right) \delta 7.47(\mathrm{~d}, J=8.3 \mathrm{~Hz}, 1 \mathrm{H}), 7.40(\mathrm{~d}, J=8.2 \mathrm{~Hz}, 1 \mathrm{H}), 7.07-6.97(\mathrm{~m}, 3 \mathrm{H})$, 6.94 (dd, $J=7.8,1.8 \mathrm{~Hz}, 2 \mathrm{H}$ ), 6.89 (dd, $J=8.3,2.4 \mathrm{~Hz}, 1 \mathrm{H}), 6.87(\mathrm{~d}, J=2.3 \mathrm{~Hz}, 1 \mathrm{H}$ ), 6.66 (dd, $J$ $=8.2,2.3 \mathrm{~Hz}, 1 \mathrm{H}), 6.42(\mathrm{~s}, 1 \mathrm{H}), 4.74(\mathrm{~s}, 1 \mathrm{H}), 4.12(\mathrm{brs}, 1 \mathrm{H}), 3.21(\mathrm{~s}, 3 \mathrm{H}) ;{ }^{13} \mathrm{C}\left\{{ }^{1} \mathrm{H}\right\} \mathrm{NMR}(125 \mathrm{MHz}$, $\left.\mathrm{C}_{6} \mathrm{D}_{6}\right) \delta 159.5,155.5,150.0,149.7,142.3,134.5,134.2,128.9,128.8,127.0,120.1,120.0,114.9$, $114.1,112.8,111.1,54.9$ (x2; gHSQC showed that the $\mathrm{CH}$ at $4.74 \mathrm{ppm}$ and the $\mathrm{CH}_{3}$ at $3.21 \mathrm{ppm}$ coincidentally have the same carbon chemical shift at $54.9 \mathrm{ppm}$ ); IR (neat, $\mathrm{cm}^{-1}$ ) 3404, 3022, 2938, 2833, 1607, 1587, 1493, 1460, 1439, 1285, 1238, 1138, 1115, 1032; HRMS (ESI $\left.{ }^{-}\right) \mathrm{m} / \mathrm{z}$ calcd for $\mathrm{C}_{20} \mathrm{H}_{15} \mathrm{O}_{2}[\mathrm{M}-\mathrm{H}]^{-}:$287.1078; found 287.1084.

7-Methoxy-9-(4-methoxyphenyl)-9H-fluoren-2-ol (5b): white solid (80 mg, 79\% yield); mp 146 $-147{ }^{\circ} \mathrm{C} ;{ }^{1} \mathrm{H}$ NMR $\left(500 \mathrm{MHz}, d_{8}\right.$-THF) $\delta 8.08(\mathrm{~s}, 1 \mathrm{H}), 7.54(\mathrm{~d}, \mathrm{~J}=8.3 \mathrm{~Hz}, 1 \mathrm{H}), 7.49(\mathrm{~d}, \mathrm{~J}=8.1 \mathrm{~Hz}$, $1 \mathrm{H}), 7.02-6.97(\mathrm{~m}, 2 \mathrm{H}), 6.85(\mathrm{dd}, J=8.3,2.4 \mathrm{~Hz}, 1 \mathrm{H}), 6.82-6.77(\mathrm{~m}, 3 \mathrm{H}), 6.72(\mathrm{dd}, J=8.2$, $2.3 \mathrm{~Hz}, 1 \mathrm{H}), 6.65-6.61(\mathrm{~m}, 1 \mathrm{H}), 4.88(\mathrm{~s}, 1 \mathrm{H}), 3.72(\mathrm{~s}, 3 \mathrm{H}), 3.71(\mathrm{~s}, 3 \mathrm{H}) ;{ }^{13} \mathrm{C}\left\{{ }^{1} \mathrm{H}\right\} \mathrm{NMR}(125 \mathrm{MHz}$, $d_{8}$ THF) $\delta 159.8,159.7,157.8,150.8,150.3,135.2,135.0,133.4,130.0,120.3,119.9,115.1$, 114.7, 113.7, 113.1, 111.6, 55.5, 55.3, 54.5; IR (neat, $\mathrm{cm}^{-1}$ ) 3418, 2100, 1653, 1634, 1585, 1506, 1464, 1283, 1250, 1223, 1117, 1036; HRMS (ESI) ${ }^{-} \mathrm{m} / \mathrm{z}$ calcd for $\mathrm{C}_{21} \mathrm{H}_{17} \mathrm{O}_{3}[\mathrm{M}-\mathrm{H}]^{-}: 317.1183$; found 317.1198.

9-(4-Chlorophenyl)-7-methoxy-9H-fluoren-2-ol (5c): off-white solid (58 mg, 86\% yield); mp 159 $-161^{\circ} \mathrm{C} ;{ }^{1} \mathrm{H}$ NMR $\left(500 \mathrm{MHz}, d_{8}\right.$-THF) $\delta 8.15(\mathrm{~s}, 1 \mathrm{H}), 7.56(\mathrm{~d}, J=8.3 \mathrm{~Hz}, 1 \mathrm{H}), 7.50(\mathrm{~d}, J=8.2 \mathrm{~Hz}$, $1 \mathrm{H}), 7.28-7.22(\mathrm{~m}, 2 \mathrm{H}), 7.10-7.02(\mathrm{~m}, 2 \mathrm{H}), 6.87(\mathrm{dd}, J=8.1,2.7 \mathrm{~Hz}, 1 \mathrm{H}), 6.80-6.76(\mathrm{~m}, 1 \mathrm{H})$, $6.74(\mathrm{dd}, J=8.2,2.3 \mathrm{~Hz}, 1 \mathrm{H}), 6.65-6.61(\mathrm{~m}, 1 \mathrm{H}), 4.94(\mathrm{~s}, 1 \mathrm{H}), 3.71(\mathrm{~s}, 3 \mathrm{H}) ;{ }^{13} \mathrm{C}\left\{{ }^{1} \mathrm{H}\right\} \mathrm{NMR}(125$ $\mathrm{MHz}, d_{8}$-THF) $\delta 159.7,157.7,149.7,149.3,142.1,135.1,133.2,132.9,130.5,129.2,120.2$, 119.9, 115.2, 113.8, 112.9, 111.4, 55.3, 54.2; IR (neat, $\mathrm{cm}^{-1}$ ) 3362, 3044, 3022, 2953, 2934, 2833, 1609, 1585, 1487, 1439, 1283, 1238, 1167, 1088, 1038, 1015; HRMS (ESI $\left.{ }^{-}\right) \mathrm{m} / \mathrm{z}$ calcd for $\mathrm{C}_{20} \mathrm{H}_{14} \mathrm{ClO}_{2}[\mathrm{M}-\mathrm{H}]^{-}:$:321.0688; found 321.0697.

9-(2-Chloro-6-fluorophenyl)-7-methoxy-9H-fluoren-2-ol (5d): colorless oil (79 $\mathrm{mg}, \mathbf{7 7 \%}$ yield); ${ }^{1} \mathrm{H}$ NMR $\left(500 \mathrm{MHz}, \mathrm{C}_{6} \mathrm{D}_{6}\right) \delta 7.50-7.45(\mathrm{~m}, 1.4 \mathrm{H}), 7.44-7.39(\mathrm{~m}, 1.4 \mathrm{H}), 6.98(\mathrm{dt}, \mathrm{J}=8.2,1.1$ $\mathrm{Hz}, 1 \mathrm{H}), 6.93-6.81(\mathrm{~m}, 3.2 \mathrm{H}), 6.78-6.63(\mathrm{~m}, 2.3 \mathrm{H}), 6.58-6.44(\mathrm{~m}, 2.6 \mathrm{H}), 6.38-6.34(\mathrm{~m}$, $0.4 \mathrm{H}), 6.28$ (ddd, $J=9.6,8.3,1.2 \mathrm{~Hz}, 1 \mathrm{H}), 5.68(\mathrm{~s}, 1 \mathrm{H}), 5.58(\mathrm{~s}, 0.5 \mathrm{H}), 4.39(\mathrm{~s}, 1.6 \mathrm{H}), 3.22-3.19$ $(\mathrm{m}, 4.7 \mathrm{H}) ;{ }^{13} \mathrm{C}\left\{{ }^{1} \mathrm{H}\right\} \mathrm{NMR}\left(125 \mathrm{MHz}, \mathrm{C}_{6} \mathrm{D}_{6}\right) \delta 161.8\left(\mathrm{~d}, J_{C F}=254.7 \mathrm{~Hz}\right), 159.5,155.5,147.4\left(\mathrm{~d}, J_{C F}\right.$ $=16.9 \mathrm{~Hz}), 146.8\left(\mathrm{~d}, J_{C F}=19.7 \mathrm{~Hz}\right), 136.2\left(\mathrm{~d}, J_{C F}=7.0 \mathrm{~Hz}\right), 136.3,135.0,134.6\left(\mathrm{~d}, J_{C F}=0.9 \mathrm{~Hz}\right)$, $134.3\left(\mathrm{~d}, J_{C F}=1.0 \mathrm{~Hz}\right), 129.0,128.94,128.87,128.6,128.4,128.3,127.0\left(\mathrm{~d}, J_{C F}=3.3 \mathrm{~Hz}\right), 125.1$ $\left(\mathrm{d}, J_{C F}=3.8 \mathrm{~Hz}\right), 120.3\left(\mathrm{~d}, J_{C F}=21.1 \mathrm{~Hz}\right), 123.3\left(\mathrm{~d}, J_{C F}=18.3 \mathrm{~Hz}\right), 115.1\left(\mathrm{~d}, J_{C F}=21.2 \mathrm{~Hz}\right), 114.9$, 114.8, 113.86, 113.85, $113.7\left(\mathrm{~d}, J_{C F}=23.7 \mathrm{~Hz}\right), 111.7,111.6,109.9,54.88,54.87,48.0\left(\mathrm{~d}, J_{C F}=\right.$ $1.0 \mathrm{~Hz}$ ); ${ }^{19} \mathrm{~F}$ NMR $\left(470 \mathrm{MHz}, \mathrm{C}_{6} \mathrm{D}_{6}\right) \delta-108.26$ (dd, $\left.J=10.0,5.8 \mathrm{~Hz}, 2.3 \mathrm{~F}\right),-115.50$ (dd, J = 9.4, 
6.2 Hz, 1.0F); IR (neat, $\mathrm{cm}^{-1}$ ) 3391, 2934, 2835, 1611, 1585, 1574, 1454, 1358, 1285, 1242, 1138, 1117, 1038, 1003; HRMS (ESI $\left.{ }^{-}\right) \mathrm{m} / \mathrm{z}$ calcd for $\mathrm{C}_{20} \mathrm{H}_{13} \mathrm{CIFO}_{2}[\mathrm{M}-\mathrm{H}]^{-}:$339.0594; found 339.0604. 5d was isolated as a $1.0: 2.3$ mixture of rotamers based on the integration of the ${ }^{19} \mathrm{~F}$ NMR signals; the data reported above is for the rotameric mixture. Some ${ }^{13} \mathrm{C}$ NMR signals of the mixture are not observed.

7-Methoxy-9-(naphthalen-2-yl)-9H-fluoren-2-ol (5e): off-white solid (94 mg, 73\% yield); mp 168 $-171{ }^{\circ} \mathrm{C} ;{ }^{1} \mathrm{H}$ NMR $\left(500 \mathrm{MHz}, d_{8}\right.$-THF) $\delta 8.23(\mathrm{~s}, 1 \mathrm{H}), 7.83(\mathrm{~d}, \mathrm{~J}=1.7 \mathrm{~Hz}, 1 \mathrm{H}), 7.80(\mathrm{~d}, J=8.0 \mathrm{~Hz}$, $1 \mathrm{H}), 7.76(\mathrm{~d}, J=8.0 \mathrm{~Hz}, 1 \mathrm{H}), 7.66(\mathrm{~d}, J=8.5 \mathrm{~Hz}, 1 \mathrm{H}), 7.60(\mathrm{~d}, J=8.3 \mathrm{~Hz}, 1 \mathrm{H}), 7.55(\mathrm{~d}, J=8.2$ $\mathrm{Hz}, 1 \mathrm{H}), 7.44-7.36(\mathrm{~m}, 2 \mathrm{H}), 6.91(\mathrm{dd}, J=8.5,1.8 \mathrm{~Hz}, 1 \mathrm{H}), 6.91-6.85(\mathrm{~m}, 1 \mathrm{H}), 6.84-6.80(\mathrm{~m}$, $1 \mathrm{H}), 6.81-6.75(\mathrm{~m}, 1 \mathrm{H}), 6.69-6.68(\mathrm{~m}, 1 \mathrm{H}), 5.11(\mathrm{~s}, 1 \mathrm{H}), 3.65(\mathrm{~s}, 3 \mathrm{H}) ;{ }^{13} \mathrm{C}\left\{{ }^{1} \mathrm{H}\right\} \mathrm{NMR}(125 \mathrm{MHz}$, $d_{8}$-THF) $\delta 159.9,157.9,150.4,149.9,140.8,135.4,134.8,133.7,133.6,129.1,128.3,128.0$, $127.1,126.7,126.2,120.4,120.1,115.4,114.1,113.3,111.7,55.5,55.4$ (one carbon signal is not observed); IR (neat, $\mathrm{cm}^{-1}$ ) 3364, 3051, 3019, 2936, 2833, 1609, 1585, 1466, 1437, 1364, 1283, 1238, 1179, 1138, 1115, 1036; HRMS $\left(E S I^{-}\right) \mathrm{m} / \mathrm{z}$ calcd for $\mathrm{C}_{24} \mathrm{H}_{17} \mathrm{O}_{2}[\mathrm{M}-\mathrm{H}]^{-}:$:337.1234; found 337.1253.

9-(Anthracen-9-yl)-7-methoxy-9H-fluoren-2-ol (5f): off-white solid (74 mg, 79\% yield); ${ }^{1} \mathrm{H}$ NMR $\left(500 \mathrm{MHz}, d_{8}\right.$-THF) $\delta 8.77(\mathrm{~d}, J=9.0 \mathrm{~Hz}, 1 \mathrm{H}), 8.49(\mathrm{~s}, 1 \mathrm{H}), 8.20-8.04(\mathrm{~m}, 1 \mathrm{H}), 7.94(\mathrm{~s}, 1 \mathrm{H}), 7.91$ $(\mathrm{d}, J=8.5 \mathrm{~Hz}, 1 \mathrm{H}), 7.75(\mathrm{~d}, J=8.4 \mathrm{~Hz}, 1 \mathrm{H}), 7.69(\mathrm{~d}, J=8.4 \mathrm{~Hz}, 1 \mathrm{H}), 7.63-7.57(\mathrm{~m}, 1 \mathrm{H}), 7.56-$ $7.48(\mathrm{~m}, 1 \mathrm{H}), 7.19(\mathrm{ddd}, J=8.3,6.4,1.2 \mathrm{~Hz}, 1 \mathrm{H}), 6.94-6.86(\mathrm{~m}, 2 \mathrm{H}), 6.86-6.75(\mathrm{~m}, 2 \mathrm{H}), 6.57$ $-6.45(\mathrm{~m}, 2 \mathrm{H}), 6.37-6.30(\mathrm{~m}, 1 \mathrm{H}), 3.53(\mathrm{~s}, 2 \mathrm{H}) ;{ }^{13} \mathrm{C}\left\{{ }^{1} \mathrm{H}\right\} \mathrm{NMR}\left(125 \mathrm{MHz}, d_{8}-\mathrm{THF}\right) \delta 159.9,157.9$, $151.4,151.2,134.5,133.3,133.2,133.1,132.9,132.7,130.6,130.3,129.5,128.4,127.2,127.0$, 125.6, 125.54, 125.48, 124.9, 120.9, 120.6, 115.1, 113.8, 112.1, 110.5, 55.4, 49.7; IR (neat, $\mathrm{cm}^{-1}$ ) $3331,3049,2900,2833,1609,1584,1522,1450,1437,1346,1281,1240,1113,1034$; HRMS $\left(\mathrm{ESI}^{-}\right) \mathrm{m} / \mathrm{z}$ calcd for $\mathrm{C}_{28} \mathrm{H}_{19} \mathrm{O}_{2}[\mathrm{M}-\mathrm{H}]^{-}: 387.1391$; found 387.1396 .

9-(tert-Butyl)-7-methoxy-9H-fluoren-2-ol (5g): off-white solid (67 mg, 74\% yield); mp $198-200$ ${ }^{\circ} \mathrm{C} ;{ }^{1} \mathrm{H}$ NMR $\left(500 \mathrm{MHz}, d_{8}-\mathrm{THF}\right) \delta 8.20(\mathrm{~s}, 1 \mathrm{H}), 7.43(\mathrm{~d}, J=8.3 \mathrm{~Hz}, 1 \mathrm{H}), 7.38(\mathrm{~d}, J=8.1 \mathrm{~Hz}, 1 \mathrm{H})$, $7.08(\mathrm{~d}, J=2.2 \mathrm{~Hz}, 1 \mathrm{H}), 6.98(\mathrm{~d}, J=2.1 \mathrm{~Hz}, 1 \mathrm{H}), 6.81(\mathrm{dd}, J=8.3,2.4 \mathrm{~Hz}, 1 \mathrm{H}), 6.70$ (dd, $J=8.1$, $2.3 \mathrm{~Hz}, 1 \mathrm{H}), 3.77(\mathrm{~s}, 3 \mathrm{H}), 3.58(\mathrm{~s}, 1 \mathrm{H}), 0.98(\mathrm{~s}, 9 \mathrm{H}) ;{ }^{13} \mathrm{C}\left\{{ }^{1} \mathrm{H}\right\}$ NMR $\left(125 \mathrm{MHz}, d_{8}-\mathrm{THF}\right) \delta 158.6$, 156.7, 148.1, 147.8, 136.4, 134.6, 119.8, 119.3, 114.8, 114.7, 113.9, 112.7, 59.2, 55.5, 35.6, 28.6; IR (neat, $\mathrm{cm}^{-1}$ ) 3368, 3017, 2951, 2884, 1605, 1584, 1466, 1354, 1317, 1283, 1225, 1138, 1111, 1024, 1003; HRMS $\left(\mathrm{ESI}^{-}\right) \mathrm{m} / \mathrm{z}$ calcd for $\mathrm{C}_{18} \mathrm{H}_{19} \mathrm{O}_{2}[\mathrm{M}-\mathrm{H}]^{-}:$: 267.1391; found 267.1395.

6-Methoxy-9-phenyl-9H-fluoren-2-ol (5h): white solid (67 mg, 52\% yield); mp $144-146{ }^{\circ} \mathrm{C}$; ${ }^{1} \mathrm{H}$ NMR (500 MHz, ds-THF) $\delta 8.20(\mathrm{~s}, 1 \mathrm{H}), 7.58(\mathrm{~d}, J=8.2 \mathrm{~Hz}, 1 \mathrm{H}), 7.23(\mathrm{~d}, J=2.4 \mathrm{~Hz}, 1 \mathrm{H}), 7.20(\mathrm{t}$, $J=7.3 \mathrm{~Hz}, 2 \mathrm{H}), 7.17-7.12(\mathrm{~m}, 1 \mathrm{H}), 7.08-7.02(\mathrm{~m}, 3 \mathrm{H}), 6.75(\mathrm{dd}, J=8.2,2.3 \mathrm{~Hz}, 1 \mathrm{H}), 6.67$ (dd, $J=8.3,2.4 \mathrm{~Hz}, 1 \mathrm{H}), 6.64(\mathrm{~d}, J=2.3 \mathrm{~Hz}, 1 \mathrm{H}), 4.90(\mathrm{~s}, 1 \mathrm{H}), 3.80(\mathrm{~s}, 3 \mathrm{H}) ;{ }^{13} \mathrm{C}\left\{{ }^{1} \mathrm{H}\right\} \mathrm{NMR}(125 \mathrm{MHz}$, $d_{8}$-THF) $\delta 160.9,158.7,151.9,143.8,143.6,140.3,133.4,129.2,129.0,127.2,126.3,121.3$, 115.3, 113.1, 112.4, 104.8, 55.5, 54.5; IR (neat, $\mathrm{cm}^{-1}$ ) 3362, 3082, 3024, 2941, 2833, 1609, 1585, 1489, 1452, 1439, 1362, 1310, 1267, 1215, 1175, 1096, 1030; HRMS $\left(\mathrm{ESI}^{+}\right) \mathrm{m} / \mathrm{z}$ calcd for $\mathrm{C}_{20} \mathrm{H}_{17} \mathrm{O}_{2}[\mathrm{M}+\mathrm{H}]^{+}:$289.1223; found 289.1225.

7-Bromo-6-methoxy-9-phenyl-9H-fluoren-2-ol (5i): white solid (32 mg, 19\% yield); ${ }^{1} \mathrm{H}$ NMR $\left(500 \mathrm{MHz}, d_{8}\right.$-THF) $\delta 8.36(\mathrm{~s}, 1 \mathrm{H}), 7.63(\mathrm{~d}, J=8.2 \mathrm{~Hz}, 1 \mathrm{H}), 7.43-7.30(\mathrm{~m}, 2 \mathrm{H}), 7.28-7.11(\mathrm{~m}$, $3 \mathrm{H}), 7.08-7.02(\mathrm{~m}, 2 \mathrm{H}), 6.77(\mathrm{dd}, J=8.2,2.3 \mathrm{~Hz}, 1 \mathrm{H}), 6.64(\mathrm{~d}, J=2.2 \mathrm{~Hz}, 1 \mathrm{H}), 4.92(\mathrm{~s}, 1 \mathrm{H})$, 3.93 (s, 3H); ${ }^{13} \mathrm{C}\left\{{ }^{1} \mathrm{H}\right\}$ NMR $\left(125 \mathrm{MHz}, d_{8}\right.$-THF) $\delta 159.1,156.8,151.7,143.2,142.8,141.5,132.7$, 130.2, 129.4, 129.0, 127.5, 121.5, 115.5, 113.1, 109.5, 103.4, 56.5, 54.4; IR (neat, $\mathrm{cm}^{-1}$ ) 3341, 3059, 3026, 2938, 2855, 1605, 1476, 1452, 1441, 1395, 1285, 1233, 1177, 1099, 1047; HRMS $\left(\mathrm{ESI}^{-}\right) \mathrm{m} / \mathrm{z}$ calcd for $\mathrm{C}_{20} \mathrm{H}_{14} \mathrm{BrO}_{2}[\mathrm{M}-\mathrm{H}]^{-}:$365.0183; found 365. 0199.

7,9-Diphenyl-9H-fluoren-2-ol (5j): white solid (113 $\mathrm{mg}, 75 \%$ yield); gradually darkens and decomposes above $215^{\circ} \mathrm{C} ;{ }^{1} \mathrm{H}$ NMR $\left(500 \mathrm{MHz}, d_{8}\right.$-THF) $\delta 8.29(\mathrm{~s}, 1 \mathrm{H}), 7.73(\mathrm{~d}, \mathrm{~J}=7.9 \mathrm{~Hz}, 1 \mathrm{H})$, $7.64(\mathrm{~d}, J=8.2 \mathrm{~Hz}, 1 \mathrm{H}), 7.58(\mathrm{dd}, J=7.9,1.7 \mathrm{~Hz}, 1 \mathrm{H}), 7.55(\mathrm{~d}, J=7.0 \mathrm{~Hz}, 2 \mathrm{H}), 7.49(\mathrm{~s}, 1 \mathrm{H}), 7.33$ 
(t, $J=7.7 \mathrm{~Hz}, 2 \mathrm{H}), 7.25-7.19(\mathrm{~m}, 3 \mathrm{H}), 7.19-7.10(\mathrm{~m}, 3 \mathrm{H}), 6.80(\mathrm{dd}, J=8.2,2.3 \mathrm{~Hz}, 1 \mathrm{H}), 6.71$ $(\mathrm{s}, 1 \mathrm{H}), 5.05$ (s, 1H); ${ }^{13} \mathrm{C}\left\{{ }^{1} \mathrm{H}\right\} \operatorname{NMR}\left(125 \mathrm{MHz}, d_{8}\right.$-THF) $\delta 158.8,151.3,149.0,143.1,142.3,141.8$, 139.6, 133.0, 129.4, 129.3, 129.2, 127.7, 127.6, 127.4, 127.0, 124.4, 121.4, 119.8, 115.5, 113.2, 55.2; IR (neat, $\mathrm{cm}^{-1}$ ) 3337, 3019, 1595, 1454, 1412, 1213, 1098, 1072; HRMS (ESI ${ }^{-}$) m/z calcd for $\mathrm{C}_{25} \mathrm{H}_{17} \mathrm{O}[\mathrm{M}-\mathrm{H}]^{-}:$333.1285; found 333.1282.

7-(tert-Butyl)-9-phenyl-9H-fluoren-2-ol (5k): white solid (192 mg, 74\% yield); mp $197-198{ }^{\circ} \mathrm{C}$; ${ }^{1} \mathrm{H}$ NMR $\left(500 \mathrm{MHz}, \mathrm{C}_{6} \mathrm{D}_{6}\right) \delta 7.60(\mathrm{~d}, J=8.0 \mathrm{~Hz}, 1 \mathrm{H}), 7.48(\mathrm{~d}, J=8.2 \mathrm{~Hz}, 1 \mathrm{H}), 7.41(\mathrm{~d}, J=1.8 \mathrm{~Hz}$, $1 \mathrm{H}), 7.36(\mathrm{dd}, J=8.1,1.8 \mathrm{~Hz}, 1 \mathrm{H}), 7.07-6.95(\mathrm{~m}, 5 \mathrm{H}), 6.65(\mathrm{dd}, J=8.2,2.3 \mathrm{~Hz}, 1 \mathrm{H}), 6.44-6.40$ $(\mathrm{m}, 1 \mathrm{H}), 4.81(\mathrm{~s}, 1 \mathrm{H}), 4.05$ (brs, $1 \mathrm{H}), 1.18(\mathrm{~s}, 9 \mathrm{H}) ;{ }^{13} \mathrm{C}\left\{{ }^{1} \mathrm{H}\right\} \mathrm{NMR}\left(125 \mathrm{MHz}, \mathrm{C}_{6} \mathrm{D}_{6}\right) \delta$ 156.0, 150.6, 149.6, 147.9, 142.5, 139.1, 134.1, 128.9, 128.8, 127.0, 124.9, 122.3, 120.7, 118.9, 114.9, 112.8, 55.0, 34.8, 31.6; IR (neat, $\mathrm{cm}^{-1}$ ) 3387, 3059, 3026, 2961, 2866, 1493, 1472, 1362, 1275, 1211, 1165, 1130, 1105, 1072, 1028; HRMS $\left(\mathrm{ESI}^{+}\right) \mathrm{m} / \mathrm{z}$ calcd for $\mathrm{C}_{23} \mathrm{H}_{22} \mathrm{ONa}[\mathrm{M}+\mathrm{Na}]^{+}$: 337.1563; found 337.1538.

7-Phenyl-7H-benzo[c]fluoren-9-ol (5I): white solid (92 mg, 75\% yield); mp $192-193{ }^{\circ} \mathrm{C} ;{ }^{1} \mathrm{H}$ NMR $\left(500 \mathrm{MHz}, \mathrm{C}_{6} \mathrm{D}_{6}\right) \delta 8.68(\mathrm{~d}, J=8.5 \mathrm{~Hz}, 1 \mathrm{H}), 8.06(\mathrm{~d}, J=8.4 \mathrm{~Hz}, 1 \mathrm{H}), 7.75(\mathrm{~d}, J=7.8 \mathrm{~Hz}$, $1 \mathrm{H}), 7.51(\mathrm{~d}, J=8.3 \mathrm{~Hz}, 1 \mathrm{H}), 7.46(\mathrm{ddd}, J=8.3,6.8,1.3 \mathrm{~Hz}, 1 \mathrm{H}), 7.34(\mathrm{t}, J=7.4 \mathrm{~Hz}, 1 \mathrm{H}), 7.27$ $(\mathrm{d}, J=8.3 \mathrm{~Hz}, 1 \mathrm{H}), 7.07-6.97(\mathrm{~m}, 3 \mathrm{H}), 6.90(\mathrm{dd}, J=7.2,2.3 \mathrm{~Hz}, 2 \mathrm{H}), 6.73(\mathrm{dd}, J=8.4,2.5 \mathrm{~Hz}$, $1 \mathrm{H}), 6.48(\mathrm{~d}, J=2.5 \mathrm{~Hz}, 1 \mathrm{H}), 4.74(\mathrm{~s}, 1 \mathrm{H}), 4.10(\mathrm{~s}, 1 \mathrm{H}) ;{ }^{13} \mathrm{C}\left\{{ }^{1} \mathrm{H}\right\} \operatorname{NMR}\left(125 \mathrm{MHz}, \mathrm{C}_{6} \mathrm{D}_{6}\right) \delta 155.5$, $151.9,146.4,141.7,136.2,135.1,134.3,129.55,129.53,128.95,128.92,127.5,127.1,126.7$, 125.4, 124.3, 124.1, 123.6, 114.8, 112.9, 55.1; IR (neat, $\mathrm{cm}^{-1}$ ) 3375, 3053, 3026, 2924, 2853, 1611, 1491, 1450, 1389, 1366, 1331, 1304, 1261, 1213, 1177, 1090, 1028; HRMS (ESI $\left.{ }^{+}\right) \mathrm{m} / \mathrm{z}$ calcd for $\mathrm{C}_{23} \mathrm{H}_{17} \mathrm{O}[\mathrm{M}+\mathrm{H}]^{+}:$309.1274; found 309.1260.

13-Phenyl-13ㅡ-indeno[1,2-/]phenanthren-11-ol (5m): off-white solid (117 $\mathrm{mg}, 77 \%$ yield); gradually darkens and decomposes above $183^{\circ} \mathrm{C} ;{ }^{1} \mathrm{H}$ NMR $\left(500 \mathrm{MHz}, d_{8}-\mathrm{THF}\right) \delta 8.94(\mathrm{~d}, \mathrm{~J}=8.0$ $\mathrm{Hz}, 1 \mathrm{H}), 8.90(\mathrm{~d}, J=8.1 \mathrm{~Hz}, 1 \mathrm{H}), 8.77(\mathrm{~d}, J=8.3 \mathrm{~Hz}, 1 \mathrm{H}), 8.31-8.25(\mathrm{~m}, 2 \mathrm{H}), 7.79-7.74(\mathrm{~m}$, 2H), 7.70 (ddd, $J=8.2,6.9,1.3 \mathrm{~Hz}, 1 \mathrm{H}$ ), 7.49 (ddd, $J=8.3,6.8,1.4 \mathrm{~Hz}, 1 \mathrm{H}$ ), 7.35 (ddd, $J=8.0$, 6.8, $1.1 \mathrm{~Hz}, 1 \mathrm{H}), 7.24-7.12(\mathrm{~m}, 5 \mathrm{H}), 6.87(\mathrm{dd}, J=8.4,2.5 \mathrm{~Hz}, 1 \mathrm{H}), 6.82(\mathrm{~d}, J=2.4 \mathrm{~Hz}, 1 \mathrm{H}), 5.39$ $(\mathrm{s}, 1 \mathrm{H}) ;{ }^{13} \mathrm{C}\left\{{ }^{1} \mathrm{H}\right\}$ NMR $\left(125 \mathrm{MHz}, d_{8}-\mathrm{THF}\right) \delta 157.9,153.3,143.5,141.9,136.6,133.8,132.4,130.9$, $130.2,129.7,129.6,128.8,127.5,127.3,127.2,126.8,126.2,126.1,125.5,124.43,124.37$, 124.1, 114.9, 113.3, 54.8; IR (neat, $\mathrm{cm}^{-1}$ ) 3358, 3076, 3026, 2924, 2853, 1607, 1493, 1478, 1454, $1360,1300,1260,1215,1179,1094,1028 ; \mathrm{HRMS}\left(\mathrm{ESI}^{-}\right) \mathrm{m} / \mathrm{z}$ calcd for $\mathrm{C}_{27} \mathrm{H}_{17} \mathrm{O}[\mathrm{M}-\mathrm{H}]^{-}: 357.1285$; found 357.1302 .

6-Phenyl-6H-benzo[b]indeno[1,2-d]thiophen-8-ol $(5 \mathrm{n})$ : off-white solid (83 $\mathrm{mg}, 80 \%$ yield); gradually darkens and decomposes above $210^{\circ} \mathrm{C} ;{ }^{1} \mathrm{H}$ NMR $\left(500 \mathrm{MHz}, d_{8}-\mathrm{THF}\right) \delta 8.27(\mathrm{~s}, 1 \mathrm{H})$, $7.88-7.82(\mathrm{~m}, 1 \mathrm{H}), 7.38-7.33(\mathrm{~m}, 1 \mathrm{H}), 7.33-7.28(\mathrm{~m}, 1 \mathrm{H}), 7.27-7.22(\mathrm{~m}, 2 \mathrm{H}), 7.22-7.12$ $(\mathrm{m}, 5 \mathrm{H}), 6.76-6.71(\mathrm{~m}, 2 \mathrm{H}), 5.05(\mathrm{~s}, 1 \mathrm{H}) ;{ }^{13} \mathrm{C}\left\{{ }^{1} \mathrm{H}\right\}$ NMR $\left(125 \mathrm{MHz}, d_{8}-\mathrm{THF}\right) \delta 158.1,154.8,144.4$, 144.3, 143.8, 141.0, 136.0 130.3, 129.4, 128.9, 127.6, 125.2, 124.5, 123.9, 121.8, 120.8, 114.8, 113.7, 52.6; IR (neat, $\mathrm{cm}^{-1}$ ) 3447, 3051, 3005, 1261, 1213; HRMS (ESI ${ }^{-}$) m/z calcd for $\mathrm{C}_{21} \mathrm{H}_{14} \mathrm{OS}$ $[\mathrm{M}-\mathrm{H}]^{-}:$:313.0693; found 313.0706.

(士)-5-Hydroxy-2-(2-hydroxyphenyl)-3-phenyl-2,3-dihydro-1H-inden-1-one (8): off-white solid (69 mg, 65\% yield); mp $173-176{ }^{\circ} \mathrm{C} ;{ }^{1} \mathrm{H}$ NMR (500 MHz, $\left.d_{8}-\mathrm{THF}\right) \delta 9.16(\mathrm{~s}, 1 \mathrm{H}), 8.30(\mathrm{~s}, 1 \mathrm{H})$, $7.63(\mathrm{~d}, J=8.3 \mathrm{~Hz}, 1 \mathrm{H}), 7.33-7.08(\mathrm{~m}, 5 \mathrm{H}), 7.03(\mathrm{td}, J=7.7,1.7 \mathrm{~Hz}, 1 \mathrm{H}), 6.88-6.81(\mathrm{~m}, 2 \mathrm{H})$, $6.72(\mathrm{~d}, J=7.7 \mathrm{~Hz}, 1 \mathrm{H}), 6.67(\mathrm{t}, J=7.6 \mathrm{~Hz}, 1 \mathrm{H}), 6.52(\mathrm{~s}, 1 \mathrm{H}), 4.63(\mathrm{~d}, J=5.4 \mathrm{~Hz}, 1 \mathrm{H}), 3.79(\mathrm{~d}, J$ $=5.5 \mathrm{~Hz}, 1 \mathrm{H}) ;{ }^{13} \mathrm{C}\left\{{ }^{1} \mathrm{H}\right\}$ NMR $\left(125 \mathrm{MHz}, d_{8}\right.$-THF) $\delta 202.6,164.6,159.7,156.3,144.4,131.9,130.0$, 129.3, 129.0, 128.7, 127.4, 127.3, 125.8, 120.0, 116.8, 116.1, 112.6, 62.8, 53.5; IR (neat, cm ${ }^{-1}$ ) 3283, 3063, 3030, 2955, 2922, 2851, 1682, 1645, 1593, 1495, 1456, 1292, 1233, 1198, 1096; HRMS $\left(\mathrm{ESI}^{+}\right) \mathrm{m} / \mathrm{z}$ calcd for $\mathrm{C}_{21} \mathrm{H}_{17} \mathrm{O}_{3}[\mathrm{M}+\mathrm{H}]^{+}: 317.1172$; found 317.1169 .

7-((9H-Fluoren-9-ylidene)methyl)-1,4-dioxaspiro[4.5]decan-8-one (10): To a stirred solution of 9-(bromomethylene)- $9 H$-fluorene ${ }^{1}(900 \mathrm{mg}, 3.5 \mathrm{mmol}, 1.1 \mathrm{eq}$ ) and ketone 1 (500 mg, $3.2 \mathrm{mmol}$, 
$1.0 \mathrm{eq})$ in THF $(16 \mathrm{~mL})$ at $0{ }^{\circ} \mathrm{C}$ was added sodium tert-butoxide $(769 \mathrm{mg}, 8.0 \mathrm{mmol}, 2.5 \mathrm{eq})$ all at once. The reaction mixture was allowed to stir at $0{ }^{\circ} \mathrm{C}$ for $30 \mathrm{~min}$ before being allowed to warm to room temperature. The reaction was quenched with $1 \mathrm{M} \mathrm{HCl}$ and the reaction mixture was extracted with diethyl ether (x3). The combined organic extracts were washed with water and brine, followed by drying over anhydrous sodium sulfate. After filtration, the solvent was removed in vacuo and the crude material was purified by flash column chromatography using a gradient of ethyl acetate in hexane as eluent (load as PhMe solution; 0, 5, 10, 15, 20, 25, 30\% ethyl acetate in hexanes; alternatively 0, 5, 10, 15, 20\% diethyl ether in PhMe can be used). Compound 10 was obtained as an off-white foam (652 $\mathrm{mg}, 61 \%$ yield): ${ }^{1} \mathrm{H}$ NMR $\left(500 \mathrm{MHz}, \mathrm{CDCl}_{3}\right) \mathrm{d} 7.89(\mathrm{~d}, J=7.6$ $\mathrm{Hz}, 1 \mathrm{H}), 7.78(\mathrm{~d}, J=8.0 \mathrm{~Hz}, 1 \mathrm{H}), 7.74(\mathrm{~d}, J=7.4 \mathrm{~Hz}, 1 \mathrm{H}), 7.68(\mathrm{~d}, J=7.0 \mathrm{~Hz}, 1 \mathrm{H}), 7.44-7.28$ $(\mathrm{m}, 4 \mathrm{H}), 7.13(\mathrm{~d}, J=8.7 \mathrm{~Hz}, 1 \mathrm{H}), 4.55(\mathrm{ddd}, J=13.9,8.7,5.5 \mathrm{~Hz}, 1 \mathrm{H}), 4.23-4.08(\mathrm{~m}, 2 \mathrm{H}), 4.08$ $-3.97(\mathrm{~m}, 2 \mathrm{H}), 2.85(\mathrm{td}, J=13.2,8.1 \mathrm{~Hz}, 1 \mathrm{H}), 2.57$ (dt, $J=14.2,4.1 \mathrm{~Hz}, 1 \mathrm{H}$ ), 2.43 (ddd, $J=13.4$, 5.3, $2.6 \mathrm{~Hz}, 1 \mathrm{H}), 2.19-2.11(\mathrm{~m}, 2 \mathrm{H}) 2.08(\mathrm{t}, J=13.5 \mathrm{~Hz}, 1 \mathrm{H}) ;{ }^{13} \mathrm{C}\left\{{ }^{1} \mathrm{H}\right\} \mathrm{NMR}\left(125 \mathrm{MHz}, \mathrm{CDCl}_{3}\right) \mathrm{d}$ 208.1, 141.2, 138.8, 138.6, 136.34, 136.25, 128.1, 127.8, 127.0, 124.4, 124.3, 120.2, 119.9, 119.3, 107.0, 64.8, 45.9, 40.3, 38.1, 34.9; IR (neat, $\mathrm{cm}^{-1}$ ) 3057, 2957, 2889, 1717, 1449, 1348, 1304, 1283, 1221, 1119, 1057, 1040; HRMS $\left(\mathrm{ESI}^{+}\right) \mathrm{m} / \mathrm{z}$ calcd for $\mathrm{C}_{22} \mathrm{H}_{21} \mathrm{O}_{3}[\mathrm{M}+\mathrm{H}]^{+}: 333.1485$; found 333.1481.

7-((9H-Fluoren-9-ylidene)methyl)-8-(4-methoxyphenyl)-1,4-dioxaspiro[4.5]decan-8-ol (11a): To a solution of 4-bromoanisole $(0.43 \mathrm{~mL}, 3.4 \mathrm{mmol}, 3.0 \mathrm{eq})$ in THF $(10 \mathrm{~mL})$ at $-78{ }^{\circ} \mathrm{C}$ was added dropwise a solution of $n$-BuLi (1.4 mL, $3.4 \mathrm{mmol}, 3.0 \mathrm{eq}, 2.4 \mathrm{M}$ in hexanes). After stirring for 30 min at this temperature, a solution of ketone $10(381 \mathrm{mg}, 1.15 \mathrm{mmol}, 1.0 \mathrm{eq})$ in THF (6 mL) was added dropwise over a period of $10 \mathrm{~min}$ at $-78^{\circ} \mathrm{C}$. The reaction mixture was allowed to stir at this temperature for 30 min before quenching with a saturated aqueous solution of $\mathrm{NH}_{4} \mathrm{Cl}$. After warming to room temperature, the reaction mixture was extracted with diethyl ether $(x 3)$. The combined organic extracts were washed with water and brine, followed by drying over anhydrous sodium sulfate. After filtration, the solvent was removed in vacuo and the crude material purified by flash column chromatography using a mixture of ethyl acetate in hexanes as eluent (gradient used: $0,10,15,20,25 \%$ ethyl acetate in hexanes). Compound 11a was obtained as a white foam and as a mixture of diastereomers $\left(436 \mathrm{mg}, 86 \%\right.$ yield): (diastereomeric mixture) ${ }^{1} \mathrm{H}$ NMR (500 $\left.\mathrm{MHz} \mathrm{CDCl}_{3}\right) \delta 8.11-8.05(\mathrm{~m}, 0.6 \mathrm{H}), 7.87(\mathrm{~d}, J=7.4 \mathrm{~Hz}, 1 \mathrm{H}), 7.76-7.69(\mathrm{~m}, 2 \mathrm{H}), 7.68-7.62$ $(\mathrm{m}, 2 \mathrm{H}), 7.55(\mathrm{dd}, J=7.5,4.9 \mathrm{~Hz}, 2 \mathrm{H}), 7.48(\mathrm{dd}, J=8.8,1.6 \mathrm{~Hz}, 2 \mathrm{H}), 7.43-7.31(\mathrm{~m}, 4 \mathrm{H}), 7.31-$ $7.18(\mathrm{~m}, 3 \mathrm{H}), 6.79-6.71(\mathrm{~m}, 4 \mathrm{H}), 6.70(\mathrm{~d}, J=10.1 \mathrm{~Hz}, 0.5 \mathrm{H}), 4.21-3.97(\mathrm{~m}, 4.5 \mathrm{H}), 3.95-3.82$ $(\mathrm{m}, 3 \mathrm{H}), 3.73-3.64(\mathrm{~m}, 6 \mathrm{H}), 2.88-2.74(\mathrm{~m}, 1 \mathrm{H}), 2.54(\mathrm{dd}, J=13.3,5.3 \mathrm{~Hz}, 1 \mathrm{H}), 2.32(\mathrm{ddd}, J=$ 17.0, 11.1, 4.0 Hz, 2H), $2.26-2.15(\mathrm{~m}, 1 \mathrm{H}), 2.15-1.67(\mathrm{~m}, 7 \mathrm{H}) ;{ }^{13} \mathrm{C}\left\{{ }^{1} \mathrm{H}\right\} \mathrm{NMR}\left(125 \mathrm{MHz}, \mathrm{CDCl}_{3}\right)$ $\delta 158.6,158.2,140.95,140.85,139.5,139.2,138.9,138.4,138.3,137.9,136.9,136.6,135.0$, $133.5,132.1$, 129.9, 127.7, 127.6, 127.3, 127.2, 127.0, 126.9, 126.79, 126.75, 126.7, 125.8, $124.74,124.67,119.9,119.8,119.7,119.21,119.20,113.59,113.55,108.4,108.1,73.8,64.6$, $64.5,64.2,64.0,55.0,45.0,43.4,39.0,35.9,35.7,31.9,31.1,31.0$; IR (neat, cm${ }^{-1}$ ) 3463, 3057, 2932, 1609, 1512, 1449, 1294, 1250, 1180, 1096, 1034; HRMS $\left(\mathrm{ESI}^{+}\right) \mathrm{m} / \mathrm{z}$ calcd for $\mathrm{C}_{29} \mathrm{H}_{28} \mathrm{O}_{4} \mathrm{Na}$ $[\mathrm{M}+\mathrm{Na}]^{+}:$463.1880; found 463.1879 .

7-((9H-Fluoren-9-ylidene)methyl)-8-(p-tolyl)-1,4-dioxaspiro[4.5]decan-8-ol (11b) was prepared according to the same procedure as $11 \mathrm{a}$ as a white foam (433 $\mathrm{mg}, 88 \%$ yield): (diastereomeric mixture) ${ }^{1} \mathrm{H}$ NMR $\left(600 \mathrm{MHz}, \mathrm{CDCl}_{3}\right) \delta 8.08-8.06(\mathrm{~m}, 0.76 \mathrm{H}), 7.86(\mathrm{~d}, \mathrm{~J}=7.4 \mathrm{~Hz}$, $1 \mathrm{H}), 7.73-7.71(\mathrm{~m}, 2 \mathrm{H}), 7.66-7.63(\mathrm{~m}, 2 \mathrm{H}), 7.55-7.53(\mathrm{~m}, 2 \mathrm{H}), 7.46(\mathrm{~d}, J=8.3 \mathrm{~Hz}, 2 \mathrm{H}), 7.39$ $-7.31(\mathrm{~m}, 6 \mathrm{H}), 7.29-7.19(\mathrm{~m}, 4 \mathrm{H}), 7.05(\mathrm{~d}, J=8.2 \mathrm{~Hz}, 2 \mathrm{H}), 7.03(\mathrm{~d}, J=7.9 \mathrm{~Hz}, 2 \mathrm{H}), 4.18-4.00$ $(\mathrm{m}, 4 \mathrm{H}), 3.90-3.85(\mathrm{~m}, 2 \mathrm{H}), 3.73-3.69(\mathrm{~m}, 2 \mathrm{H}), 2.84(\mathrm{ddd}, \mathrm{J}=14.0,11.9,4.2 \mathrm{~Hz}, 1 \mathrm{H}), 2.53(\mathrm{dd}$, $J=13.3,5.5 \mathrm{~Hz}, 1 \mathrm{H}), 2.36-2.29(\mathrm{~m}, 2 \mathrm{H}), 2.23(\mathrm{~s}, 3 \mathrm{H}), 2.21(\mathrm{~s}, 2.35 \mathrm{H}), 2.14-2.00(3.6 \mathrm{H}), 1.92$ - $1.79(\mathrm{~m}, 4.5 \mathrm{H}) ;{ }^{13} \mathrm{C}\left\{{ }^{1} \mathrm{H}\right\}$ NMR $\left(150 \mathrm{MHz}, \mathrm{CDCl}_{3}\right) \delta 143.8,142.7,140.94,140.86,139.6,139.2$, $138.4,138.3,137.1,136.9,136.7,136.4,135.1,133.6,132.1,129.8,129.2,129.1,127.7,127.6$, $127.3,127.2,127.1,127.0,126.74,126.69,125.5,125.1,124.8,124.7,124.5,120.0,119.8$, $119.7,119.21,119.19,108.5,108.1,74.0,74.0,64.6,64.5,64.3,64.0,63.7,45.0,43.4,39.0$, 
36.0, 35.7, 31.6, 31.1, 31.0, 22.6, 20.9, 20.8, 14.1; IR (neat, $\mathrm{cm}^{-1}$ ) 3448, 3058, 2957, 2930, 2884, 1449, 1368, 1291, 1265, 1223, 1190, 1097, 1058, 1035; HRMS (ESI $\left.{ }^{+}\right) \mathrm{m} / \mathrm{z}$ calcd for $\mathrm{C}_{29} \mathrm{H}_{28} \mathrm{O}_{3} \mathrm{Na}$ $[\mathrm{M}+\mathrm{Na}]^{+}:$447.1931; found: 447.1945 .

14-Methoxydibenzo[g,p]chrysen-3-ol (12a): To a solution of alcohol 11a (684 mg, $1.55 \mathrm{mmol}$, $1.0 \mathrm{eq})$ in $\mathrm{MeCN}(137 \mathrm{~mL}$ ) was added a drop of conc. $\mathrm{HBr}$ at room temperature and allowed to stir for $30 \mathrm{~min}$ before $\mathrm{CuBr}_{2}(693 \mathrm{mg}, 3.10 \mathrm{mmol}, 2.0 \mathrm{eq})$ was added all at once. After $10 \mathrm{~min}$, the reaction mixture was heated to $60{ }^{\circ} \mathrm{C}$ and additional $\mathrm{CuBr}_{2}(693 \mathrm{mg}, 3.10 \mathrm{mmol}, 2.0 \mathrm{eq})$ was added. After heating for $18 \mathrm{~h}$, the reaction mixture was cooled to room temperature and quenched with a sat. aq. solution of $\mathrm{NH}_{4} \mathrm{Cl}$ and allowed to stir vigorously for $30 \mathrm{~min}$. The reaction mixture was extracted with diethyl ether (x3). The combined organic extracts were washed with a sat. aq. solution of $\mathrm{NaHCO}_{3}(\mathrm{x} 2)$, water, and brine, followed by drying over anhydrous sodium sulfate. After filtration, the solvent was removed in vacuo and the crude material was purified by flash column chromatography using diethyl ether in toluene as eluent $(0,2,5,10 \%$ diethyl ether in toluene) to afford dibenzo[g,p]chrysene $12 \mathrm{a}$ as an off-white solid (319 mg, $55 \%$ yield): ${ }^{1} \mathrm{H}$ NMR $\left(500 \mathrm{MHz}, d_{8}-\mathrm{THF}\right) \delta 8.80(\mathrm{t}, J=7.8 \mathrm{~Hz}, 2 \mathrm{H}), 8.75(\mathrm{~d}, J=8.0 \mathrm{~Hz}, 2 \mathrm{H}), 8.60(\mathrm{~s}, 1 \mathrm{H}), 8.55(\mathrm{~d}, J=$ $9.0 \mathrm{~Hz}, 1 \mathrm{H}), 8.52(\mathrm{~d}, J=8.8 \mathrm{~Hz}, 1 \mathrm{H}), 8.12(\mathrm{~d}, J=2.6 \mathrm{~Hz}, 1 \mathrm{H}), 8.03(\mathrm{~d}, J=2.5 \mathrm{~Hz}, 1 \mathrm{H}), 7.71-$ $7.56(\mathrm{~m}, 4 \mathrm{H}), 7.26(\mathrm{dd}, J=9.0,2.6 \mathrm{~Hz}, 1 \mathrm{H}), 7.16(\mathrm{dd}, J=8.8,2.5 \mathrm{~Hz}, 1 \mathrm{H}), 3.89(\mathrm{~s}, 3 \mathrm{H}) ;{ }^{13} \mathrm{C}\left\{{ }^{1} \mathrm{H}\right\}$ NMR $\left(125 \mathrm{MHz}, d_{8}\right.$-THF) $\delta 158.5,156.9,131.74,131.72,130.6,130.4,130.4,130.1,129.2,129.0$, 128.7, 127.3, 127.2, 127.1, 126.5, 125.5, 125.2, 124.54, 124.47, 117.4, 116.6, 113.5, 111.5, 55.6; IR (neat, $\left.\mathrm{cm}^{-1}\right)$ 3428, 3065, 2995, 2953, 2932, 2833, 1612, 1495, 1481, 1452, 1441, 1423, 1383, 1300, 1267, 1234, 1196, 1173, 1130, 1045; HRMS (ESI $\left.{ }^{-}\right) \mathrm{m} / \mathrm{z}$ calcd for $\mathrm{C}_{27} \mathrm{H}_{17} \mathrm{O}_{2}[\mathrm{M}-\mathrm{H}]^{-}: 373.1234$; found 373.1242 .

14-Methyldibenzo[g,p]chrysen-3-ol (12b) was prepared in the same was as 12 a using Sc(OTf) 3 (1.0 eq) in place of conc. HBr: Off-white solid (92 mg, $41 \%$ yield); mp $209-211{ }^{\circ} \mathrm{C} ;{ }^{1} \mathrm{H}$ NMR $(600$ $\left.\mathrm{MHz}_{\mathrm{CDCl}}\right) \delta 8.71-8.67(\mathrm{~m}, 4 \mathrm{H}), 8.54(\mathrm{~d}, \mathrm{~J}=8.8 \mathrm{~Hz}, 1 \mathrm{H}), 8.47(\mathrm{~d}, J=8.3 \mathrm{~Hz}, 1 \mathrm{H}), 8.44(\mathrm{~s}, 1 \mathrm{H})$, $8.08(\mathrm{~d}, J=2.5 \mathrm{~Hz}, 1 \mathrm{H}), 7.69-7.62(\mathrm{~m}, 3 \mathrm{H}), 7.60(\mathrm{td}, J=7.6,1.4 \mathrm{~Hz}, 1 \mathrm{H}), 7.47$ (dd, $J=8.3,2.0$ $\mathrm{Hz}, 1 \mathrm{H}), 7.20$ (dd, $J=8.8,2.6 \mathrm{~Hz}, 1 \mathrm{H}), 5.10$ (brs, $1 \mathrm{H}), 2.56(\mathrm{~s}, 3 \mathrm{H}) ;{ }^{13} \mathrm{C}\left\{{ }^{1} \mathrm{H}\right\} \mathrm{NMR}(150 \mathrm{MHz}$, $\left.\mathrm{CDCl}_{3}\right) \delta 153.9,135.3,130.8,130.6,130.3,129.3,129.3,128.9,128.70,128.66,128.4,128.22$, 128.19, 127.0, 126.6, 126.55, 126.51, 125.22, 125.17, 123.6, 123.5, 123.0, 115.8, 113.2, 21.8; IR (neat, $\mathrm{cm}^{-1}$ ) 3369, 3066, 2917, 1612, 1482, 1452, 1428, 1265, 1231, 1209, 1178; HRMS (ESI $)$ $\mathrm{m} / \mathrm{z}$ calcd for $\mathrm{C}_{27} \mathrm{H}_{17} \mathrm{O}[\mathrm{M}-\mathrm{H}]^{-}:$357.1285; found: 357.1291 .

7-((9H-Fluoren-9-ylidene)methyl)-8-(4-ethylphenyl)-1,4-dioxaspiro[4.5]dec-7-ene (13a): To a solution of ketone $10(318 \mathrm{mg}, 0.96 \mathrm{mmol}, 1.0 \mathrm{eq})$ in $\mathrm{DCM}(10 \mathrm{~mL})$ at room temperature was added $t$-BuONa (192 mg, $2.0 \mathrm{mmol}$ ) all at once. After $5 \mathrm{~min}$, triflic anhydride $(0.25 \mathrm{~mL}, 1.5 \mathrm{mmol})$ was added dropwise. Caution! An exotherm was observed during the addition of triflic anhydride. The reaction mixture was quenched with $\mathrm{NaHCO}_{3}$ (sat. aq.) and extracted with $\mathrm{DCM}(\times 3)$. The combined organic extracts were dried over anhydrous magnesium sulfate, filtered, and concentrated in vacuo. The crude material was used immediately in the next step without purification. A degassed solution of enol triflate, 4-ethylbenzeneboronic acid (300 mg, $2.0 \mathrm{mmol})$, CsF (456 mg, $3.0 \mathrm{mmol})$, and $\mathrm{PdCl}_{2} \cdot \mathrm{dppf}(18 \mathrm{mg}, 0.025 \mathrm{mmol}, 2.5 \mathrm{~mol} \%$ ) in THF (15 mL) was heated at $60{ }^{\circ} \mathrm{C}$ for $2.5 \mathrm{~h}$. The reaction mixture was cooled to room temperature and quenched by the addition of $\mathrm{NH}_{4} \mathrm{Cl}$ (sat. aq.) and extracted with diethyl ether (x3). The combined organic extracts were dried over $\mathrm{Na}_{2} \mathrm{SO}_{4}$, filtered, and concentrated in vacuo. The crude oil was purified by silica gel chromatography $(0,5,8 \%$ ethyl acetate in hexanes as eluent) which afforded styrene $13 \mathrm{a}$ as a pale-yellow foam ( $351 \mathrm{mg}, 86 \%$ yield over 2 steps). The styrene was used immediately in the next step without characterization.

7-((9H-fluoren-9-ylidene)methyl)-8-(4-(trifluoromethyl)phenyl)-1,4-dioxaspiro[4.5]dec-7ene (13b) was prepared according to the same procedure as 13a as an off-white foam (328 $\mathrm{mg}$, $73 \%$ over 2 steps). The styrene was used immediately in the next step without characterization. 
2-((9H-Fluoren-9-ylidene)methyl)-4'-ethyl-[1,1'-biphenyl]-4-ol (14a): To a stirred solution of $13 \mathrm{a}(334 \mathrm{mg}, 0.79 \mathrm{mmol}, 1.0 \mathrm{eq})$ in $\mathrm{MeCN}(33 \mathrm{~mL}, 10 \mathrm{mg} / \mathrm{mL})$ under air at room temperature was added $\mathrm{CuBr}_{2}(352 \mathrm{mg}, 1.58 \mathrm{mmol}, 2.0 \mathrm{eq}$ ) all at once. After stirring for $15 \mathrm{~min}, 1-2$ drops of $\mathrm{HBr}(48 \%$ aq.) were added. The reaction was complete within $1 \mathrm{~h}$. The reaction mixture was quenched by the addition of a sat. aq. solution of $\mathrm{NH}_{4} \mathrm{Cl}$ and stirred for $20 \mathrm{~min}$. The phases were separated and the aqueous phase was extracted with diethyl ether $(x 3)$. The combined organic extracts were washed with a saturated aqueous solution of $\mathrm{NaHCO}_{3}$ ( $\mathrm{x} 2$ or until the blue color subsided in the aqueous phase) and brine. The combined organic extracts were dried over anhydrous sodium sulfate. After filtration, the solvent was removed in vacuo and the crude oil was purified by flash column chromatography on silica gel using a gradient of $0,2,5,10 \%$ ethyl acetate in hexanes as eluent to afford $14 \mathrm{a}$ as a yellow-orange foam $\left(288 \mathrm{mg}, 97 \%\right.$ yield): ${ }^{1} \mathrm{H}$ NMR (300 $\left.\mathrm{MHz} \mathrm{CDCl}_{3}\right) \delta 7.80-7.75(\mathrm{~m}, 3 \mathrm{H}), 7.60(\mathrm{~d}, J=7.6 \mathrm{~Hz}, 1 \mathrm{H}), 7.46-7.33(\mathrm{~m}, 6 \mathrm{H}), 7.28(\mathrm{td}, J=$ 7.4, $1.2 \mathrm{~Hz}, 1 \mathrm{H}), 7.21-7.14(\mathrm{~m}, 4 \mathrm{H}), 6.99(\mathrm{dd}, J=8.4,2.7 \mathrm{~Hz}, 1 \mathrm{H}), 4.95(\mathrm{brs}, 1 \mathrm{H}), 2.68(\mathrm{q}, J=$ $7.5 \mathrm{~Hz}, 2 \mathrm{H}), 1.28$ (t, J = 7.5 Hz, 3H); ${ }^{13} \mathrm{C}\left\{{ }^{1} \mathrm{H}\right\}$ NMR $\left(75 \mathrm{MHz}, \mathrm{CDCl}_{3}\right) \delta 154.3,143.0,141.3,139.5$, 139.0, 137.6, 136.8, 136.3, 136.0, 134.5, 131.5, 129.6, 128.5, 128.13, 128.10, 127.7, 127.0, 126.9, 124.7, 120.6, 119.9, 119.6, 117.4, 115.9, 28.5, 15.4; IR (neat, $\mathrm{cm}^{-1}$ ) 3373, 3058, 3024, 2965, 2931, 2872, 1602, 1574, 1487, 1448, 1350, 1294, 1228, 1173, 1109; HRMS (ESI $\left.{ }^{-}\right) \mathrm{m} / \mathrm{z}$ calcd for $\mathrm{C}_{28} \mathrm{H}_{21} \mathrm{O}[\mathrm{M}-\mathrm{H}]^{-}:$: 373.1598; found: 373.1611 .

2-((9H-Fluoren-9-ylidene)methyl)-4'-(trifluoromethyl)-[1,1'-biphenyl]-4-ol (14b) was prepared in the same way as $14 \mathrm{a}$ as a pale yellow foam $\left(241 \mathrm{mg}, 82 \%\right.$ yield): ${ }^{1} \mathrm{H}$ NMR $(600 \mathrm{MHz}$, $\left.\mathrm{CDCl}_{3}\right) \delta 7.75(\mathrm{~d}, J=7.7 \mathrm{~Hz}, 1 \mathrm{H}), 7.72(\mathrm{~d}, J=7.5 \mathrm{~Hz}, 1 \mathrm{H}), 7.65(\mathrm{~d}, J=7.7 \mathrm{~Hz}, 1 \mathrm{H}), 7.57(\mathrm{~d}, J=$ $7.6 \mathrm{~Hz}, 1 \mathrm{H}), 7.55(\mathrm{~d}, J=8.1 \mathrm{~Hz}, 2 \mathrm{H}), 7.49(\mathrm{~d}, J=8.1 \mathrm{~Hz}, 2 \mathrm{H}), 7.41(\mathrm{~d}, J=8.4 \mathrm{~Hz}, 1 \mathrm{H}), 7.39-$ $7.35(\mathrm{~m}, 2 \mathrm{H}), 7.31(\mathrm{~s}, 1 \mathrm{H}), 7.27(\mathrm{td}, J=7.5,1.2 \mathrm{~Hz}, 1 \mathrm{H}), 7.20(\mathrm{~d}, J=2.6 \mathrm{~Hz}, 1 \mathrm{H}), 7.1(\mathrm{td}, J=7.7$, $1.2 \mathrm{~Hz}, 1 \mathrm{H}), 7.01(\mathrm{dd}, J=8.4,2.6 \mathrm{~Hz}, 1 \mathrm{H}), 5.08(\mathrm{brs}, 1 \mathrm{H}) ;{ }^{13} \mathrm{C}\left\{{ }^{1} \mathrm{H}\right\} \mathrm{NMR}\left(150 \mathrm{MHz}, \mathrm{CDCl}_{3}\right) \delta 155.2$, $143.8\left(\mathrm{q}, J_{C F}=1.1 \mathrm{~Hz}\right), 141.4,139.03,139.01,137.2,136.5,136.4,132.8,131.5,130.0,129.0$ $\left(\mathrm{q}, J_{C F}=32.4 \mathrm{~Hz}\right), 128.8,128.4,127.1,126.9,126.6,125.1\left(\mathrm{q}, J_{C F}=4.0 \mathrm{~Hz}\right), 124.5,124.2\left(\mathrm{q}, J_{C F}\right.$ $=272.2 \mathrm{~Hz}$ ), 120.4, 120.0, 119.6, 117.5, 115.9; $\left.{ }^{19} \mathrm{~F} \mathrm{NMR} \mathrm{(565} \mathrm{MHz,} \mathrm{CDCl}\right) \delta-62.4$ (s); IR (neat, $\mathrm{cm}^{-1}$ ) 3388, 3059, 2931, 1615, 1602, 1487, 1448, 1405, 1325, 1292, 1230, 1167, 1123, 1069, 1019, 1007; HRMS (ESI $\left.{ }^{-}\right) \mathrm{m} / \mathrm{z}$ calcd for $\mathrm{C}_{27} \mathrm{H}_{16} \mathrm{~F}_{3} \mathrm{O}[\mathrm{M}-\mathrm{H}]^{-}:$: 413.1159; found 413.1167.

9-((4,4'-Diethyl-[1,1'-biphenyl]-2-yl)methylene)-9H-fluorene (15a). To a solution of phenol 14a (189 mg, $0.51 \mathrm{mmol}, 1.0 \mathrm{eq})$ and pyridine $(0.1 \mathrm{~mL}, 1.01 \mathrm{mmol}, 2.0 \mathrm{eq})$ in DCM $(10 \mathrm{~mL})$ at $0{ }^{\circ} \mathrm{C}$ was added triflic anhydride $(0.13 \mathrm{~mL}, 0.75 \mathrm{mmol}, 1.5 \mathrm{eq})$. The reaction mixture was quenched by the addition of $1 \mathrm{M} \mathrm{HCl}$ and extracted with $\mathrm{DCM}(\mathrm{x} 3)$. The combined organic extracts were washed with $1 \mathrm{M} \mathrm{HCl}$ and dried over anhydrous magnesium sulfate. After filtration, the solvent was removed in vacuo and the crude oil was used immediately in the next step. To a degassed solution of crude aryl triflate, $\mathrm{Pd}(\mathrm{OAc})_{2}$ ( $6 \mathrm{mg}, 0.03 \mathrm{mmol}, 5 \mathrm{~mol} \%$ ), and DavePhos (24 mg, $0.06 \mathrm{mmol}, 10$ $\mathrm{mol} \%)$ in THF $(8 \mathrm{~mL})$ was added a solution of freshly prepared $\mathrm{EtZnl} \cdot \mathrm{LiCl}(1.4 \mathrm{~mL}, 1.01 \mathrm{mmol} .0 .7$ $\mathrm{M}$ soln in THF, $2.0 \mathrm{eq})$. The reaction mixture was quenched by the addition of a sat. aq. solution of $\mathrm{NH}_{4} \mathrm{Cl}$ and extracted with diethyl ether (x3). The combined organic extracts were dried over anhydrous sodium sulfate, filtered, and concentrated in vacuo. The crude oil was purified using silica gel flash column chromatography (dry load, $0,2 \%$ diethyl ether in hexanes) to afford compound $15 \mathrm{a}$ a pale-yellow foam $\left(165 \mathrm{mg}, 84 \%\right.$ yield over the 2 steps): ${ }^{1} \mathrm{H}$ NMR $(300 \mathrm{MHz}$, $\left.\mathrm{CDCl}_{3}\right) \delta 7.90-7.82(\mathrm{~m}, 3 \mathrm{H}), 7.76(\mathrm{~d}, J=1.8 \mathrm{~Hz}, 1 \mathrm{H}), 7.69(\mathrm{dt}, J=7.6,1.0 \mathrm{~Hz}, 1 \mathrm{H}), 7.58-7.56$ $(\mathrm{m}, 2 \mathrm{H}), 7.49-7.40(\mathrm{~m}, 5 \mathrm{H}), 7.34(\mathrm{td}, J=7.5,1.2 \mathrm{~Hz}, 1 \mathrm{H}), 7.27-7.20(\mathrm{~m}, 3 \mathrm{H}), 2.81(\mathrm{q}, J=7.6$ $\mathrm{Hz}, 2 \mathrm{H}), 2.75(\mathrm{q}, J=7.6 \mathrm{~Hz}, 2 \mathrm{H}), 1.39(\mathrm{t}, J=7.6 \mathrm{~Hz}, 3 \mathrm{H}), 1.35(\mathrm{t}, J=7.6 \mathrm{~Hz}, 3 \mathrm{H}) ;{ }^{13} \mathrm{C}\left\{{ }^{1} \mathrm{H}\right\} \mathrm{NMR}$ $\left(75 \mathrm{MHz}, \mathrm{CDCl}_{3}\right) \delta 143.3,143.0,141.3,139.7,139.1,139.0,138.0,137.2,136.0,134.7,130.8$, $130.1,129.7,129.1,128.50,128.46,128.0,127.8,127.0,126.7,124.64,120.57,119.9,119.6$, 28.7, 28.6, 16.0, 15.5; IR (neat, $\mathrm{cm}^{-1}$ ) 3058, 3020, 2964, 2930, 2871, 1484, 1449; HRMS (EI) m/z calcd for $\mathrm{C}_{30} \mathrm{H}_{26}[\mathrm{M}]^{+}$386.2029; found 386.2034. 

prepared in the same way as $15 \mathrm{a}$ as an off-white foam $\left(195 \mathrm{mg}, 79 \%\right.$ yield over 2 steps): ${ }^{1} \mathrm{H}$ NMR $\left(600 \mathrm{MHz}, \mathrm{CDCl}_{3}\right) \delta 7.78(\mathrm{dt}, J=7.5,1.0 \mathrm{~Hz}, 1 \mathrm{H}), 7.76(\mathrm{dt}, J=7.6,0.9 \mathrm{~Hz}, 1 \mathrm{H}), 7.69-7.67(\mathrm{~m}$, $2 \mathrm{H}), 7.61(\mathrm{~d}, J=7.6 \mathrm{~Hz}, 1 \mathrm{H}), 7.60(\mathrm{~d}, J=8.0 \mathrm{~Hz}, 2 \mathrm{H}), 7.55(\mathrm{~d}, J=8.0 \mathrm{~Hz}, 2 \mathrm{H}), 7.47(\mathrm{~d}, J=7.9$ $\mathrm{Hz}, 1 \mathrm{H}), 7.40-7.36(\mathrm{~m}, 4 \mathrm{H}), 7.29(\mathrm{td}, J=7.5,1.1 \mathrm{~Hz}, 1 \mathrm{H}), 7.14(\mathrm{td}, J=7.5,1.2 \mathrm{~Hz}, 1 \mathrm{H}), 2.75(\mathrm{q}$, $J=7.6 \mathrm{~Hz}, 2 \mathrm{H}), 1.32(\mathrm{t}, J=7.6 \mathrm{~Hz}, 3 \mathrm{H}) ;{ }^{13} \mathrm{C}\left\{{ }^{1} \mathrm{H}\right\}$ NMR $\left(150 \mathrm{MHz}, \mathrm{CDCl}_{3}\right) \delta 144.2\left(\mathrm{q}, J_{C F}=1.5 \mathrm{~Hz}\right)$, 144.1, 141.3, 139.2, 139.0, 137.4, 136.8, 136.7, 134.8, 130.8, 129.9, 129.8, 129.1 (q, J J $=32.4$ $\mathrm{Hz}), 128.6,128.5,128.2,127.4,127.0,126.7,125.1\left(\mathrm{q}, J_{C F}=3.8 \mathrm{~Hz}\right), 124.4,124.2$ (q, $J_{C F}=272.2$ $\mathrm{Hz}$ ), 120.4, 119.9, 119.6, 28.5, 15.7; ${ }^{19} \mathrm{~F}$ NMR (565 MHz, CDCl 3 ) $\delta-62.36$ (s); IR (neat, cm ${ }^{-1}$ ) 3061, 3018, 2965, 2931, 2872, 1615, 1449, 1400, 1324, 1166, 1124, 1008; HRMS (EI) calcd for $\mathrm{C}_{29} \mathrm{H}_{21} \mathrm{~F}_{3}[\mathrm{M}]^{+}$426.1590; found 426.1595.

3,14-Diethyldibenzo[g,p]chrysene (16a). To a solution of biaryl 15a (162 mg, $0.42 \mathrm{mmol}, 1.0$ eq) in $\mathrm{MeCN}(16 \mathrm{~mL})$ was added $\mathrm{CuBr}_{2}(187 \mathrm{mg}, 0.84 \mathrm{mmol}, 2.0 \mathrm{eq})$. The reaction mixture was heated to $60{ }^{\circ} \mathrm{C}$ for $2 \mathrm{~h}$. The reaction mixture was cooled to room temperature and quenched with a sat. aq. solution of $\mathrm{NH}_{4} \mathrm{Cl}$ and diluted with diethyl ether. The phases were separated and the aqueous phase was extracted with diethyl ether $(x 2)$. The combined organic extracts were washed with a sat. aq. solution of $\mathrm{NaHCO}_{3}$, water, and brine, and dried over anhydrous sodium sulfate. After filtration, the solvent was removed in vacuo and the crude oil purified on silica gel chromatography using hexanes as eluent to provide compound 16a as a white foam (140 mg, $87 \%$ yield): ${ }^{1} \mathrm{H}$ NMR $\left(300 \mathrm{MHz} \mathrm{CDCl}_{3}\right) \delta 8.81-8.74(\mathrm{~m}, 4 \mathrm{H}), 8.63(\mathrm{~d}, \mathrm{~J}=8.4 \mathrm{~Hz}, 2 \mathrm{H}), 8.58(\mathrm{~d}, J$ $=1.7 \mathrm{~Hz}, 2 \mathrm{H}), 7.75-7.66(\mathrm{~m}, 4 \mathrm{H}), 7.57(\mathrm{dd}, J=8.4,1.7 \mathrm{~Hz}, 2 \mathrm{H}), 2.93(\mathrm{q}, J=7.6 \mathrm{~Hz}, 4 \mathrm{H}), 1.42$ $(\mathrm{t}, J=7.6 \mathrm{~Hz}, 6 \mathrm{H}) ;{ }^{13} \mathrm{C}\left\{{ }^{1} \mathrm{H}\right\} \mathrm{NMR}\left(75 \mathrm{MHz} \mathrm{CDCl}_{3}\right) \delta 142.2,130.9,129.5,129.14,129.07,128.9$, 127.7, 127.6, 127.0, 126.54, 126.48, 123.7, 123.5, 29.3, 16.0; IR (neat, $\mathrm{cm}^{-1}$ ) 3064, 3022, 2964, 2930, 2870, 1614, 1481, 1450, 1428, 1386, 1316, 1234, 1050; HRMS (ESI ${ }^{+}$) calcd for $\mathrm{C}_{30} \mathrm{H}_{25}$ $[\mathrm{M}+\mathrm{H}]^{+} 385.1951$; found 385.1937.

3-Ethyl-14-(trifluoromethyl)dibenzo[g,p]chrysene (16b) was prepared in the same way as 16a except that 4.0 eq of $\mathrm{CuBr}_{2}$ was needed to achieve complete conversion over $18 \mathrm{~h}$ : white solid (118 mg, 81\% yield); mp $173-174{ }^{\circ} \mathrm{C}(\mathrm{MeOH}) ;{ }^{1} \mathrm{H}$ NMR $\left(600 \mathrm{MHz}, \mathrm{CDCl}_{3}\right) \delta 8.96(\mathrm{~s}, 1 \mathrm{H}), 8.75-$ $8.68(\mathrm{~m}, 4 \mathrm{H}), 8.61(\mathrm{~d}, J=8.3 \mathrm{~Hz}, 1 \mathrm{H}), 8.56(\mathrm{dd}, J=8.0,1.5 \mathrm{~Hz}, 1 \mathrm{H}), 8.52(\mathrm{~d}, J=1.8 \mathrm{~Hz}, 1 \mathrm{H})$, 7.85 (dd, $J=8.6,1.8 \mathrm{~Hz}, 1 \mathrm{H}), 7.73-7.64(\mathrm{~m}, 4 \mathrm{H}), 7.57(\mathrm{dd}, J=8.3,1.7 \mathrm{~Hz}, 1 \mathrm{H}), 2.89$ (q, $J=7.6$ $\mathrm{Hz}, 2 \mathrm{H}), 1.36(\mathrm{t}, J=7.6 \mathrm{~Hz}, 3 \mathrm{H}) ;{ }^{13} \mathrm{C}\left\{{ }^{1} \mathrm{H}\right\} \mathrm{NMR}\left(150 \mathrm{MHz}, \mathrm{CDCl}_{3}\right) \delta 143.8,133.0\left(\mathrm{q}, J_{C F}=1.4 \mathrm{~Hz}\right)$, $131.0,130.9,130.0,129.0,128.78,128.75,128.44,128.35,128.31,127.9,127.8\left(q, J_{C F}=32.3\right.$ $\mathrm{Hz}), 127.7,127.3,127.04,126.97,126.95,126.8,126.7,126.1\left(\mathrm{q}, J_{C F}=4.3 \mathrm{~Hz}\right), 124.6\left(\mathrm{q}, J_{C F}=\right.$ $272.3 \mathrm{~Hz}), 124.2,123.9,123.74\left(\mathrm{q}, J_{C F}=0.9 \mathrm{~Hz}\right), 123.69,122.4\left(\mathrm{q}, J_{C F}=3.4 \mathrm{~Hz}\right), 29.2,15.7 ;{ }^{19} \mathrm{~F}$ NMR (565 MHz, CDCl ${ }_{3}$ ) $\delta-61.8$ (s); IR (neat, $\mathrm{cm}^{-1}$ ) 3067, 2966, 2932, 2873, 1615, 1483, 1418, 1387, 1334, 1298, 1263, 1251, 1237, 1173, 148, 1121, 1085, 1051; HRMS $\left(\mathrm{ESI}^{+}\right)$calcd for $\mathrm{C}_{29} \mathrm{H}_{19} \mathrm{~F}_{3}[\mathrm{M}]^{+}$424.1433; found 424.1430.

2,4-Dichloro-14-ethyldibenzo[g,p]chrysen-3-ol (16c). To a stirred solution of $14 a$ (62 $\mathrm{mg}, 0.17$ $\mathrm{mmol}, 1.0 \mathrm{eq})$ in $\mathrm{MeCN}(12.4 \mathrm{~mL}, 5 \mathrm{mg} / \mathrm{mL})$ under air at room temperature was added $\mathrm{CuCl}_{2}(137$ $\mathrm{mg}, 1.02 \mathrm{mmol}, 6.0 \mathrm{eq}$ ) all at once. The reaction mixture was heated to $60^{\circ} \mathrm{C}$ for $24 \mathrm{~h}$. The reaction mixture was quenched by the addition of a sat. aq. solution of $\mathrm{NH}_{4} \mathrm{Cl}$ and stirred for $20 \mathrm{~min}$. The phases were separated and the aqueous phase was extracted with diethyl ether $(x 3)$. The combined organic extracts were washed with a saturated aqueous solution of $\mathrm{NaHCO}_{3}(\mathrm{x} 2$ or until the blue color subsided in the aqueous phase) and brine. The combined organic extracts were dried over anhydrous sodium sulfate. After filtration, the solvent was removed in vacuo and the crude oil was purified by flash column chromatography on silica gel using a gradient of $0,2,4$, $8 \%$ diethyl ether in hexanes as eluent to afford $16 \mathrm{c}$ as an off-white solid (55 mg, $75 \%$ yield): ${ }^{1} \mathrm{H}$ NMR $\left(600 \mathrm{MHz}, \mathrm{CDCl}_{3}\right) \delta 8.70-8.68(\mathrm{~m}, 2 \mathrm{H}), 8.67(\mathrm{~s}, 1 \mathrm{H}), 8.56(\mathrm{dd}, J=8.2,1.3 \mathrm{~Hz}, 1 \mathrm{H}), 8.51$ (d, $J=1.7 \mathrm{~Hz}, 1 \mathrm{H}), 8.45(\mathrm{~d}, J=8.4 \mathrm{~Hz}, 1 \mathrm{H}), 7.81$ (dd, $J=8.2,1.2 \mathrm{~Hz}, 1 \mathrm{H}), 7.74$ (ddd, $J=8.1,7.0$, $1.3 \mathrm{~Hz}, 1 \mathrm{H}), 7.68$ (ddd, $J=8.3,7.0,1.3 \mathrm{~Hz}, 1 \mathrm{H}), 7.60$ (ddd, $J=8.1,6.9,1.3 \mathrm{~Hz}, 1 \mathrm{H}), 7.52-7.49$ 
(m, 2H), 6.34 (brs, 1H), 2.84 (q, J = $7.5 \mathrm{~Hz}, 2 \mathrm{H}), 1.32(\mathrm{t}, J=7.5 \mathrm{~Hz}, 3 \mathrm{H}) ;{ }^{13} \mathrm{C}\left\{{ }^{1} \mathrm{H}\right\} \mathrm{NMR}(150 \mathrm{MHz}$, $\left.\mathrm{CDCl}_{3}\right) \delta 147.2,143.3,131.8,131.6,129.9,129.5,129.10,129.06,128.8,128.7,128.6,127.5$, $127.1,127.0,126.5,126.4,126.3,125.8,125.7,125.1,123.7,123.3,123.3,123.0,120.8,117.7$, 29.2, 15.7; IR (neat, $\mathrm{cm}^{-1}$ ) 3500, 3064, 2963, 2930, 2870, 1612, 1588, 1533, 1479, 1463, 1449, 1428, 1415, 1389, 1326, 1266, 1244, 1192, 1022; HRMS $\left(\mathrm{ESI}^{-}\right) \mathrm{m} / \mathrm{z}$ calcd for $\mathrm{C}_{28} \mathrm{H}_{17} \mathrm{Cl}_{2} \mathrm{O}[\mathrm{M}-\mathrm{H}]^{-}$: 439.0662; found: 439.0673 .

2,5,7-Trimethoxybenzo[p]indeno[1,2,3,4-defg]chrysene (17a). To a stirred solution of dibenzo[g,p]chrysene 12a (86 mg, $0.23 \mathrm{mmol}, 1.0 \mathrm{eq})$ and DDQ (208 mg, $0.92 \mathrm{mmol}, 4.0 \mathrm{eq})$ in $\mathrm{MeOH}(77 \mathrm{~mL})$ was added 2 drops of trifluoroacetic acid from a Pasteur pipet under air in a glass pressure tube. The vessel was sealed and heated to $100{ }^{\circ} \mathrm{C}$ overnight. After cooling to room temperature, additional DDQ (104 $\mathrm{mg}, 0.46 \mathrm{mmol})$ was added. In subsequent runs it was found to be beneficial to add DDQ in portions throughout the reaction rather than all at once at the beginning of the reaction. The reaction mixture was heated at $100{ }^{\circ} \mathrm{C}$ for an additional $5 \mathrm{~h}$. After cooling to room temperature, the reaction mixture was quenched by the addition of water and $\mathrm{NaHCO}_{3}$ (sat. aq.). The reaction mixture was extracted with diethyl ether (x3). The combined organic extracts were washed with $\mathrm{NaHCO}_{3}$ (sat. aq.), water, and brine, followed by drying over anhydrous sodium sulfate. After filtration, the solvent was removed in vacuo and the crude oil purified by flash column chromatography on silica gel using a gradient of ethyl acetate in hexanes as eluent $(5,7,9,11 \%)$. Compound 17 a was isolated as a yellow-reddish solid ( $45 \mathrm{mg}, 44 \%$ yield). Compound 17a was contaminated with a small amount of the corresponding hemiketal 17b (reddish color) of which red-orange crystals suitable for X-ray crystallography were grown by slow evaporation from a toluene solution of the material isolated from column chromatography. 17a: ${ }^{1} \mathrm{H}$ NMR $\left(500 \mathrm{MHz}, d_{8}\right.$-THF) $\delta 9.69(\mathrm{~d}, J=9.5 \mathrm{~Hz}, 1 \mathrm{H}), 9.08(\mathrm{~d}, J=8.1 \mathrm{~Hz}, 1 \mathrm{H}), 8.83(\mathrm{~d}, J=7.9$ $\mathrm{Hz}, 1 \mathrm{H}), 8.45-8.37(\mathrm{~m}, 2 \mathrm{H}), 7.82-7.68(\mathrm{~m}, 3 \mathrm{H}), 7.42(\mathrm{~d}, J=7.8 \mathrm{~Hz}, 1 \mathrm{H}), 7.37$ (dd, J = 9.5, 2.7 $\mathrm{Hz}, 1 \mathrm{H}), 5.99(\mathrm{~s}, 1 \mathrm{H}), 4.02(\mathrm{~s}, 3 \mathrm{H}), 3.98(\mathrm{~s}, 3 \mathrm{H}), 3.25(\mathrm{~s}, 3 \mathrm{H}) ;{ }^{13} \mathrm{C}\left\{{ }^{1} \mathrm{H}\right\} \mathrm{NMR}\left(125 \mathrm{MHz}, d_{8}-\mathrm{THF}\right) \delta$ 186.7, 167.8, 159.5, 149.6, 133.0, 130.8, 130.6, 130.1, 129.93, 129.86, 129.1, 128.7, 128.0, 127.6, 126.2, 125.3, 123.6, 120.9, 119.3, 117.3, 115.7, 108.7, 105.7, 93.4, 56.5, 55.5, 52.6; IR (neat, $\mathrm{cm}^{-1}$ ) 2938, 2835, 1812, 1651, 1614, 1514, 1456, 1361, 1304, 1232, 1200, 1161, 1096, 1036; HRMS $\left(\mathrm{El}^{+}\right) \mathrm{m} / \mathrm{z}$ calcd for $\mathrm{C}_{27} \mathrm{H}_{17} \mathrm{O}_{4}$ [M-OMe] $]^{+}: 417.1121$; found 417.1127.

"COSY, HSQC, HMBC are provided in the NMR Spectra section of the SI. Two carbon signals are not observed in the ${ }^{13} \mathrm{C}\left\{{ }^{1} \mathrm{H}\right\}$ NMR spectrum. 


\section{Optimization of Reaction Conditions}

Our initial reaction screening started with tertiary alcohol 2 (Table S1). Treatment of 2 with stoichiometric $\mathrm{CuBr}_{2}$ led to a very rapid reaction $(<10 \mathrm{~min})$ that resulted in a mixture of the 4-aryl phenol $\mathbf{3 a}$ and 4-aryl ether $\mathbf{3 b}$ (3:1 mixture, respectively). The formation of 4-arylether $\mathbf{3 b}$ was a result of competitive ketal hydrolysis with aromatization of the enol ether precursor. These initial results were encouraging, however, we desired to optimize the reaction to limit the amount of 4arylether formed.

Initial reaction optimization led us to screen copper salts (Table S1). Only $\mathrm{CuBr}_{2}$ and $\mathrm{CuCl}_{2}$ efficiently reacted (entries 1-2) while other copper (II) salts either failed to react or led to other unidentified compounds (entries 3-6). $\mathrm{CuCl}_{2}$ provided a better ratio of 4-aryl phenol to 4-aryl ether (8:1, respectively), but the reaction was initially heterogenous only becoming homogenous after prolonged stirring. From subsequent reactions, we were able to determine that this was due to the production of $\mathrm{HCl}$ in the reaction mixture. The slower oxidation reaction with copper chloride allowed more time for ketal hydrolysis to take place prior to aromatization, leading to higher selectivity for the phenol.

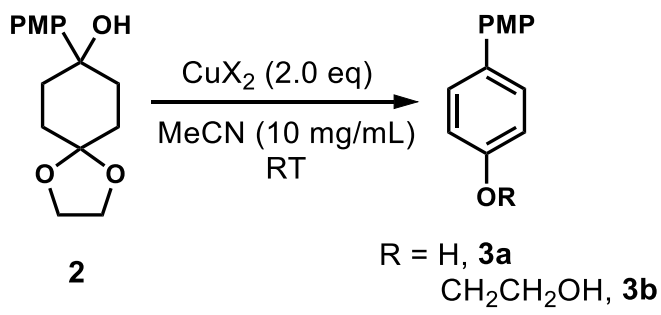

\begin{tabular}{|c|c|c|c|c|c|}
\hline Entry & $\mathrm{CuX}_{2}$ & Time & $\% \mathrm{v} / \mathbf{v ~ H}_{2} \mathrm{O}^{[a]}$ & $3 a: 3 b^{[b]}$ & Isolated Yield of $3 a$ \\
\hline 1 & $\mathrm{CuBr}_{2}$ & $<10 \mathrm{~min}$ & 0 & $3: 1$ & n.d. \\
\hline 2 & $\mathrm{CuCl}_{2}$ & $2 \mathrm{~h}$ & 0 & $8: 1$ & $86 \%$ \\
\hline 3 & $\mathrm{Cu}(\mathrm{OTf})_{2}$ & $2 \mathrm{~h}$ & 0 & $-[c]$ & - \\
\hline 4 & $\mathrm{CuSO}_{4}$ & $2 d$ & 0 & [dd] & - \\
\hline 5 & $\mathrm{Cu}(\mathrm{acac})_{2}$ & $2 d$ & 0 & [-[e] & - \\
\hline 6 & $\mathrm{Cu}(\mathrm{OAc})_{2}$ & $2 d$ & 0 & [-[e] & - \\
\hline 7 & $\mathrm{CuBr}_{2}$ & $18 \mathrm{~h}$ & 10 & [-[e] & - \\
\hline 8 & $\mathrm{CuBr}_{2}$ & $<25 \mathrm{~min}$ & 1.0 & $>20: 1$ & $84 \%$ \\
\hline 9 & $\mathrm{CuBr}_{2}^{[f]}$ & $2 \mathrm{~h}$ & 1.0 & n.d. & $69 \%$ \\
\hline 10 & $\mathrm{CuCl}_{2}^{[\mathrm{gg}]}$ & $24 \mathrm{~h}$ & 0 & n.d. & $54 \%$ \\
\hline
\end{tabular}

Table S1: Optimization of the reaction conditions. [a] Refers only to added water (no attempt was made to remove adventitious water). [b] Determined by analysis of the crude ${ }^{1} \mathrm{H}$ NMR spectrum. [c] An unidentified compound was produced. [d] Reaction was slow and produced only intermediates. [e] No reaction occurred. [f] 20 mol\% was used. [g] 30 mol\% was used. n.d. $=$ not determined. PMP $=4-$ methoxyphenyl.

Ketal hydrolysis could be promoted by the addition water to the acetonitrile $(1 \% \mathrm{v} / \mathrm{v})$. Using stoichiometric $\mathrm{CuBr}_{2}$ with $1 \% \mathrm{v} / \mathrm{v} \mathrm{H}_{2} \mathrm{O}$ led to the 4-arylphenol $3 \mathrm{a}$ in $84 \%$ yield with small amounts $(<5 \%)$ of 4 -arylether $\mathbf{3 b}$ (entry 8 ). Using larger amounts of water led to either reduced (2 or $3 \%$ $\mathrm{v} / \mathrm{v})$ or no $(>10 \% \mathrm{v} / \mathrm{v}$, entry 7$)$ reactivity of $\mathrm{CuBr}_{2}$. The reaction can also be carried out using substoichiometric $\mathrm{CuBr}_{2}\left(20 \mathrm{~mol} \%\right.$ ) or $\mathrm{CuCl}_{2}(30 \mathrm{~mol} \%)$ to give $69 \%$ and $54 \%$ of 4 -arylphenol $3 \mathrm{a}$, respectively. Because of the rapid reaction and higher yield, the use of stoichiometric $\mathrm{CuBr}_{2}$ was optimal. 
The scope of the reaction is outlined in Figure S1. The aromatization reaction is general for electron rich and neutral aromatics. The reaction was particularly fast for the tertiary benzylic alcohol which provided 4-(4-methoxyphenyl)phenol 3a in less than 25 minutes. Other less electron rich benzylic alcohols did not react as quickly to provide the corresponding phenols (B59) since elimination of the alcohol was rendered much slower. In fact, $\mathrm{CuBr}_{2}$ in acetonitrile at room temperature failed to eliminate the alcohol for a majority of cases. This was remedied by the addition a catalytic amount of aqueous $\mathrm{HBr}$.

The method is not limited to the addition products of $\mathbf{1}$; exposure of $\mathbf{1}$ to LHMDS in THF at -78 ${ }^{\circ} \mathrm{C}$, followed by 4-tert-butylbenzyl bromide, led to the expected $\alpha$-benzylcyclohexanone which was converted to $\mathbf{B} 4 \mathbf{a}$ by sequential treatment of $p$-methoxyphenyllithium and $\mathrm{CuBr}_{2}$. Prolonged exposure of $\mathbf{B 4 a}$ to $\mathrm{CuBr}_{2}$ resulted in partial $\alpha$-bromination of the phenol ( $9 \%$ isolated).

3,5-Bistrifluoromethylbenzylic alcohol $\mathbf{A} 9$ failed to produce the desired 4-aryl phenol $\mathbf{B 1 0}$ in acetonitrile with stoichiometric $\mathrm{CuBr}_{2}$ and catalytic $\mathrm{HBr}$, rather a mixture of $\alpha$-bromoketal $\mathbf{A} 11$ and ketone A12 were isolated. Elimination of the benzylic alcohol A9 could be achieved by prior treatment with catalytic $p$ - $\mathrm{TsOH}$ in refluxing toluene. Following a solvent exchange, treatment of styrene S1, prepared in situ, with $\mathrm{CuBr}_{2}$ provided the 4-aryl phenol B10 in moderate yield (46\% over two sequential steps, $86 \%$ from isolated styrene) (Scheme S1). The styrene could also be accessed by means of a Suzuki cross coupling reaction from the enol triflate T1. Phenols B1014 were prepared in this way from the corresponding styrenes. The aromatization reaction is general provided that the styrene can be accessed in situ or in a prior step.

Insight into the mechanism of the cascade of reactions that culminate in the creation of the aromatic ring was provided by the examples described above. A plausible course for conversion of 2 to $\mathbf{3}$ involved the following four steps: $\alpha$-bromination of the ketal, hydrolysis of the ketal, dehydration of the alcohol to form a styrene, and dehydrobromination. The sequence of these reactions cannot be determined with certainty from the evidence at hand and is likely to be substrate dependent. However, since the phenol is the major product in all cases, hydrolytic cleavage of the ethylene ketal must precede dehydrobromination under the reaction conditions, otherwise a 4-aryl-2-hydroxyethyl ether would have formed as a major product. In some cases, small amounts of this byproduct could be identified. If alcohol dehydration is rendered slow, as in the case of $\mathbf{A 9}$, the bromide can be isolated. It is plausible that dehydration of the alcohol may be a rate limiting process for the conversion of less activated benzylic alcohols. Further evidence is provided by the necessity for added acid in order to prepare 4-aryl phenols B5-9. Since elimination to the styrene for less activated benzylic alcohols is a slow process in the absence of sufficiently strong acid, bromination and hydrolysis of the ketal are rendered fast. In the case of activated benzylic alcohols, elimination may be competitive with ketal hydrolysis and can lead to a mixture of 4-arylphenol and 4-arylether in the absence of added water. 


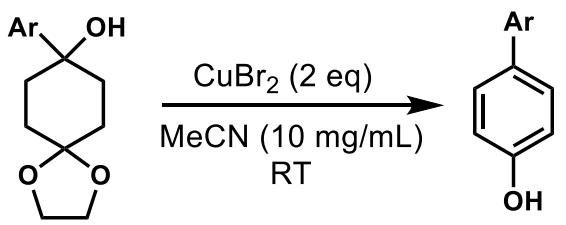<smiles>COc1ccc(-c2ccc(O)cc2)cc1</smiles><smiles>Oc1ccc(-c2ccccc2)cc1</smiles>

$3 a$ $84 \%^{[a]}$

B1

$69 \%{ }^{[a]}$<smiles>Oc1ccc(-c2ccc3ccccc3c2)cc1</smiles>
B6
$85 \%^{[\mathrm{b}]}$

B5 $80 \%{ }^{[\mathrm{b}]}$<smiles>Oc1ccc(-c2cccc3ccccc23)cc1</smiles><smiles>COc1cccc(-c2ccc(O)cc2)c1</smiles>

B2 $45 \%$ [a]<smiles>Cc1ccc(-c2ccc(O)cc2)cc1</smiles>

B7<smiles>COc1ccccc1-c1ccc(O)cc1</smiles><smiles>[R]c1cc(-c2ccc(OC)cc2)c(C[14CH2]c2ccccc2)cc1O</smiles>

\section{B3} $94 \%{ }^{[a]}$

$\mathrm{R}=\mathrm{H}, 80 \%^{[\mathrm{a}]}, \mathbf{B} 4 \mathbf{a}$

$=\mathrm{Br}, 9 \%$, B4b<smiles>Oc1ccc(-c2ccc(-c3ccccc3)cc2)cc1</smiles>

B8<smiles>Oc1ccc(-c2ccc(Br)cc2)cc1</smiles>

B9<smiles>Oc1ccc(-c2cc(C(F)(F)F)cc(C(F)(F)F)c2)cc1</smiles><smiles>Oc1ccc(-c2ccc(F)cc2)cc1</smiles>

B10

$86 \%$ [c]

$$
\begin{gathered}
\text { B11 } \\
67 \% \text { [c] }
\end{gathered}
$$

$92 \%$ [b]

Quant. ${ }^{[b]}$

$48 \%{ }^{[b]}$

Figure S1: Scope of the $\mathrm{CuBr}_{2}$-mediated aromatization reaction. [a] $1 \% \mathrm{v} / \mathrm{v}$ $\mathrm{H}_{2} \mathrm{O} / \mathrm{MeCN}$ was used as solvent. [b] A catalytic amount of $48 \% \mathrm{HBr}$ was added to the reaction mixture after $15 \mathrm{~min}$. [c] Prepared from the corresponding styrene 
<smiles>OC1(c2cc(C(F)(F)F)cc(C(F)(F)F)c2)CCC2(CC1)OCCO2</smiles>

A9

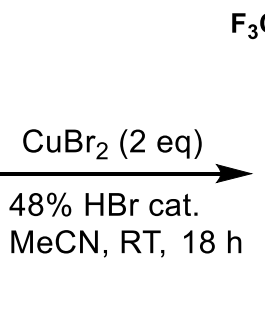<smiles>O=C1CCC(O)(c2cc(C(F)(F)F)cc(C(F)(F)F)c2)CC1Br</smiles>

A12

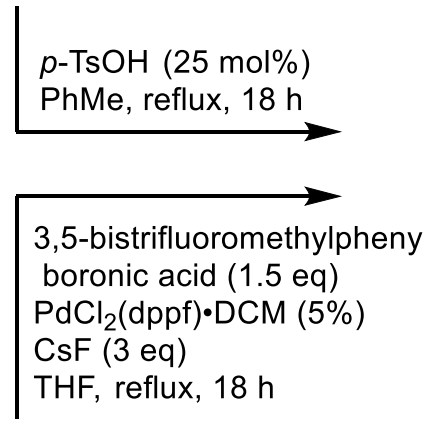

(1)

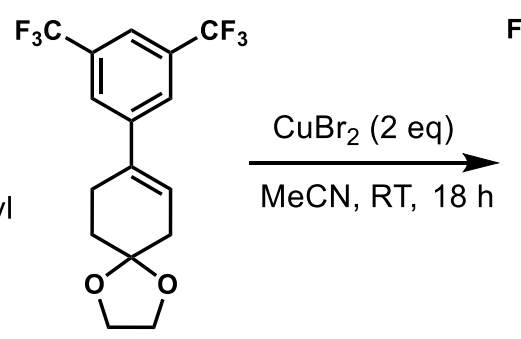

S1<smiles>Oc1ccc(-c2cc(C(F)(F)F)cc(C(F)(F)F)c2)cc1</smiles>

B10

$46 \%$ over 2 steps $^{\text {[a] }}$

$63 \%$ over 2 steps ${ }^{[b]}$

T1

Scheme S1: Synthetic routes to compound B10. [a] From tertiary alcohol 21; [b] From enol triflate T1

We sought to apply this methodology as a mild and simple approach to the synthesis of substituted fluorenes and other functionalized polyaromatics. We surmised that benzylic allylic alcohols, such as, 4a would be suitable precursors to substituted fluorenes. Formation of the fluorene from this type of benzylic allylic alcohol requires an initial aromatic Nazarov cyclization to construct the five-membered ring, followed by a similar series of cascade reactions involving $\mathrm{CuBr}_{2}$ to provide the phenol.

Alcohol $\mathbf{4 a}$ is prepared by aldol condensation of 1 with benzaldehyde, followed by 1,2-addition of 4-anisyllithium. Our initial treatment of alcohol $4 \mathrm{a}$ with stoichiometric $\mathrm{CuBr}_{2}$ in acetonitrile provided a mixture of phenol $\mathbf{5 a}$ and aryl ether $\mathbf{5 a 2}$ as a 1:1 mixture (this ratio was irreproducible). We optimized the reaction conditions to favor the formation of $5 \mathbf{a}$ (Table S2). 


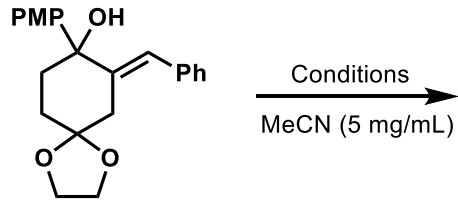

4

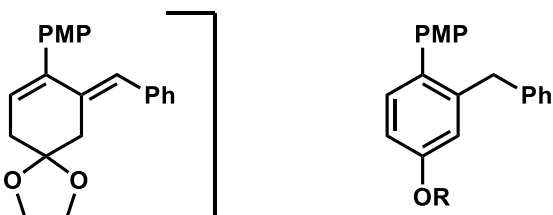

$6 \mathrm{R}=\mathrm{H}$ 6a R $=\mathrm{CH}_{2} \mathrm{CH}_{2} \mathrm{OH}$

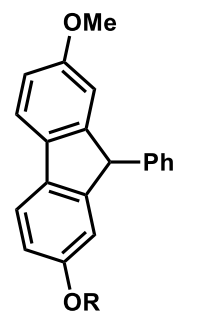

5 a $\mathrm{R}=\mathrm{H}$ 5 a2 $\mathrm{R}=\mathrm{CH}_{2} \mathrm{CH}_{2} \mathrm{OH}$

\begin{tabular}{|c|c|c|}
\hline Entry & Conditions & Result \\
\hline 1 & $\mathrm{CuBr}_{2}(2.0 \mathrm{eq}), 30 \mathrm{~min}$ & $1: 15 \mathbf{a}: 5 \mathbf{a} 2$ \\
\hline 2 & $\mathrm{CuBr}_{2}(2.0 \mathrm{eq}), 1 \% \mathrm{v} / \mathrm{v} \mathrm{H}_{2} \mathrm{O}, 18 \mathrm{~h}$ & -[a] \\
\hline 3 & $\mathrm{CuBr}_{2}(0.5 \mathrm{eq}), 18 \mathrm{~h}$ & Mix of biaryls 6 and $6 a$ \\
\hline 4 & $\mathrm{HBr}$ cat., $5 \mathrm{~min}$ & $1: 16: 6 a$ \\
\hline 5 & $\begin{array}{l}\mathrm{Yb}(\mathrm{OTf})_{3}(10 \mathrm{~mol} \%), 30 \mathrm{~min} \\
\text { then } \mathrm{CuBr}_{2}(2.0 \mathrm{eq}), 15 \mathrm{~min}\end{array}$ & $5: 15 a: 6$ \\
\hline 6 & $\begin{array}{l}\mathrm{Sc}(\mathrm{OTf})_{3}(10 \mathrm{~mol} \%), 30 \mathrm{~min} \\
\text { then } \mathrm{CuBr}_{2}(2.0 \mathrm{eq}), 15 \mathrm{~min}\end{array}$ & $5 \mathbf{a}, 78 \%$ yield \\
\hline
\end{tabular}

We first attempted to favor the phenol $\mathbf{5 a}$ by addition of $1 \% \mathrm{v} / \mathrm{v}$ water to the reaction mixture, but this led to longer reaction times and resulted in more complicated reaction mixtures that did not provide the desired phenol $\mathbf{5 a}$ as a major component (entry 2). Use of catalytic $\mathrm{CuBr}_{2}(0.5 \mathrm{eq}$ ) initially led to the dehydration of the alcohol to the corresponding styrene $\mathbf{6 b}$ (detected by ${ }^{1} \mathrm{H} \mathrm{NMR}$ analysis of an aliquot of the reaction mixture) which eventually converted to a mixture of $\mathbf{6}$ and 6a. Treatment of alcohol $4 a$ with catalytic $\mathrm{HBr}$ in acetonitrile resulted in a fast reaction that provided a mixture of 4-aryl phenol $\mathbf{6}$ and 4-aryl ether $\mathbf{6 a}$ in good isolated yields. Based on these observations, it became clear that dehydration of the alcohol would not provide a route to the fluorene. We hypothesized that the generation of small amounts of $\mathrm{HBr}$ under the reaction conditions would allow for an undesired, fast acid-catalyzed isomerization to the biaryl byproducts of 6. We decided to determine whether the use of other strong, non-oxidizing Lewis acids would provide a more efficient route to cyclization. When alcohol 4 a was treated sequentially with $\mathrm{Yb}(\mathrm{OTf})_{3}(10 \mathrm{~mol} \%)$ and $\mathrm{CuBr}_{2}(2.0 \mathrm{eq})$ a mixture of biaryl 6 and fluorene 5 a were formed in a 1:5 ratio, respectively. However, when alcohol 4a was first treated with $\mathrm{Sc}(\mathrm{OTf})_{3}(10 \mathrm{~mol} \%)$ in acetonitrile at room temperature followed by stoichiometric $\mathrm{CuBr}_{2}$, the fluorene $\mathbf{5 a}$ was isolated as the sole product in $78 \%$ yield.

The aromatic Nazarov cyclization tolerates electron rich to neutral alcohols well; however, the cyclization fails with electron poor alcohols (e.g., $\mathrm{Ar}^{\prime}=4-\mathrm{CF}_{3} \mathrm{Ph}$, Figure S2). Under the optimized reaction conditions diarylmethanols are formed in moderate yields $\mathbf{( 5 0 - 5 q )}$. 

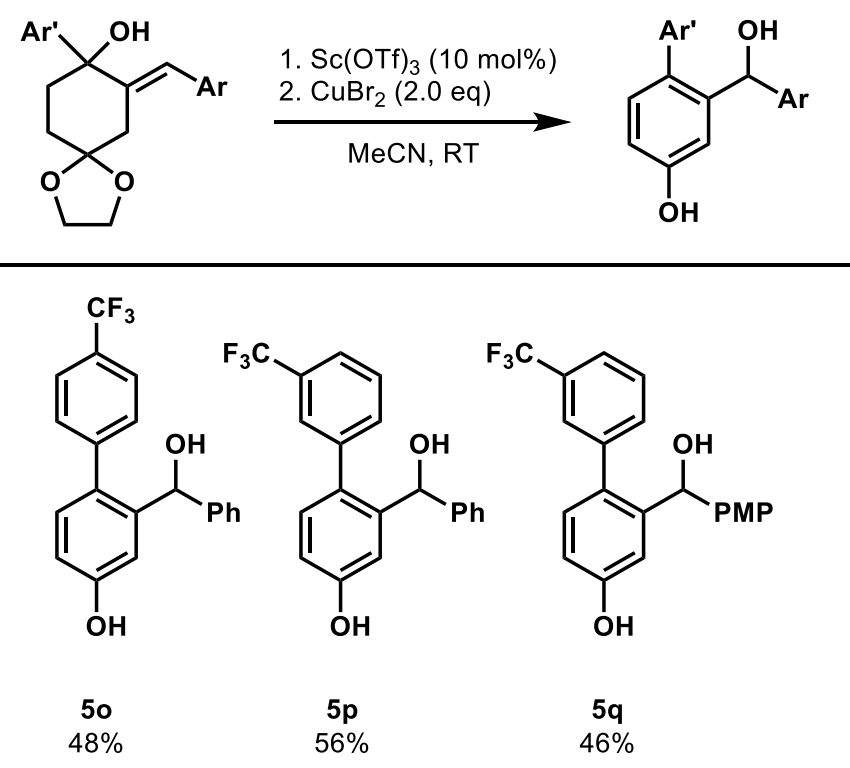

Figure S2: List of diarylmethanols.

1,3-Transposition of the allylic alcohol with $\mathrm{Sc}(\mathrm{OTf})_{3}$ and subsequent oxidative aromatization with $\mathrm{CuBr}_{2}$ provided the diarylmethanols (Scheme S2). Heating the reaction mixture prior to addition of $\mathrm{CuBr}_{2}$ did not lead to formation of the 5-membered ring. Other attempts to promote 5-membered ring formation using $\mathrm{Sc}(\mathrm{OTf})_{3}$ in toluene at $60^{\circ} \mathrm{C}$ led to the dehydration product S6. Introduction of a $p$-methoxyphenyl group (5q), which we presumed would facilitate the formation of the cation also failed to induce cyclization.<smiles>OC1(c2ccc(C(F)(F)F)cc2)CCC2(CC1=CP)OCCO2</smiles>

40

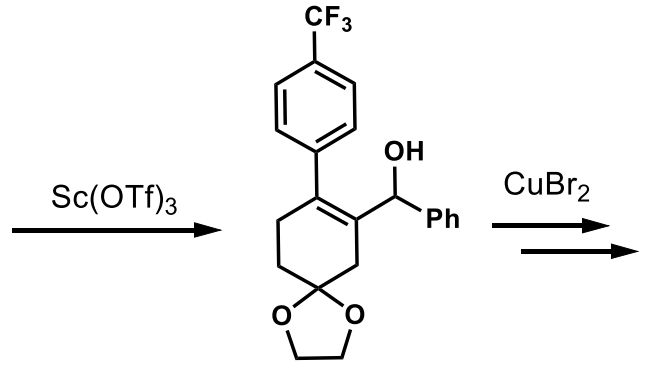

Scheme S2<smiles>Oc1ccc(-c2ccc(C(F)(F)F)cc2)c(C(O)c2ccccc2)c1</smiles>

50<smiles>FC(F)(F)c1ccc(C2=CCC3(CC2=Cc2ccccc2)OCCO3)cc1</smiles>

S6

Diarylmethanol 50 could be converted to its corresponding fluorene by treatment with triflic acid in DCM at room temperature within 3 hours in $>95 \%$ yield. This suggests that diarylmethanols $\mathbf{5 p}$ and $\mathbf{5 q}$ should also provide the fluorene in a similar reaction. 


\section{Synthesis of Aldol Adducts}

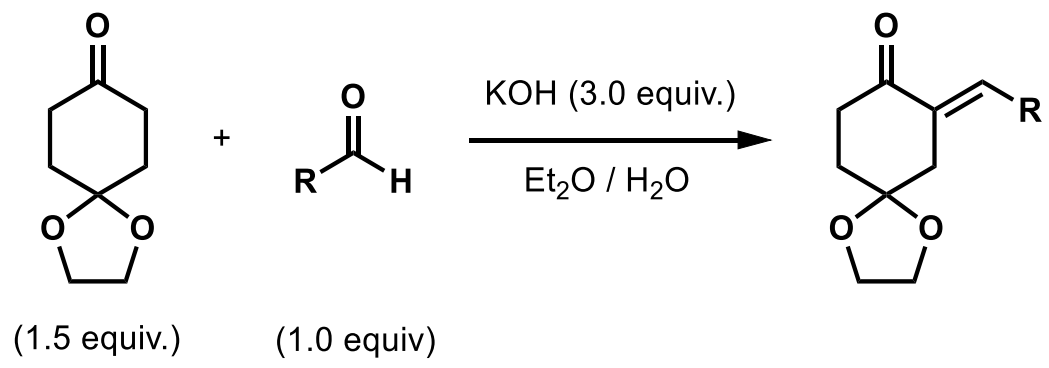

Representative Procedure:

To a stirred solution of 1,4-cyclohexanedione monoethylene ketal ( $2.26 \mathrm{~g}, 15.0 \mathrm{mmol}, 1.5$ equiv.) in diethyl ether $(20 \mathrm{~mL})$ was added benzaldehyde $(1.0 \mathrm{~mL}, 10.0 \mathrm{mmol}, 1.0$ equiv.). An aqueous $\mathrm{KOH}$ solution ( $8.3 \mathrm{~mL}$ of a $3.6 \mathrm{M}$ solution, 3.0 equiv.) was added at once to the mixture and the reaction was vigorously stirred overnight. The reaction mixture was poured into a separatory funnel and the phases separated. The aqueous phase was extracted with diethyl ether ( $\times 3$ ). The combined organic extracts were washed with water and brine, and dried over anhydrous sodium sulfate. After filtration, the combined organic extracts were concentrated in vacuo to afford a crude solid. The condensation product $\mathbf{K} \mathbf{1}$ was purified by recrystallization from the crude material using hexanes/diethyl ether to afford $\mathbf{K} \mathbf{1}$ as colorless needles (1.76 g, 72\%).

Note: The success of some of these reactions was found to be dependent on the purity of the starting materials. Some reactions did not proceed to completion despite prolonged stirring at room temperature (e.g. $>2$ days), with heating of the reaction mixture, or addition of more 1,4cyclohexanedione monoethylene ketal. In some cases, only small amounts of the double aldol product were detected by TLC.<smiles>O=C1CCC2(C/C1=C\c1ccccc1)OCCO2</smiles>

(E)-7-benzylidene-1,4-dioxaspiro[4.5]decan-8-one (K1)

White needles (1.76 g, $72 \%$ yield); m.p. $=102-103{ }^{\circ} \mathrm{C}\left(\mathrm{Hex} / \mathrm{Et}_{2} \mathrm{O}\right) ;{ }^{1} \mathrm{H} \mathrm{NMR}\left(500 \mathrm{MHz}, \mathrm{CDCl}_{3}\right)$ $7.58(t, J=2.3 \mathrm{~Hz}, 1 \mathrm{H}), 7.41-7.37(\mathrm{~m}, 4 \mathrm{H}), 7.37-7.32(\mathrm{~m}, 1 \mathrm{H}), 4.01-3.93(\mathrm{~m}, 4 \mathrm{H}), 3.08-3.03$ (m, 2H), $2.71(\mathrm{t}, J=7.1 \mathrm{~Hz}, 2 \mathrm{H}), 2.13(\mathrm{t}, J=7.1 \mathrm{~Hz}, 2 \mathrm{H}) ;{ }^{13} \mathrm{C}\left\{{ }^{1} \mathrm{H}\right\} \quad \mathrm{NMR}\left(125 \mathrm{MHz}, \mathrm{CDCl}_{3}\right) 199.5$, 137.9, 135.1, 132.6, 130.3, 128.8, 128.4, 107.3, 64.7, 38.3, 36.6, 31.5; IR (neat, $\mathrm{cm}^{-1}$ ) 2960, 2889, $1682,1597,1492,1447,1276,1178,1112,1057$; HRMS $\left(E S I^{+}\right) \mathrm{m} / z$ calcd for $\mathrm{C}_{15} \mathrm{H}_{17} \mathrm{O}_{3}[\mathrm{M}+\mathrm{H}]^{+}$: 245.1172; found 245.1175 . 
<smiles>COc1ccc(/C=C2\CC3(CCC2=O)OCCO3)cc1</smiles>

(E)-7-(4-methoxybenzylidene)-1,4-dioxaspiro[4.5]decan-8-one (K2)

Pale yellow needles (4.65 g, 85\% yield); m.p. = $104-106{ }^{\circ} \mathrm{C}\left(\mathrm{Hex}_{\mathrm{Et}} \mathrm{O}\right) ;{ }^{1} \mathrm{H} \mathrm{NMR}(500 \mathrm{MHz}$, $\left.\mathrm{CDCl}_{3}\right) 7.56(\mathrm{t}, J=2.3 \mathrm{~Hz}, 1 \mathrm{H}), 7.41-7.36(\mathrm{~m}, 2 \mathrm{H}), 6.96-6.88(\mathrm{~m}, 2 \mathrm{H}), 4.02-3.95(\mathrm{~m}, 4 \mathrm{H})$, $3.83(\mathrm{~s}, 3 \mathrm{H}), 3.09-3.03(\mathrm{~m}, 2 \mathrm{H}), 2.70(\mathrm{t}, J=7.1 \mathrm{~Hz}, 2 \mathrm{H}), 2.12(\mathrm{tt}, J=7.2,1.1 \mathrm{~Hz}, 2 \mathrm{H}) ;{ }^{3} \mathrm{C}\left\{{ }^{1} \mathrm{H}\right\}$ $\operatorname{NMR}\left(125 \mathrm{MHz}, \mathrm{CDCl}_{3}\right)$ 199.2, 160.2, 138.0, 132.4, 130.4, 127.7, 114.0, 107.3, 64.7, 55.3, 38.5, 36.5, 31.4; IR (neat, $\mathrm{cm}^{-1}$ ) 2997, 2893, 2835, 1678, 1582, 1510, 1454, 1418, 1373, 1304, 1260, $1171,1115,1076,1057,1030,1009 ; \mathrm{HRMS}\left(\mathrm{ESI}^{+}\right) \mathrm{m} / \mathrm{z}$ calc for $\mathrm{C}_{16} \mathrm{H}_{19} \mathrm{O}_{4}[\mathrm{M}+\mathrm{H}]^{+}: 275.1278$; found 275.1274.<smiles>O=C1CCC2(C/C1=C\c1ccc(Cl)cc1)OCCO2</smiles>

(E)-7-(4-chlorobenzylidene)-1,4-dioxaspiro[4.5]decan-8-one (K3)

Pale yellow solid (902 mg, 65\% yield); m.p. = $101-102{ }^{\circ} \mathrm{C}\left(\mathrm{Hex}_{/} \mathrm{Et}_{2} \mathrm{O}\right) ;{ }^{1} \mathrm{H} \mathrm{NMR}\left(500 \mathrm{MHz}, \mathrm{CDCl}_{3}\right)$ $7.50(\mathrm{t}, J=2.3 \mathrm{~Hz}, 1 \mathrm{H}), 7.39-7.33(\mathrm{~m}, 2 \mathrm{H}) ; 7.33-7.28(\mathrm{~m}, 2 \mathrm{H}), 4.02-3.91(\mathrm{~m}, 4 \mathrm{H}) ; 3.04-2.96$ $(\mathrm{m}, 2 \mathrm{H}), 2.70$ (t, $J=7.1 \mathrm{~Hz}, 2 \mathrm{H}), 2.12$ (t, $J=7.1 \mathrm{~Hz}, 2 \mathrm{H}) ;{ }^{3} \mathrm{C}\left\{{ }^{1} \mathrm{H}\right\} \mathrm{NMR}\left(125 \mathrm{MHz}, \mathrm{CDCl}_{3}\right)$ 199.3, 136.4, 134.8, 133.5, 133.1, 131.5, 128.7, 107.2, 64.7, 38.3, 36.5, 31.4; IR (neat, cm ${ }^{-1}$ ) 2956, 2887, $\left.1682,1598,1489,1425,1406,1365,1312,1274,1176,1115,1072,1055,1012 ; \mathrm{HRMS}^{(E S I}{ }^{+}\right)$ $\mathrm{m} / \mathrm{z}$ calcd for $\mathrm{C}_{15} \mathrm{H}_{16} \mathrm{ClO}_{3}[\mathrm{M}+\mathrm{H}]^{+}: 279.0783$; found 279.0784 .<smiles>O=C1CCC2(CC1=Cc1c(F)cccc1Cl)OCCO2</smiles>

(E)-7-(2-chloro-6-fluorobenzylidene)-1,4-dioxaspiro[4.5]decan-8-one (K4)

Pale yellow solid $\left(3.50 \mathrm{~g}, 79 \%\right.$ yield); m.p. $=105-107^{\circ} \mathrm{C}\left(\mathrm{Hex} / \mathrm{Et}_{2} \mathrm{O}\right) ;{ }^{1} \mathrm{H} \mathrm{NMR}\left(500 \mathrm{MHz}, \mathrm{CDCl}_{3}\right)$ $7.31-7.28(\mathrm{~m}, 1 \mathrm{H}), 7.28-7.21(\mathrm{~m}, 2 \mathrm{H}), 7.02$ (ddd, $J=9.3,7.9,1.4 \mathrm{~Hz}, 1 \mathrm{H}), 3.98-3.87(\mathrm{~m}, 4 \mathrm{H})$, $2.72(\mathrm{t}, J=7.1 \mathrm{~Hz}, 2 \mathrm{H}), 2.68-2.65(\mathrm{~m}, 2 \mathrm{H}), 2.11(\mathrm{t}, J=7.1 \mathrm{~Hz}, 2 \mathrm{H}) ;{ }^{13} \mathrm{C}\left\{{ }^{1} \mathrm{H}\right\} \mathrm{NMR}(125 \mathrm{MHz}$, $\left.\mathrm{CDCl}_{3}\right)$ 198.7, $159.6\left(\mathrm{~d}, J_{C F}=251.4 \mathrm{~Hz}\right), 137.7\left(\mathrm{~d}, J_{C F}=1.3 \mathrm{~Hz}\right), 135.3\left(\mathrm{~d}, J_{C F}=5.2 \mathrm{~Hz}\right), 130.2(\mathrm{~d}$, $\left.J_{C F}=9.7 \mathrm{~Hz}\right), 128.5,125.3\left(\mathrm{~d}, J_{C F}=3.3 \mathrm{~Hz}\right), 122.6\left(\mathrm{~d}, J_{C F}=18.9 \mathrm{~Hz}\right), 114.3\left(\mathrm{~d}, J_{C F}=22.8 \mathrm{~Hz}\right)$, $107.2\left(\mathrm{~d}, J_{C F}=1.4 \mathrm{~Hz}\right), 64.0,38.1(\mathrm{~d}, J=4.9 \mathrm{~Hz}), 36.8,31.7 ;{ }^{19} \mathrm{~F} \mathrm{NMR}\left(470 \mathrm{MHz}, \mathrm{CDCl}_{3}\right)-106.89$ -106.9 (m); IR (neat, $\mathrm{cm}^{-1}$ ) 2957, 2887, 1694, 1620, 1568, 1445, 1420, 1364, 1346, 1312, 1271, $1246,1207,1184,1117,1076,1057,1038,1001 ; \mathrm{HRMS}\left(\mathrm{ESI}{ }^{+}\right) \mathrm{m} / z$ calcd for $\mathrm{C}_{15} \mathrm{H}_{15} \mathrm{ClFO}_{3}[\mathrm{M}+\mathrm{H}]^{+}$ 297.0688; found 297.0682. 
<smiles>O=C1CCC2(CC1=Cc1ccc3ccccc3c1)OCCO2</smiles>

(E)-7-(naphthalen-2-ylmethylene)-1,4-dioxaspiro[4.5]decan-8-one (K5)

Pale yellow solid $(771 \mathrm{mg}, 52 \%$ yield $)$; m.p. $=116-118{ }^{\circ} \mathrm{C}\left(\mathrm{Hex}_{\mathrm{Et}} \mathrm{O}\right) ;{ }^{1} \mathrm{H} \mathrm{NMR}\left(500 \mathrm{MHz}, \mathrm{CDCl}_{3}\right)$ $7.90-7.83(\mathrm{~m}, 4 \mathrm{H}), 7.77-7.74(\mathrm{~m}, 1 \mathrm{H}), 7.56-7.49(\mathrm{~m}, 3 \mathrm{H}), 4.02-3.93(\mathrm{~m}, 4 \mathrm{H}), 3.20-3.17$ $(\mathrm{m}, 2 \mathrm{H}), 2.77(\mathrm{t}, J=7.1 \mathrm{~Hz}, 2 \mathrm{H}), 2.17(\mathrm{t}, J=7.1 \mathrm{~Hz}, 2 \mathrm{H}) ;{ }^{13} \mathrm{C}\left\{{ }^{1} \mathrm{H}\right\} \mathrm{NMR}\left(125 \mathrm{MHz}, \mathrm{CDCl}_{3}\right)$ 199.5, 138.1, 133.2, 133.0, 132.9, 132.6, 130.3, 128.5, 128.1, 127.7, 127.6, 127.1, 126.5, 107.4, 64.7, 38.5, 36.7, 31.6; IR (neat, $\mathrm{cm}^{-1}$ ) 3053, 2955, 2886, 1682, 1587, 1503, 1423, 1358, 1314, 1275, 1173, 1117, 1055, 1003; HRMS (ESI $) \mathrm{m} / z$ calcd for $\mathrm{C}_{19} \mathrm{H}_{19} \mathrm{O}_{3}[\mathrm{M}+\mathrm{H}]^{+}$295.1329; found 295.1331.<smiles>O=C1CCOC2(CC1)C/C(=C\c1c3ccccc3cc3ccccc13)CO2</smiles>

(E)-7-(anthracen-9-ylmethylene)-1,4-dioxaspiro[4.5]decan-8-one (K6)

Yellow solid (1.05 g, 61\% yield); m.p. = $162-163{ }^{\circ} \mathrm{C} ;{ }^{1} \mathrm{H}$ NMR $\left(300 \mathrm{MHz}, \mathrm{CDCl}_{3}\right) 8.47(\mathrm{~s}, 1 \mathrm{H})$, $8.27-8.23(\mathrm{~m}, 1 \mathrm{H}), 8.09-7.96(\mathrm{~m}, 4 \mathrm{H}), 7.57-7.43(\mathrm{~m}, 4 \mathrm{H}), 3.90-3.80(\mathrm{~m}, 2 \mathrm{H}), 3.68-3.57$ $(\mathrm{m}, 2 \mathrm{H}), 2.89(\mathrm{t}, J=7.2 \mathrm{~Hz}, 2 \mathrm{H}), 2.47-2.39(\mathrm{~m}, 2 \mathrm{H}), 2.17(\mathrm{t}, J=7.2 \mathrm{~Hz}, 2 \mathrm{H}) ;{ }^{13} \mathrm{C}\left\{{ }^{1} \mathrm{H}\right\} \mathrm{NMR}(75$ $\left.\mathrm{MHz}, \mathrm{CDCl}_{3}\right)$ 199.0, 138.2, 135.8, 131.2, 129.6, 128.9, 128.8, 127.4, 126.0, 125.7, 125.4, 107.3, 64.5, 37.5, 37.2, 32.3; IR (neat, $\mathrm{cm}^{-1}$ ) 3049, 2955, 2886, 1694, 1624, 1518, 1420, 1356, 1310, 1261, 1180, 1111, 1057, 1007; HRMS $\left(E S I^{+}\right) \mathrm{m} / z$ calcd for $\mathrm{C}_{23} \mathrm{H}_{21} \mathrm{O}_{3}[\mathrm{M}+\mathrm{H}]^{+}:$345.1485; found 345.1473.<smiles>CC(C)(C)C=C1CC2(CCC1=O)OCCO2</smiles>

(E)-7-(2,2-dimethylpropylidene)-1,4-dioxaspiro[4.5]decan-8-one (K7)

Colorless oil (100 mg, 29\% yield); ${ }^{1} \mathrm{H}$ NMR $\left(300 \mathrm{MHz}, \mathrm{CDCl}_{3}\right) 6.68(\mathrm{t}, \mathrm{J}=2.1 \mathrm{~Hz}, 1 \mathrm{H}), 4.05-4.00$ (m, 4H), 2.91 (dt, $J=2.1,1.0 \mathrm{~Hz}, 2 \mathrm{H}), 2.60(\mathrm{t}, J=7.1 \mathrm{~Hz}, 2 \mathrm{H}), 2.05$ (tt, $J=7.1,1.0,2 \mathrm{H}), 1.17$ (s, $9 \mathrm{H}) ;{ }^{13} \mathrm{C}\left\{{ }^{1} \mathrm{H}\right\} \mathrm{NMR}\left(75 \mathrm{MHz}, \mathrm{CDCl}_{3}\right)$ 200.2, 150.6, 131.8, 107.5, 64.6, 37.0, 36.5, 33.0, 31.5, 30.04; IR (neat, $\mathrm{cm}^{-1}$ ) 2957, 2872, 1690, 1609, 1481, 1458, 1364, 1263, 1209, 1117, 1084, 1057, 1038, 1005; HRMS $\left(\mathrm{ESI}^{+}\right) \mathrm{m} / z$ calcd for $\mathrm{C}_{13} \mathrm{H}_{21} \mathrm{O}_{3}[\mathrm{M}+\mathrm{H}]^{+}:$: 225.1485; found 225.1482. 


\section{Synthesis of Tertiary Alcohols}<smiles>O=C1CCC2(CC1)OCCO2</smiles>

$$
\underset{\text { THF, }-78^{\circ} \mathrm{C}}{\stackrel{\operatorname{Ar}-\mathrm{X}(1.5 \text { equiv. })}{n \text {-BuLi }(1.5 \text { equiv. })}}
$$<smiles>OC1([Al])CCC2(CC1)OCCO2</smiles><smiles>[R]C=C1CC2(CCC1=O)OCCO2</smiles>

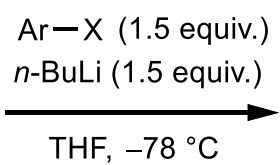<smiles>[R]C=C1CC2(CCC1(O)[Al])OCCO2</smiles>

Representative procedure:

To a stirred solution of 4-bromoanisole $(0.19 \mathrm{~mL}, 1.5 \mathrm{mmol}, 1.5$ equiv. $)$ in THF $(10 \mathrm{~mL})$ at $-78^{\circ} \mathrm{C}$ was added a solution of $n$-BuLi (3.8 mL of a $2.5 \mathrm{M}$ solution in hexanes, $1.5 \mathrm{mmol}$, 1.5 equiv.) dropwise. The reaction mixture was stirred at this temperature for $40 \mathrm{~min}$ before a THF solution $(5 \mathrm{~mL})$ of 1,4-cyclohexanedione monoethylene ketal (156 mg, $1.0 \mathrm{mmol}, 1.0$ equiv.) was added dropwise at $-78^{\circ} \mathrm{C}$ over a period of $5 \mathrm{~min}$. After $20 \mathrm{~min}$, the reaction mixture was removed from the cooling bath and allowed to warm for 10 min before quenching with sat. aq. $\mathrm{NH}_{4} \mathrm{Cl}(10 \mathrm{~mL})$. Water $(5 \mathrm{~mL})$ was added to dissolve any precipitated salts. The aqueous phase was extracted with diethyl ether $(x 3)$. The combined organic extracts were washed with water and brine and dried over anhydrous sodium sulfate. After filtration, the solvent was removed in vacuo and the crude oil was purified by flash column chromatography on silica gel using a gradient of ethyl acetate in hexanes $(5,10,15,20 \%)$ as eluent to afford 2 as a white solid (200 mg, 76\%).

Note: Some of the alcohol products, particularly those prepared from $\alpha, \beta$-unsaturated ketones, have identical $R_{f}$ values with the ketone they were prepared from in most eluents examined. In these cases, the alcohol was purified by taking advantage of a very slight mobility difference in hexane/DCM (for reference, using the correct ratio of hexane/DCM, separation could be achieved on TLC after six consecutive elutions). After sufficient elution using hexane/DCM, the solvent system was changed to hexane/ethyl acetate to elute the two compounds off the column. Consequently, some of the alcohols isolated in this way have $\leq 5 \%$ contamination by the corresponding ketone.<smiles>COc1ccc(C2(O)CCC3(CC2)OCCO3)cc1</smiles>

\section{8-(4-methoxyphenyl)-1,4-dioxaspiro[4.5]decan-8-ol (2)}

White solid $\left(1.00 \mathrm{~g}, 76 \%\right.$ yield); m.p. $=121-123{ }^{\circ} \mathrm{C} ;{ }^{1} \mathrm{H} \mathrm{NMR}\left(500 \mathrm{MHz}, \mathrm{CDCl}_{3}\right) 7.54-7.36(\mathrm{~m}$, $2 \mathrm{H}), 6.97-6.77(\mathrm{~m}, 2 \mathrm{H}), 3.88-3.69(\mathrm{~m}, 4 \mathrm{H}), 3.80(\mathrm{~s}, 3 \mathrm{H}), 2.24-1.98(\mathrm{~m}, 4 \mathrm{H}), 1.82-1.75(\mathrm{~m}$, 2H) $1.71-1.65(\mathrm{~m}, 2 \mathrm{H}), 1.50(\mathrm{~s}, 1 \mathrm{H}) ;{ }^{13} \mathrm{C}\left\{{ }^{1} \mathrm{H}\right\} \mathrm{NMR}\left(125 \mathrm{MHz}, \mathrm{CDCl}_{3}\right)$ 158.5, 140.6, 125.7, 113.5, 108.5, 72.0, 64.3, 64.2, 55.3, 36.6, 30.8; IR (neat, $\mathrm{cm}^{-1}$ ) 3430, 3054, 2987, 2936, 2887, 1512, 
1423, 1265, 1180, 1101, 1037; HRMS $\left(E S I^{+}\right) \mathrm{m} / z$ calcd for $\mathrm{C}_{15} \mathrm{H}_{21} \mathrm{O}_{4}[\mathrm{M}+\mathrm{H}]^{+}:$265.1434; found 265.1434.

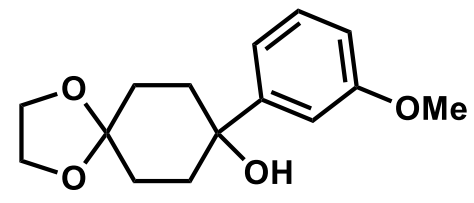

8-(3-methoxyphenyl)-1,4-dioxaspiro[4.5]decan-8-ol (A1)

Colorless oil $\left(1.20 \mathrm{~g}, 92 \%\right.$ yield); ${ }^{1} \mathrm{H}$ NMR $\left(500 \mathrm{MHz}, \mathrm{CDCl}_{3}\right) 7.29-7.23(\mathrm{~m}, 1 \mathrm{H}), 7.12-7.06(\mathrm{~m}$, $2 \mathrm{H}), 6.83-6.76(\mathrm{~m}, 1 \mathrm{H}), 4.03-3.94(\mathrm{~m}, 4 \mathrm{H}), 3.82(\mathrm{~s}, 3 \mathrm{H}), 2.23-2.06(\mathrm{~m}, 4 \mathrm{H}), 1.86-1.77(\mathrm{~m}$, $2 \mathrm{H}), 1.73-1.66(\mathrm{~m}, 2 \mathrm{H}), 1.52(\mathrm{~s}, 1 \mathrm{H}) ;{ }^{13} \mathrm{C}\left\{{ }^{1} \mathrm{H}\right\} \mathrm{NMR}\left(125 \mathrm{MHz}, \mathrm{CDCl}_{3}\right)$ 159.6, 150.3, 129.3, 116.9, 112.1, 110.6, 108.4, 72.5, 64.3, 64.2, 55.2, 36.6, 30.7; IR (neat, $\mathrm{cm}^{-1}$ ) 3460, 2957, 2930, 2856, 1601, 1584, 1485, 1466, 1431, 1368, 1318, 1288, 1256, 1198, 1177, 1138, 1099, 1034; HRMS $(\mathrm{ESI})^{+} \mathrm{m} / \mathrm{z}$ calcd for $\mathrm{C}_{15} \mathrm{H}_{19} \mathrm{O}_{3}(\mathrm{M}+\mathrm{H})^{+}-\left[\mathrm{H}_{2} \mathrm{O}\right]: 247.1329$; found 247.1323.

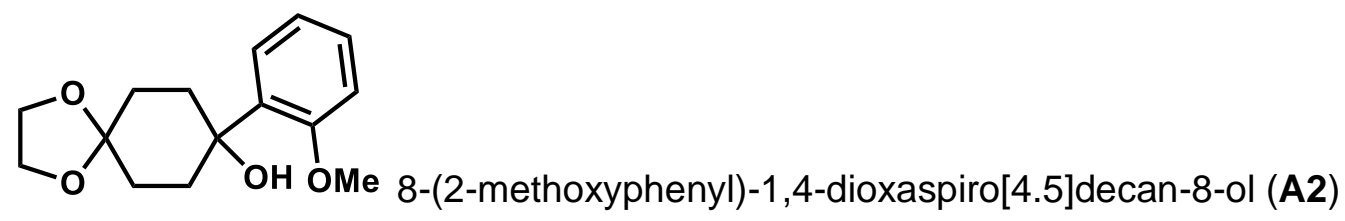

White solid $\left(1.00 \mathrm{~g}, 76 \%\right.$ yield); m.p. $=79-81^{\circ} \mathrm{C} ;{ }^{1} \mathrm{H}$ NMR $\left(500 \mathrm{MHz}, \mathrm{CDCl}_{3}\right) 7.30$ (dd, $J=7.7$, $1.7 \mathrm{~Hz}, 1 \mathrm{H}), 7.23(\mathrm{td}, J=7.7,1.7 \mathrm{~Hz}, 1 \mathrm{H}), 6.97-6.90(\mathrm{~m}, 2 \mathrm{H}), 4.12(\mathrm{~s}, 1 \mathrm{H}), 4.01-3.92(\mathrm{~m}, 4 \mathrm{H})$, $3.90(\mathrm{~s}, 3 \mathrm{H}), 2.19(\mathrm{td}, J=13.0,4.4 \mathrm{~Hz}, 2 \mathrm{H}), 2.14-2.01(\mathrm{~m}, 4 \mathrm{H}), 1.70-1.60(\mathrm{~m}, 2 \mathrm{H}) ;{ }^{13} \mathrm{C}\left\{{ }^{1} \mathrm{H}\right\}$ $\operatorname{NMR}\left(125 \mathrm{MHz}, \mathrm{CDCl}_{3}\right)$ 157.2, 135.1, 128.2, 125.7, 121.1, 111.3, 108.8, 72.1, 64.3, 64.2, 55.3, 34.2, 30.5; IR (neat, $\mathrm{cm}^{-1}$ ) 3519, 2958, 2930, 2877, 1600, 1583, 1491, 1489, 1439, 1400, 1361, 1252, 1232, 1106, 1035; HRMS (ESI) ${ }^{+} \mathrm{m} / \mathrm{z}$ calcd for $\mathrm{C}_{15} \mathrm{H}_{20} \mathrm{O}_{4} \mathrm{Na}[\mathrm{M}+\mathrm{Na}]^{+}:$287.1254; found 287.1248.

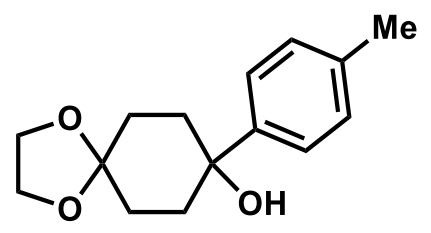

8-(p-tolyl)-1,4-dioxaspiro[4.5]decan-8-ol (A3)

White solid (1.15 g, 93\% yield); m.p. $=111-112{ }^{\circ} \mathrm{C} ;{ }^{1} \mathrm{H}$ NMR $\left(500 \mathrm{MHz}, \mathrm{CDCl}_{3}\right) 7.41$ (d, J=8.2, $2 \mathrm{H}), 7.16(\mathrm{~d}, J=8.0 \mathrm{~Hz}, 2 \mathrm{H}), 4.03-3.95(\mathrm{~m}, 4 \mathrm{H}), 2.34(\mathrm{~s}, 3 \mathrm{H}), 2.22-2.05(\mathrm{~m}, 4 \mathrm{H}), 1.86-1.76$ $(\mathrm{m}, 2 \mathrm{H}), 1.73-1.65(\mathrm{~m}, 2 \mathrm{H}), 1.47(\mathrm{~s}, 1 \mathrm{H}) ;{ }^{3} \mathrm{C}\left\{{ }^{1} \mathrm{H}\right\} \mathrm{NMR}\left(125 \mathrm{MHz}, \mathrm{CDCl}_{3}\right)$ 145.5, 136.4, 128.8, 124.4, 108.4, 72.1, 64.2, 64.1, 36.5, 30.7, 20.9; IR (neat, $\mathrm{cm}^{-1}$ ) 3464, 3022, 2961, 2924, 2893, $1514,1435,1371,1337,1315,1281,1254,1225,1209,1184,1140,1101,1032$; HRMS (ESI) ${ }^{+}$ $\mathrm{m} / \mathrm{z}$ calcd for $\mathrm{C}_{15} \mathrm{H}_{19} \mathrm{O}_{2}(\mathrm{M}+\mathrm{H})^{+}-\left[\mathrm{H}_{2} \mathrm{O}\right]: 231.1380$; found 231.1374.<smiles>OC1(c2ccc(Br)cc2)CCC2(CC1)OCCO2</smiles>

\section{8-(4-bromophenyl)-1,4-dioxaspiro[4.5]decan-8-ol (A4)}

White solid (614 mg, 65\% yield); m.p. = $122-125{ }^{\circ} \mathrm{C} ;{ }^{1} \mathrm{H}$ NMR $\left(300 \mathrm{MHz}, \mathrm{CDCl}_{3}\right) 7.49-7.34$ (m, $4 \mathrm{H}), 4.00-3.92(\mathrm{~m}, 4 \mathrm{H}), 2.21-1.93(\mathrm{~m}, 4 \mathrm{H}), 1.89-1.59(\mathrm{~m}, 5 \mathrm{H}) ;{ }^{13} \mathrm{C}\left\{{ }^{1} \mathrm{H}\right\} \mathrm{NMR}\left(75 \mathrm{MHz} \mathrm{CDCl}_{3}\right)$ 147.6, 131.3, 126.5, 120.8, 108.3, 72.3, 64.4, 64.3, 36.5, 30.7; IR (neat, $\mathrm{cm}^{-1}$ ) 3583, 3054, 2987, 
2887, 2686, 1423, 1266, 1100, 1034, 1009; HRMS (ESI) ${ }^{+} \mathrm{m} / \mathrm{z}$ calcd for $\mathrm{C}_{14} \mathrm{H}_{16} \mathrm{BrO}_{2}(\mathrm{M}+\mathrm{H})^{+}-\left[\mathrm{H}_{2} \mathrm{O}\right]$ : 295.0328; found 295.0325.

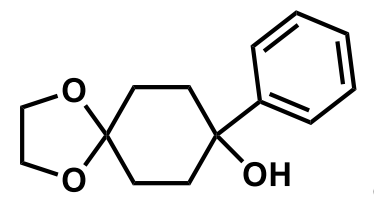

8-phenyl-1,4-dioxaspiro[4.5]decan-8-ol (A5)

White solid (415 mg, 60\% yield); ${ }^{1} \mathrm{H}$ NMR (300 MHz, $\left.\mathrm{CDCl}_{3}\right) 7.57-7.48(\mathrm{~m}, 2 \mathrm{H}), 7.34(\mathrm{t}, \mathrm{J}=7.5$ $\mathrm{Hz}, 2 \mathrm{H}), 7.29-7.20(\mathrm{~m}, 1 \mathrm{H}), 3.95(\mathrm{~s}, 4 \mathrm{H}), 2.31(\mathrm{~s}, 1 \mathrm{H}), 2.25-2.00(\mathrm{~m}, 4 \mathrm{H}), 1.90-1.60(\mathrm{~m}, 4 \mathrm{H})$; ${ }^{13} \mathrm{C}\left\{{ }^{1} \mathrm{H}\right\} \operatorname{NMR}\left(75 \mathrm{MHz}, \mathrm{CDCl}_{3}\right) \delta 148.6,128.2,126.9,124.6,108.6,72.3,64.3,64.2,36.5,30.7$; IR (neat, $\mathrm{cm}^{-1}$ ) 3453, 3057, 3024, 2930, 2884, 1601, 1493, 1445, 1369, 1337, 1252, 1217, 1144, 1099, 1034; HRMS $\left(E S{ }^{+}\right) \mathrm{m} / z$ calcd for $\mathrm{C}_{14} \mathrm{H}_{17} \mathrm{O}_{2}[\mathrm{M}+\mathrm{H}]^{+}-\left(\mathrm{H}_{2} \mathrm{O}\right): 217.1223$; found 217.1216.<smiles>OC1(c2cccc3ccccc23)CCC2(CC1)OCCO2</smiles>

8-(naphthalen-1-yl)-1,4-dioxaspiro[4.5]decan-8-ol (A6)

Off-white solid (1.24g, 87\% yield); m.p. = $161-162{ }^{\circ} \mathrm{C} ;{ }^{1} \mathrm{H} \mathrm{NMR}\left(500 \mathrm{MHz}, \mathrm{CDCl}_{3}\right) 8.87(\mathrm{~d}, \mathrm{~J}=$ $8.8 \mathrm{~Hz}, 1 \mathrm{H}), 7.86(\mathrm{dd}, J=8.0,1.7 \mathrm{~Hz}, 1 \mathrm{H}), 7.77(\mathrm{~d}, J=8.1 \mathrm{~Hz}, 1 \mathrm{H}), 7.64$ (dd, $J=7.4,1.3 \mathrm{~Hz}, 1 \mathrm{H})$, 7.51 (ddd, $J=8.7,6.8,1.7 \mathrm{~Hz}, 1 \mathrm{H}$ ), 7.47 (ddd, $J=8.0,6.8,1.3 \mathrm{~Hz}, 1 \mathrm{H}$ ), 7.41 (dd, $J=8.1,7.4 \mathrm{~Hz}$, $1 \mathrm{H}), 4.06-3.98(\mathrm{~m}, 4 \mathrm{H}), 2.50-2.40(\mathrm{~m}, 2 \mathrm{H}), 2.32-2.20(\mathrm{~m}, 4 \mathrm{H}), 1.80-1.73(\mathrm{~m}, 2 \mathrm{H}), 1.71(\mathrm{~s}$, $1 \mathrm{H}) ;{ }^{13} \mathrm{C}\left\{{ }^{1} \mathrm{H}\right\}$ NMR $\left(125 \mathrm{MHz}, \mathrm{CDCl}_{3}\right) 143.0134 .9,131.1,129.2,128.8,127.1,125.3,125.2,124.9$, 122.7, 108.7, 73.7, 64.5, 64.3 35.8, 30.7; IR (neat, $\mathrm{cm}^{-1}$ ) 3456, 3048, 2957, 2930, 2884, 1597, 1508, 1443, 1371, 1248, 1101, 1032; HRMS $(E S I)^{+} \mathrm{m} / \mathrm{z}$ calcd for $\mathrm{C}_{18} \mathrm{H}_{19} \mathrm{O}_{2}(\mathrm{M}+\mathrm{H})^{+}-\left[\mathrm{H}_{2} \mathrm{O}\right]$ : 267.1380; found 267.1379.<smiles>OC1(c2ccc3ccccc3c2)CCC2(CC1)OCCO2</smiles>

8-(naphthalen-2-yl)-1,4-dioxaspiro[4.5]decan-8-ol (A7)

White solid (1.13 g, 79\% yield); m.p. = $105-108{ }^{\circ} \mathrm{C} ;{ }^{1} \mathrm{H}$ NMR $\left(500 \mathrm{MHz}, \mathrm{CDCl}_{3}\right) 7.98(\mathrm{~d}, \mathrm{~J}=1.9$ $\mathrm{Hz}, 1 \mathrm{H}), 7.87-7.78(\mathrm{~m}, 3 \mathrm{H}), 7.65(\mathrm{dd}, J=8.7,2.0 \mathrm{~Hz}, 1 \mathrm{H}), 7.52-7.42(\mathrm{~m}, 2 \mathrm{H}), 4.06-3.97(\mathrm{~m}$, 4H), 2.31 (td, $J=13.7,4.1 \mathrm{~Hz}, 2 \mathrm{H}), 2.16(\mathrm{td}, J=13.3,4.1 \mathrm{~Hz}, 2 \mathrm{H}), 1.93-1.85(\mathrm{~m}, 2 \mathrm{H}), 1.78-$ $1.71(\mathrm{~m}, 2 \mathrm{H}), 1.68(\mathrm{~s}, 1 \mathrm{H}) ;{ }^{13} \mathrm{C}\left\{{ }^{1} \mathrm{H}\right\}$ NMR $\left(125 \mathrm{MHz}, \mathrm{CDCl}_{3}\right)$ 145.8, 133.2, 132.4, 128.1, 127.9, 127.4, 126.1, 125.8, 123.4, 122.8, 108.4, 72.6, 64.4, 64.3, 36.5, 30.8; IR (neat, cm ${ }^{-1}$ ) 3447, 3055, 2959, 2930, 2884, 1437, 1369, 1256, 1186, 1138, 1099, 1034; HRMS (ESI) ${ }^{+} \mathrm{m} / \mathrm{z}$ calcd for $\mathrm{C}_{18} \mathrm{H}_{19} \mathrm{O}_{2}(\mathrm{M}+\mathrm{H})^{+}-\left[\mathrm{H}_{2} \mathrm{O}\right]: 267.1380$; found 267.1384 .<smiles>OC1(c2ccc(-c3ccccc3)cc2)CCC2(CC1)OCCO2</smiles>

8-([1,1'-biphenyl]-4-yl)-1,4-dioxaspiro[4.5]decan-8-ol (A8)

White solid (569 mg, 61\% yield); m.p. = $131-133{ }^{\circ} \mathrm{C} ;{ }^{1} \mathrm{H} \mathrm{NMR}\left(500 \mathrm{MHz}, \mathrm{CDCl}_{3}\right) 7.36-7.56(\mathrm{~m}$, $6 \mathrm{H}), 7.44(\mathrm{t}, J=7.7 \mathrm{~Hz}, 2 \mathrm{H}), 7.34(\mathrm{t}, J=7.4 \mathrm{~Hz}, 1 \mathrm{H}), 4.04-3.97(\mathrm{~m}, 4 \mathrm{H}), 2.23(\mathrm{td}, J=13.5,3.9$ $\mathrm{Hz}, 2 \mathrm{H}), 2.13(\mathrm{td}, J=13.2,3.8 \mathrm{~Hz}, 2 \mathrm{H}), 1.90-1.82(\mathrm{~m}, 2 \mathrm{H}), 1.76-1.68(\mathrm{~m}, 2 \mathrm{H}) ;{ }^{13} \mathrm{C}\left\{{ }^{1} \mathrm{H}\right\} \mathrm{NMR}$ 
$\left(125 \mathrm{MHz}, \mathrm{CDCl}_{3}\right)$ 147.5, 140.7, 139.8, 128.7, 127.2, 127.1, 127.0, 125.0, 108.4, 72.4, 64.4, 64.2, 36.6, 30.7; IR (neat, $\mathrm{cm}^{-1}$ ) 3473, 3054, 2986, 2962, 2933, 2887, 1487, 1423, 1265, 1101, 1034; HRMS $(\mathrm{ESI})^{+} \mathrm{m} / \mathrm{z}$ calcd for $\mathrm{C}_{20} \mathrm{H}_{22} \mathrm{O}_{3} \mathrm{Na}[\mathrm{M}+\mathrm{Na}]^{+}: 333.1461$; found 333.1456 .<smiles>OC1(c2cc(C(F)(F)F)cc(C(F)(F)F)c2)CCC2(CC1)OCCO2</smiles>

8-(3,5-bis(trifluoromethyl)phenyl)-1,4-dioxaspiro[4.5]decan-8-ol (A9)

White solid ( $1.56 \mathrm{~g}, 86 \%$ yield); m.p. $=116-117^{\circ} \mathrm{C} ;{ }^{1} \mathrm{H}$ NMR $\left(500 \mathrm{MHz}, \mathrm{CDCl}_{3}\right) 7.98(\mathrm{~s}, 2 \mathrm{H})$, $7.77(\mathrm{~s}, 1 \mathrm{H}), 4.04-3.98(\mathrm{~m}, 4 \mathrm{H}), 2.19(\mathrm{td}, J=13.6,3.8 \mathrm{~Hz}, 2 \mathrm{H}), 2.09$ (td, $J=13.2,3.6 \mathrm{~Hz}), 2 \mathrm{H}$ ), $1.91(\mathrm{brd} \mathrm{s}, 1 \mathrm{H}), 1.85-1.77(\mathrm{~m}, 2 \mathrm{H}), 1.77-1.68(\mathrm{~m}, 2 \mathrm{H}) ;{ }^{13} \mathrm{C}\left\{{ }^{1} \mathrm{H}\right\} \mathrm{NMR}\left(125 \mathrm{MHz}, \mathrm{CDCl}_{3}\right)$ 151.3, $131.5\left(\mathrm{q}, J_{C F}=33.0 \mathrm{~Hz}\right), 125.2-125.0(\mathrm{~m}), 123.4\left(\mathrm{q}, J_{C F}=272.8 \mathrm{~Hz}\right), 121.1-120.7(\mathrm{~m}), 107.8$, 72.5, 64.5, 64.3, 36.6, 30.5; ${ }^{19} \mathrm{~F} \mathrm{NMR}\left(470 \mathrm{MHz}, \mathrm{CDCl}_{3}\right)$-63.6; IR (neat, $\left.\mathrm{cm}^{-1}\right) 3460,2961,2932$, $2891,2855,1375,1279,1177,1130,1107,1036$; HRMS $\left(\mathrm{ESI}^{+}\right) \mathrm{m} / \mathrm{z}$ calcd for $\mathrm{C}_{16} \mathrm{H}_{15} \mathrm{~F}_{6} \mathrm{O}_{2}(\mathrm{M}+\mathrm{H})^{+}-$ $\left[\mathrm{H}_{2} \mathrm{O}\right] 353.0971$; found 353.0971 .

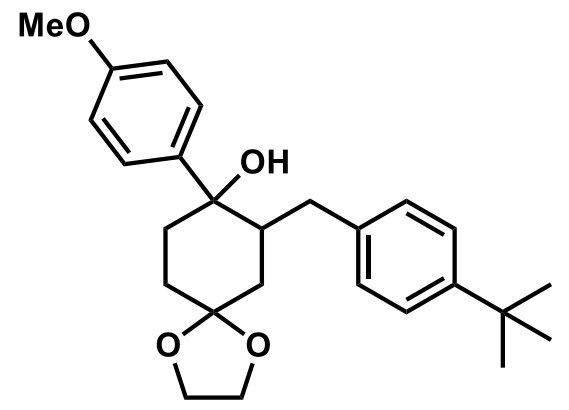

7-(4-(tert-butyl)benzyl)-8-(4-methoxyphenyl)-1,4-dioxaspiro[4.5]decan-8-ol (A10)

White solid (249 mg, 54\% yield, 70\% BRSM); ${ }^{1} \mathrm{H} \mathrm{NMR}^{*}\left(300 \mathrm{MHz}, \mathrm{CDCl}_{3}\right) 7.53-7.42(\mathrm{~m}, 2 \mathrm{H})$, $7.25-7.17(\mathrm{~m}, 2 \mathrm{H}), 6.98-6.84(\mathrm{~m}, 4 \mathrm{H}), 3.98-3.76(\mathrm{~m}, 7 \mathrm{H}), 2.50-2.28(\mathrm{~m}, 2 \mathrm{H}), 2.25-1.93$ (m, 3H), $1.86-1.57(\mathrm{~m}, 4 \mathrm{H}), 1.27(\mathrm{~s}, 9 \mathrm{H}) ;{ }^{13} \mathrm{C}\left\{{ }^{1} \mathrm{H}\right\} \mathrm{NMR}^{*}\left(75 \mathrm{MHz}, \mathrm{CDCl}_{3}\right)$ 158.3, 148.5, 139.5, 137.2, 128.6, 125.9, 125.1, 113.7, 108.8, 75.4, 64.23, 64.20, 55.3, 44.9, 39.1, 35.6, 35.5, 34.3, 31.4, 30.7; IR (neat, $\mathrm{cm}^{-1}$ ) 3487, 2989, 2932, 2874, 1611, 1510, 1464, 1443, 1364, 1300, 1250, 1215, 1179, 1140, 1049, 1038; HRMS $(E S I)^{+} \mathrm{m} / z$ calcd for $\mathrm{C}_{26} \mathrm{H}_{33} \mathrm{O}_{3}(\mathrm{M}+\mathrm{H})^{+}-\left[\mathrm{H}_{2} \mathrm{O}\right]: 393.2424$; found 393.2432.

*isolated as a mixture of two diasteromers; the major diasteromer's data is reported here 
<smiles></smiles>

(E)-7-benzylidene-8-(4-methoxyphenyl)-1,4-dioxaspiro[4.5]decan-8-ol (4a)

White foam (454 mg, 79\% yield); ${ }^{1} \mathrm{H}$ NMR (500 MHz, $\left.\mathrm{CDCl}_{3}\right) 7.51-7.44(\mathrm{~m}, 2 \mathrm{H}), 7.35-7.27$ (m, 4H), $7.22(\mathrm{tt}, J=6.5,1.7 \mathrm{~Hz}, 1 \mathrm{H}), 6.94-6.87(\mathrm{~m}, 2 \mathrm{H}), 6.67(\mathrm{~s}, 1 \mathrm{H}), 4.00-3.84(\mathrm{~m}, 4 \mathrm{H}), 3.82(\mathrm{~s}$, $3 \mathrm{H}$ ), 2.91 (ddd, $J=13.9,2.1,1.1 \mathrm{~Hz}, 1 \mathrm{H}$ ), 2.59 (ddd, $J=14.4,7.4,4.1 \mathrm{~Hz}, 1 \mathrm{H}), 2.36$ (dt, $J=13.9$, $1.4 \mathrm{~Hz}, 1 \mathrm{H}), 2.09-1.97(\mathrm{~m}, 2 \mathrm{H}), 1.81-1.69(\mathrm{~m}, 2 \mathrm{H}) ;{ }^{13} \mathrm{C}\left\{{ }^{1} \mathrm{H}\right\} \operatorname{NMR}\left(125 \mathrm{MHz}, \mathrm{CDCl}_{3}\right) 159.0$, $141.7,137.5,135.3,129.00,128.96,128.0,127.9,126.8,126.4,113.8,109.3,76.3,64.4,55.3$, 36.2, 36.1, 32.2; IR (neat, $\mathrm{cm}^{-1}$ ) 3440, 2955, 2933, 2885, 2837, 1609, 1571, 1494, 1463, 1444, $1305,1301,1251,1179,1105,1072,1036$; HRMS $\left(E S I^{+}\right) \mathrm{m} / z$ calcd for $\mathrm{C}_{22} \mathrm{H}_{23} \mathrm{O}_{3}[\mathrm{M}+\mathrm{H}]^{+}-\left(\mathrm{H}_{2} \mathrm{O}\right)$ : 335.1642; found 335.1643.

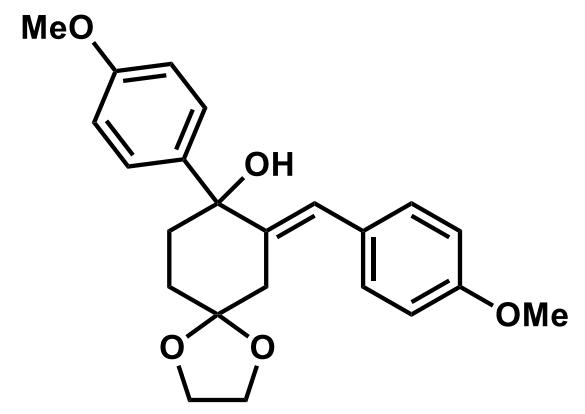

(E)-7-(4-methoxybenzylidene)-8-(4-methoxyphenyl)-1,4-dioxaspiro[4.5]decan-8-ol (4b)

White foam (263 mg, 72\% yield, 88\% BRSM); ${ }^{1} \mathrm{H}$ NMR (500 MHz, $\left.\mathrm{CDCl}_{3}\right) 7.46$ (d, $J=8.8 \mathrm{~Hz}$, $2 \mathrm{H}), 7.20(\mathrm{~d}, J=8.4 \mathrm{~Hz}, 2 \mathrm{H}), 6.89(\mathrm{~d}, J=8.8 \mathrm{~Hz}, 2 \mathrm{H}), 6.85(\mathrm{~d}, J=8.8 \mathrm{~Hz}, 2 \mathrm{H}), 6.57(\mathrm{~s}, 1 \mathrm{H}), 3.97$ $-3.85(\mathrm{~m}, 3 \mathrm{H}), 3.80(\mathrm{~s}, 2 \mathrm{H}), 3.79(\mathrm{~s}, 3 \mathrm{H}), 2.94-2.88(\mathrm{~m}, 1 \mathrm{H}), 2.60-2.52(\mathrm{~m}, 1 \mathrm{H}), 2.39(\mathrm{~d}, J=$ $13.9 \mathrm{~Hz}, 1 \mathrm{H}), 2.06-1.97(\mathrm{~m}, 2 \mathrm{H}), 1.78-1.67(\mathrm{~m}, 1 \mathrm{H}) ;{ }^{13} \mathrm{C}\left\{{ }^{1} \mathrm{H}\right\} \mathrm{NMR}\left(125 \mathrm{MHz}, \mathrm{CDCl}_{3}\right) 158.8$, 158.1, 140.6, 135.5, 130.1, 127.8, 126.3, 125.6, 113.6, 113.4, 109.3, 76.2, 64.3, 55.2, 55.1, 36.08, 36.07, 32.0; IR (neat, $\mathrm{cm}^{-1}$ ) 3443, 2934, 1605, 1512, 1258, 1169, 1088, 1030; HRMS (ESI $) \mathrm{m} / \mathrm{z}$ calcd for $\mathrm{C}_{23} \mathrm{H}_{25} \mathrm{O}_{4}(\mathrm{M}+\mathrm{H})^{+}-\left[\mathrm{H}_{2} \mathrm{O}\right]: 365.1747$; found 365.1750. 
<smiles>COc1ccc(C2(O)CCC3(OCCO)C/C(=C\c4ccc(Cl)cc4)C2(O)OCCO3)cc1</smiles>

(E)-7-(4-chlorobenzylidene)-8-(4-methoxyphenyl)-1,4-dioxaspiro[4.5]decan-8-ol (4c)

White foam (148 mg, 38\% yield, 84\% BRSM); ${ }^{1} \mathrm{H}$ NMR (500 MHz, $\left.\mathrm{CDCl}_{3}\right) 7.47-7.40(\mathrm{~m}, 2 \mathrm{H})$, $7.30-7.26(\mathrm{~m}, 2 \mathrm{H}), 7.23-7.18(\mathrm{~m}, 2 \mathrm{H}), 6.94-6.86(\mathrm{~m}, 2 \mathrm{H}), 6.59(\mathrm{~s}, 1 \mathrm{H}), 3.97-3.82(\mathrm{~m}, 4 \mathrm{H})$, $3.80(\mathrm{~s}, 3 \mathrm{H}), 2.84(\mathrm{dt}, J=13.6,1.5 \mathrm{~Hz}, 1 \mathrm{H}), 2.59-2.52(\mathrm{~m}, 1 \mathrm{H}), 2.31$ (dt, $J=13.9,1.3 \mathrm{~Hz}, 1 \mathrm{H}$ ), 2.15 (brs, $1 \mathrm{H}), 2.05-1.96(\mathrm{~m}, 2 \mathrm{H}), 1.77-1.69(\mathrm{~m}, 1 \mathrm{H}) ;{ }^{13} \mathrm{C}\left\{{ }^{1} \mathrm{H}\right\} \mathrm{NMR}\left(125 \mathrm{MHz}, \mathrm{CDCl}_{3}\right)$ 158.9, 142.5, 135.9, 135.1, 132.1, 130.3, 128.1, 127.8, 125.5, 113.8, 109.3, 76.2, 64.3, 55.2, 36.1, 35.9, 32.1; IR (neat, cm ${ }^{-1}$ ) 3447, 2955, 2932, 1609, 1489, 1510, 1464, 1354, 1300, 1250, 1103, 1072, 1036, 1015; HRMS (ESI $\left.{ }^{+}\right) \mathrm{m} / z$ calcd for $\mathrm{C}_{22} \mathrm{H}_{23} \mathrm{ClO}_{4} \mathrm{Na}[\mathrm{M}+\mathrm{Na}]^{+}: 409.1177$; found 409.1176 .<smiles>COc1ccc(C2(O)CCC3(CC2=Cc2c(F)cccc2Cl)OCCO3)cc1</smiles>

(E)-7-(2-chloro-6-fluorobenzylidene)-8-(4-methoxyphenyl)-1,4-dioxaspiro[4.5]decan-8-ol (4d)

White foam (299 mg, 76\% yield, 96\% BRSM); ${ }^{1} \mathrm{H}$ NMR (500 MHz, $\left.\mathrm{CDCl}_{3}\right) 7.57-7.52(\mathrm{~m}, 2 \mathrm{H})$, $7.24-7.14(\mathrm{~m}, 2 \mathrm{H}), 7.00$ (ddd, $J=9.2,8.0,1.6 \mathrm{~Hz}, 1 \mathrm{H}), 6.95-6.89(\mathrm{~m}, 2 \mathrm{H}), 6.36(\mathrm{~s}, 1 \mathrm{H}), 3.92-$ $3.78(\mathrm{~m}, 5 \mathrm{H}), 3.75-3.63(\mathrm{~m}, 2 \mathrm{H}), 2.57(\mathrm{ddd}, J=13.6,6.9,4.1 \mathrm{~Hz}, 1 \mathrm{H}), 2.53-2.45(\mathrm{~m}, 1 \mathrm{H}), 2.19$ $-2.11(\mathrm{~m}, 1 \mathrm{H}), 2.08(\mathrm{ddd}, J=13.9,10.3,4.0 \mathrm{~Hz}, 1 \mathrm{H}), 2.03-1.93(\mathrm{~m}, 2 \mathrm{H}), 1.78-1.66(\mathrm{~m}, 1 \mathrm{H})$; ${ }^{13} \mathrm{C}\left\{{ }^{1} \mathrm{H}\right\} \operatorname{NMR}\left(125 \mathrm{MHz}, \mathrm{CDCl}_{3}\right) 159.8\left(\mathrm{~d}, J_{C F}=247.7 \mathrm{~Hz}\right), 159.0,146.2\left(\mathrm{~d}, J_{C F}=1.1 \mathrm{~Hz}\right), 135.3$ $\left(\mathrm{d}, J_{C F}=5.2 \mathrm{~Hz}\right), 135.0,128.6\left(\mathrm{~d}, J_{C F}=9.2 \mathrm{~Hz}\right), 128.0,124.9\left(\mathrm{~d}, J_{C F}=3.6 \mathrm{~Hz}\right), 124.7,117.4$, $113.82\left(\mathrm{~d}, J_{C F}=23.0 \mathrm{~Hz}\right), 113.79,108.9,76.464 .23,62.15,55.3,37.5,36.0,32.2 ;{ }^{19} \mathrm{~F}$ NMR $(470$

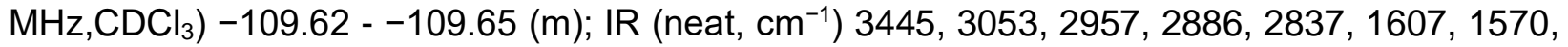
1512 , 1462, 1445, 1360, 1302, 1250, 1180, 1069, 1036; HRMS $\left(\mathrm{ESI}^{+}\right) \mathrm{m} / \mathrm{z}$ calcd for $\mathrm{C}_{22} \mathrm{H}_{22} \mathrm{ClFO}_{4} \mathrm{Na}[\mathrm{M}+\mathrm{Na}]^{+}:$427.1083; found 427.1087. 
<smiles>COc1ccc(C2(O)CCC3(CC2=Cc2ccc4ccccc4c2)OCCO3)cc1</smiles>

(E)-8-(4-methoxyphenyl)-7-(naphthalen-2-ylmethylene)-1,4-dioxaspiro[4.5]decan-8-ol (4e)

White foam (230 mg, 57\% yield); ${ }^{1} \mathrm{H}$ NMR $\left(500 \mathrm{MHz}, \mathrm{CDCl}_{3}\right) 7.86-7.75(\mathrm{~m}, 4 \mathrm{H}), 7.52(\mathrm{~d}, J=8.8$ $\mathrm{Hz}, 2 \mathrm{H}), 7.51-7.39(\mathrm{~m}, 3 \mathrm{H}), 6.94(\mathrm{~d}, J=8.8 \mathrm{~Hz}, 2 \mathrm{H}), 6.82(\mathrm{~s}, 1 \mathrm{H}), 3.99-3.84(\mathrm{~m}, 4 \mathrm{H}), 3.82(\mathrm{~s}$, 3H), 2.99 (dt, $J=14.0,1.5 \mathrm{~Hz}, 1 \mathrm{H}), 2.63$ (ddd, $J=14.4,7.5,4.2 \mathrm{~Hz}, 1 \mathrm{H}), 2.45$ (dt, $J=13.9,1.4$ $\mathrm{Hz}, 1 \mathrm{H}), 2.20-2.02(\mathrm{~m}, 2 \mathrm{H}), 1.84-1.74(\mathrm{~m}, 1 \mathrm{H}) ;{ }^{13} \mathrm{C}\left\{{ }^{1} \mathrm{H}\right\} \mathrm{NMR}\left(125 \mathrm{MHz}, \mathrm{CDCl}_{3}\right)$ 158.9, 142.2, 135.3, 135.1, 133.2, 132.1, 127.9, 127.8, 127.6, 127.54, 127.53, 127.4, 126.7, 125.9, 125.5, 113.8, 109.4, 76.3, 64.4, 55.2, 36.2, 36.1, 32.2; IR (neat, $\mathrm{cm}^{-1}$ ) 3453, 3053, 2955, 2884, 2835, 1609, 1582, 1510, 1443, 1360, 1300, 1250, 1179, 1105, 1070, 1036; HRMS (ESI $\left.{ }^{+}\right) \mathrm{m} / z$ calcd for $\mathrm{C}_{26} \mathrm{H}_{26} \mathrm{O}_{4} \mathrm{Na}[\mathrm{M}+\mathrm{Na}]^{+}:$425.1723; found 425.1716.<smiles></smiles>

(E)-7-(anthracen-9-ylmethylene)-8-(4-methoxyphenyl)-1,4-dioxaspiro[4.5]decan-8-ol (4f)

Pale yellow solid (258 mg, 68\% yield, 84\% BRSM) ${ }^{1} \mathrm{H}$ NMR $\left(300 \mathrm{MHz}, \mathrm{CDCl}_{3}\right) 8.38(\mathrm{~s}, 1 \mathrm{H}), 8.23$ - $8.15(\mathrm{~m}, 2 \mathrm{H}), 8.05-7.95(\mathrm{~m}, 2 \mathrm{H}), 7.79-7.71(\mathrm{~m}, 2 \mathrm{H}), 7.56-7.42(\mathrm{~m}, 4 \mathrm{H}), 7.05-6.97(\mathrm{~m}$, $2 \mathrm{H}), 6.92(\mathrm{~s}, 1 \mathrm{H}), 3.83(\mathrm{~s}, 3 \mathrm{H}), 3.66-3.51(\mathrm{~m}, 2 \mathrm{H}), 3.20-3.01(\mathrm{~m}, 2 \mathrm{H}), 2.77$ (ddd, J = 13.1, 8.7, $4.2 \mathrm{~Hz}, 1 \mathrm{H}), 2.43-2.33(\mathrm{~m}, 2 \mathrm{H}), 2.30-2.09(\mathrm{~m}, 2 \mathrm{H}), 1.99(\mathrm{dt}, J=14.0,1.3 \mathrm{~Hz}, 1 \mathrm{H}), 1.89-1.76$ $(\mathrm{m}, 1 \mathrm{H}) ;{ }^{13} \mathrm{C}\left\{{ }^{1} \mathrm{H}\right\}$ NMR $\left(75 \mathrm{MHz} \mathrm{CDCl}_{3}\right)$ 159.1, 146.1, 136.4, 132.3, 131.4, 131.3, 129.7, 129.6, 128.5, 128.3, 128.0, 126.8, 126.6, 126.0, 125.3, 125.14, 125.11, 124.0, 113.9, 109.0, 76.6, 63.9, 55.4, 37.0, 36.4, 32.5; IR (neat, $\mathrm{cm}^{-1}$ ) 3443, 3051, 2959, 2887, 2837, 1609, 1510, 1462, 1441, 1366, 1300, 1265, 1252, 1179, 1109, 1070, 1036; HRMS (ESI $\left.{ }^{-}\right) \mathrm{m} / \mathrm{z}$ calcd for $\mathrm{C}_{30} \mathrm{H}_{27} \mathrm{O}_{4}[\mathrm{M}-\mathrm{H}]^{-}$: 451.1915; found 451.1908 . 
<smiles>COc1ccc(C2(O)CCC3(CC2=CC(C)(C)C)OCCO3)cc1</smiles>

(E)-7-(2,2-dimethylpropylidene)-8-(4-methoxyphenyl)-1,4-dioxaspiro[4.5]decan-8-ol (4g)

Colorless oil (145 mg, 45\% yield, 79\% BRSM); ${ }^{1} \mathrm{H}$ NMR (300 MHz, $\left.\mathrm{CDCl}_{3}\right) 7.43-7.33(\mathrm{~m}, 2 \mathrm{H})$, $6.91-6.82(\mathrm{~m}, 2 \mathrm{H}), 5.66(\mathrm{t}, J=1.3 \mathrm{~Hz}, 1 \mathrm{H}), 4.01-3.88(\mathrm{~m}, 4 \mathrm{H}), 3.80(\mathrm{~s}, 3 \mathrm{H}), 2.92(\mathrm{dq}, J=14.0$, $1.0 \mathrm{~Hz}, 1 \mathrm{H}$ ), 2.48 (ddd, $J=12.6,5.5,4.0 \mathrm{~Hz}, 1 \mathrm{H}), 2.29(\mathrm{dt}, J=14.1,1.3 \mathrm{~Hz}, 1 \mathrm{H}), 1.97-1.86(\mathrm{~m}$, $2 \mathrm{H}), 1.73-1.57(\mathrm{~m}, 1 \mathrm{H}), 1.16(\mathrm{~s}, 9 \mathrm{H}) ;{ }^{13} \mathrm{C}\left\{{ }^{1} \mathrm{H}\right\} \mathrm{NMR}\left(75 \mathrm{MHz}, \mathrm{CDCl}_{3}\right)$ 158.8, 138.0, 136.9, 135.9, 127.9, 113.7, 109.3, 76.2, 64.52, 64.51, 55.3, 36.7, 36.1, 32.3, 31.8, 31.7; IR (neat, $\mathrm{cm}^{-1}$ ) 3462, 2955, 2874, 1609, 1582, 1510, 1464, 1364, 1300, 1250, 1209, 1179, 1117, 1105, 1069, 1038; HRMS $\left(\mathrm{ESI}^{+}\right) \mathrm{m} / z$ calcd for $\mathrm{C}_{20} \mathrm{H}_{27} \mathrm{O}_{3}(\mathrm{M}+\mathrm{H})^{+}-\left[\mathrm{H}_{2} \mathrm{O}\right]: 315.1955$; found 315.1956.<smiles>COc1cccc(C2(O)CCC3(CCO/C(=C/c4ccccc4)C3)C2)c1</smiles>

(E)-7-benzylidene-8-(3-methoxyphenyl)-1,4-dioxaspiro[4.5]decan-8-ol (4h)

White foam (228 mg, 65\% yield); ${ }^{1} \mathrm{H}$ NMR (300 MHz, $\left.\mathrm{CDCl}_{3}\right) 7.40-7.20(\mathrm{~m}, 6 \mathrm{H}), 7.19-7.12(\mathrm{~m}$, 2H), 6.87 (ddd, J = 8.1, 2.5, $1.0 \mathrm{~Hz}, 1 \mathrm{H}), 6.64(\mathrm{~s}, 1 \mathrm{H}), 4.02-3.86(\mathrm{~m}, 4 \mathrm{H}), 3.84(\mathrm{~s}, 3 \mathrm{H}), 2.92(\mathrm{dt}$, $J=14.0,1.5 \mathrm{~Hz}, 1 \mathrm{H}), 2.69-2.52(\mathrm{~m}, 1 \mathrm{H}), 2.45(\mathrm{dt}, J=14.0,1.4 \mathrm{~Hz}, 1 \mathrm{H}), 2.17-1.99(\mathrm{~m}, 3 \mathrm{H})$, $1.88-1.68(\mathrm{~m}, 1 \mathrm{H}) ;{ }^{13} \mathrm{C}\left\{{ }^{1} \mathrm{H}\right\}$ NMR $\left(75 \mathrm{MHz} \mathrm{CDCl}_{3}\right)$ 159.8, 145.2, 141.4, 137.5, 129.5, 129.0, 128.1, 127.2, 126.5, 119.1, 112.8, 112.7, 109.3, 76.7, 64.5, 64.4, 55.3, 36.14, 36.12, 32.1; IR (neat, $\mathrm{cm}^{-1}$ ) 3453, 3055, 2955, 2884, 2835, 1599, 1584, 1487, 1462, 1447, 1433, 1360, 1315, 1288, 1250, 1153, 1111, 1094, 1074, 1049; HRMS $\left(\mathrm{ESI}^{+}\right) \mathrm{m} / \mathrm{z}$ calcd for $\mathrm{C}_{22} \mathrm{H}_{25} \mathrm{O}_{4}[\mathrm{M}+\mathrm{H}]^{+}$ 353.1747; found 353.1739. 
<smiles>OC1(c2ccc(-c3ccccc3)cc2)CCC2(CC1=Cc1ccccc1)OCCO2</smiles>

(E)-8-([1,1'-biphenyl]-4-yl)-7-benzylidene-1,4-dioxaspiro[4.5]decan-8-ol (4j)

White foam (242 mg, 61\% yield); ${ }^{1} \mathrm{H}$ NMR (500 MHz, $\left.\mathrm{CDCl}_{3}\right) 7.67-7.58(\mathrm{~m}, 6 \mathrm{H}), 7.45(\mathrm{t}, J=7.7$ $\mathrm{Hz}, 2 \mathrm{H}), 7.40-7.28(\mathrm{~m}, 5 \mathrm{H}), 7.25-7.20(\mathrm{~m}, 1 \mathrm{H}), 6.67(\mathrm{~s}, 1 \mathrm{H}), 4.01-3.85(\mathrm{~m}, 4 \mathrm{H}), 2.94(\mathrm{~d}, J=$ $14.0 \mathrm{~Hz}, 1 \mathrm{H}), 2.69-2.61(\mathrm{~m}, 1 \mathrm{H}), 2.46(\mathrm{~d}, J=14.0 \mathrm{~Hz}, 1 \mathrm{H}), 2.14-2.03(\mathrm{~m}, 2 \mathrm{H}), 1.93(\mathrm{~s}, 1 \mathrm{H})$, $1.87-1.77(\mathrm{~m}, 1 \mathrm{H}) ;{ }^{13} \mathrm{C}\left\{{ }^{1} \mathrm{H}\right\} \mathrm{NMR}^{*}\left(125 \mathrm{MHz} \mathrm{CDCl}_{3}\right) 142.4,141.5,140.7,140.5,137.4,129.0$, 128.8, 128.1, 127.4, 127.3, 127.2, 127.1, 126.5, 109.3, 76.6, 64.5, 36.23, 36.16, 32.1; IR (neat, $\mathrm{cm}^{-1}$ ) 3444, 3055, 3028, 2955, 2930, 2883, 1599, 1486, 1446, 1356, 1266, 1221, 1134, 1105, 1073; HRMS $\left(\mathrm{ESI}^{+}\right) \mathrm{m} / z$ calcd for $\mathrm{C}_{27} \mathrm{H}_{26} \mathrm{NaO}_{3}[\mathrm{M}+\mathrm{Na}]^{+} 421.1774$; found 421.1788 .

${ }^{*}$ One carbon signal is not observed in the ${ }^{13} \mathrm{C}\left\{{ }^{1} \mathrm{H}\right\}$ NMR.<smiles></smiles>

(E)-7-benzylidene-8-(4-(tert-butyl)phenyl)-1,4-dioxaspiro[4.5]decan-8-ol (4k)

White foam (359 mg, 95\% yield); ${ }^{1} \mathrm{H}$ NMR (300 MHz, $\left.\mathrm{CDCl}_{3}\right) 7.55$ - $7.46(\mathrm{~m}, 2 \mathrm{H}), 7.45$ - 7.39 (m, $2 \mathrm{H}), 7.38-7.29(\mathrm{~m}, 4 \mathrm{H}), 7.29-7.21(\mathrm{~m}, 1 \mathrm{H}), 6.73(\mathrm{~s}, 1 \mathrm{H}), 4.00-3.85(\mathrm{~m}, 4 \mathrm{H}), 2.94(\mathrm{ddd}, J=$ 14.0, 2.1, 1.0 Hz, 1H), 2.65 (ddd, $J=14.1,7.1,4.1 \mathrm{~Hz}, 1 \mathrm{H}$ ), 2.38 (dt, $J=13.9,1.3 \mathrm{~Hz}, 1 \mathrm{H}$ ), 2.14 - $1.97(\mathrm{~m}, 2 \mathrm{H}), 1.87-1.69(\mathrm{~m}, 1 \mathrm{H}), 1.36(\mathrm{~s}, 9 \mathrm{H}) ;{ }^{13} \mathrm{C}\left\{{ }^{1} \mathrm{H}\right\} \mathrm{NMR}\left(75 \mathrm{MHz}, \mathrm{CDCl}_{3}\right)$ 150.6, 141.6, 140.1, 137.7, 129.1, 128.1, 126.7, 126.5, 126.4, 125.5, 109.4, 76.5, 64.4, 36.2, 36.0, 34.5, 32.3, 31.5; IR (neat, $\mathrm{cm}^{-1}$ ) 3443, 3055, 3022, 2961, 2903, 2878, 1493, 1462, 1445, 1395, 1362, 1269 , 1107, 1072, 1030; HRMS $\left(\mathrm{ESI}^{+}\right) \mathrm{m} / \mathrm{z}$ calcd for $\mathrm{C}_{25} \mathrm{H}_{30} \mathrm{O}_{3} \mathrm{Na}[\mathrm{M}+\mathrm{Na}]^{+} 401.2087$; found 401.2089. 


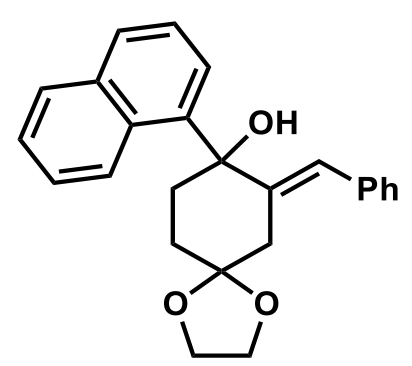

(E)-7-benzylidene-8-(naphthalen-1-yl)-1,4-dioxaspiro[4.5]decan-8-ol (4I)

White solid (349 mg, 94\%); m.p. = $148-150^{\circ} \mathrm{C} ;{ }^{1} \mathrm{H}$ NMR $\left(300 \mathrm{MHz}, \mathrm{CDCl}_{3}\right) 8.46-8.33(\mathrm{~m}, 1 \mathrm{H})$, $8.00(\mathrm{dd}, J=7.3,1.3 \mathrm{~Hz}, 1 \mathrm{H}), 7.93-7.81(\mathrm{~m}, 2 \mathrm{H}), 7.58-7.35(\mathrm{~m}, 3 \mathrm{H}), 7.32-7.14(\mathrm{~m}, 3 \mathrm{H}), 7.07$ $-6.98(\mathrm{~m}, 2 \mathrm{H}), 6.06(\mathrm{~d}, J=2.3 \mathrm{~Hz}, 1 \mathrm{H}), 4.10-3.94(\mathrm{~m}, 4 \mathrm{H}), 3.14-2.81(\mathrm{~m}, 3 \mathrm{H}), 2.40(\mathrm{td}, J=$ 13.1, 4.7 Hz, 1H), $2.08(\mathrm{~d}, J=2.3 \mathrm{~Hz}, 1 \mathrm{H}), 1.99$ (ddd, $J=14.3,4.8,2.8 \mathrm{~Hz}, 1 \mathrm{H}$ ), 1.86 (ddt, $J=$ 13.1, 5.0, $2.7 \mathrm{~Hz}, 1 \mathrm{H}) ;{ }^{13} \mathrm{C}\left\{{ }^{1} \mathrm{H}\right\} \mathrm{NMR}^{*}\left(75 \mathrm{MHz}, \mathrm{CDCl}_{3}\right) 141.7,140.9,137.1,134.6,130.9,130.1$, 128.84, 128.77, 128.73, 128.4, 128.0, 126.6, 125.10, 125.07, 125.02, 124.1, 109.4, 77.4, 64.6, 36.2, 35.7, 30.9; IR (neat, $\mathrm{cm}^{-1}$ ) 3441, 3051, 3022, 2955, 2934, 2884, 1599, 1508, 1493, 1445, $1360,1312,1163,1136,1111,1082,1057,1045,1011 ; \mathrm{HRMS}\left(\mathrm{ESI}{ }^{+}\right) \mathrm{m} / \mathrm{z}$ calcd for $\mathrm{C}_{25} \mathrm{H}_{24} \mathrm{NaO}_{3}$ $[\mathrm{M}+\mathrm{Na}]^{+}$395.1618; found 395.1621.

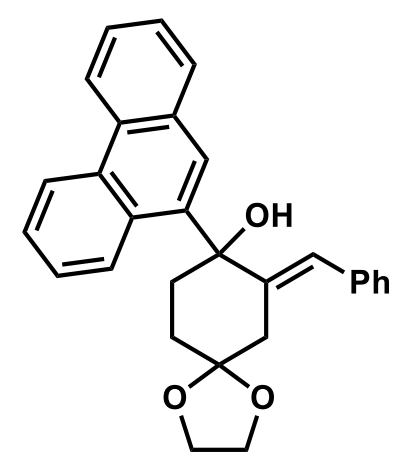

(E)-7-benzylidene-8-(phenanthren-9-yl)-1,4-dioxaspiro[4.5]decan-8-ol (4m)

White solid (218 mg, 52\% yield, 72\% BRSM); m.p. $=98-100{ }^{\circ} \mathrm{C} ;{ }^{1} \mathrm{H}$ NMR $\left(500 \mathrm{MHz}, d_{8}\right.$-THF) $8.79(\mathrm{~d}, J=8.3 \mathrm{~Hz}, 1 \mathrm{H}), 8.73(\mathrm{~d}, J=8.2 \mathrm{~Hz}, 1 \mathrm{H}), 8.58(\mathrm{~d}, J=8.2 \mathrm{~Hz}, 1 \mathrm{H}), 8.33(\mathrm{brs}, 1 \mathrm{H}), 7.93(\mathrm{~d}$, $J=7.8 \mathrm{~Hz}, 1 \mathrm{H}), 7.66-7.43(\mathrm{~m}, 4 \mathrm{H}), 7.17(\mathrm{t}, J=7.5 \mathrm{~Hz}, 2 \mathrm{H}), 7.08(\mathrm{t}, J=7.4 \mathrm{~Hz}, 1 \mathrm{H}), 7.00(\mathrm{~d}, J=$ $7.6 \mathrm{~Hz}, 2 \mathrm{H}), 6.01(\mathrm{~s}, 1 \mathrm{H}), 4.75$ (brs, $1 \mathrm{H}), 4.08-3.82(\mathrm{~m}, 4 \mathrm{H}), 3.10-2.84(\mathrm{~m}, 3 \mathrm{H}), 2.39$ (td, $J=$ 13.1, $4.8 \mathrm{~Hz}, 1 \mathrm{H}), 1.96-1.69(\mathrm{~m}, 2 \mathrm{H}) ;{ }^{3} \mathrm{C}\left\{{ }^{1} \mathrm{H}\right\} \mathrm{NMR}\left(125 \mathrm{MHz}, \mathrm{d}_{8}\right.$-THF) 142.5, $142.3(\mathrm{br}), 138.6$, 132.7, 132.3, 131.4, 131.3, 130.9 (br), 129.8 (br), 129.62, 129.59, 128.6, 127.3, 127.2, 127.0, 126.4, 126.3, 125.9, 123.6, 123.2, 110.4, 77.0, 65.3, 65.2, 37.1 (br), 36.7, 31.7; HRMS (ESI+) m/z calcd for $\mathrm{C}_{29} \mathrm{H}_{27} \mathrm{O}_{3}[\mathrm{M}+\mathrm{H}]^{+}:$423.1955; found 423.1956. 
<smiles>OC1(c2csc3ccccc23)CCC2(C/C1=C\c1ccccc1)OCCO2</smiles>

(E)-8-(benzo[b]thiophen-3-yl)-7-benzylidene-1,4-dioxaspiro[4.5]decan-8-ol (4n)

White solid (348 mg, 92\% yield); m.p. $=99-101{ }^{\circ} \mathrm{C} ;{ }^{1} \mathrm{H}$ NMR $\left(500 \mathrm{MHz}, \mathrm{CDCl}_{3}\right) 7.82(\mathrm{~d}, \mathrm{~J}=7.0$ $\mathrm{Hz}, 1 \mathrm{H}), 7.74(\mathrm{~d}, J=7.2 \mathrm{~Hz}, 1 \mathrm{H}), 7.38-7.29(\mathrm{~m}, 7 \mathrm{H}), 7.26-7.24(\mathrm{~m}, 1 \mathrm{H}), 6.92(\mathrm{~s}, 1 \mathrm{H}), 3.98-$ $3.83(\mathrm{~m}, 4 \mathrm{H}), 2.96(\mathrm{dd}, J=14.1,2.2 \mathrm{~Hz}, 1 \mathrm{H}), 2.59$ (ddd, $J=13.7,6.5,4.3 \mathrm{~Hz}, 1 \mathrm{H}), 2.52(\mathrm{~d}, J=$ $14.2 \mathrm{~Hz}, 1 \mathrm{H}$ ), 2.30 (s, 1H), 2.25 (ddd, $J=13.6,10.6,4.3 \mathrm{~Hz}, 1 \mathrm{H}), 2.08-2.01(\mathrm{~m}, 1 \mathrm{H}), 1.91$ (ddd, $J=14.2,10.5,4.2 \mathrm{~Hz}, 1 \mathrm{H}) ;{ }^{13} \mathrm{C}\left\{{ }^{1} \mathrm{H}\right\} \operatorname{NMR}\left(125 \mathrm{MHz}, \mathrm{CDCl}_{3}\right)$ 149.9, 140.4, 139.7, 137.1, 129.0, 128.1, 126.9, 126.7, 124.5, 124.3, 123.7, 122.4, 122.0, 108.8, 75.1, 64.5, 37.6, 36.1, 32.3; IR (neat, $\mathrm{cm}^{-1}$ ) 3449, 3053, 2955, 2884, 1493, 1456, 1360, 1304, 1265, 1213, 1152, 1101, 1034; HRMS $\left(\mathrm{ESI}^{+}\right) \mathrm{m} / \mathrm{z}$ calcd for $\mathrm{C}_{23} \mathrm{H}_{23} \mathrm{O}_{3} \mathrm{~S}[\mathrm{M}+\mathrm{H}]^{+} 379.1362$; found 379.1362.<smiles>OC1(c2cc3ccccc3o2)CCC2(C/C1=C\c1ccccc1)OCCO2</smiles>

(E)-8-(benzofuran-2-yl)-7-benzylidene-1,4-dioxaspiro[4.5]decan-8-ol (7)

White foam (257 mg, 71\% yield); ${ }^{1} \mathrm{H}$ NMR $\left(500 \mathrm{MHz}, \mathrm{CDCl}_{3}\right) 7.57(\mathrm{~d}, J=7.6 \mathrm{~Hz}, 1 \mathrm{H}), 7.50(\mathrm{~d}, J=$ $8.2 \mathrm{~Hz}, 1 \mathrm{H}), 7.37-7.20(\mathrm{~m}, 8 \mathrm{H}), 6.76(\mathrm{~s}, 1 \mathrm{H}), 6.63(\mathrm{~s}, 1 \mathrm{H}), 4.01-3.83(\mathrm{~m}, 4 \mathrm{H}), 2.94(\mathrm{~d}, J=14.1$ $\mathrm{Hz}, 1 \mathrm{H}), 2.74-2.65(\mathrm{~m}, 1 \mathrm{H}), 2.63(\mathrm{~d}, J=14.0 \mathrm{~Hz}, 1 \mathrm{H}), 2.38(\mathrm{~s}, 1 \mathrm{H}), 2.18-2.06(\mathrm{~m}, 2 \mathrm{H}), 1.86-$ $1.77(\mathrm{~m}, 1 \mathrm{H}) ;{ }^{13} \mathrm{C}\left\{{ }^{1} \mathrm{H}\right\} \mathrm{NMR}\left(125 \mathrm{MHz}, \mathrm{CDCl}_{3}\right)$ 159.4, 154.8, 139.0, 136.9, 129.0, 128.12, 128.07, $127.1,126.7,124.3,122.9,121.1,111.4,109.0,104.4,73.9,64.5,35.8,34.9$, 31.5; IR (neat, $\mathrm{cm}^{-1}$ ) 3429, 3055, 3022, 2955, 2926, 2886, 2855, 1454, 1358, 1306, 1256, 1113, 1103; HRMS $\left(\mathrm{ESI}^{+}\right) \mathrm{m} / \mathrm{z}$ calcd for $\mathrm{C}_{23} \mathrm{H}_{22} \mathrm{O}_{4} \mathrm{Na}[\mathrm{M}+\mathrm{Na}]^{+}:$385.1410; found 385.1414. 


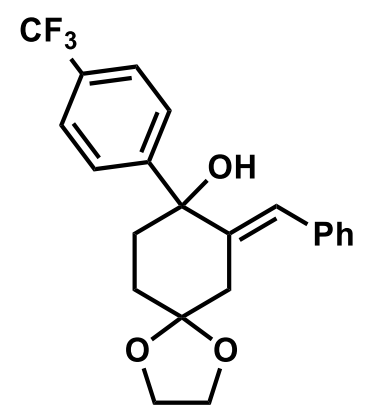

(E)-7-benzylidene-8-(4-(trifluoromethyl)phenyl)-1,4-dioxaspiro[4-5]decan-8-ol (40)

Thick colorless oil (245 mg, 63\% yield); ${ }^{1} \mathrm{H}$ NMR (500 MHz, $\left.\mathrm{CDCl}_{3}\right) 7.69$ (d, J=8.3 Hz, 2H), 7.64 $(\mathrm{d}, J=8.3 \mathrm{~Hz}, 2 \mathrm{H}), 7.32(\mathrm{t}, J=7.6 \mathrm{~Hz}, 2 \mathrm{H}), 7.27-7.20(\mathrm{~m}, 3 \mathrm{H}), 6.43(\mathrm{~s}, 1 \mathrm{H}), 4.00-3.85(\mathrm{~m}, 4 \mathrm{H})$, $2.88(\mathrm{~d}, J=14.2 \mathrm{~Hz}, 1 \mathrm{H}), 2.58-2.49(\mathrm{~m}, 2 \mathrm{H}), 2.37$ (brs, $1 \mathrm{H}), 2.13$ (ddd, $J=13.2,9.4,4.0 \mathrm{~Hz}$, 1H) 2.03 (ddd, $J=13.7,8.2,4.2 \mathrm{~Hz}, 1 \mathrm{H}), 1.84-1.70(\mathrm{~m}, 1 \mathrm{H}) ;{ }^{13} \mathrm{C}\left\{{ }^{1} \mathrm{H}\right\} \mathrm{NMR}\left(125 \mathrm{MHz}, \mathrm{CDCl}_{3}\right)$ 147.9, 141.1, 136.9, $129.6\left(\mathrm{q}, J_{C F}=32.5 \mathrm{~Hz}\right), 128.9,128.12,128.08,127.1,126.7,125.2\left(\mathrm{q}, J_{C F}\right.$ $=3.6 \mathrm{~Hz}), 124.1\left(\mathrm{q}, J_{C F}=272.0 \mathrm{~Hz}\right), 109.0,76.5,64.4,36.5,35.9,31.7 ;{ }^{19} \mathrm{~F} \mathrm{NMR}(470 \mathrm{MHz}$, $\mathrm{CDCl}_{3}$ ) -63.0; IR (neat, $\mathrm{cm}^{-1}$ ) 3429, 3055, 2957, 2886, 1618, 1493, 1445, 1408, 1165, 1121, 1070, 1016; HRMS (ESI $\left.{ }^{+}\right) \mathrm{m} / z$ calcd for $\mathrm{C}_{22} \mathrm{H}_{21} \mathrm{~F}_{3} \mathrm{O}_{3} \mathrm{Na}[\mathrm{M}+\mathrm{Na}]^{+}:$413.1335; found 413.1340.<smiles>OC1(c2cccc(C(F)(F)F)c2)CCC2(CC1=Cc1ccccc1)OCCO2</smiles>

(E)-7-benzylidene-8-(3-(trifluoromethyl)phenyl)-1,4-dioxaspiro[4-5]decan-8-ol (4p)

White foam (330 mg, 85\%); ${ }^{1} \mathrm{H}$ NMR $\left(300 \mathrm{MHz}, \mathrm{CDCl}_{3}\right) 7.85$ (td, $\left.J=1.8,0.9 \mathrm{~Hz}, 1 \mathrm{H}\right), 7.75$ (dt, $J$ $=7.7,1.6 \mathrm{~Hz}, 1 \mathrm{H}), 7.59(\mathrm{dt}, J=7.0,1.3 \mathrm{~Hz}, 1 \mathrm{H}), 7.51(\mathrm{t}, J=7.7 \mathrm{~Hz}, 1 \mathrm{H}), 7.40-7.18(\mathrm{~m}, 5 \mathrm{H})$, $6.45(\mathrm{~s}, 1 \mathrm{H}), 4.02-3.83(\mathrm{~m}, 4 \mathrm{H}), 2.89(\mathrm{dt}, J=14.1,1.5 \mathrm{~Hz}, 1 \mathrm{H}), 2.62-2.46(\mathrm{~m}, 2 \mathrm{H}), 2.21-1.98$ $(\mathrm{m}, 3 \mathrm{H}), 1.84-1.70(\mathrm{~m}, 1 \mathrm{H}) ;{ }^{13} \mathrm{C}\left\{{ }^{1} \mathrm{H}\right\} \operatorname{NMR}\left(75 \mathrm{MHz}, \mathrm{CDCl}_{3}\right) 145.0,141.2,137.0,130.8\left(\mathrm{q}, J_{C F}=\right.$ $32.2 \mathrm{~Hz}$ ), $130.3\left(\mathrm{q}, J_{C F}=1.4 \mathrm{~Hz}\right), 129.0,128.8,128.19,128.16,126.8,124.5$ (q, $\left.J_{C F}=3.8 \mathrm{~Hz}\right)$, $124.2\left(\mathrm{q}, J_{C F}=275.5 \mathrm{~Hz}\right.$ ), $123.4\left(\mathrm{q}, J_{C F}=3.9 \mathrm{~Hz}\right), 109.1,76.6,64.5,36.6,36.0,31.8 ;{ }^{19} \mathrm{~F}$ NMR (282 MHz, $\mathrm{CDCl}_{3}$ ) -62.34; IR (neat, $\mathrm{cm}^{-1}$ ) 3445, 3024, 2959, 2886, 1599, 1491, 1443, 1329, 1165 , 1125, 1099, 1076; HRMS (ESI $\left.{ }^{+}\right) \mathrm{m} / z$ calcd for $\mathrm{C}_{22} \mathrm{H}_{21} \mathrm{~F}_{3} \mathrm{O}_{3} \mathrm{Na}[\mathrm{M}+\mathrm{Na}]^{+}:$413.1335; found 413.1340. 


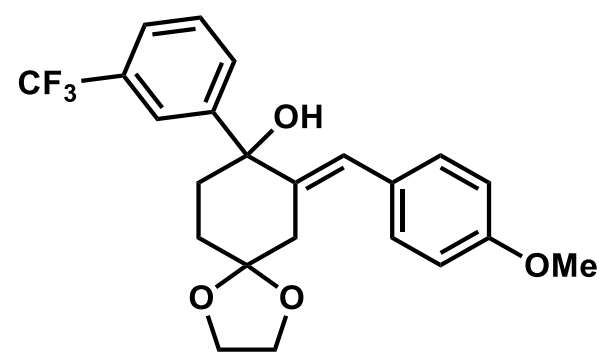

(E)-7-(4-methoxybenzylidene)-8-(3-(trifluoromethyl)phenyl)-1,4-dioxaspiro[4.5]decan-8-ol (4q)

Thick colorless oil ( $320 \mathrm{mg}, 76 \%$ yield); ${ }^{1} \mathrm{H}$ NMR $\left(500 \mathrm{MHz}, \mathrm{CDCl}_{3}\right) 7.86(\mathrm{~s}, 1 \mathrm{H}), 7.73$ (d, $J=7.9$ $\mathrm{Hz}, 1 \mathrm{H}), 7.57(\mathrm{~d}, J=7.8 \mathrm{~Hz}, 1 \mathrm{H}), 7.48(\mathrm{t}, J=7.8 \mathrm{~Hz}, 1 \mathrm{H}), 7.15(\mathrm{~d}, J=8.4 \mathrm{~Hz}, 2 \mathrm{H}), 6.85(\mathrm{~d}, J=$ $8.8 \mathrm{~Hz}, 2 \mathrm{H}), 6.34(\mathrm{~s}, 1 \mathrm{H}), 3.97-3.85(\mathrm{~m}, 4 \mathrm{H}), 3.78(\mathrm{~s}, 3 \mathrm{H}), 2.89(\mathrm{~d}, J=14.2 \mathrm{~Hz}, 1 \mathrm{H}), 2.65-2.47$ (m, 3H), 2.13 (ddd, $J=13.6,9.4,4.4 \mathrm{~Hz}, 1 \mathrm{H}$ ), 2.01 (ddt, $J=13.6,7.4,4.2 \mathrm{~Hz}, 1 \mathrm{H}$ ), $1.78-1.70$ $(\mathrm{m}, 1 \mathrm{H}) ;{ }^{13} \mathrm{C}\left\{{ }^{1} \mathrm{H}\right\} \mathrm{NMR}\left(125 \mathrm{MHz}, \mathrm{CDCl}_{3}\right) 158.3,145.2,140.0,132.3,130.5\left(\mathrm{q}, \mathrm{J}_{\mathrm{CF}}=32.1 \mathrm{~Hz}\right)$, $133.3\left(q, J_{C F}=1.1 \mathrm{~Hz}\right), 129.2,128.6,127.6,124.3\left(q, J_{C F}=272.4 \mathrm{~Hz}\right), 124.2\left(q, J_{C F}=3.7 \mathrm{~Hz}\right)$, $123.3\left(q, J_{C F}=3.7 \mathrm{~Hz}\right), 113.5,109.0,76.4,64.3,55.1,36.5,35.9,31.5$; IR (neat, $\mathrm{cm}^{-1}$ ) 3445, 2957, 2936, 2889, 2837, 1607, 1510, 1441, 1329, 1250, 1207, 1123, 1074, 1034; HRMS (ESI $\left.{ }^{+}\right)$ $\mathrm{m} / z$ calcd for $\mathrm{C}_{23} \mathrm{H}_{23} \mathrm{~F}_{3} \mathrm{O}_{4} \mathrm{Na}[\mathrm{M}+\mathrm{Na}]^{+}: 443.1441$; found 443.1441 . 


\section{Synthesis of Styrenes}

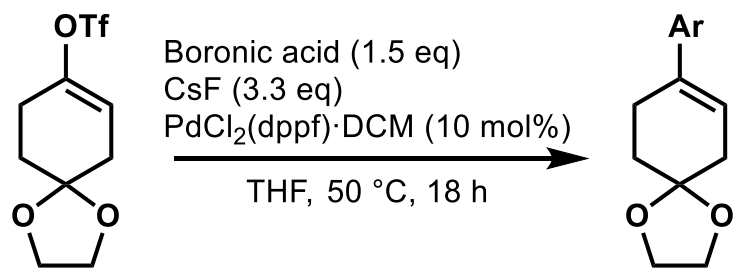

T1

Representative Procedure:

Triflate $\mathbf{T} 1$ was prepared according to a procedure from the literature. ${ }^{2}$

A vigorously stirred suspension of 3,5-bis(trifluoromethyl)phenylboronic acid (387 mg, $1.5 \mathrm{mmol}$, 1.5 equiv.), CsF (501 mg, $3.3 \mathrm{mmol}, 3.3$ equiv.), and $\mathrm{PdCl}_{2}$ (dppf)·DCM ( $82 \mathrm{mg}, 0.1 \mathrm{mmol}, 10$ mol\%) in THF (5 mL) was gently degassed. Triflate T1 (288 mg, $1.0 \mathrm{mmol}, 1.0$ equiv.) was added to the suspension as a THF solution $(1 \mathrm{~mL})$ at once. The reaction was stirred at $50{ }^{\circ} \mathrm{C}$ until completion of the reaction. The reaction was quenched with sat. aq. $\mathrm{NH}_{4} \mathrm{Cl}$ at room temperature and stirred for $20 \mathrm{~min}$ before filtration over Celite. The mixture was extracted with diethyl ether (x3) and the combined organic extracts were washed with water and brine and dried over anhydrous sodium sulfate. After filtration, the solvent was removed in vacuo and the crude material was purified by flash column chromatography on silica gel using a gradient of ethyl acetate in hexanes as eluent $(0,2,5,7,10 \%)$ to afford styrene $\mathbf{S 1}$ as a white solid $(261 \mathrm{mg}, 74 \%$ yield).

Styrenes $\mathbf{~} 1-\mathbf{5}$ were purified and used immediately in the next step.<smiles>FC(F)(F)c1cc(C2=CCC3(CC2)OCCO3)cc(C(F)(F)F)c1</smiles>

8-(3,5-bis(trifluoromethyl)phenyl)-1,4-dioxaspiro[4.5]dec-7-ene (S1)

White solid (261 mg, 74\% yield).<smiles>Fc1ccc(C2=CCC3(CC2)OCCO3)cc1</smiles>
8-(4-fluorophenyl)-1,4-dioxaspiro[4.5]dec-7-ene (S2)

White solid (129 mg, 55\% yield).

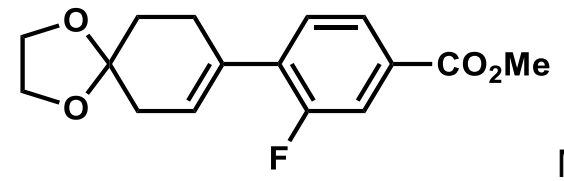

(S3)

Methyl 3-fluoro-4-(1,4-dioxaspiro[4.5]dec-7-en-8-yl)benzoate

Colorless oil (153 mg, 68\% yield). 


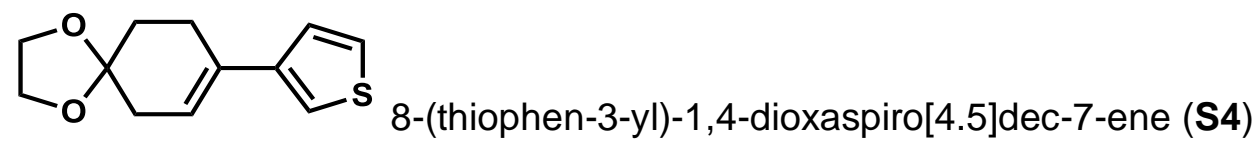

White solid (165 mg, 74\% yield).

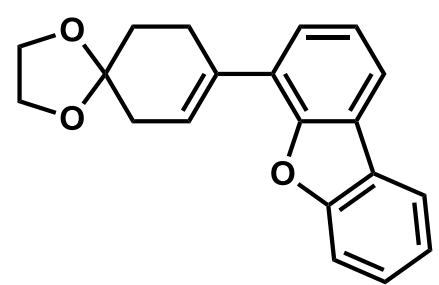

4-(1,4-dioxaspiro[4.5]dec-7-en-8-yl)dibenzo[b,d]furan (S5)

Colorless oil (107 mg, 45\% yield). 


\section{Synthesis of Biaryls}

Route A:<smiles>OC1([Al])CCC2(CC1)OCCO2</smiles>

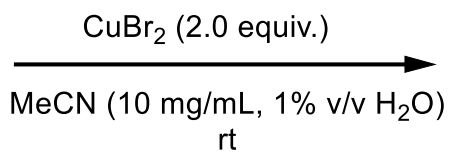<smiles>Oc1ccc(Br)cc1</smiles>

Route B:<smiles>OC1([Al])CCC2(CC1)OCCO2</smiles>

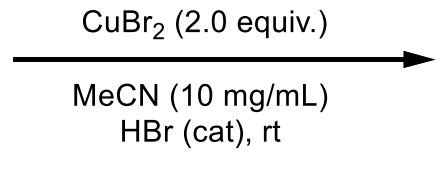<smiles>Oc1ccc(Br)cc1</smiles>

Route C:<smiles>BrC1=CCC2(CC1)OCCO2</smiles><smiles>C[N+](C)=O</smiles><smiles>Oc1ccc(Br)cc1</smiles>

Route D:<smiles>OC1([Al])CCC2(CC1)OCCO2</smiles>

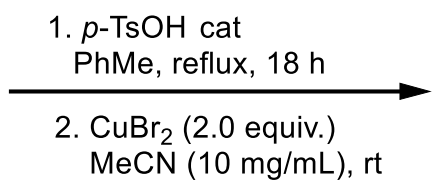<smiles>Oc1ccc(Br)cc1</smiles>

\section{Representative procedures:}

Note: Route $A$ is the preferred route for electron rich biphenyls, while route $B, C$, or $D$ are the preferred routes for electron neutral to poor biphenyls. 


\section{Route A:}

To a stirred solution of 2 (265 mg, 1.0 mmol, 1.0 equiv.) in $\mathrm{MeCN}\left(27 \mathrm{~mL}\right.$ of $1 \% \mathrm{v} / \mathrm{v} \mathrm{H} \mathrm{H}_{2} \mathrm{O}, 10$ $\mathrm{mg} / \mathrm{mL}$ ) under air at room temperature was added $\mathrm{CuB}_{2}(224 \mathrm{mg}, 1.0 \mathrm{mmol}, 1.0$ equiv.) at once. Within 15 minutes, the dark green colored solution suddenly became colorless. The solution slowly regained the dark green color over 10 minutes. The reaction was quenched by the addition of a sat. aq. solution of $\mathrm{NH}_{4} \mathrm{Cl}$ and stirred for 20 minutes. The phases were separated and the aqueous phase was extracted with diethyl ether $(x 3)$. The combined organic extracts were washed with a saturated aqueous solution of $\mathrm{NaHCO}_{3}$ (x2 or until the blue color subsided in the aqueous phase) and brine. The combined organic extracts dried over anhydrous sodium sulfate. After filtration, the solvent was removed in vacuo and the crude oil was purified by flash column chromatography on silica gel using a gradient of ethyl acetate in hexanes as eluent $(0,5,10,15$, $20 \%$ ) to afford 3 as a white solid ( $173 \mathrm{mg}, 84 \%$ yield).

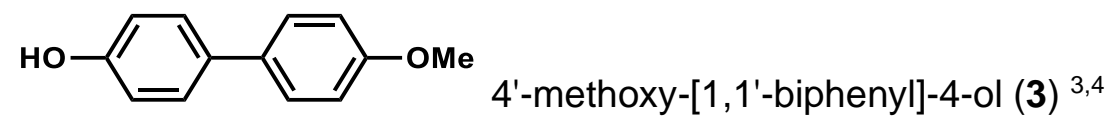

White solid (173 mg, 84\% yield); ${ }^{1} \mathrm{H}$ NMR (500 MHz, $\left.\mathrm{CDCl}_{3}\right) 7.50-7.46(\mathrm{~m}, 2 \mathrm{H}), 7.46-7.39$ (m, $2 \mathrm{H}), 6.99-6.92(\mathrm{~m}, 2 \mathrm{H}), 6.92-6.85(\mathrm{~m}, 2 \mathrm{H}), 4.78(\mathrm{~s}, 1 \mathrm{H}), 3.84(\mathrm{~s}, 3 \mathrm{H}) ;{ }^{13} \mathrm{C}\left\{{ }^{1} \mathrm{H}\right\} \mathrm{NMR}(125 \mathrm{MHz}$, $\left.\mathrm{CDCl}_{3}\right)$ 158.7, 154.6, 133.7, 133.4, 128.0, 127.7, 115.6, 114.2, 55.3.

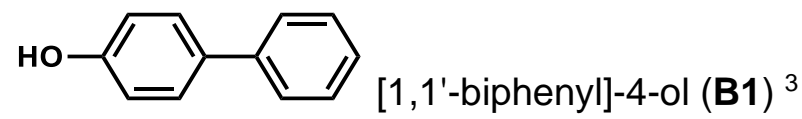

White solid (183 mg, 61\% yield); ${ }^{1} \mathrm{H}$ NMR (300 MHz, DMSO- $\left.d_{6}\right) 9.57(\mathrm{~s}, 1 \mathrm{H}), 7.61-7.53(\mathrm{~m}, 2 \mathrm{H})$, $7.53-7.45(\mathrm{~m}, 2 \mathrm{H}), 7.40(\mathrm{t}, J=7.6 \mathrm{~Hz}, 2 \mathrm{H}), 7.32-7.23(\mathrm{~m}, 1 \mathrm{H}), 6.90-6.80(\mathrm{~m}, 2 \mathrm{H}) ;{ }^{13} \mathrm{C}\left\{{ }^{1} \mathrm{H}\right\}$ NMR $\left(75 \mathrm{MHz}\right.$, DMSO-d $\left.d_{6}\right) 157.6,140.7,131.4,129.3,128.2,126.8,126.4,116.2$.

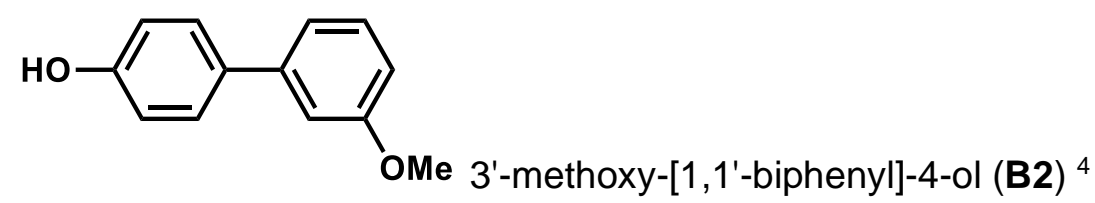

Off-white solid (87 mg, $45 \%$ yield); ${ }^{1} \mathrm{H}$ NMR $\left(300 \mathrm{MHz}, \mathrm{CDCl}_{3}\right) 7.54-7.45(\mathrm{~m}, 2 \mathrm{H}), 7.35$ (t, $\mathrm{J}=$ $7.9 \mathrm{~Hz}, 1 \mathrm{H}), 7.15$ (ddd, $J=7.6,1.7,1.0 \mathrm{~Hz}, 1 \mathrm{H}), 7.11(\mathrm{dd}, J=2.6,1.6 \mathrm{~Hz}, 1 \mathrm{H}), 6.98-6.85(\mathrm{~m}$, $3 \mathrm{H}), 5.87$ (brs, $1 \mathrm{H}), 3.88(\mathrm{~s}, 3 \mathrm{H}) ;{ }^{13} \mathrm{C}\left\{{ }^{1} \mathrm{H}\right\} \mathrm{NMR}\left(75 \mathrm{MHz}, \mathrm{CDCl}_{3}\right)$ 159.8, 155.4, 142.4, 133.7, 129.8 , 128.5, 119.4, 115.7, 112.5, 112.1, 55.4; HRMS (ESI+) $\mathrm{m} / z$ calcd for $\mathrm{C}_{13} \mathrm{H}_{13} \mathrm{O}_{2}[\mathrm{M}+\mathrm{H}]^{+}:$201.0910; found 201.0912 .

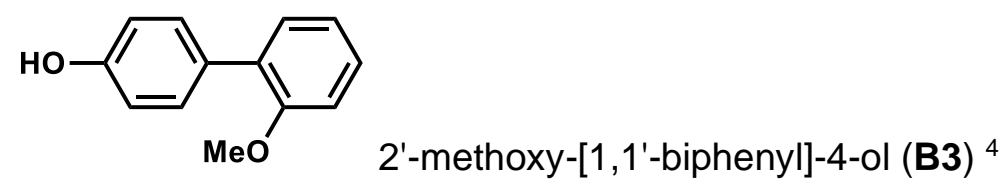

White solid (143 mg, 94\% yield); ${ }^{1} \mathrm{H} \mathrm{NMR}\left(300 \mathrm{MHz}, \mathrm{CDCl}_{3}\right) 7.49-7.40(\mathrm{~m}, 2 \mathrm{H}), 7.36-7.28(\mathrm{~m}$, 2H), $7.09-6.97(\mathrm{~m}, 2 \mathrm{H}), 6.93-6.85(\mathrm{~m}, 2 \mathrm{H}), 5.15(\mathrm{~s}, 1 \mathrm{H}), 3.83(\mathrm{~s}, 3 \mathrm{H}) ;{ }^{13} \mathrm{C}\left\{{ }^{1} \mathrm{H}\right\} \mathrm{NMR}(75 \mathrm{MHz}$, $\left.\mathrm{CDCl}_{3}\right)$ 156.4, 154.6, 131.1, 130.9, 130.7, 130.3, 128.3, 120.9, 115.0, 111.3, 55.6. 


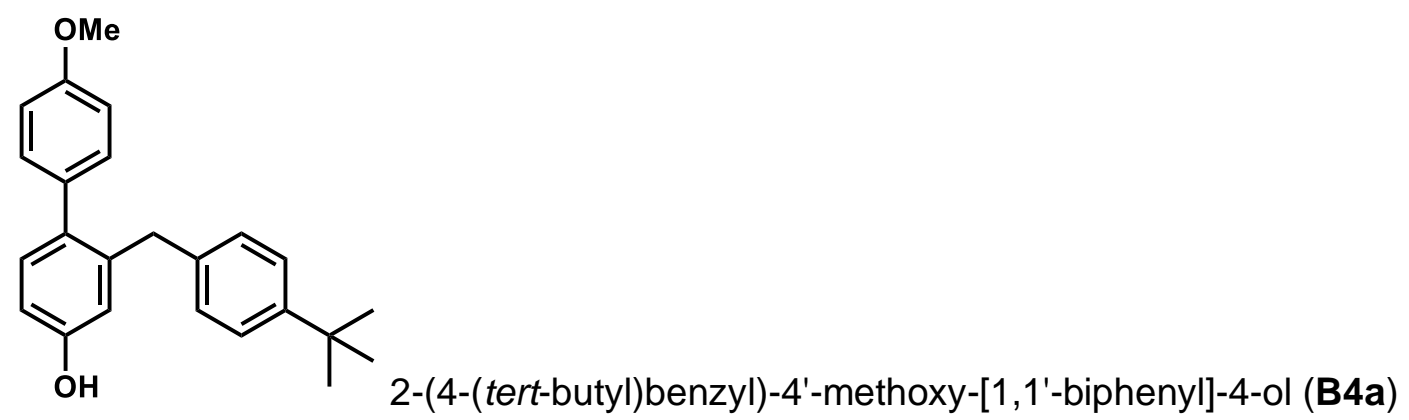

Off-white solid (128 mg, 80\% yield); ${ }^{1} \mathrm{H}$ NMR (300 MHz, $\left.\mathrm{CDCl}_{3}\right) 7.34-7.26(\mathrm{~m}, 2 \mathrm{H}), 7.26-7.20$ $(\mathrm{m}, 2 \mathrm{H}), 7.19-7.14(\mathrm{~m}, 1 \mathrm{H}), 7.04-6.89(\mathrm{~m}, 4 \mathrm{H}), 6.76(\mathrm{dd}, J=8.2,2.7 \mathrm{~Hz}, 1 \mathrm{H}), 6.68(\mathrm{~d}, J=2.6$ $\mathrm{Hz}, 1 \mathrm{H}), 5.16$ (brs, $1 \mathrm{H}), 3.91(\mathrm{~s}, 2 \mathrm{H}), 3.88(\mathrm{~s}, 3 \mathrm{H}), 1.34(\mathrm{~s}, 9 \mathrm{H}) ;{ }^{13} \mathrm{C}\left\{{ }^{1} \mathrm{H}\right\} \mathrm{NMR}\left(75 \mathrm{MHz}, \mathrm{CDCl}_{3}\right)$ 158.4, 154.7, 148.7, 140.3, 138.2, 134.5, 133.9, 131.5, 130.7, 128.6, 125.3, 116.8, 113.5, 113.2, 55.4, 38.5, 34.4, 31.5; IR (neat, $\mathrm{cm}^{-1}$ ) 3406, 2961, 2866, 1607, 1582, 1489, 1462, 1441, 1362, $1269,1177,1107,1038,1018,1001$; HRMS $\left(\mathrm{ESI}^{+}\right) \mathrm{m} / \mathrm{z}$ calcd for $\mathrm{C}_{24} \mathrm{H}_{26} \mathrm{O}_{2} \mathrm{Na}[\mathrm{M}+\mathrm{Na}]^{+}: 369.1825$; found 369.1830 .<smiles>COc1ccc(-c2cc(Br)c(O)cc2Cc2ccc(C(C)(C)C)cc2)cc1</smiles>

$(B 4 b)^{*}$

5-bromo-2-(4-(tert-butyl)benzyl)-4'-methoxy-[1,1'-biphenyl]-4-ol

Off-white solid (17 mg, 9\% yield); ${ }^{1} \mathrm{H}$ NMR (300 MHz, $\left.\mathrm{CDCl}_{3}\right) 7.34(\mathrm{~s}, 1 \mathrm{H}), 7.26-7.21(\mathrm{~m}, 2 \mathrm{H})$, $7.19-7.12(\mathrm{~m}, 2 \mathrm{H}), 6.95-6.87(\mathrm{~m}, 4 \mathrm{H}), 6.85(\mathrm{~s}, 1 \mathrm{H}), 5.40$ (brs, 1H), $3.84(\mathrm{~s}, 3 \mathrm{H}), 3.83(\mathrm{~s}, 2 \mathrm{H})$, 1.29 (s, 9H); HRMS (ESI $\left.{ }^{-}\right) \mathrm{m} / z$ calcd for $\mathrm{C}_{24} \mathrm{H}_{24} \mathrm{BrO}_{2}[\mathrm{M}-\mathrm{H}]^{-}: 423.0965$; found 423.0984.

*This compound was not fully characterized. 


\section{Route B:}

To a stirred solution of $\mathbf{A 6}$ (156 mg, $0.55 \mathrm{mmol}, 1.0$ equiv.) in $\mathrm{MeCN}$ (15 mL, $10 \mathrm{mg} / \mathrm{mL}$ ) under air at room temperature was added $\mathrm{CuBr}_{2}(235 \mathrm{mg}, 1.06 \mathrm{mmol}, 2.0$ equiv.) at once. After stirring for 15 minutes, $1-2$ drops of $\mathrm{HBr}$ (48\% aq.) were added. The reaction was complete within $1 \mathrm{~h}$. The reaction mixture was quenched by the addition of a sat. aq. solution of $\mathrm{NH}_{4} \mathrm{Cl}$ and stirred for 20 minutes. The phases were separated and the aqueous phase was extracted with diethyl ether (x3). The combined organic extracts were washed with a saturated aqueous solution of $\mathrm{NaHCO}_{3}$ (x2 or until the blue color subsided in the aqueous phase) and brine. The combined organic extracts were dried over anhydrous sodium sulfate. After filtration, the solvent was removed in vacuo and the crude oil was purified by flash column chromatography on silica gel using a gradient of ethyl acetate in hexane as eluent $(0,5,10,15,20,25 \%)$ to afford B5 as a white solid (96 mg, $80 \%$ yield).

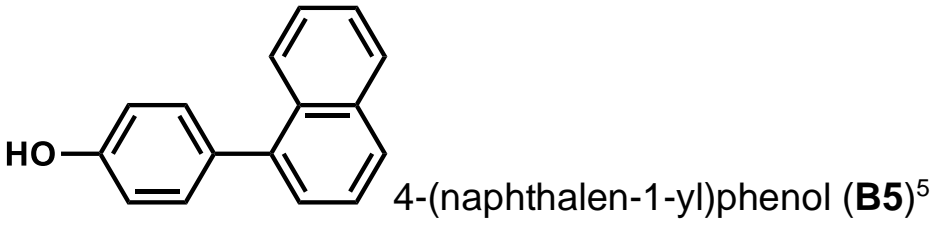

White solid (96 mg, 80\% yield); ${ }^{1} \mathrm{H}$ NMR (300 MHz, $\left.\mathrm{CDCl}_{3}\right) 7.99-7.90(\mathrm{~m}, 2 \mathrm{H}), 7.87$ (dt, J= 8.3, $1.2 \mathrm{~Hz}, 1 \mathrm{H}), 7.58-7.36(\mathrm{~m}, 6 \mathrm{H}), 7.03-6.94(\mathrm{~m}, 2 \mathrm{H}), 5.31$ (brs, $1 \mathrm{H}) ;{ }^{13} \mathrm{C}\left\{{ }^{1} \mathrm{H}\right\}$ NMR $(75 \mathrm{MHz}$, $\left.\mathrm{CDCl}_{3}\right) 154.9,139.9,133.9,133.4,131.8,131.4,128.3,127.4,127.0,126.1,126.0,125.8,125.5$, 115.2 .

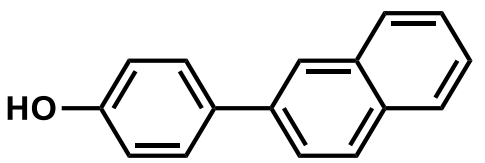

4-(naphthalen-2-yl)phenol (B6) ${ }^{6}$

White solid (131 mg, 85\% yield); ${ }^{1} \mathrm{H}$ NMR (500 MHz, $\left.\mathrm{CDCl}_{3}\right) 7.98(\mathrm{~d}, \mathrm{~J}=1.3 \mathrm{~Hz}, 1 \mathrm{H}), 7.93-7.83$ $(\mathrm{m}, 3 \mathrm{H}), 7.71(\mathrm{dd}, J=8.5,1.9 \mathrm{~Hz}, 1 \mathrm{H}), 7.65-7.60(\mathrm{~m}, 2 \mathrm{H}), 7.53-7.43(\mathrm{~m}, 2 \mathrm{H}), 6.99-6.92(\mathrm{~m}$, 2H), 4.92 (brs, $1 \mathrm{H}) ;{ }^{13} \mathrm{C}\left\{{ }^{1} \mathrm{H}\right\} \mathrm{NMR}\left(125 \mathrm{MHz}, \mathrm{CDCl}_{3}\right)$ 155.1, 138.1, 133.9, 133.7, 132.3, 128.7, 128.3, 128.0, 127.6, 126.2, 125.7, 125.4, 125.0, 115.7.

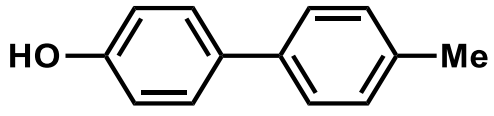

4'-methyl-[1,1'-biphenyl]-4-ol (B7) ${ }^{7}$

White solid (68 mg, 92\% yield); ${ }^{1} \mathrm{H}$ NMR (300 MHz, $\left.\mathrm{CDCl}_{3}\right) 7.55-7.40(\mathrm{~m}, 4 \mathrm{H}), 7.26(\mathrm{~d}, J=7.9$ $\mathrm{Hz}, 2 \mathrm{H}), 6.92(\mathrm{~d}, J=8.6 \mathrm{~Hz}, 2 \mathrm{H}), 5.06$ (brs, $1 \mathrm{H}), 2.42(\mathrm{~s}, 3 \mathrm{H}) ;{ }^{13} \mathrm{C}\left\{{ }^{1} \mathrm{H}\right\} \mathrm{NMR}\left(75 \mathrm{MHz}, \mathrm{CDCl}_{3}\right)$ 154.9, 137.9, 136.5, 134.0, 129.5, 128.2, 126.6, 115.6, 21.1.

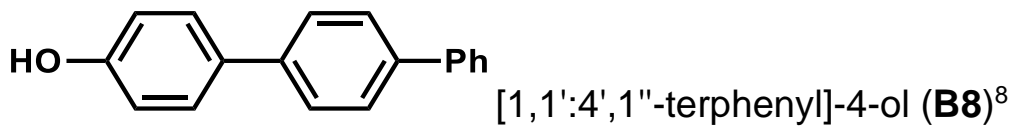

White solid (82 mg, quant. yield); ${ }^{1} \mathrm{H}$ NMR (300 MHz, DMSO-d6) 9.61 (brs, $\left.1 \mathrm{H}\right), 7.75-7.63$ (m, $6 \mathrm{H}), 7.55$ (d, $J=8.6 \mathrm{~Hz}, 2 \mathrm{H}), 7.47(\mathrm{t}, J=7.5 \mathrm{~Hz}, 2 \mathrm{H}), 7.36(\mathrm{t}, J=7.3 \mathrm{~Hz}, 1 \mathrm{H}), 6.89(\mathrm{~d}, J=8.6 \mathrm{~Hz}$, 
$2 \mathrm{H}) ;{ }^{13} \mathrm{C}\left\{{ }^{1} \mathrm{H}\right\}$ NMR $\left(75 \mathrm{MHz}\right.$, DMSO- $\left.d_{6}\right) 157.7,140.2,139.7,138.5,130.8,129.4,128.1,127.8$, $127.5,126.90,126.86,116.3$.

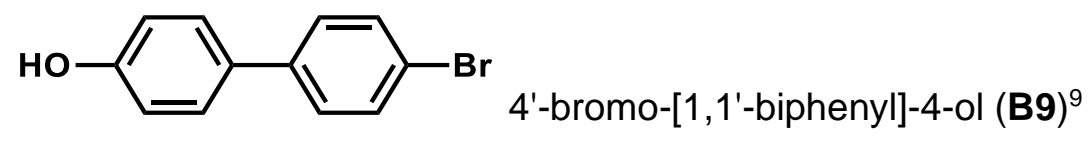

Off-white solid (76 mg, 48\% yield); ${ }^{1} \mathrm{H}$ NMR (300 MHz, $\left.\mathrm{CDCl}_{3}\right) 7.60-7.35(\mathrm{~m}, 6 \mathrm{H}), 6.91$ (dd, $\mathrm{J}=$ 8.6, $1.9 \mathrm{~Hz}, 2 \mathrm{H}) ;{ }^{13} \mathrm{C}\left\{{ }^{1} \mathrm{H}\right\} \mathrm{NMR}\left(75 \mathrm{MHz}, \mathrm{CDCl}_{3}\right)$ 155.4, 139.7, 131.8, 128.8, 128.3, 126.7, 120.9, 115.8. 


\section{Route C:}

To a stirred solution of styrene $\mathbf{S 1}$ (125 mg, $0.36 \mathrm{mmol}, 1.0$ equiv.) in MeCN (12.5 mL, $10 \mathrm{mg} / \mathrm{mL}$ ) under air at room temperature is added $\mathrm{CuBr}_{2}(161 \mathrm{mg}, 0.72 \mathrm{mmol}, 2.0$ equiv.) at once. Once complete, the reaction mixture is quenched by the addition of sat. aq. solution of $\mathrm{NH}_{4} \mathrm{Cl}$ and stirred for 20 minutes. The phases were separated and the aqueous phase was extracted with diethyl ether (x3). The combined organic extracts were washed with a saturated aqueous solution of $\mathrm{NaHCO}_{3}$ (x2 or until the blue color subsided in the aqueous phase) and brine. The combined organic extracts were dried over anhydrous sodium sulfate. After filtration, the solvent was removed in vacuo and the crude oil was purified by flash column chromatography on silica gel using a gradient of ethyl acetate in hexane as eluent $(0,5,10,15,20,25 \%)$ to afford $\mathbf{B 1 0}$ as a white solid (95 $\mathrm{mg}, 86 \%$ yield).

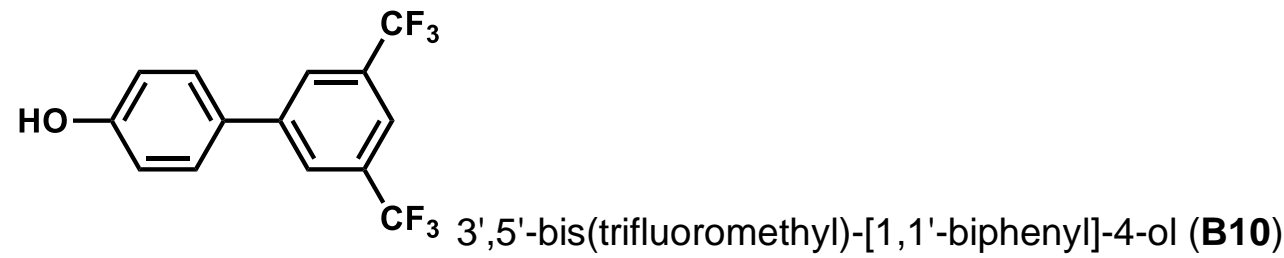

m.p. $=110-111^{\circ} \mathrm{C} ;{ }^{1} \mathrm{H}$ NMR $\left(300 \mathrm{MHz}, \mathrm{CDCl}_{3}\right) 8.00-7.90(\mathrm{~m}, 2 \mathrm{H}), 7.85-7.78(\mathrm{~m}, 1 \mathrm{H}), 7.60-$ $7.42(\mathrm{~m}, 2 \mathrm{H}), 7.05-6.89(\mathrm{~m}, 2 \mathrm{H}), 5.36$ (brs, $1 \mathrm{H}) ;{ }^{13} \mathrm{C}\left\{{ }^{1} \mathrm{H}\right\} \mathrm{NMR}^{*}\left(100 \mathrm{MHz}, \mathrm{CDCl}_{3}\right)$ 156.3, 142.7, $132.0\left(\mathrm{q}, J_{C F}=33.2 \mathrm{~Hz}\right), 128.7-128.6(\mathrm{~m}), 127.8-126.5(\mathrm{~m}), 123.4\left(\mathrm{q}, J_{C F}=272.8 \mathrm{~Hz}\right), 120.4$ - $120.1(\mathrm{~m}), 116.2 ;{ }^{19} \mathrm{~F} \mathrm{NMR}\left(282 \mathrm{MHz}, \mathrm{CDCl}_{3}\right.$ ) -62.85; IR (neat, $\mathrm{cm}^{-1}$ ) 3374, 3103, 3040, 2926, 1610, 1595, 1521, 1466, 1379, 1283, 1265, 1190, 1159, 1126, 1055, 897, 847, 823; HRMS (ESI $\left.{ }^{-}\right)$ $\mathrm{m} / \mathrm{z}$ calcd for $\mathrm{C}_{14} \mathrm{H}_{7} \mathrm{~F}_{6} \mathrm{O}[\mathrm{M}-\mathrm{H}]^{-}: 305.0407$; found 305.0405 .

${ }^{*}$ One carbon signal is not observed in the ${ }^{13} \mathrm{C}$ NMR.

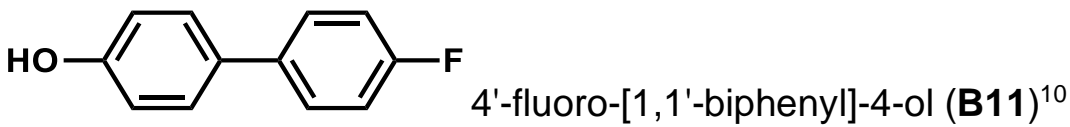

White solid (59 mg, 67\% yield); m.p. = $167-168{ }^{\circ} \mathrm{C} ;{ }^{1} \mathrm{H}$ NMR $(500 \mathrm{MHz}$, DMSO-d $) 9.54(\mathrm{~s}, 1 \mathrm{H})$, $7.60-7.55(\mathrm{~m}, 2 \mathrm{H}), 7.51-7.39(\mathrm{~m}, 2 \mathrm{H}), 7.25-7.16(\mathrm{~m}, 2 \mathrm{H}), 6.87-6.79(\mathrm{~m}, 2 \mathrm{H}) ;{ }^{13} \mathrm{C}\left\{{ }^{1} \mathrm{H}\right\} \mathrm{NMR}$ $\left(125 \mathrm{MHz}, \mathrm{DMSO}-d_{6}\right) 161.2\left(\mathrm{~d}, J_{C F}=242.7 \mathrm{~Hz}\right), 157.1,136.7\left(\mathrm{~d}, J_{C F}=2.9 \mathrm{~Hz}\right), 129.9,127.8$ (d, $\left.J_{C F}=7.9 \mathrm{~Hz}\right), 127.7,115.5\left(\mathrm{~d}, J_{C F}=21.1 \mathrm{~Hz}\right) ;{ }^{19} \mathrm{~F}$ NMR $(282 \mathrm{MHz}$, DMSO-d $)-117.1$; HRMS m/z calcd for $\mathrm{C}_{12} \mathrm{H}_{8} \mathrm{FO}[\mathrm{M}-\mathrm{H}]^{-}:$187.0565; found 187.0562 .

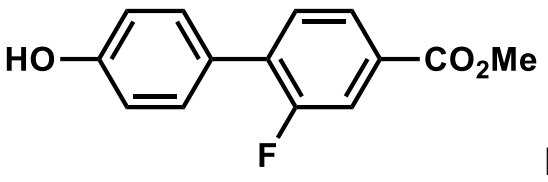

Methyl 2-fluoro-4'-hydroxy-[1,1'-biphenyl]-4-carboxylate (B12)

White solid (119 mg, 66\% yield); m.p. $=135-136{ }^{\circ} \mathrm{C} ;{ }^{1} \mathrm{H}$ NMR $\left(500 \mathrm{MHz}, \mathrm{CDCl}_{3}\right) 7.86$ (dd, $J=$ 8.0, 1.7 Hz, 1H), 7.79 (dd, $J=11.2,1.7 \mathrm{~Hz}, 1 \mathrm{H}), 7.52-7.44(\mathrm{~m}, 3 \mathrm{H}), 6.97-6.92(\mathrm{~m}, 2 \mathrm{H}), 3.95$ $(\mathrm{s}, 3 \mathrm{H}) ;{ }^{13} \mathrm{C}\left\{{ }^{1} \mathrm{H}\right\} \mathrm{NMR}\left(125 \mathrm{MHz}, \mathrm{CDCl}_{3}\right) 166.2\left(\mathrm{~d}, J_{C F}=2.7 \mathrm{~Hz}\right), 159.3\left(\mathrm{~d}, J_{C F}=248.2 \mathrm{~Hz}\right), 156.0$, $133.3\left(\mathrm{~d}, J_{C F}=13.3 \mathrm{~Hz}\right), 130.5\left(\mathrm{~d}, J_{C F}=3.4 \mathrm{~Hz}\right), 130.3\left(\mathrm{~d}, J_{C F}=3.6 \mathrm{~Hz}\right), 130.1\left(\mathrm{~d}, J_{C F}=7.9 \mathrm{~Hz}\right)$, $127.1\left(\mathrm{~d}, J_{C F}=1.4 \mathrm{~Hz}\right), 125.5\left(\mathrm{~d}, J_{C F}=3.5 \mathrm{~Hz}\right), 117.3\left(\mathrm{~d}, J_{C F}=25.1 \mathrm{~Hz}\right), 115.6,52.4 ;{ }^{19} \mathrm{~F} \mathrm{NMR}$ (282 MHz, $\mathrm{CDCl}_{3}$ ) -117.4 (m); IR (neat, $\mathrm{cm}^{-1}$ ) 3406, 2955, 2922, 2853, 1707, 1609, 1437, 1406, 1300, 1236, 1211, 1126, 1096; HRMS $\left(\mathrm{ESI}^{+}\right) \mathrm{m} / \mathrm{z}$ calcd for $\mathrm{C}_{13} \mathrm{H}_{12} \mathrm{FO}_{3}[\mathrm{M}+\mathrm{H}]^{+} 235.0765$; found 247.0772 . 


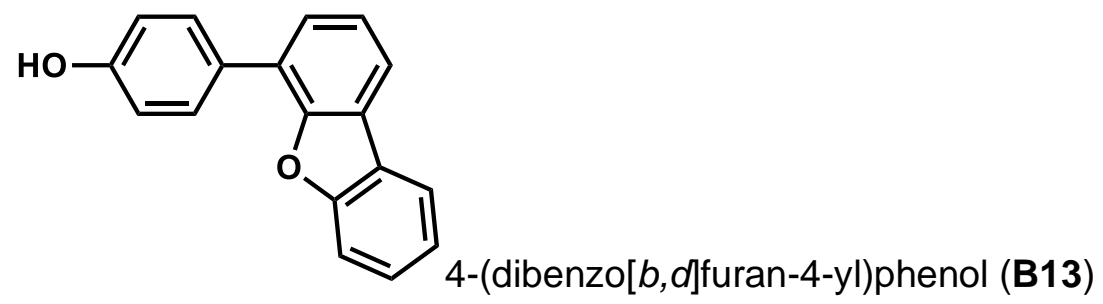

White solid (129 mg, 83\% yield); m.p. = $126-127{ }^{\circ} \mathrm{C} ;{ }^{1} \mathrm{H}$ NMR $\left(500 \mathrm{MHz}, \mathrm{CDCl}_{3}\right) 7.99$ (d, J = 7.6 $\mathrm{Hz}, 1 \mathrm{H}), 7.91(\mathrm{dd}, J=7.6,1.3 \mathrm{~Hz}, 1 \mathrm{H}), 7.86-7.81(\mathrm{~m}, 2 \mathrm{H}), 7.61(\mathrm{~d}, J=8.2 \mathrm{~Hz}, 1 \mathrm{H}), 7.57$ (dd, $J$ $=7.6,1.3 \mathrm{~Hz}, 1 \mathrm{H}), 7.47(\mathrm{ddd}, J=8.4,7.3,1.3 \mathrm{~Hz}, 1 \mathrm{H}), 7.41(\mathrm{t}, J=7.6 \mathrm{~Hz}, 1 \mathrm{H}), 7.37(\mathrm{td}, J=7.5$, $1.0 \mathrm{~Hz}, 1 \mathrm{H}), 7.05-6.99(\mathrm{~m}, 2 \mathrm{H}), 5.26$ (brs, $1 \mathrm{H}) ;{ }^{13} \mathrm{C}\left\{{ }^{1} \mathrm{H}\right\} \mathrm{NMR}\left(125 \mathrm{MHz}, \mathrm{CDCl}_{3}\right)$ 156.1, 155.3, 153.2, 130.1, 129.0, 127.1, 126.4, 125.4, 124.8, 124.2, 123.2, 122.7, 120.6, 119.1, 115.6, 111.8; IR (neat, $\mathrm{cm}^{-1}$ ) 3347, 3053, 2922, 1612, 1518, 1487, 1450, 1402, 1261, 1233, 1190, 1099, 1061; HRMS $\mathrm{m} / \mathrm{z}$ calcd for $\mathrm{C}_{18} \mathrm{H}_{11} \mathrm{O}_{2}[\mathrm{M}-\mathrm{H}]^{-}: 259.0765$; found 259.0758 .

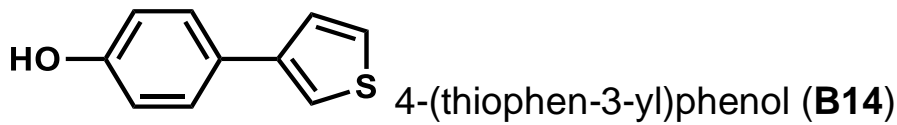

White solid (48 mg, 64\% yield); m.p. = $168-169{ }^{\circ} \mathrm{C} ;{ }^{1} \mathrm{H}$ NMR $\left(500 \mathrm{MHz}, \mathrm{DMSO}-\mathrm{d}_{6}\right) 9.49(\mathrm{~s}, 1 \mathrm{H})$, 7.62 (dd, $J=2.9,1.4 \mathrm{~Hz}, 1 \mathrm{H}), 7.56(\mathrm{dd}, J=5.0,2.9 \mathrm{~Hz}, 1 \mathrm{H}), 7.53-7.48(\mathrm{~m}, 2 \mathrm{H}), 7.44$ (dd, $J=$ 5.0, $1.4 \mathrm{~Hz}, 1 \mathrm{H}), 6.81-6.75(\mathrm{~m}, 2 \mathrm{H}) ;{ }^{13} \mathrm{C}\left\{{ }^{1} \mathrm{H}\right\}$ NMR (100 MHz, DMSO-d 6 ) 156.68, 141.60, 127.28, 126.68, 126.38, 125.98, 118.52, 115.56; IR (neat, $\mathrm{cm}^{-1}$ ) 3348, 3096, 2957, 2922, 2852, 1892, 1612, 1599, 1535, 1504, 1468, 1435, 1377, 1258, 1200, 1109; HRMS (ESI $\left.{ }^{-}\right) \mathrm{m} / \mathrm{z}$ calcd for $\mathrm{C}_{10} \mathrm{H}_{7} \mathrm{OS}[\mathrm{M}-\mathrm{H}]^{-}$175.0223; found 175.0222 . 


\section{Route D:}

A stirred solution of tertiary alcohol 2 l $(283 \mathrm{mg}, 0.76 \mathrm{mmol}, 1.0$ equiv.) and $p$ - $\mathrm{TsOH}$ ( $33 \mathrm{mg}, 0.19$ $\mathrm{mmol}, 25 \mathrm{~mol} \%$ ) in toluene $(5 \mathrm{~mL})$ was heated to reflux for $18 \mathrm{~h}$. After cooling to room temperature, the solvent was removed in vacuo and the crude oil diluted with MeCN $(28 \mathrm{~mL})$, followed by addition of $\mathrm{CuBr}_{2}$ (339 mg, $1.52 \mathrm{mmol}, 2.0$ equiv.) at once. Once complete, the reaction mixture was quenched by the addition of sat. aq. solution of $\mathrm{NH}_{4} \mathrm{Cl}$, diluted with diethyl ether, and stirred for 20 minutes. The phases were separated and the aqueous phase was extracted with diethyl ether (x3). The combined organic extracts were washed with a saturated aqueous solution of $\mathrm{NaHCO}_{3}$ ( $\times 2$ or until the blue color subsided in the aqueous phase) and brine. The combined organic extracts were dried over anhydrous sodium sulfate. After filtration, the solvent was removed in vacuo and the crude oil was purified by flash column chromatography on silica gel using a gradient of ethyl acetate in hexanes as eluent $(0,5,10,15,20 \%)$ to afford B10 as a white solid (116 mg, 46\% yield over the two steps). 
Biaryls $50-5 q$ were prepared in the same manner as fluorene $\mathbf{5 a}$.

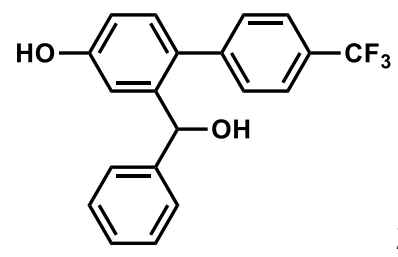

2-(hydroxy(phenyl)methyl)-4'-(trifluoromethyl)-[1,1'-biphenyl]-4-ol (50)

White solid (42 mg, 48\% yield); m.p. = $156-161{ }^{\circ} \mathrm{C} ;{ }^{1} \mathrm{H}$ NMR $\left(500 \mathrm{MHz}, d_{8}\right.$-THF) 8.38 (s, $\left.1 \mathrm{H}\right)$, $7.65(\mathrm{~d}, J=7.9 \mathrm{~Hz}, 2 \mathrm{H}), 7.50(\mathrm{~d}, J=7.9 \mathrm{~Hz}, 2 \mathrm{H}), 7.22-7.06(\mathrm{~m}, 5 \mathrm{H}), 7.04(\mathrm{~d}, J=8.3 \mathrm{~Hz}, 1 \mathrm{H})$, $6.91(\mathrm{~d}, J=2.6 \mathrm{~Hz}, 1 \mathrm{H}), 6.72(\mathrm{dd}, J=8.3,2.7 \mathrm{~Hz}, 1 \mathrm{H}), 5.73(\mathrm{~d}, J=4.0 \mathrm{~Hz}, 1 \mathrm{H}), 4.85(\mathrm{~d}, J=4.1$ $\mathrm{Hz}, 1 \mathrm{H}) ;{ }^{13} \mathrm{C}\left\{{ }^{1} \mathrm{H}\right\} \operatorname{NMR}\left(125 \mathrm{MHz}, d_{8}\right.$-THF) $158.1,146.1\left(\mathrm{q}, J_{C F}=1.4 \mathrm{~Hz}\right), 145.7,144.1,131.2$, 130.8, 130.5, $128.6\left(\mathrm{q}, J_{C F}=32.1 \mathrm{~Hz}\right), 127.8,126.7,126.6,125.0$ (q, $\left.J_{C F}=271.5 \mathrm{~Hz}\right), 124.9$ (q, $J_{C F}=3.8 \mathrm{~Hz}$ ), 114.9, 114.5, 71.6; ${ }^{19} \mathrm{~F}$ NMR (470 MHz, $d_{8}$-THF) -63.55; IR (neat, $\mathrm{cm}^{-1}$ ) 3418, 3264, 3063, 3028, 2955, 2920, 2849, 1607, 1445, 1321, 1225, 1163, 1111, 1015; HRMS (ESI $\left.{ }^{+}\right) \mathrm{m} / \mathrm{z}$ calcd for $\mathrm{C}_{20} \mathrm{H}_{15} \mathrm{~F}_{3} \mathrm{O}_{2} \mathrm{Na}[\mathrm{M}+\mathrm{Na}]^{+}: 367.0916$; found 367.0915.

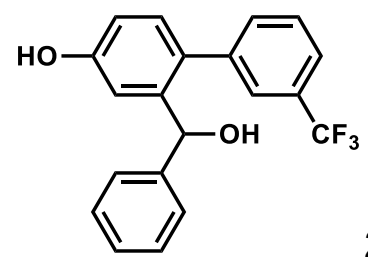

\section{2-(hydroxy(phenyl)methyl)-3'-(trifluoromethyl)-[1,1'-biphenyl]-4-ol (5p)}

White foam (56 mg, 56\% yield); ${ }^{1} \mathrm{H}$ NMR (500 MHz, $d_{8}$-THF) $8.46(\mathrm{~s}, 1 \mathrm{H}), 7.63-7.59(\mathrm{~m}, 2 \mathrm{H})$, $7.58-7.49(\mathrm{~m}, 2 \mathrm{H}), 7.22-7.15(\mathrm{~m}, 2 \mathrm{H}), 7.16-7.09(\mathrm{~m}, 3 \mathrm{H}), 7.05(\mathrm{~d}, J=8.2 \mathrm{~Hz}, 1 \mathrm{H}), 7.00(\mathrm{~d}, J$ $=2.6 \mathrm{~Hz}, 1 \mathrm{H}), 6.74(\mathrm{dd}, J=8.3,2.6 \mathrm{~Hz}, 1 \mathrm{H}), 5.72(\mathrm{~d}, J=3.6 \mathrm{~Hz}, 1 \mathrm{H}), 4.94(\mathrm{~d}, J=4.0 \mathrm{~Hz}, 1 \mathrm{H})$; ${ }^{13} \mathrm{C}\left\{{ }^{1} \mathrm{H}\right\} \operatorname{NMR}\left(125 \mathrm{MHz}, d_{8}\right.$-THF) 158.7, 146.2, 144.8, 143.6, 134.3 (q, $\left.J_{C F}=1.3 \mathrm{~Hz}\right), 131.7,131.6$, $130.9\left(\mathrm{q}, J_{C F}=31.9 \mathrm{~Hz}\right), 129.5,128.5,127.5,127.34\left(\mathrm{q}, J_{C F}=3.8 \mathrm{~Hz}\right), 127.32,125.4\left(\mathrm{q}, J_{C F}=\right.$ $272.1 \mathrm{~Hz}), 124.0\left(\mathrm{q}, J_{C F}=3.8 \mathrm{~Hz}\right), 115.4,115.1,72.5 ;{ }^{9} \mathrm{~F}$ NMR (470 MHz, $\left.d_{8}-\mathrm{THF}\right)-63.67$; IR (neat, $\mathrm{cm}^{-1}$ ) 3348, 3065, 3032, 2957, 2926, 2857, 1611, 1427, 1335, 1165, 1125, 1092, 1074, 1030; HRMS (ESI $\left.{ }^{-}\right) \mathrm{m} / z$ calcd for $\mathrm{C}_{20} \mathrm{H}_{15} \mathrm{~F}_{3} \mathrm{O}_{2} \mathrm{Na}[\mathrm{M}+\mathrm{H}]^{+}:$367.0916; found 367.0913.

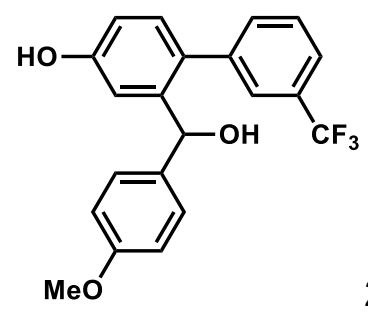

2-(hydroxy(4-methoxyphenyl)methyl)-3'-(trifluoromethyl)-[1,1'-biphenyl]-4-ol

$(5 \mathbf{q})$

White solid (64 mg, 46\%); m.p. = $169-171^{\circ} \mathrm{C} ;{ }^{1} \mathrm{H}$ NMR $\left(500 \mathrm{MHz}, d_{8}\right.$-THF) $8.40(\mathrm{~s}, 1 \mathrm{H}), 7.62-$ $7.46(\mathrm{~m}, 4 \mathrm{H}), 7.06-6.97(\mathrm{~m}, 4 \mathrm{H}), 6.77-6.69(\mathrm{~m}, 3 \mathrm{H}), 5.64(\mathrm{~d}, J=3.4 \mathrm{~Hz}, 1 \mathrm{H}), 4.78(\mathrm{~d}, J=3.9$ $\mathrm{Hz}, 1 \mathrm{H}), 3.69(\mathrm{~s}, 3 \mathrm{H}) ;{ }^{13} \mathrm{C}\left\{{ }^{1} \mathrm{H}\right\}$ NMR $\left(125 \mathrm{MHz}, d_{8}\right.$-THF) 159.6, 158.6, 145.1, 143.6, 138.1, 134.3 $\left(q, J_{C F}=1.2 \mathrm{~Hz}\right), 131.6,131.5,130.8\left(\mathrm{q}, J_{C F}=31.9 \mathrm{~Hz}\right), 129.4,128.6,127.3\left(\mathrm{q}, J_{C F}=4.0 \mathrm{~Hz}\right)$, $125.4\left(\mathrm{q}, J_{C F}=272.2 \mathrm{~Hz}\right), 123.9\left(\mathrm{q}, J_{C F}=3.6 \mathrm{~Hz}\right), 115.2,114.9,113.9,72.2,55.3 ;{ }^{19} \mathrm{~F}$ NMR $(470$ $\mathrm{MHz}, d_{8}$-THF) -63.64; IR (neat, $\mathrm{cm}^{-1}$ ) 3400, 2955, 2924, 2853, 1609, 1510, 1333, 1250, 1165, 1125, 1093, 1074, 1032; HRMS $\left(\mathrm{ESI}^{-}\right) \mathrm{m} / \mathrm{z}$ calcd for $\mathrm{C}_{21} \mathrm{H}_{16} \mathrm{~F}_{3} \mathrm{O}_{3}[\mathrm{M}-\mathrm{H}]^{-}:$:373.1057; found 373.1073. 


\section{Compound 16c Structure Assignment}

We were able to determine the position of the two $\mathrm{Cl}$ atoms in $16 \mathrm{c}$ by comparison of its ${ }^{1} \mathrm{H} \mathrm{NMR}$ spectrum to that of $\mathbf{1 2 b}$. Compound $\mathbf{1 2 b}$ has $\mathrm{C} 2$ and $\mathrm{C} 4$ hydrogen resonances at $7.20 \mathrm{ppm}$ (dd, $J=8.8,2.6 \mathrm{~Hz})$ and $8.08 \mathrm{ppm}(\mathrm{d}, J=2.6 \mathrm{~Hz})$, respectively. The absence of $\mathrm{C} 2$ and $\mathrm{C} 4$ hydrogen resonances in $16 \mathrm{c}$ indicated that the two $\mathrm{Cl}$ atoms are substituted on $\mathrm{C} 2$ and $\mathrm{C} 4$.
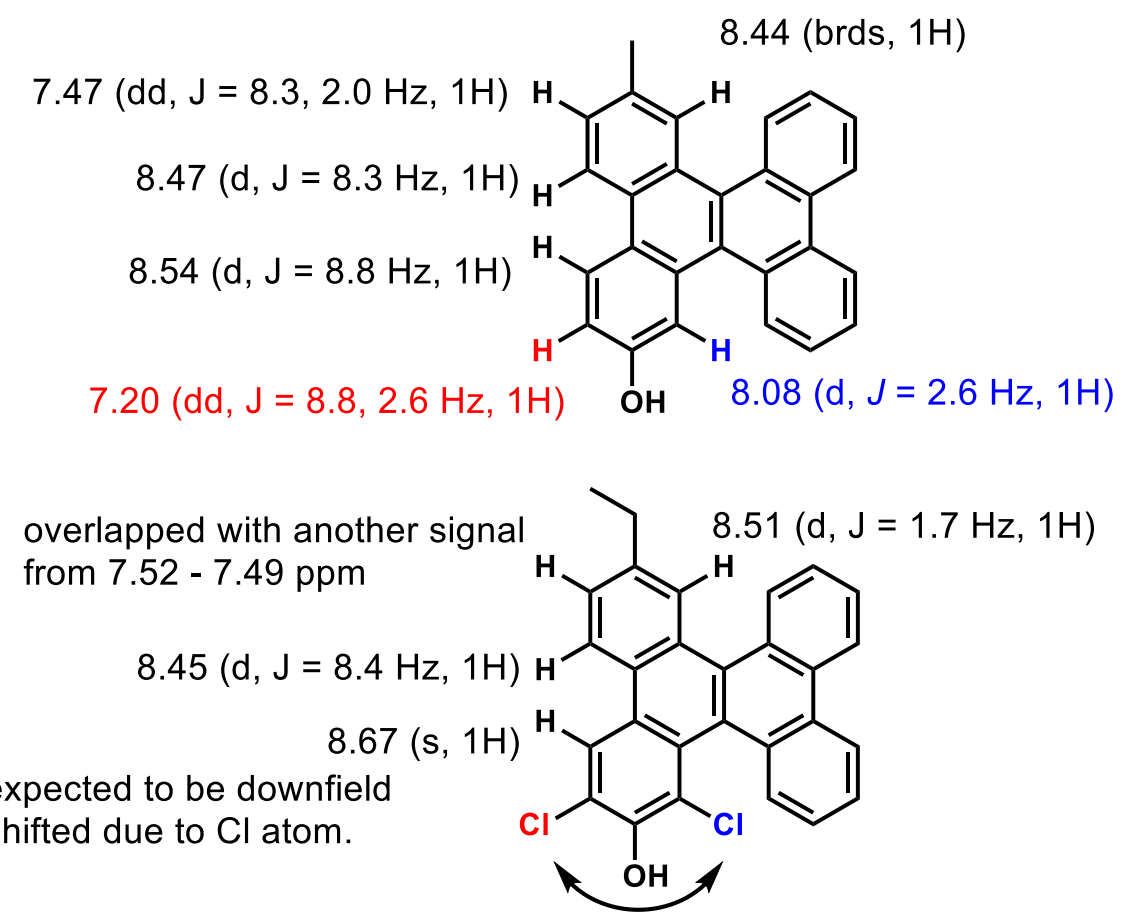

corresponding $1 \mathrm{H}$ signals are absent 
9. Postulated Reaction Mechanism for the Formation of 17a<smiles>COc1ccc2c3ccc(O)cc3c3c4ccccc4c4ccccc4c3c2c1</smiles><smiles>COC1(OC)C=CC2=c3c2c2ccc(=O)cc-2c2c4ccccc4c4ccccc4c2c3=C1</smiles><smiles></smiles><smiles></smiles>

$\downarrow \begin{gathered}\text { TFA } \\ -\mathrm{H}_{2} \mathrm{O}\end{gathered}$

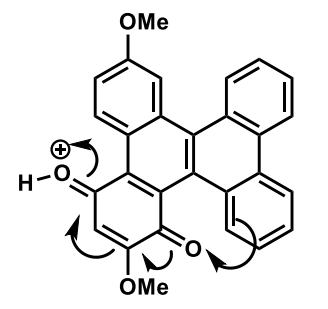<smiles>COC1=CC(=O)c2c(c3c4ccccc4c4ccccc4c3c3cc(OC)ccc23)C1=O</smiles>

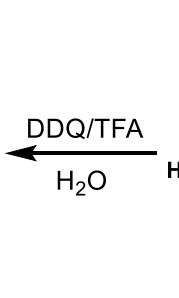<smiles>COc1ccc2c(c1)c1cc(OC)cc(O)c1c1c3ccccc3c3ccccc3c21</smiles><smiles></smiles>

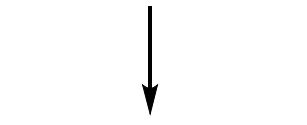<smiles></smiles><smiles></smiles>

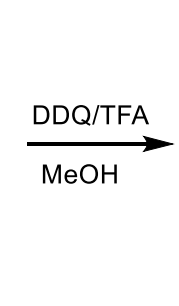<smiles></smiles> 


\section{X-Ray Crystallographic Data for 17b}

Crystals were obtained from a slow evaporation of a solution in toluene of the commingled products $17 \mathrm{a}$ and $\mathbf{1 7 b}$. Although two crystal habits were observed, only one type was suitable. The selected crystals were mounted using viscous oil onto a plastic mesh and cooled to the data collection temperature. Data were collected on a Bruker-AXS APEX II DUO CCD diffractometer with $\mathrm{Cu}$-Ka radiation $(\lambda=1.54178 \AA)$ focused with Goebel mirrors. Unit cell parameters were obtained from 48 data frames, $0.5^{\circ} \mathrm{\omega}$, from different sections of the Ewald sphere. The unit-cell dimensions, equivalent reflections and systematic absences in the diffraction data are consistent with $C c$, and $C 2 / c$ with the centrosymmetric space group option, $C 2 / c$, yielding chemically reasonable and computationally stable results of refinement. Because of the closeness of the $\beta$ angle to $90^{\circ}$, orthorhombic space groups were initially explored but were eventually discarded. No higher symmetry was suggested by ADDSYM. ${ }^{11}$ The data were treated with multi-scan absorption corrections. ${ }^{12}$ Structures were solved using intrinsic phasing methods ${ }^{13}$ and refined with full-matrix, least-squares procedures on $F^{2} .^{14}$

A toluene molecule of solvation was located disordered with a refined site occupancy of 90/10. Non-hydrogen atoms were refined with anisotropic displacement parameters. Hydrogen atoms on the compound molecule were located from the difference map and refined with isotropic parameters. Hydrogen atoms on the toluene molecule were treated as idealized contributions with geometrically calculated positions and with $U_{\text {iso }}$ equal to $1.2 U_{e q}\left(1.5 U_{e q}\right.$ for methyl) of the attached atom. Atomic scattering factors are contained in the SHELXTL program library. ${ }^{12}$ The structure has been deposited at the Cambridge Structural Database under CCDC 2098884.

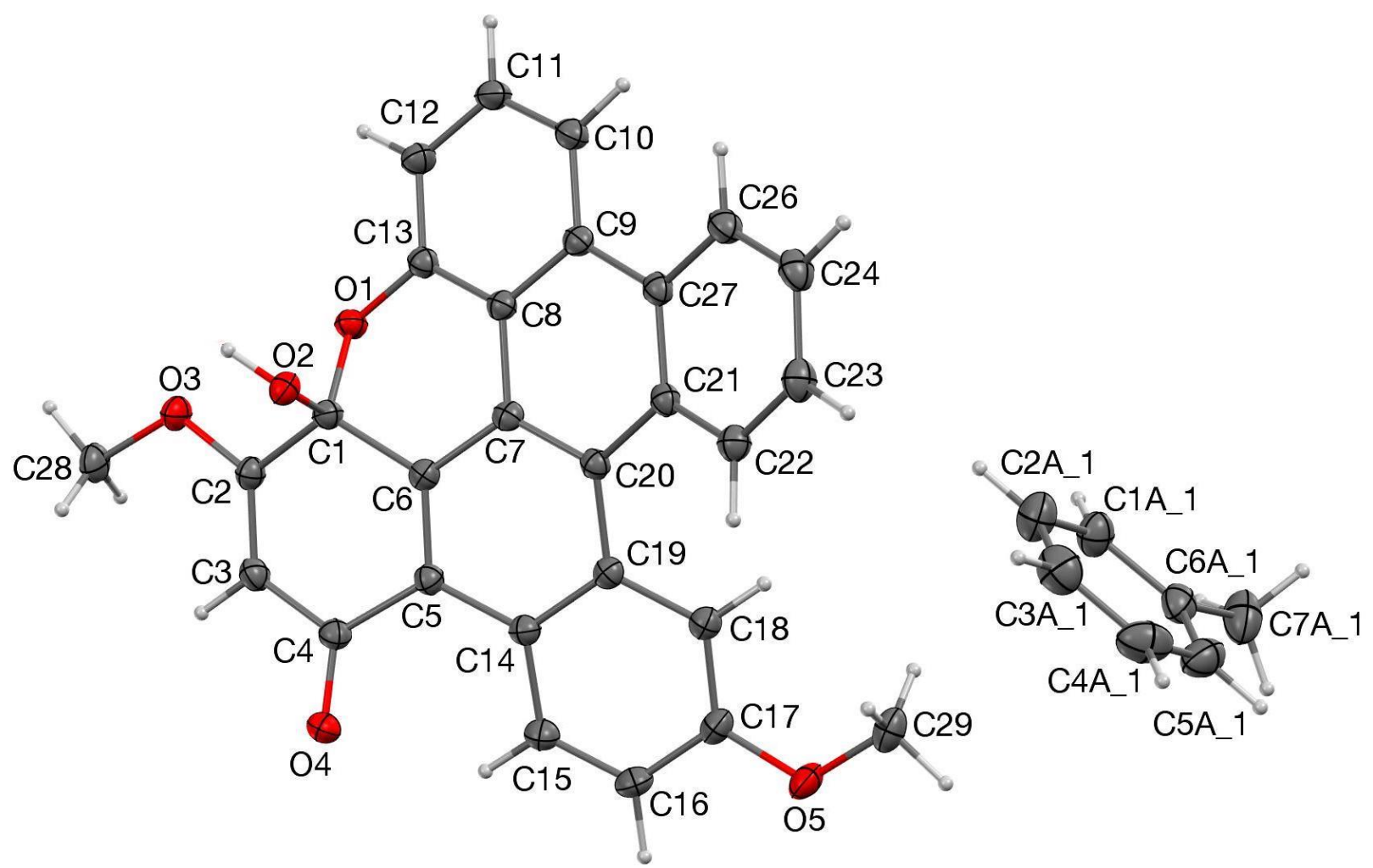

Figure S3: Molecular diagram and labelling scheme with $30 \%$ probability ellipsoids. $\mathrm{H}$-atoms depicted with arbitrary radius. Minor disordered contribution of the toluene solvent molecule is omitted for clarity. 
Table S3.

Crystal data and structure refinement for $\mathbf{1 7 b}$.

\begin{tabular}{|c|c|}
\hline \multicolumn{2}{|l|}{ Identification code } \\
\hline \multicolumn{2}{|r|}{ C35 H26 O5 } \\
\hline Formula weight & 526.56 \\
\hline Temperature & $100(2) \mathrm{K}$ \\
\hline Wavelength & $1.54178 \mathrm{~A}$ \\
\hline Crystal system, space group & Monoclinic, $\mathrm{C} 2 / \mathrm{c}$ \\
\hline \multicolumn{2}{|l|}{ Unit cell dimensions } \\
\hline \multicolumn{2}{|c|}{$\begin{array}{l}a=19.9030(8) \text { A } \quad \text { alpha }=90 \mathrm{deg} . \\
b=11.3141(5) A \quad \text { beta }=90.020(2) \mathrm{deg} . \\
c=22.0976(9) \text { A gamma }=90 \mathrm{deg} .\end{array}$} \\
\hline Volume & $4976.0(4) A^{\wedge} 3$ \\
\hline Z, Calculated density & $8,1.406 \mathrm{Mg} / \mathrm{m}^{\wedge} 3$ \\
\hline Absorption coefficient & $0.753 \mathrm{~mm}^{\wedge}-1$ \\
\hline$F(000)$ & 2208 \\
\hline Crystal size & $0.195 \times 0.184 \times 0.180 \mathrm{~mm}$ \\
\hline Theta range for data collection & 4.443 to $75.481 \mathrm{deg}$. \\
\hline Limiting indices & $-24<=h<=24,-14<=k<=14,-26<=\mid<=27$ \\
\hline Reflections collected / unique & $35825 / 5111[R($ int $)=0.0320]$ \\
\hline Completeness to theta $=67.679$ & $99.8 \%$ \\
\hline Absorption correction & Semi-empirical from equivalents \\
\hline Max. and min. transmission & 0.7539 and 0.7072 \\
\hline Refinement method & Full-matrix least-squares on $\mathrm{F}^{\wedge} 2$ \\
\hline Data / restraints / parameters & $5111 / 0 / 445$ \\
\hline Goodness-of-fit on $\mathrm{F}^{\wedge} 2$ & 1.040 \\
\hline Final $R$ indices [l>2sigma $(\mathrm{I})]$ & $\mathrm{R} 1=0.0356, \mathrm{wR} 2=0.0973$ \\
\hline $\mathrm{R}$ indices (all data) & $R 1=0.0378, w R 2=0.0994$ \\
\hline Extinction coefficient & $\mathrm{n} / \mathrm{a}$ \\
\hline Largest diff. peak and hole & 0.293 and -0.227 e. $A^{\wedge}-3$ \\
\hline
\end{tabular}

\section{Table S4.}

Atomic coordinates ( $\left.\times 10^{\wedge} 4\right)$ and equivalent isotropic displacement parameters $A^{\wedge} 2 \times 10^{\wedge} 3$ ) for 17b.

$\mathrm{U}(\mathrm{eq})$ is defined as one third of the trace of the orthogonalized Uij tensor.

\begin{tabular}{lllll}
\hline & $\mathrm{x}$ & $\mathrm{y}$ & $\mathrm{U}(\mathrm{eq})$ \\
& & & \\
\hline $\mathrm{O}(1)$ & $3332(1)$ & $5307(1)$ & $1798(1)$ & $19(1)$ \\
$\mathrm{O}(2)$ & $2648(1)$ & $4856(1)$ & $2619(1)$ & $19(1)$ \\
$\mathrm{O}(3)$ & $2453(1)$ & $3674(1)$ & $1471(1)$ & $21(1)$ \\
$\mathrm{O}(4)$ & $3496(1)$ & $1039(1)$ & $2820(1)$ & $24(1)$ \\
$\mathrm{O}(5)$ & $6170(1)$ & $2339(1)$ & $4305(1)$ & $25(1)$ \\
$\mathrm{C}(1)$ & $3157(1)$ & $4427(1)$ & $2238(1)$ & $17(1)$ \\
$\mathrm{C}(2)$ & $2897(1)$ & $3363(1)$ & $1898(1)$ & $18(1)$
\end{tabular}




$\begin{array}{lcccc}\text { C(3) } & 3051(1) & 2263(1) & 2072(1) & 20(1) \\ \text { C(4) } & 3492(1) & 2032(1) & 2585(1) & 19(1) \\ \text { C(5) } & 3918(1) & 3024(1) & 2812(1) & 17(1) \\ \text { C(6) } & 3755(1) & 4149(1) & 2633(1) & 16(1) \\ \text { C(7) } & 4098(1) & 5154(1) & 2861(1) & 16(1) \\ \text { C(8) } & 3959(1) & 6287(1) & 2590(1) & 17(1) \\ \text { C(9) } & 4232(1) & 7333(1) & 2832(1) & 18(1) \\ \text { C(10) } & 4117(1) & 8402(1) & 2524(1) & 21(1) \\ \text { C(11) } & 3751(1) & 8421(1) & 1995(1) & 22(1) \\ \text { C(12) } & 3482(1) & 7385(1) & 1749(1) & 21(1) \\ \text { C(13) } & 3586(1) & 6334(1) & 2050(1) & 18(1) \\ \text { C(14) } & 4489(1) & 2861(1) & 3205(1) & 18(1) \\ \text { C(15) } & 4763(1) & 1726(1) & 3328(1) & 23(1) \\ \text { C(16) } & 5311(1) & 1582(1) & 3694(1) & 24(1) \\ \text { C(17) } & 5629(1) & 2577(1) & 3946(1) & 21(1) \\ \text { C(18) } & 5397(1) & 3694(1) & 3820(1) & 19(1) \\ \text { C(19) } & 4811(1) & 3871(1) & 3465(1) & 17(1) \\ \text { C(20) } & 4560(1) & 5043(1) & 3333(1) & 16(1) \\ \text { C(21) } & 4737(1) & 6125(1) & 3663(1) & 17(1) \\ \text { C(22) } & 4985(1) & 6099(1) & 4263(1) & 21(1) \\ \text { C(23) } & 5125(1) & 7125(1) & 4577(1) & 24(1) \\ \text { C(24) } & 5017(1) & 8222(1) & 4309(1) & 25(1) \\ \text { C(26) } & 4743(1) & 8277(1) & 3739(1) & 23(1) \\ \text { C(27) } & 4586(1) & 7248(1) & 3408(1) & 18(1) \\ \text { C(28) } & 2122(1) & 2718(1) & 1160(1) & 24(1) \\ \text { C(29) } & 6452(1) & 3319(1) & 4630(1) & 27(1) \\ \text { C1A1 } & 7460(1) & 6011(1) & 4596(1) & 29(1) \\ \text { C2A1 } & 6852(1) & 6244(1) & 4876(1) & 34(1) \\ \text { C3A1 } & 6769(1) & 6031(2) & 5487(1) & 36(1) \\ \text { C4A1 } & 7303(1) & 5579(1) & 5819(1) & 38(1) \\ \text { C5A1 } & 7911(1) & 5350(1) & 5536(1) & 34(1) \\ \text { C6A1 } & 8001(1) & 5569(1) & 4923(1) & 27(1) \\ \text { C7A1 } & 8667(1) & 5370(2) & 4619(1) & 40(1) \\ \text { C1B1 } & 7329(4) & 6243(9) & 4741(4) & 29(1) \\ \text { C2B1 } & 7839(5) & 6124(9) & 4316(3) & 34(1) \\ \text { C3B1 } & 8425(4) & 5520(9) & 4465(4) & 36(1) \\ \text { C4B1 } & 8500(4) & 5035(9) & 5039(4) & 38(1) \\ \text { C5B1 } & 7989(5) & 5154(9) & 5464(3) & 34(1) \\ \text { C6B1 } & 7404(4) & 5758(9) & 5315(4) & 27(1) \\ \text { C7B1 } & 6902(9) & 5912(17) & 5745(9) & 40(1)\end{array}$


Table S5.

Bond lengths [A] and angles [deg] for 17b.

\begin{tabular}{|c|c|}
\hline $\mathrm{O}(1)-\mathrm{C}(13)$ & $1.3836(12)$ \\
\hline $\mathrm{O}(1)-\mathrm{C}(1)$ & $1.4343(12)$ \\
\hline $\mathrm{O}(2)-\mathrm{C}(1)$ & $1.4044(12)$ \\
\hline $\mathrm{O}(2)-\mathrm{H}(1)$ & $0.895(18)$ \\
\hline $\mathrm{O}(3)-\mathrm{C}(2)$ & $1.3389(13)$ \\
\hline $\mathrm{O}(3)-\mathrm{C}(28)$ & $1.4408(13)$ \\
\hline $\mathrm{O}(4)-\mathrm{C}(4)$ & $1.2377(13)$ \\
\hline $\mathrm{O}(5)-\mathrm{C}(17)$ & $1.3641(13)$ \\
\hline $\mathrm{O}(5)-\mathrm{C}(29)$ & $1.4345(15)$ \\
\hline$C(1)-C(2)$ & $1.5100(14)$ \\
\hline$C(1)-C(6)$ & $1.5105(14)$ \\
\hline$C(2)-C(3)$ & $1.3393(15)$ \\
\hline$C(3)-C(4)$ & $1.4558(15)$ \\
\hline $\mathrm{C}(3)-\mathrm{H}(2)$ & $0.963(16)$ \\
\hline$C(4)-C(5)$ & $1.4938(14)$ \\
\hline$C(5)-C(6)$ & $1.3721(14)$ \\
\hline$C(5)-C(14)$ & $1.4397(14)$ \\
\hline$C(6)-C(7)$ & $1.4185(14)$ \\
\hline$C(7)-C(20)$ & $1.3959(14)$ \\
\hline $\mathrm{C}(7)-\mathrm{C}(8)$ & $1.4418(14)$ \\
\hline$C(8)-C(13)$ & $1.4064(15)$ \\
\hline$C(8)-C(9)$ & $1.4072(14)$ \\
\hline$C(9)-C(10)$ & $1.4063(15)$ \\
\hline$C(9)-C(27)$ & $1.4588(15)$ \\
\hline$C(10)-C(11)$ & $1.3780(16)$ \\
\hline $\mathrm{C}(10)-\mathrm{H}(3)$ & $0.980(15)$ \\
\hline$C(11)-C(12)$ & $1.3983(16)$ \\
\hline $\mathrm{C}(11)-\mathrm{H}(4)$ & $0.972(14)$ \\
\hline$C(12)-C(13)$ & $1.3780(15)$ \\
\hline $\mathrm{C}(12)-\mathrm{H}(5)$ & $0.982(15)$ \\
\hline$C(14)-C(15)$ & $1.4211(15)$ \\
\hline$C(14)-C(19)$ & $1.4308(14)$ \\
\hline$C(15)-C(16)$ & $1.3668(16)$ \\
\hline $\mathrm{C}(15)-\mathrm{H}(6)$ & $0.983(15)$ \\
\hline$C(16)-C(17)$ & $1.4062(16)$ \\
\hline $\mathrm{C}(16)-\mathrm{H}(7)$ & $1.014(16)$ \\
\hline$C(17)-C(18)$ & $1.3748(15)$ \\
\hline$C(18)-C(19)$ & $1.4209(14)$ \\
\hline $\mathrm{C}(18)-\mathrm{H}(8)$ & $0.945(14)$ \\
\hline$C(19)-C(20)$ & $1.4458(14)$ \\
\hline$C(20)-C(21)$ & 1.4684(14) \\
\hline$C(21)-C(22)$ & $1.4135(15)$ \\
\hline$C(21)-C(27)$ & 1.4218(14) \\
\hline$C(22)-C(23)$ & $1.3815(15)$ \\
\hline
\end{tabular}




\begin{tabular}{|c|c|}
\hline $\mathrm{C}(22)-\mathrm{H}(9)$ & $0.977(14)$ \\
\hline$C(23)-C(24)$ & $1.3919(17)$ \\
\hline $\mathrm{C}(23)-\mathrm{H}(10)$ & $0.984(15)$ \\
\hline$C(24)-C(26)$ & $1.3742(17)$ \\
\hline $\mathrm{C}(24)-\mathrm{H}(11)$ & $0.985(16)$ \\
\hline$C(26)-C(27)$ & $1.4097(15)$ \\
\hline $\mathrm{C}(26)-\mathrm{H}(12)$ & $0.980(15)$ \\
\hline $\mathrm{C}(28)-\mathrm{H}(13)$ & $0.993(16)$ \\
\hline $\mathrm{C}(28)-\mathrm{H}(14)$ & $0.960(15)$ \\
\hline $\mathrm{C}(28)-\mathrm{H}(15)$ & $0.974(15)$ \\
\hline $\mathrm{C}(29)-\mathrm{H}(16)$ & $0.981(15)$ \\
\hline $\mathrm{C}(29)-\mathrm{H}(17)$ & $0.978(15)$ \\
\hline $\mathrm{C}(29)-\mathrm{H}(18)$ & $0.990(15)$ \\
\hline C1A1-C2A1 & $1.383(2)$ \\
\hline C1A1-C6A1 & $1.3889(19)$ \\
\hline $\mathrm{C} 1 \mathrm{~A} 1-\mathrm{H} 1 \mathrm{~A} 1$ & 0.9500 \\
\hline C2A1-C3A1 & $1.382(2)$ \\
\hline $\mathrm{C} 2 \mathrm{~A} 1-\mathrm{H} 2 \mathrm{~A} 1$ & 0.9500 \\
\hline C3A1-C4A1 & $1.389(3)$ \\
\hline C3A1-H3A1 & 0.9500 \\
\hline C4A1-C5A1 & $1.388(3)$ \\
\hline $\mathrm{C} 4 \mathrm{~A} 1-\mathrm{H} 4 \mathrm{~A} 1$ & 0.9500 \\
\hline C5A1-C6A1 & $1.388(2)$ \\
\hline C5A1-H5A1 & 0.9500 \\
\hline C6A1-C7A1 & $1.503(2)$ \\
\hline C7A1-H7A1 & 0.9800 \\
\hline C7A1-H7B1 & 0.9800 \\
\hline C7A1-H7C1 & 0.9800 \\
\hline C1B1-C2B1 & 1.3900 \\
\hline C1B1-C6B1 & 1.3900 \\
\hline C1B1-H1B1 & 0.9500 \\
\hline C2B1-C3B1 & 1.3900 \\
\hline C2B1-H2B1 & 0.9500 \\
\hline C3B1-C4B1 & 1.3900 \\
\hline C3B1-H3B1 & 0.9500 \\
\hline C4B1-C5B1 & 1.3900 \\
\hline C4B1-H4B1 & 0.9500 \\
\hline C5B1-C6B1 & 1.3900 \\
\hline C5B1-H5B1 & 0.9500 \\
\hline C6B1-C7B1 & $1.390(19)$ \\
\hline C7B1-H7D1 & 0.9800 \\
\hline C7B1-H7E1 & 0.9800 \\
\hline C7B1-H7F1 & 0.9800 \\
\hline $\mathrm{C}(13)-\mathrm{O}(1)-\mathrm{C}(1)$ & $113.50(8)$ \\
\hline $\mathrm{C}(1)-\mathrm{O}(2)-\mathrm{H}(1)$ & $108.5(11)$ \\
\hline$C(2)-O(3)-C(28)$ & $116.15(8)$ \\
\hline $\mathrm{C}(17)-\mathrm{O}(5)-\mathrm{C}(29)$ & $116.56(9)$ \\
\hline
\end{tabular}




$\begin{array}{lc}\mathrm{O}(2)-\mathrm{C}(1)-\mathrm{O}(1) & 110.03(8) \\ \mathrm{O}(2)-\mathrm{C}(1)-\mathrm{C}(2) & 109.10(8) \\ \mathrm{O}(1)-\mathrm{C}(1)-\mathrm{C}(2) & 107.42(8) \\ \mathrm{O}(2)-\mathrm{C}(1)-\mathrm{C}(6) & 107.01(8) \\ \mathrm{O}(1)-\mathrm{C}(1)-\mathrm{C}(6) & 110.20(8) \\ \mathrm{C}(2)-\mathrm{C}(1)-\mathrm{C}(6) & 113.08(8) \\ \mathrm{O}(3)-\mathrm{C}(2)-\mathrm{C}(3) & 126.75(10) \\ \mathrm{O}(3)-\mathrm{C}(2)-\mathrm{C}(1) & 111.54(9) \\ \mathrm{C}(3)-\mathrm{C}(2)-\mathrm{C}(1) & 121.28(9) \\ \mathrm{C}(2)-\mathrm{C}(3)-\mathrm{C}(4) & 121.93(10) \\ \mathrm{C}(2)-\mathrm{C}(3)-\mathrm{H}(2) & 122.1(9) \\ \mathrm{C}(4)-\mathrm{C}(3)-\mathrm{H}(2) & 115.6(9) \\ \mathrm{O}(4)-\mathrm{C}(4)-\mathrm{C}(3) & 119.56(10) \\ \mathrm{O}(4)-\mathrm{C}(4)-\mathrm{C}(5) & 122.44(10) \\ \mathrm{C}(3)-\mathrm{C}(4)-\mathrm{C}(5) & 118.00(9) \\ \mathrm{C}(6)-\mathrm{C}(5)-\mathrm{C}(14) & 118.60(9) \\ \mathrm{C}(6)-\mathrm{C}(5)-\mathrm{C}(4) & 117.73(9) \\ \mathrm{C}(14)-\mathrm{C}(5)-\mathrm{C}(4) & 123.67(9) \\ \mathrm{C}(5)-\mathrm{C}(6)-\mathrm{C}(7) & 121.86(9) \\ \mathrm{C}(5)-\mathrm{C}(6)-\mathrm{C}(1) & 123.14(9) \\ \mathrm{C}(7)-\mathrm{C}(6)-\mathrm{C}(1) & 114.68(9) \\ \mathrm{C}(20)-\mathrm{C}(7)-\mathrm{C}(6) & 120.65(9) \\ \mathrm{C}(20)-\mathrm{C}(7)-\mathrm{C}(8) & 121.12(9) \\ \mathrm{C}(6)-\mathrm{C}(7)-\mathrm{C}(8) & 118.23(9) \\ \mathrm{C}(13)-\mathrm{C}(8)-\mathrm{C}(9) & 119.63(9) \\ \mathrm{C}(13)-\mathrm{C}(8)-\mathrm{C}(7) & 119.17(9) \\ \mathrm{C}(9)-\mathrm{C}(8)-\mathrm{C}(7) & 121.03(9) \\ \mathrm{C}(10)-\mathrm{C}(9)-\mathrm{C}(8) & 118.52(10) \\ \mathrm{C}(10)-\mathrm{C}(9)-\mathrm{C}(27) & 123.82(10) \\ \mathrm{C}(8)-\mathrm{C}(9)-\mathrm{C}(27) & 117.56(9) \\ \mathrm{C}(11)-\mathrm{C}(10)-\mathrm{C}(9) & 120.59(10) \\ \mathrm{C}(11)-\mathrm{C}(10)-\mathrm{H}(3) & 117.8(8) \\ \mathrm{C}(9)-\mathrm{C}(10)-\mathrm{H}(3) & 121.6(8) \\ \mathrm{C}(10)-\mathrm{C}(11)-\mathrm{C}(12) & 121.28(10) \\ \mathrm{C}(10)-\mathrm{C}(11)-\mathrm{H}(4) & 119.3(8) \\ \mathrm{C}(12)-\mathrm{C}(11)-\mathrm{H}(4) & 119.4(8) \\ \mathrm{C}(13)-\mathrm{C}(12)-\mathrm{C}(11) & 118.62(10) \\ \mathrm{C}(13)-\mathrm{C}(12)-\mathrm{H}(5) & 120.1(9) \\ \mathrm{C}(11)-\mathrm{C}(12)-\mathrm{H}(5) & 121.2(9) \\ \mathrm{C}(12)-\mathrm{C}(13)-\mathrm{O}(1) & 118.43(9) \\ \mathrm{C}(12)-\mathrm{C}(13)-\mathrm{C}(8) & 121.36(10) \\ \mathrm{O}(1)-\mathrm{C}(13)-\mathrm{C}(8) & 120.18(9) \\ \mathrm{C}(15)-\mathrm{C}(14)-\mathrm{C}(19) & 118.21(9) \\ \mathrm{C}(15)-\mathrm{C}(14)-\mathrm{C}(5) & 122.25(10) \\ \mathrm{C}(19)-\mathrm{C}(14)-\mathrm{C}(5) & 119.50(9) \\ \mathrm{C}(16)-\mathrm{C}(15)-\mathrm{C}(14) & 121.83(10) \\ \mathrm{C}(16)-\mathrm{C}(15)-\mathrm{H}(6) & 121.1(9) \\ \mathrm{C}(14)-\mathrm{C}(15)-\mathrm{H}(6) & 117.1(9) \\ & \end{array}$




$\begin{array}{lc}\mathrm{C}(15)-\mathrm{C}(16)-\mathrm{C}(17) & 119.90(10) \\ \mathrm{C}(15)-\mathrm{C}(16)-\mathrm{H}(7) & 121.0(9) \\ \mathrm{C}(17)-\mathrm{C}(16)-\mathrm{H}(7) & 119.1(9) \\ \mathrm{O}(5)-\mathrm{C}(17)-\mathrm{C}(18) & 124.34(10) \\ \mathrm{O}(5)-\mathrm{C}(17)-\mathrm{C}(16) & 115.39(10) \\ \mathrm{C}(18)-\mathrm{C}(17)-\mathrm{C}(16) & 120.26(10) \\ \mathrm{C}(17)-\mathrm{C}(18)-\mathrm{C}(19) & 121.16(10) \\ \mathrm{C}(17)-\mathrm{C}(18)-\mathrm{H}(8) & 119.5(8) \\ \mathrm{C}(19)-\mathrm{C}(18)-\mathrm{H}(8) & 119.3(8) \\ \mathrm{C}(18)-\mathrm{C}(19)-\mathrm{C}(14) & 118.49(9) \\ \mathrm{C}(18)-\mathrm{C}(19)-\mathrm{C}(20) & 121.55(9) \\ \mathrm{C}(14)-\mathrm{C}(19)-\mathrm{C}(20) & 119.85(9) \\ \mathrm{C}(7)-\mathrm{C}(20)-\mathrm{C}(19) & 117.37(9) \\ \mathrm{C}(7)-\mathrm{C}(20)-\mathrm{C}(21) & 117.01(9) \\ \mathrm{C}(19)-\mathrm{C}(20)-\mathrm{C}(21) & 125.60(9) \\ \mathrm{C}(22)-\mathrm{C}(21)-\mathrm{C}(27) & 117.65(9) \\ \mathrm{C}(22)-\mathrm{C}(21)-\mathrm{C}(20) & 122.15(9) \\ \mathrm{C}(27)-\mathrm{C}(21)-\mathrm{C}(20) & 119.80(9) \\ \mathrm{C}(23)-\mathrm{C}(22)-\mathrm{C}(21) & 121.62(10) \\ \mathrm{C}(23)-\mathrm{C}(22)-\mathrm{H}(9) & 119.3(8) \\ \mathrm{C}(21)-\mathrm{C}(22)-\mathrm{H}(9) & 119.0(8) \\ \mathrm{C}(22)-\mathrm{C}(23)-\mathrm{C}(24) & 120.24(10) \\ \mathrm{C}(22)-\mathrm{C}(23)-\mathrm{H}(10) & 120.2(8) \\ \mathrm{C}(24)-\mathrm{C}(23)-\mathrm{H}(10) & 119.5(8) \\ \mathrm{C}(26)-\mathrm{C}(24)-\mathrm{C}(23) & 119.47(10) \\ \mathrm{C}(26)-\mathrm{C}(24)-\mathrm{H}(11) & 119.4(9) \\ \mathrm{C}(23)-\mathrm{C}(24)-\mathrm{H}(11) & 121.1(9) \\ \mathrm{C}(24)-\mathrm{C}(26)-\mathrm{C}(27) & 121.74(11) \\ \mathrm{C}(24)-\mathrm{C}(26)-\mathrm{H}(12) & 117.4(8) \\ \mathrm{C}(27)-\mathrm{C}(26)-\mathrm{H}(12) & 120.8(8) \\ \mathrm{C}(26)-\mathrm{C}(27)-\mathrm{C}(21) & 119.02(10) \\ \mathrm{C}(26)-\mathrm{C}(27)-\mathrm{C}(9) & 120.36(10) \\ \mathrm{C}(21)-\mathrm{C}(27)-\mathrm{C}(9) & 120.48(9) \\ \mathrm{O}(3)-\mathrm{C}(28)-\mathrm{H}(13) & 104.4(9) \\ \mathrm{O}(3)-\mathrm{C}(28)-\mathrm{H}(14) & 109.7(9) \\ \mathrm{H}(13)-\mathrm{C}(28)-\mathrm{H}(14) & 112.0(12) \\ \mathrm{O}(3)-\mathrm{C}(28)-\mathrm{H}(15) & 110.2(8) \\ \mathrm{H}(13)-\mathrm{C}(28)-\mathrm{H}(15) & 109.6(12) \\ \mathrm{H}(14)-\mathrm{C}(28)-\mathrm{H}(15) & 110.8(12) \\ \mathrm{O}(5)-\mathrm{C}(29)-\mathrm{H}(16) & 110.5(8) \\ \mathrm{O}(5)-\mathrm{C}(29)-\mathrm{H}(17) & 110.7(8) \\ \mathrm{H}(16)-\mathrm{C}(29)-\mathrm{H}(17) & 111.2(12) \\ \mathrm{O}(5)-\mathrm{C}(29)-\mathrm{H}(18) & 104.6(9) \\ \mathrm{H}(16)-\mathrm{C}(29)-\mathrm{H}(18) & 109.6(12) \\ \mathrm{H}(17)-\mathrm{C}(29)-\mathrm{H}(18) & 110.0(12) \\ \mathrm{C} 2 \mathrm{~A} 1-\mathrm{C} 1 \mathrm{~A} 1-\mathrm{C} 6 \mathrm{~A} 1 & 121.00(14) \\ \mathrm{C} 2 \mathrm{~A} 1-\mathrm{C} 1 \mathrm{~A} 1-\mathrm{H} 1 \mathrm{~A} 1 & 119.5 \\ \mathrm{C} 6 \mathrm{~A} 1-\mathrm{C} 1 \mathrm{~A} 1-\mathrm{H} 1 \mathrm{~A} 1 & 119.5 \\ & \end{array}$




\begin{tabular}{|c|c|}
\hline C3A1-C2A1-C1A1 & $120.53(14)$ \\
\hline $\mathrm{C} 3 \mathrm{~A} 1-\mathrm{C} 2 \mathrm{~A} 1-\mathrm{H} 2 \mathrm{~A} 1$ & 119.7 \\
\hline $\mathrm{C} 1 \mathrm{~A} 1-\mathrm{C} 2 \mathrm{~A} 1-\mathrm{H} 2 \mathrm{~A} 1$ & 119.7 \\
\hline C2A1-C3A1-C4A1 & $119.22(15)$ \\
\hline C2A1-C3A1-H3A1 & 120.4 \\
\hline C4A1-C3A1-H3A1 & 120.4 \\
\hline C5A1-C4A1-C3A1 & $119.90(14)$ \\
\hline $\mathrm{C} 5 \mathrm{~A} 1-\mathrm{C} 4 \mathrm{~A} 1-\mathrm{H} 4 \mathrm{~A} 1$ & 120.0 \\
\hline C3A1-C4A1-H4A1 & 120.0 \\
\hline C4A1-C5A1-C6A1 & $121.23(15)$ \\
\hline C4A1-C5A1-H5A1 & 119.4 \\
\hline C6A1-C5A1-H5A1 & 119.4 \\
\hline C5A1-C6A1-C1A1 & $118.10(14)$ \\
\hline C5A1-C6A1-C7A1 & $121.49(14)$ \\
\hline C1A1-C6A1-C7A1 & $120.39(13)$ \\
\hline C6A1-C7A1-H7A1 & 109.5 \\
\hline C6A1-C7A1-H7B1 & 109.5 \\
\hline H7A1-C7A1-H7B1 & 109.5 \\
\hline C6A1-C7A1-H7C1 & 109.5 \\
\hline H7A1-C7A1-H7C1 & 109.5 \\
\hline H7B1-C7A1-H7C1 & 109.5 \\
\hline C2B1-C1B1-C6B1 & 120.0 \\
\hline C2B1-C1B1-H1B1 & 120.0 \\
\hline C6B1-C1B1-H1B1 & 120.0 \\
\hline C1B1-C2B1-C3B1 & 120.0 \\
\hline C1B1-C2B1-H2B1 & 120.0 \\
\hline C3B1-C2B1-H2B1 & 120.0 \\
\hline $\mathrm{C} 4 \mathrm{~B} 1-\mathrm{C} 3 \mathrm{~B} 1-\mathrm{C} 2 \mathrm{~B} 1$ & 120.0 \\
\hline C4B1-C3B1-H3B1 & 120.0 \\
\hline C2B1-C3B1-H3B1 & 120.0 \\
\hline C5B1-C4B1-C3B1 & 120.0 \\
\hline C5B1-C4B1-H4B1 & 120.0 \\
\hline C3B1-C4B1-H4B1 & 120.0 \\
\hline $\mathrm{C} 4 \mathrm{~B} 1-\mathrm{C} 5 \mathrm{~B} 1-\mathrm{C} 6 \mathrm{~B} 1$ & 120.0 \\
\hline C4B1-C5B1-H5B1 & 120.0 \\
\hline C6B1-C5B1-H5B1 & 120.0 \\
\hline C5B1-C6B1-C1B1 & 120.0 \\
\hline C5B1-C6B1-C7B1 & $120.1(11)$ \\
\hline C1B1-C6B1-C7B1 & $119.9(11)$ \\
\hline C6B1-C7B1-H7D1 & 109.5 \\
\hline C6B1-C7B1-H7E1 & 109.5 \\
\hline H7D1-C7B1-H7E1 & 109.5 \\
\hline C6B1-C7B1-H7F1 & 109.5 \\
\hline H7D1-C7B1-H7F1 & 109.5 \\
\hline H7E1-C7B1-H7F1 & 109.5 \\
\hline
\end{tabular}

Symmetry transformations used to generate equivalent atoms: 
Table S6.

Anisotropic displacement parameters $\left(\mathrm{A}^{\wedge} 2 \times 10^{\wedge} 3\right)$ for $\mathbf{1 7 b}$.

The anisotropic displacement factor exponent takes the form:

$-2 \mathrm{pi}^{\wedge} 2\left[\mathrm{~h}^{\wedge} 2 \mathrm{a}^{\star \wedge} 2 \mathrm{U} 11+\ldots+2 \mathrm{~h} \mathrm{k} \mathrm{a}^{*} \mathrm{~b}^{*} \mathrm{U} 12\right.$ ]

\begin{tabular}{|c|c|c|c|c|c|c|}
\hline & U11 & U22 & U33 & U23 & U13 & U12 \\
\hline $\mathrm{O}(1)$ & $23(1)$ & $16(1)$ & $17(1)$ & $1(1)$ & $-3(1)$ & $-1(1)$ \\
\hline $\mathrm{O}(2)$ & $17(1)$ & $20(1)$ & $19(1)$ & $-1(1)$ & $-2(1)$ & $3(1)$ \\
\hline $\mathrm{O}(3)$ & $22(1)$ & $21(1)$ & $19(1)$ & $-1(1)$ & $-7(1)$ & $-1(1)$ \\
\hline $\mathrm{O}(4)$ & $26(1)$ & $16(1)$ & $32(1)$ & $3(1)$ & $-7(1)$ & $-2(1)$ \\
\hline $\mathrm{O}(5)$ & $22(1)$ & $29(1)$ & $26(1)$ & $2(1)$ & $-8(1)$ & $6(1)$ \\
\hline$C(1)$ & $18(1)$ & $16(1)$ & $17(1)$ & $1(1)$ & $-1(1)$ & $1(1)$ \\
\hline $\mathrm{C}(2)$ & $17(1)$ & $20(1)$ & $16(1)$ & $0(1)$ & $-1(1)$ & $0(1)$ \\
\hline$C(3)$ & $21(1)$ & $18(1)$ & $21(1)$ & $-2(1)$ & $-2(1)$ & $-4(1)$ \\
\hline$C(4)$ & $19(1)$ & $16(1)$ & $22(1)$ & $-1(1)$ & $0(1)$ & $1(1)$ \\
\hline$C(5)$ & $17(1)$ & $17(1)$ & $18(1)$ & $-1(1)$ & $-1(1)$ & $0(1)$ \\
\hline$C(6)$ & $16(1)$ & $17(1)$ & $16(1)$ & $0(1)$ & $0(1)$ & $0(1)$ \\
\hline $\mathrm{C}(7)$ & $16(1)$ & $16(1)$ & $17(1)$ & $-1(1)$ & $2(1)$ & $1(1)$ \\
\hline $\mathrm{C}(8)$ & $15(1)$ & $16(1)$ & $19(1)$ & $0(1)$ & $2(1)$ & $1(1)$ \\
\hline $\mathrm{C}(9)$ & $16(1)$ & $16(1)$ & $22(1)$ & $0(1)$ & $2(1)$ & $1(1)$ \\
\hline$C(10)$ & $21(1)$ & $16(1)$ & $27(1)$ & $0(1)$ & $0(1)$ & $-1(1)$ \\
\hline$C(11)$ & $22(1)$ & $17(1)$ & $27(1)$ & $6(1)$ & $0(1)$ & $2(1)$ \\
\hline $\mathrm{C}(12)$ & $22(1)$ & $21(1)$ & $20(1)$ & $3(1)$ & $-1(1)$ & $1(1)$ \\
\hline$C(13)$ & $17(1)$ & $16(1)$ & $20(1)$ & $-1(1)$ & 1(1) & $0(1)$ \\
\hline$C(14)$ & $18(1)$ & $16(1)$ & $18(1)$ & 1(1) & $0(1)$ & $1(1)$ \\
\hline$C(15)$ & $23(1)$ & $17(1)$ & $27(1)$ & $0(1)$ & $-3(1)$ & $1(1)$ \\
\hline$C(16)$ & $24(1)$ & $19(1)$ & $29(1)$ & $4(1)$ & $-3(1)$ & $5(1)$ \\
\hline $\mathrm{C}(17)$ & $17(1)$ & $26(1)$ & $19(1)$ & 2(1) & $-2(1)$ & $4(1)$ \\
\hline$C(18)$ & $17(1)$ & $21(1)$ & $18(1)$ & $0(1)$ & $0(1)$ & $0(1)$ \\
\hline $\mathrm{C}(19)$ & $16(1)$ & $18(1)$ & $16(1)$ & $0(1)$ & $1(1)$ & $1(1)$ \\
\hline$C(20)$ & $15(1)$ & $17(1)$ & $17(1)$ & $0(1)$ & 1(1) & $-1(1)$ \\
\hline $\mathrm{C}(21)$ & $15(1)$ & $18(1)$ & $19(1)$ & $-2(1)$ & $1(1)$ & $-1(1)$ \\
\hline $\mathrm{C}(22)$ & $20(1)$ & $22(1)$ & $20(1)$ & $-1(1)$ & $0(1)$ & $0(1)$ \\
\hline$C(23)$ & $22(1)$ & $28(1)$ & $20(1)$ & $-6(1)$ & $-1(1)$ & $-1(1)$ \\
\hline$C(24)$ & $25(1)$ & $23(1)$ & $28(1)$ & $-9(1)$ & $0(1)$ & $-3(1)$ \\
\hline$C(26)$ & $22(1)$ & $18(1)$ & $29(1)$ & $-3(1)$ & $0(1)$ & $-1(1)$ \\
\hline $\mathrm{C}(27)$ & $15(1)$ & $18(1)$ & $22(1)$ & $-2(1)$ & $1(1)$ & $-1(1)$ \\
\hline$C(28)$ & $23(1)$ & $27(1)$ & $23(1)$ & $-4(1)$ & $-6(1)$ & $-3(1)$ \\
\hline$C(29)$ & $21(1)$ & $37(1)$ & $23(1)$ & $-1(1)$ & $-5(1)$ & $2(1)$ \\
\hline $\mathrm{C} 1 \mathrm{~A} 1$ & $28(1)$ & $36(1)$ & $22(1)$ & $-1(1)$ & $-4(1)$ & $-1(1)$ \\
\hline $\mathrm{C} 2 \mathrm{~A} 1$ & $26(1)$ & $44(1)$ & $34(1)$ & $0(1)$ & $-4(1)$ & $1(1)$ \\
\hline C3A1 & $34(1)$ & $37(1)$ & $36(1)$ & $-3(1)$ & $5(1)$ & $-2(1)$ \\
\hline C4A1 & $59(1)$ & $32(1)$ & $23(1)$ & $2(1)$ & $2(1)$ & $0(1)$ \\
\hline C5A1 & $44(1)$ & $28(1)$ & $31(1)$ & $-1(1)$ & $-12(1)$ & $6(1)$ \\
\hline C6A1 & $27(1)$ & $23(1)$ & $30(1)$ & $-7(1)$ & $-6(1)$ & $-1(1)$ \\
\hline C7A1 & $27(1)$ & $44(1)$ & $49(1)$ & $-16(1)$ & $-6(1)$ & $3(1)$ \\
\hline
\end{tabular}




$\begin{array}{llllllc}\text { C1B1 } & 28(1) & 36(1) & 22(1) & -1(1) & -4(1) & -1(1) \\ \text { C2B1 } & 26(1) & 44(1) & 34(1) & 0(1) & -4(1) & 1(1) \\ \text { C3B1 } & 34(1) & 37(1) & 36(1) & -3(1) & 5(1) & -2(1) \\ \text { C4B1 } & 59(1) & 32(1) & 23(1) & 2(1) & 2(1) & 0(1) \\ \text { C5B1 } & 44(1) & 28(1) & 31(1) & -1(1) & -12(1) & 6(1) \\ \text { C6B1 } & 27(1) & 23(1) & 30(1) & -7(1) & -6(1) & -1(1) \\ \text { C7B1 } & 27(1) & 44(1) & 49(1) & -16(1) & -6(1) & 3(1)\end{array}$

Table S7.

Hydrogen coordinates ( $\left.\times 10^{\wedge} 4\right)$ and isotropic displacement parameters $\left(A^{\wedge} 2 \times 10^{\wedge} 3\right)$ for $\mathbf{1 7 b}$.

\begin{tabular}{|c|c|c|c|c|}
\hline & $x$ & $z$ & $\mathrm{U}(\mathrm{eq})$ & \\
\hline$H(1)$ & $2294(9)$ & $5054(16)$ & 2392(8) & $47(5)$ \\
\hline $\mathrm{H}(2)$ & $2840(8)$ & $1578(14)$ & $1896(7)$ & $30(4)$ \\
\hline $\mathrm{H}(3)$ & $4290(7)$ & $9155(13)$ & $2678(6)$ & $26(3)$ \\
\hline $\mathrm{H}(4)$ & $3677(7)$ & $9169(13)$ & $1790(6)$ & $23(3)$ \\
\hline $\mathrm{H}(5)$ & $3220(7)$ & $7399(13)$ & $1373(7)$ & $29(4)$ \\
\hline$H(6)$ & $4546(8)$ & $1045(13)$ & $3133(7)$ & $31(4)$ \\
\hline $\mathrm{H}(7)$ & $5497(8)$ & $767(14)$ & $3782(7)$ & $33(4)$ \\
\hline $\mathrm{H}(8)$ & $5648(7)$ & $4358(13)$ & $3949(6)$ & $22(3)$ \\
\hline $\mathrm{H}(9)$ & $5030(7)$ & $5339(13)$ & $4468(6)$ & $24(3)$ \\
\hline$H(10)$ & $5288(7)$ & $7087(12)$ & $4998(7)$ & $26(3)$ \\
\hline$H(11)$ & $5119(8)$ & $8961(14)$ & $4526(7)$ & $34(4)$ \\
\hline$H(12)$ & $4636(7)$ & $9062(13)$ & $3578(7)$ & $29(4)$ \\
\hline$H(13)$ & $1825(8)$ & $3116(14)$ & $861(7)$ & $34(4)$ \\
\hline$H(14)$ & $1872(7)$ & $2253(13)$ & $1444(7)$ & $30(4)$ \\
\hline$H(15)$ & $2451(7)$ & $2233(13)$ & $948(6)$ & $25(3)$ \\
\hline$H(16)$ & $6112(7)$ & $3686(12)$ & $4890(6)$ & $24(3)$ \\
\hline $\mathrm{H}(17)$ & $6646(7)$ & $3895(12)$ & $4349(6)$ & $24(3)$ \\
\hline $\mathrm{H}(18)$ & $6811(8)$ & $2967(13)$ & $4883(7)$ & $31(4)$ \\
\hline H1A1 & 7508 & 6155 & 4175 & 35 \\
\hline $\mathrm{H} 2 \mathrm{~A} 1$ & 6489 & 6553 & 4645 & 41 \\
\hline H3A1 & 6351 & 6192 & 5677 & 43 \\
\hline $\mathrm{H} 4 \mathrm{~A} 1$ & 7251 & 5426 & 6239 & 46 \\
\hline H5A1 & 8274 & 5038 & 5766 & 41 \\
\hline H7A1 & 8595 & 5220 & 4187 & 60 \\
\hline H7B1 & 8890 & 4687 & 4803 & 60 \\
\hline $\mathrm{H} 7 \mathrm{C} 1$ & 8949 & 6073 & 4669 & 60 \\
\hline H1B1 & 6929 & 6655 & 4639 & 35 \\
\hline H2B1 & 7788 & 6455 & 3924 & 41 \\
\hline H3B1 & 8773 & 5439 & 4175 & 43 \\
\hline H4B1 & 8900 & 4623 & 5141 & 46 \\
\hline H5B1 & 8040 & 4823 & 5856 & 41 \\
\hline H7D1 & 7095 & 5834 & 6151 & 60 \\
\hline
\end{tabular}




$\begin{array}{lllll}\text { H7E1 } & 6554 & 5311 & 5688 & 60 \\ \text { H7F1 } & 6704 & 6700 & 5700 & 60\end{array}$

Table S8.

Torsion angles [deg] for $\mathbf{1 7 b}$.

\begin{tabular}{lc}
\hline$C(13)-O(1)-C(1)-O(2)$ & $61.95(10)$ \\
$C(13)-O(1)-C(1)-C(2)$ & $-179.41(8)$ \\
$C(13)-O(1)-C(1)-C(6)$ & $-55.82(11)$ \\
$C(28)-O(3)-C(2)-C(3)$ & $-1.52(15)$ \\
$C(28)-O(3)-C(2)-C(1)$ & $-173.93(9)$ \\
$O(2)-C(1)-C(2)-O(3)$ & $72.62(10)$ \\
$O(1)-C(1)-C(2)-O(3)$ & $-46.63(11)$ \\
$C(6)-C(1)-C(2)-O(3)$ & $-168.43(8)$ \\
$O(2)-C(1)-C(2)-C(3)$ & $-100.27(11)$ \\
$O(1)-C(1)-C(2)-C(3)$ & $140.48(10)$ \\
$C(6)-C(1)-C(2)-C(3)$ & $18.68(14)$ \\
$O(3)-C(2)-C(3)-C(4)$ & $-173.62(10)$ \\
$C(1)-C(2)-C(3)-C(4)$ & $-1.88(16)$ \\
$C(2)-C(3)-C(4)-O(4)$ & $162.84(11)$ \\
$C(2)-C(3)-C(4)-C(5)$ & $-16.34(15)$ \\
$O(4)-C(4)-C(5)-C(6)$ & $-163.20(10)$ \\
$C(3)-C(4)-C(5)-C(6)$ & $15.96(14)$ \\
$O(4)-C(4)-C(5)-C(14)$ & $17.01(16)$ \\
$C(3)-C(4)-C(5)-C(14)$ & $-163.84(10)$ \\
$C(14)-C(5)-C(6)-C(7)$ & $-4.99(15)$ \\
$C(4)-C(5)-C(6)-C(7)$ & $175.21(9)$ \\
$C(14)-C(5)-C(6)-C(1)$ & $-178.18(9)$ \\
$C(4)-C(5)-C(6)-C(1)$ & $2.02(15)$ \\
$O(2)-C(1)-C(6)-C(5)$ & $101.41(11)$ \\
$O(1)-C(1)-C(6)-C(5)$ & $-138.97(10)$ \\
$C(2)-C(1)-C(6)-C(5)$ & $-18.74(14)$ \\
$O(2)-C(1)-C(6)-C(7)$ & $-72.23(11)$ \\
$O(1)-C(1)-C(6)-C(7)$ & $47.39(11)$ \\
$C(2)-C(1)-C(6)-C(7)$ & $167.62(8)$ \\
$C(5)-C(6)-C(7)-C(20)$ & $-7.97(15)$ \\
$C(1)-C(6)-C(7)-C(20)$ & $165.76(9)$ \\
$C(5)-C(6)-C(7)-C(8)$ & $171.64(9)$ \\
$C(1)-C(6)-C(7)-C(8)$ & $-14.63(13)$ \\
$C(20)-C(7)-C(8)-C(13)$ & $168.70(9)$ \\
$C(6)-C(7)-C(8)-C(13)$ & $-10.92(14)$ \\
$C(20)-C(7)-C(8)-C(9)$ & $-6.61(15)$ \\
$C(6)-C(7)-C(8)-C(9)$ & $0.42(15)$ \\
$C(13)-C(8)-C(9)-C(10)$ & \\
$C(7)-C(8)-C(9)-C(10)$ & \\
& \\
$O$ & \\
$O$ &
\end{tabular}




\begin{tabular}{|c|c|}
\hline$C(13)-C(8)-C(9)-C(27)$ & $176.99(9)$ \\
\hline$C(7)-C(8)-C(9)-C(27)$ & $-7.72(15)$ \\
\hline$C(8)-C(9)-C(10)-C(11)$ & $-0.63(16)$ \\
\hline$C(27)-C(9)-C(10)-C(11)$ & $-176.97(10)$ \\
\hline$C(9)-C(10)-C(11)-C(12)$ & $0.15(17)$ \\
\hline$C(10)-C(11)-C(12)-C(13)$ & $0.55(17)$ \\
\hline$C(11)-C(12)-C(13)-O(1)$ & $-178.61(9)$ \\
\hline$C(11)-C(12)-C(13)-C(8)$ & $-0.76(16)$ \\
\hline$C(1)-O(1)-C(13)-C(12)$ & $-150.21(10)$ \\
\hline $\mathrm{C}(1)-\mathrm{O}(1)-\mathrm{C}(13)-\mathrm{C}(8)$ & $31.92(13)$ \\
\hline$C(9)-C(8)-C(13)-C(12)$ & $0.28(16)$ \\
\hline$C(7)-C(8)-C(13)-C(12)$ & $-175.09(9)$ \\
\hline $\mathrm{C}(9)-\mathrm{C}(8)-\mathrm{C}(13)-\mathrm{O}(1)$ & $178.09(9)$ \\
\hline $\mathrm{C}(7)-\mathrm{C}(8)-\mathrm{C}(13)-\mathrm{O}(1)$ & $2.71(15)$ \\
\hline$C(6)-C(5)-C(14)-C(15)$ & $-168.99(10)$ \\
\hline$C(4)-C(5)-C(14)-C(15)$ & $10.80(16)$ \\
\hline$C(6)-C(5)-C(14)-C(19)$ & $8.63(15)$ \\
\hline$C(4)-C(5)-C(14)-C(19)$ & $-171.57(9)$ \\
\hline$C(19)-C(14)-C(15)-C(16)$ & $1.24(16)$ \\
\hline$C(5)-C(14)-C(15)-C(16)$ & $178.89(11)$ \\
\hline$C(14)-C(15)-C(16)-C(17)$ & $-1.55(18)$ \\
\hline $\mathrm{C}(29)-\mathrm{O}(5)-\mathrm{C}(17)-\mathrm{C}(18)$ & $8.61(15)$ \\
\hline $\mathrm{C}(29)-\mathrm{O}(5)-\mathrm{C}(17)-\mathrm{C}(16)$ & $-172.42(10)$ \\
\hline$C(15)-C(16)-C(17)-O(5)$ & $179.86(10)$ \\
\hline$C(15)-C(16)-C(17)-C(18)$ & $-1.12(17)$ \\
\hline $\mathrm{O}(5)-\mathrm{C}(17)-\mathrm{C}(18)-\mathrm{C}(19)$ & $-176.99(10)$ \\
\hline$C(16)-C(17)-C(18)-C(19)$ & $4.08(16)$ \\
\hline$C(17)-C(18)-C(19)-C(14)$ & $-4.29(15)$ \\
\hline$C(17)-C(18)-C(19)-C(20)$ & $179.49(10)$ \\
\hline$C(15)-C(14)-C(19)-C(18)$ & $1.63(15)$ \\
\hline$C(5)-C(14)-C(19)-C(18)$ & $-176.09(9)$ \\
\hline$C(15)-C(14)-C(19)-C(20)$ & $177.91(9)$ \\
\hline$C(5)-C(14)-C(19)-C(20)$ & $0.19(15)$ \\
\hline$C(6)-C(7)-C(20)-C(19)$ & $16.52(14)$ \\
\hline$C(8)-C(7)-C(20)-C(19)$ & $-163.09(9)$ \\
\hline$C(6)-C(7)-C(20)-C(21)$ & $-161.90(9)$ \\
\hline$C(8)-C(7)-C(20)-C(21)$ & $18.49(14)$ \\
\hline$C(18)-C(19)-C(20)-C(7)$ & $163.57(10)$ \\
\hline$C(14)-C(19)-C(20)-C(7)$ & $-12.59(14)$ \\
\hline$C(18)-C(19)-C(20)-C(21)$ & $-18.16(15)$ \\
\hline$C(14)-C(19)-C(20)-C(21)$ & $165.68(10)$ \\
\hline$C(7)-C(20)-C(21)-C(22)$ & $156.12(10)$ \\
\hline$C(19)-C(20)-C(21)-C(22)$ & $-22.16(16)$ \\
\hline$C(7)-C(20)-C(21)-C(27)$ & $-16.44(14)$ \\
\hline$C(19)-C(20)-C(21)-C(27)$ & $165.29(10)$ \\
\hline$C(27)-C(21)-C(22)-C(23)$ & $-4.74(16)$ \\
\hline$C(20)-C(21)-C(22)-C(23)$ & $-177.44(10)$ \\
\hline$C(21)-C(22)-C(23)-C(24)$ & $0.58(17)$ \\
\hline
\end{tabular}




$\begin{array}{lc}\mathrm{C}(22)-\mathrm{C}(23)-\mathrm{C}(24)-\mathrm{C}(26) & 2.88(17) \\ \mathrm{C}(23)-\mathrm{C}(24)-\mathrm{C}(26)-\mathrm{C}(27) & -2.03(18) \\ \mathrm{C}(24)-\mathrm{C}(26)-\mathrm{C}(27)-\mathrm{C}(21) & -2.22(17) \\ \mathrm{C}(24)-\mathrm{C}(26)-\mathrm{C}(27)-\mathrm{C}(9) & 173.56(10) \\ \mathrm{C}(22)-\mathrm{C}(21)-\mathrm{C}(27)-\mathrm{C}(26) & 5.46(15) \\ \mathrm{C}(20)-\mathrm{C}(21)-\mathrm{C}(27)-\mathrm{C}(26) & 178.35(9) \\ \mathrm{C}(22)-\mathrm{C}(21)-\mathrm{C}(27)-\mathrm{C}(9) & -170.31(10) \\ \mathrm{C}(20)-\mathrm{C}(21)-\mathrm{C}(27)-\mathrm{C}(9) & 2.58(15) \\ \mathrm{C}(10)-\mathrm{C}(9)-\mathrm{C}(27)-\mathrm{C}(26) & 10.13(16) \\ \mathrm{C}(8)-\mathrm{C}(9)-\mathrm{C}(27)-\mathrm{C}(26) & -166.25(10) \\ \mathrm{C}(10)-\mathrm{C}(9)-\mathrm{C}(27)-\mathrm{C}(21) & -174.16(10) \\ \mathrm{C}(8)-\mathrm{C}(9)-\mathrm{C}(27)-\mathrm{C}(21) & 9.46(15) \\ \text { C6A1-C1A1-C2A1-C3A1 } & -0.6(2) \\ \text { C1A1-C2A1-C3A1-C4A1 } & 0.0(2) \\ \text { C2A1-C3A1-C4A1-C5A1 } & 0.2(2) \\ \text { C3A1-C4A1-C5A1-C6A1 } & 0.2(2) \\ \text { C4A1-C5A1-C6A1-C1A1 } & -0.8(2) \\ \text { C4A1-C5A1-C6A1-C7A1 } & 177.48(15) \\ \text { C2A1-C1A1-C6A1-C5A1 } & 1.0(2) \\ \text { C2A1-C1A1-C6A1-C7A1 } & -177.33(14) \\ \text { C6B1-C1B1-C2B1-C3B1 } & 0.0 \\ \text { C1B1-C2B1-C3B1-C4B1 } & 0.0 \\ \text { C2B1-C3B1-C4B1-C5B1 } & 0.0 \\ \text { C3B1-C4B1-C5B1-C6B1 } & 0.0 \\ \text { C4B1-C5B1-C6B1-C1B1 } & 0.0 \\ \text { C4B1-C5B1-C6B1-C7B1 } & -177.8(11) \\ \text { C2B1-C1B1-C6B1-C5B1 } & 0.0 \\ \text { C2B1-C1B1-C6B1-C7B1 } & 177.8(11) \\ & \end{array}$

Symmetry transformations used to generate equivalent atoms:

Table S9.

Hydrogen bonds for 17b [A and deg.].

\begin{tabular}{lrrrrr}
\hline $\mathrm{D}-\mathrm{H} \ldots \mathrm{A}$ & $\mathrm{d}(\mathrm{D}-\mathrm{H})$ & $\mathrm{d}(\mathrm{H} \ldots \mathrm{A})$ & $\mathrm{d}(\mathrm{D} \ldots \mathrm{A})$ & $<(\mathrm{DHA})$ \\
$\mathrm{O}(2)-\mathrm{H}(1) \ldots \mathrm{O}(4) \# 1$ & $0.895(18)$ & $1.983(19)$ & $2.8143(11)$ & $153.9(16)$
\end{tabular}

Symmetry transformations used to generate equivalent atoms:

$\# 1-x+1 / 2, y+1 / 2,-z+1 / 2$ 


\section{References}

1. Alcarazo, M.; Suarez, R. M.; Goddard, R.; Fürstner, A. A New Class of Singlet Carbenes. Chem. Eur. J. 2010, 16, 9746.

2. Zhang, S.; Neumann, H.; Beller, M. Pd-Catalyzed Carbonylation of VInyl Triflates To Afford $\alpha, \beta$-Unsaturated Aldehydes, Esters, and Amides under Mild Conditions. Org. Lett. 2019, 21, 3528.

3. Scheurermann, G. M. R., L.; Steurer, P.; Bannwarth, W.; Mulhaupt, R. Palladium Nanoparticles on Graphite Oxide and Its Functionalized Graphene Derivative as Highly Active Catalysts for the Suzuki-Miyaura Coupling Reaction. J. Am. Chem. Soc. 2009, 8262.

4. Schmidt, B.; Riemer, M. Suzuki-Miyaura Coupling of Halophenols and Phenol Boronic Acids: Systematic Investigation of Positional Isomer Effects and Conclusions for the Synthesis of Phytoalexins from Pyrinae. J. Org. Chem. 2014, 79, 4104.

5. Huang, Z.; Kwon, O.; Esguerra, K. N.; Lumb, J. A Divergent and Selective Synthesis of Ortho- and Para-Quinones from Phenols. Tetrahedron 2015, 71, 5871.

6. Imahori, T.; Tokuda, T.; Toguchi, T.; Takahata, H. An Alternative Approach to para-C-H Arylations of Phenol: Palladium-Catatlyzed Tandem y-Arylation/Aromazation of 2Cyclohexen-1-one Derivatives. Org. Lett. 2012, 14, 1172.

7. Ji, H.; Wu, L.-Y.; Cai, J.-H.; Li, G.-R.; Gan, N.-N.; Wang, Z.-H. Room-temperature Borylation and One-pot Two-step Borylation/Suzuki-Mlyaura Cross-coupling Reaction of Aryl Chlorides. RSC Adv. 2018, 8, 13643.

8. Jansa, J.; Řezníček, T.; Jambor, R.; Bureš, F.; Lyčka, A. Synthesis of HydroxySubstituted $p$-Terphenyls and some Larger Oligophenylenes via Palladium on Charcoal Catalyzed Suzuki-Miyaura Reaction. Adv. Synth. Catal. 2017, 359, 339.

9. Sen, C. P.; Valiyaveettil, S. Solvent Dependent Isomerization of Photochromic Diethienylethenes: Synthesis, Photochromism, and Self-assembly. RSC Adv. 2016, 6, 95137.

10. Tang, T.; Harned, A. M. Experimental Evidence for the Formation of Cationic Intermediates During lodine(III)-Mediated Oxidative Dearomatization of Phenols. Org. Biomol. Chem. 2018, 16, 8249.

11. Spek, A. L. PLATON SQUEEZE: A Tool for the Calculation of the Disordered SOlvent Contribution to the Calculated Structure Factors. Acta. Cryst., 2015, C71, 9.

12. Apex3, Bruker AXS Inc. Madison, WI, 2015.

13. Sheldrick, G. M. SHELXT - Integrated Space-Group and Crystal-Structure Determination. Acta. Cryst., 2015, A71, 3.

14. Sheldrick, G. M. Crystal Structure Refinement with SHELXL. Acta. Cryst. 2015, C71, 3. 


\section{NMR Spectra}

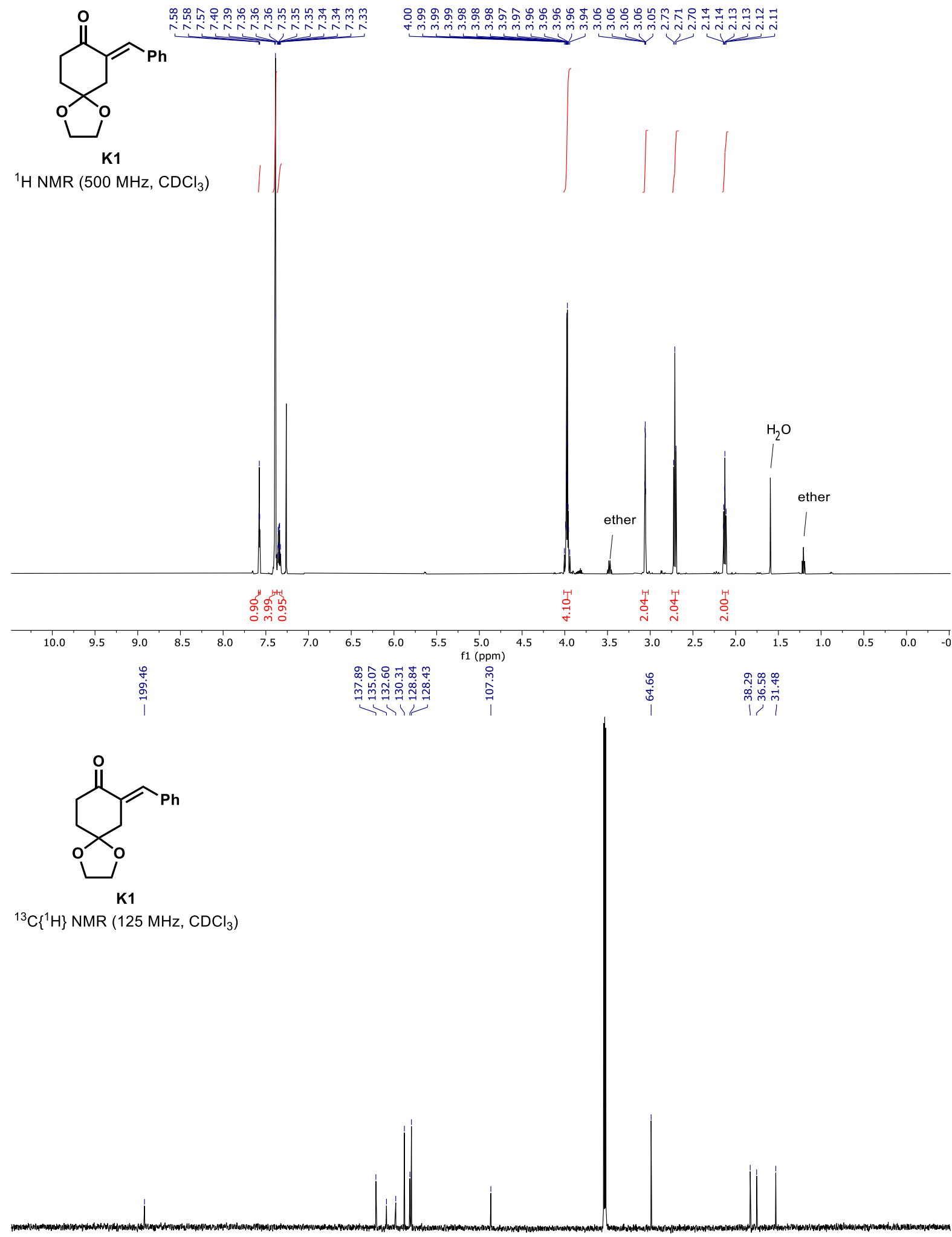

$\begin{array}{lllllllllllllllllllllllll}1 & 1 \\ 230 & 220 & 210 & 200 & 190 & 180 & 170 & 160 & 150 & 140 & 130 & 120 & 110 & 100 & 90 & 80 & 70 & 60 & 50 & 40 & 30 & 20 & 10 & 0 & -10\end{array}$ 


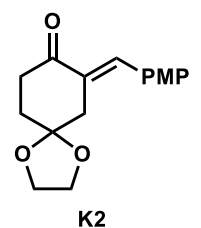

${ }^{1} \mathrm{H} \mathrm{NMR}\left(500 \mathrm{MHz}, \mathrm{CDCl}_{3}\right)$

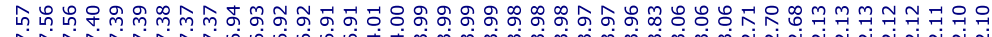

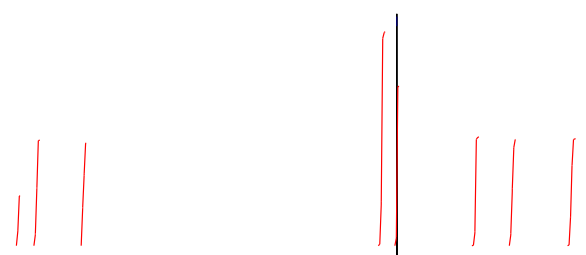

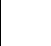

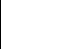

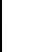
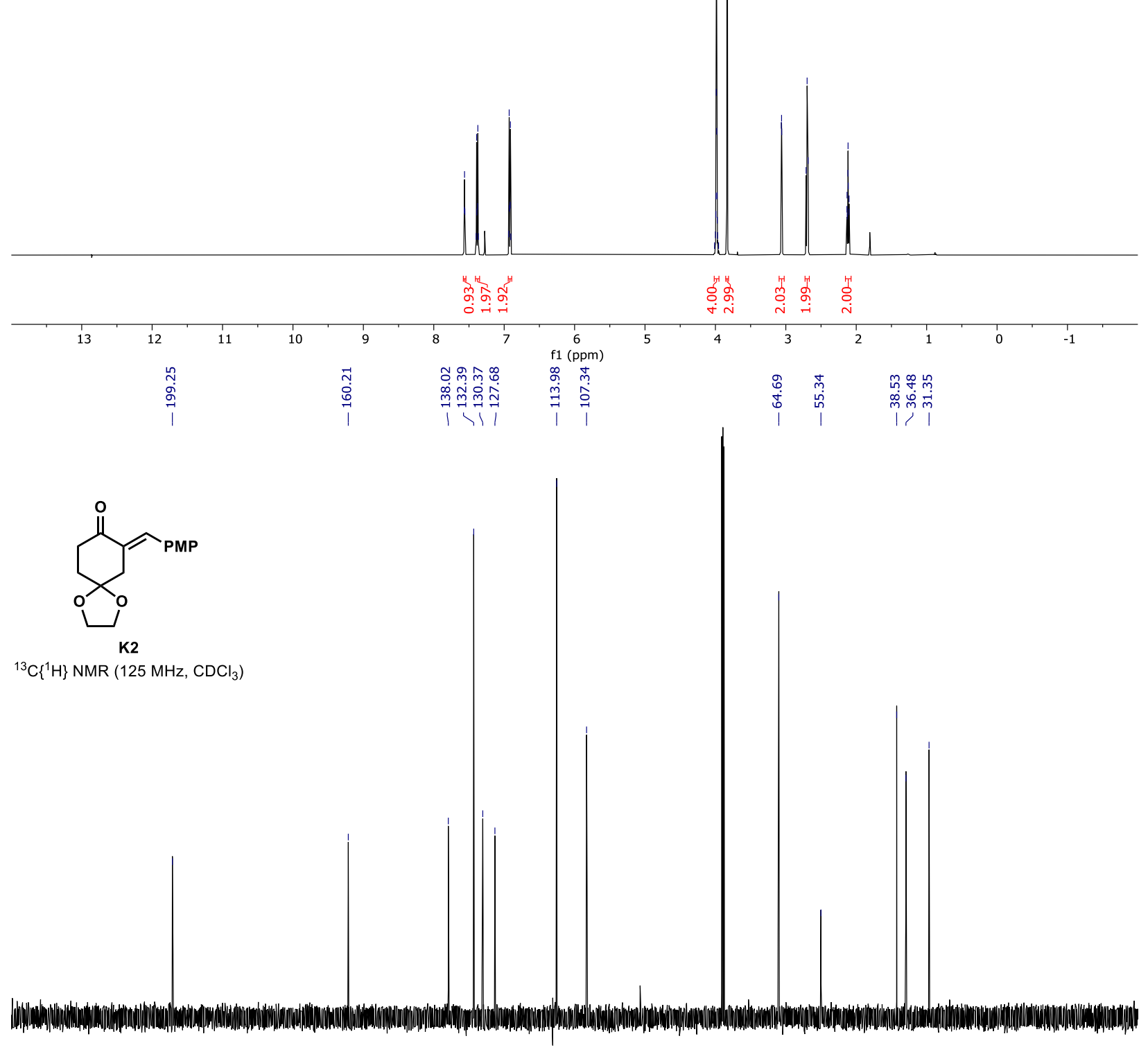

${ }^{3} \mathrm{C}\left\{{ }^{1} \mathrm{H}\right\}$ NMR (125 MHz, CDCin

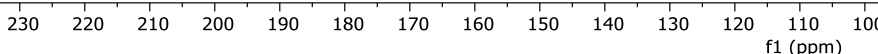




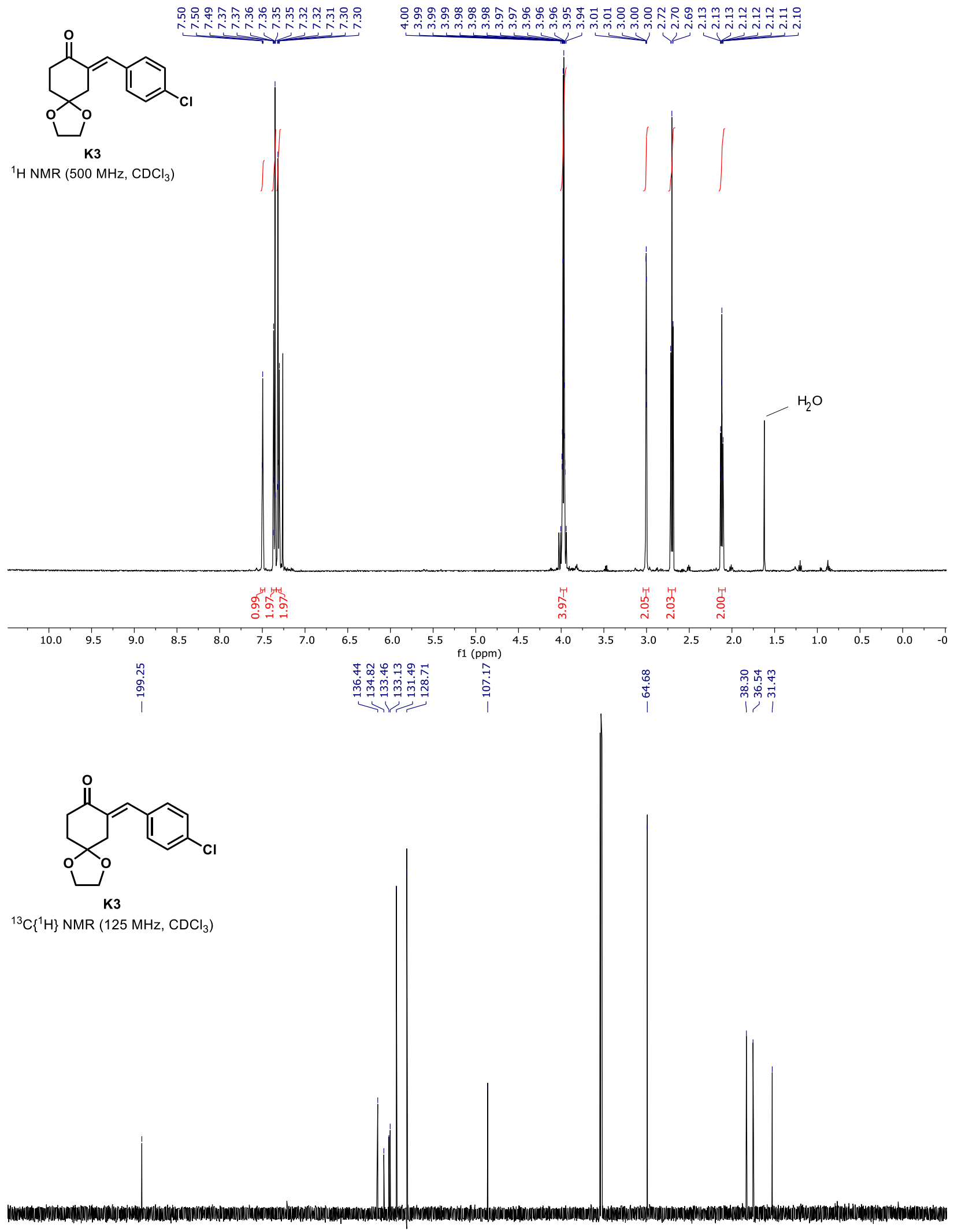

$\begin{array}{llllllllllllllllllllllllll}1 & 230 & 220 & 210 & 200 & 190 & 180 & 170 & 160 & 150 & 140 & 130 & 120 & 110 & 100 & 90 & 80 & 70 & 60 & 50 & 40 & 30 & 20 & 10 & 0 & -10\end{array}$ 


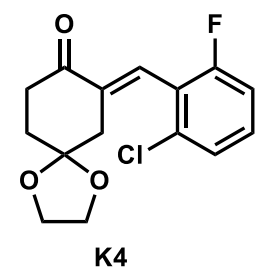

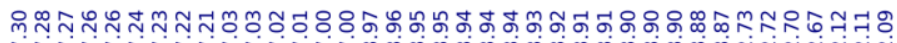

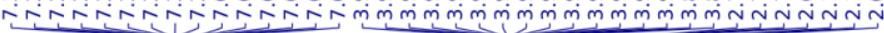

${ }^{1} \mathrm{H}$ NMR $\left(500 \mathrm{MHz}, \mathrm{CDCl}_{3}\right)$
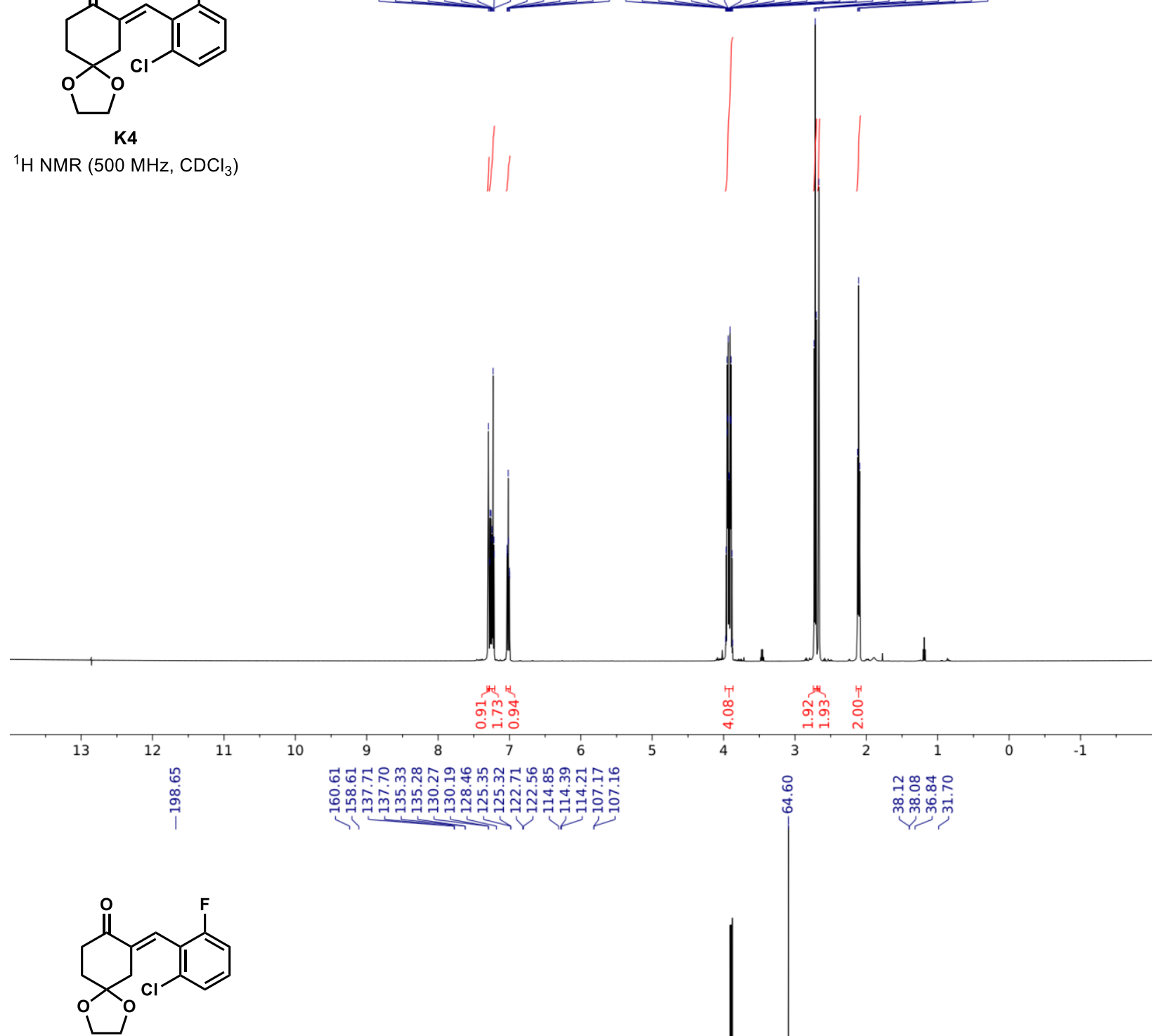

K4

${ }^{13} \mathrm{C}\left\{{ }^{1} \mathrm{H}\right\} \mathrm{NMR}\left(125 \mathrm{MHz}, \mathrm{CDCl}_{3}\right)$

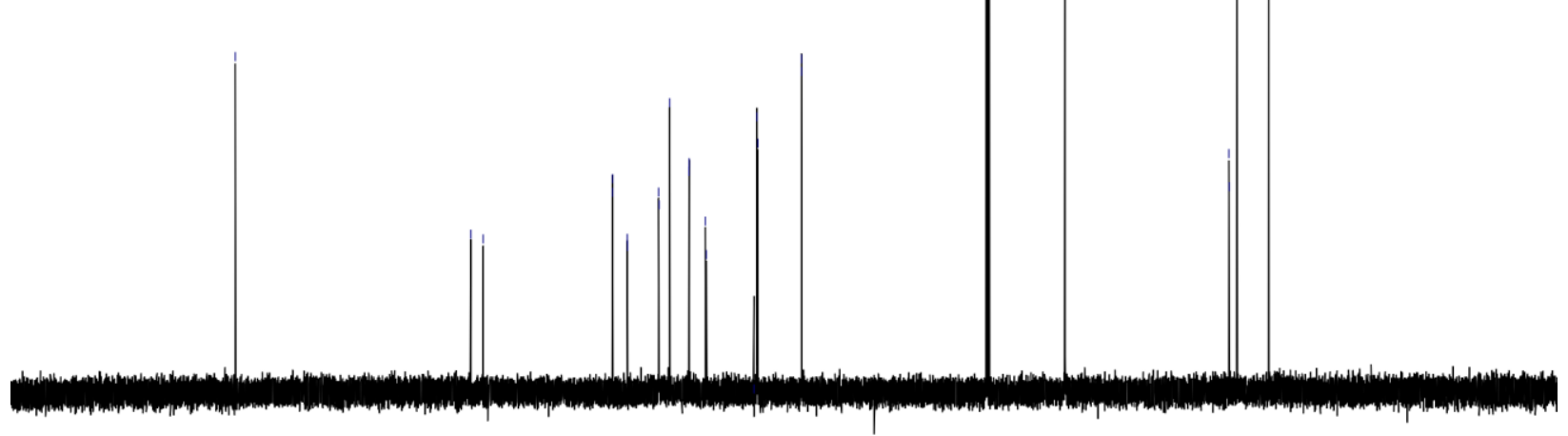

$\begin{array}{lllllllllllllllllllllllll}230 & 220 & 210 & 200 & 190 & 180 & 170 & 160 & 150 & 140 & 130 & 120 & 110 & 100 & 90 & 80 & 70 & 60 & 50 & 40 & 30 & 20 & 10 & 0 & -10\end{array}$ 


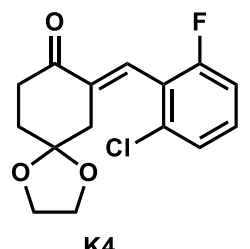

${ }^{19} \mathrm{~F} \mathrm{NMR}\left(470 \mathrm{MHz}, \mathrm{CDCl}_{3}\right)$

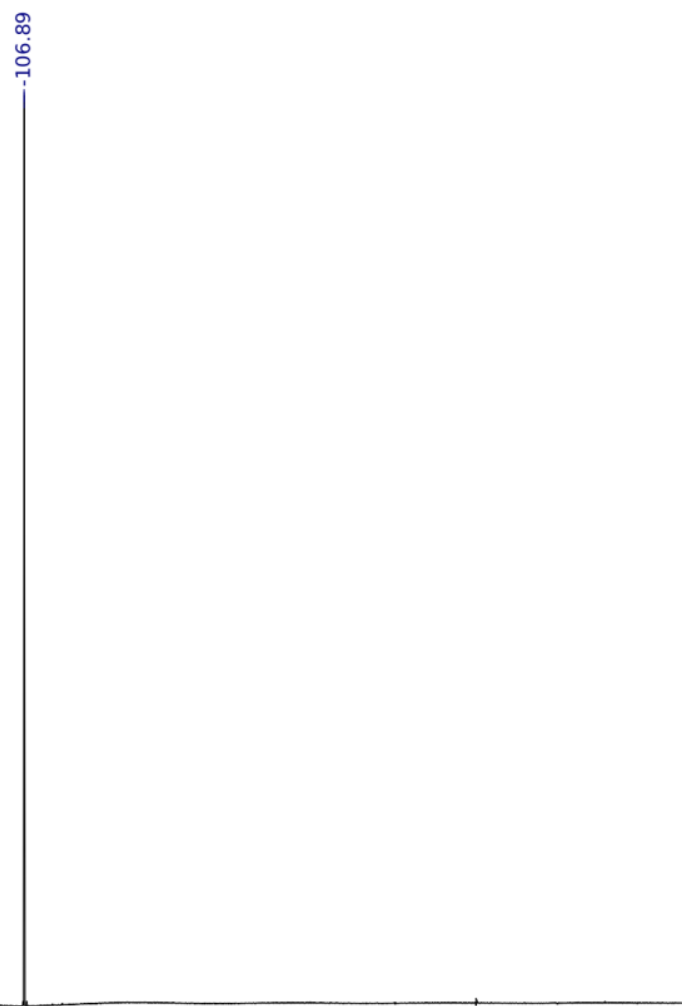

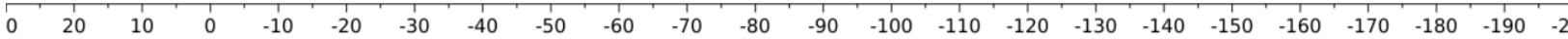


œ

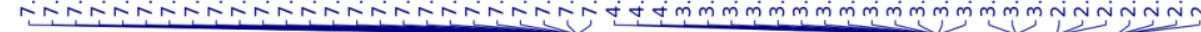<smiles>O=C1CCC2(C/C1=C\c1ccc3ccccc3c1)OCCO2</smiles>

K5

${ }^{1} \mathrm{H}$ NMR $\left(500 \mathrm{MHz}, \mathrm{CDCl}_{3}\right)$
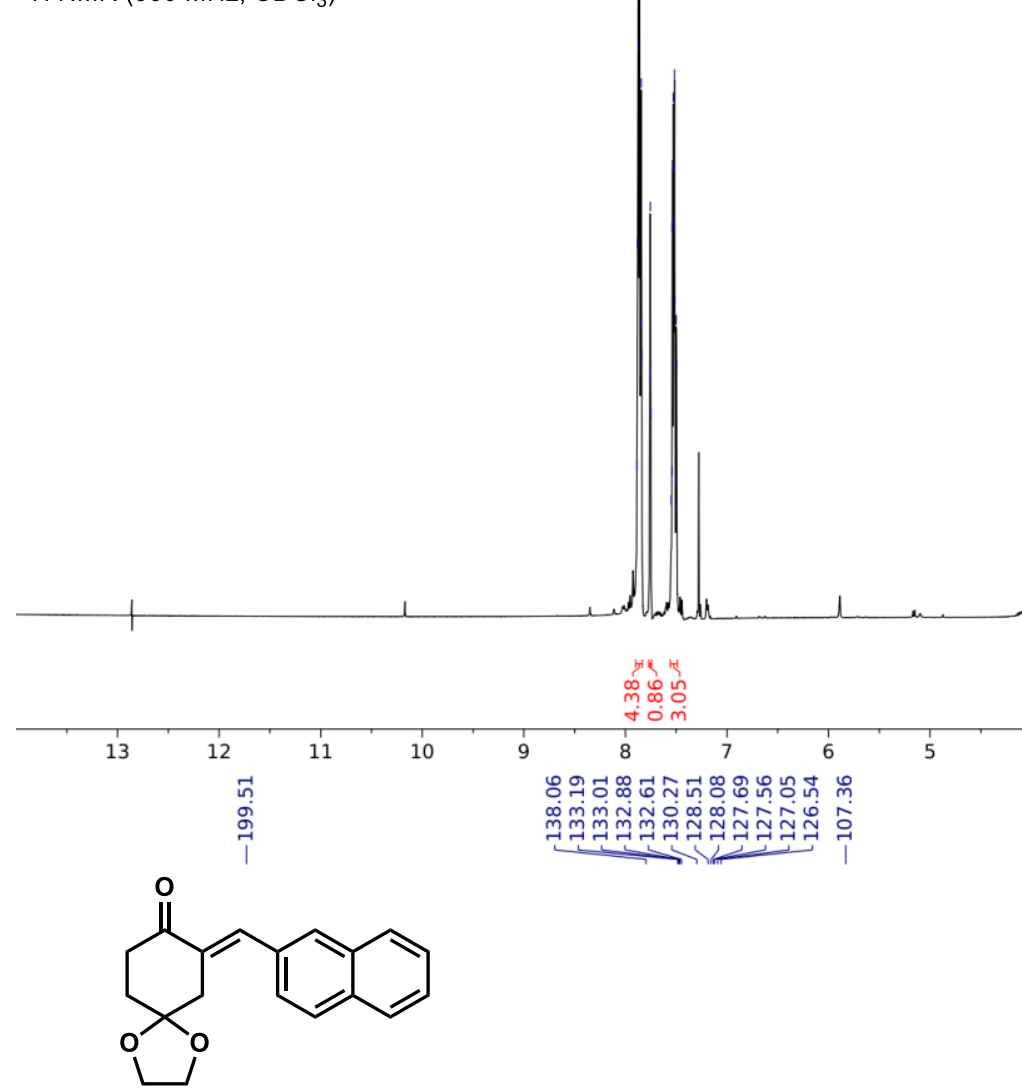

K5

${ }^{13} \mathrm{C}\left\{{ }^{1} \mathrm{H}\right\}$ NMR $\left(125 \mathrm{MHz}, \mathrm{CDCl}_{3}\right)$

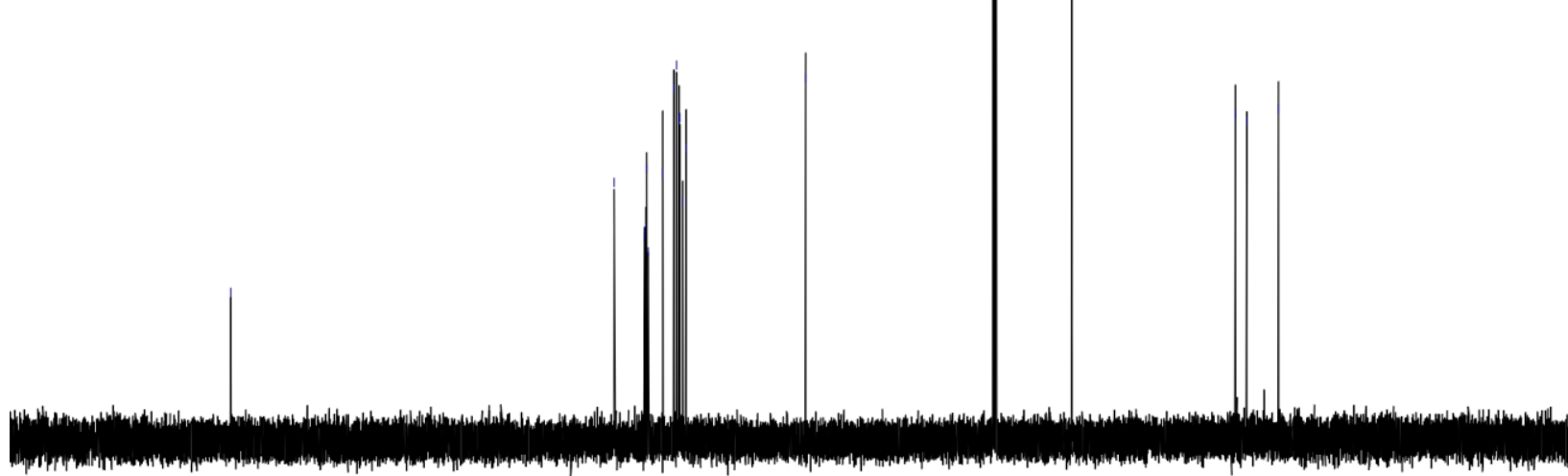

$\begin{array}{lllllllllllllllllllllllll}230 & 220 & 210 & 200 & 190 & 180 & 170 & 160 & 150 & 140 & 130 & 120 & 110 & 100 & 90 & 80 & 70 & 60 & 50 & 40 & 30 & 20 & 10 & 0 & -10\end{array}$ 


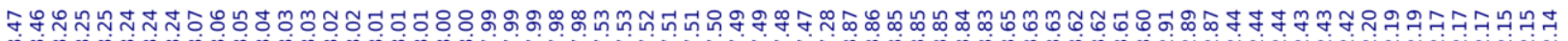
我<smiles>O=C1CCC2(CC1=Cc1c3ccccc3cc3ccccc13)OCCO2</smiles>

K6

${ }^{1} \mathrm{H}$ NMR $\left(300 \mathrm{MHz}, \mathrm{CDCl}_{3}\right)$
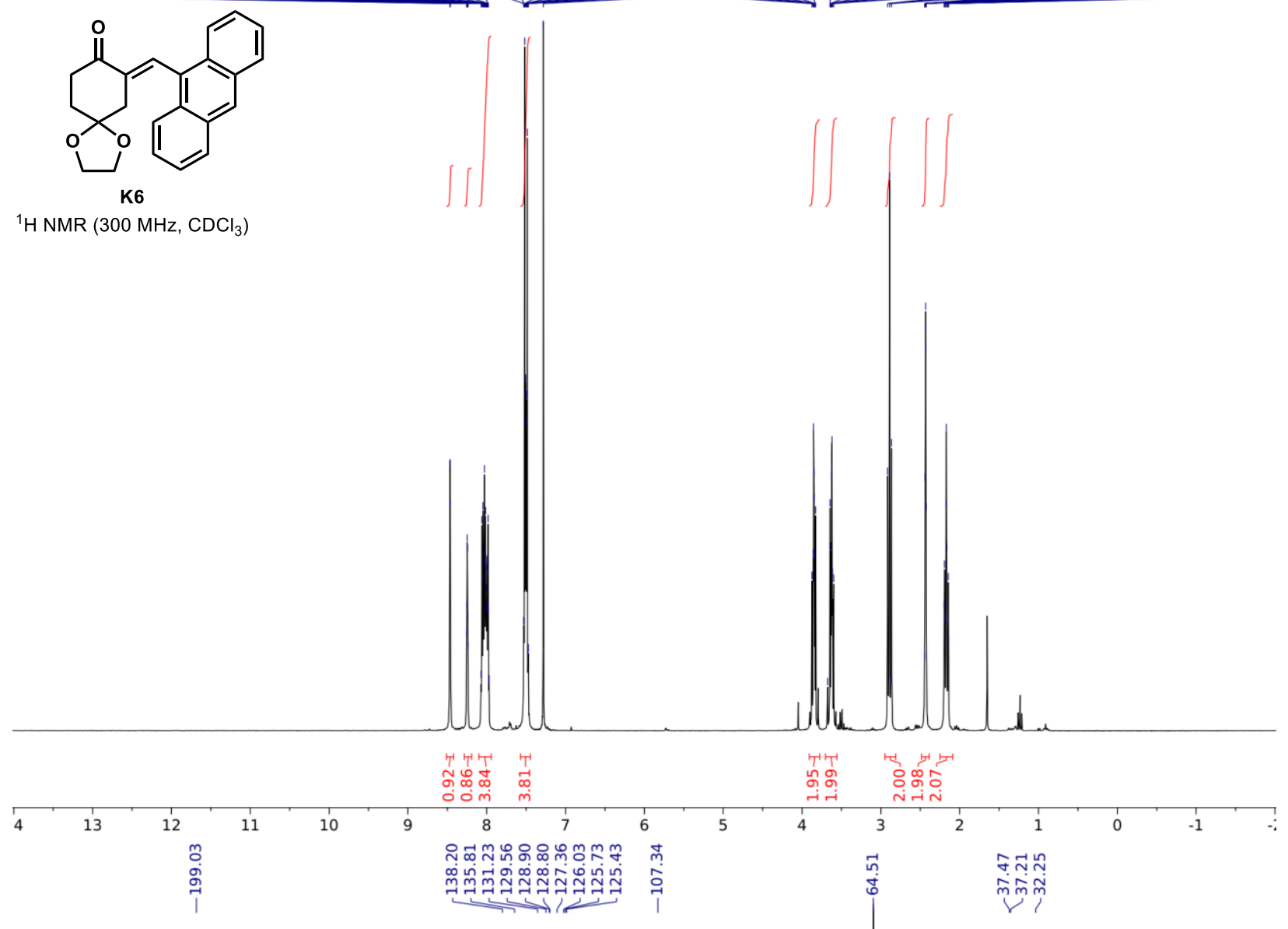<smiles>O=C1CCOC2(CC1)C/C(=C\c1c3ccccc3cc3ccccc13)CO2</smiles>

K6

${ }^{13} \mathrm{C}\left\{{ }^{1} \mathrm{H}\right\}$ NMR $\left(75 \mathrm{MHz}, \mathrm{CDCl}_{3}\right)$

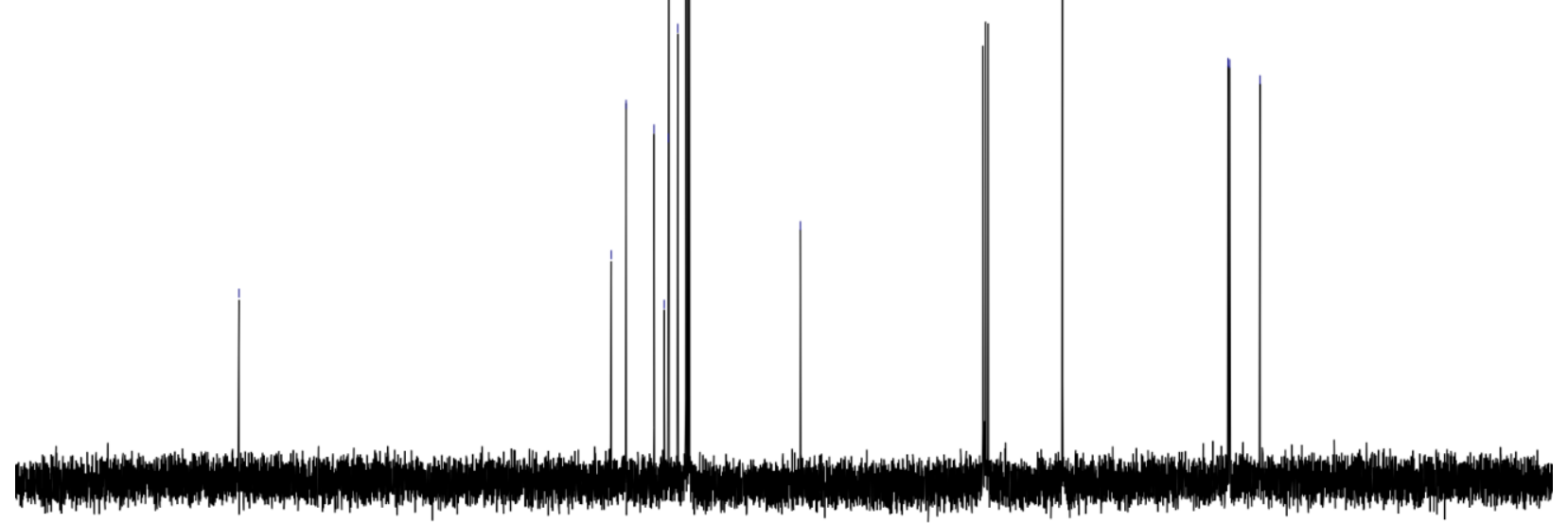

$\begin{array}{lllllllllllllllllllllllll}230 & 220 & 210 & 200 & 190 & 180 & 170 & 160 & 150 & 140 & 130 & 120 & 110 & 100 & 90 & 80 & 70 & 60 & 50 & 40 & 30 & 20 & 10 & 0 & -10\end{array}$ 


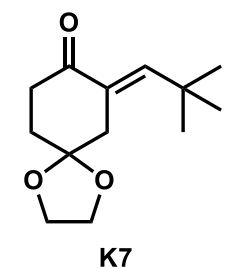

${ }^{1} \mathrm{H}$ NMR $\left(300 \mathrm{MHz}, \mathrm{CDCl}_{3}\right)$
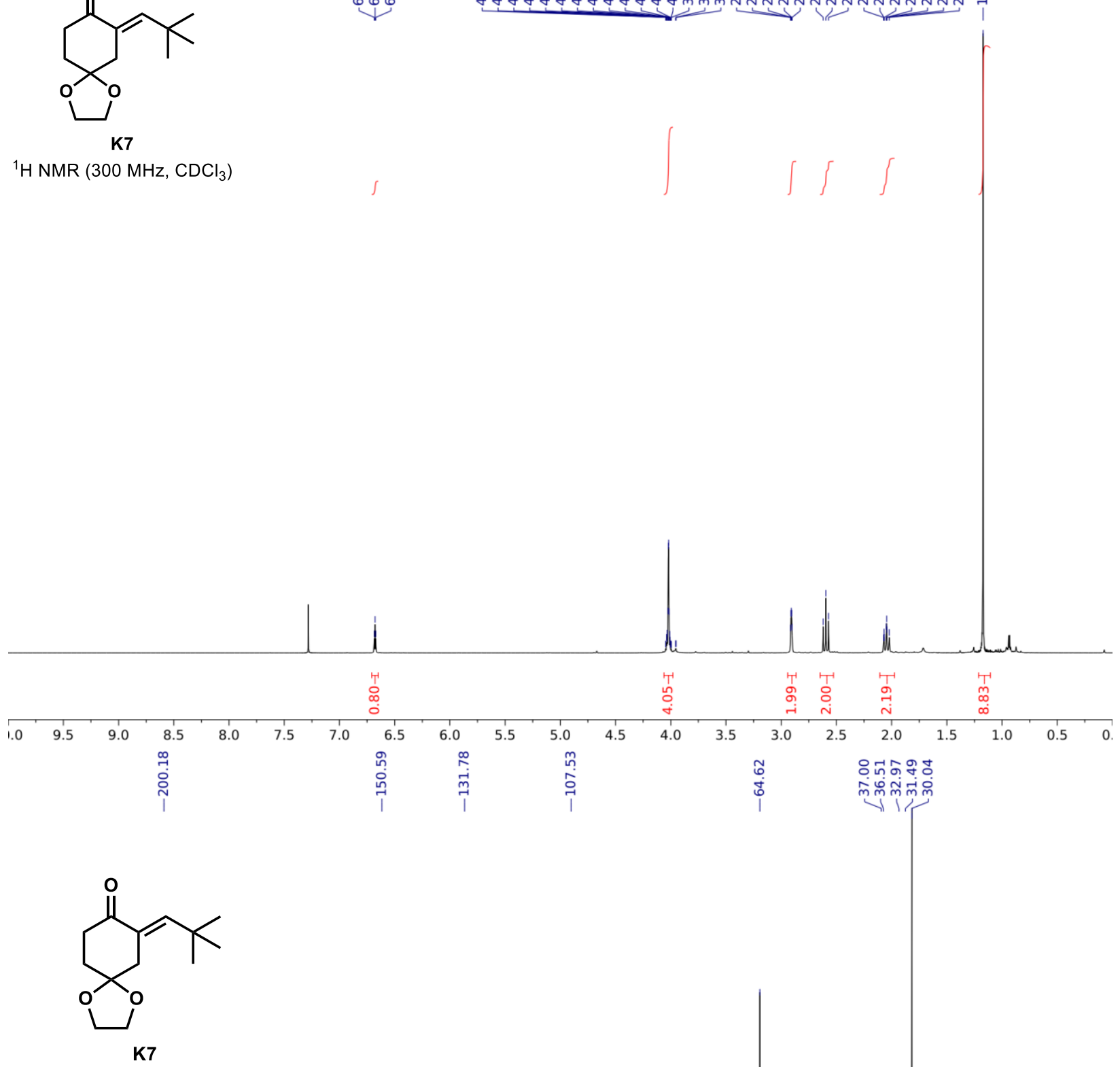

${ }^{13} \mathrm{C}\left\{{ }^{1} \mathrm{H}\right\} \operatorname{NMR}\left(75 \mathrm{MHz}, \mathrm{CDCl}_{3}\right)$

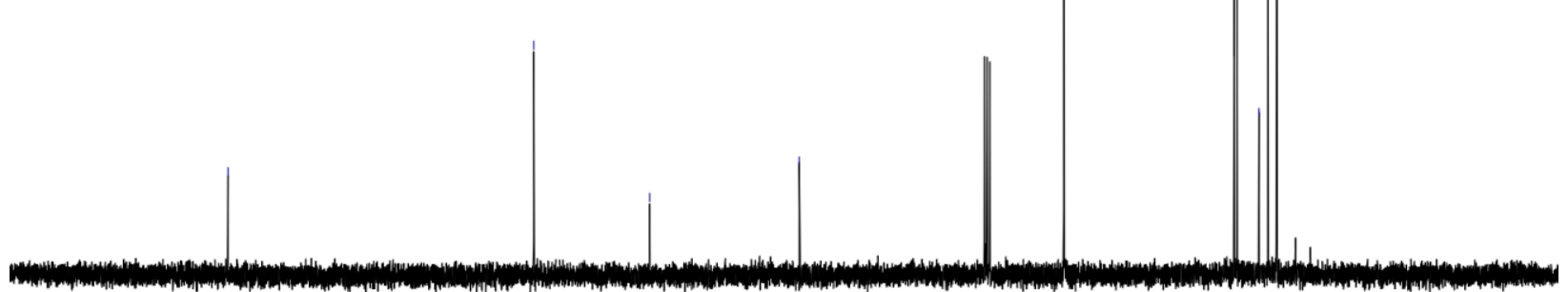

$\begin{array}{lllllllllllllllllllllllll}230 & 220 & 210 & 200 & 190 & 180 & 170 & 160 & 150 & 140 & 130 & 120 & 110 & 100 & 90 & 80 & 70 & 60 & 50 & 40 & 30 & 20 & 10 & 0 & -10\end{array}$ 

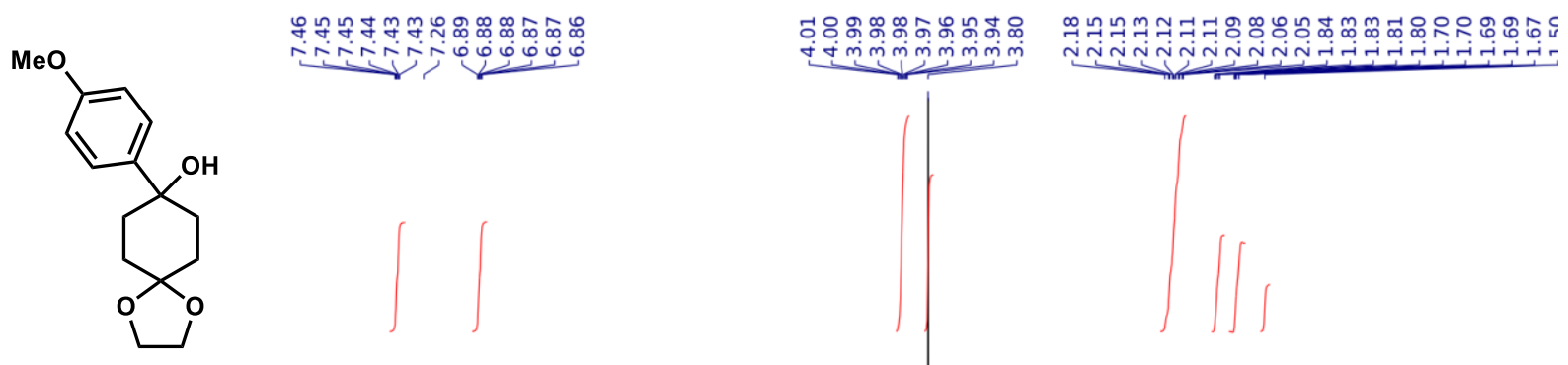

${ }^{1} \mathrm{H}$ NMR $\left(500 \mathrm{MHz}, \mathrm{CDCl}_{3}\right)$
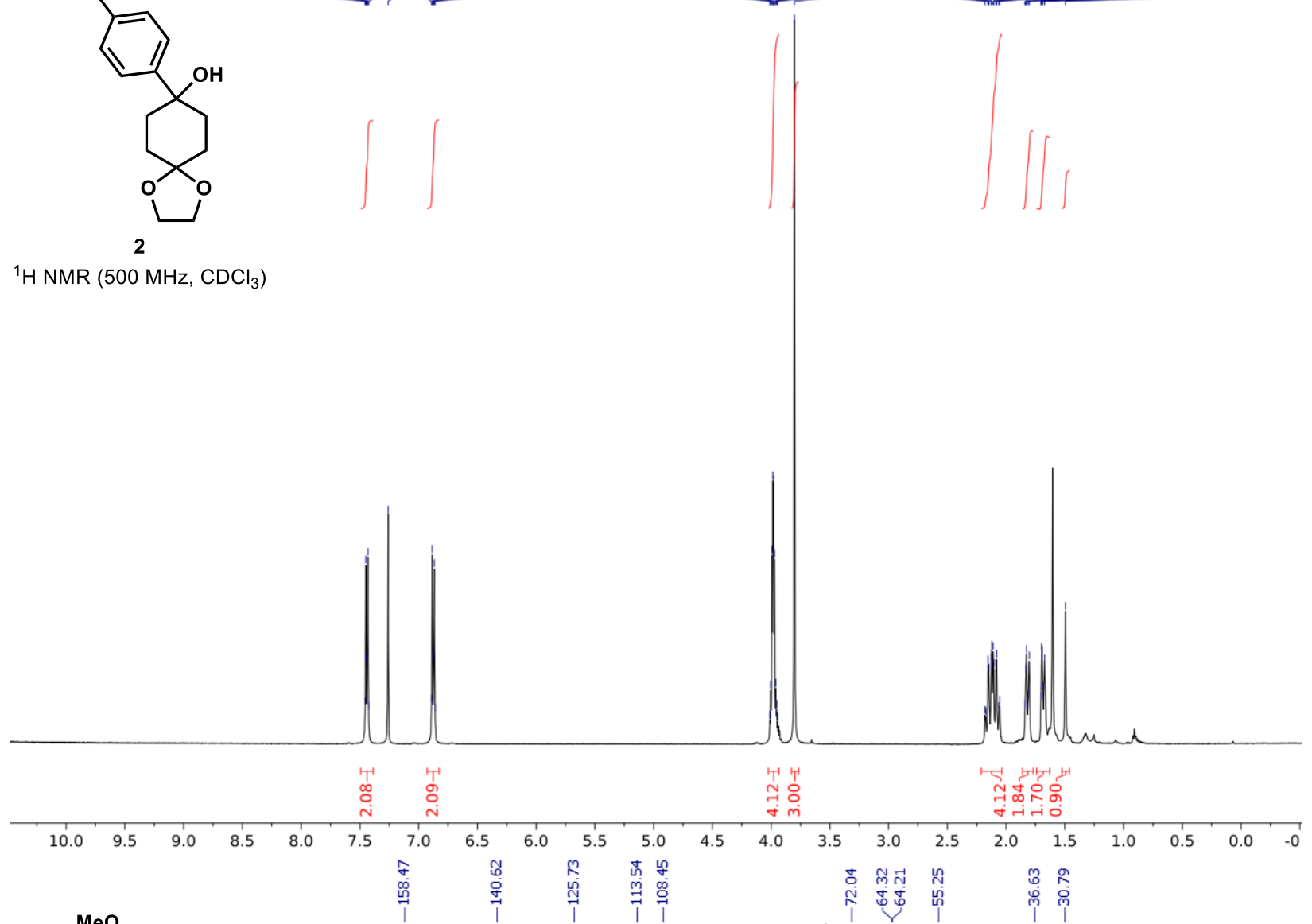

$\mathrm{MeO}$<smiles>Cc1ccc(C2(O)CCC3(CC2)OCCO3)cc1</smiles>

2

${ }^{13} \mathrm{C}\left\{{ }^{1} \mathrm{H}\right\}$ NMR $\left(125 \mathrm{MHz}, \mathrm{CDCl}_{3}\right)$
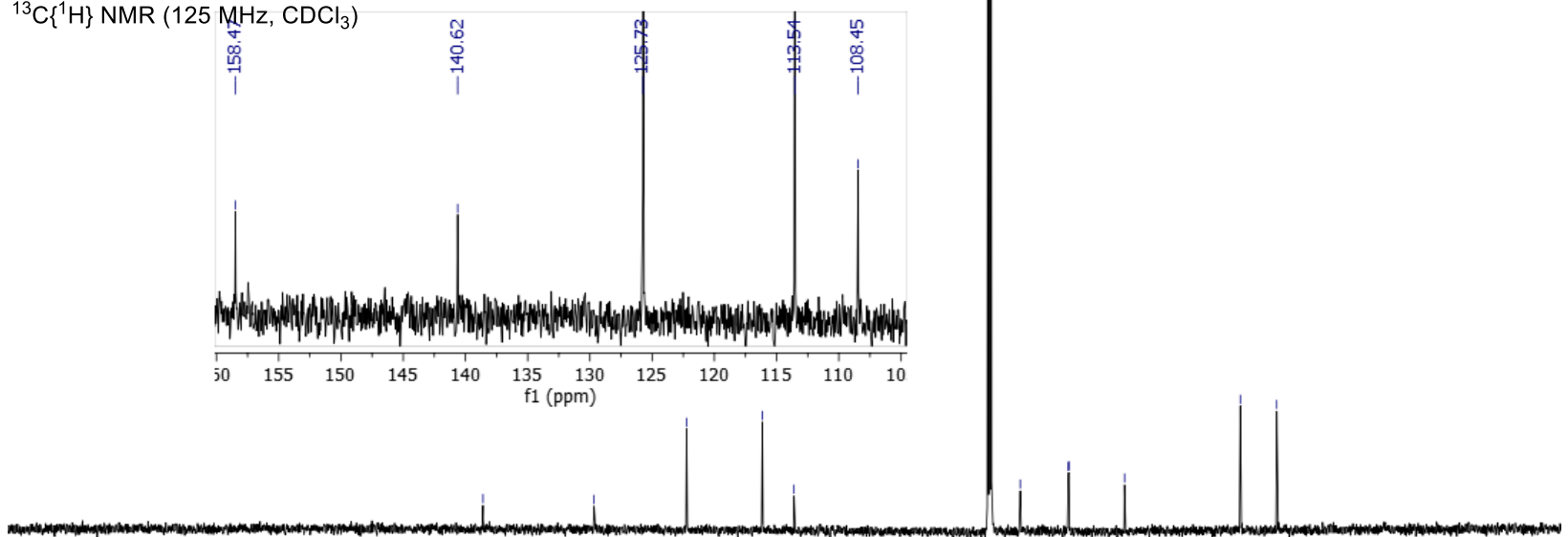

$\begin{array}{llllllllllllllllllllllllll}230 & 220 & 210 & 200 & 190 & 180 & 170 & 160 & 150 & 140 & 130 & 120 & 110 & 100 & 90 & 80 & 70 & 60 & 50 & 40 & 30 & 20 & 10 & 0 & -10\end{array}$ 


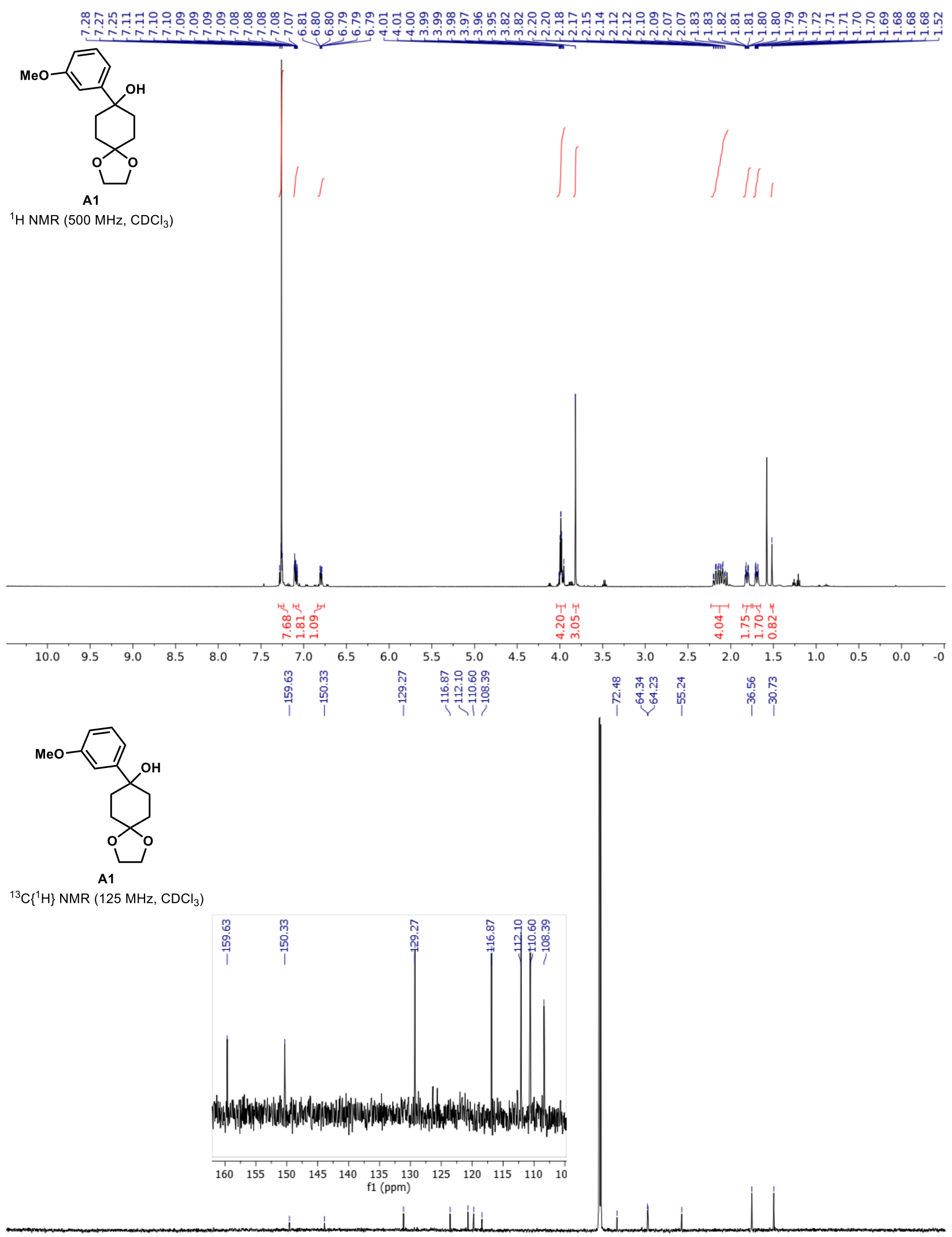

NMR (500 MHz, CDC 


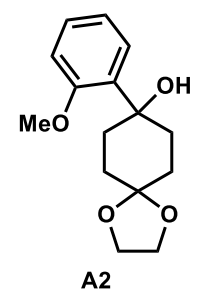

mై

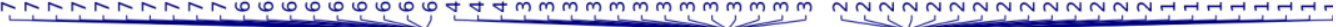

${ }^{1} \mathrm{H} \mathrm{NMR}\left(500 \mathrm{MHz}, \mathrm{CDCl}_{3}\right)$
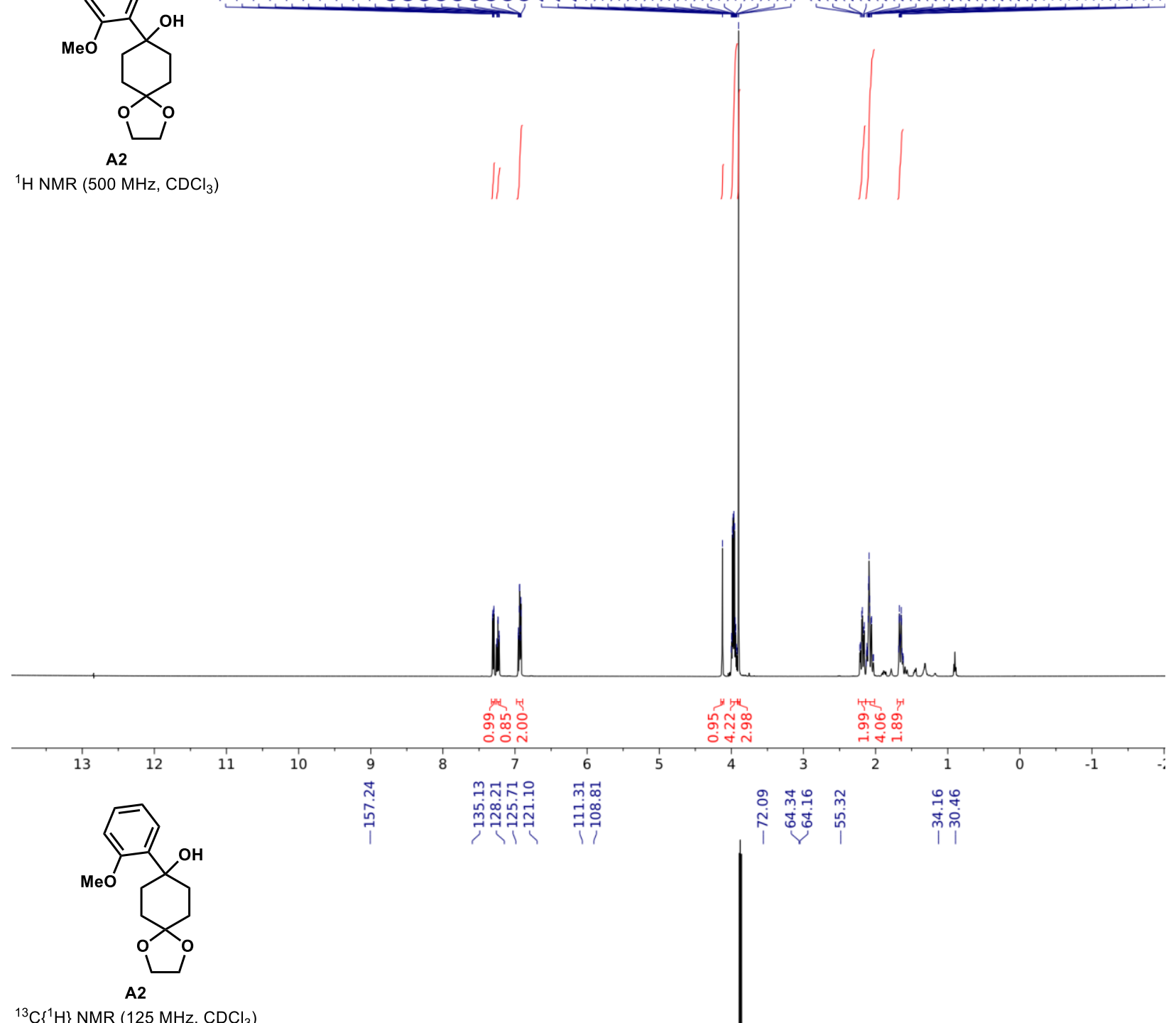

${ }^{13} \mathrm{C}\left\{{ }^{1} \mathrm{H}\right\} \mathrm{NMR}\left(125 \mathrm{MHz}, \mathrm{CDCl}_{3}\right)$

Whin

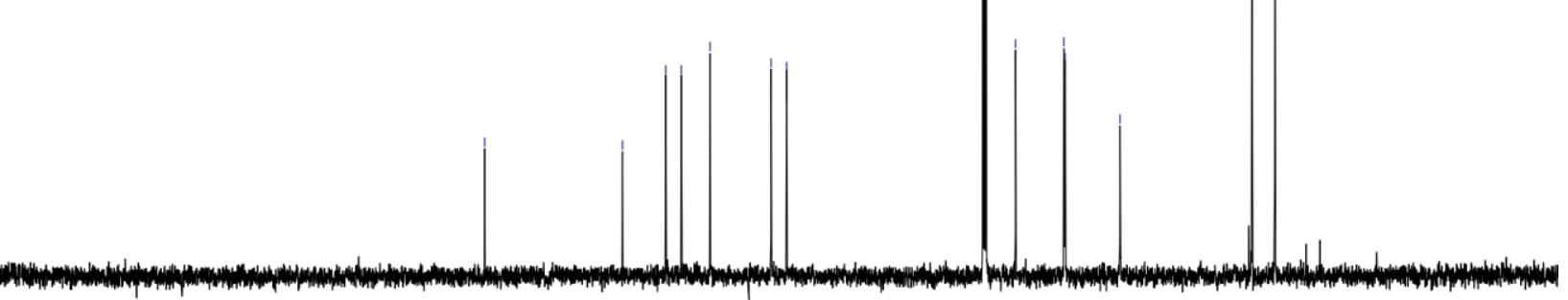

$\begin{array}{lllllllllllllllllllllllll}230 & 220 & 210 & 200 & 190 & 180 & 170 & 160 & 150 & 140 & 130 & 120 & 110 & 100 & 90 & 80 & 70 & 60 & 50 & 40 & 30 & 20 & 10 & 0 & -10\end{array}$ 


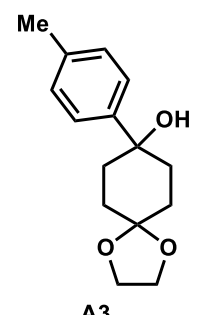

${ }^{1} \mathrm{H}$ NMR $\left(500 \mathrm{MHz}, \mathrm{CDCl}_{3}\right)$

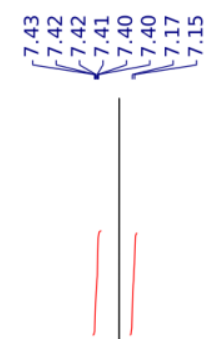

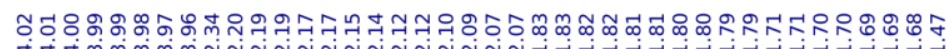

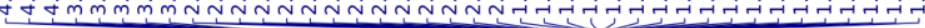
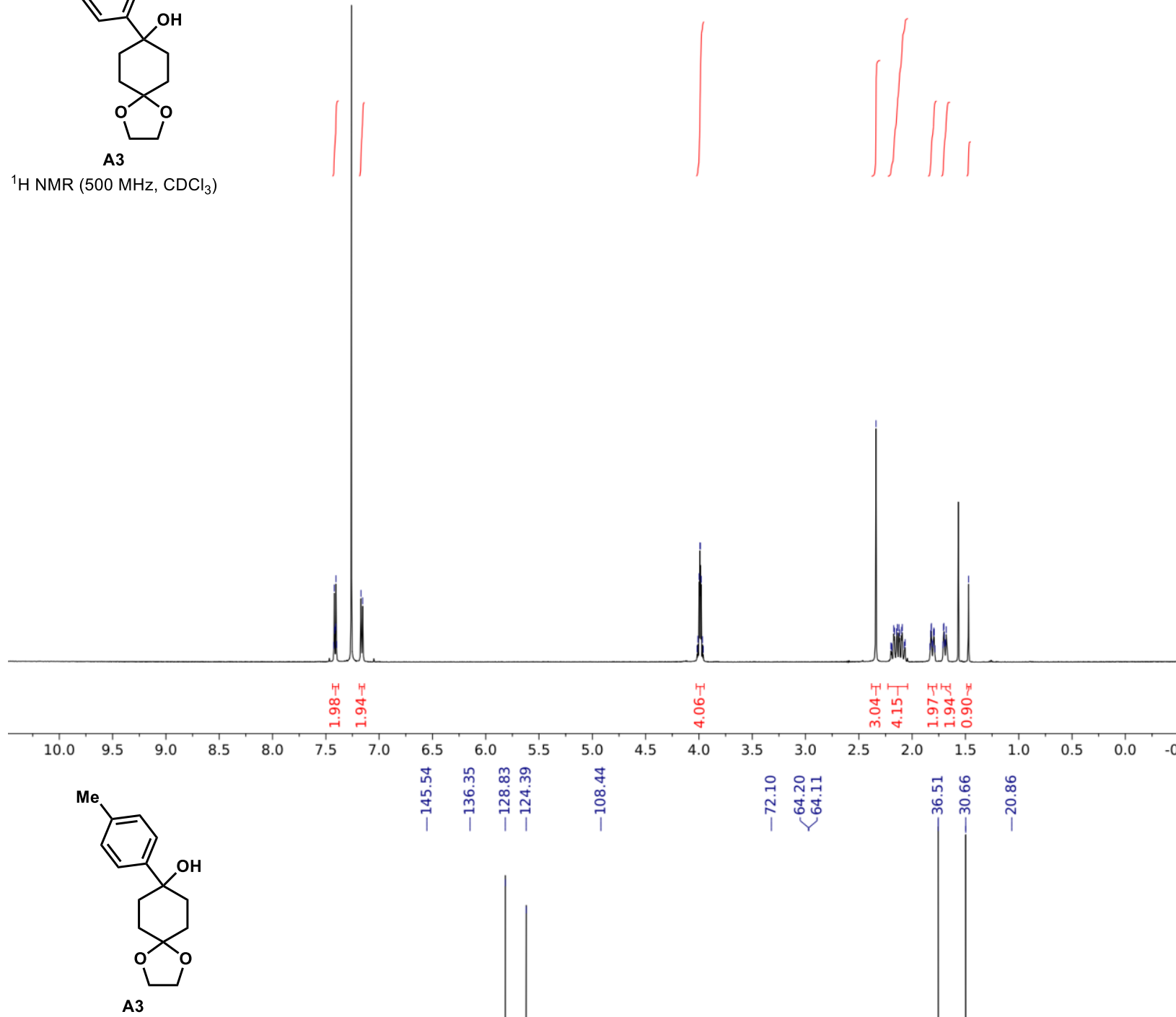

${ }^{13} \mathrm{C}\left\{{ }^{1} \mathrm{H}\right\}$ NMR $\left(125 \mathrm{MHz}, \mathrm{CDCl}_{3}\right)$

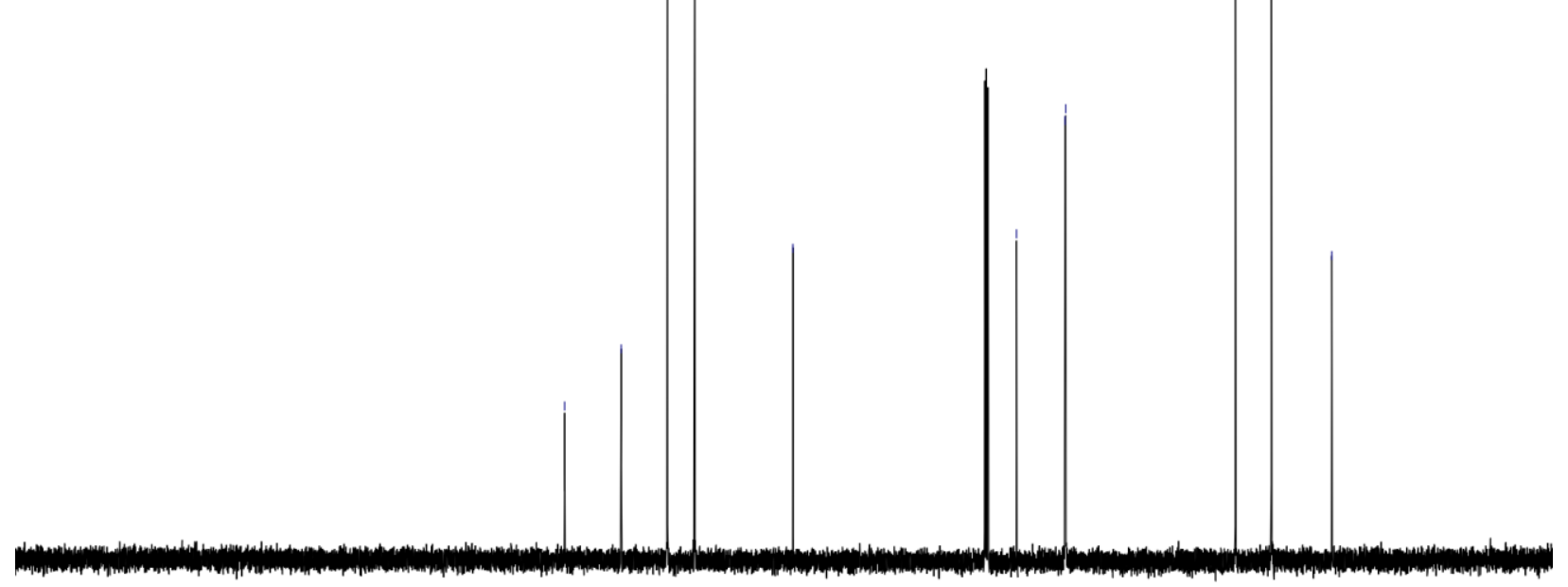

$\begin{array}{lllllllllllllllllllllllllllllllll}230 & 220 & 210 & 200 & 190 & 180 & 170 & 160 & 150 & 140 & 130 & 120 & 110 & 100 & 90 & 80 & 70 & 60 & 50 & 40 & 30 & 20 & 10 & 0 & -10\end{array}$ 


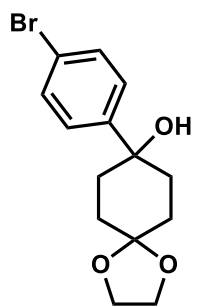

A4

${ }^{1} \mathrm{H}$ NMR $\left(300 \mathrm{MHz}, \mathrm{CDCl}_{3}\right)$
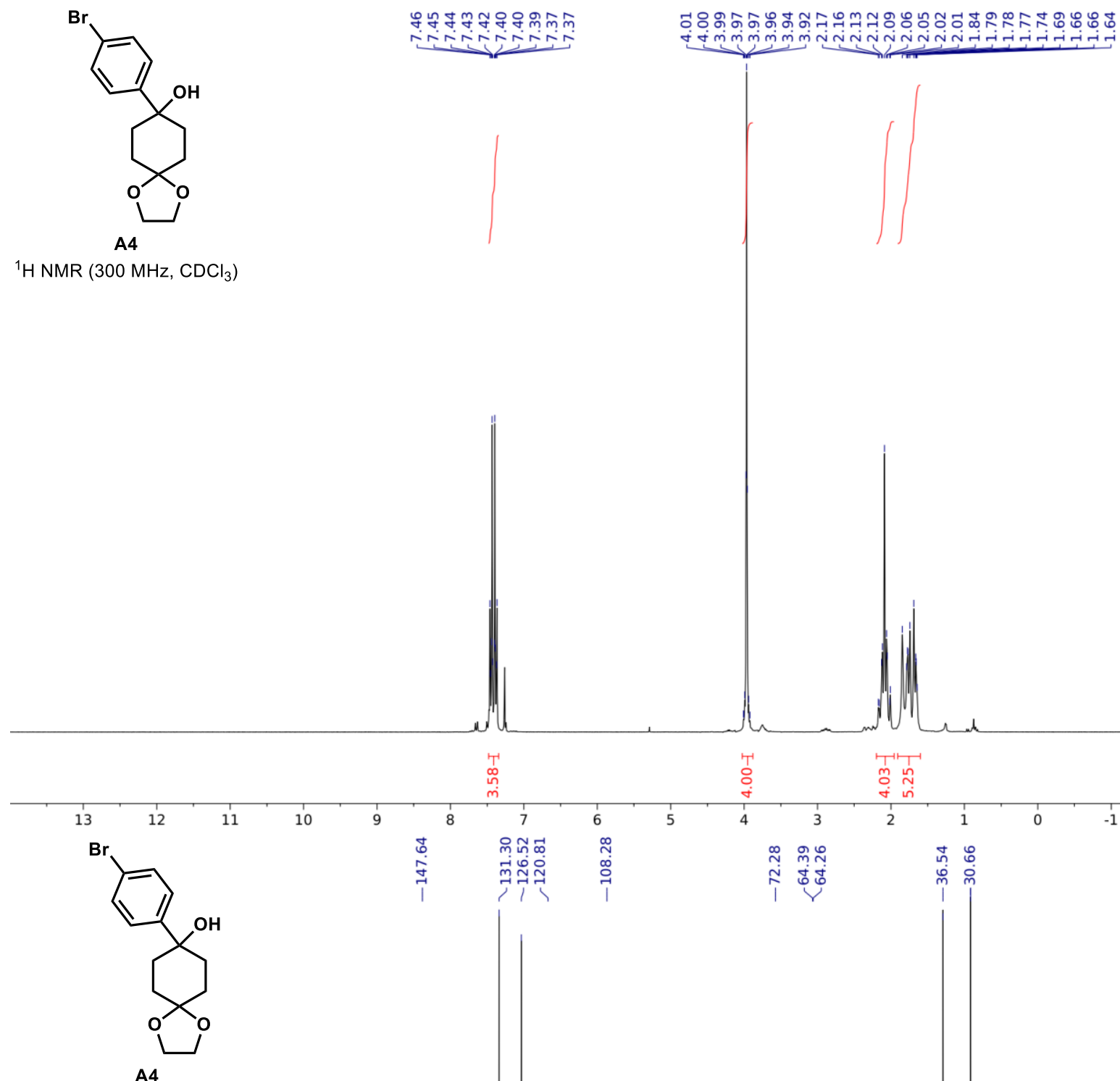

${ }^{13} \mathrm{C}\left\{{ }^{1} \mathrm{H}\right\}$ NMR $\left(125 \mathrm{MHz}, \mathrm{CDCl}_{3}\right)$

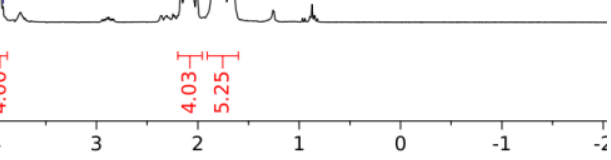

$\stackrel{\infty}{\sim} \stackrel{\sim}{\sim} \stackrel{\sim}{\oplus}$

i

लำำ
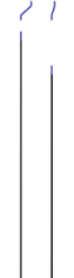

疍




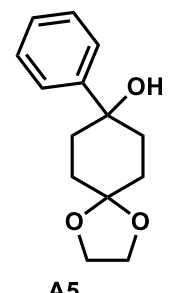

${ }^{1} \mathrm{H}$ NMR $\left(500 \mathrm{MHz}, \mathrm{CDCl}_{3}\right)$

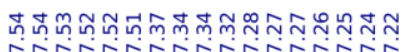

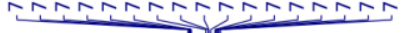

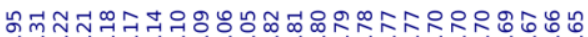

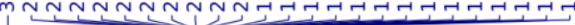
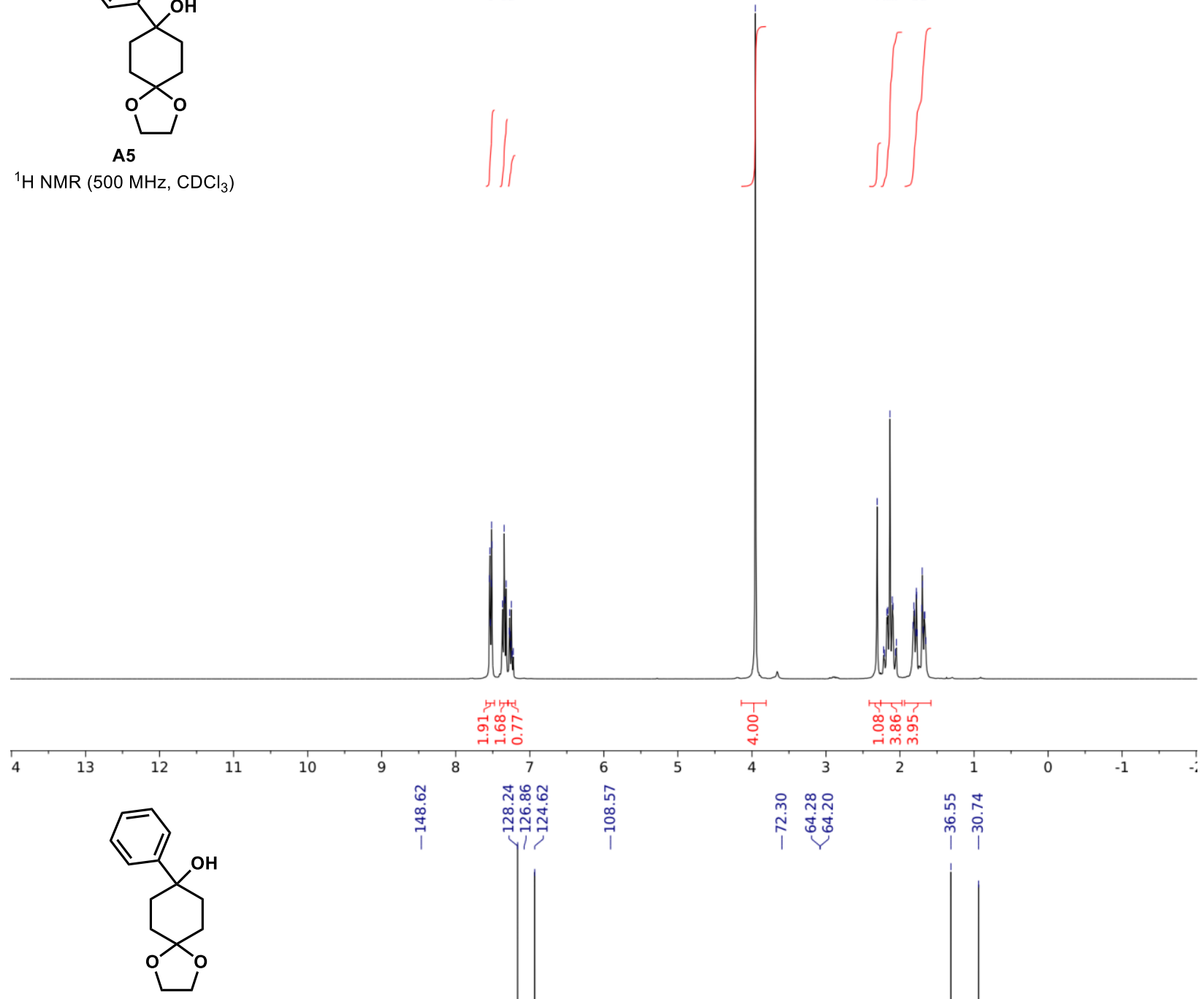

A5

${ }^{13} \mathrm{C}\left\{{ }^{1} \mathrm{H}\right\}$ NMR $\left(125 \mathrm{MHz}, \mathrm{CDCl}_{3}\right)$
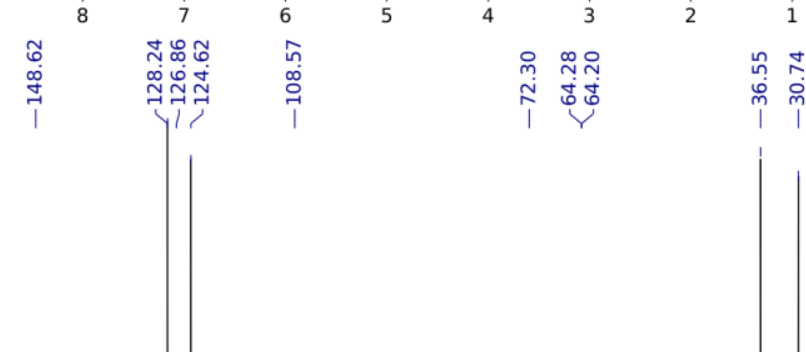

(3)

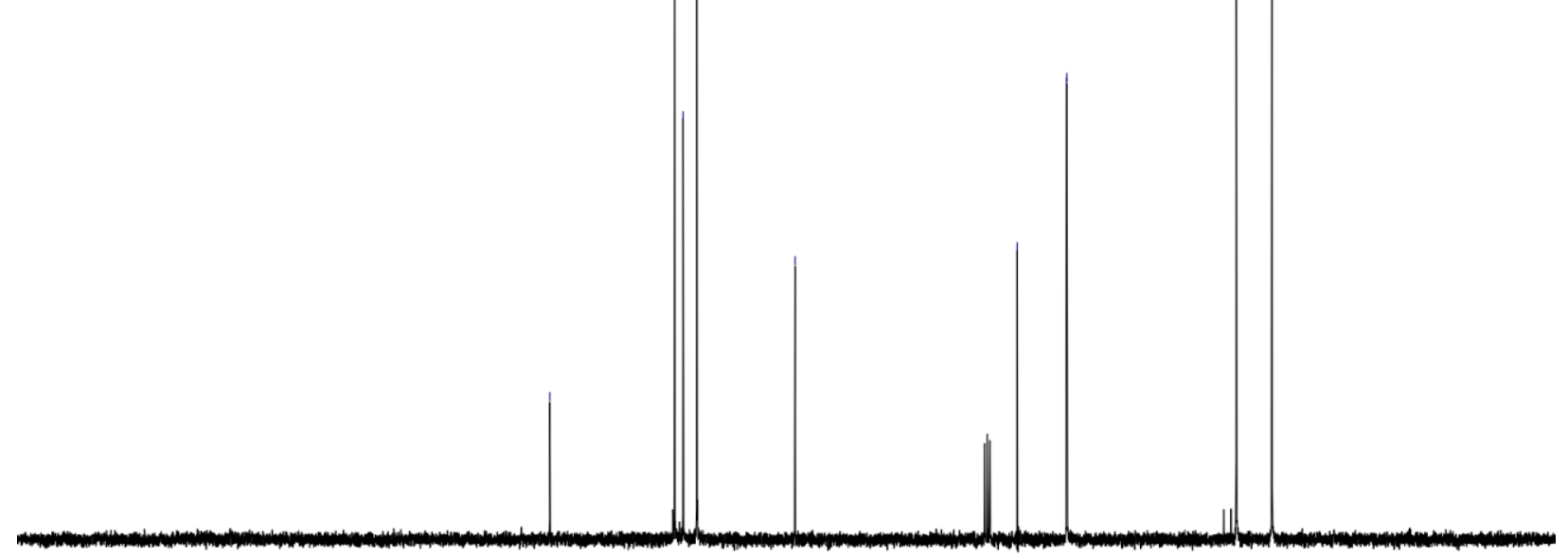

$\begin{array}{llllllllllllllllllllllllll}230 & 220 & 210 & 200 & 190 & 180 & 170 & 160 & 150 & 140 & 130 & 120 & 110 & 100 & 90 & 80 & 70 & 60 & 50 & 40 & 30 & 20 & 10 & 0 & -10\end{array}$ 
œ œ

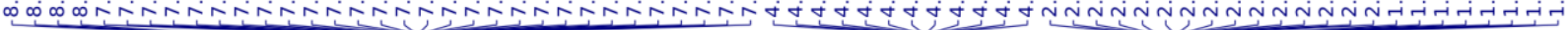
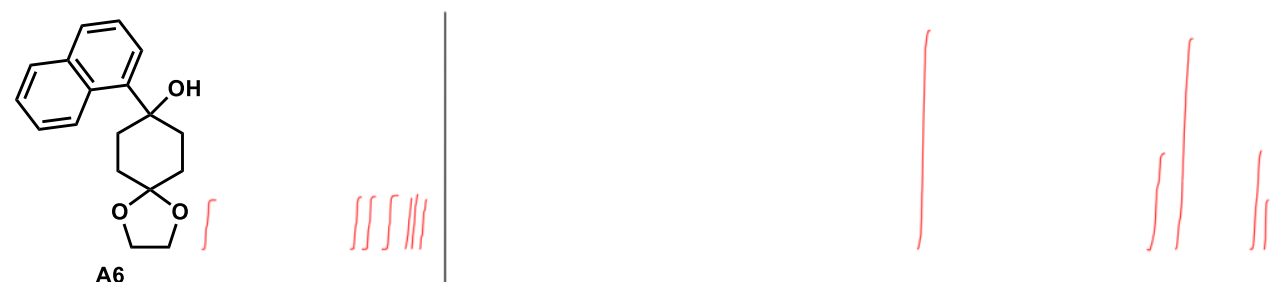

${ }^{1} \mathrm{H}$ NMR $\left(500 \mathrm{MHz}, \mathrm{CDCl}_{3}\right)$

'HNR (500
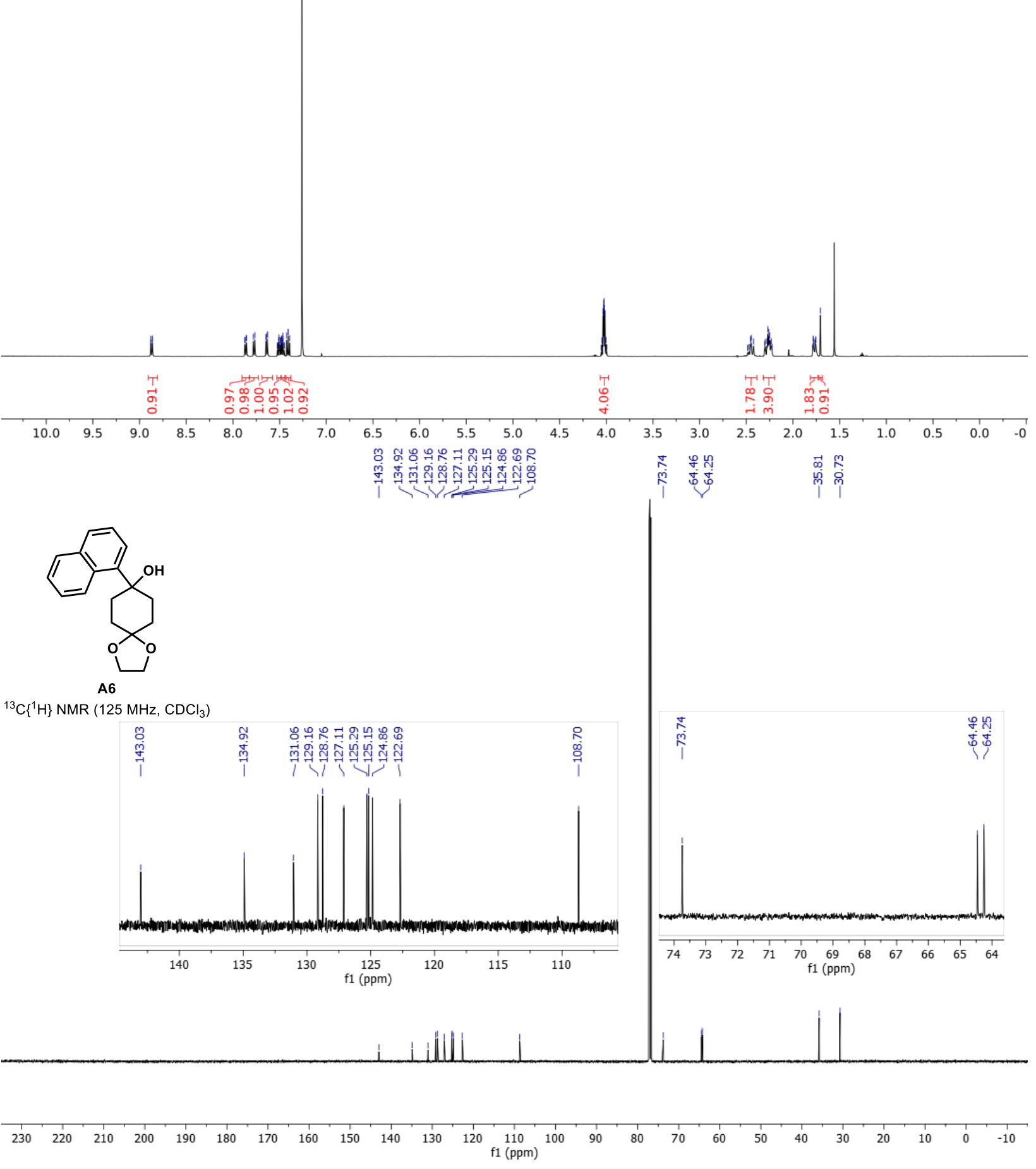


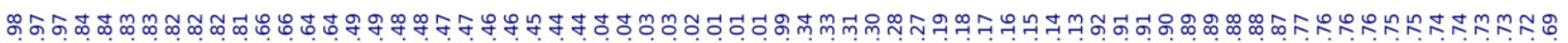

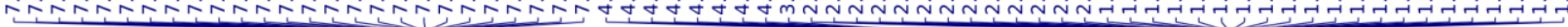
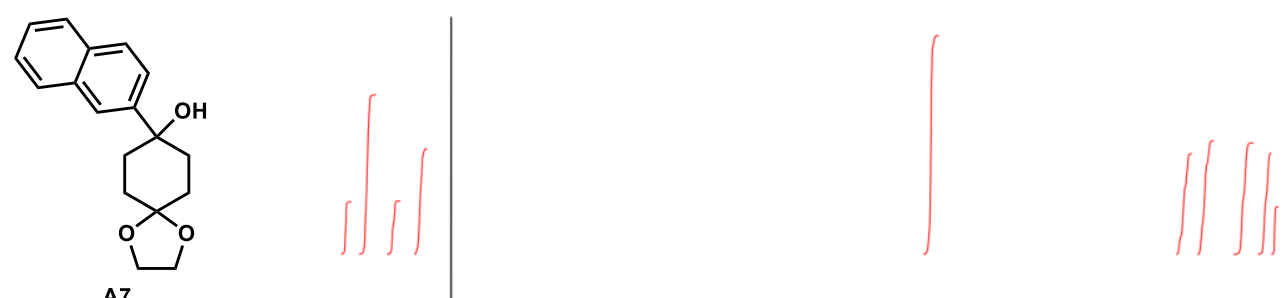

${ }^{1} \mathrm{H} \mathrm{NMR}\left(500 \mathrm{MHz}, \mathrm{CDCl}_{3}\right)$

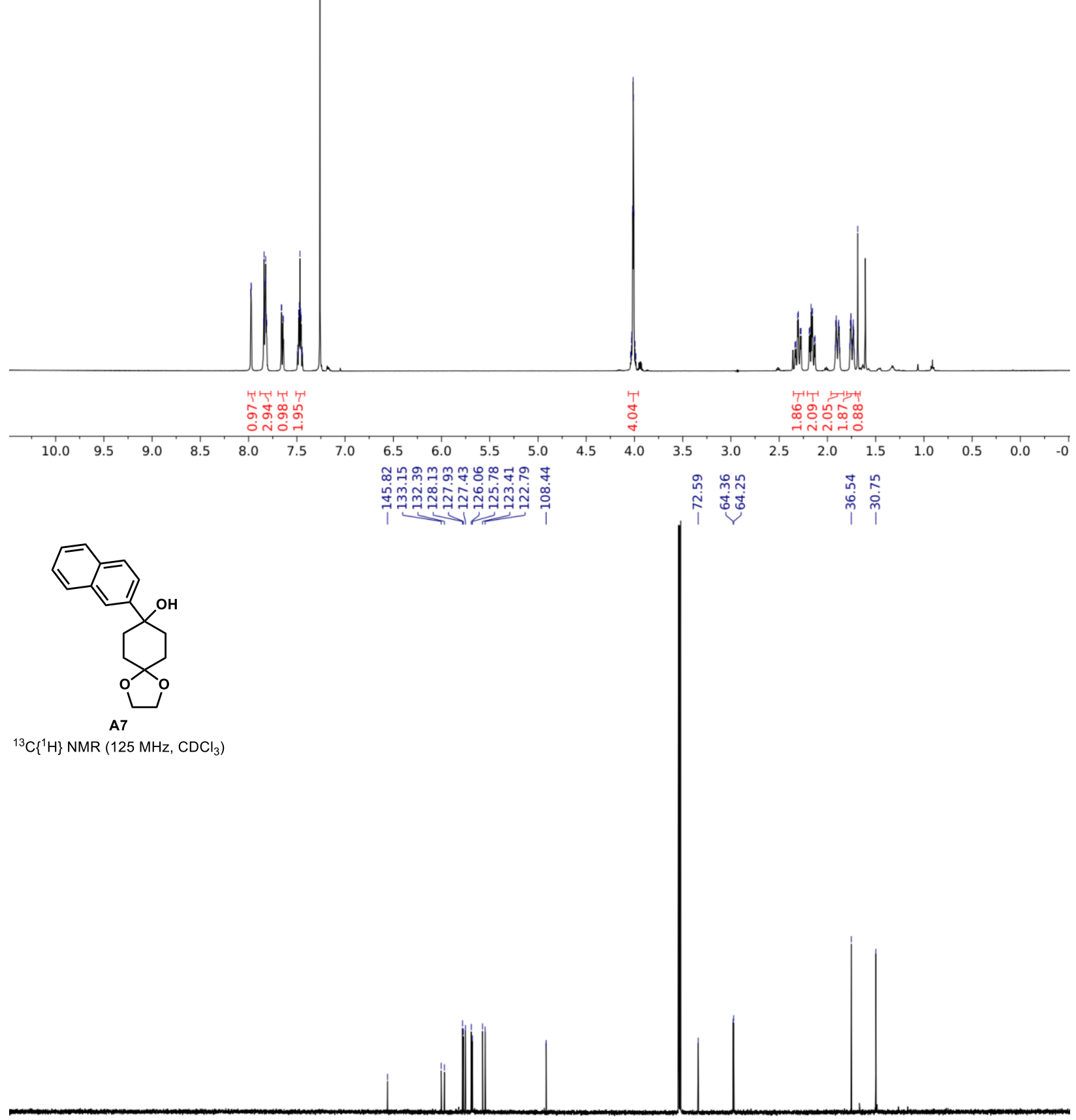

$\begin{array}{lllllllllllllllllllllllll}230 & 220 & 210 & 200 & 190 & 180 & 170 & 160 & 150 & 140 & 130 & 120 & 110 & 100 & 90 & 80 & 70 & 60 & 50 & 40 & 30 & 20 & 10 & 0 & -10\end{array}$ 


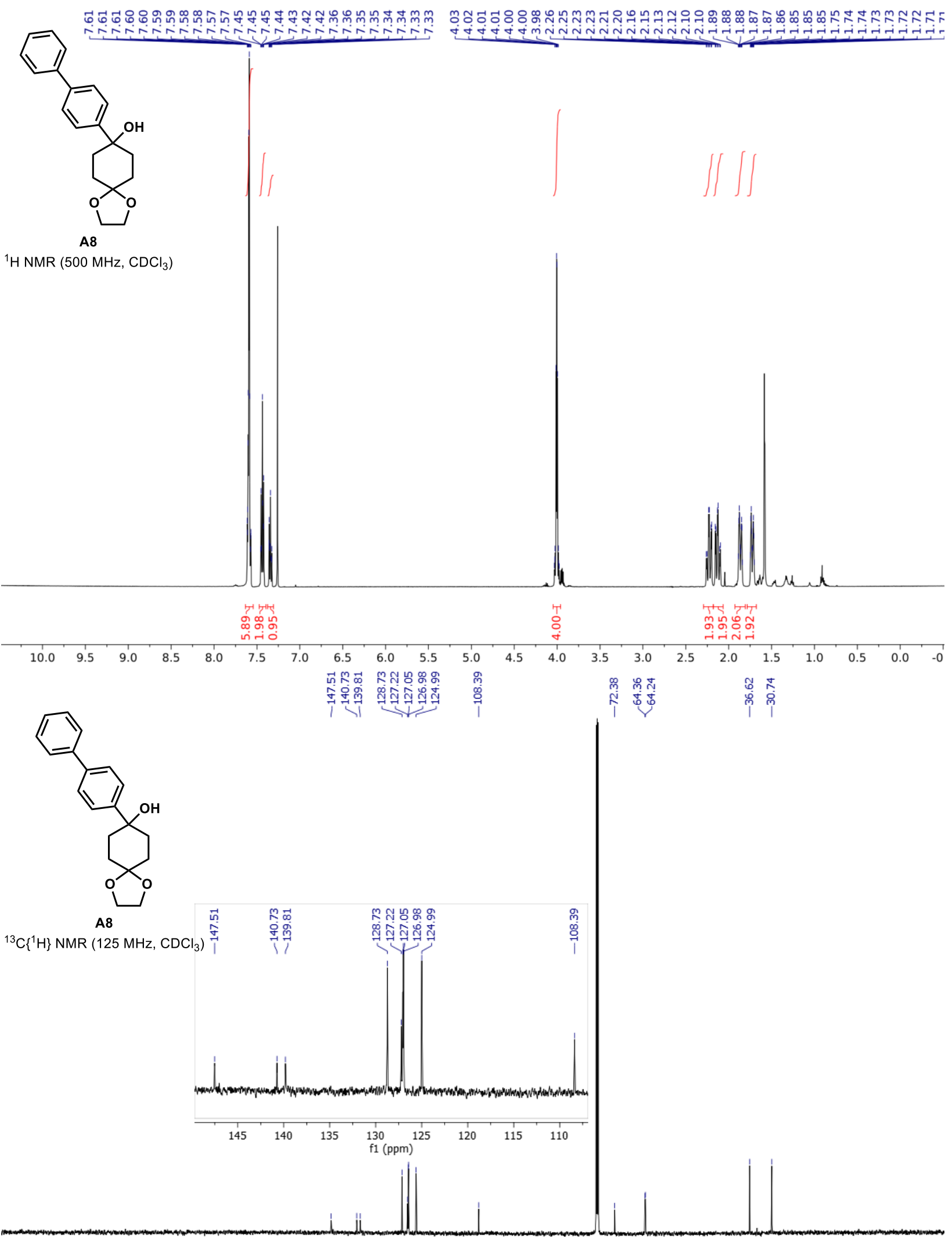

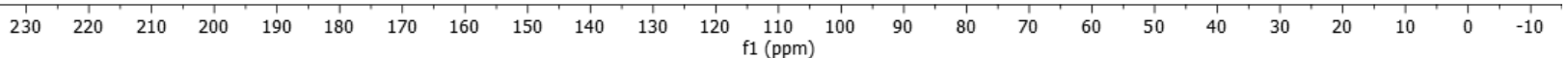



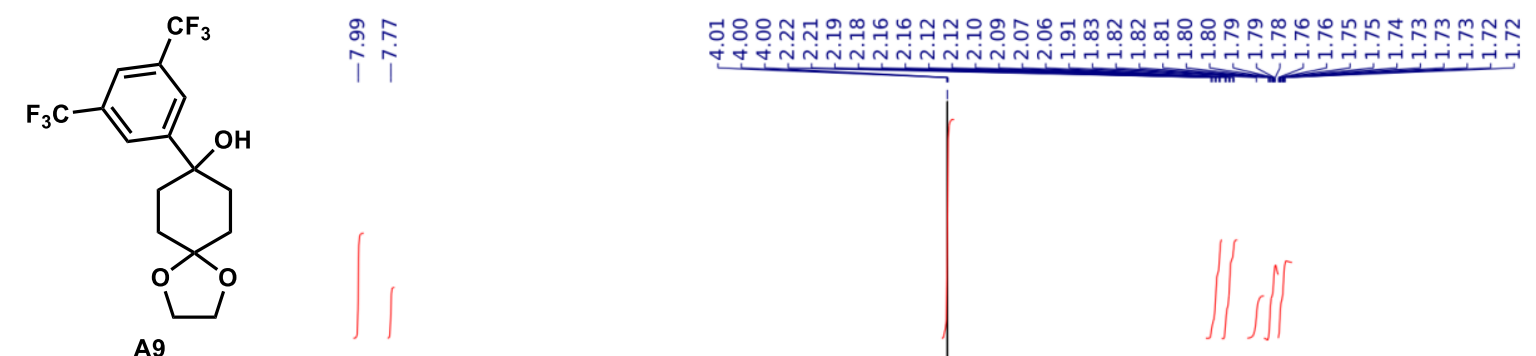

${ }^{1} \mathrm{H} \mathrm{NMR}\left(500 \mathrm{MHz}, \mathrm{CDCl}_{3}\right)$
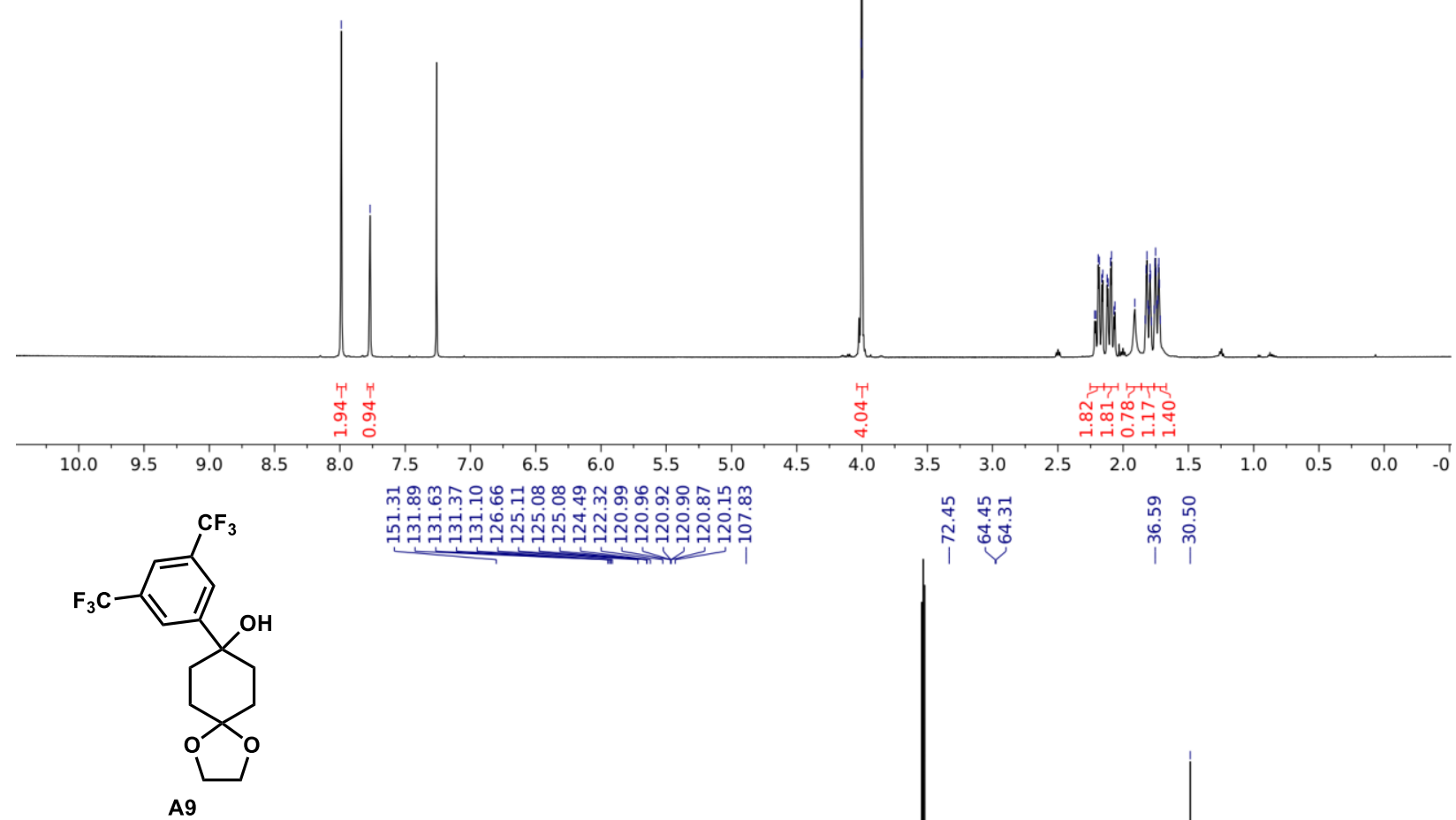

そे ๆे

i

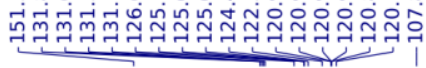

${ }^{13} \mathrm{C}\left\{{ }^{1} \mathrm{H}\right\}$ NMR $\left(125 \mathrm{MHz}, \mathrm{CDCl}_{3}\right)$

$\begin{array}{llllllllllllllllllllllllllllllllll}230 & 220 & 210 & 200 & 190 & 180 & 170 & 160 & 150 & 140 & 130 & 120 & 110 & 100 & 90 & 80 & 70 & 60 & 50 & 40 & 30 & 20 & 10 & 0 & -10\end{array}$ 


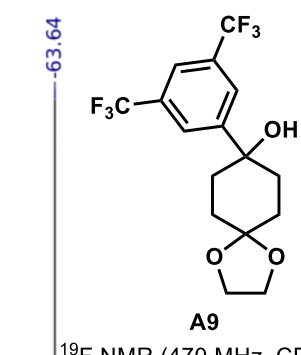

${ }^{19} \mathrm{~F} \mathrm{NMR}\left(470 \mathrm{MHz}, \mathrm{CDCl}_{3}\right)$

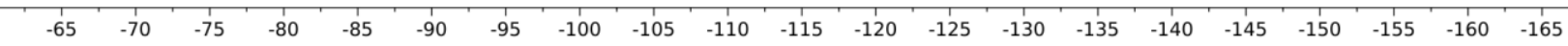


MeO<smiles>Cc1ccc(CO)cc1</smiles><smiles>CC(C)(C)c1ccc(CC2CC3(CCC2(C)C)OCCO3)cc1</smiles>

A10

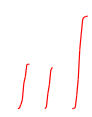

${ }^{1} \mathrm{H}$ NMR $\left(300 \mathrm{MHz}, \mathrm{CDCl}_{3}\right)$

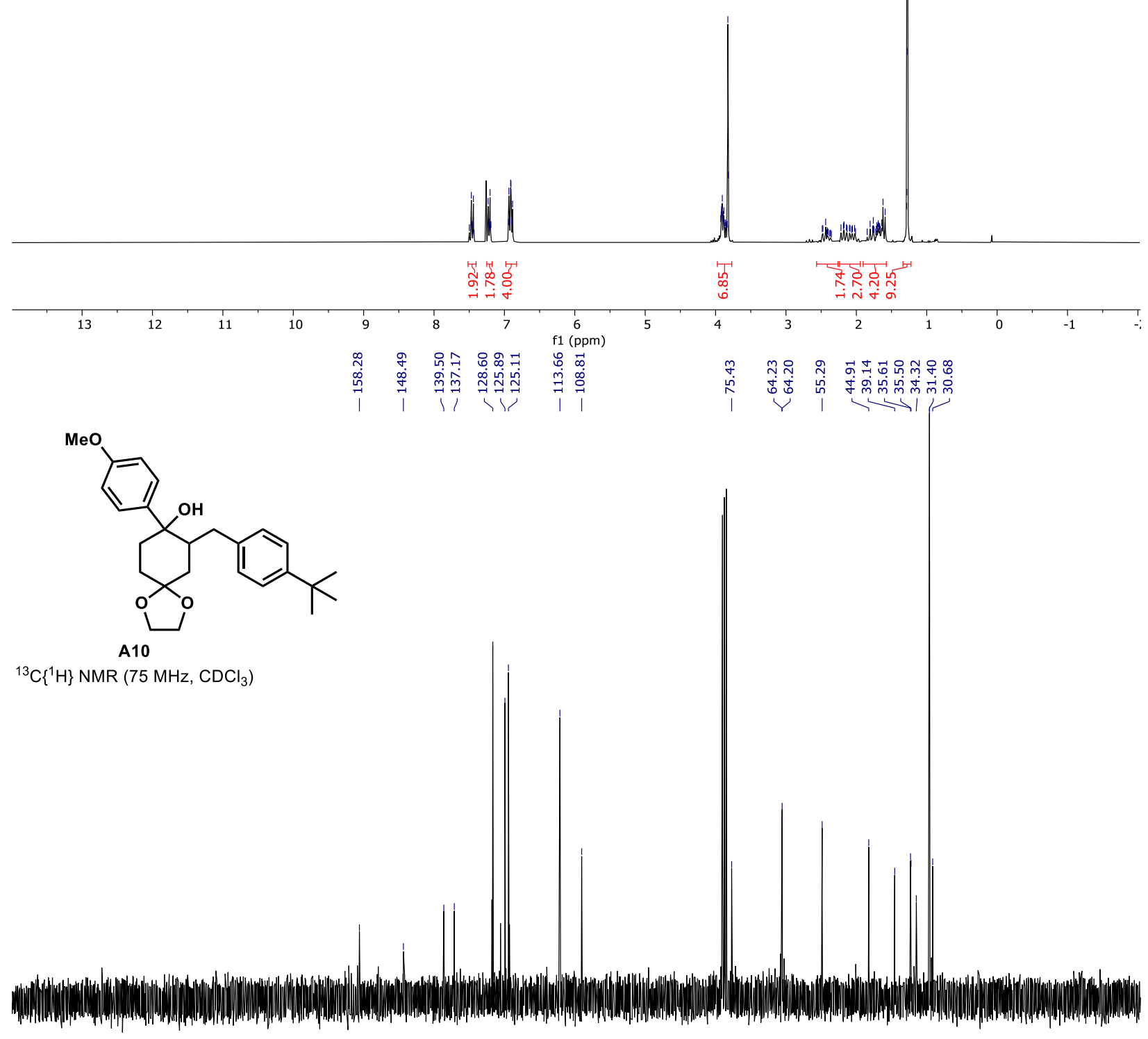

$\begin{array}{lllllllllllllllllllllllllllll}130 & 220 & 210 & 200 & 190 & 180 & 170 & 160 & 150 & 140 & 130 & 120 & 110 & 100 & 90 & 80 & 70 & 60 & 50 & 40 & 30 & 20 & 10 & 0 & -10\end{array}$ 


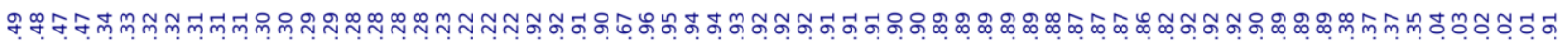

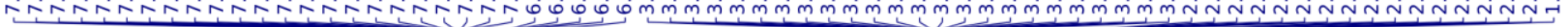

$\mathrm{MeO}$<smiles></smiles>

4a

${ }^{1} \mathrm{H}$ NMR $\left(500 \mathrm{MHz}, \mathrm{CDCl}_{3}\right)$
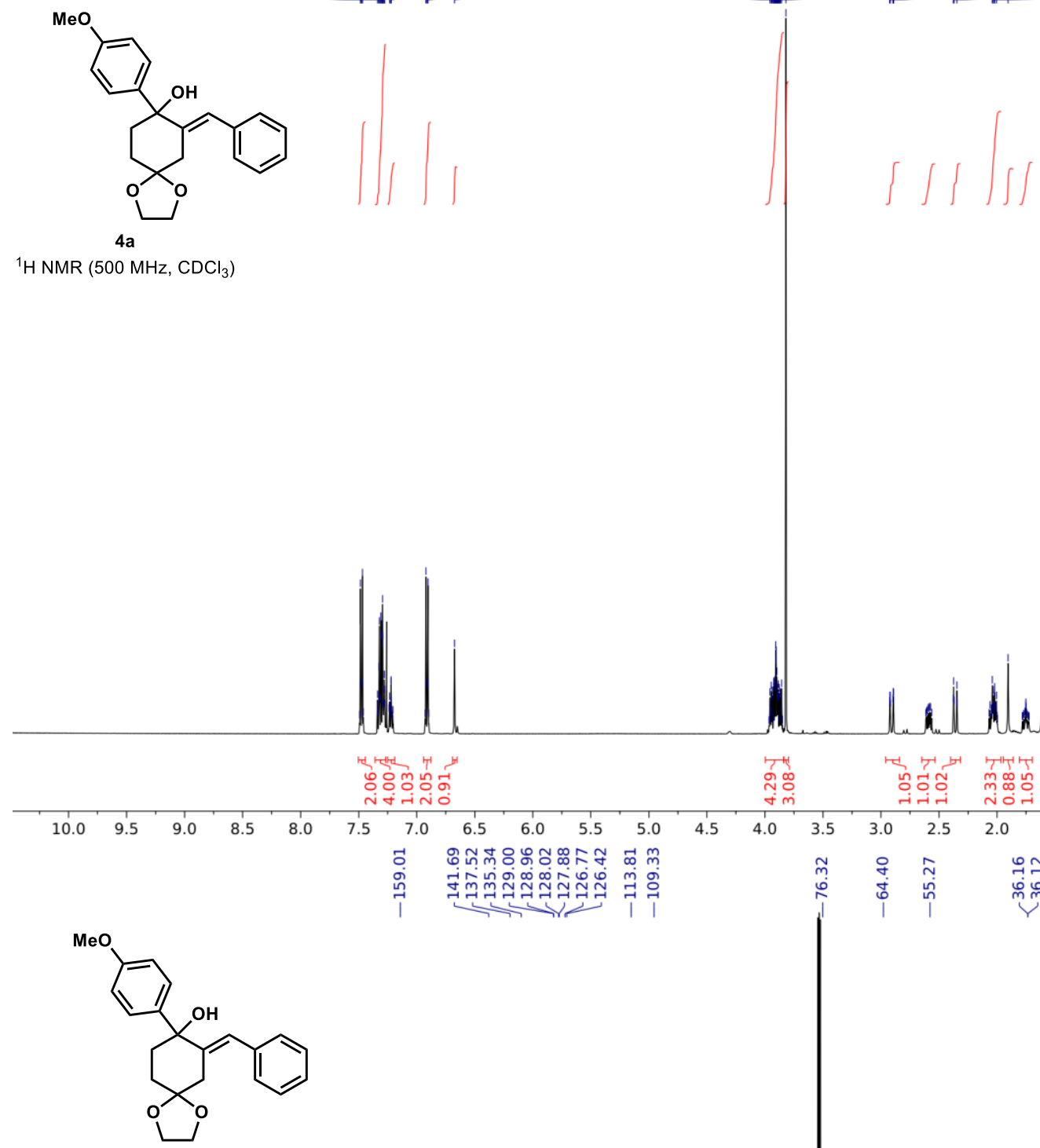

$4 a$

${ }^{13} \mathrm{C}\left\{{ }^{1} \mathrm{H}\right\}$ NMR $\left(125 \mathrm{MHz}, \mathrm{CDCl}_{3}\right)$

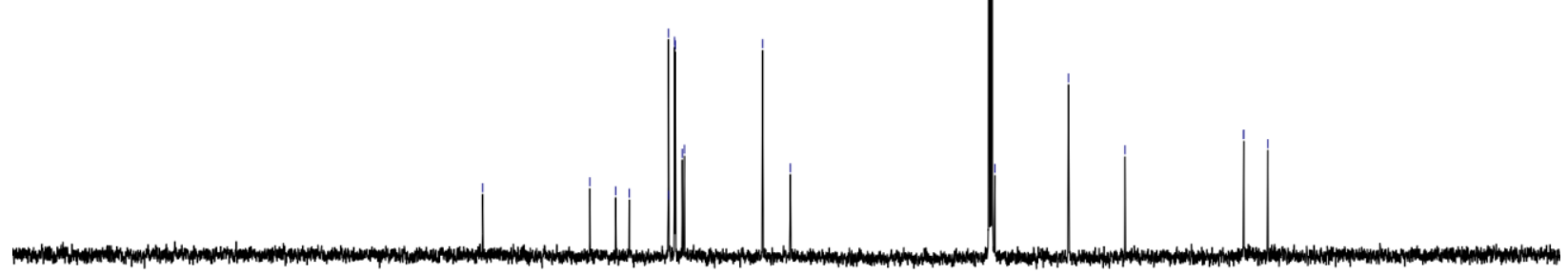

$\begin{array}{lllllllllllllllllllllllll}230 & 220 & 210 & 200 & 190 & 180 & 170 & 160 & 150 & 140 & 130 & 120 & 110 & 100 & 90 & 80 & 70 & 60 & 50 & 40 & 30 & 20 & 10 & 0 & -10\end{array}$ 
$\mathrm{MeO}$<smiles>COc1ccc(C=C2CC3(CCC2(O)c2ccc(C)cc2)OCCO3)cc1</smiles>

4b

${ }^{1} \mathrm{H}$ NMR $\left(500 \mathrm{MHz}, \mathrm{CDCl}_{3}\right)$

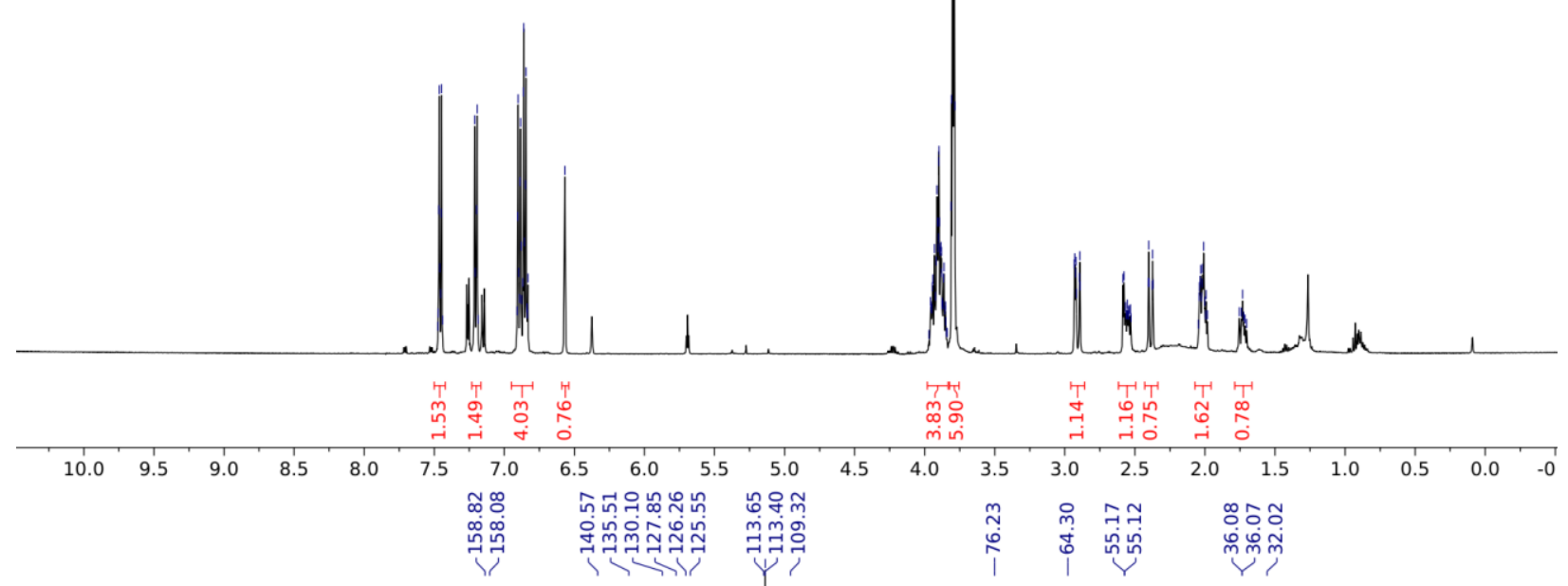<smiles>COc1ccc(/C=C2\CC3(CCC2(O)c2ccc(OC)cc2)OCCO3)cc1</smiles>

4b

${ }^{13} \mathrm{C}\left\{{ }^{1} \mathrm{H}\right\}$ NMR $\left(125 \mathrm{MHz}, \mathrm{CDCl}_{3}\right)$

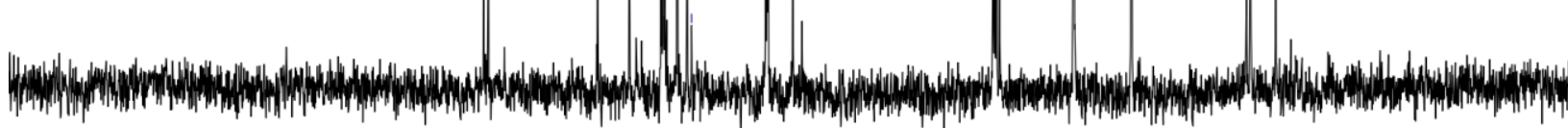

$\begin{array}{lllllllllllllllllllllllll}230 & 220 & 210 & 200 & 190 & 180 & 170 & 160 & 150 & 140 & 130 & 120 & 110 & 100 & 90 & 80 & 70 & 60 & 50 & 40 & 30 & 20 & 10 & 0 & -10\end{array}$ 


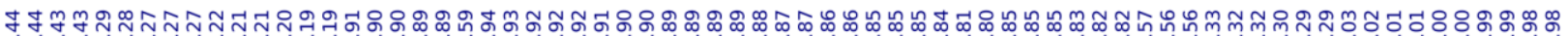

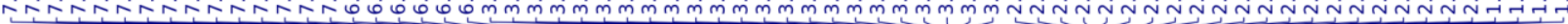

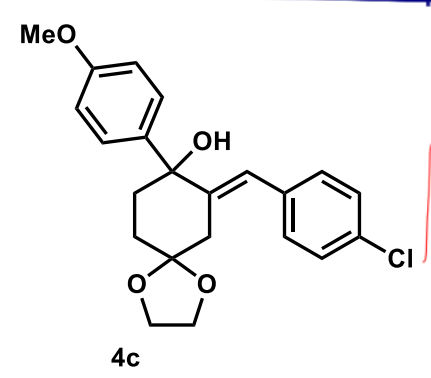

${ }^{1} \mathrm{H} \operatorname{NMR}\left(500 \mathrm{MHz}, \mathrm{CDCl}_{3}\right)$
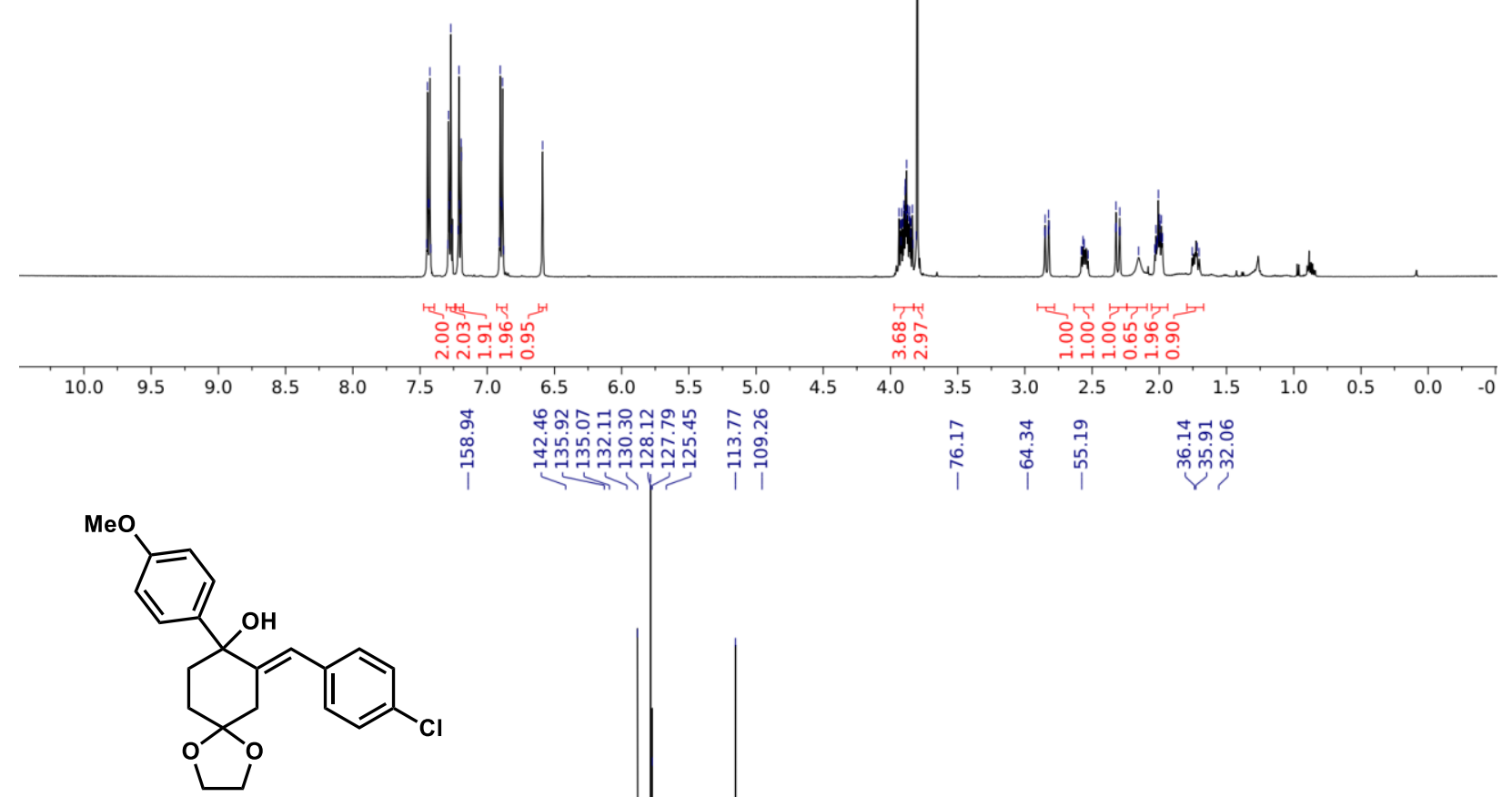

4c

${ }^{13} \mathrm{C}\left\{{ }^{1} \mathrm{H}\right\}$ NMR $\left(125 \mathrm{MHz}, \mathrm{CDCl}_{3}\right)$

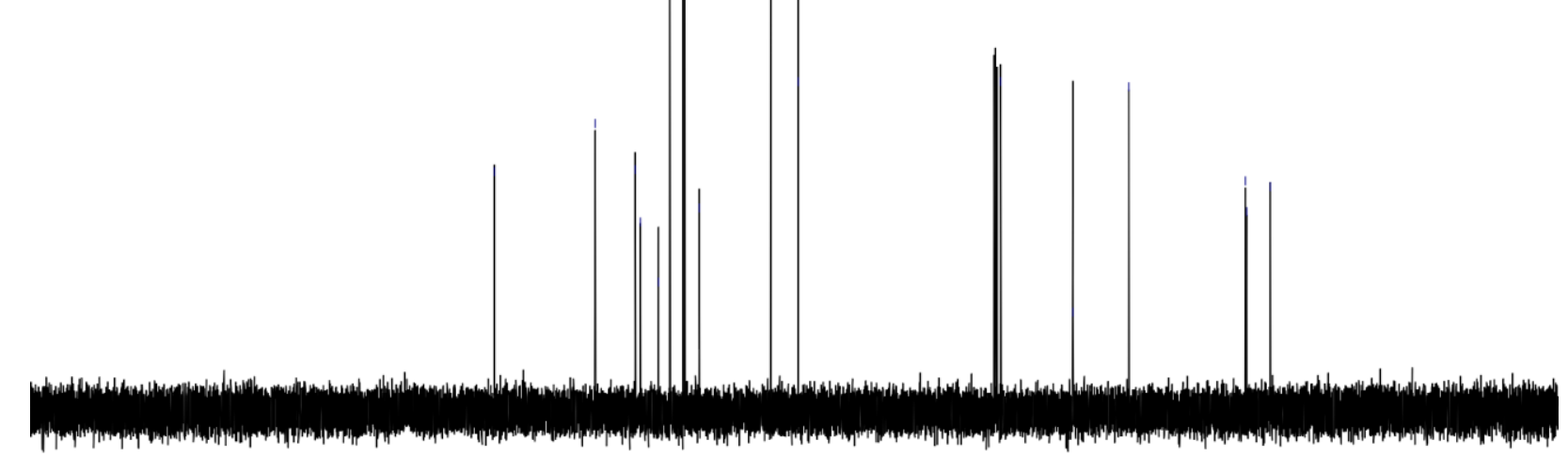

$\begin{array}{lllllllllllllllllllllllll}230 & 220 & 210 & 200 & 190 & 180 & 170 & 160 & 150 & 140 & 130 & 120 & 110 & 100 & 90 & 80 & 70 & 60 & 50 & 40 & 30 & 20 & 10 & 0 & -10\end{array}$ 


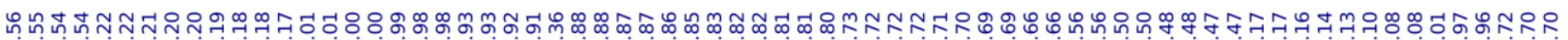
NNNNNNNNNNNNNNNー $\mathrm{MeO}$

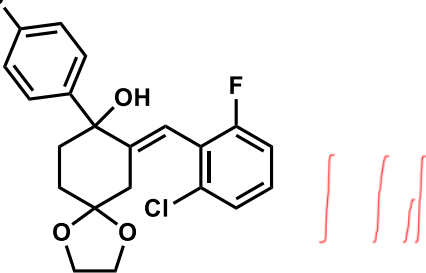

4d

${ }^{1} \mathrm{H}$ NMR $\left(500 \mathrm{MHz}, \mathrm{CDCl}_{3}\right)$
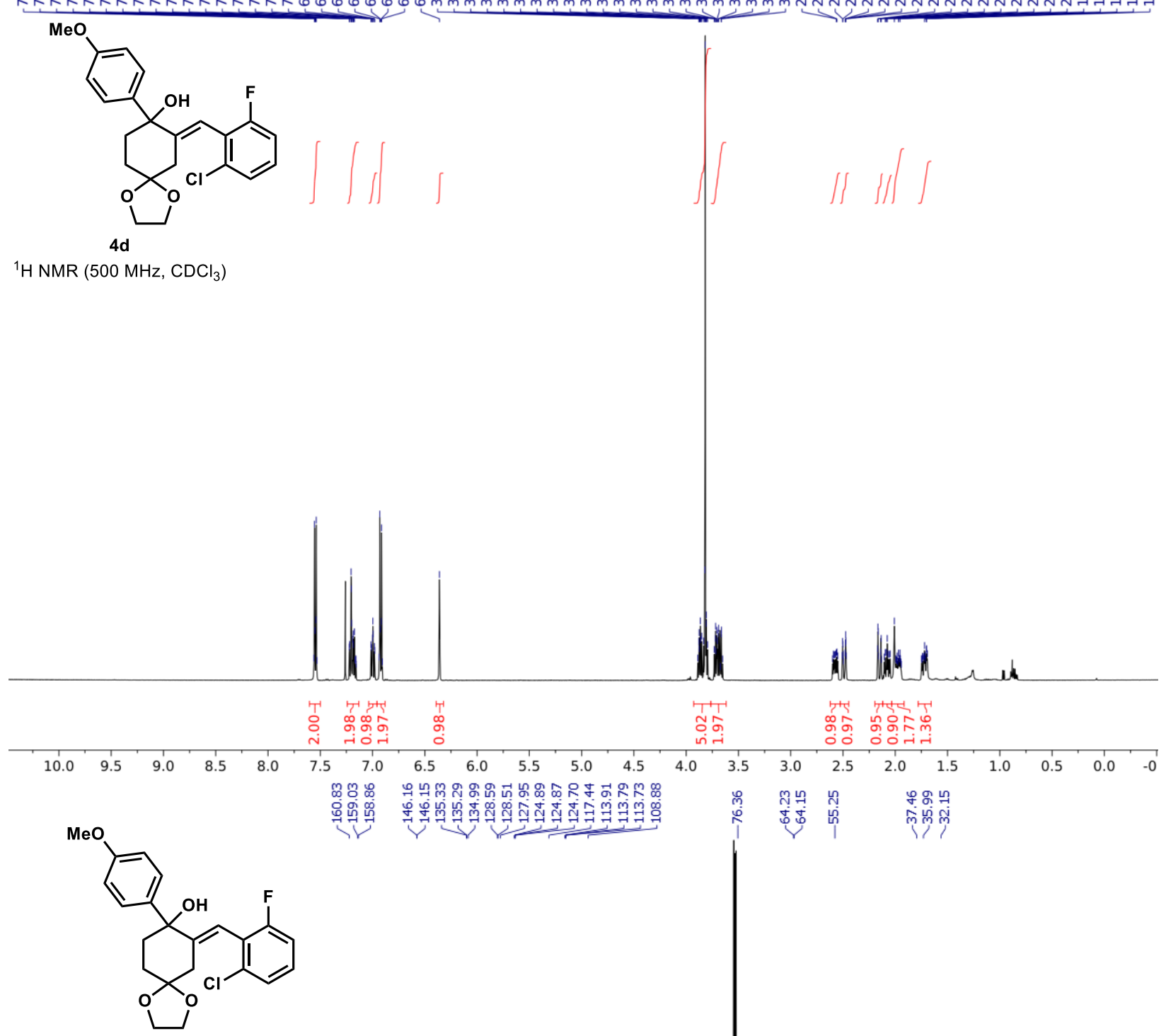

4d

${ }^{13} \mathrm{C}\left\{{ }^{1} \mathrm{H}\right\} \mathrm{NMR}\left(125 \mathrm{MHz}, \mathrm{CDCl}_{3}\right)$

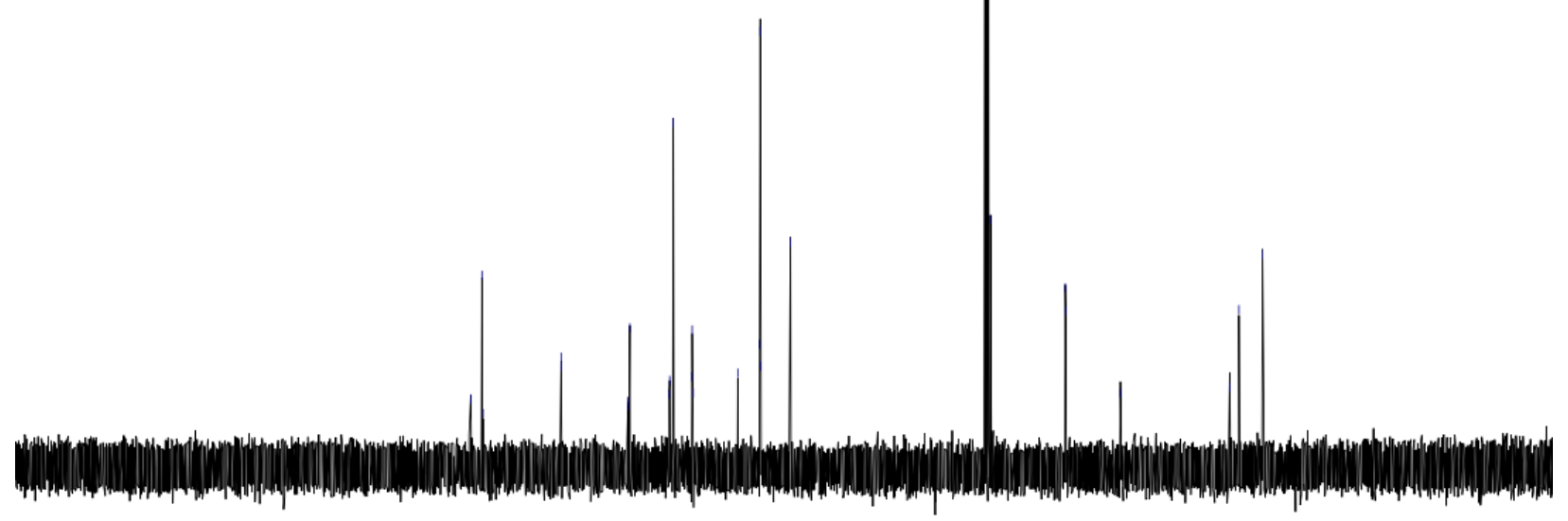

$\begin{array}{rlllllllllllllllllllllllllll}230 & 220 & 210 & 200 & 190 & 180 & 170 & 160 & 150 & 140 & 130 & 120 & 110 & 100 & 90 & 80 & 70 & 60 & 50 & 40 & 30 & 20 & 10 & 0 & -10\end{array}$ 
$\mathrm{MeO}$

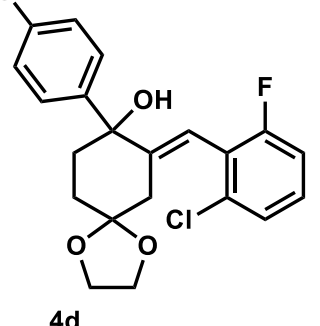

${ }^{19} \mathrm{~F}$ NMR $\left(470 \mathrm{MHz}, \mathrm{CDCl}_{3}\right)$
ธ్య

8.

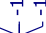

\begin{tabular}{lllllllllllllllllllllllll}
\hline 20 & 10 & 0 & -10 & -20 & -30 & -40 & -50 & -60 & -70 & -80 & -90 & -100 & -110 & -120 & -130 & -140 & -150 & -160 & -170 & -180 & -190 & -2
\end{tabular}




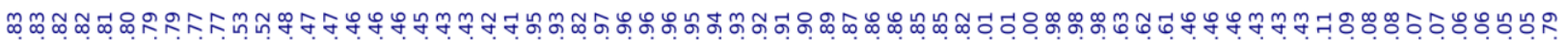

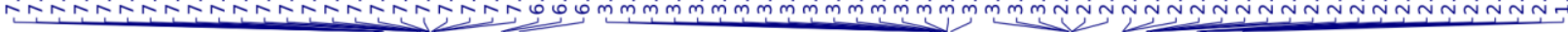

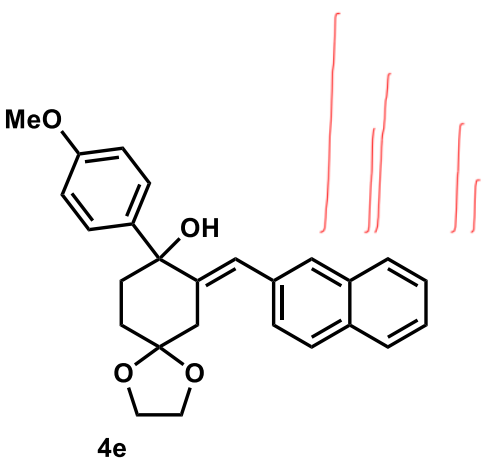

${ }^{1} \mathrm{H}$ NMR $\left(500 \mathrm{MHz}, \mathrm{CDCl}_{3}\right)$
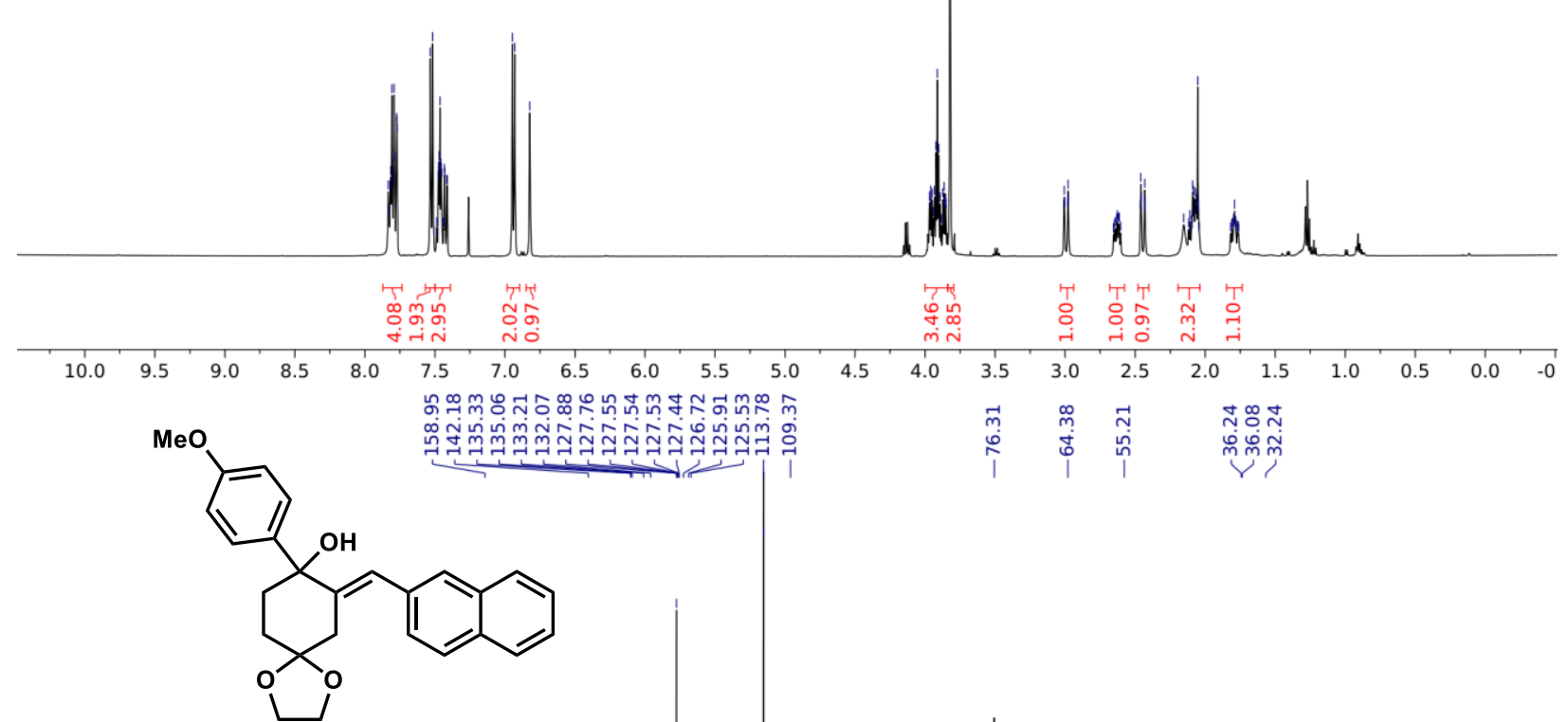

$4 e$

${ }^{13} \mathrm{C}\left\{{ }^{1} \mathrm{H}\right\}$ NMR $\left(125 \mathrm{MHz}, \mathrm{CDCl}_{3}\right)$

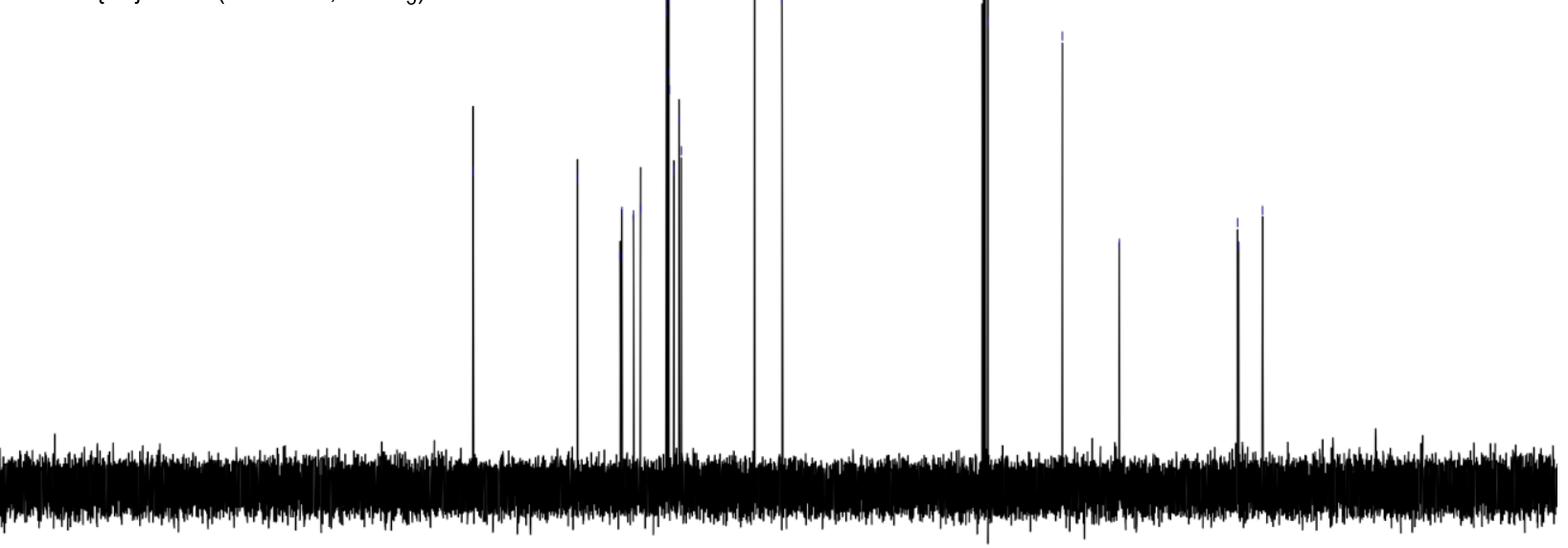

$\begin{array}{lllllllllllllllllllllllll}230 & 220 & 210 & 200 & 190 & 180 & 170 & 160 & 150 & 140 & 130 & 120 & 110 & 100 & 90 & 80 & 70 & 60 & 50 & 40 & 30 & 20 & 10 & 0 & -10\end{array}$ 


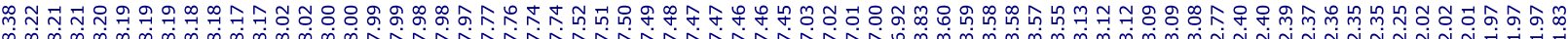

$\mathrm{MeO}$<smiles>Cc1ccc(C2(O)CCC3(CC(=Cc4c5ccccc5cc5ccccc45)C3)OCCO2)cc1</smiles>

$4 f$

${ }^{1} \mathrm{H}$ NMR $\left(300 \mathrm{MHz}, \mathrm{CDCl}_{3}\right)$
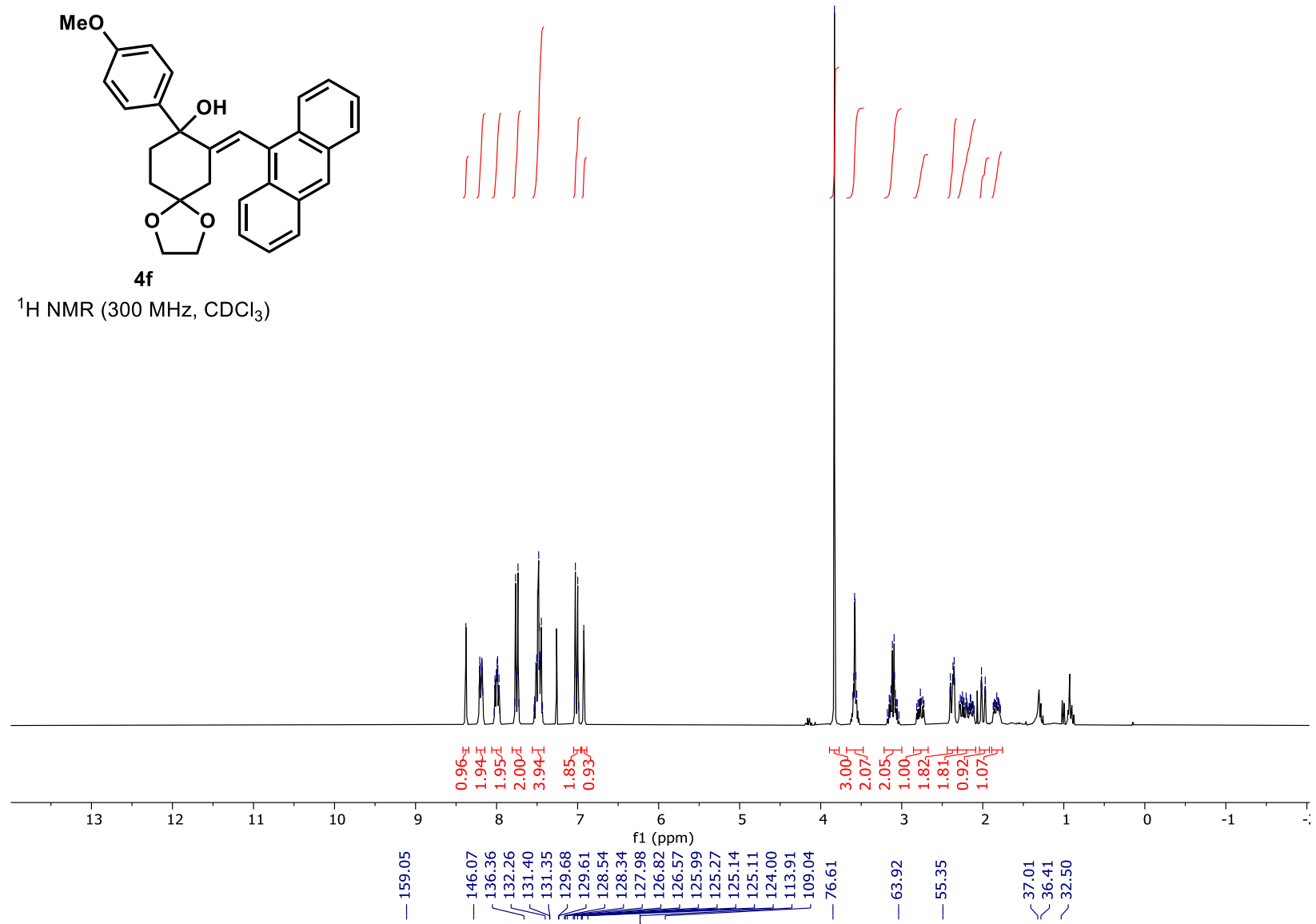

$\mathrm{MeO}$<smiles>Cc1ccc(C2(O)CCC3(CC(=Cc4c5ccccc5cc5ccccc45)C3)OCCO2)cc1</smiles>

4f

${ }^{13} \mathrm{C}\left\{{ }^{1} \mathrm{H}\right\} \mathrm{NMR}\left(75 \mathrm{MHz}, \mathrm{CDCl}_{3}\right)$

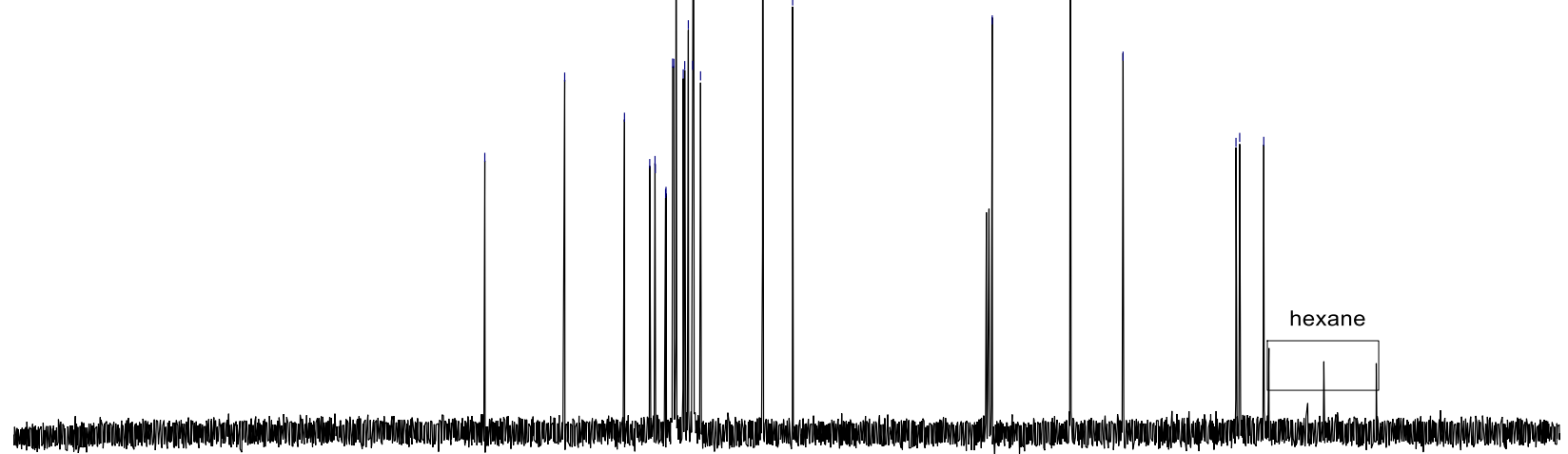

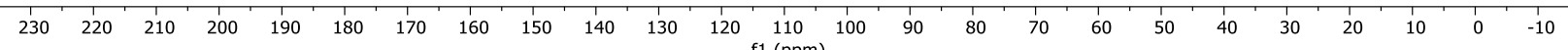


mి N-Nヘอ

$\mathrm{MeO}$<smiles>Cc1ccc(C2(O)CCC3(CC2=CC(C)(C)C)OCCO3)cc1</smiles>

$4 \mathrm{~g}$

${ }^{1} \mathrm{H}$ NMR $\left(300 \mathrm{MHz}, \mathrm{CDCl}_{3}\right)$

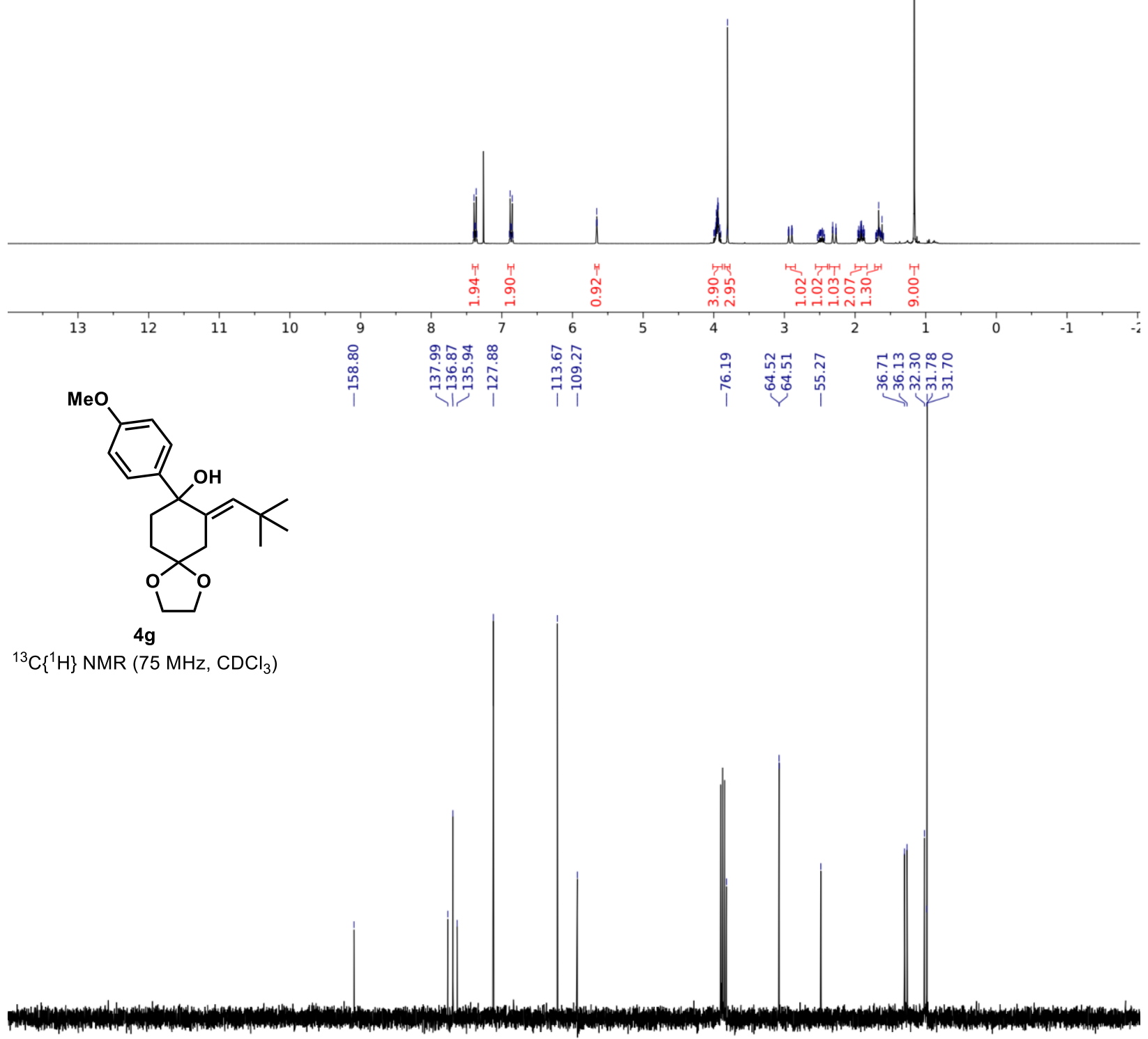

$\begin{array}{lllllllllllllllllllllllll}230 & 220 & 210 & 200 & 190 & 180 & 170 & 160 & 150 & 140 & 130 & 120 & 110 & 100 & 90 & 80 & 70 & 60 & 50 & 40 & 30 & 20 & 10 & 0 & -10\end{array}$ 


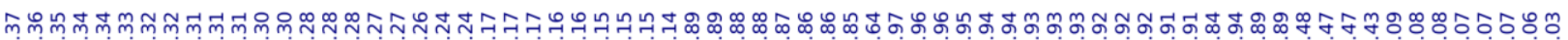

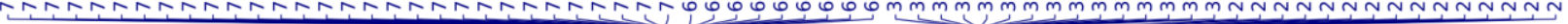<smiles>COc1cccc(C2(O)CCC3(CC2=Cc2ccccc2)OCCO3)c1</smiles>

4h

${ }^{1} \mathrm{H}$ NMR $\left(300 \mathrm{MHz}, \mathrm{CDCl}_{3}\right)$$$
\text { (300 } \left.\mathrm{MHZ}, \mathrm{CDCl}_{3}\right)
$$
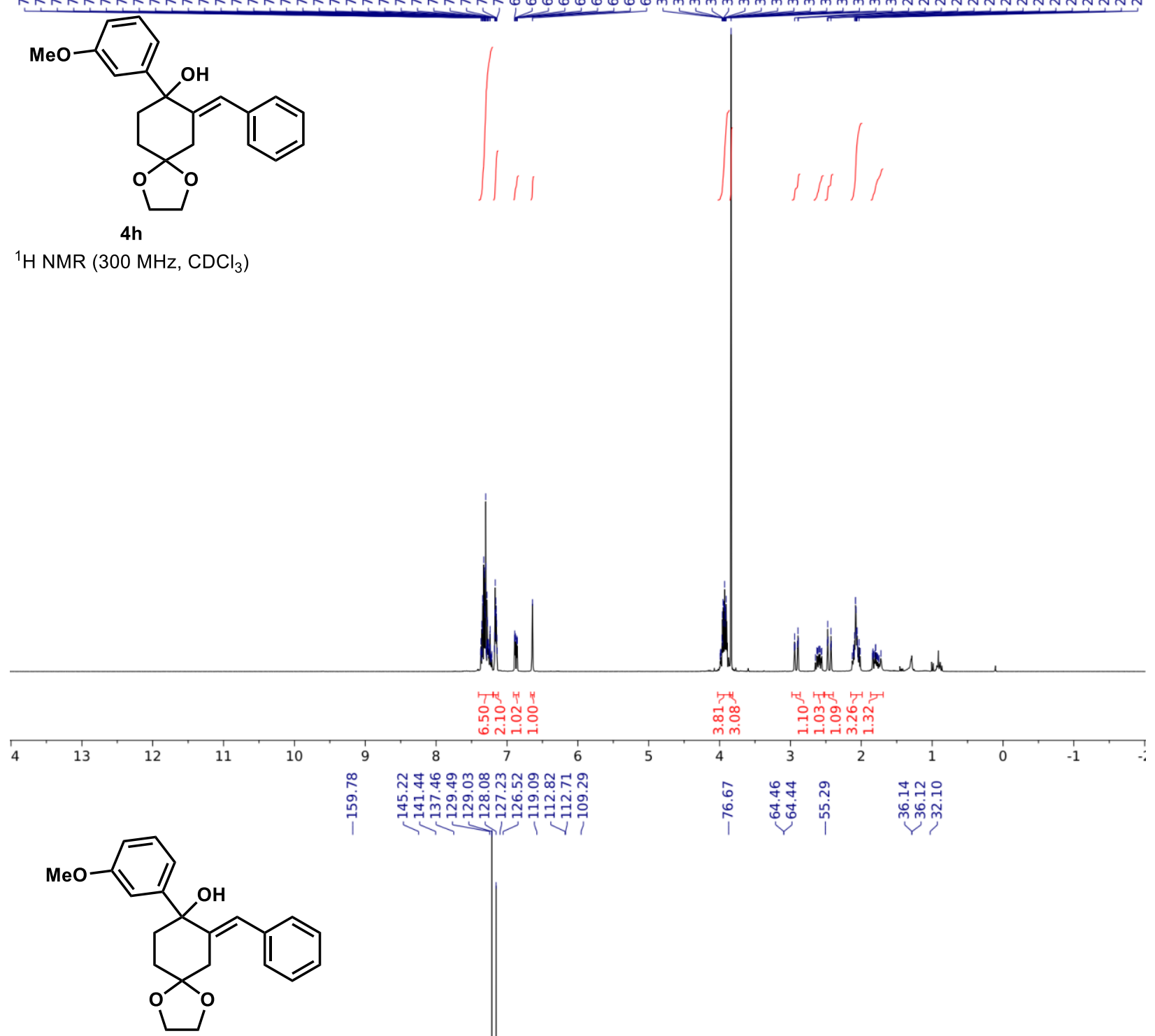

4h

${ }^{13} \mathrm{C}\left\{{ }^{1} \mathrm{H}\right\}$ NMR $\left(75 \mathrm{MHz}, \mathrm{CDCl}_{3}\right)$

$\begin{array}{llllllllllllllllllllllllll}230 & 220 & 210 & 200 & 190 & 180 & 170 & 160 & 150 & 140 & 130 & 120 & 110 & 100 & 90 & 80 & 70 & 60 & 50 & 40 & 30 & 20 & 10 & 0 & -10\end{array}$ 
ดั

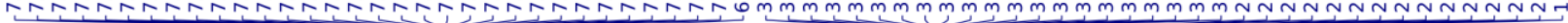
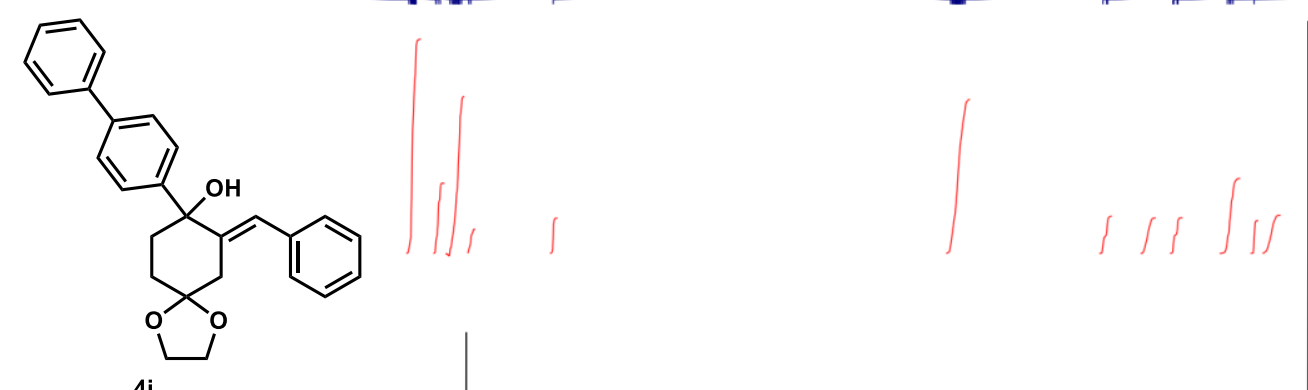

${ }^{1} \mathrm{H} \mathrm{NMR}\left(500 \mathrm{MHz}, \mathrm{CDCl}_{3}\right)$

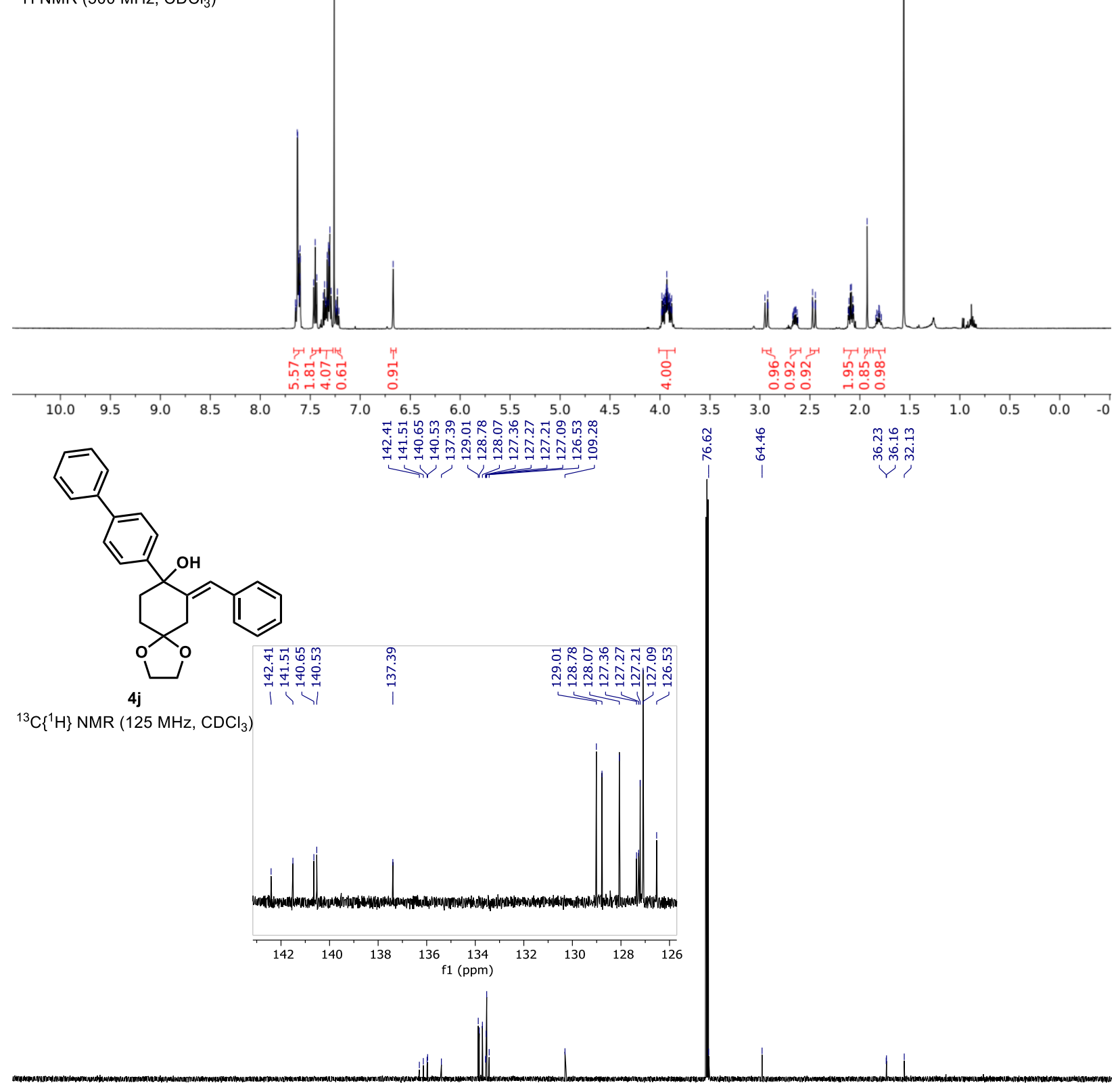

230

$\begin{array}{llll}20 & 210 & 200 & 190\end{array}$

$80 \quad 170$

$120 \quad 110 \quad 100$

$90 \quad 80$

$70 \quad 60$

40 
<smiles>Cc1ccccc1</smiles><smiles>CCCCCCCC</smiles>

${ }^{1} \mathrm{H}$ NMR $\left(300 \mathrm{MHz}, \mathrm{CDCl}_{3}\right)$
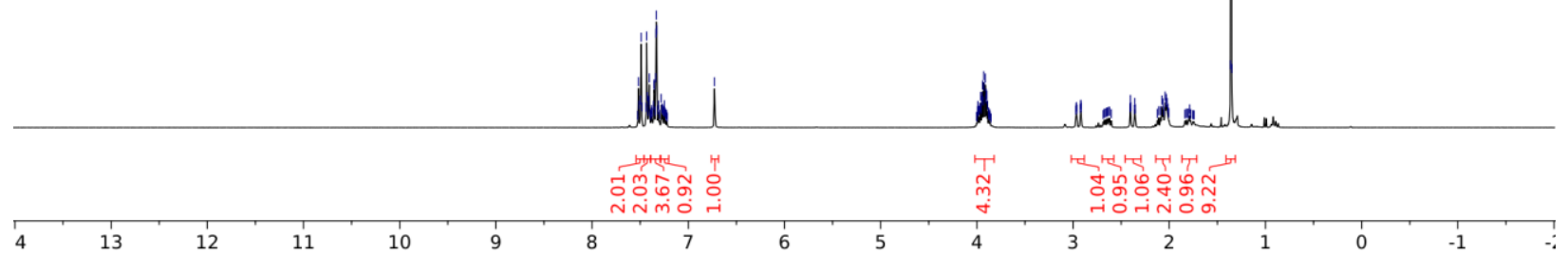

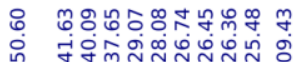

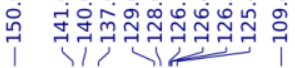

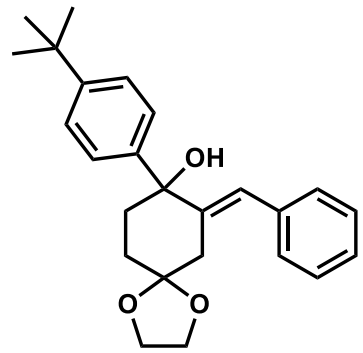

4k

${ }^{13} \mathrm{C}\left\{{ }^{1} \mathrm{H}\right\} \mathrm{NMR}\left(75 \mathrm{MHz}, \mathrm{CDCl}_{3}\right)$

$\begin{array}{lllllllllllllllllllllllll}230 & 220 & 210 & 200 & 190 & 180 & 170 & 160 & 150 & 140 & 130 & 120 & 110 & 100 & 90 & 80 & 70 & 60 & 50 & 40 & 30 & 20 & 10 & 0 & -10\end{array}$ 
위ำ 要<smiles>OC1(c2cccc3ccccc23)CCC2(CC1=Cc1ccccc1)OCCO2</smiles>

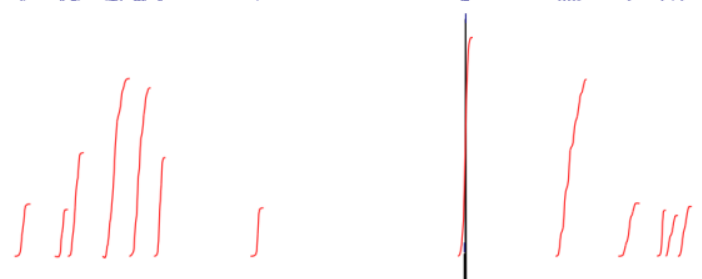

${ }^{1} \mathrm{H}$ NMR $\left(300 \mathrm{MHz}, \mathrm{CDCl}_{3}\right)$

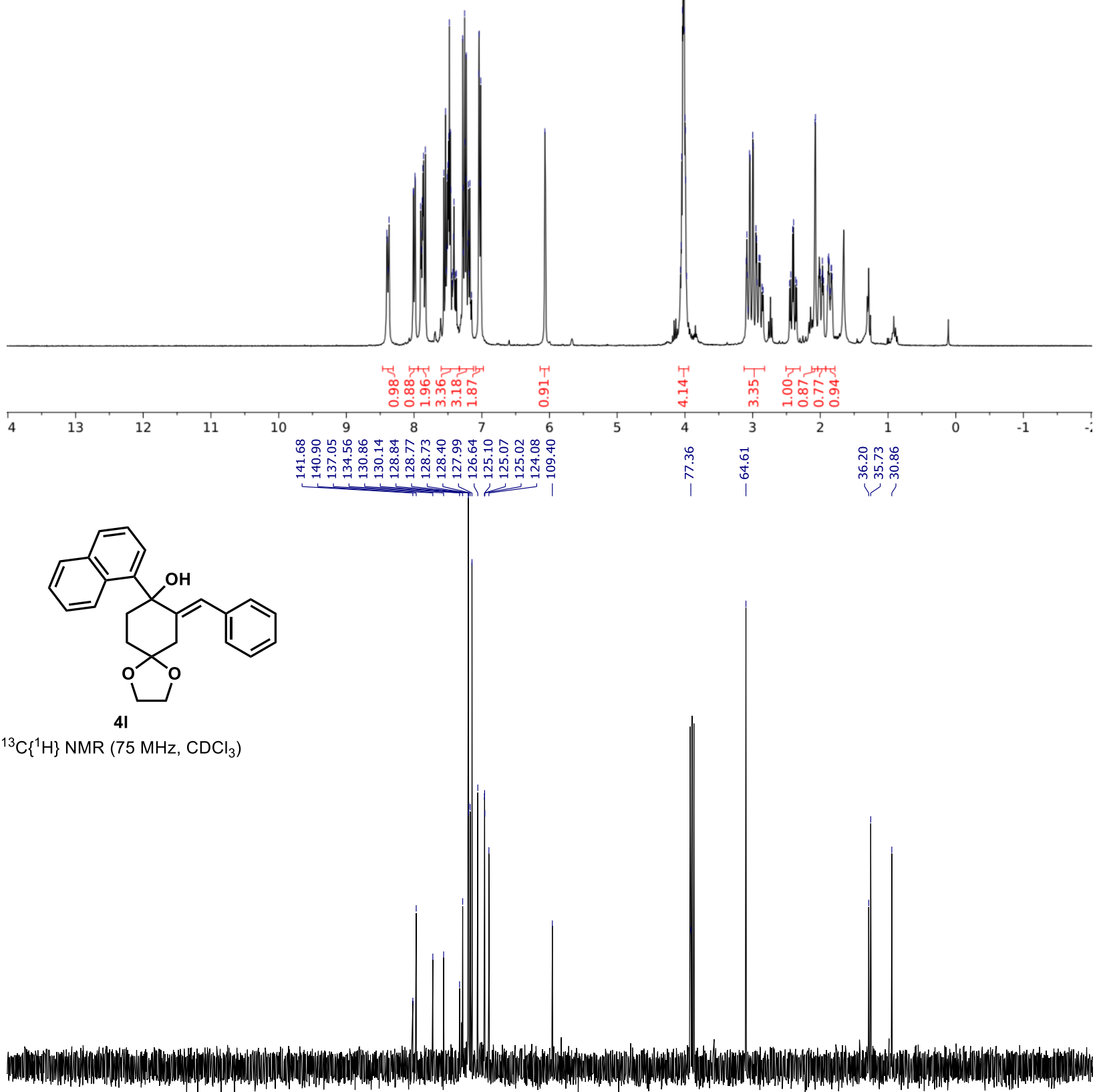

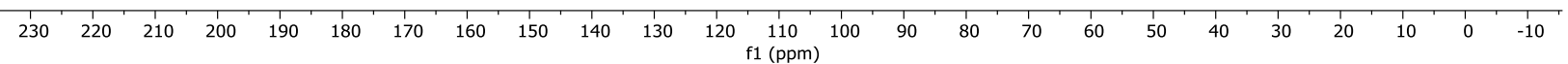




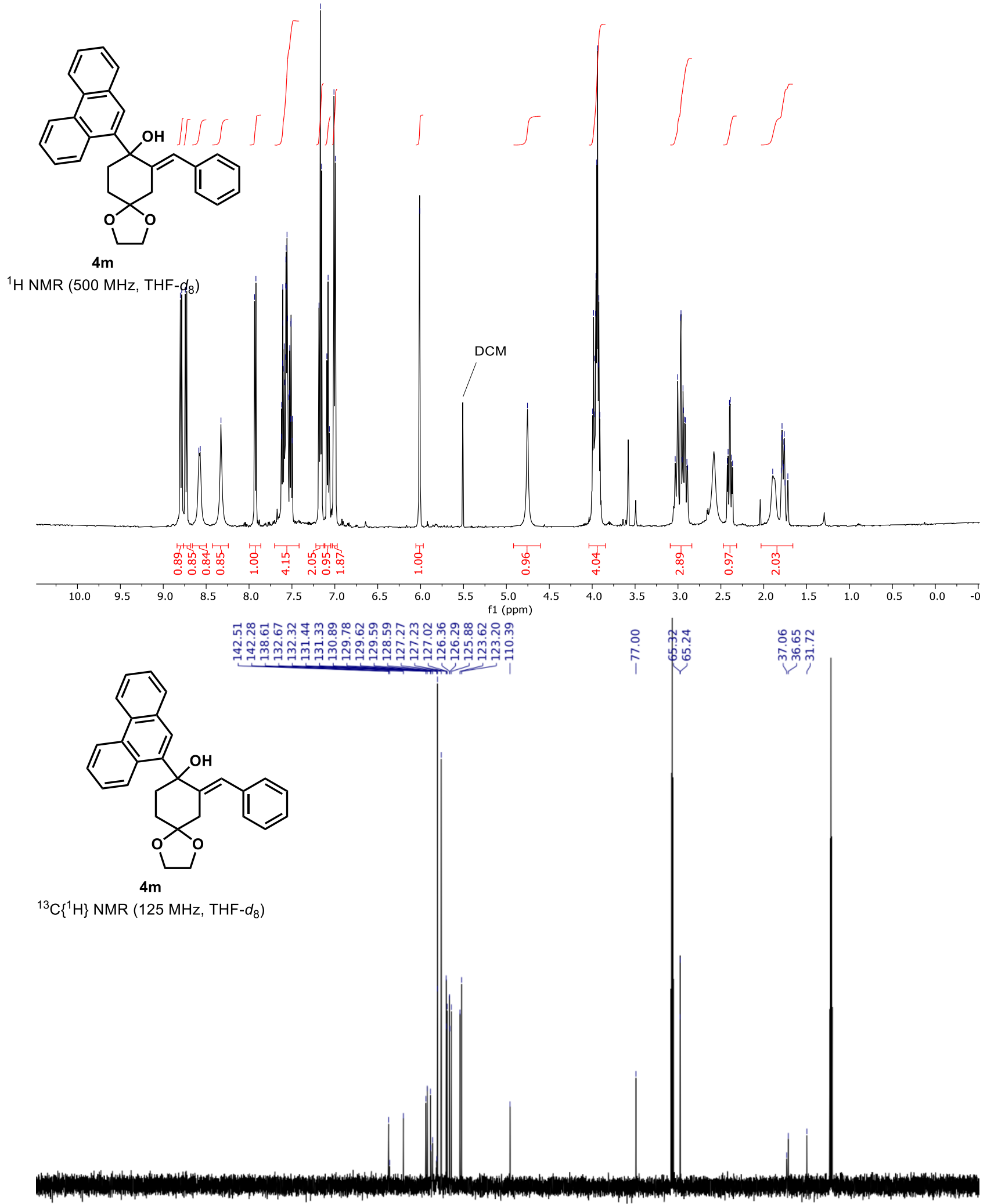

$\begin{array}{lllllllllllllllllllllllllll}230 & 220 & 210 & 200 & 190 & 180 & 170 & 160 & 150 & 140 & 130 & 120 & 110 & 100 & 90 & 80 & 70 & 60 & 50 & 40 & 30 & 20 & 10 & 0 & -10\end{array}$ 
<smiles>OC1(c2csc3ccccc23)CCC2(C/C1=C\c1ccccc1)OCCO2</smiles>

4n

${ }^{1} \mathrm{H}$ NMR $\left(500 \mathrm{MHz}, \mathrm{CDCl}_{3}\right)$

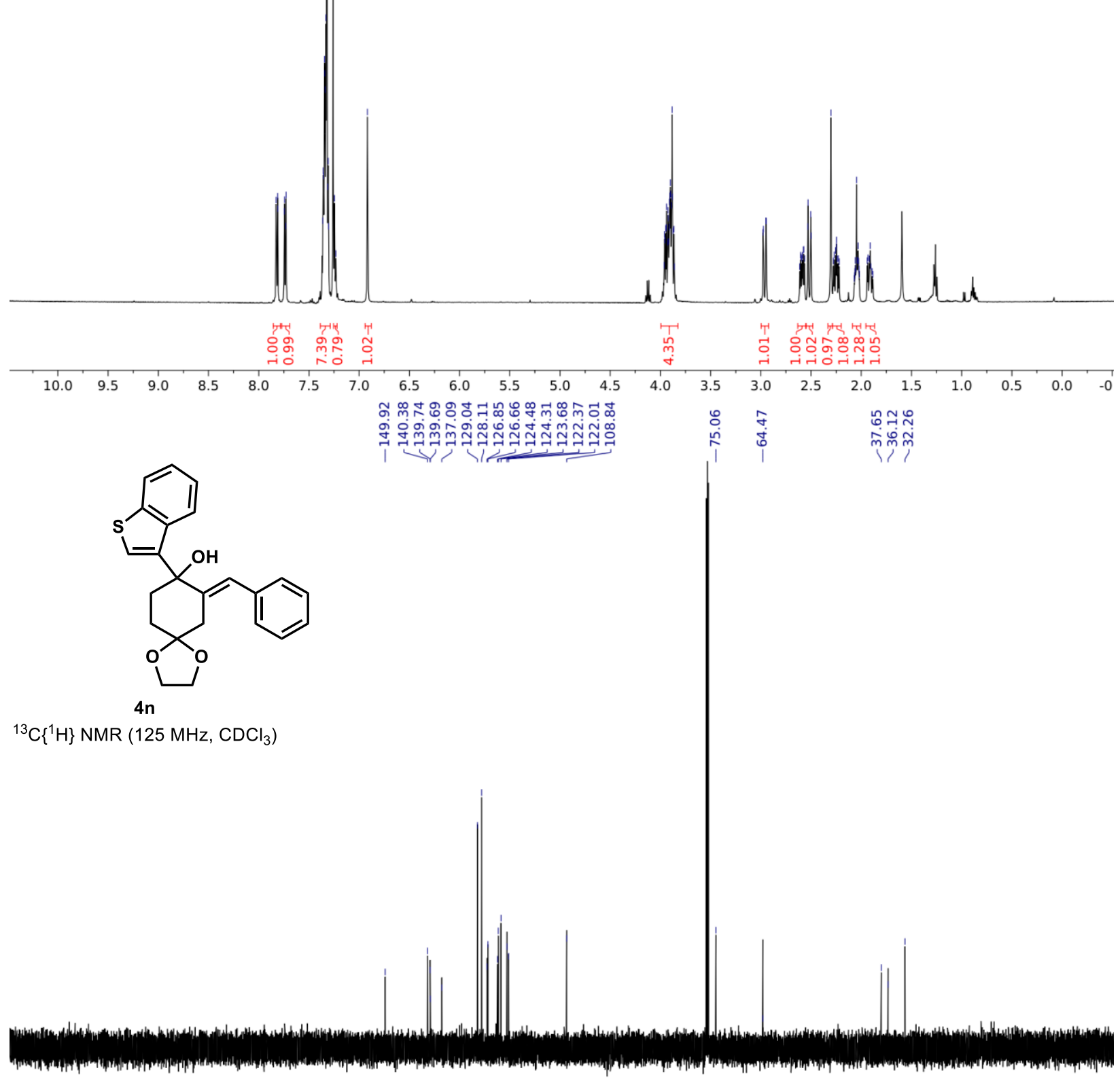

$\begin{array}{lllllllllllllllllllllllll}230 & 220 & 210 & 200 & 190 & 180 & 170 & 160 & 150 & 140 & 130 & 120 & 110 & 100 & 90 & 80 & 70 & 60 & 50 & 40 & 30 & 20 & 10 & 0 & -10\end{array}$ 


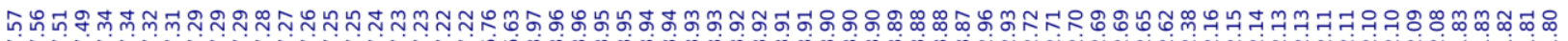

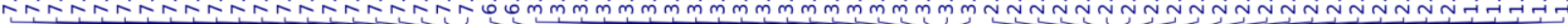<smiles>OC1(c2cc3ccccc3o2)CCC2(CC1=Cc1ccccc1)OCCO2</smiles>

7

${ }^{1} \mathrm{H}$ NMR $\left(500 \mathrm{MHz}, \mathrm{CDCl}_{3}\right)$
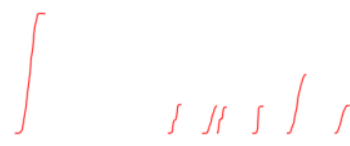

J

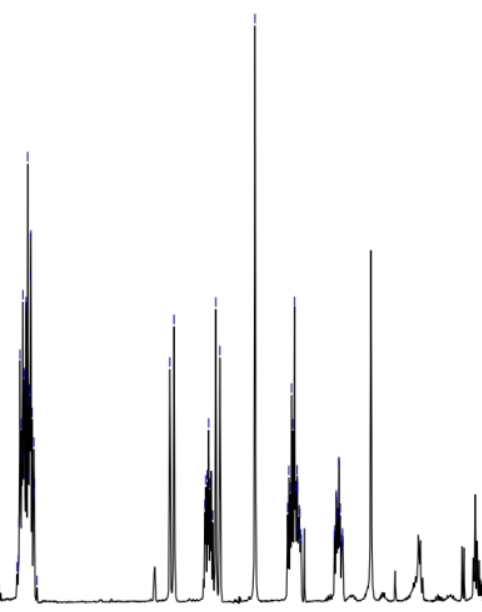

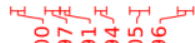
iononio<smiles>OC1(c2cc3ccccc3o2)CCC2(C/C1=C\c1ccccc1)OCCO2</smiles>

7

${ }^{13} \mathrm{C}\left\{{ }^{1} \mathrm{H}\right\}$ NMR $\left(125 \mathrm{MHz}, \mathrm{CDCl}_{3}\right)$

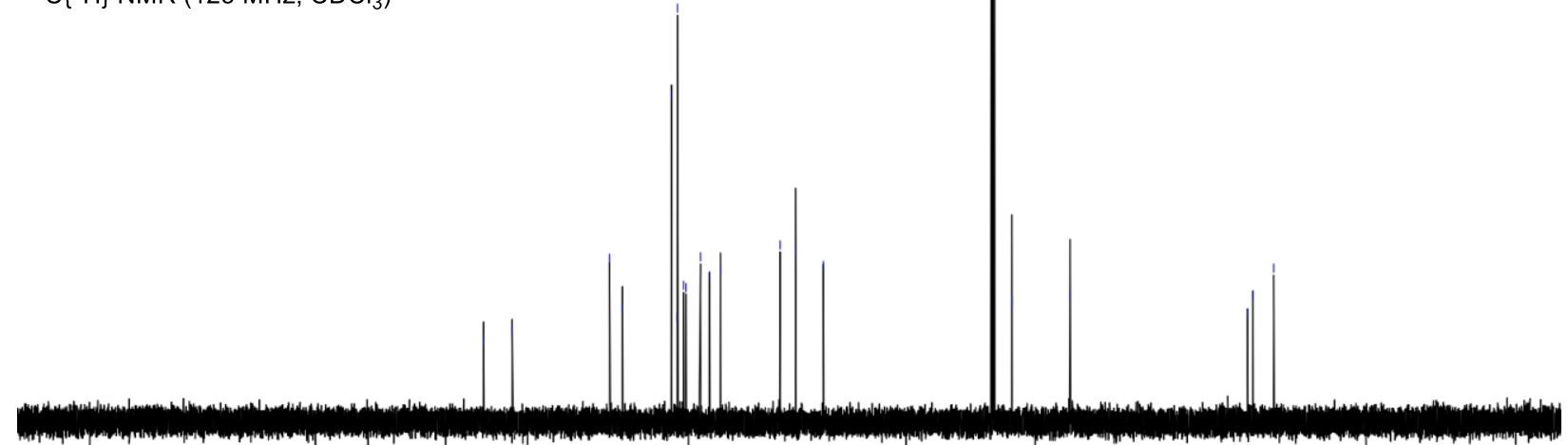

$\begin{array}{lllllllllllllllllllllllll}230 & 220 & 210 & 200 & 190 & 180 & 170 & 160 & 150 & 140 & 130 & 120 & 110 & 100 & 90 & 80 & 70 & 60 & 50 & 40 & 30 & 20 & 10 & 0 & -10\end{array}$ 


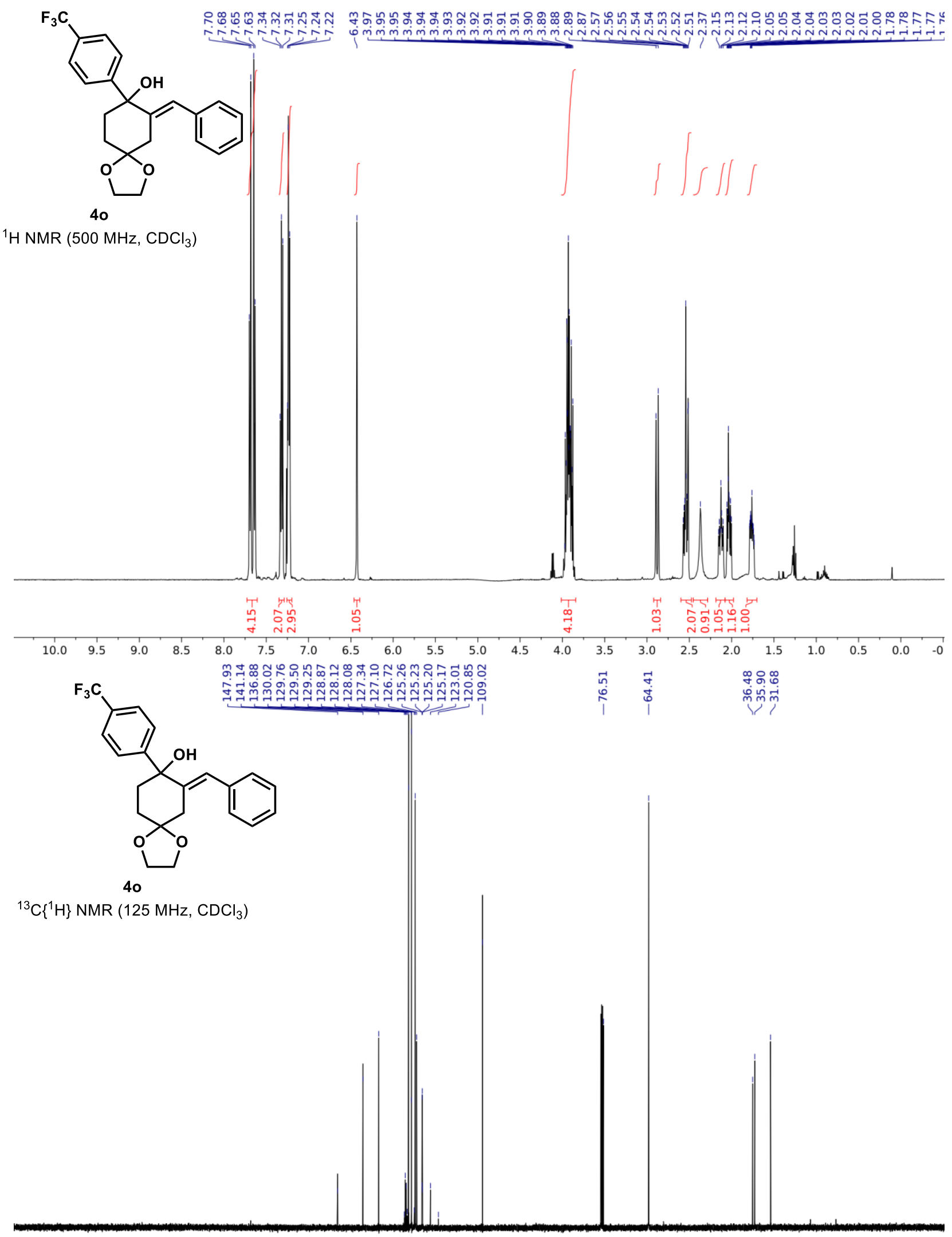

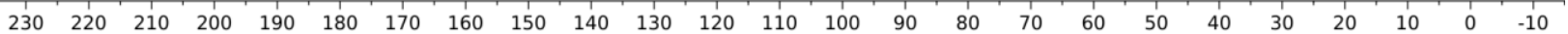




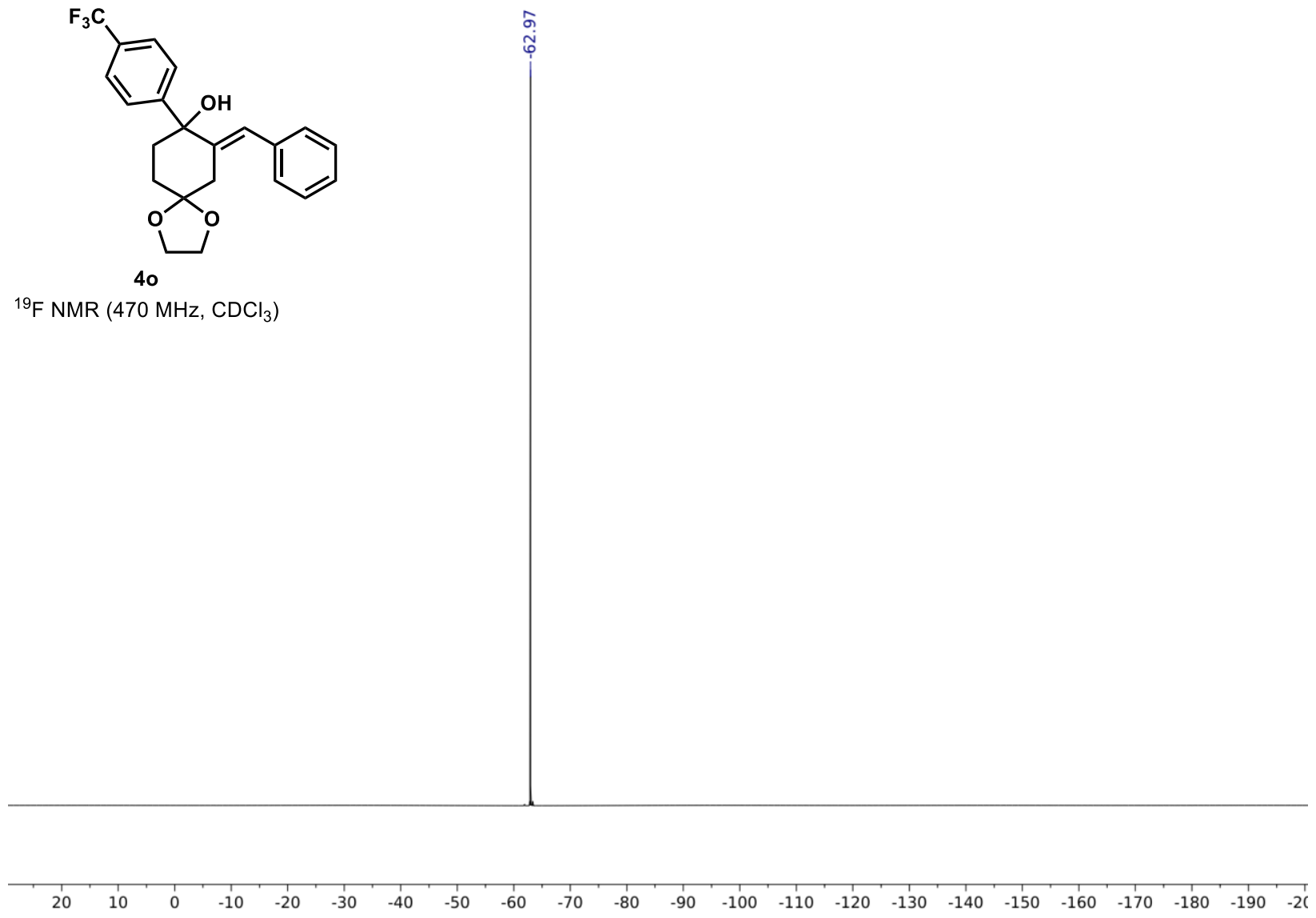




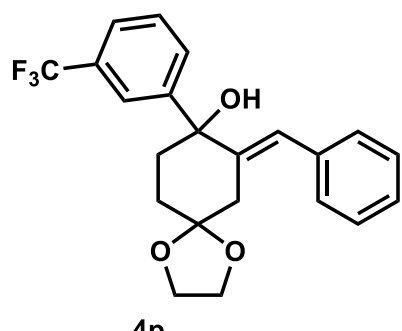

$4 p$

${ }^{1} \mathrm{H}$ NMR $\left(300 \mathrm{MHz}, \mathrm{CDCl}_{3}\right)$

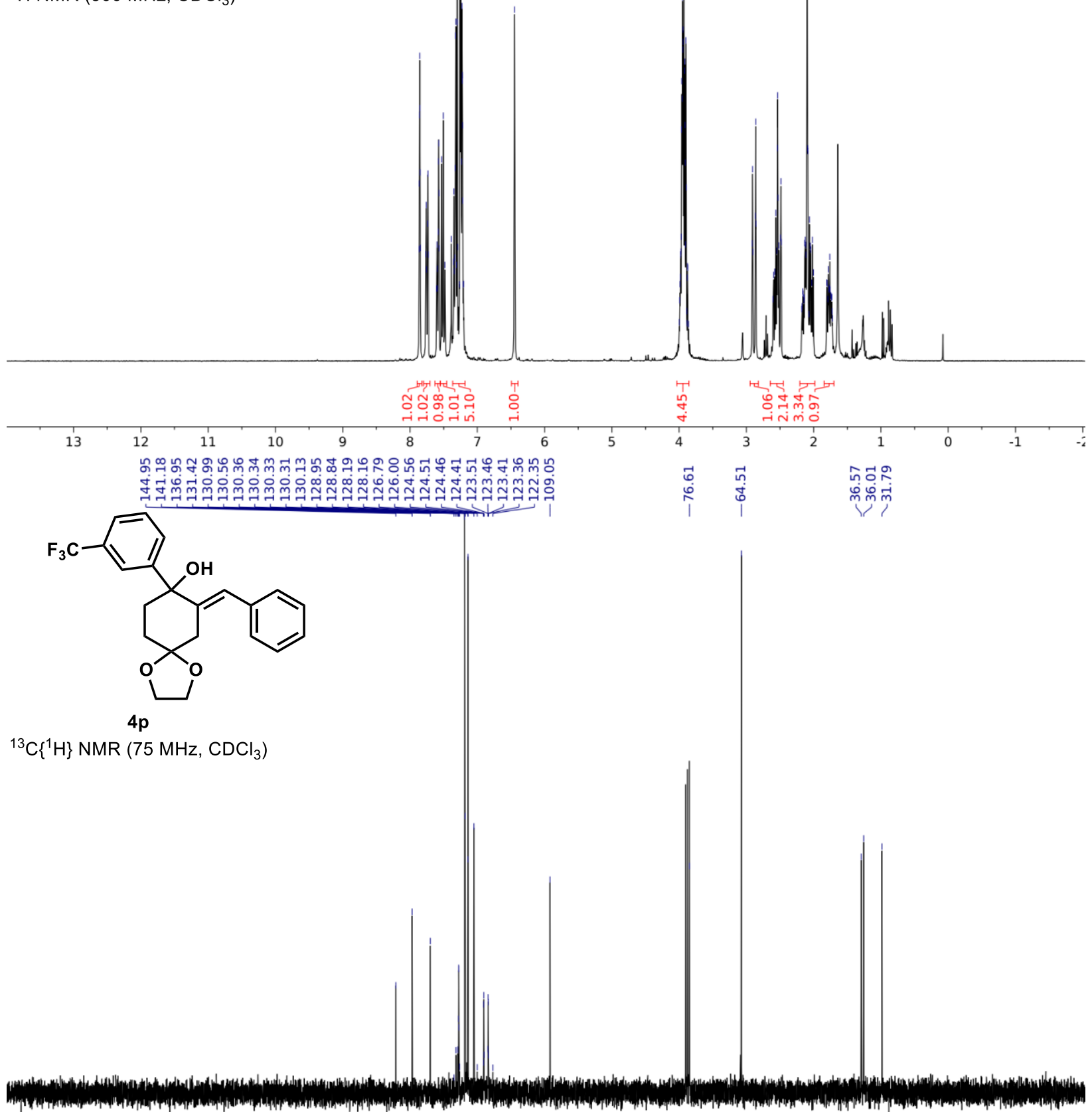

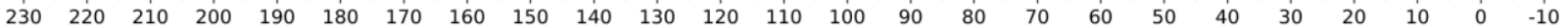




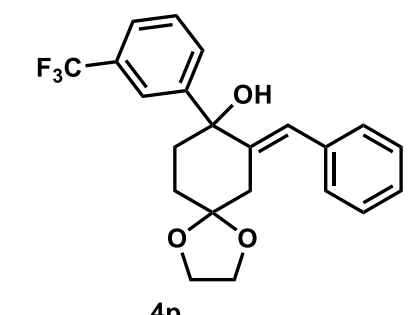

${ }^{19} \mathrm{~F} \mathrm{NMR}\left(282 \mathrm{MHz}, \mathrm{CDCl}_{3}\right)$

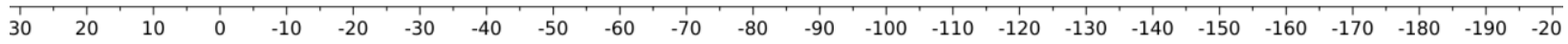




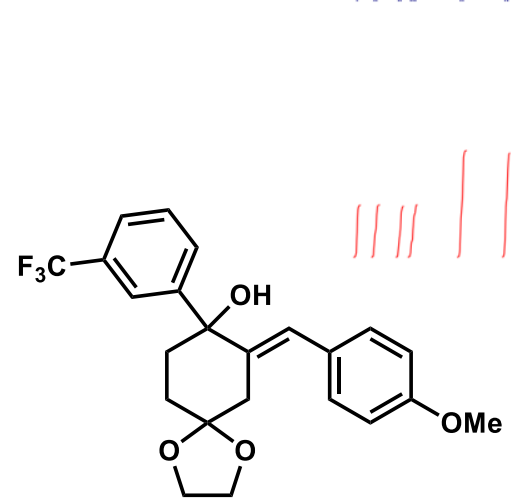

$4 q$

${ }^{1} \mathrm{H} \mathrm{NMR}\left(500 \mathrm{MHz}, \mathrm{CDCl}_{3}\right)$

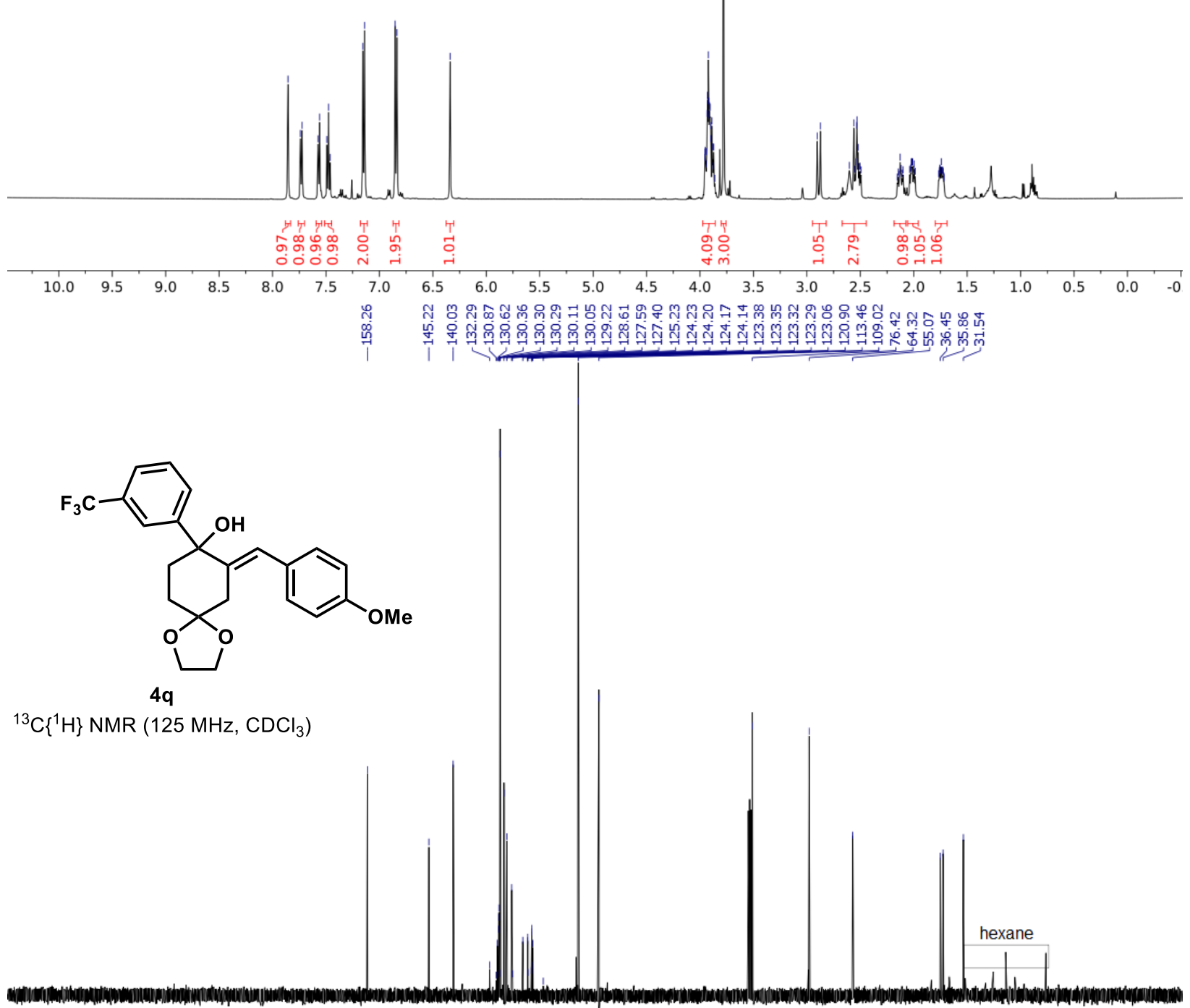

$\begin{array}{lllllllllllllllllllllllll}230 & 220 & 210 & 200 & 190 & 180 & 170 & 160 & 150 & 140 & 130 & 120 & 110 & 100 & 90 & 80 & 70 & 60 & 50 & 40 & 30 & 20 & 10 & 0 & -10\end{array}$ 


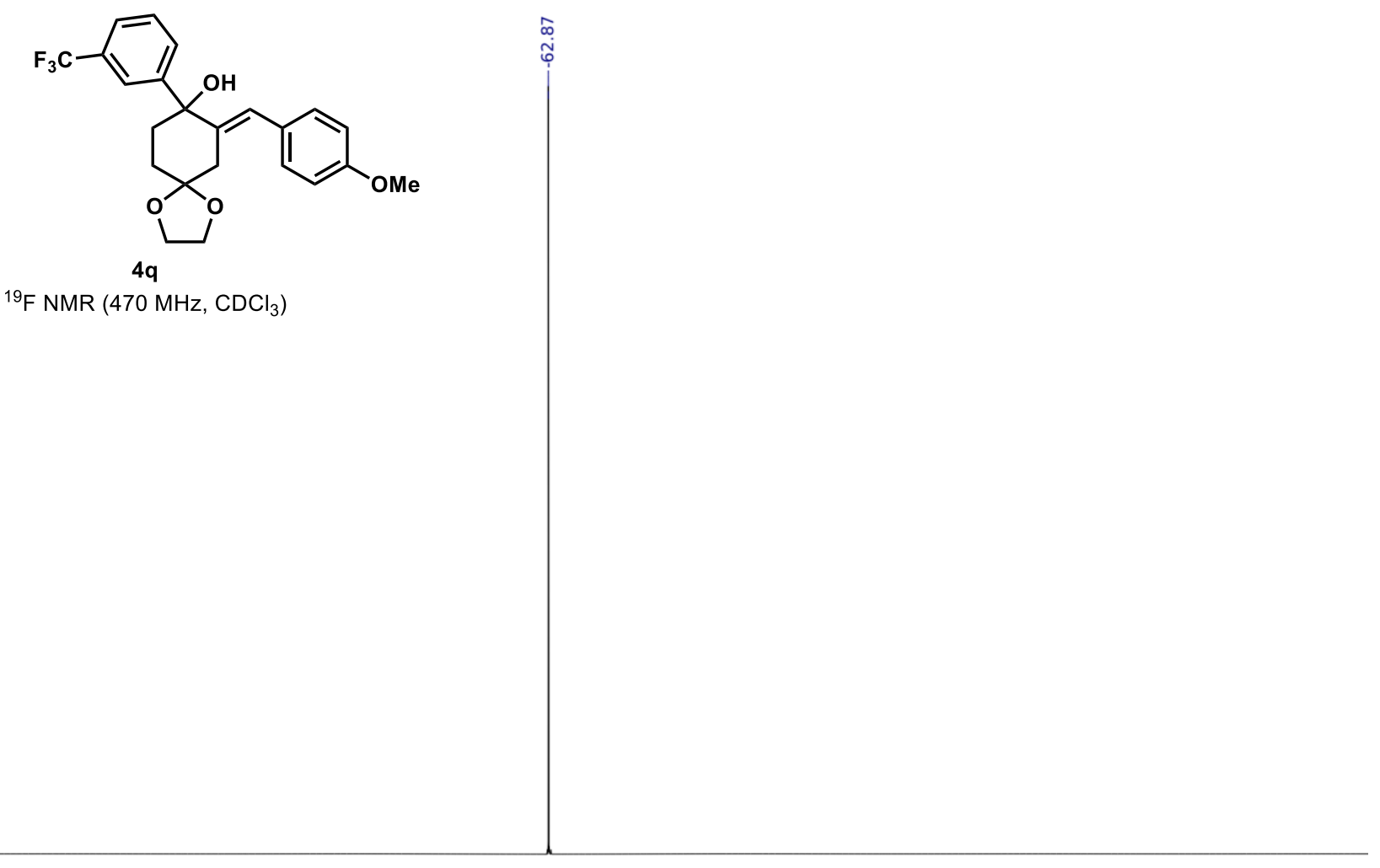

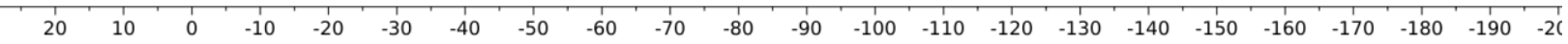




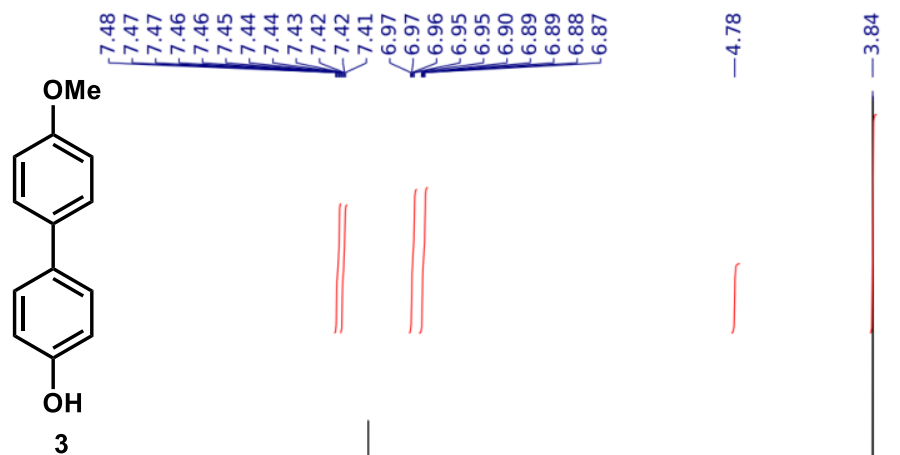

${ }^{1} \mathrm{H} \mathrm{NMR}\left(500 \mathrm{MHz}, \mathrm{CDCl}_{3}\right)$
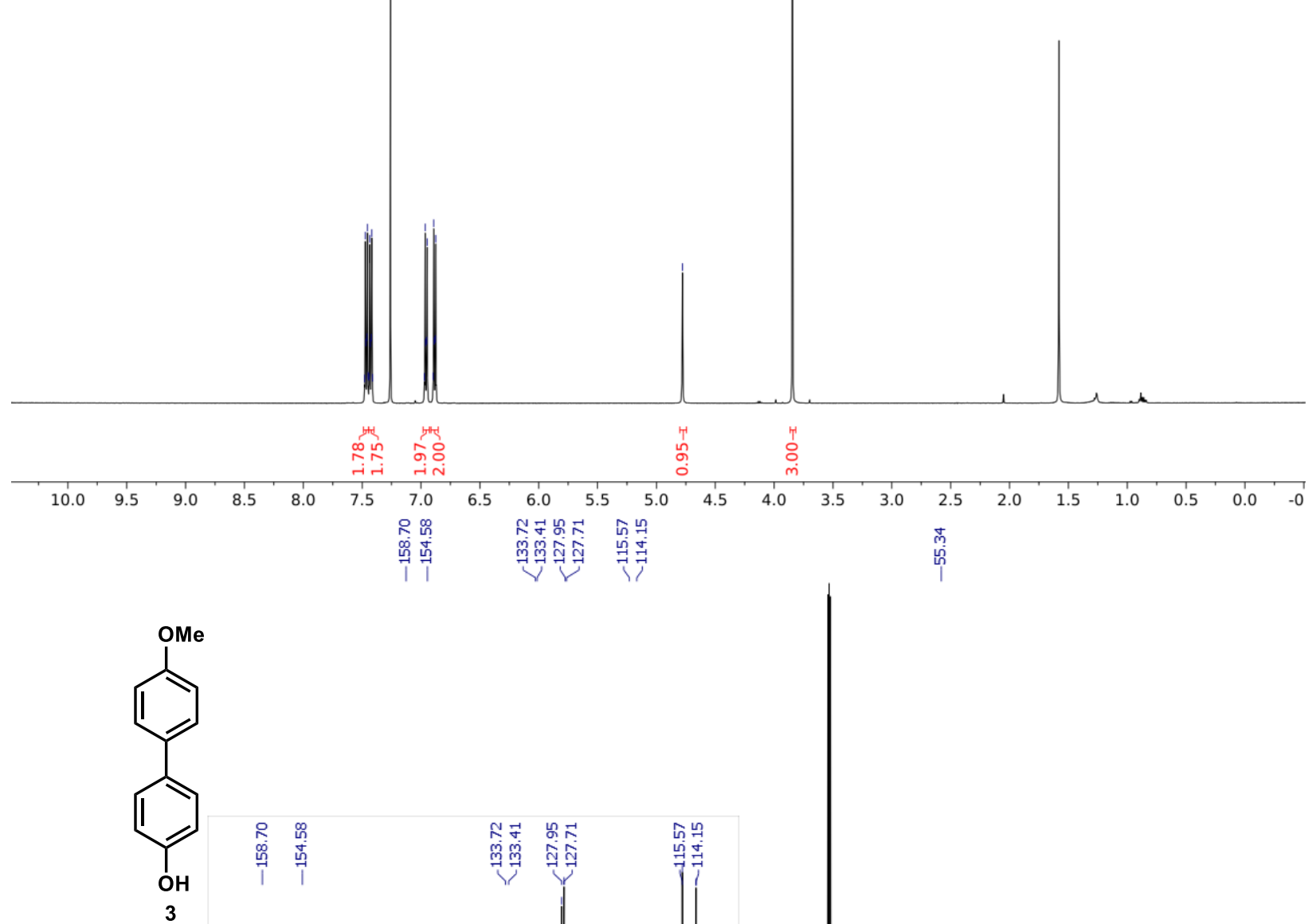

${ }^{13} \mathrm{C}\left\{{ }^{1} \mathrm{H}\right\}$ NMR $\left(125 \mathrm{MHz}, \mathrm{CDCl}_{3}\right)$

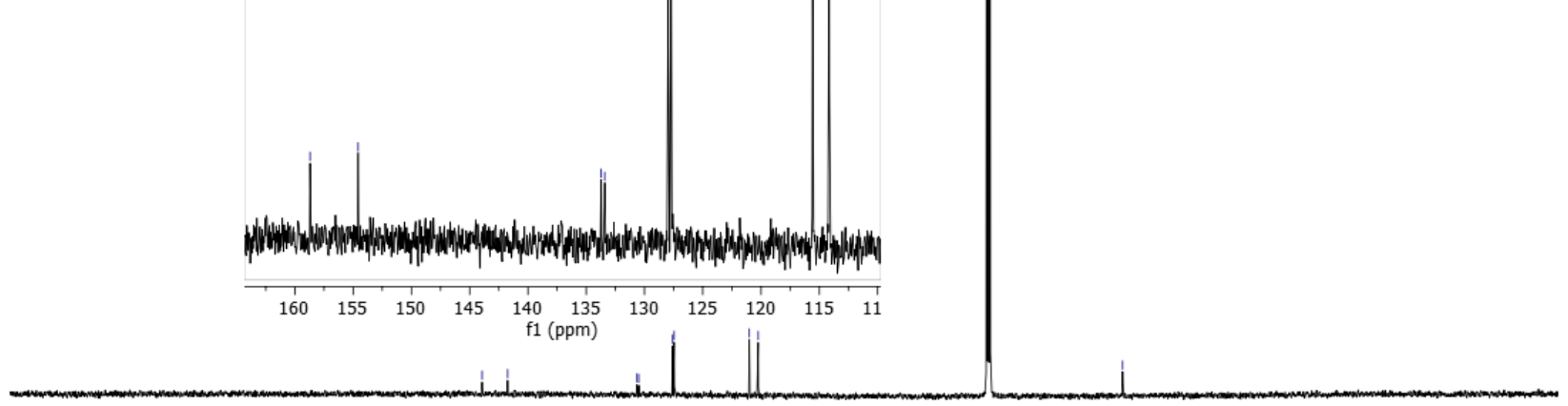

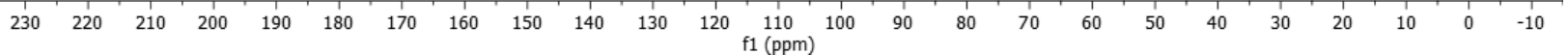




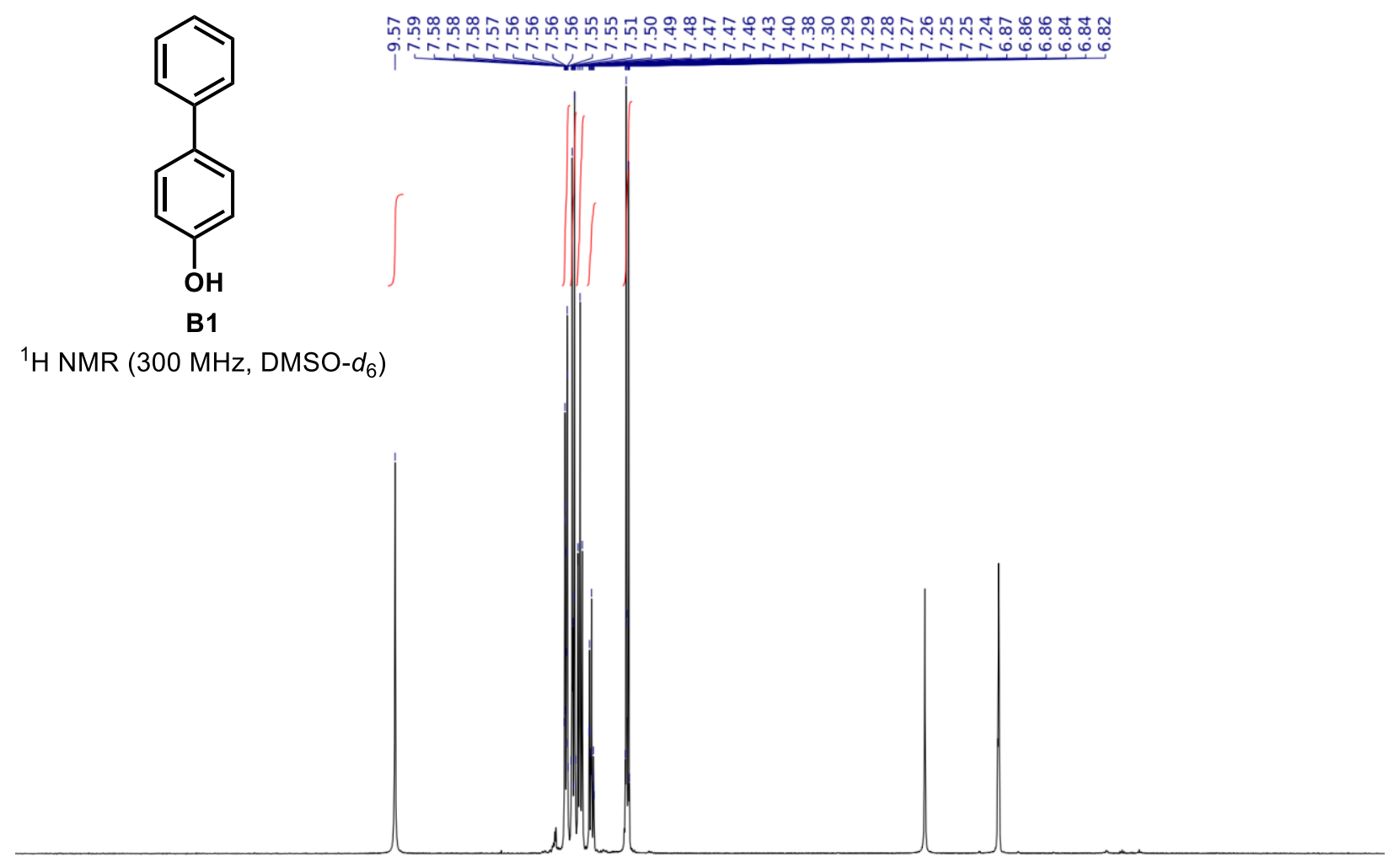

${ }^{1} \mathrm{HNMR}\left(300 \mathrm{MHz}, \mathrm{DMSO}-d_{6}\right)$

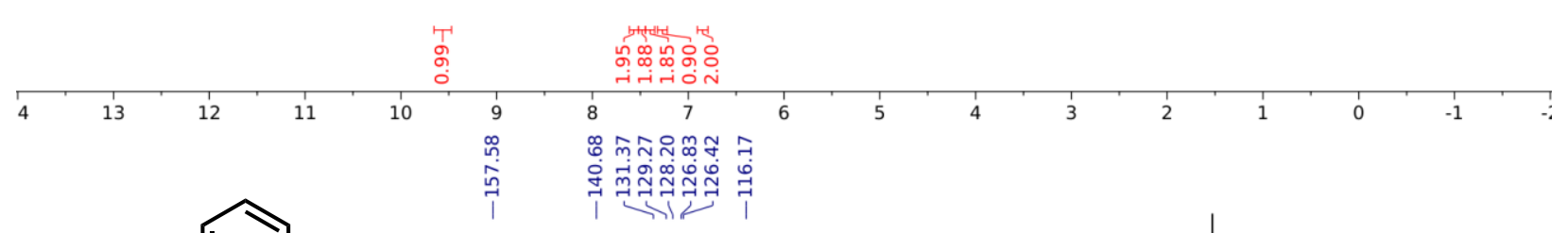<smiles>Oc1ccc(-c2ccccc2)cc1</smiles>

B1

${ }^{13} \mathrm{C}\left\{{ }^{1} \mathrm{H}\right\}$ NMR $\left(75 \mathrm{MHz}\right.$, DMSO- $\left.d_{6}\right)$

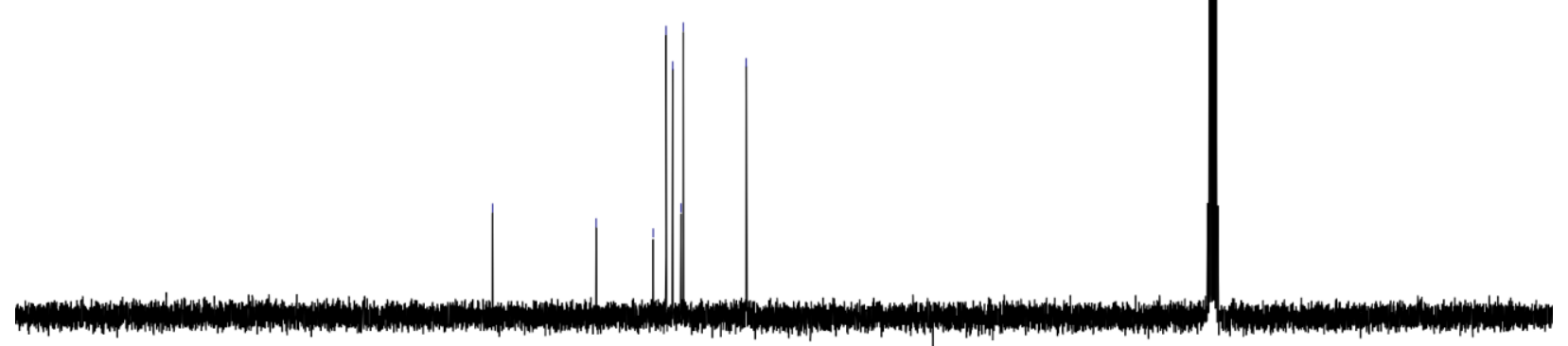

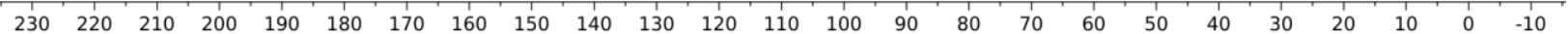




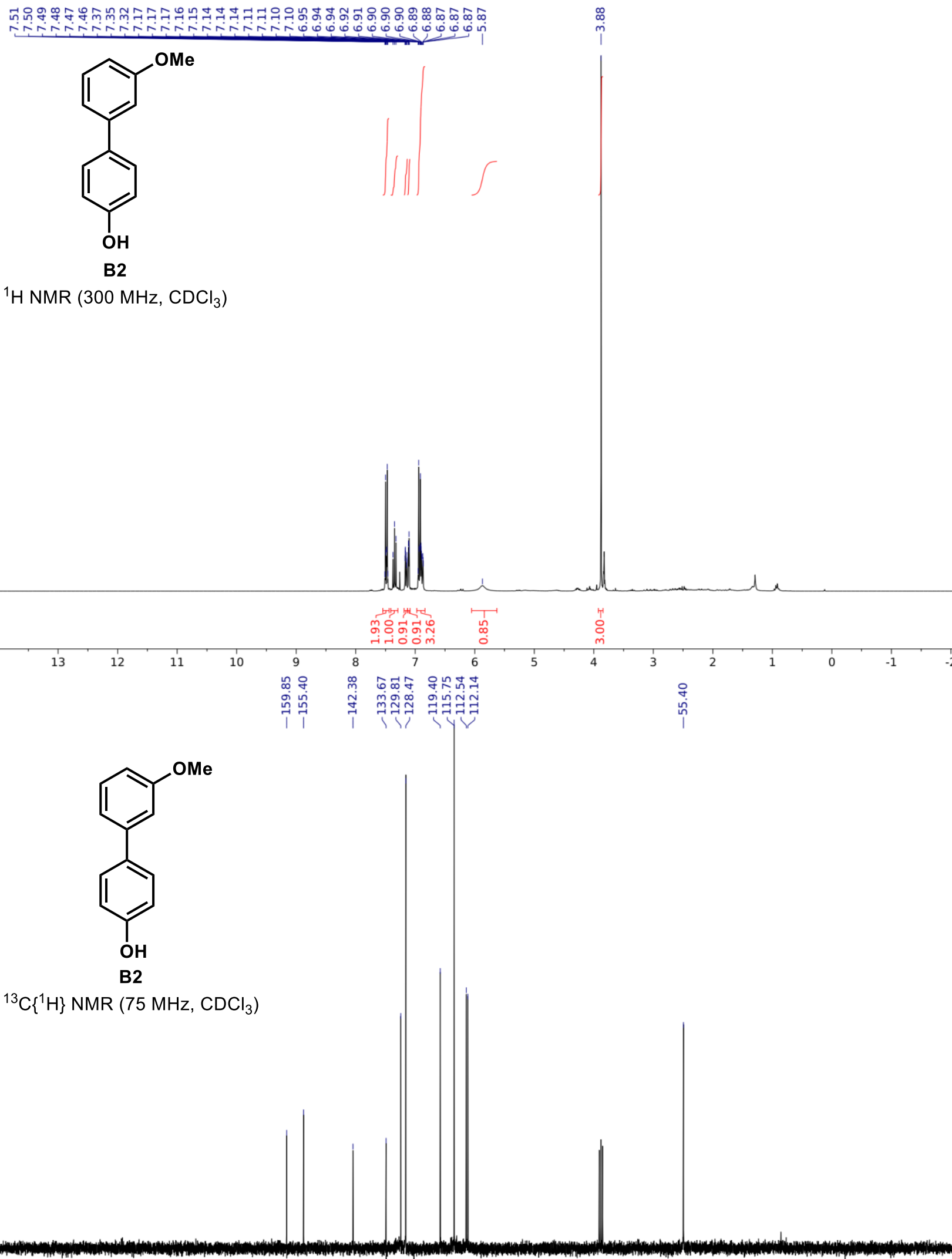

$\begin{array}{llllllllllllllllllllllllllllll}230 & 220 & 210 & 200 & 190 & 180 & 170 & 160 & 150 & 140 & 130 & 120 & 110 & 100 & 90 & 80 & 70 & 60 & 50 & 40 & 30 & 20 & 10 & 0 & -10\end{array}$ 

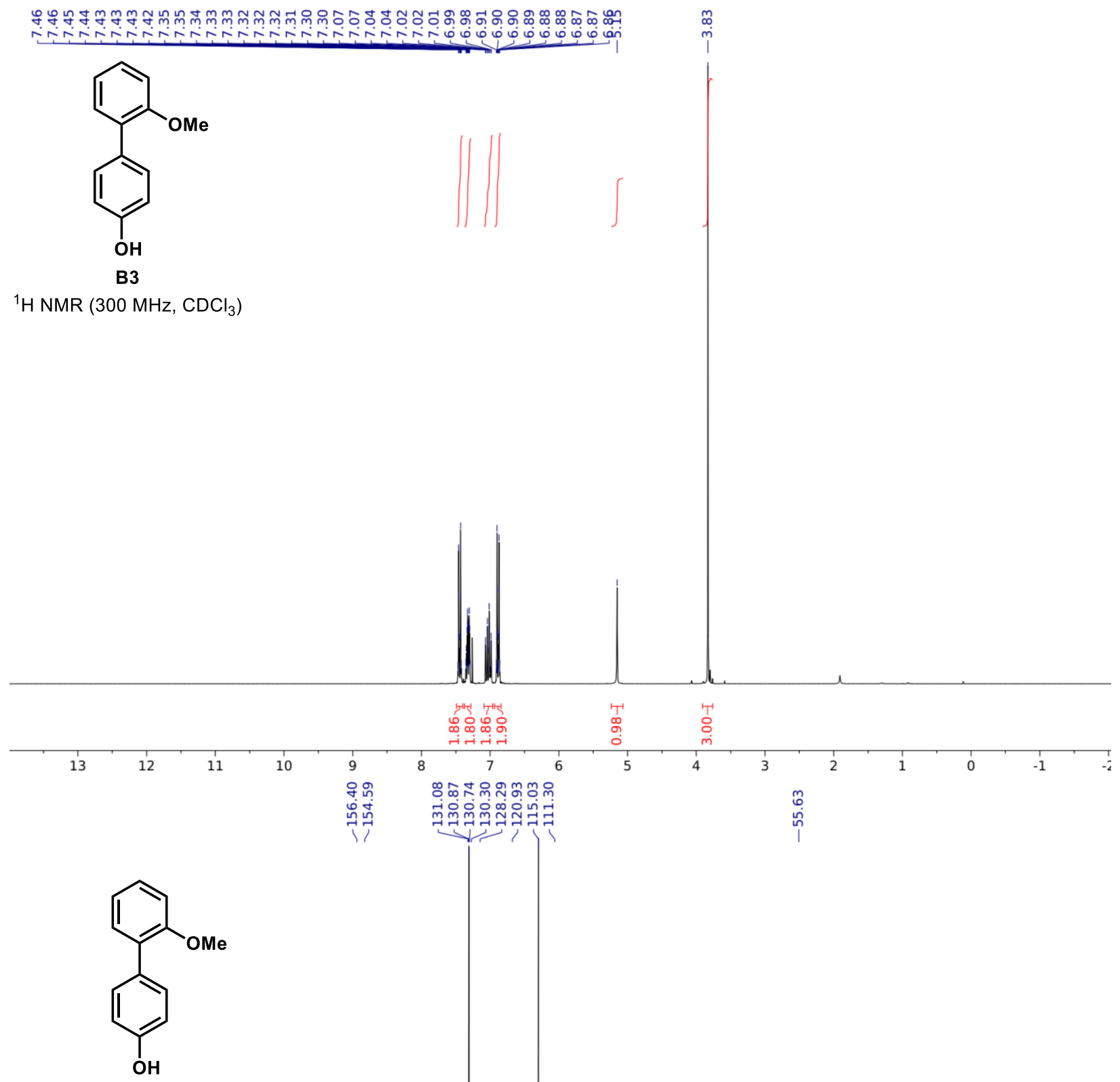

B3

${ }^{13} \mathrm{C}\left\{{ }^{1} \mathrm{H}\right\}$ NMR $\left(75 \mathrm{MHz}, \mathrm{CDCl}_{3}\right)$

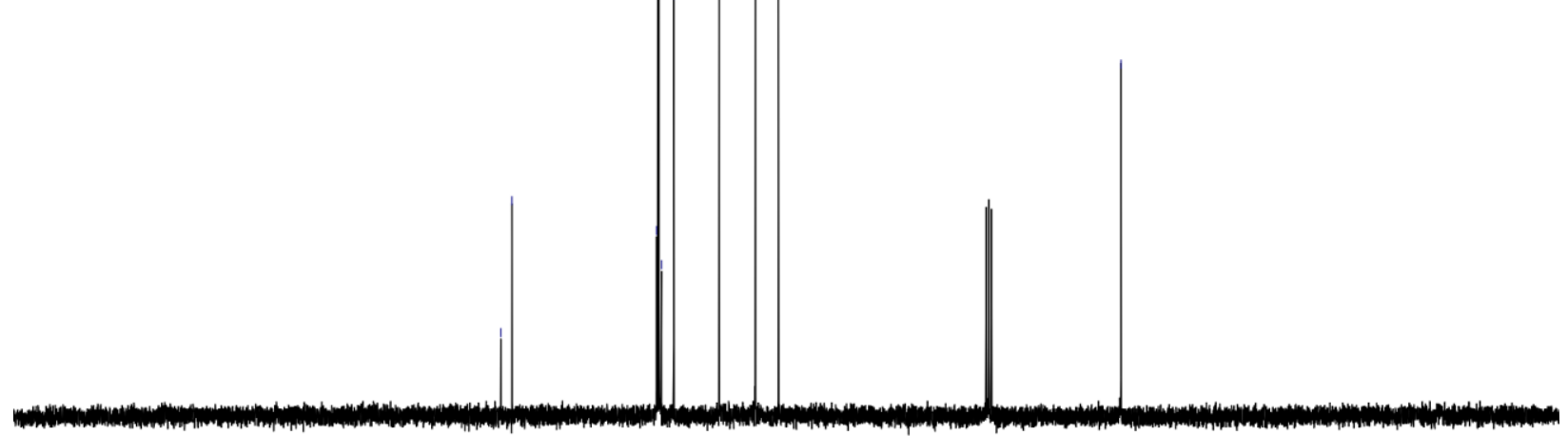

$\begin{array}{lllllllllllllllllllllllllllllllll}230 & 220 & 210 & 200 & 190 & 180 & 170 & 160 & 150 & 140 & 130 & 120 & 110 & 100 & 90 & 80 & 70 & 60 & 50 & 40 & 30 & 20 & 10 & 0 & -10\end{array}$ 


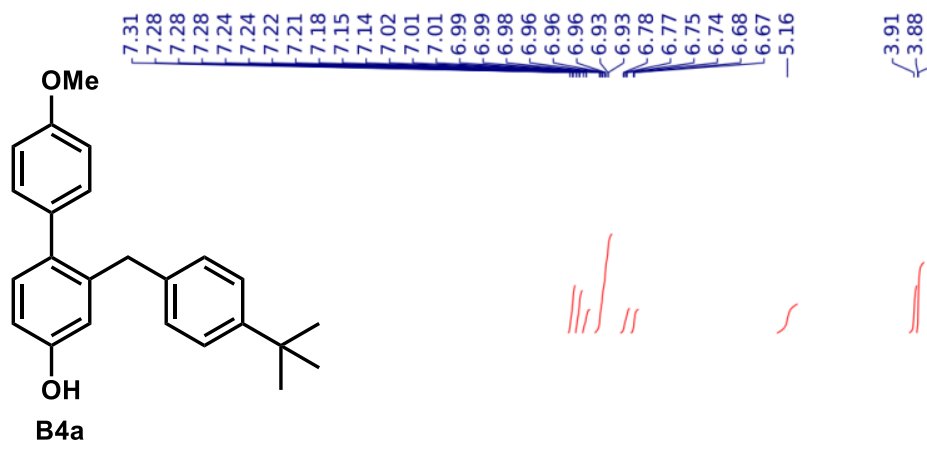

${ }^{1} \mathrm{H}$ NMR $\left(300 \mathrm{MHz}, \mathrm{CDCl}_{3}\right)$

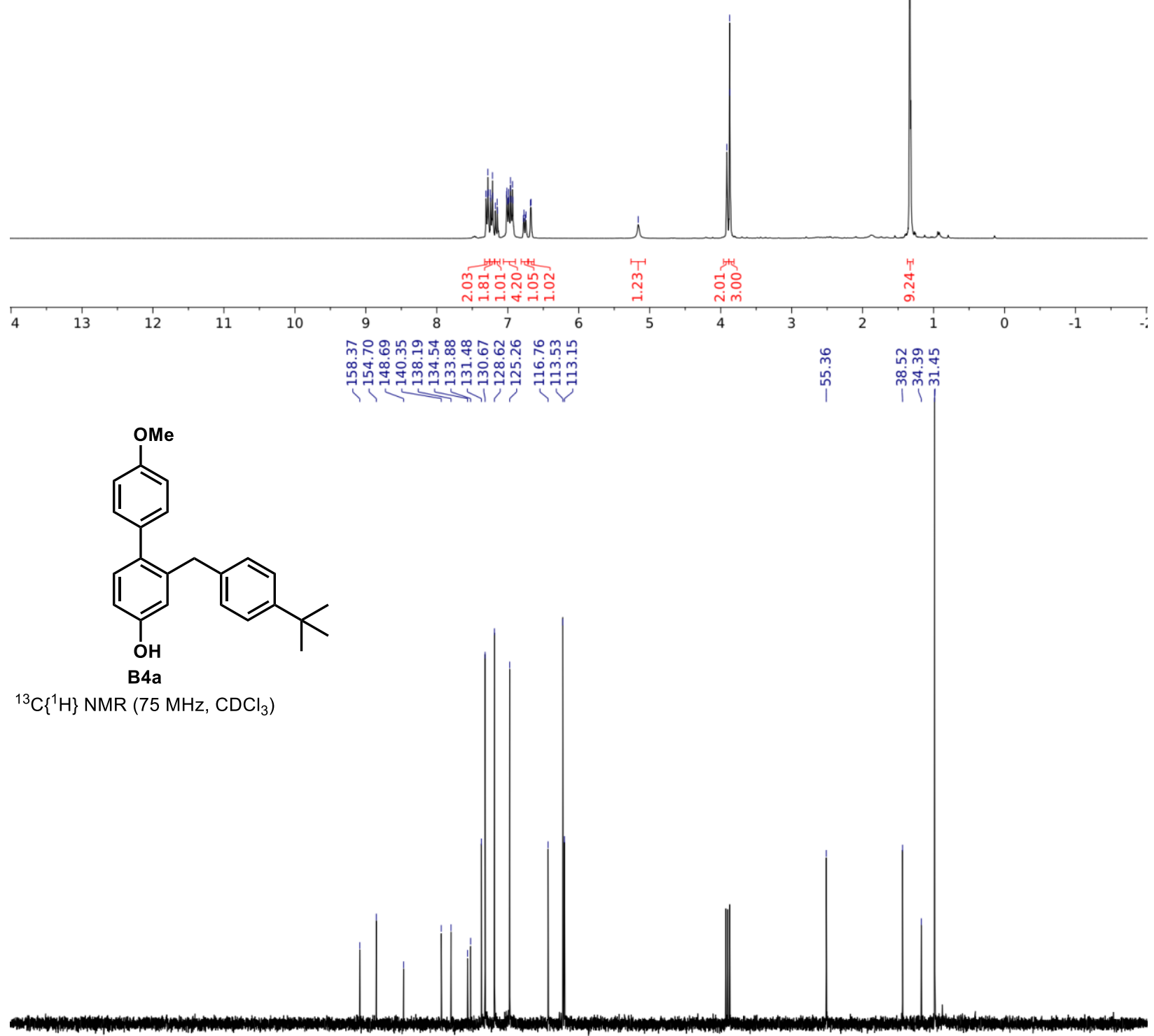

$\begin{array}{lllllllllllllllllllllllll}230 & 220 & 210 & 200 & 190 & 180 & 170 & 160 & 150 & 140 & 130 & 120 & 110 & 100 & 90 & 80 & 70 & 60 & 50 & 40 & 30 & 20 & 10 & 0 & -10\end{array}$ 
<smiles>Oc1ccc(-c2ccc(Br)c(O)c2)cc1</smiles>

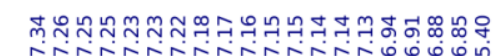

志

$\stackrel{\infty}{n}^{\infty}$

III

B4b

${ }^{1} \mathrm{H}$ NMR $\left(300 \mathrm{MHz}, \mathrm{CDCl}_{3}\right)$

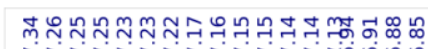

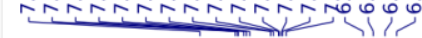

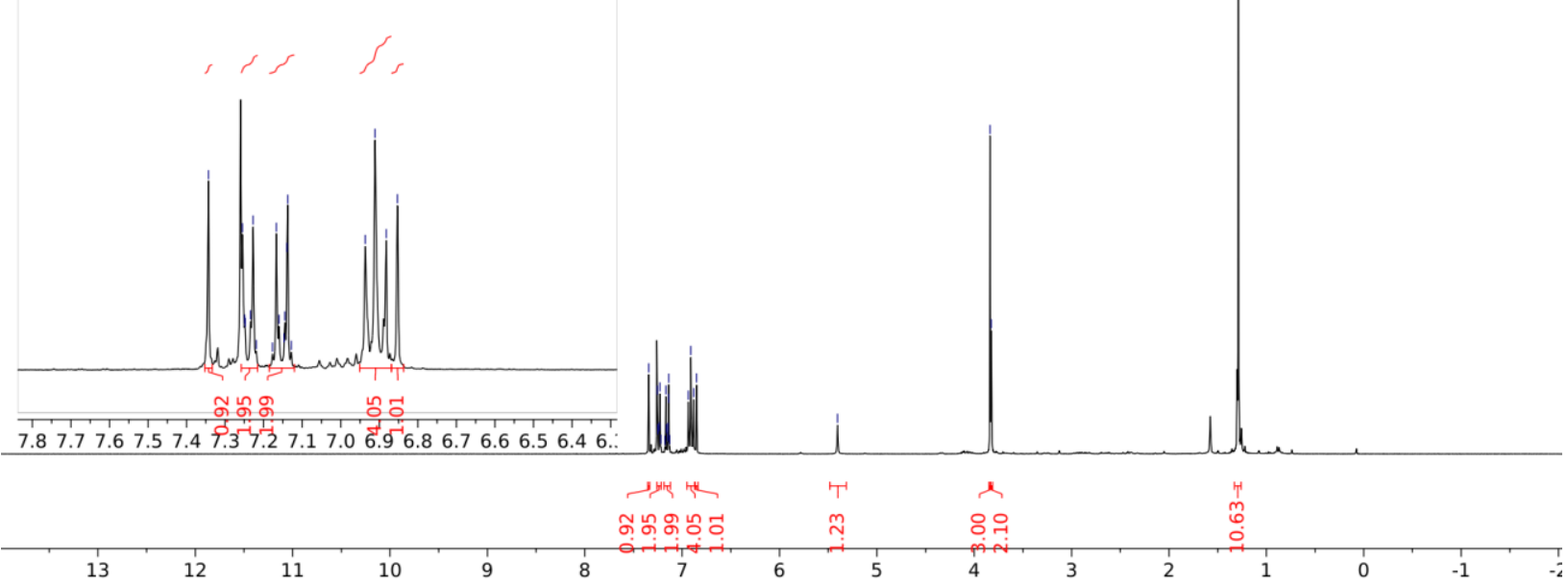



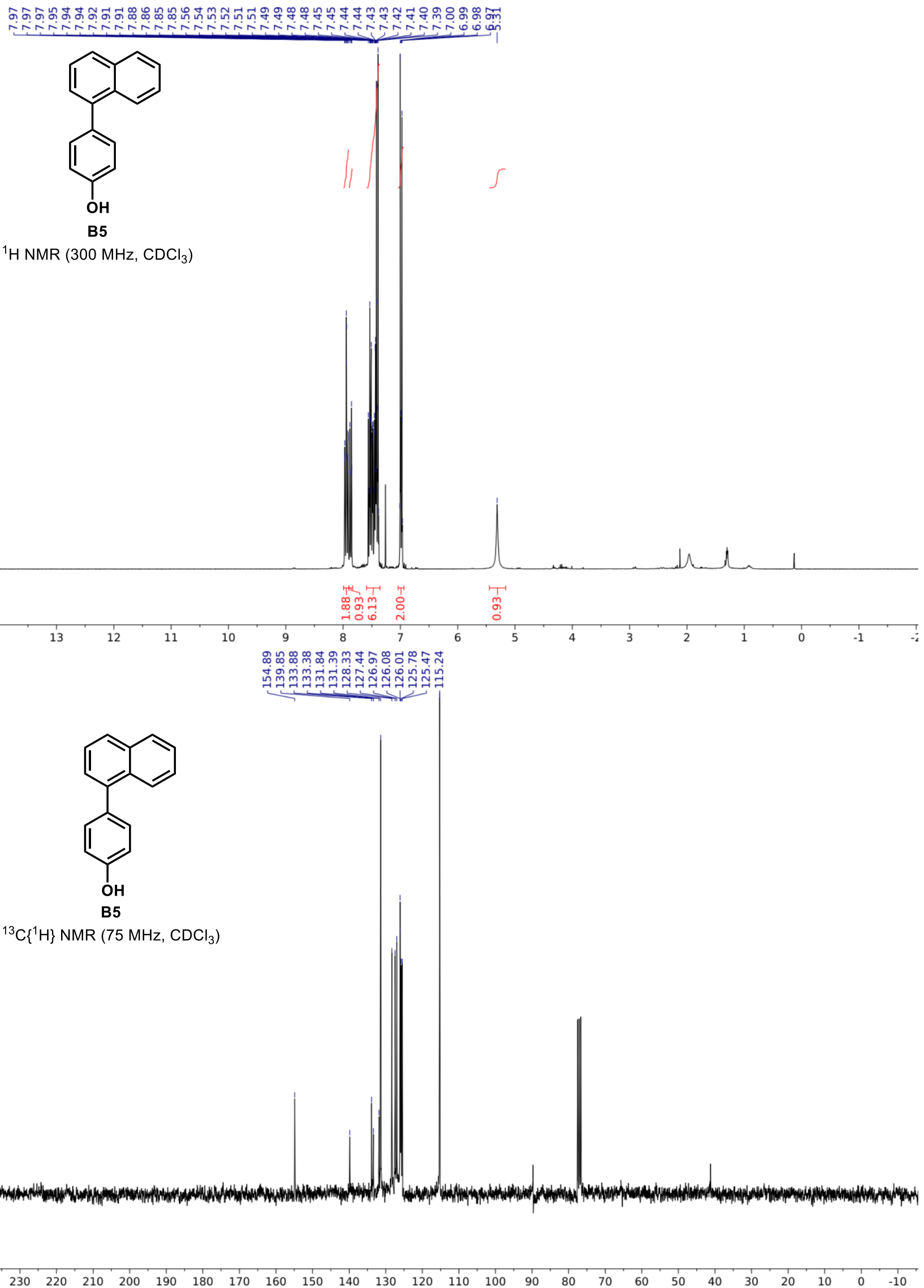

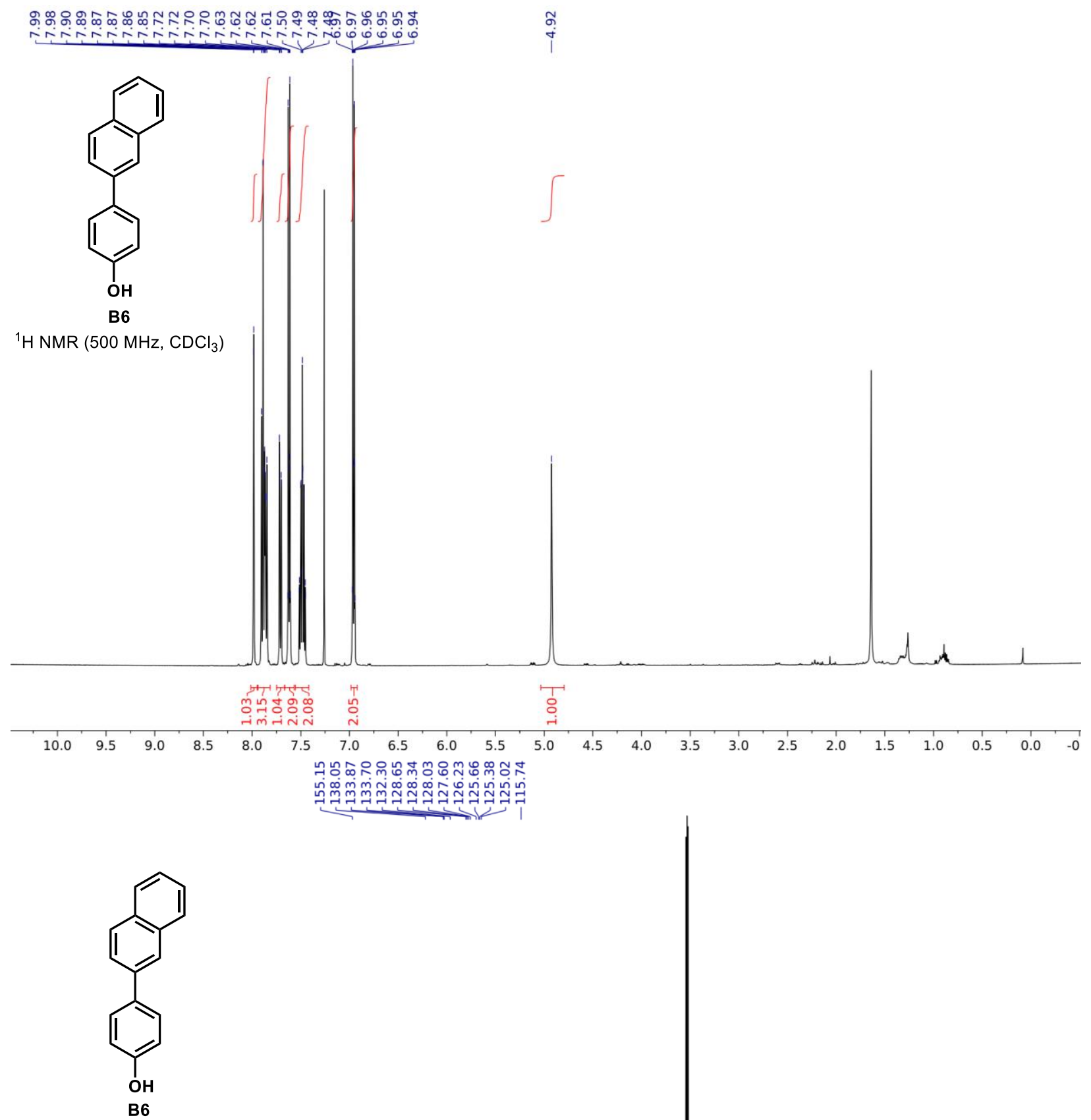

${ }^{13} \mathrm{C}\left\{{ }^{1} \mathrm{H}\right\}$ NMR (125 MHz, $\left.\mathrm{CDCl}_{3}\right)$

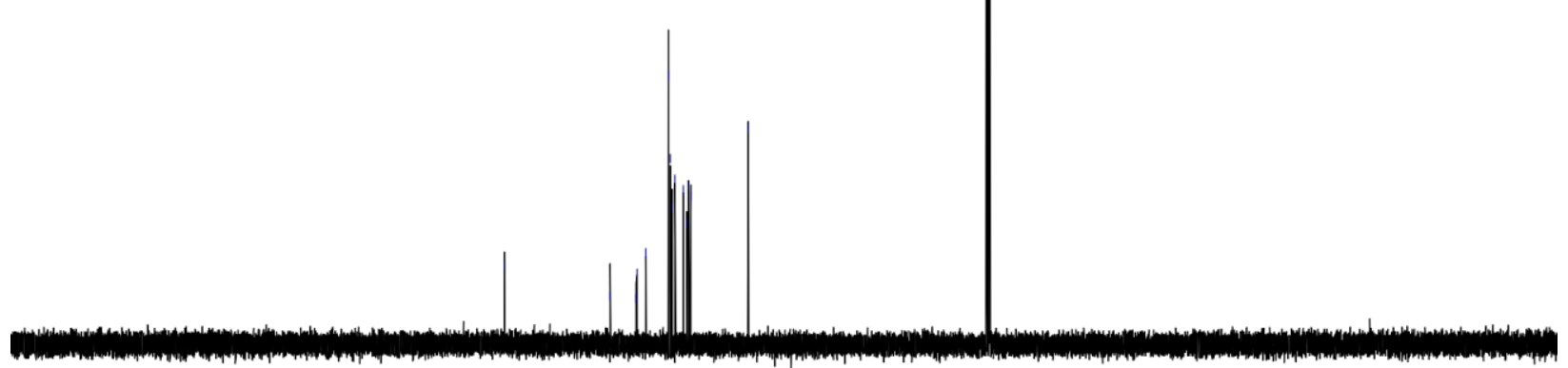

$\begin{array}{lllllllllllllllllllllllll}230 & 220 & 210 & 200 & 190 & 180 & 170 & 160 & 150 & 140 & 130 & 120 & 110 & 100 & 90 & 80 & 70 & 60 & 50 & 40 & 30 & 20 & 10 & 0 & -10\end{array}$ 
<smiles>Cc1ccc(-c2ccc(O)cc2)cc1</smiles>

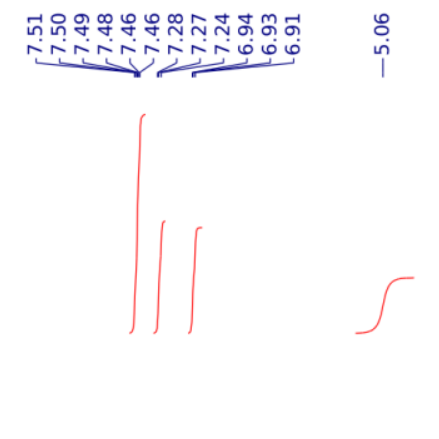

${ }^{1} \mathrm{H} \mathrm{NMR}\left(300 \mathrm{MHz}, \mathrm{CDCl}_{3}\right)$
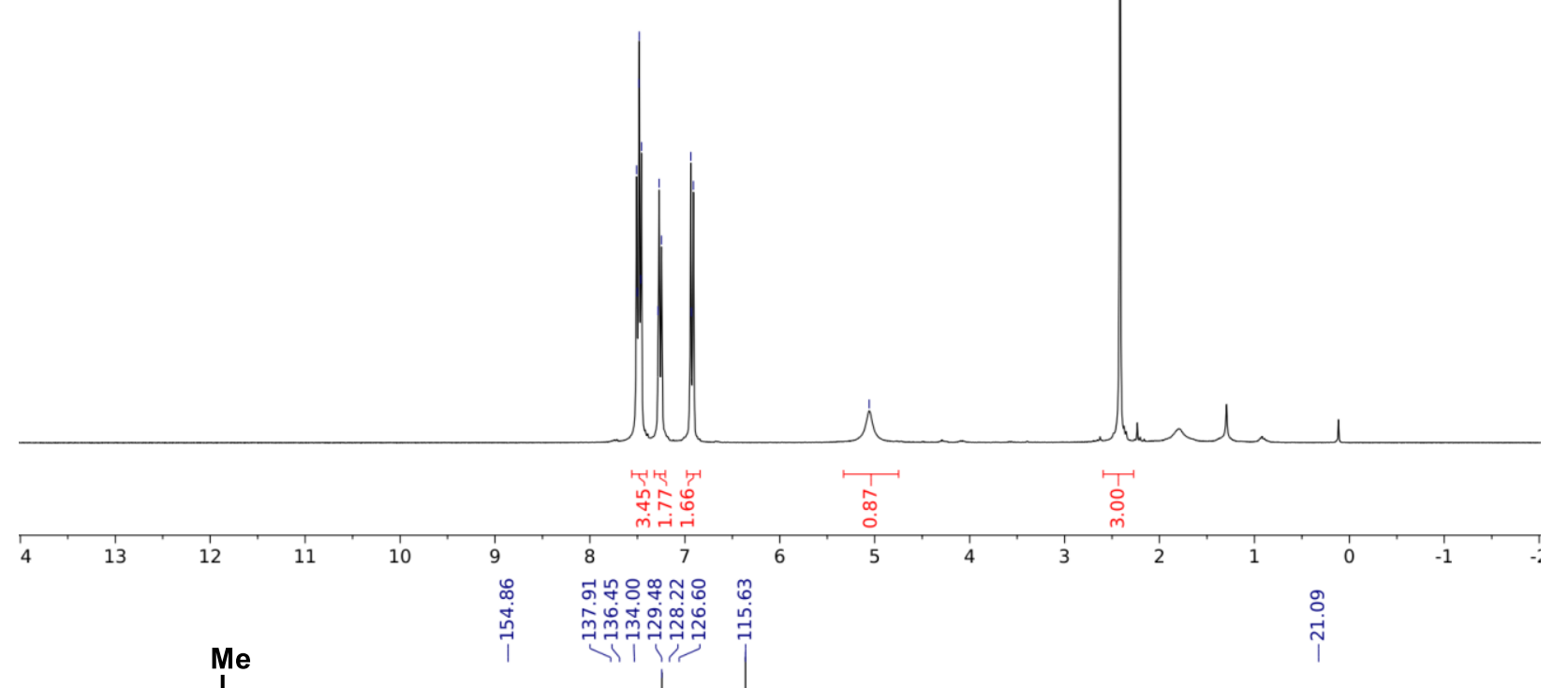<smiles>Cc1ccc(-c2ccc(O)cc2)cc1</smiles>

B7

${ }^{13} \mathrm{C}\left\{{ }^{1} \mathrm{H}\right\}$ NMR $\left(75 \mathrm{MHz}, \mathrm{CDCl}_{3}\right)$

$\begin{array}{llllllllllllllllllllllllll}230 & 220 & 210 & 200 & 190 & 180 & 170 & 160 & 150 & 140 & 130 & 120 & 110 & 100 & 90 & 80 & 70 & 60 & 50 & 40 & 30 & 20 & 10 & 0 & -10\end{array}$ 

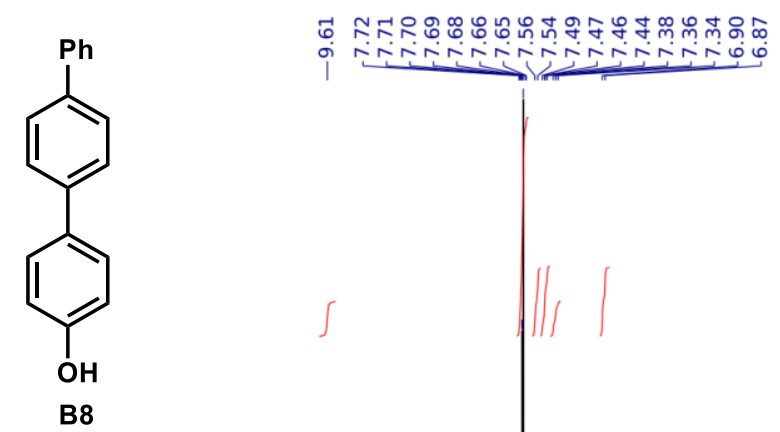

${ }^{1} \mathrm{H}$ NMR $\left(300 \mathrm{MHz}, \mathrm{DMSO}-d_{6}\right)$

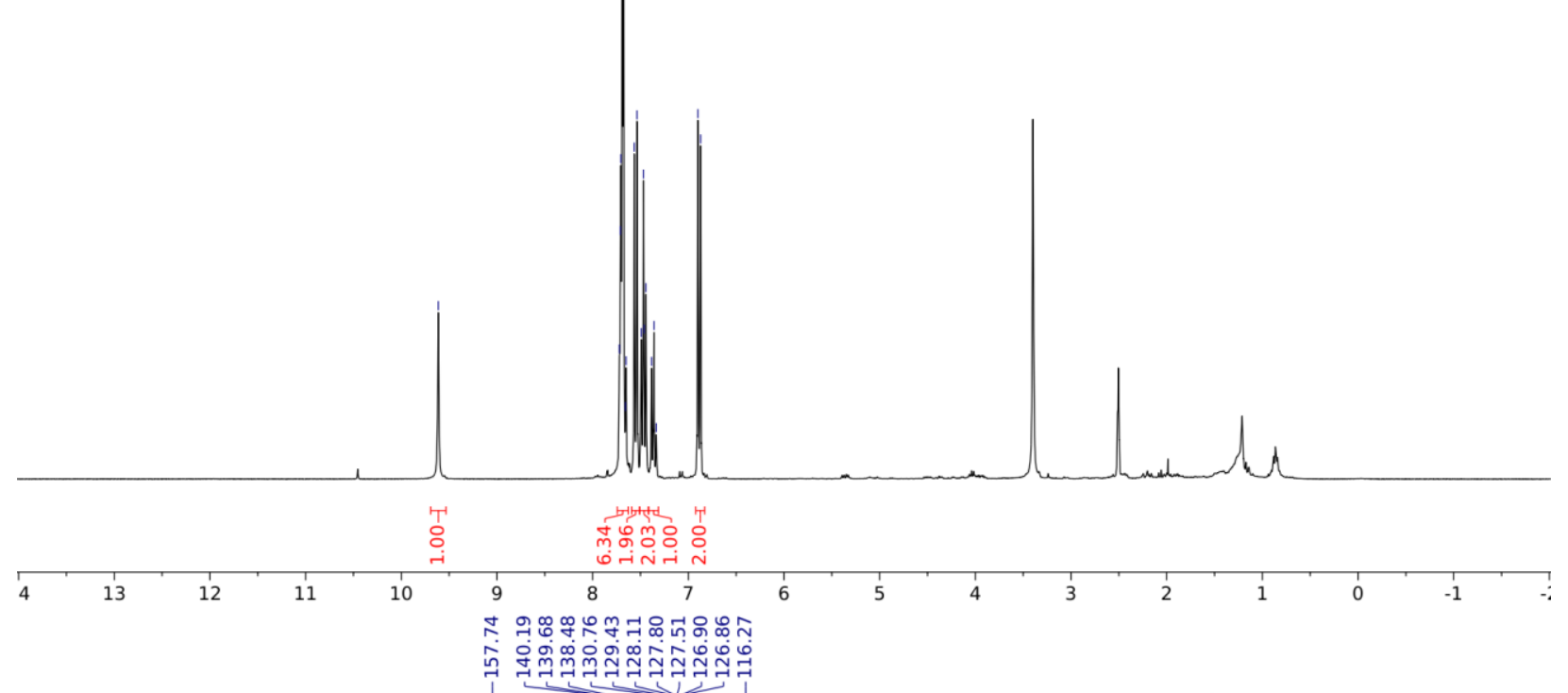<smiles>Oc1ccc(-c2ccc(-c3ccccc3)cc2)cc1</smiles>

${ }^{13} \mathrm{C}\left\{{ }^{1} \mathrm{H}\right\}$ NMR (75 MHz, DMSO- $\left.d_{6}\right)$

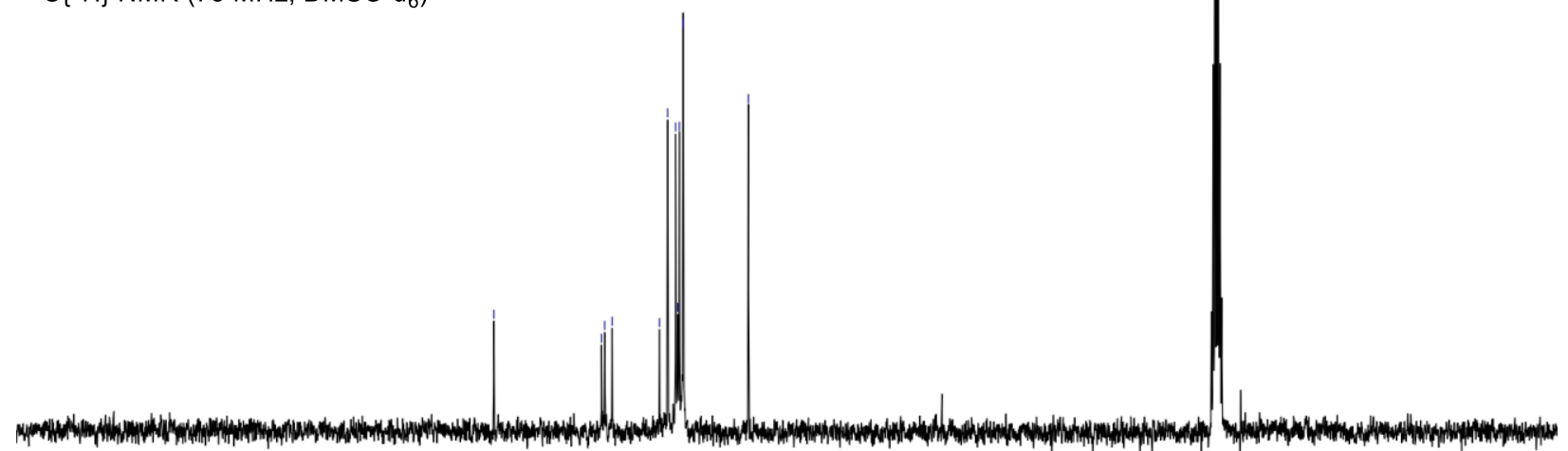

$\begin{array}{llllllllllllllllllllllllll}230 & 220 & 210 & 200 & 190 & 180 & 170 & 160 & 150 & 140 & 130 & 120 & 110 & 100 & 90 & 80 & 70 & 60 & 50 & 40 & 30 & 20 & 10 & 0 & -10\end{array}$ 
<smiles>Oc1ccc(-c2ccc(Br)cc2)cc1</smiles>

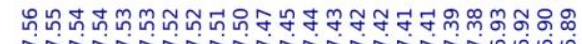

B9

${ }^{1} \mathrm{H}$ NMR $\left(300 \mathrm{MHz}, \mathrm{CDCl}_{3}\right)$
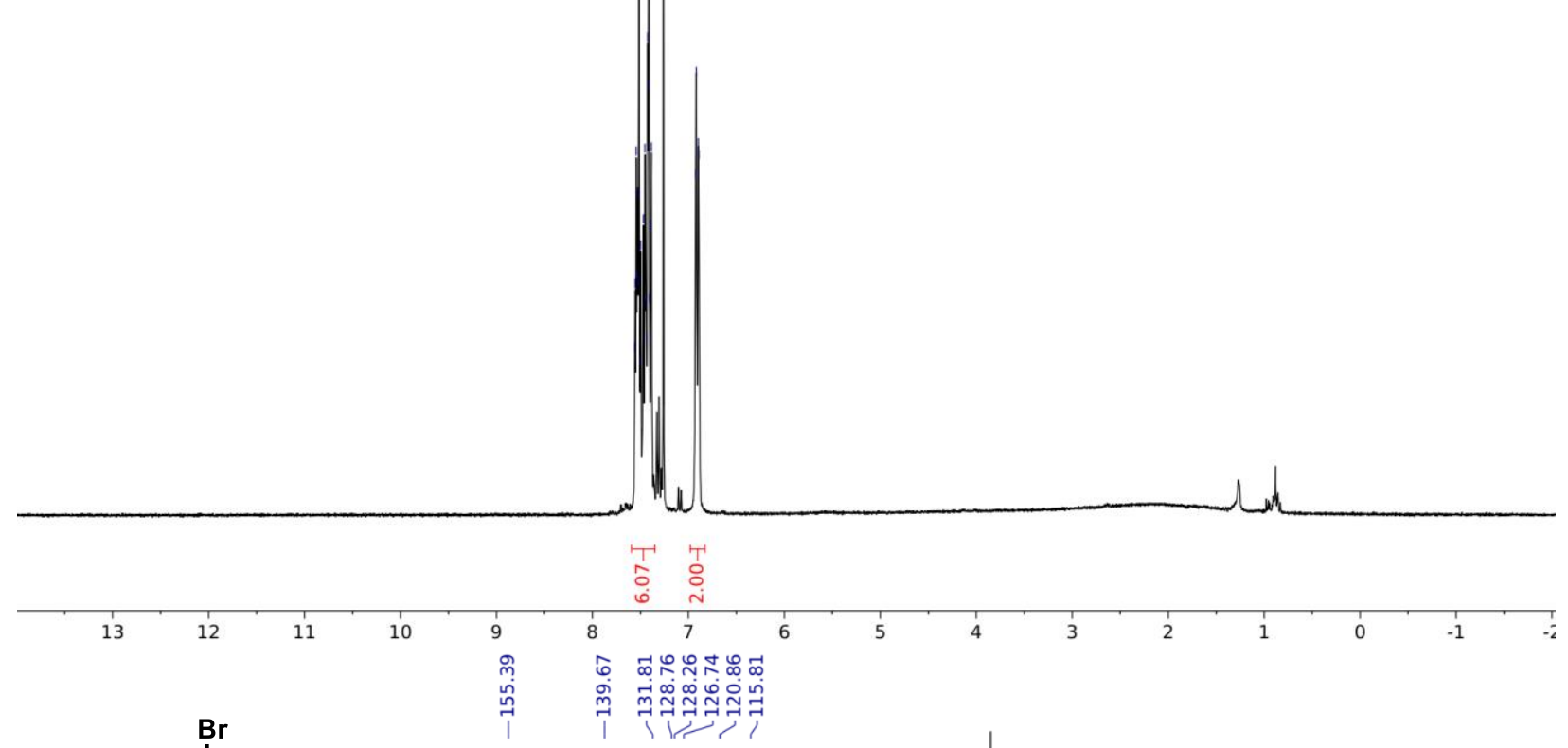<smiles>Oc1ccc(-c2ccc(Br)cc2)cc1</smiles>

${ }^{13} \mathrm{C}\left\{{ }^{1} \mathrm{H}\right\}$ NMR $\left(75 \mathrm{MHz}, \mathrm{CDCl}_{3}\right)$

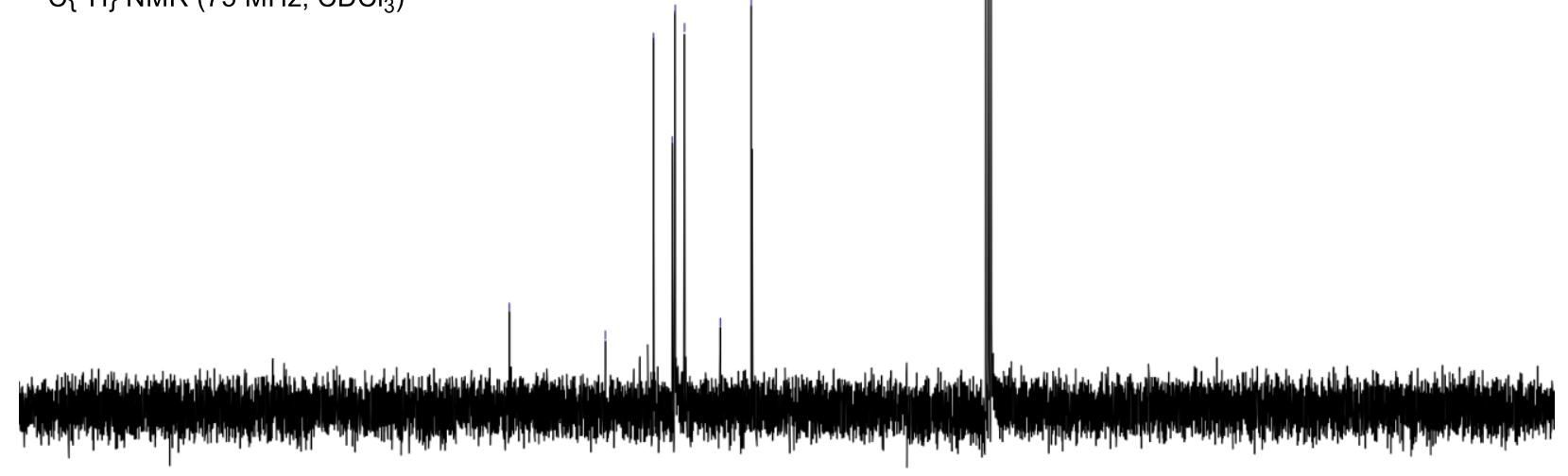

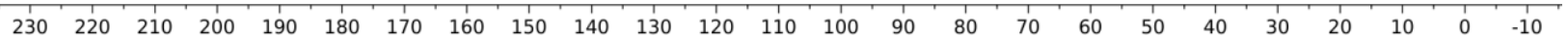




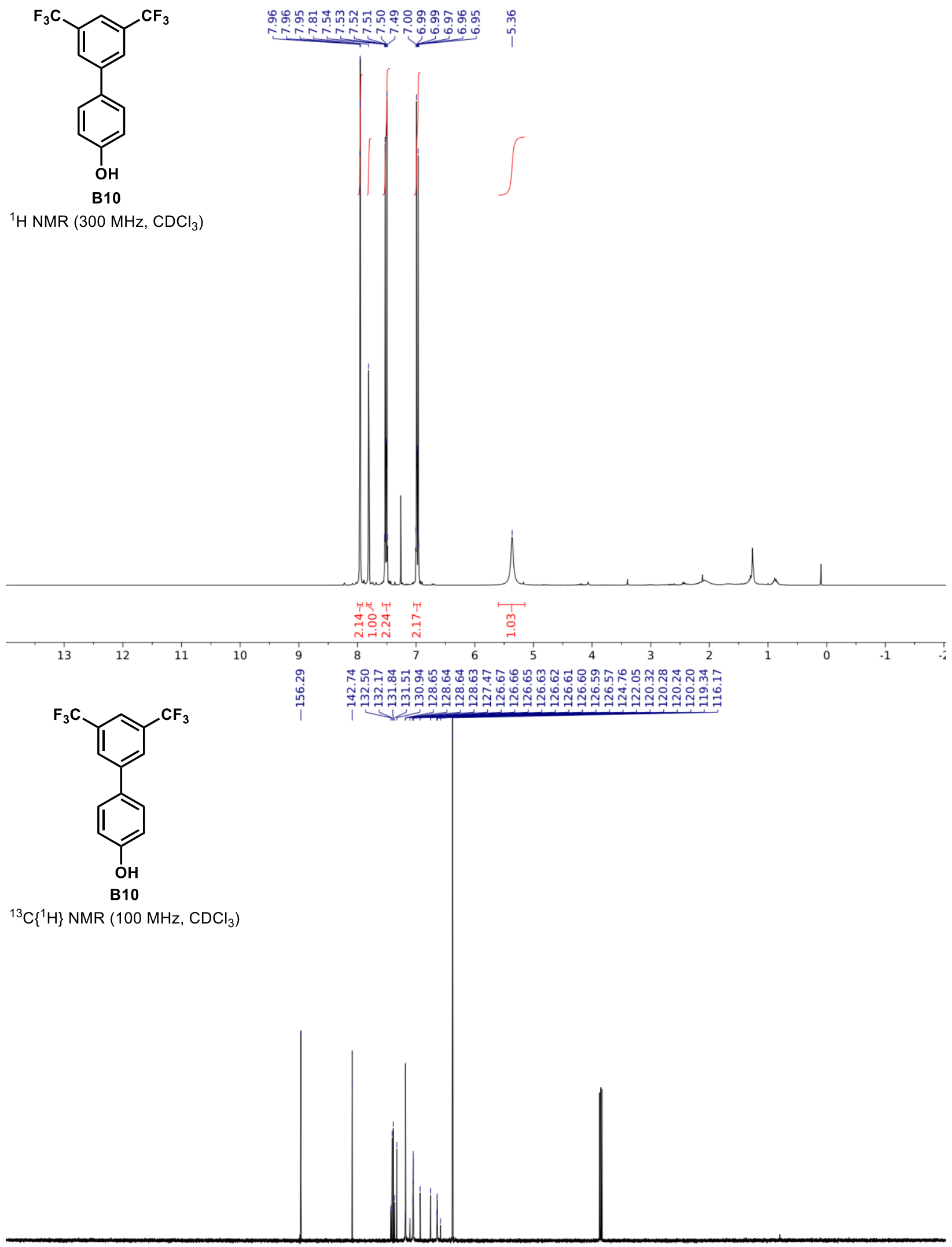

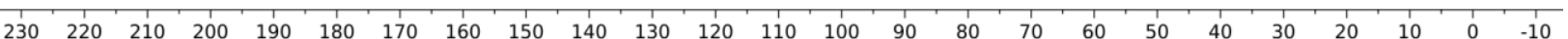




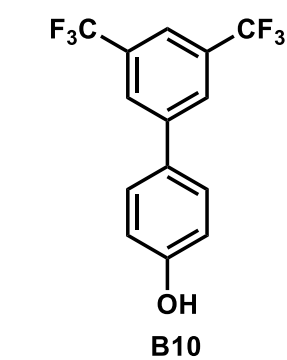

${ }^{19} \mathrm{~F} \mathrm{NMR}\left(282 \mathrm{MHz}, \mathrm{CDCl}_{3}\right)$

\begin{tabular}{lllllllllllllllllllllllll}
\hline & 10 & 20 & 10 & 0 & -10 & -20 & -30 & -40 & -50 & -60 & -70 & -80 & -90 & -100 & -110 & -120 & -130 & -140 & -150 & -160 & -170 & -180 & -190 & -20
\end{tabular} 

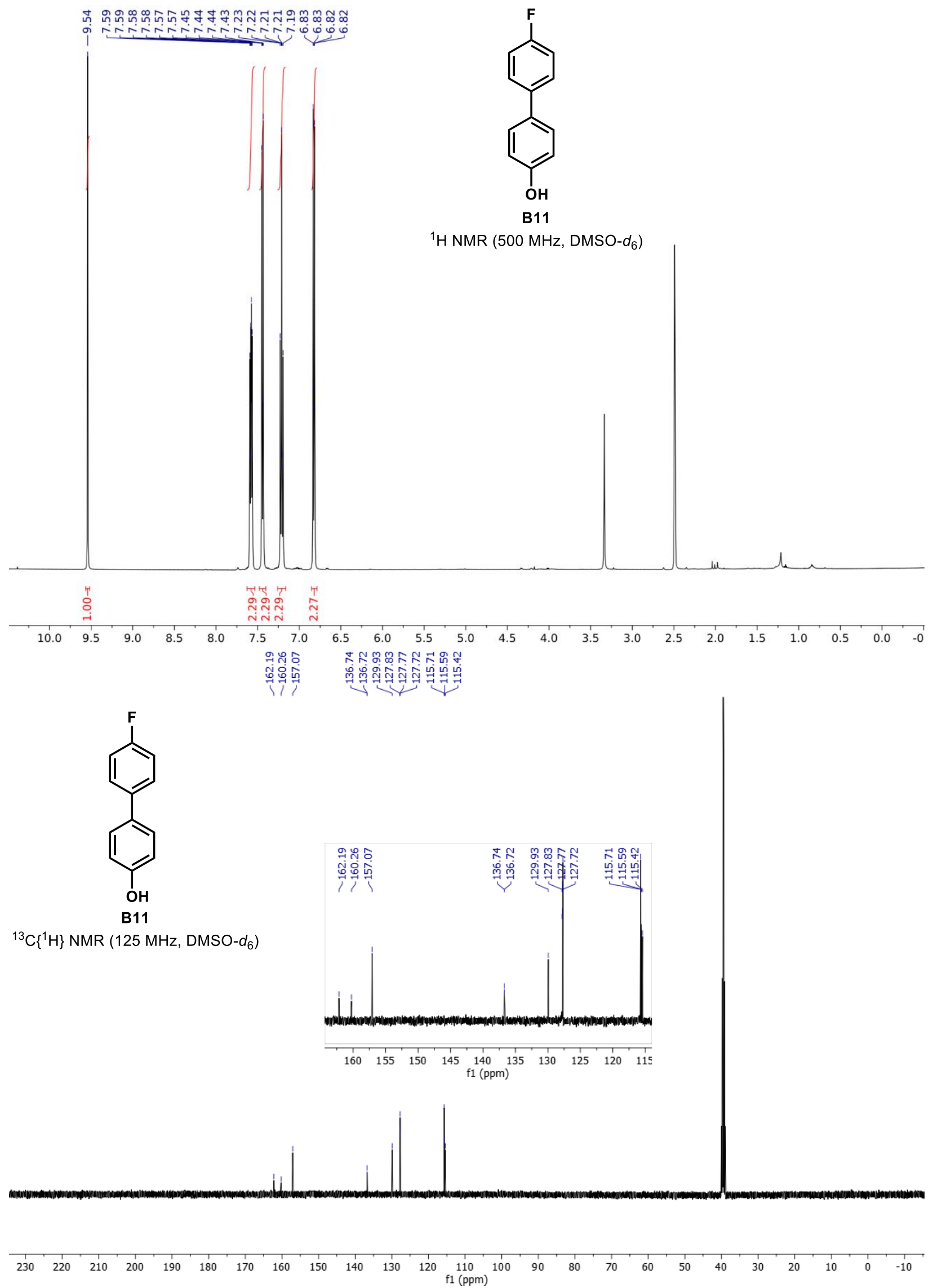


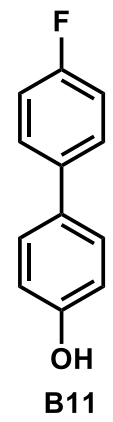

${ }^{19} \mathrm{~F}$ NMR $\left(282 \mathrm{MHz}\right.$, DMSO- $\left.d_{6}\right)$

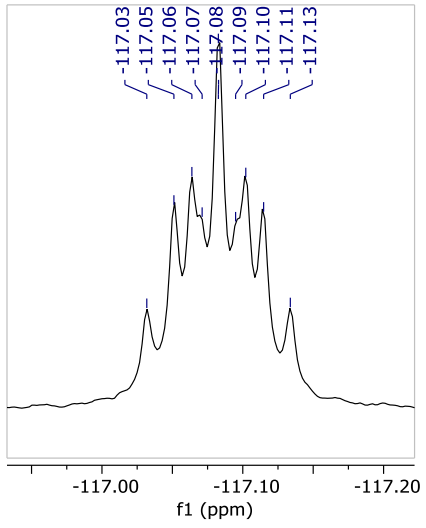

$\begin{array}{lllllllllllllllllllllllll}10 & 20 & 10 & 0 & -10 & -20 & -30 & -40 & -50 & -60 & -70 & -80 & -90 & -100 & -110 & -120 & -130 & -140 & -150 & -160 & -170 & -180 & -190 & -20\end{array}$ 


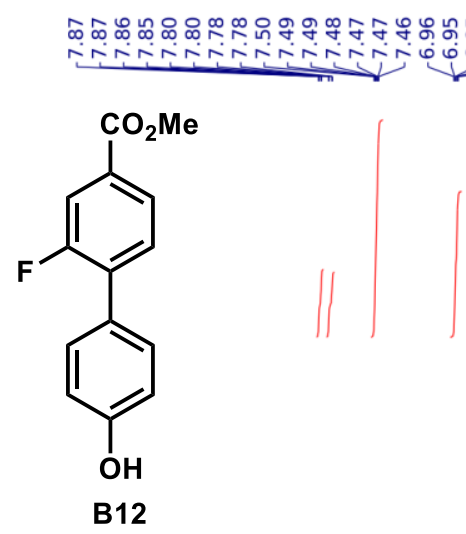

${ }^{1} \mathrm{H} \mathrm{NMR}\left(500 \mathrm{MHz}, \mathrm{CDCl}_{3}\right)$

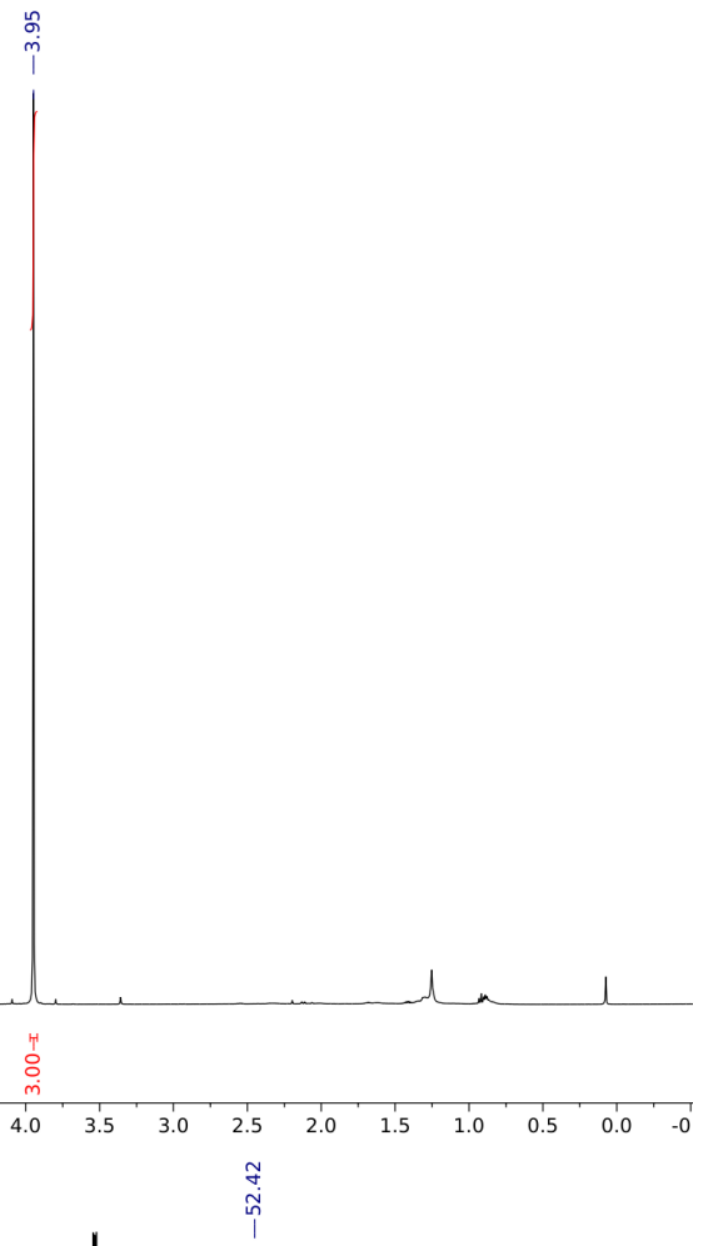<smiles>CC(=O)c1ccc(-c2ccc(O)cc2)c(F)c1</smiles>

B12

$\left.{ }^{13} \mathrm{C}\left\{{ }^{1} \mathrm{H}\right\} \mathrm{NMR}(125 \mathrm{MHz}, \mathrm{CDCl})_{3}\right)$

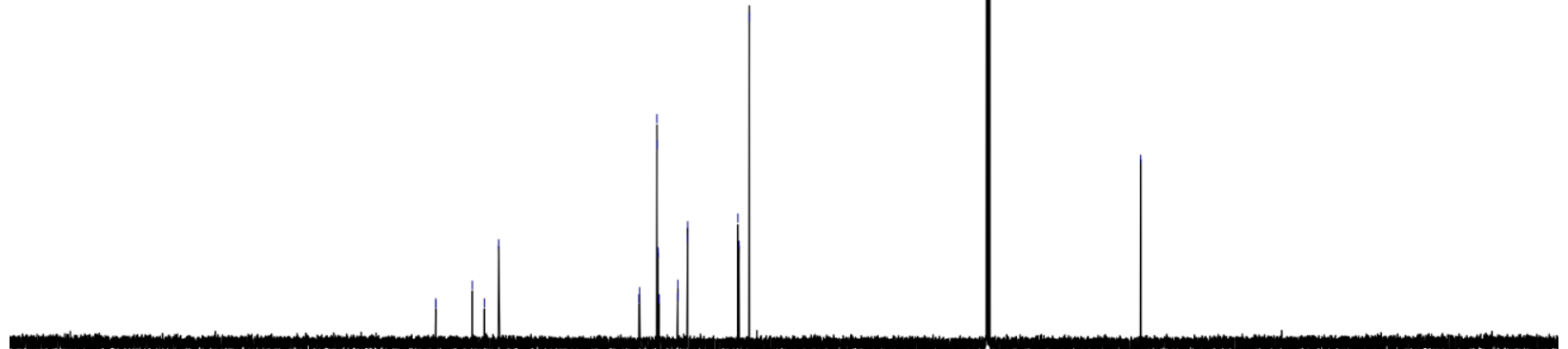

$\begin{array}{lllllllllllllllllllllllll}230 & 220 & 210 & 200 & 190 & 180 & 170 & 160 & 150 & 140 & 130 & 120 & 110 & 100 & 90 & 80 & 70 & 60 & 50 & 40 & 30 & 20 & 10 & 0 & -10\end{array}$ 


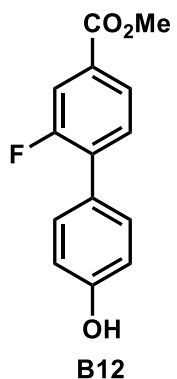

${ }^{19} \mathrm{~F}$ NMR $\left(282 \mathrm{MHz}, \mathrm{CDCl}_{3}\right)$
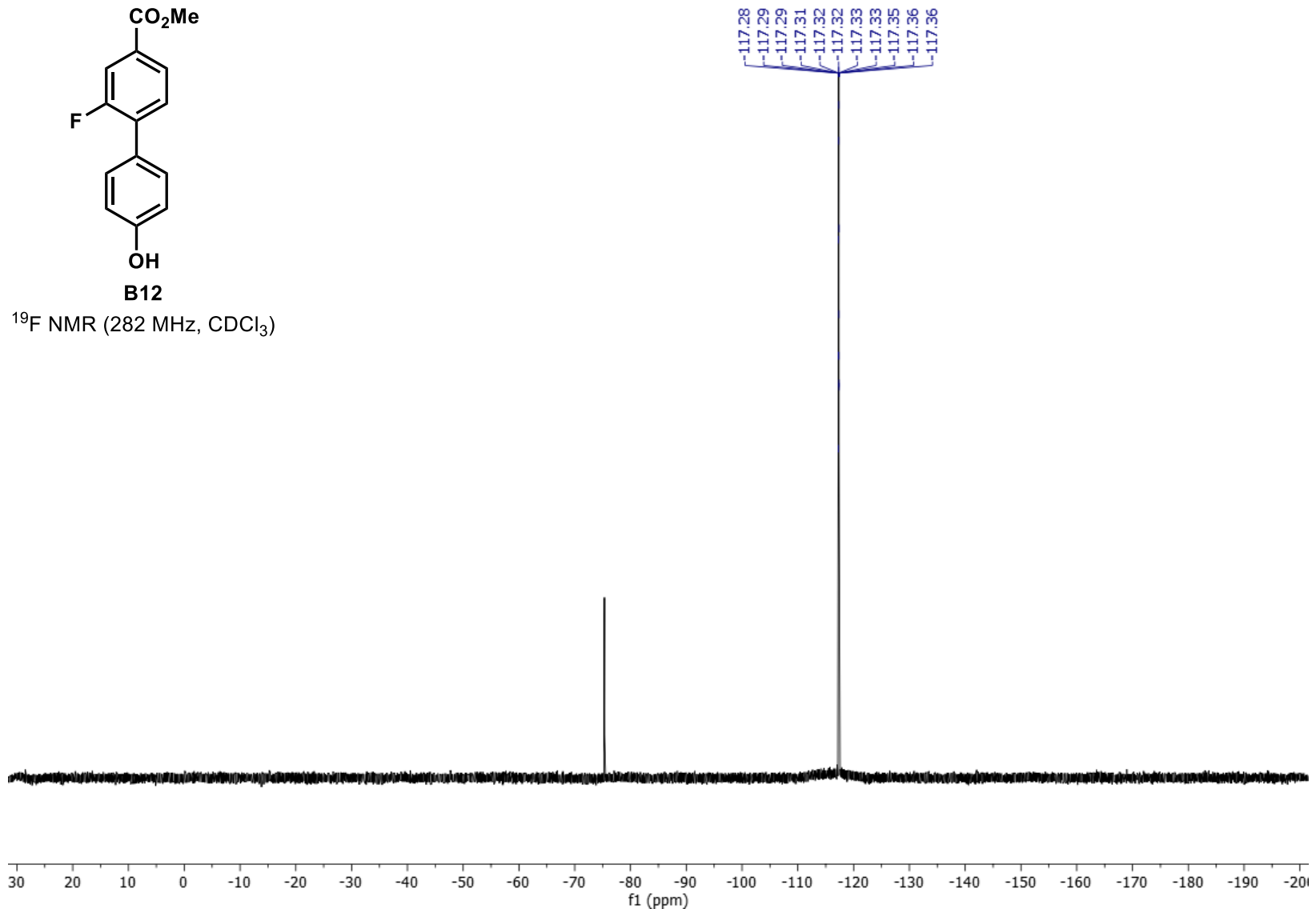


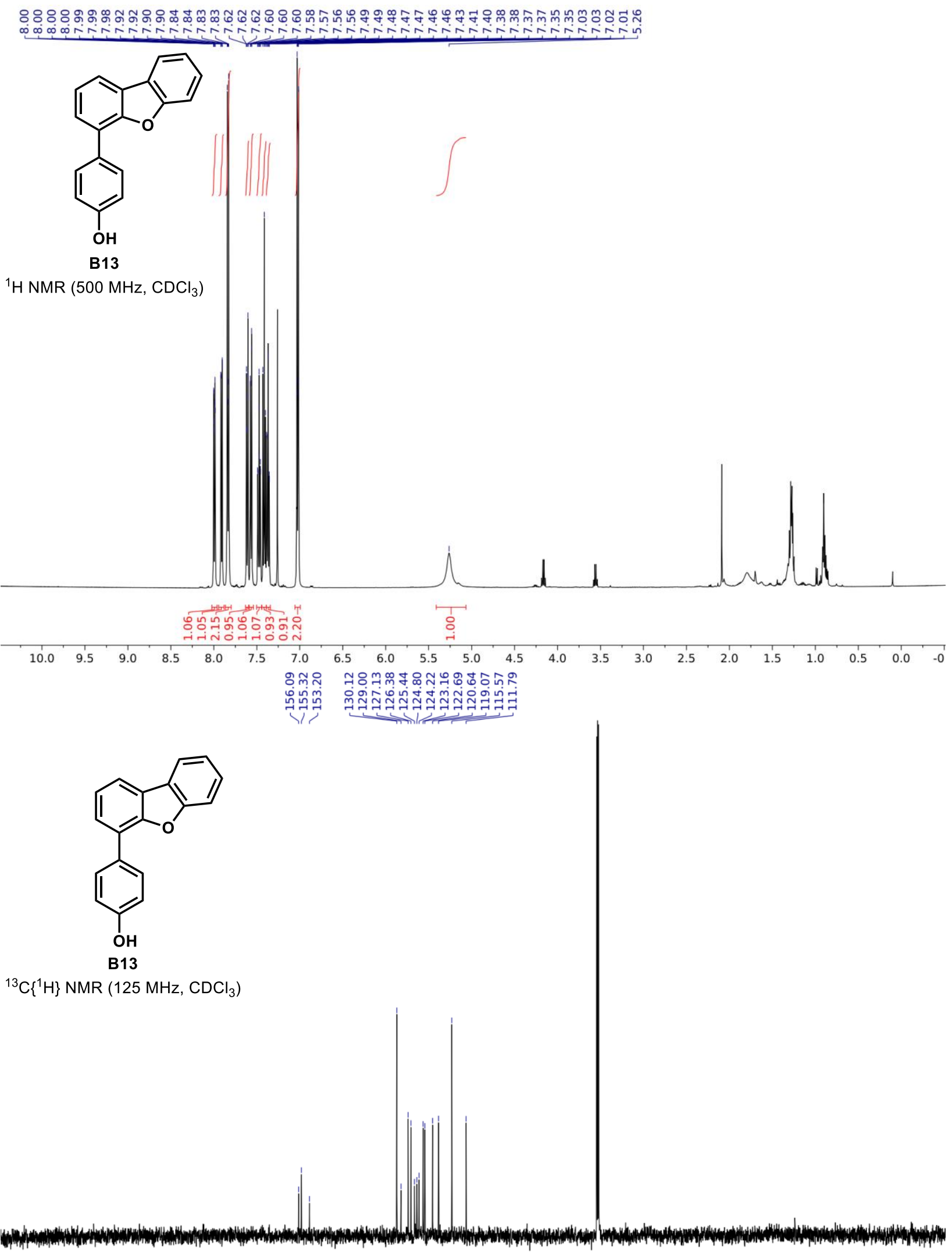

$\begin{array}{lllllllllllllllllllllllllllllll}230 & 220 & 210 & 200 & 190 & 180 & 170 & 160 & 150 & 140 & 130 & 120 & 110 & 100 & 90 & 80 & 70 & 60 & 50 & 40 & 30 & 20 & 10 & 0 & -10\end{array}$ 

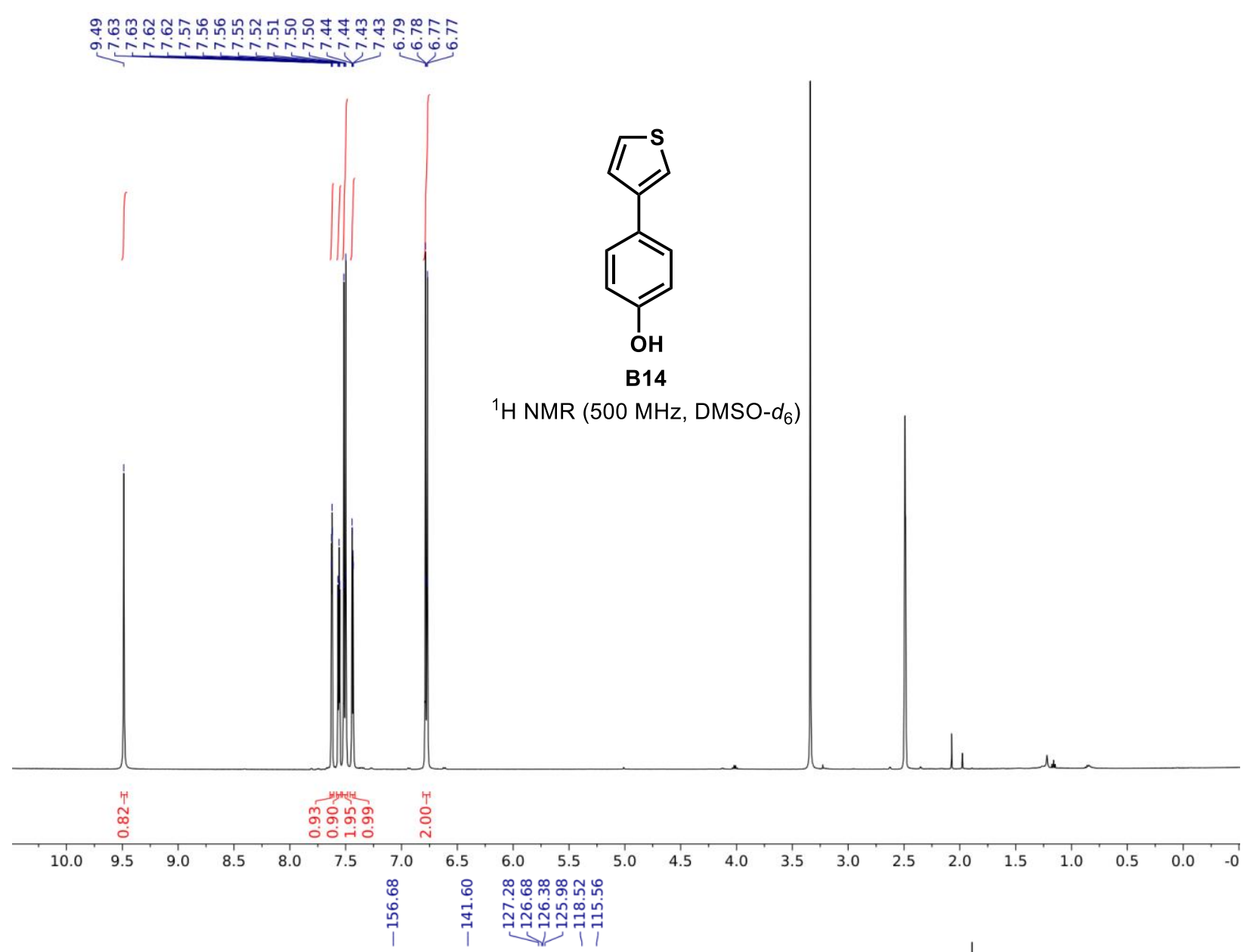<smiles>Oc1ccc(-c2ccsc2)cc1</smiles>

B14
${ }^{13} \mathrm{C}\left\{{ }^{1} \mathrm{H}\right\}$ NMR $\left(100 \mathrm{MHz}\right.$, DMSO- $\left.d_{6}\right)$
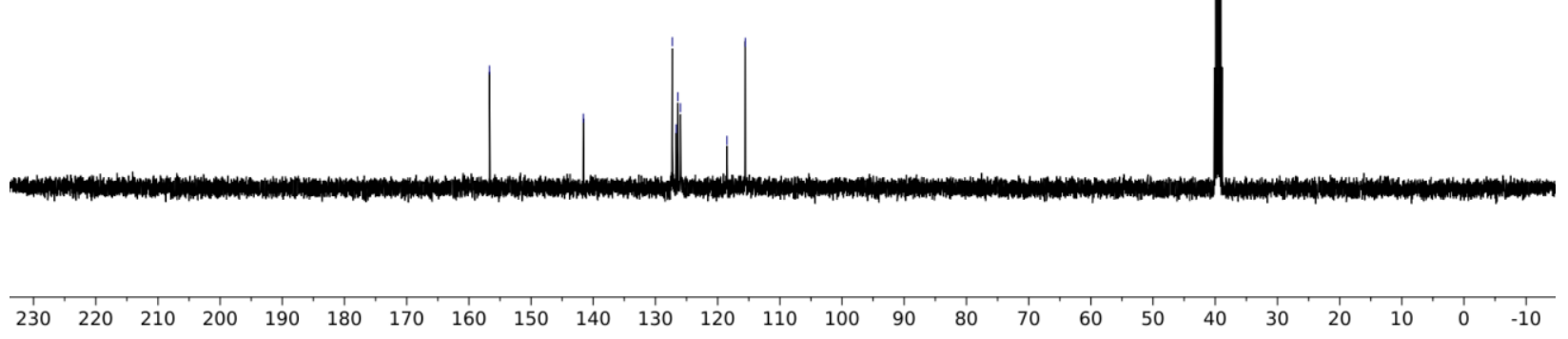

S-119 

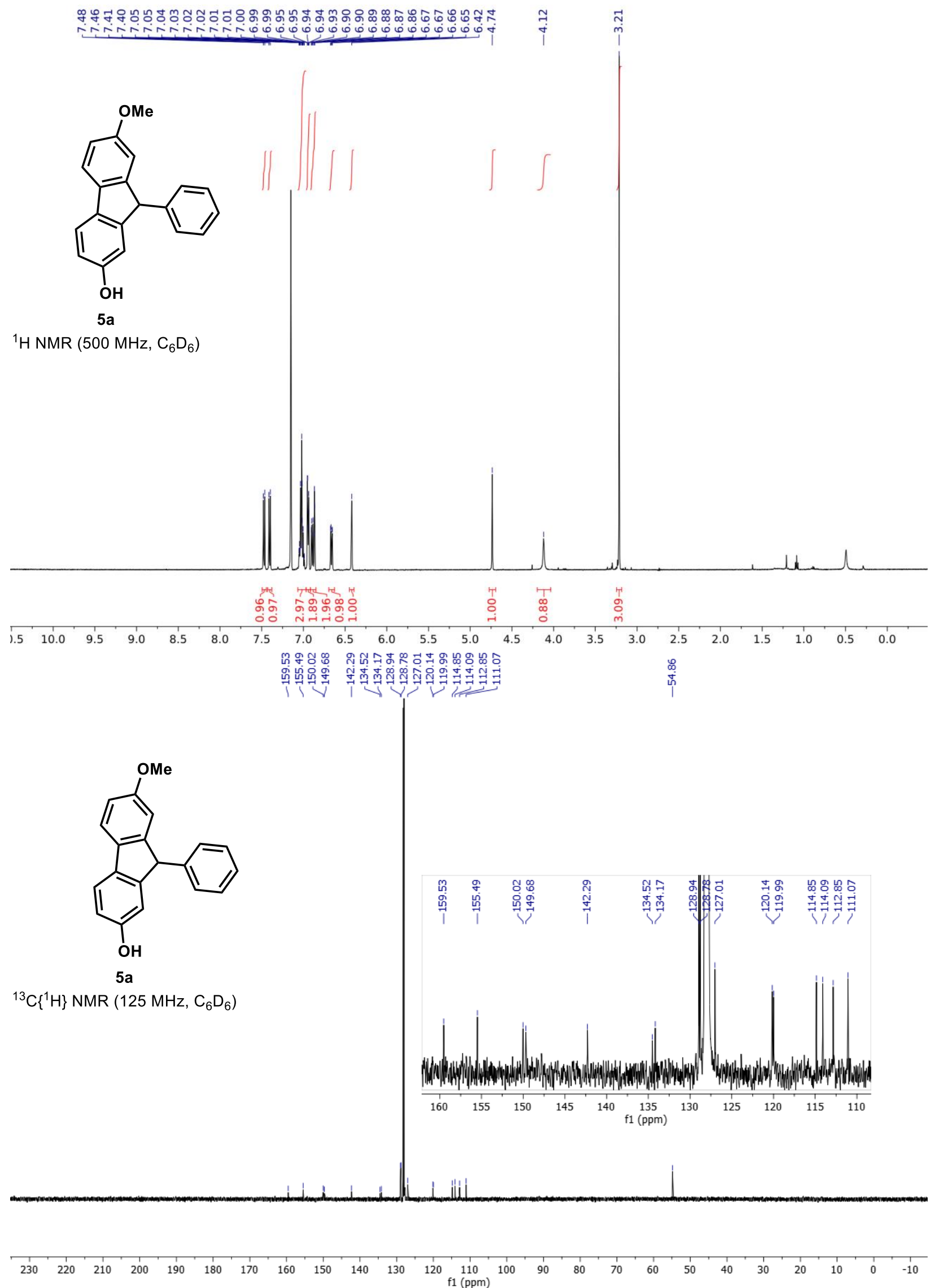


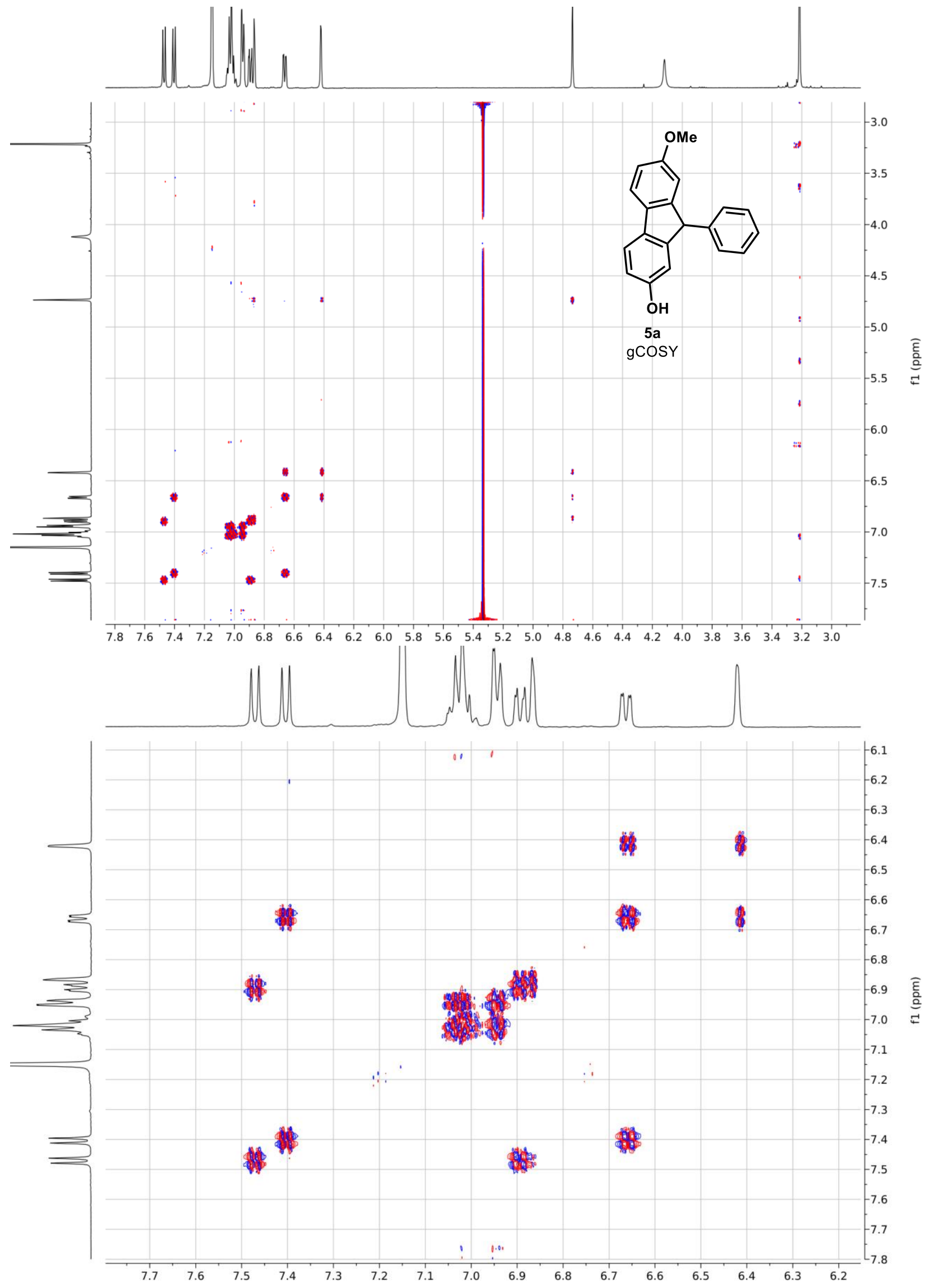



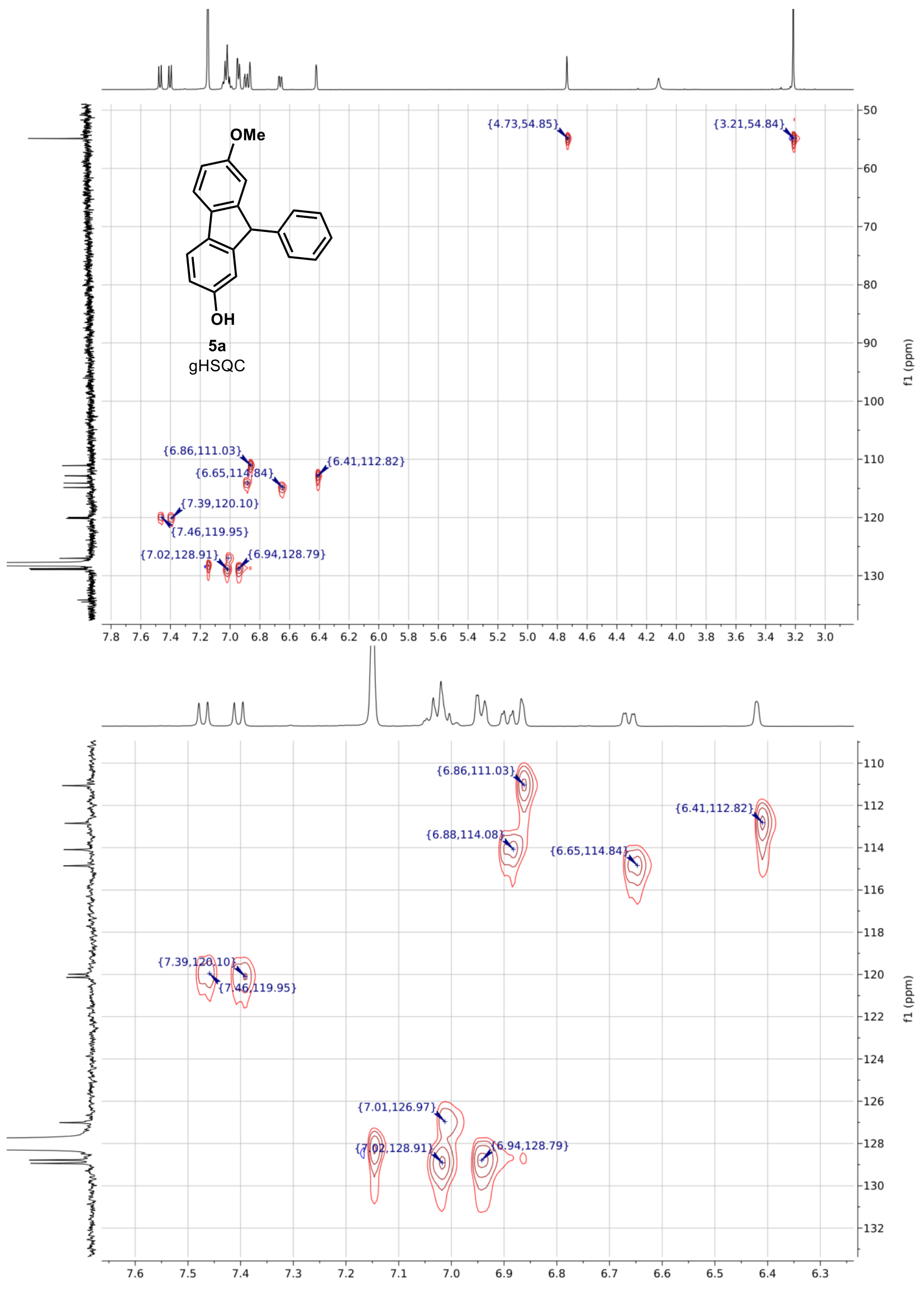

S-122 

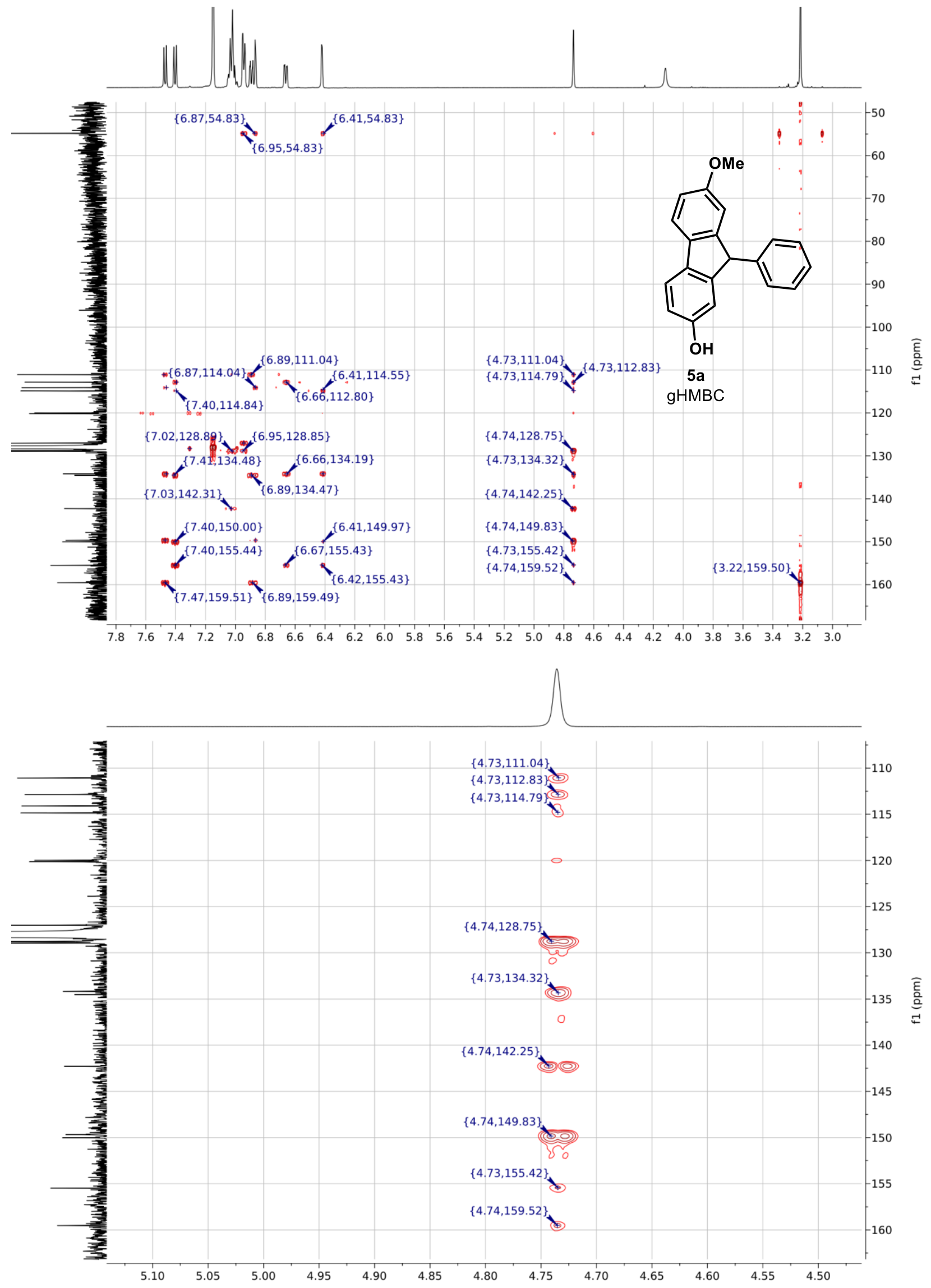


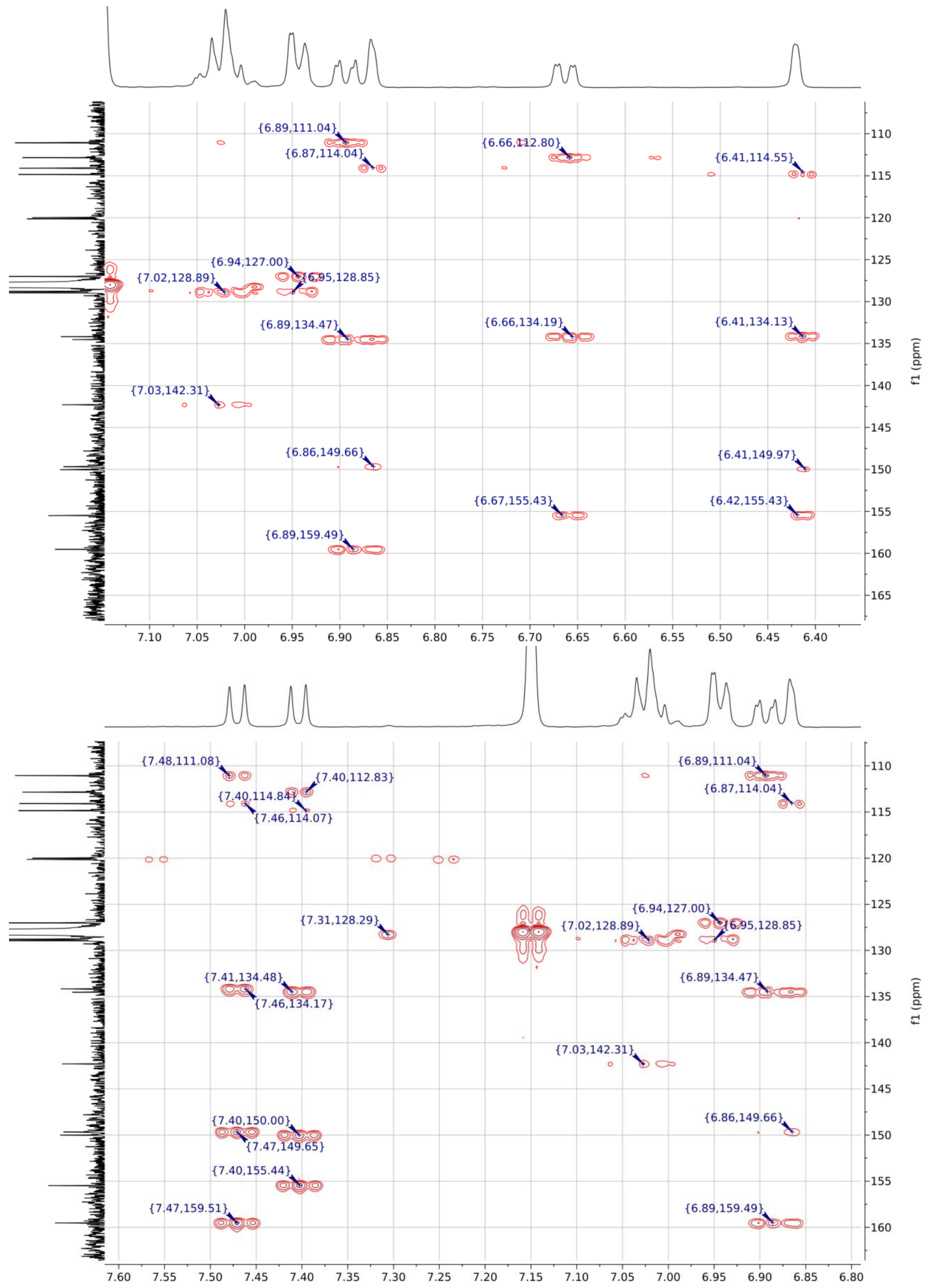




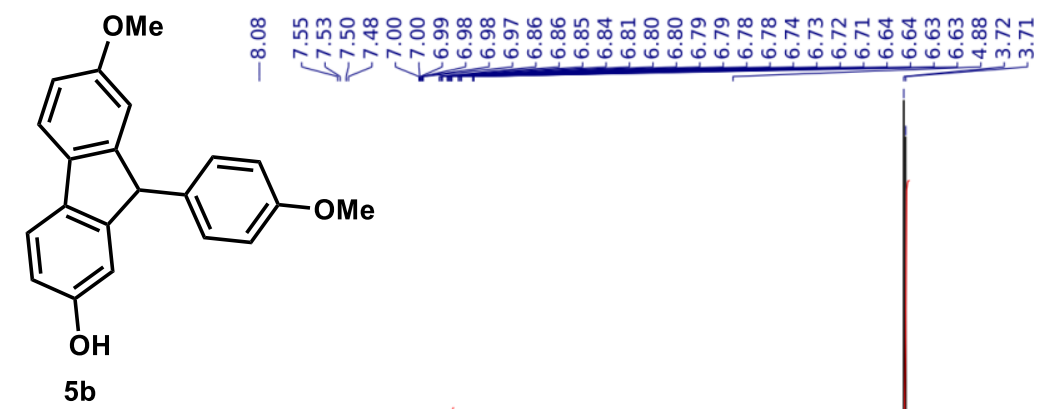

${ }^{1} \mathrm{H}$ NMR $\left(500 \mathrm{MHz}, \mathrm{THF}-\mathrm{d}_{8}\right)$
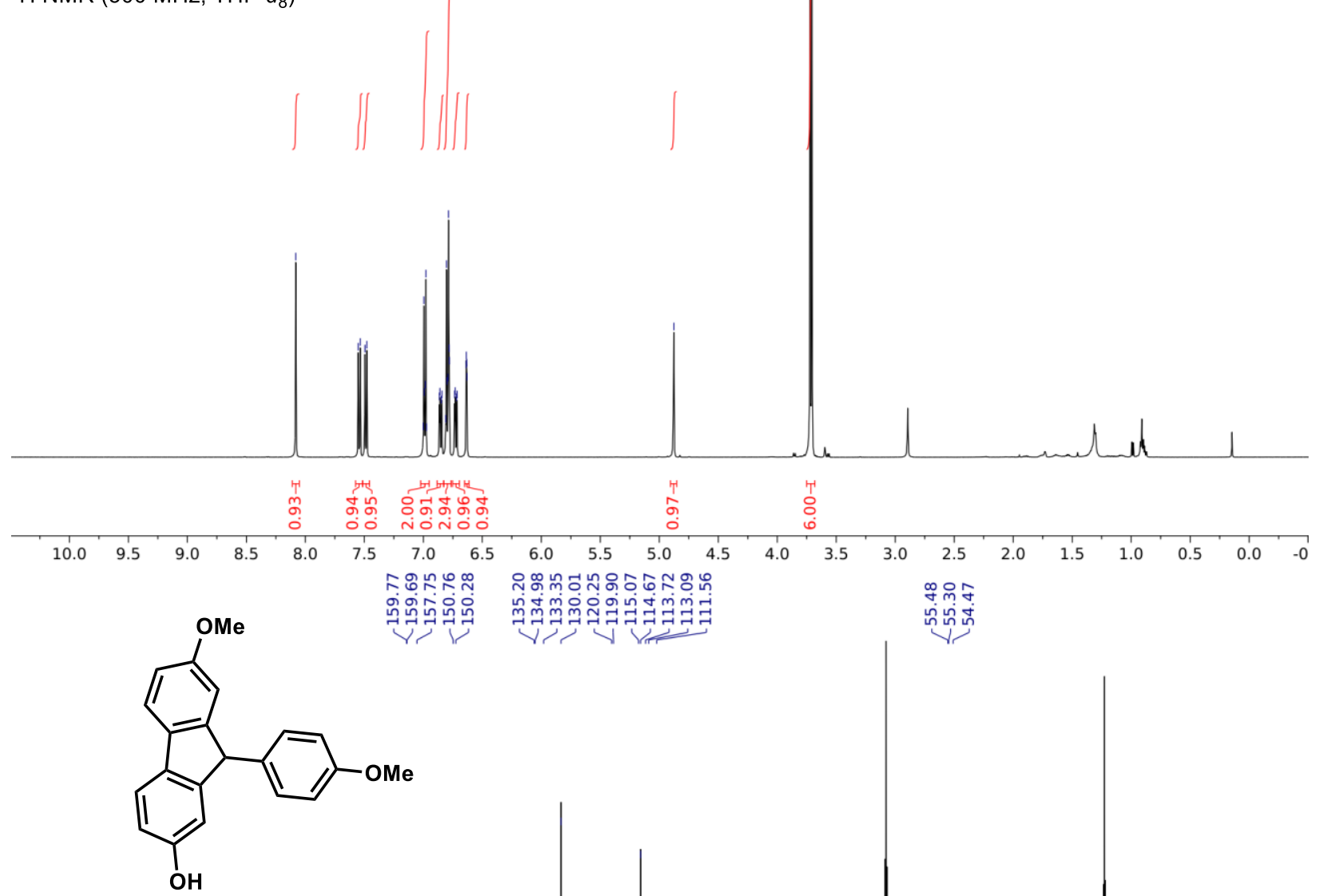

$5 b$

${ }^{13} \mathrm{C}\left\{{ }^{1} \mathrm{H}\right\}$ NMR $\left(125 \mathrm{MHz}, \mathrm{THF}-\mathrm{d}_{8}\right)$

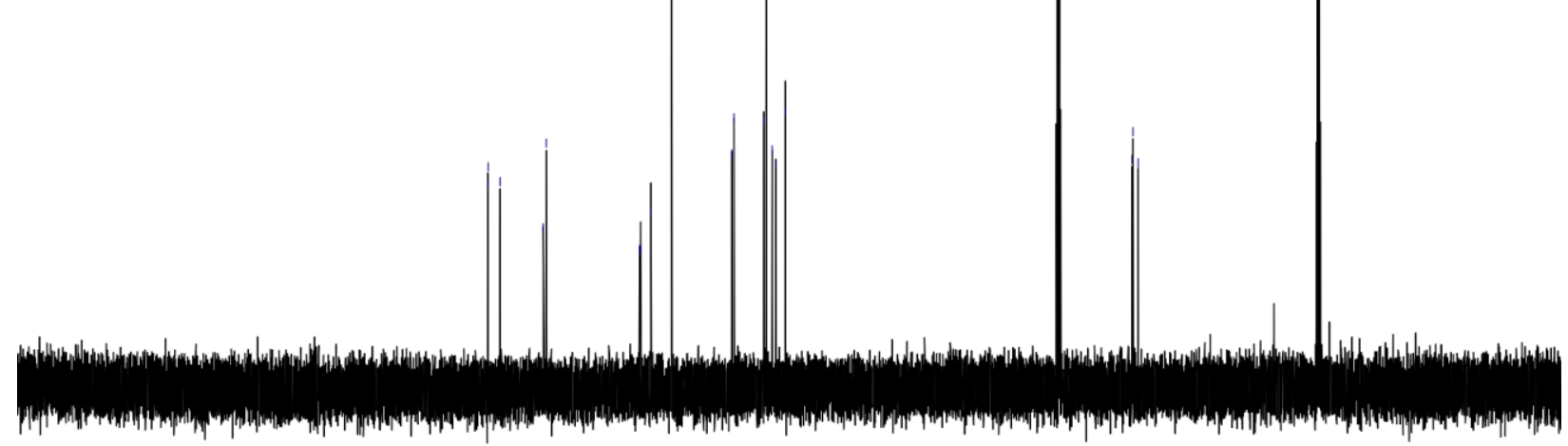

$\begin{array}{lllllllllllllllllllllllll}230 & 220 & 210 & 200 & 190 & 180 & 170 & 160 & 150 & 140 & 130 & 120 & 110 & 100 & 90 & 80 & 70 & 60 & 50 & 40 & 30 & 20 & 10 & 0 & -10\end{array}$ 


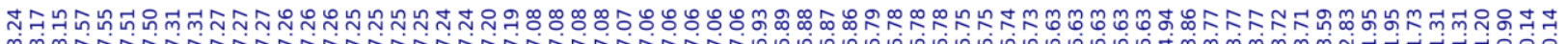

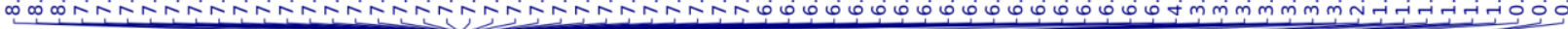

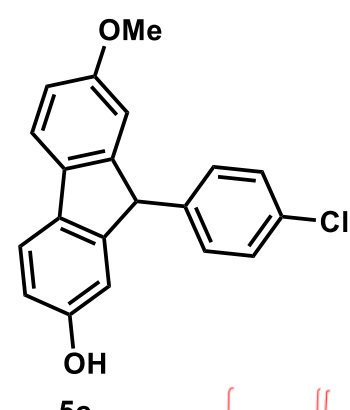

${ }^{1} \mathrm{H}$ NMR $\left(500 \mathrm{MHz}, \mathrm{THF}-d_{8}\right)$
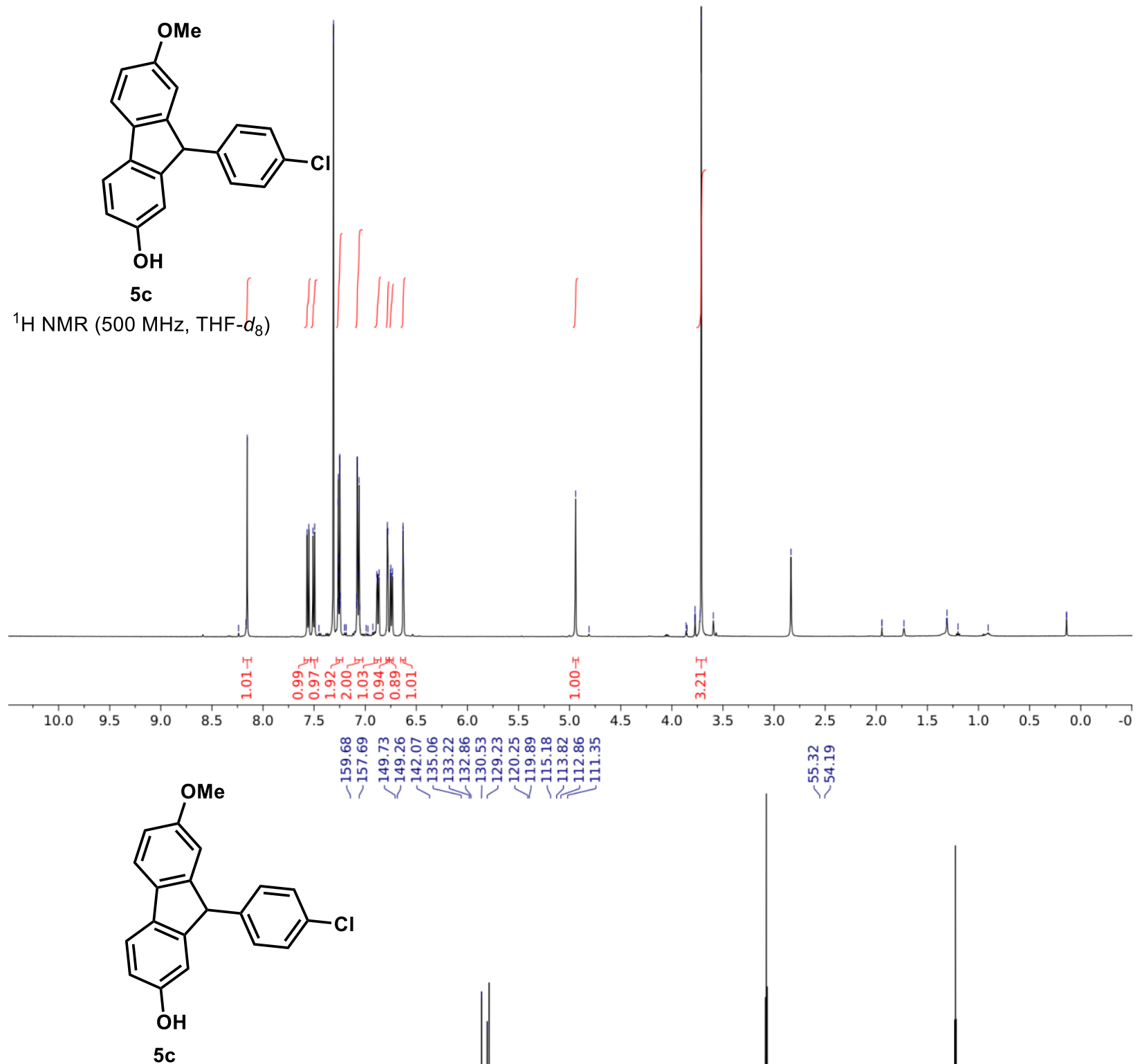

${ }^{13} \mathrm{C}\left\{{ }^{1} \mathrm{H}\right\}$ NMR $\left(125 \mathrm{MHz}, \mathrm{THF}-d_{8}\right)$

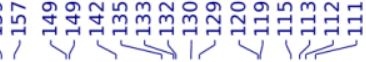

约

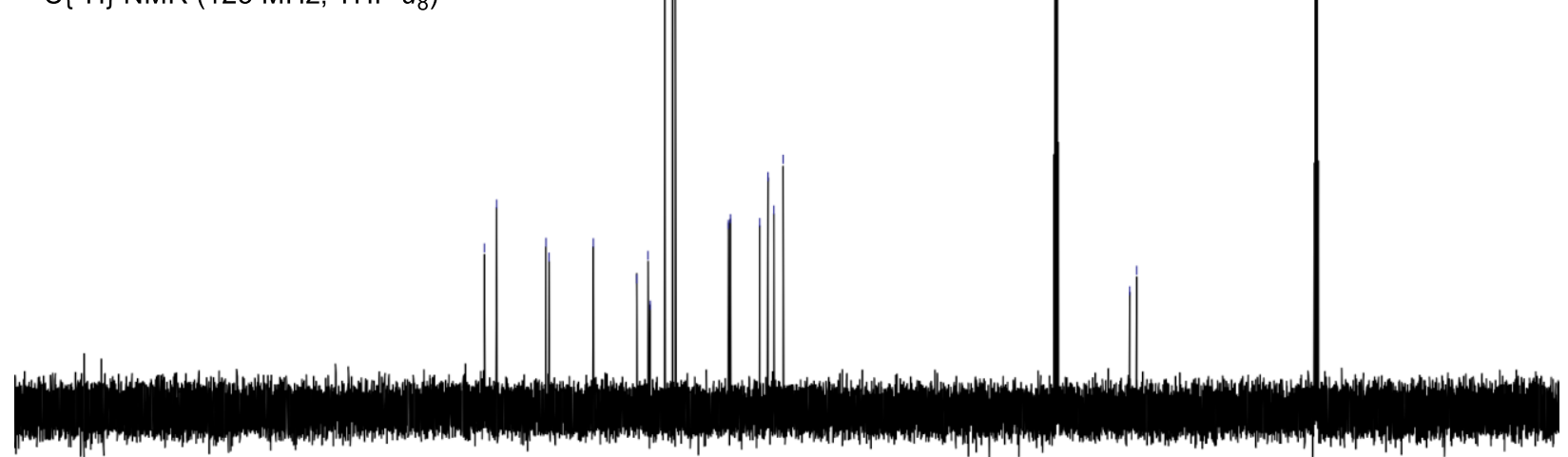

$\begin{array}{lllllllllllllllllllllllll}230 & 220 & 210 & 200 & 190 & 180 & 170 & 160 & 150 & 140 & 130 & 120 & 110 & 100 & 90 & 80 & 70 & 60 & 50 & 40 & 30 & 20 & 10 & 0 & -10\end{array}$ 
<smiles>COc1ccc2c(c1)C(c1c(F)cccc1Cl)c1cc(O)ccc1-2</smiles>

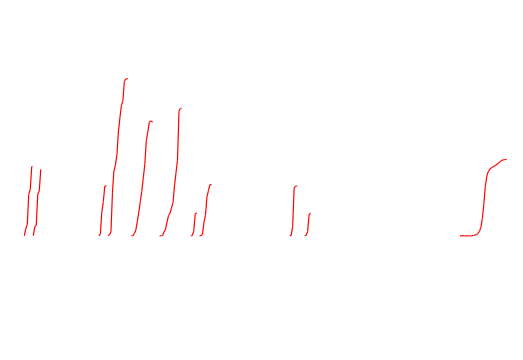

5d

${ }^{1} \mathrm{H}$ NMR $\left(500 \mathrm{MHz}, \mathrm{C}_{6} \mathrm{D}_{6}\right)$

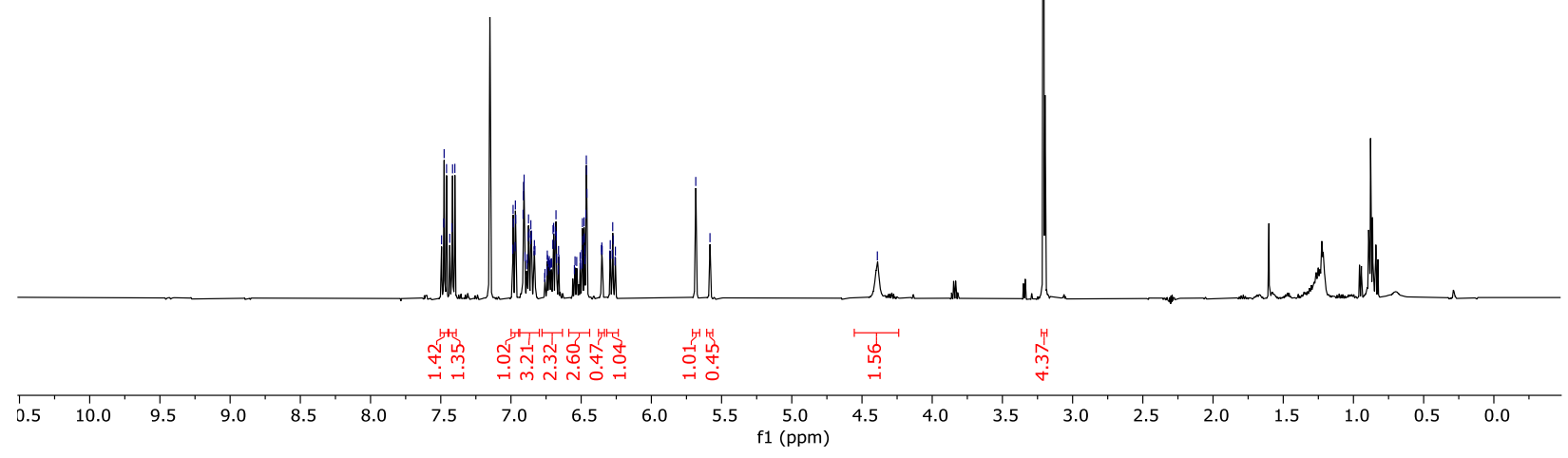

以ू.

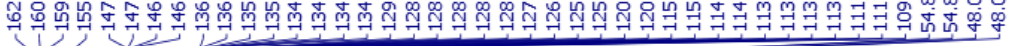

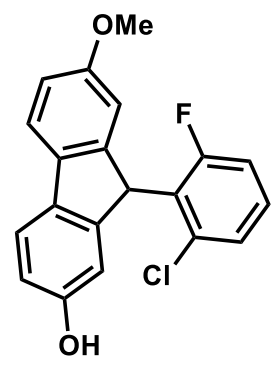

5d

${ }^{13} \mathrm{C}\left\{{ }^{1} \mathrm{H}\right\}$ NMR $\left(125 \mathrm{MHz}, \mathrm{C}_{6} \mathrm{D}_{6}\right)$
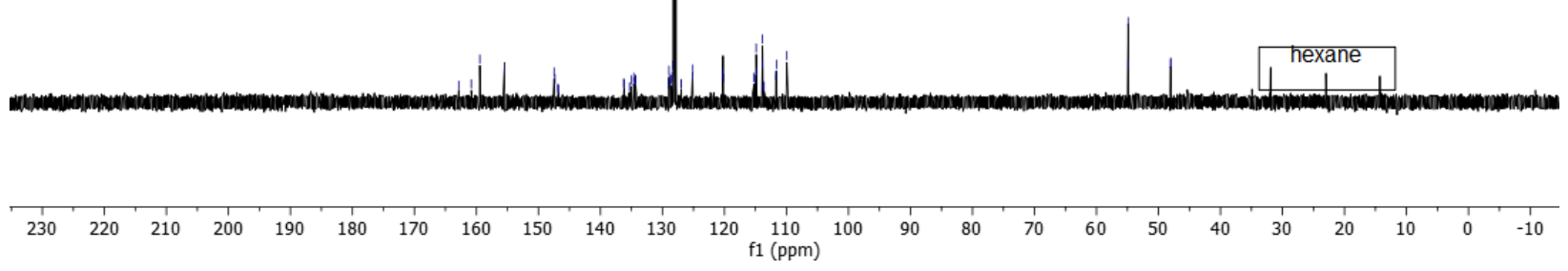

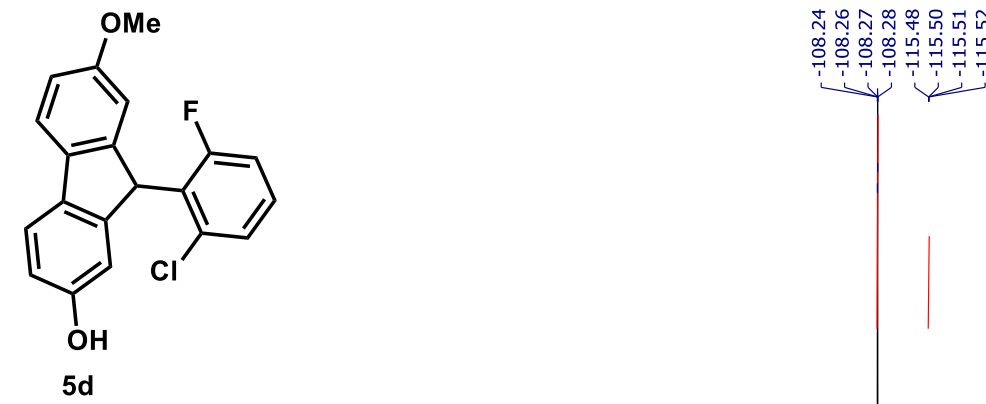

${ }^{19} \mathrm{~F}$ NMR $\left(470 \mathrm{MHz}, \mathrm{C}_{6} \mathrm{D}_{6}\right)$

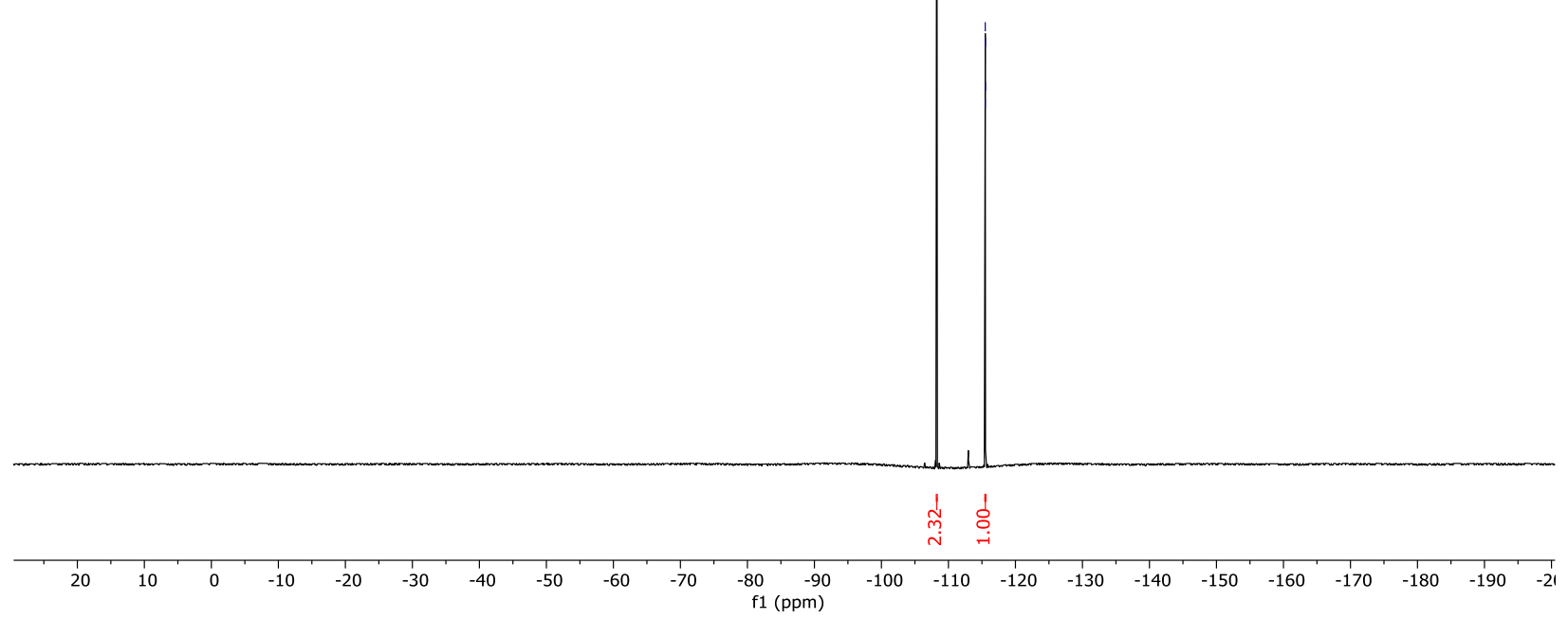



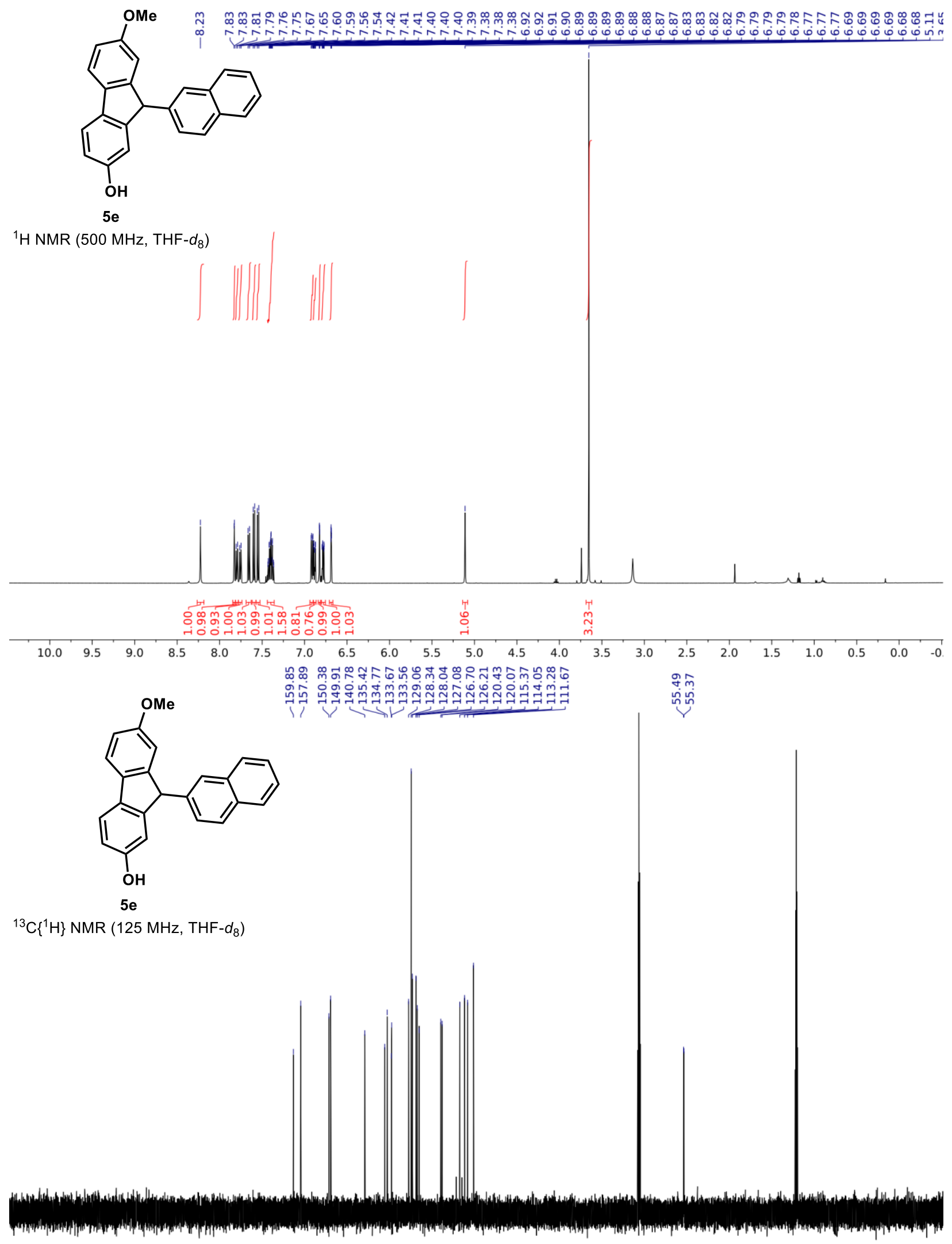

$\begin{array}{lllllllllllllllllllllllll}230 & 220 & 210 & 200 & 190 & 180 & 170 & 160 & 150 & 140 & 130 & 120 & 110 & 100 & 90 & 80 & 70 & 60 & 50 & 40 & 30 & 20 & 10 & 0 & -10\end{array}$ 

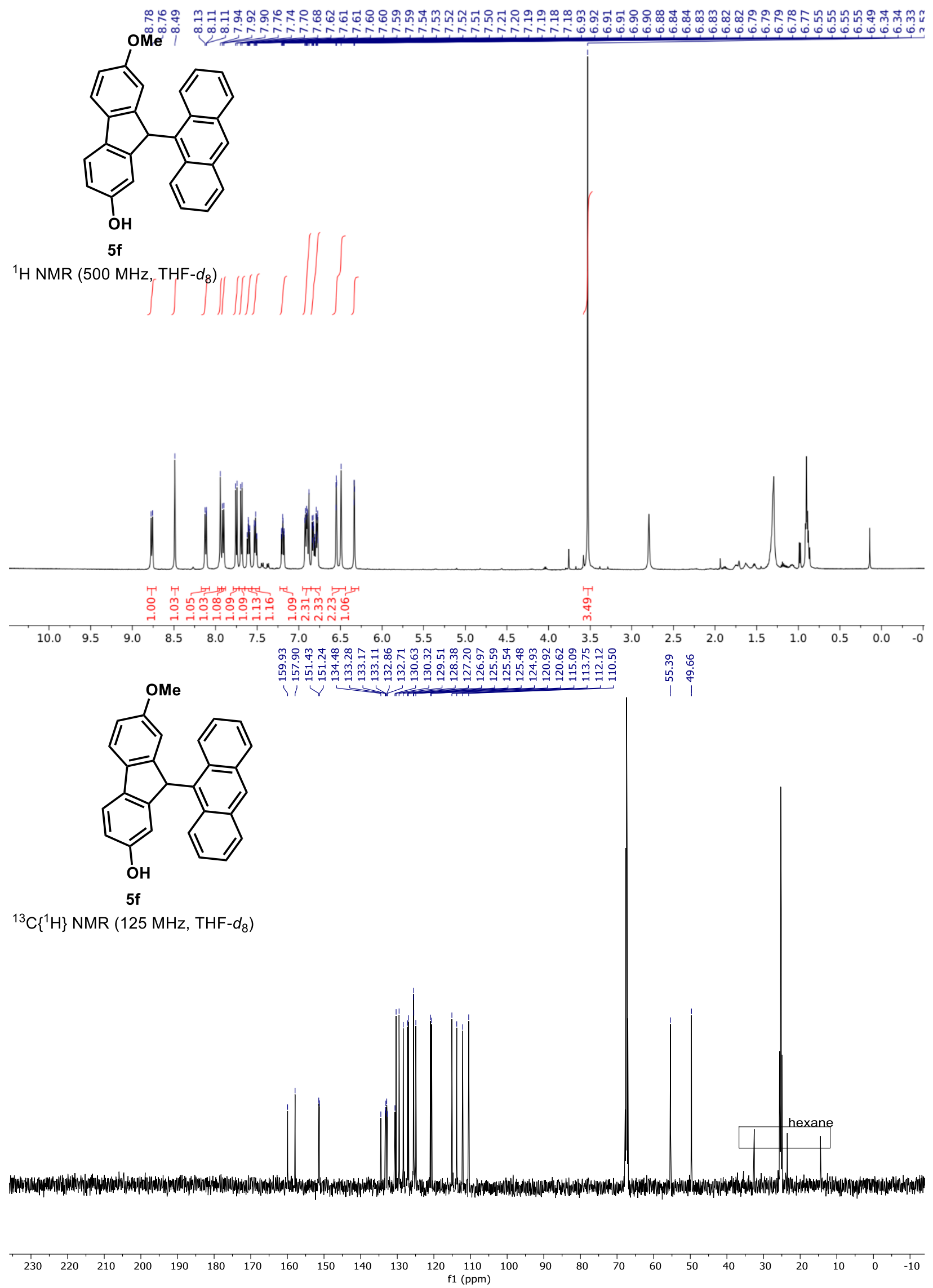


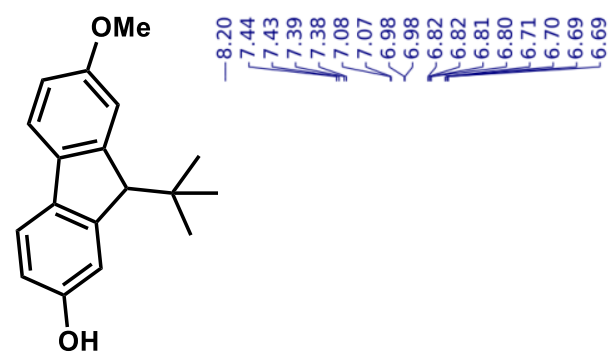



$5 \mathrm{~g}$

${ }^{1} \mathrm{H}$ NMR $\left(500 \mathrm{MHz}, \mathrm{THF}-d_{8}\right)$
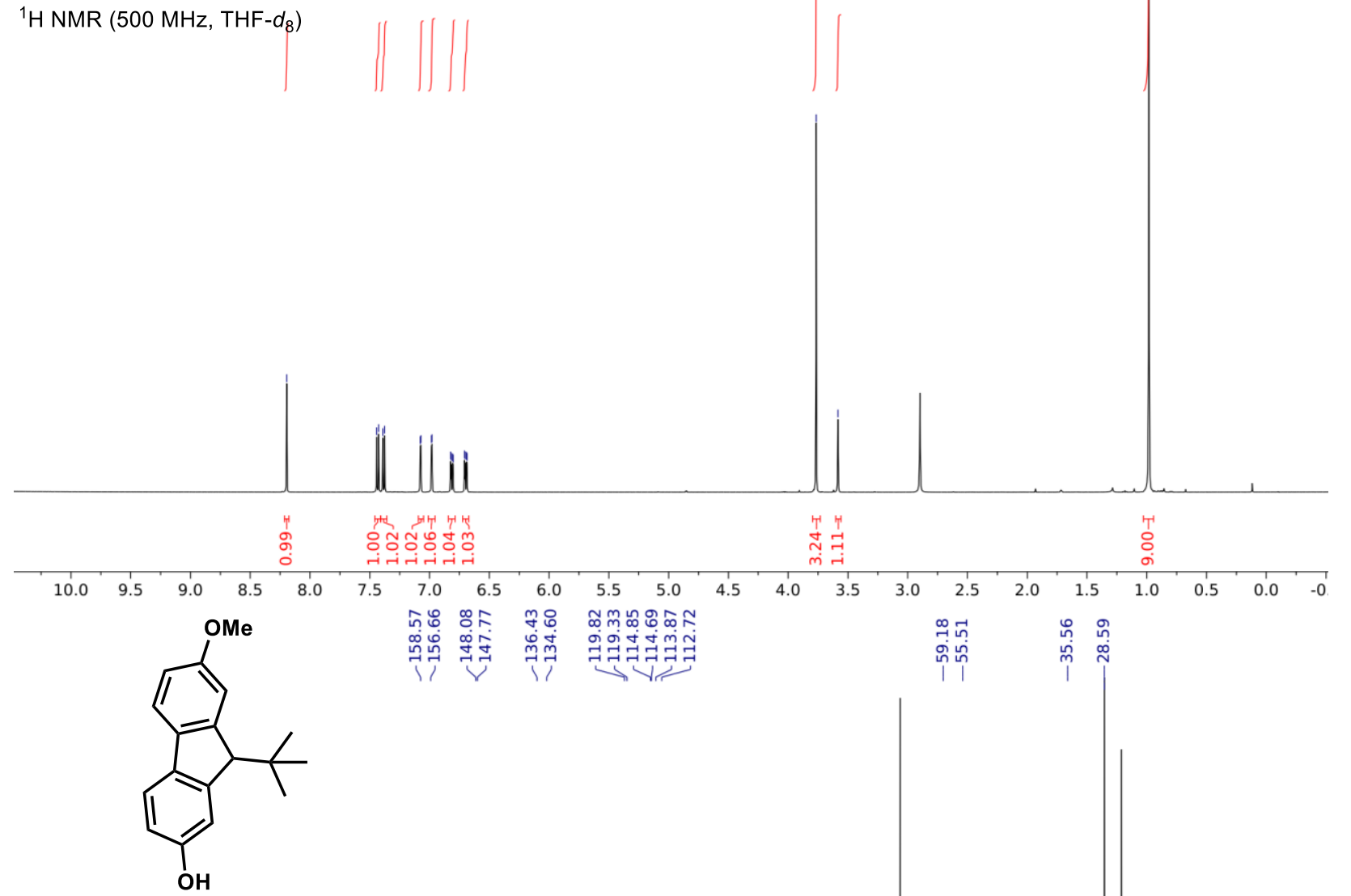

$5 \mathrm{~g}$

${ }^{13} \mathrm{C}\left\{{ }^{1} \mathrm{H}\right\}$ NMR $\left(125 \mathrm{MHz}\right.$, THF- $\left.d_{8}\right)$

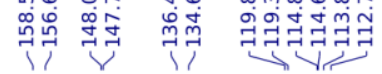




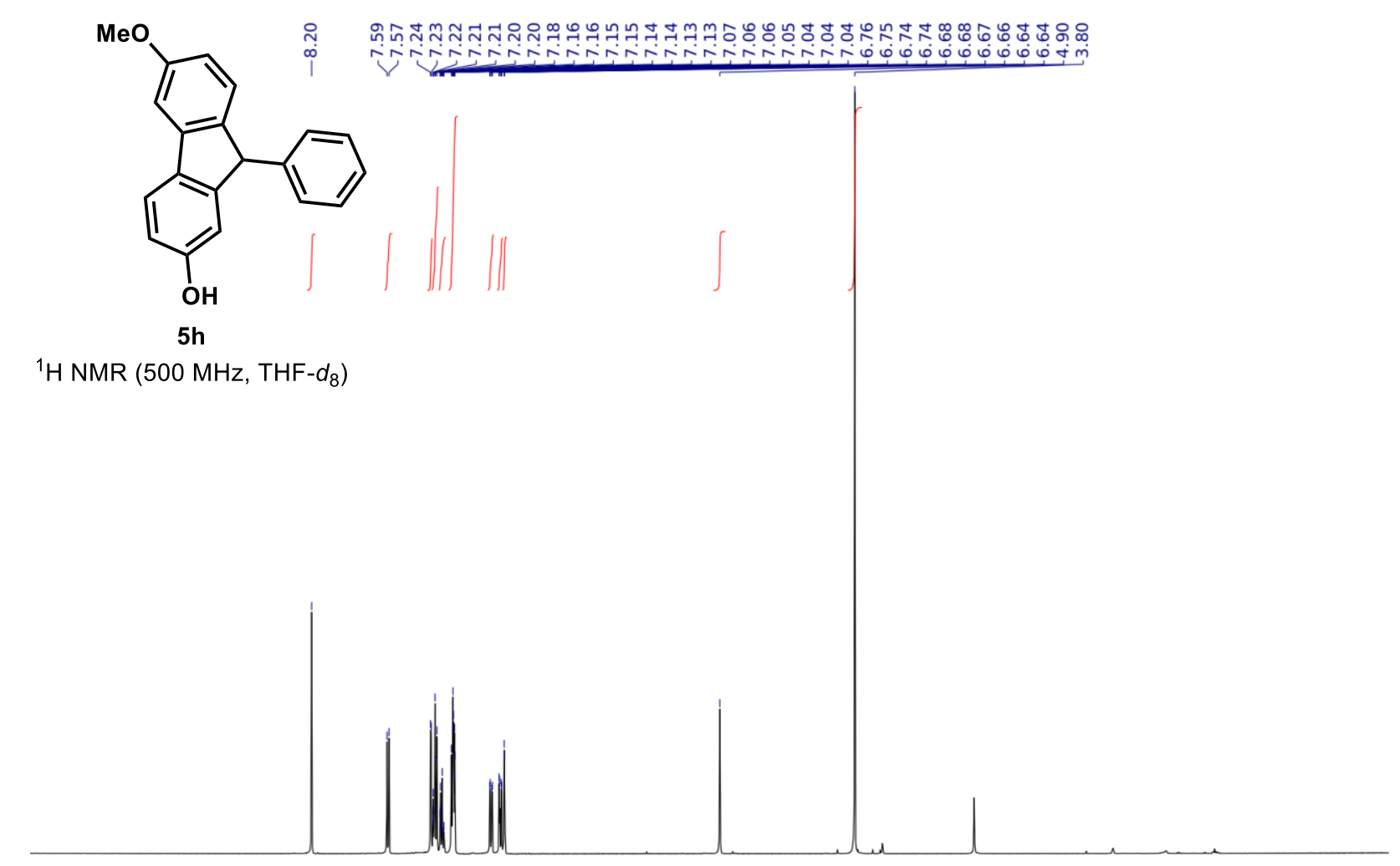

${ }^{1} \mathrm{H}$ NMR $\left(500 \mathrm{MHz}\right.$, THF- $\left.d_{8}\right)$

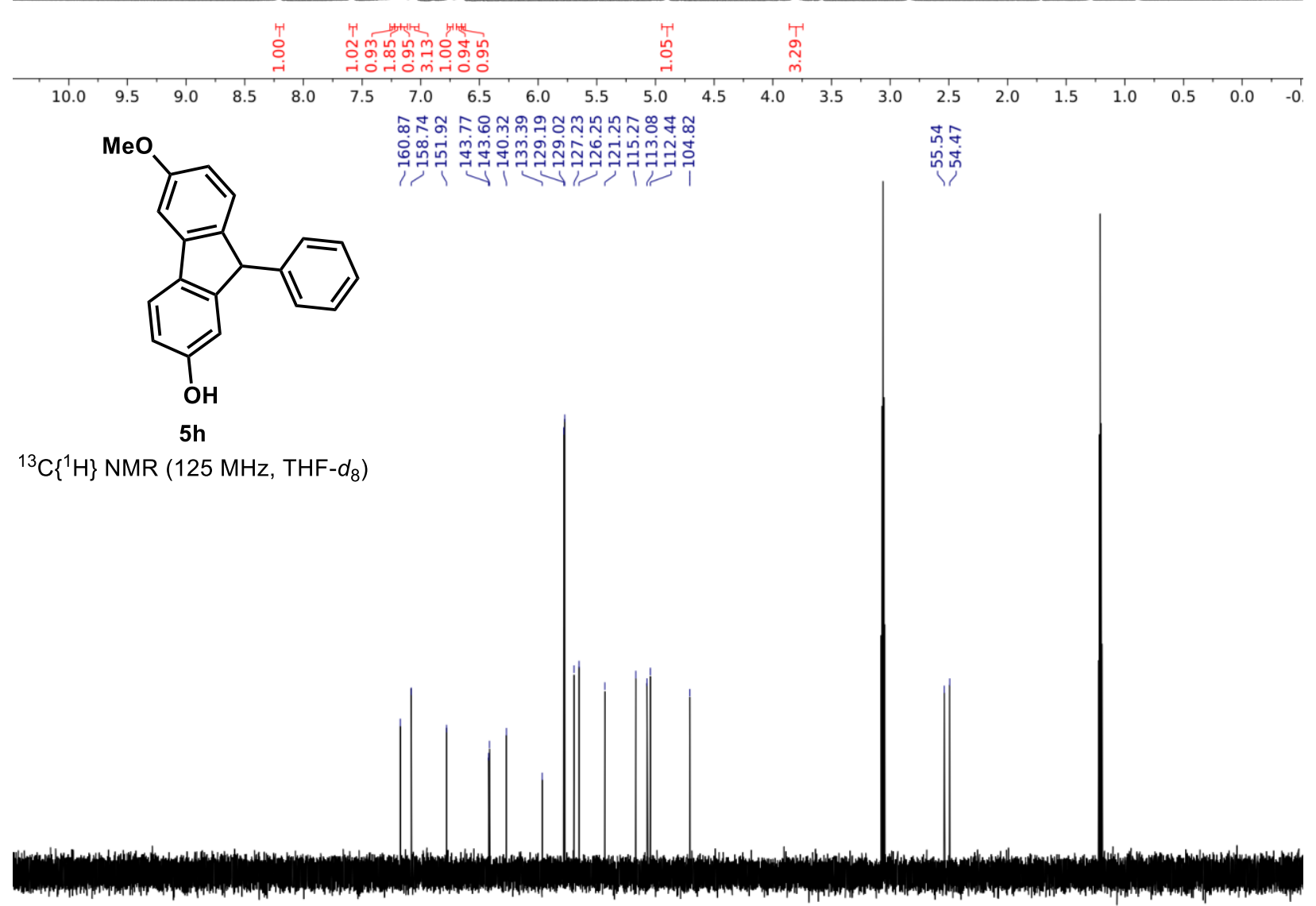

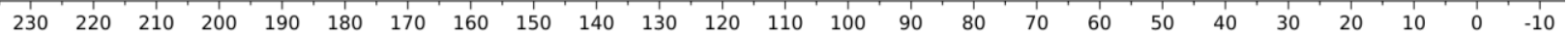




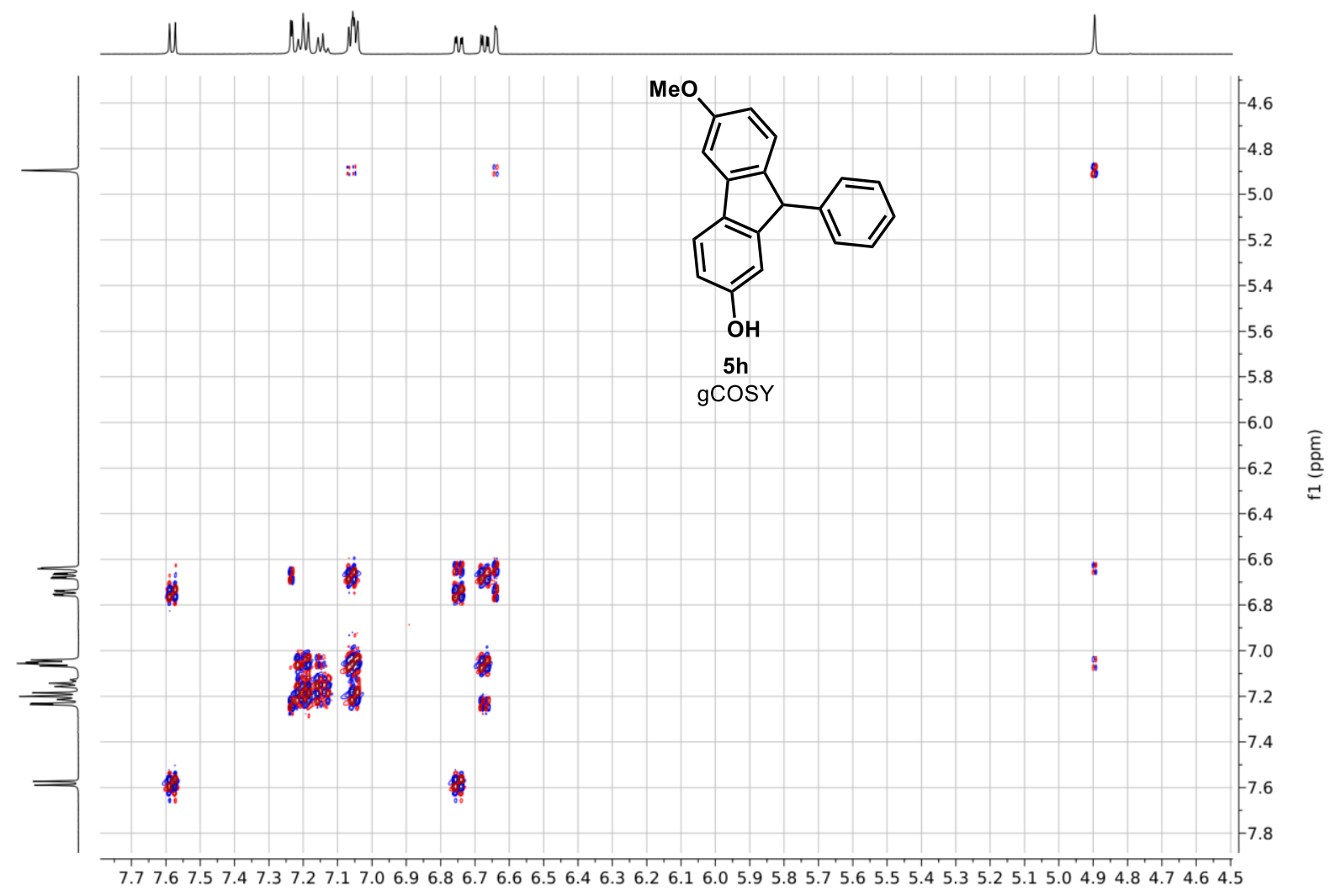




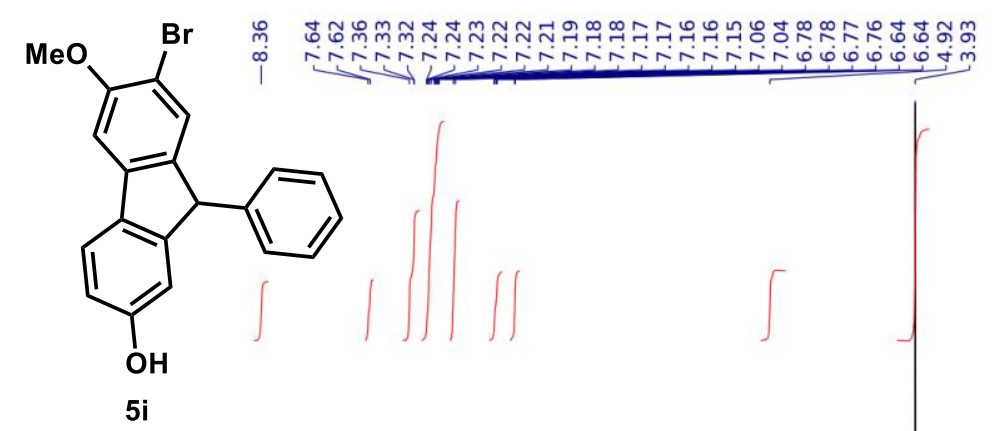

${ }^{1} \mathrm{H}$ NMR $\left(500 \mathrm{MHz}, \mathrm{THF}-d_{8}\right)$
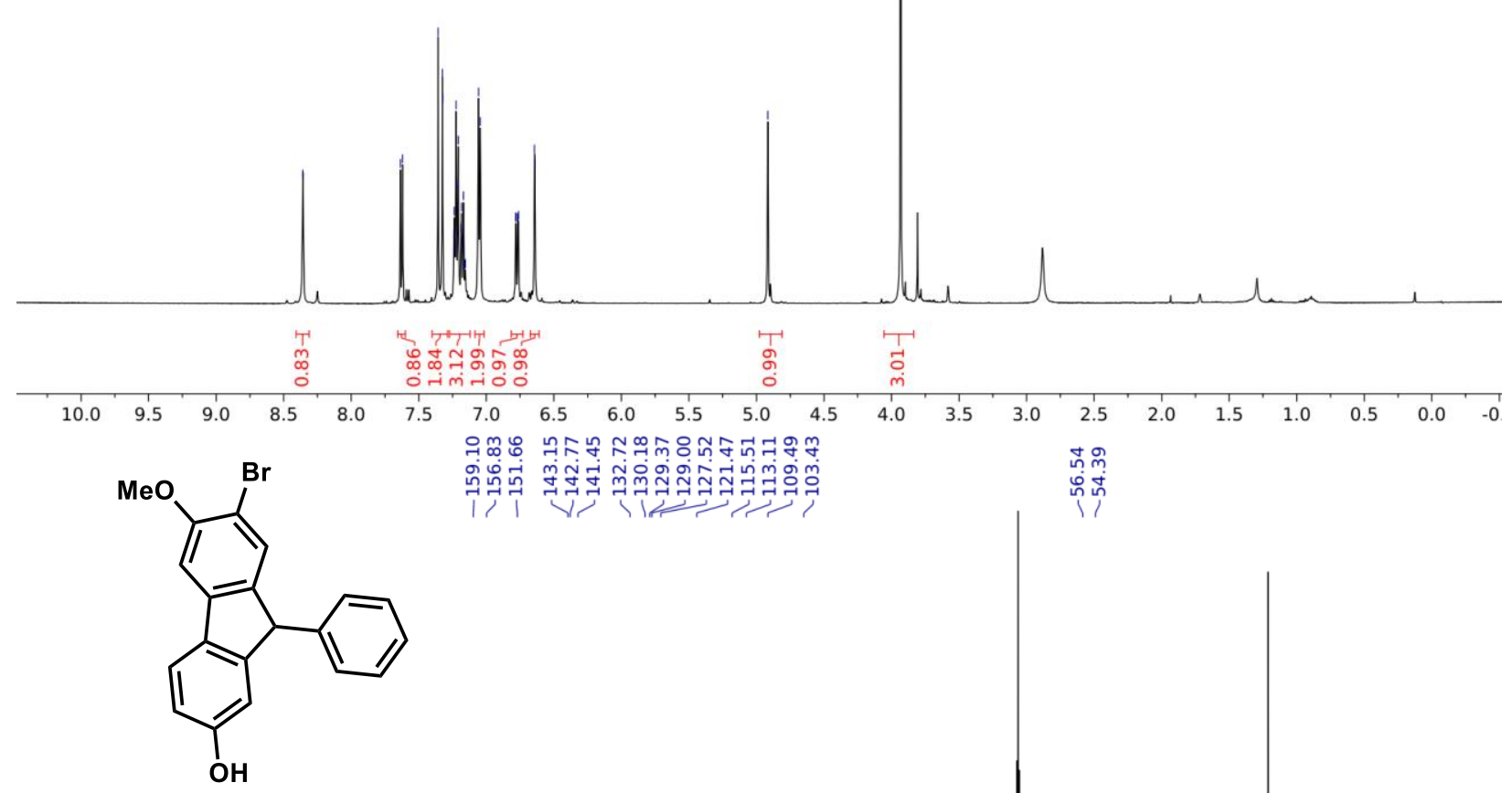

$5 \mathbf{i}$

${ }^{13} \mathrm{C}\left\{{ }^{1} \mathrm{H}\right\}$ NMR $\left(125 \mathrm{MHz}\right.$, THF- $\left.d_{8}\right)$

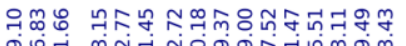

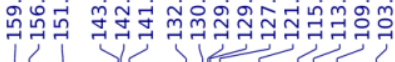

กั้

น้ำ
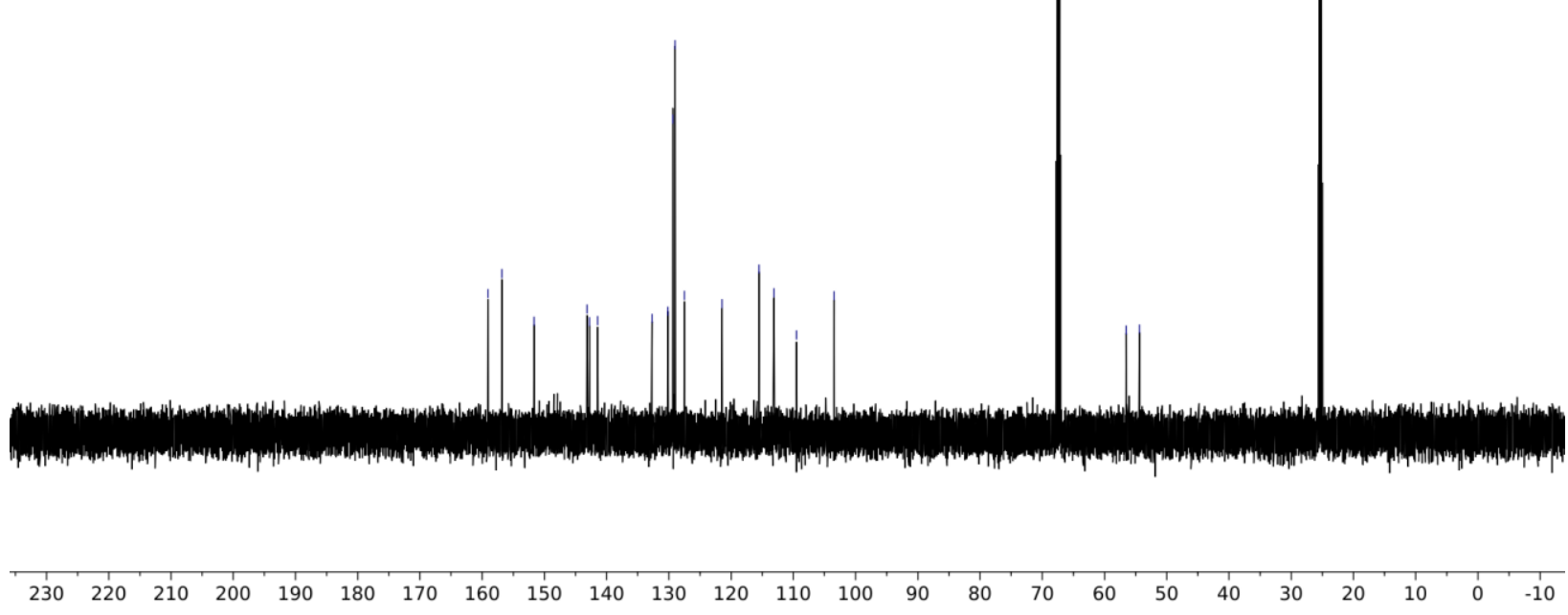


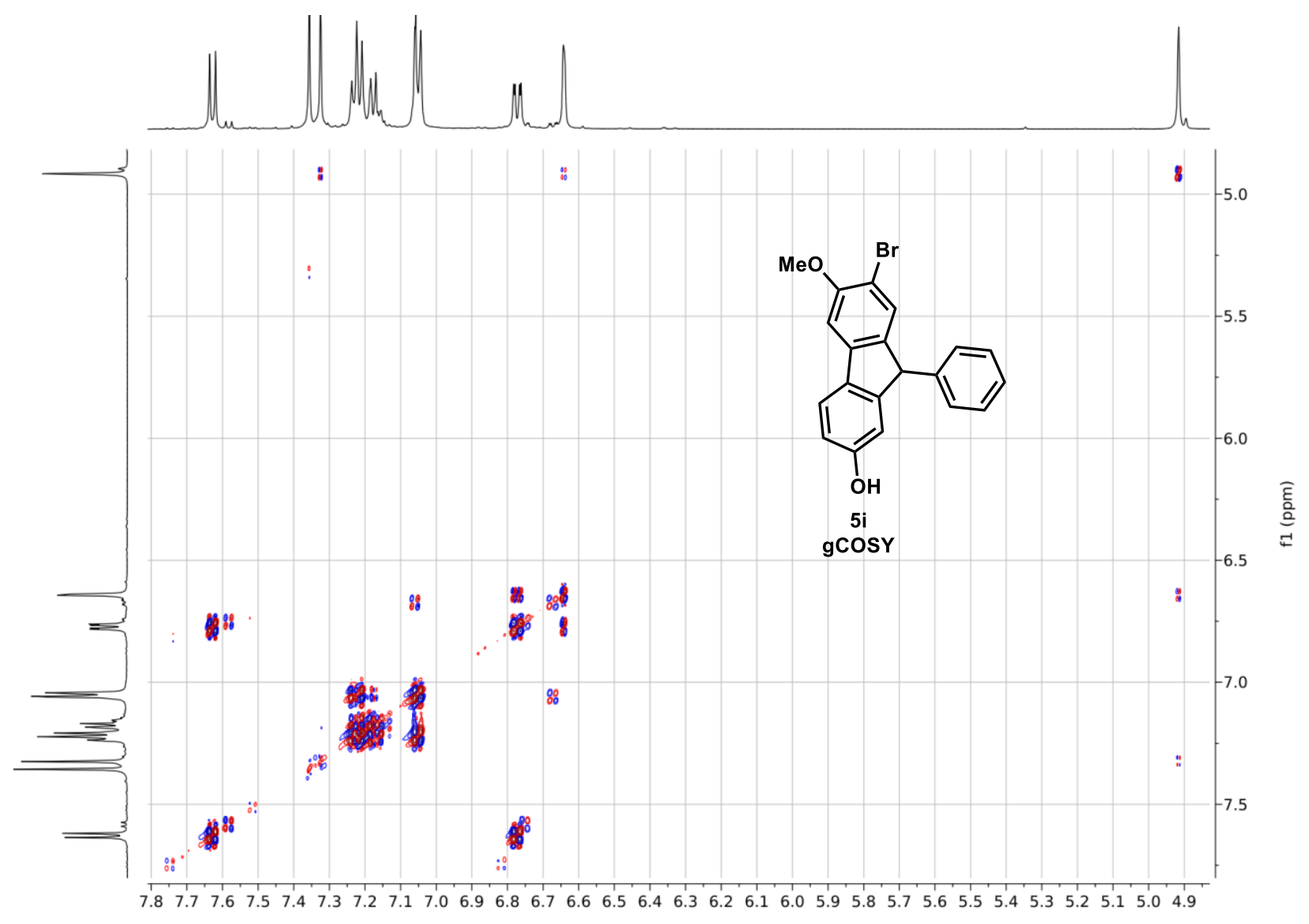




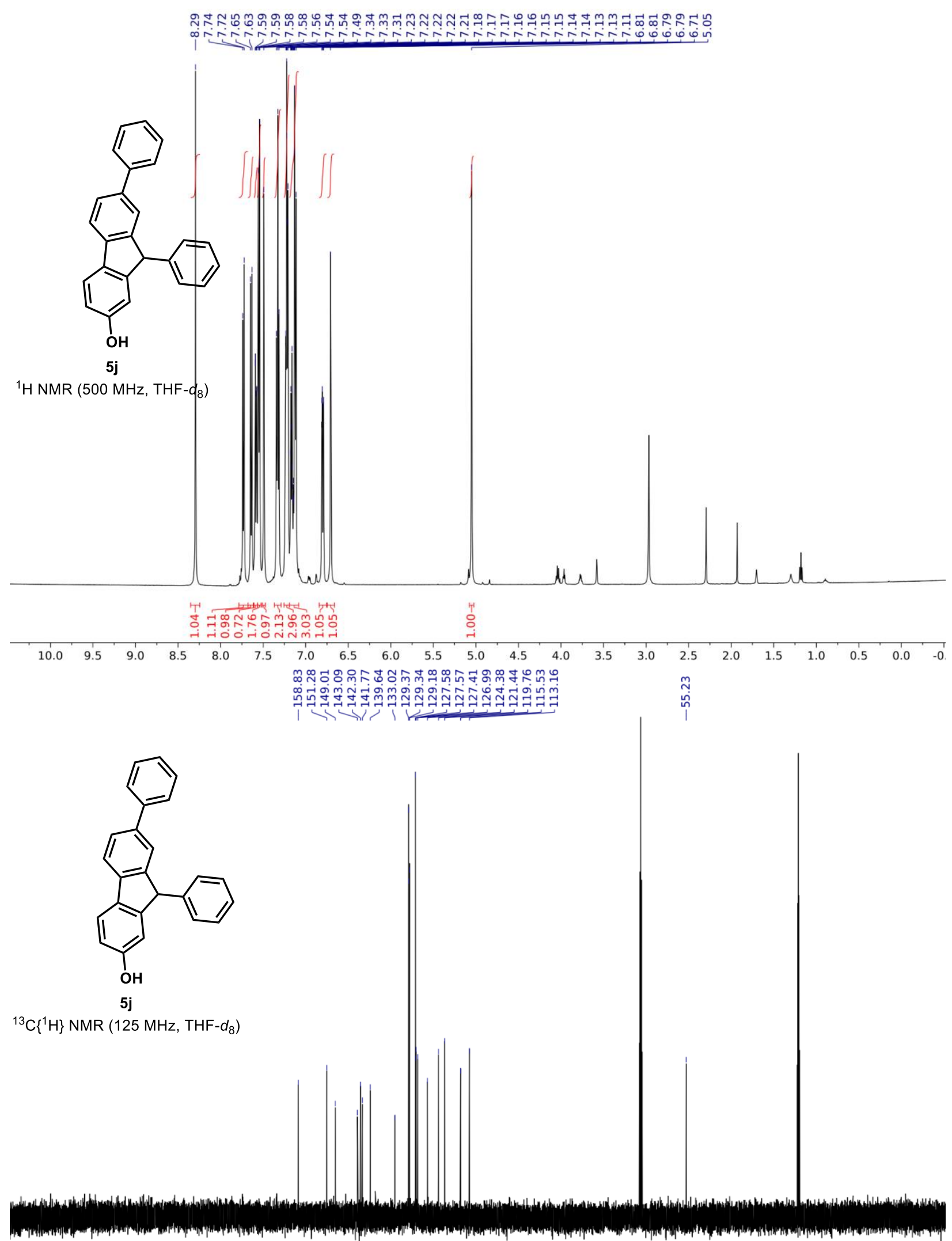

$\begin{array}{lllllllllllllllllllllllllllllllllll}1 & 230 & 220 & 210 & 200 & 190 & 180 & 170 & 160 & 150 & 140 & 130 & 120 & 110 & 100 & 90 & 80 & 70 & 60 & 50 & 40 & 30 & 20 & 10 & 0 & -10\end{array}$ 


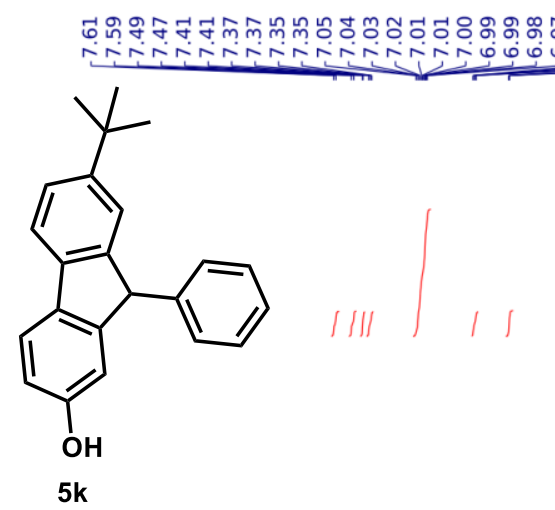

${ }^{1} \mathrm{H}$ NMR $\left(500 \mathrm{MHz}, \mathrm{C}_{6} \mathrm{D}_{6}\right)$

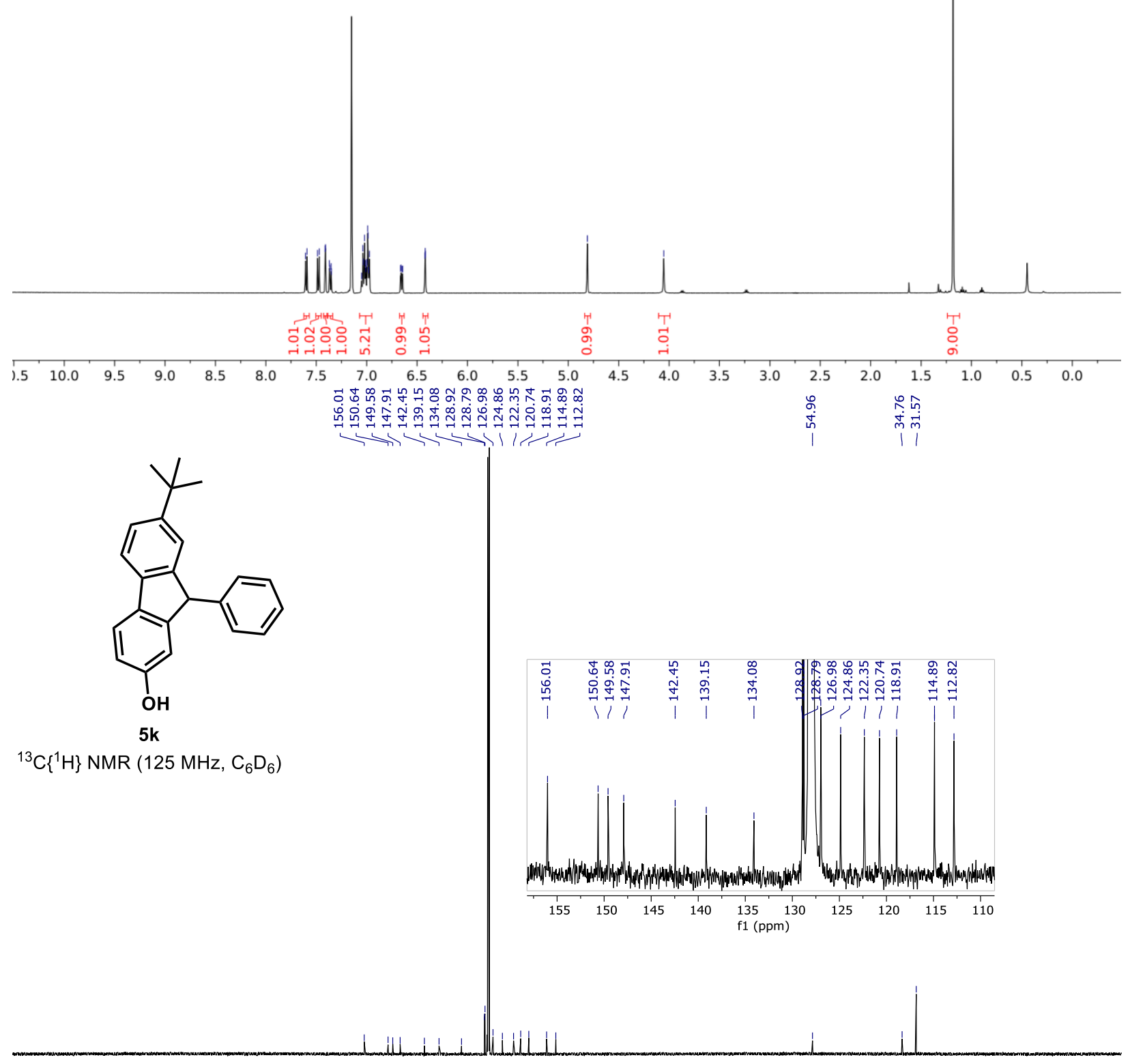

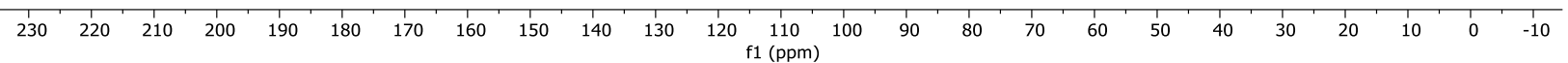




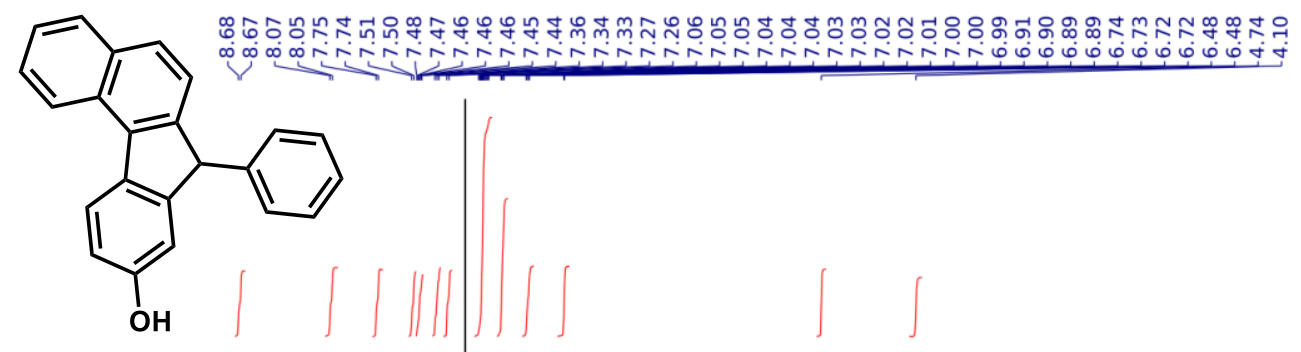

51

${ }^{1} \mathrm{H}$ NMR $\left(500 \mathrm{MHz}, \mathrm{C}_{6} \mathrm{D}_{6}\right)$

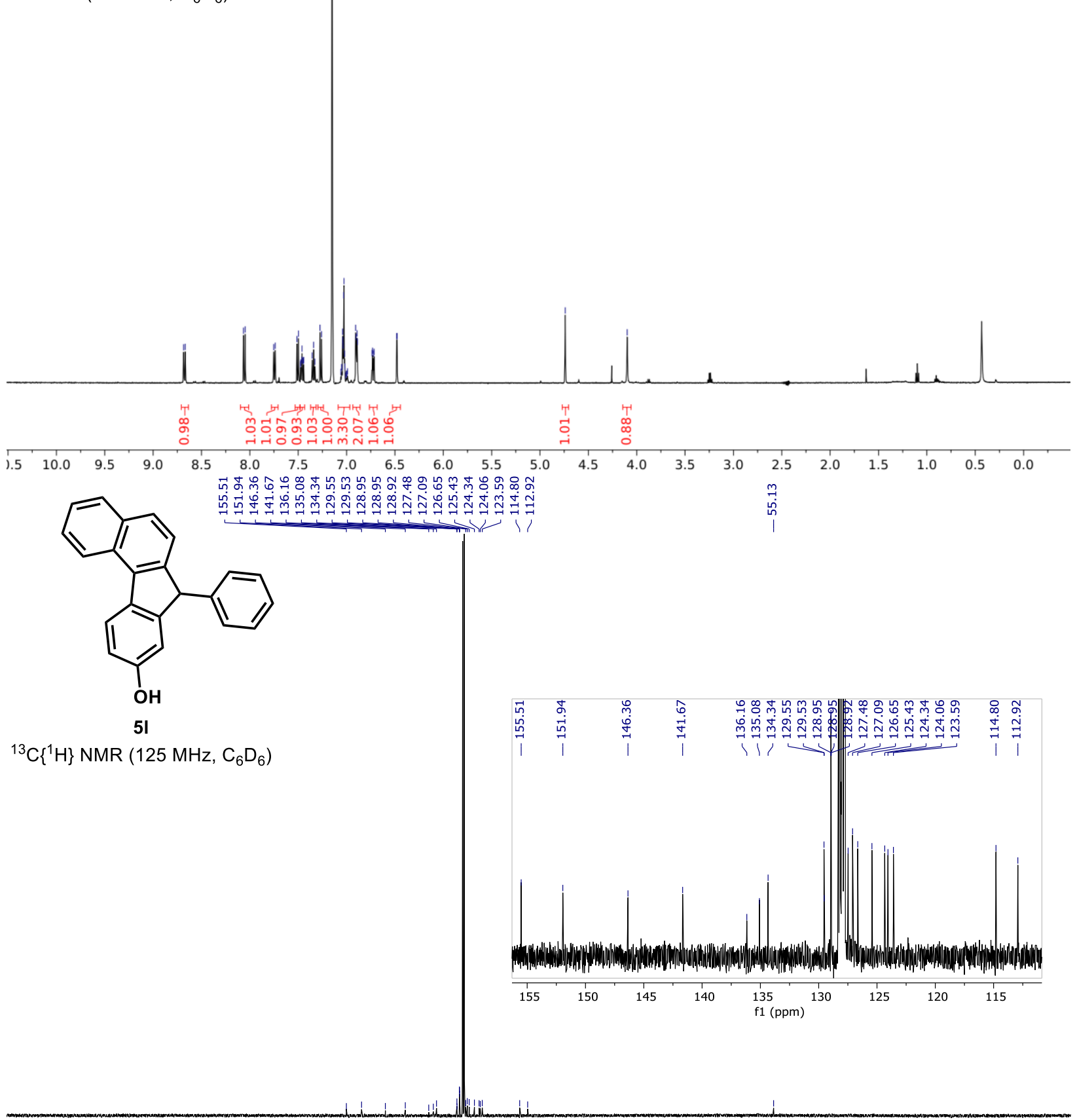

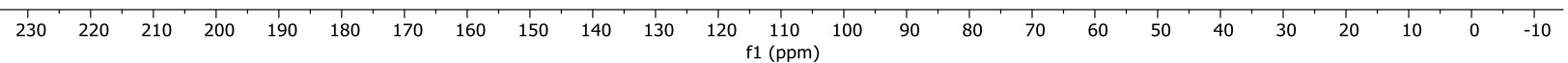




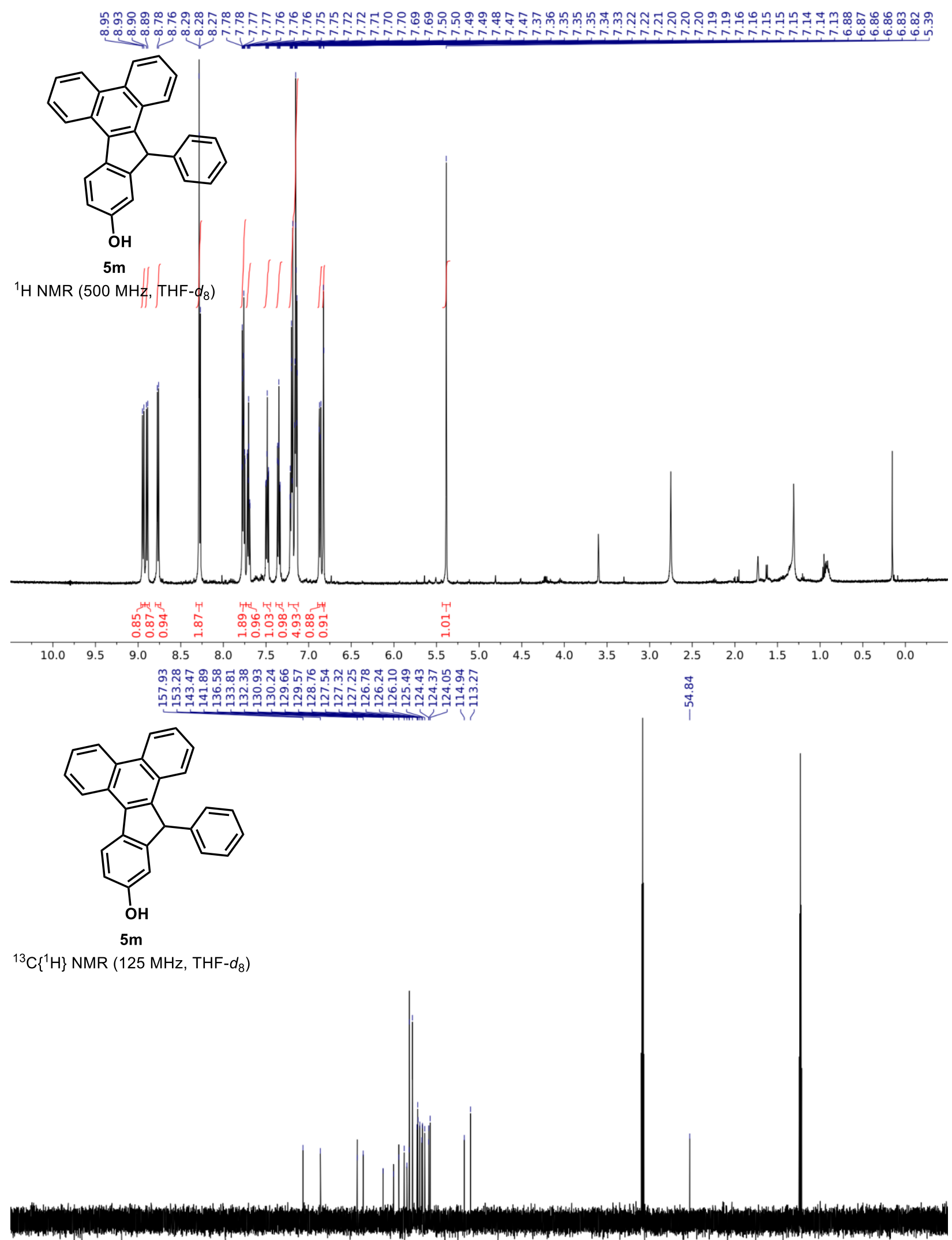

$\begin{array}{lllllllllllllllllllllllll}230 & 220 & 210 & 200 & 190 & 180 & 170 & 160 & 150 & 140 & 130 & 120 & 110 & 100 & 90 & 80 & 70 & 60 & 50 & 40 & 30 & 20 & 10 & 0 & -10\end{array}$ 

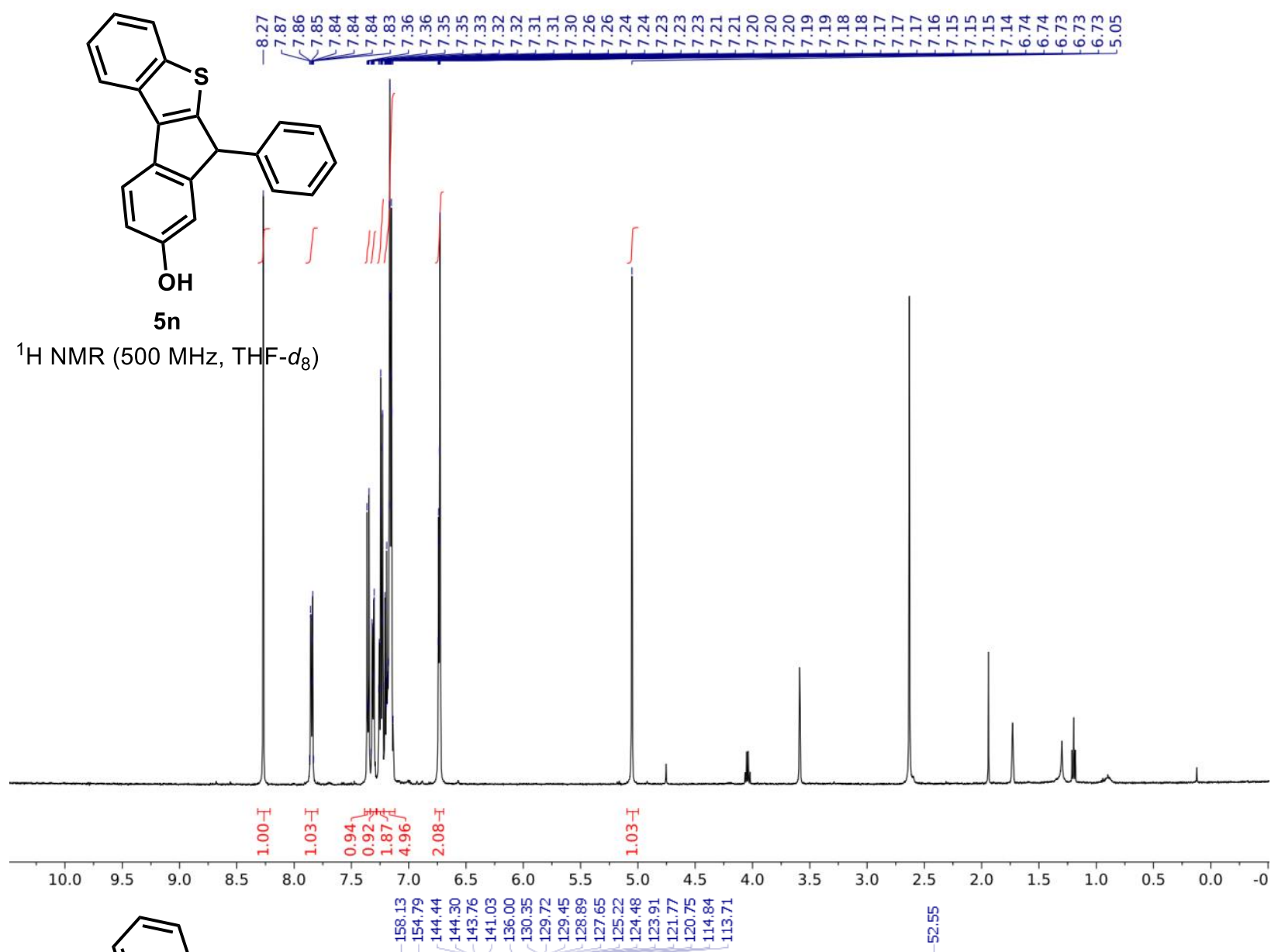<smiles>Oc1ccc2c(c1)C(c1ccccc1)c1sc3ccccc3c1-2</smiles>

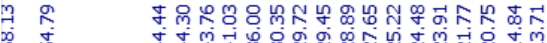
$5 n$

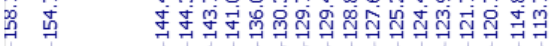

${ }^{13} \mathrm{C}\left\{{ }^{1} \mathrm{H}\right\}$ NMR $\left(125 \mathrm{MHz}\right.$, THF- $\left.d_{8}\right)$
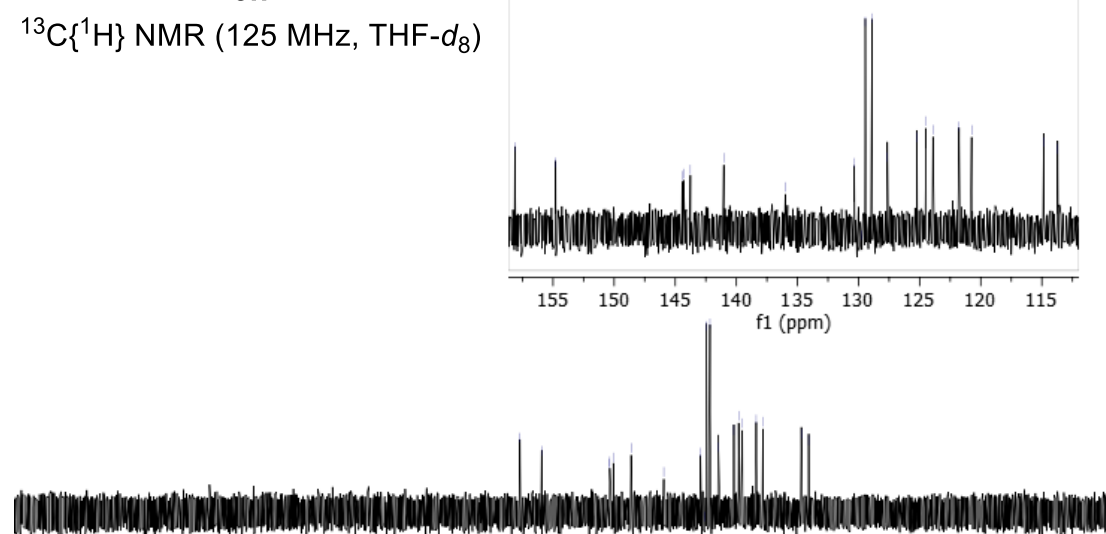

$\begin{array}{llllllllllllllllllllllllllll}230 & 220 & 210 & 200 & 190 & 180 & 170 & 160 & 150 & 140 & 130 & 120 & 110 & 100 & 90 & 80 & 70 & 60 & 50 & 40 & 30 & 20 & 10 & 0 & -10\end{array}$ 

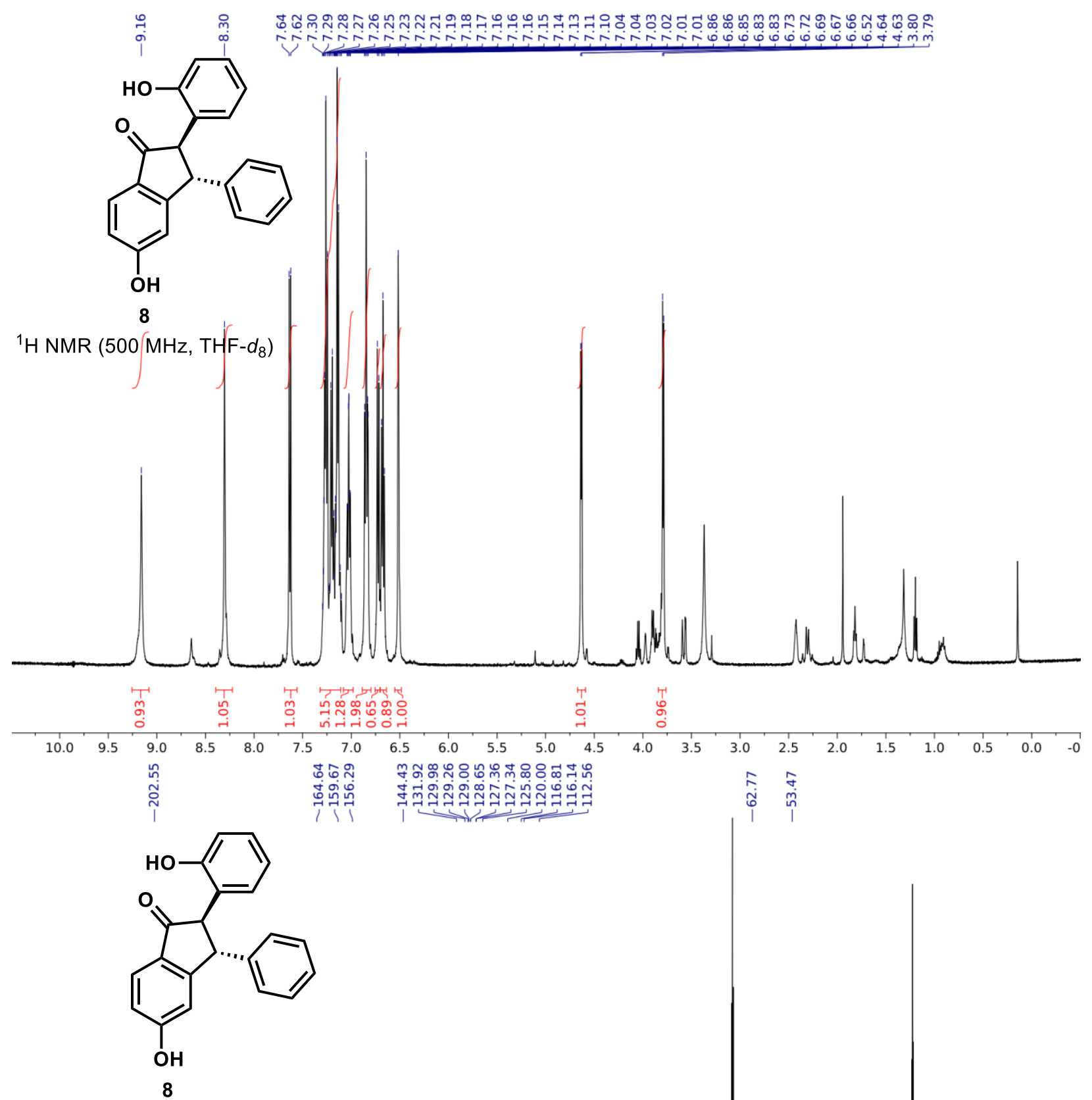

${ }^{13} \mathrm{C}\left\{{ }^{1} \mathrm{H}\right\}$ NMR $\left(125 \mathrm{MHz}\right.$, THF- $\left.d_{8}\right)$

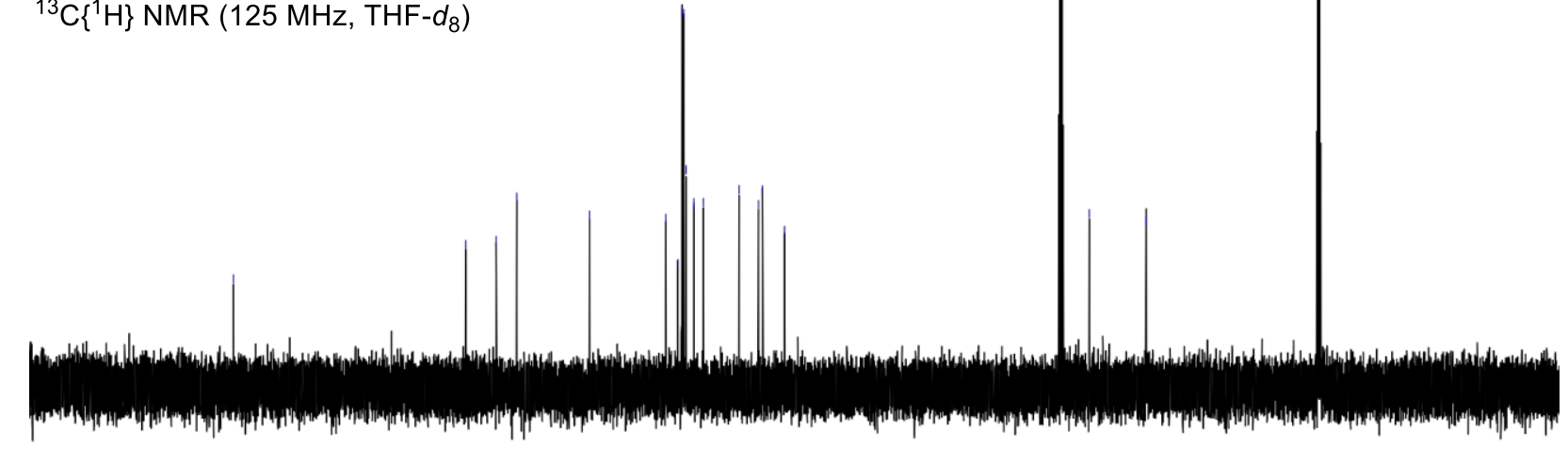

$\begin{array}{llllllllllllllllllllllllll}230 & 220 & 210 & 200 & 190 & 180 & 170 & 160 & 150 & 140 & 130 & 120 & 110 & 100 & 90 & 80 & 70 & 60 & 50 & 40 & 30 & 20 & 10 & 0 & -10\end{array}$ 

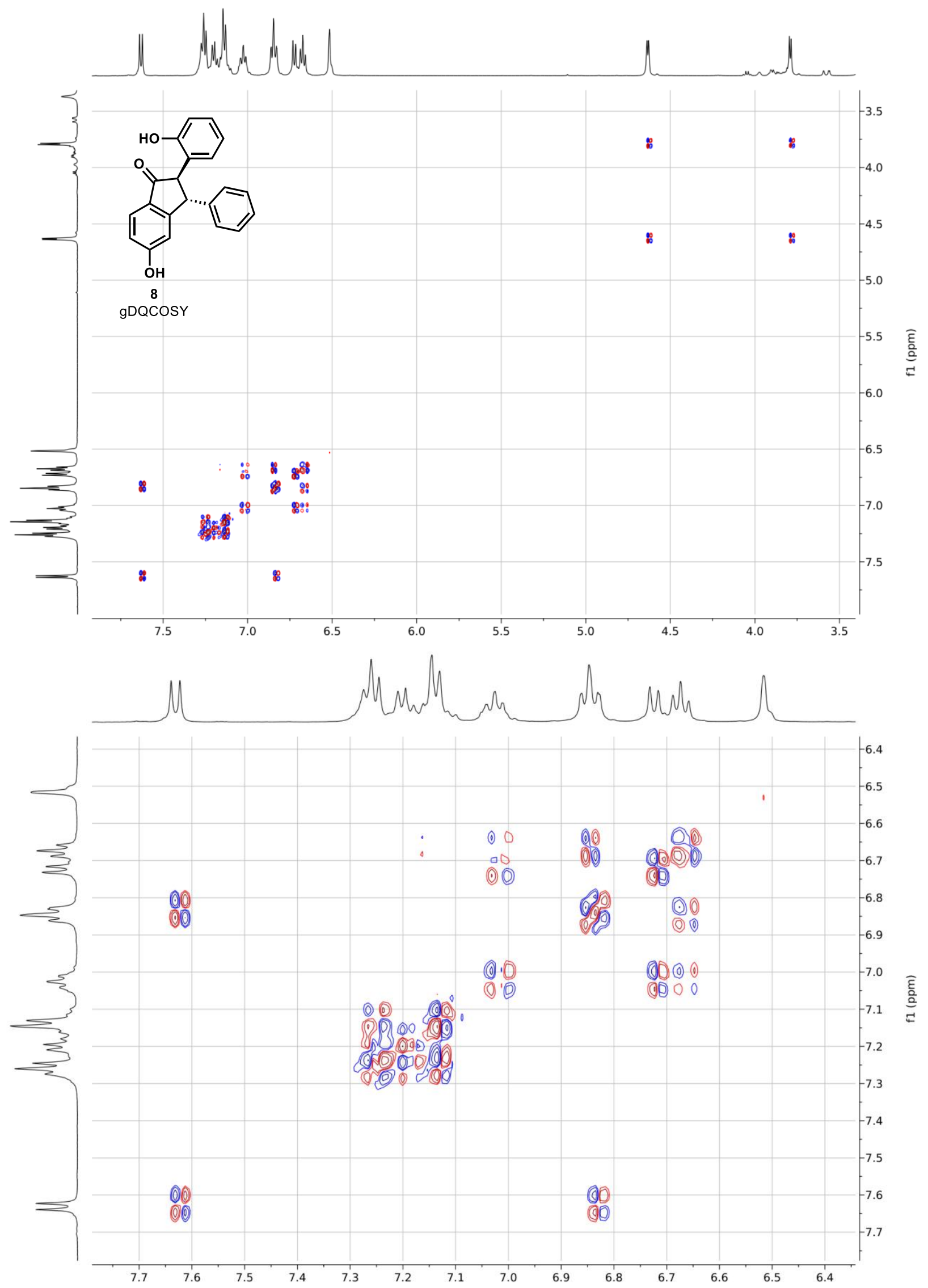


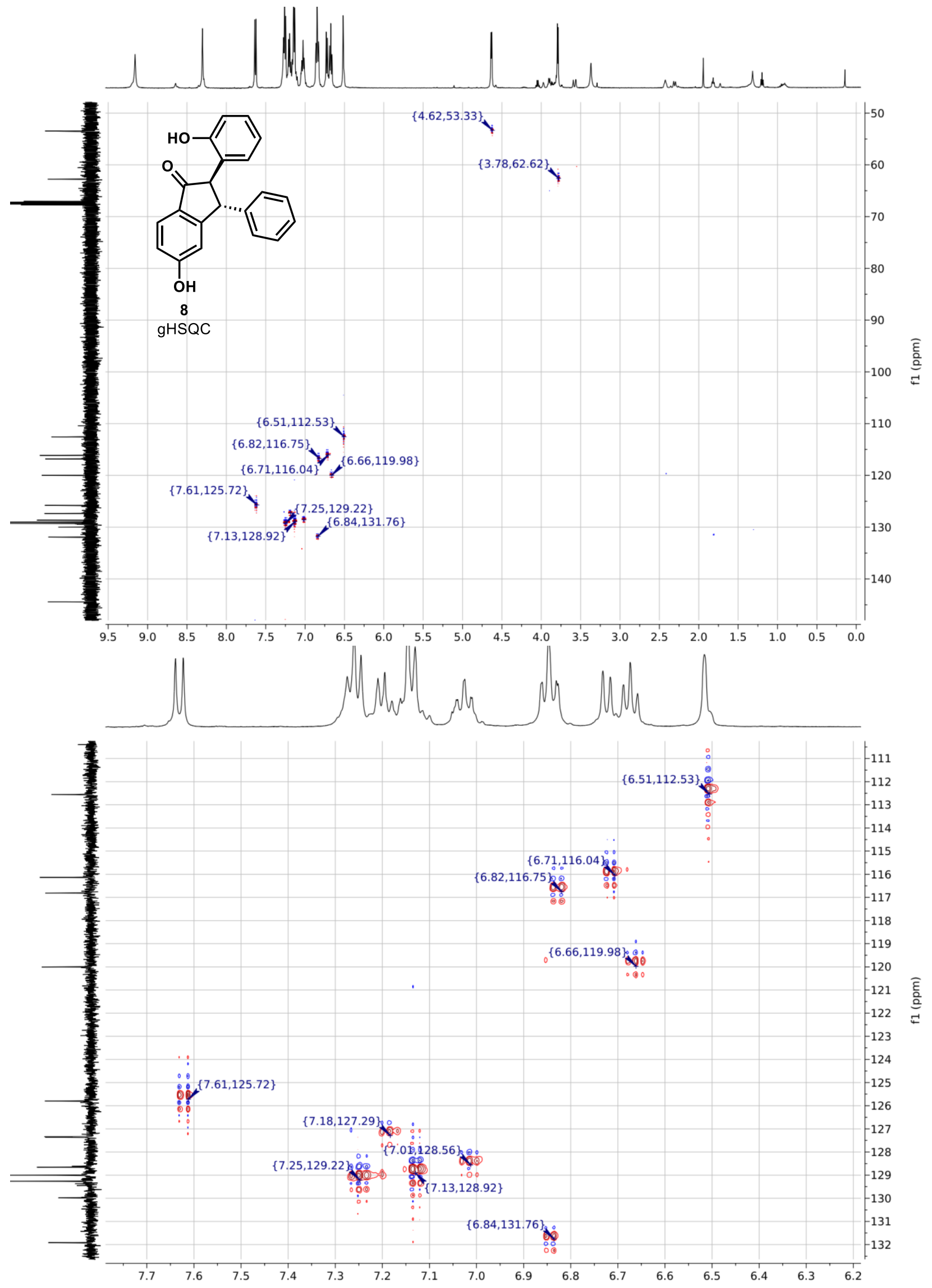



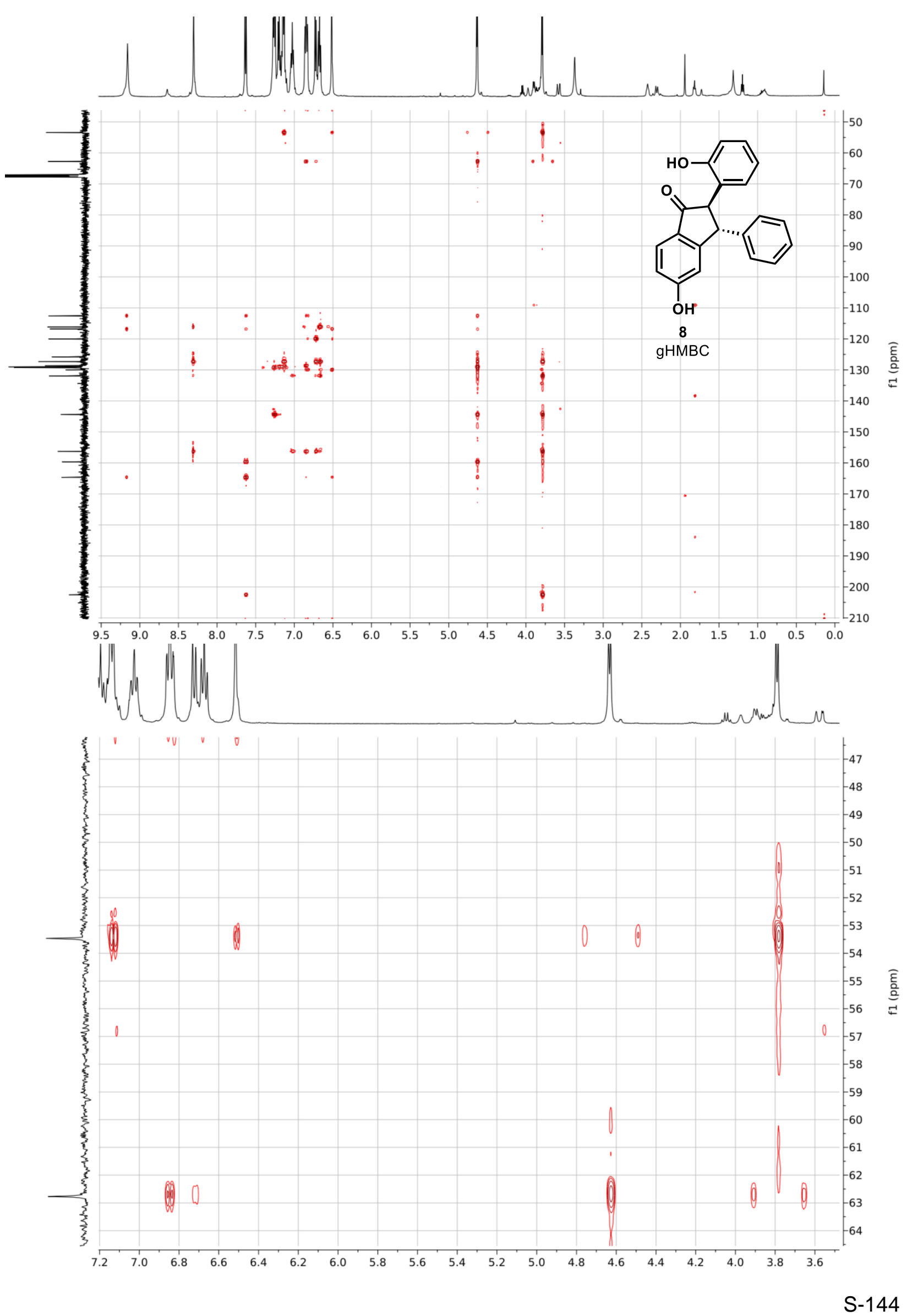


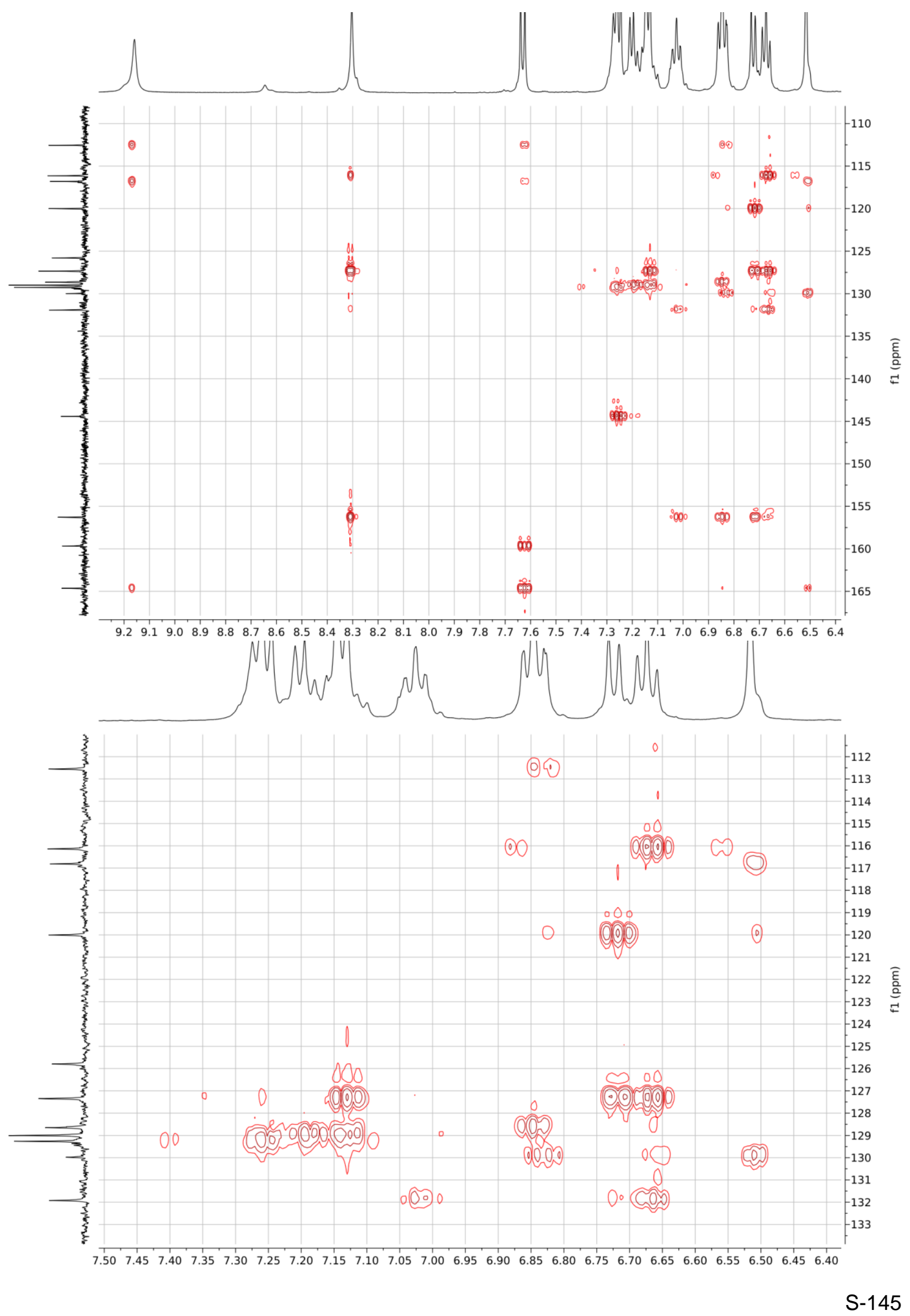




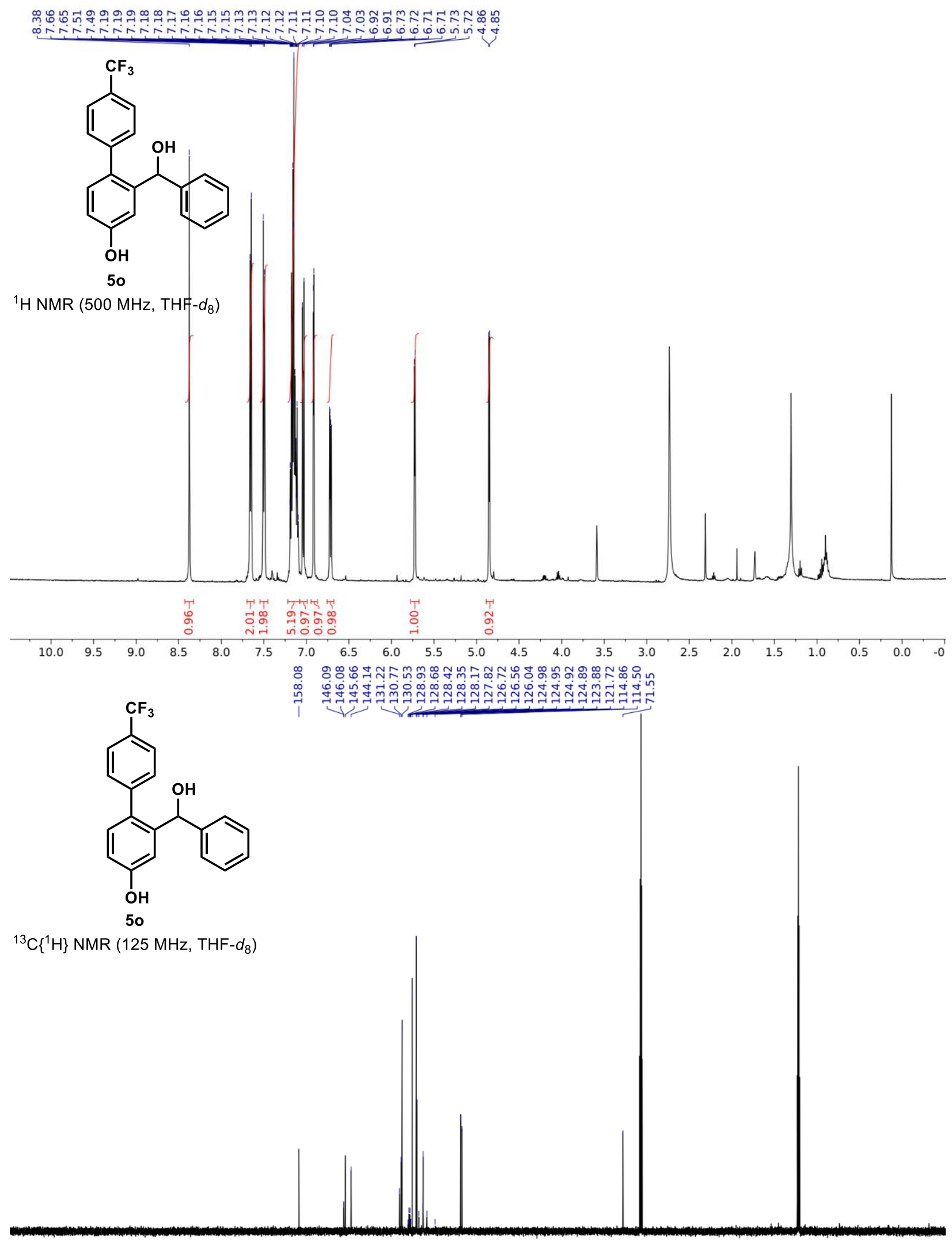

$\begin{array}{lllllllllllllllllllllllllllllllllll}230 & 220 & 210 & 200 & 190 & 180 & 170 & 160 & 150 & 140 & 130 & 120 & 110 & 100 & 90 & 80 & 70 & 60 & 50 & 40 & 30 & 20 & 10 & 0 & -10\end{array}$ 


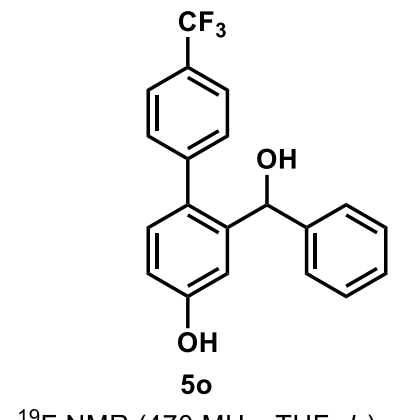

น

亗

${ }^{19} \mathrm{~F}$ NMR $\left(470 \mathrm{MHz}, \mathrm{THF}-\mathrm{d}_{8}\right)$

$\begin{array}{lllllllllllllllllllllll}20 & 10 & 0 & -10 & -20 & -30 & -40 & -50 & -60 & -70 & -80 & -90 & -100 & -110 & -120 & -130 & -140 & -150 & -160 & -170 & -180 & -190 & -26\end{array}$




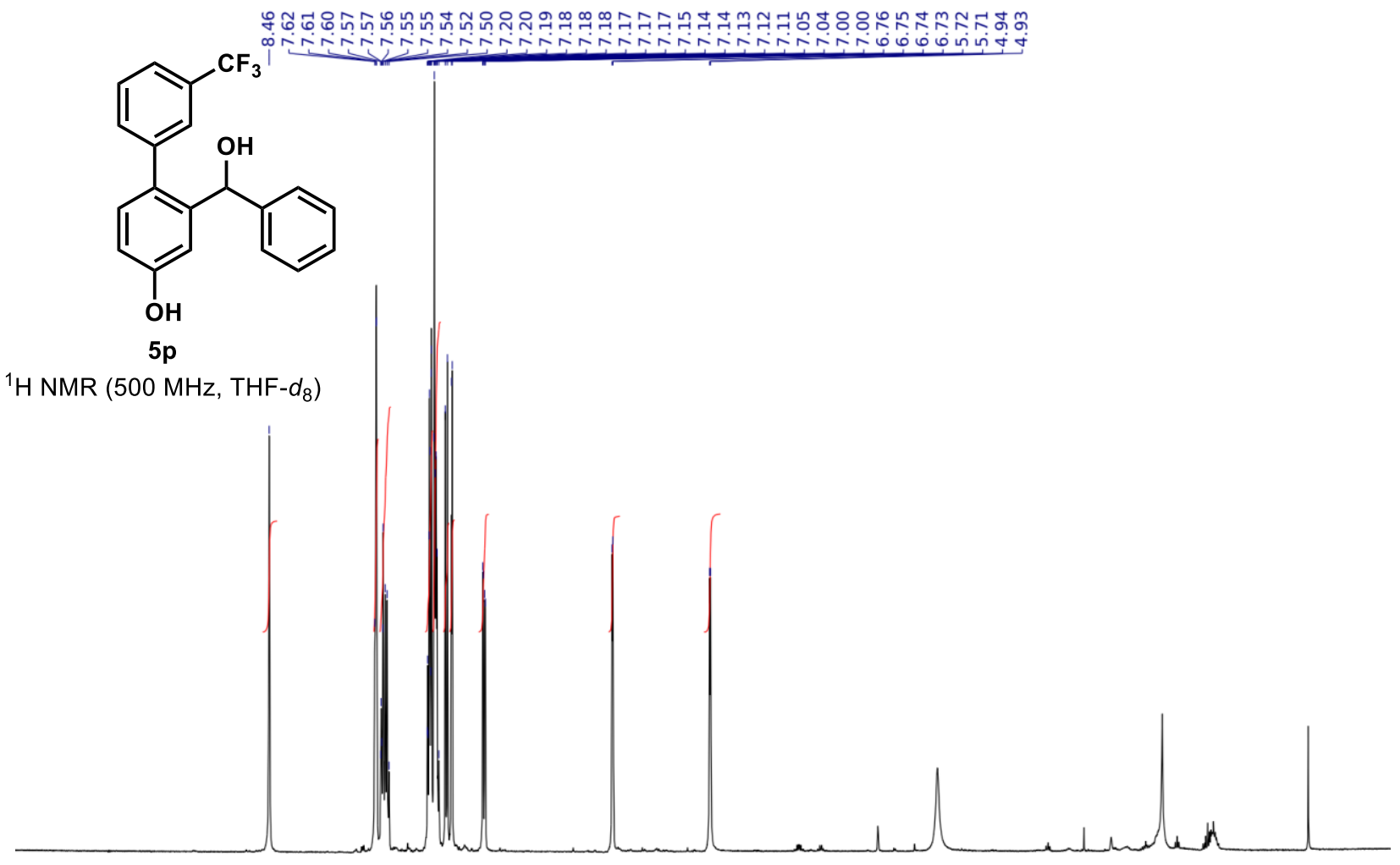

${ }^{1} \mathrm{H}$ NMR $\left(500 \mathrm{MHz}, \mathrm{THF}-d_{8}\right)$
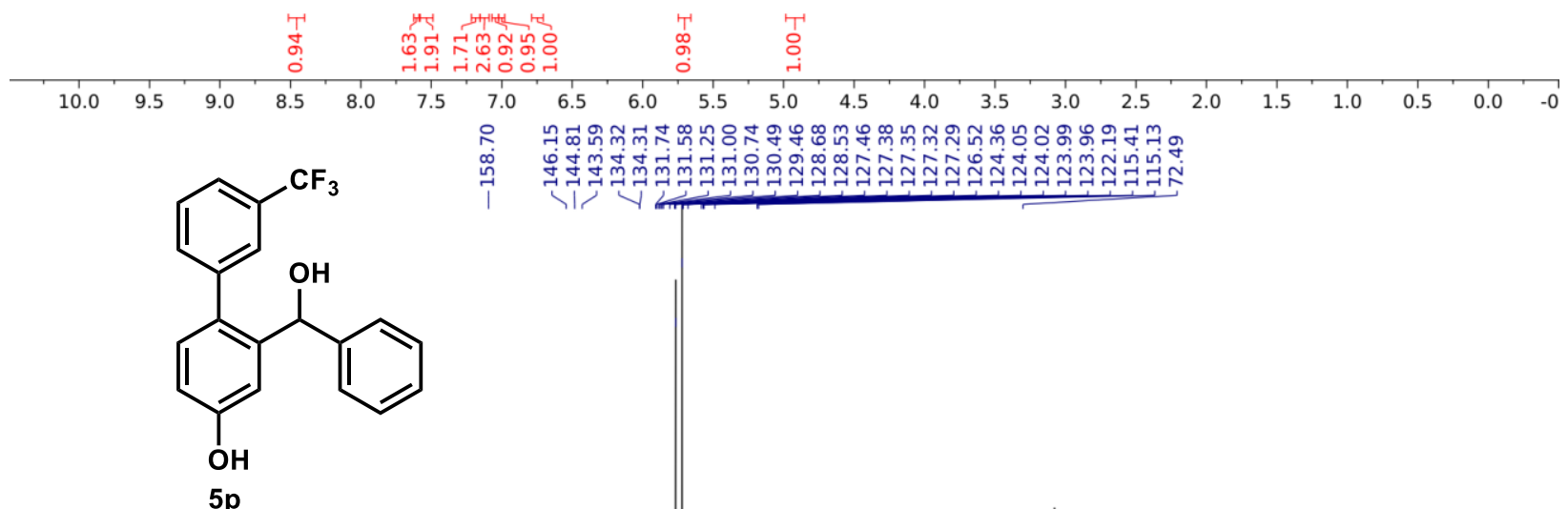

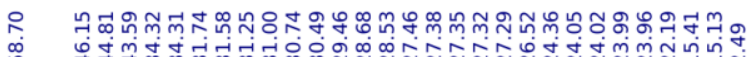

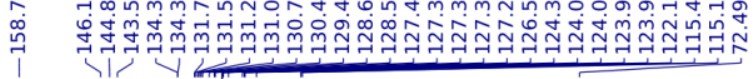

$5 p$

${ }^{13} \mathrm{C}\left\{{ }^{1} \mathrm{H}\right\}$ NMR $\left(125 \mathrm{MHz}\right.$, THF- $\left.d_{8}\right)$

$\begin{array}{llllllllllllllllllllllllll}230 & 220 & 210 & 200 & 190 & 180 & 170 & 160 & 150 & 140 & 130 & 120 & 110 & 100 & 90 & 80 & 70 & 60 & 50 & 40 & 30 & 20 & 10 & 0 & -10\end{array}$ 


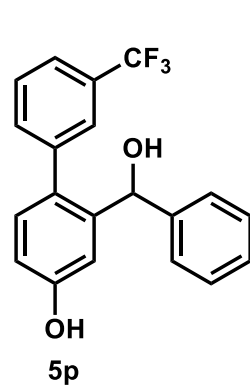

${ }^{19} \mathrm{~F}$ NMR (470 MHz, THF- $\left.d_{8}\right)$

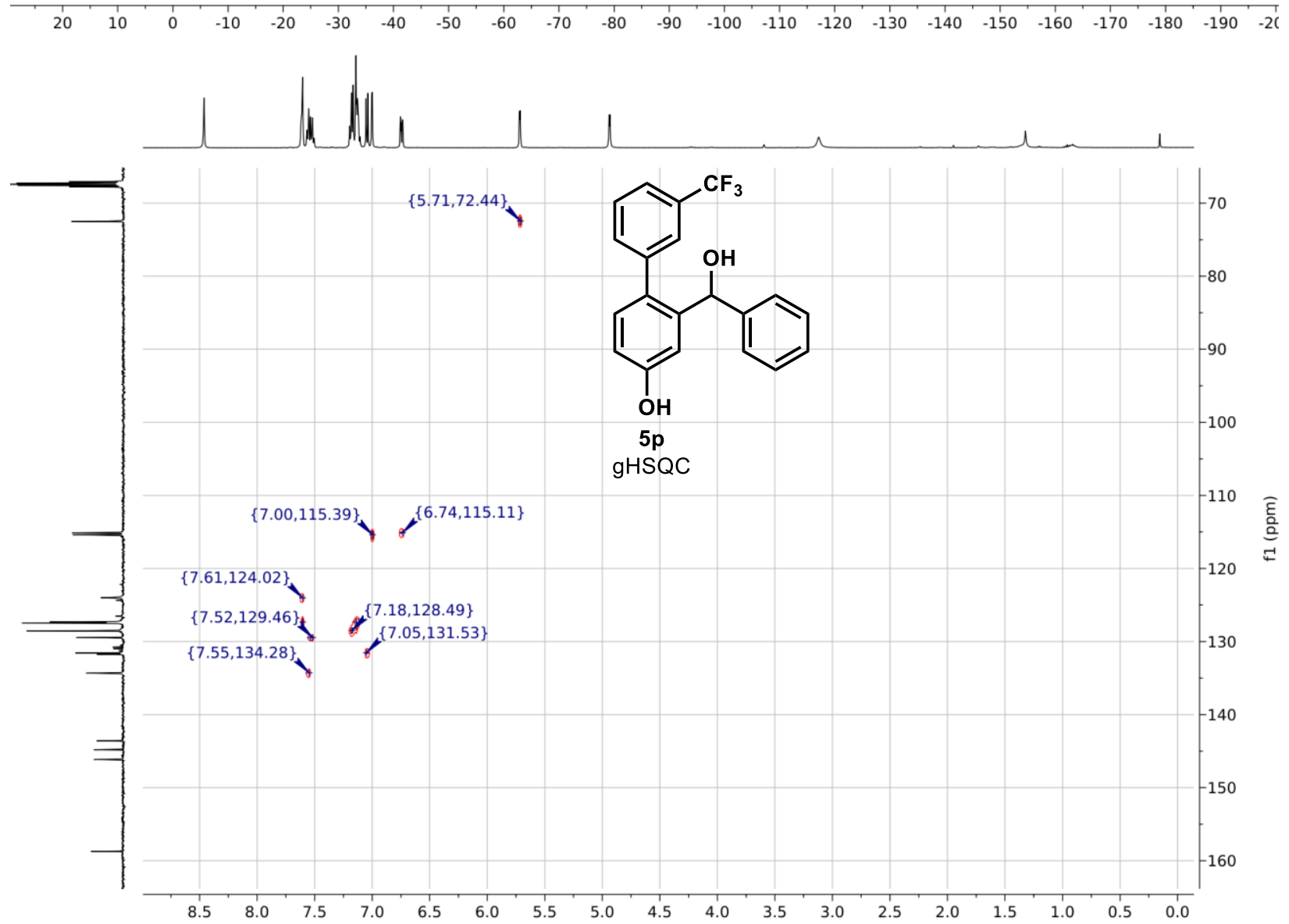




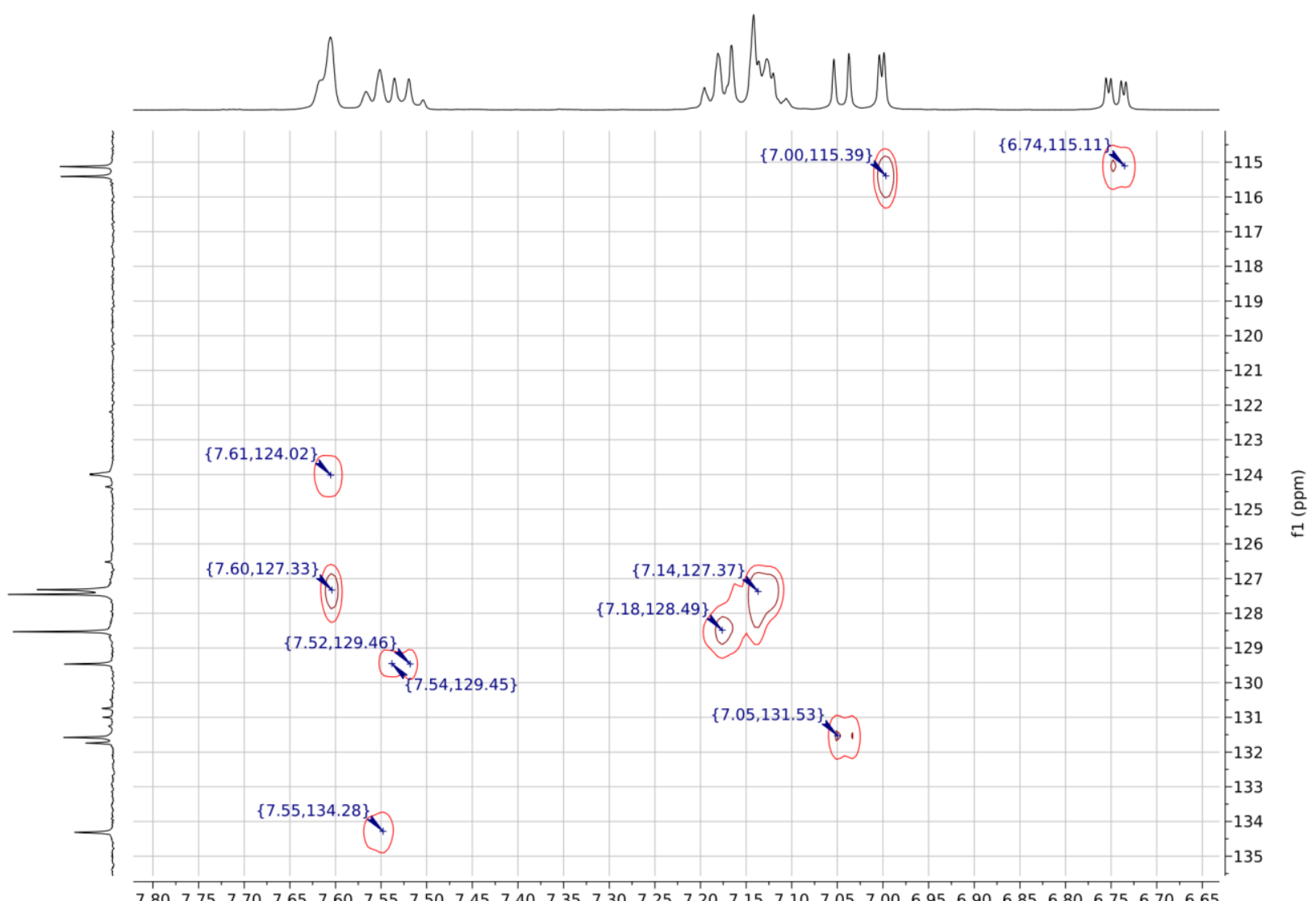

$\begin{array}{lllllllllllllllllllllllll}7.80 & 7.75 & 7.70 & 7.65 & 7.60 & 7.55 & 7.50 & 7.45 & 7.40 & 7.35 & 7.30 & 7.25 & 7.20 & 7.15 & 7.10 & 7.05 & 7.00 & 6.95 & 6.90 & 6.85 & 6.80 & 6.75 & 6.70 & 6.65\end{array}$ 


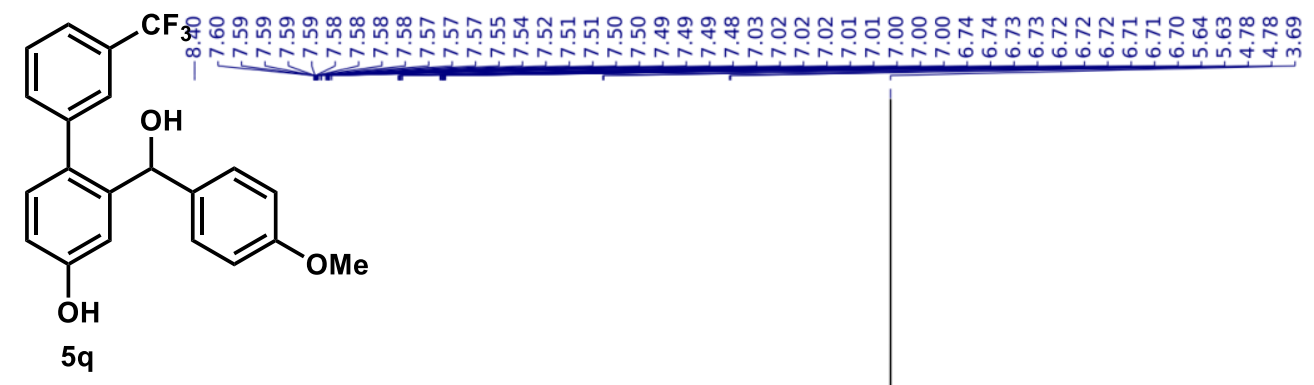

${ }^{1} \mathrm{H}$ NMR $\left(500 \mathrm{MHz}, \mathrm{THF}-d_{8}\right)$
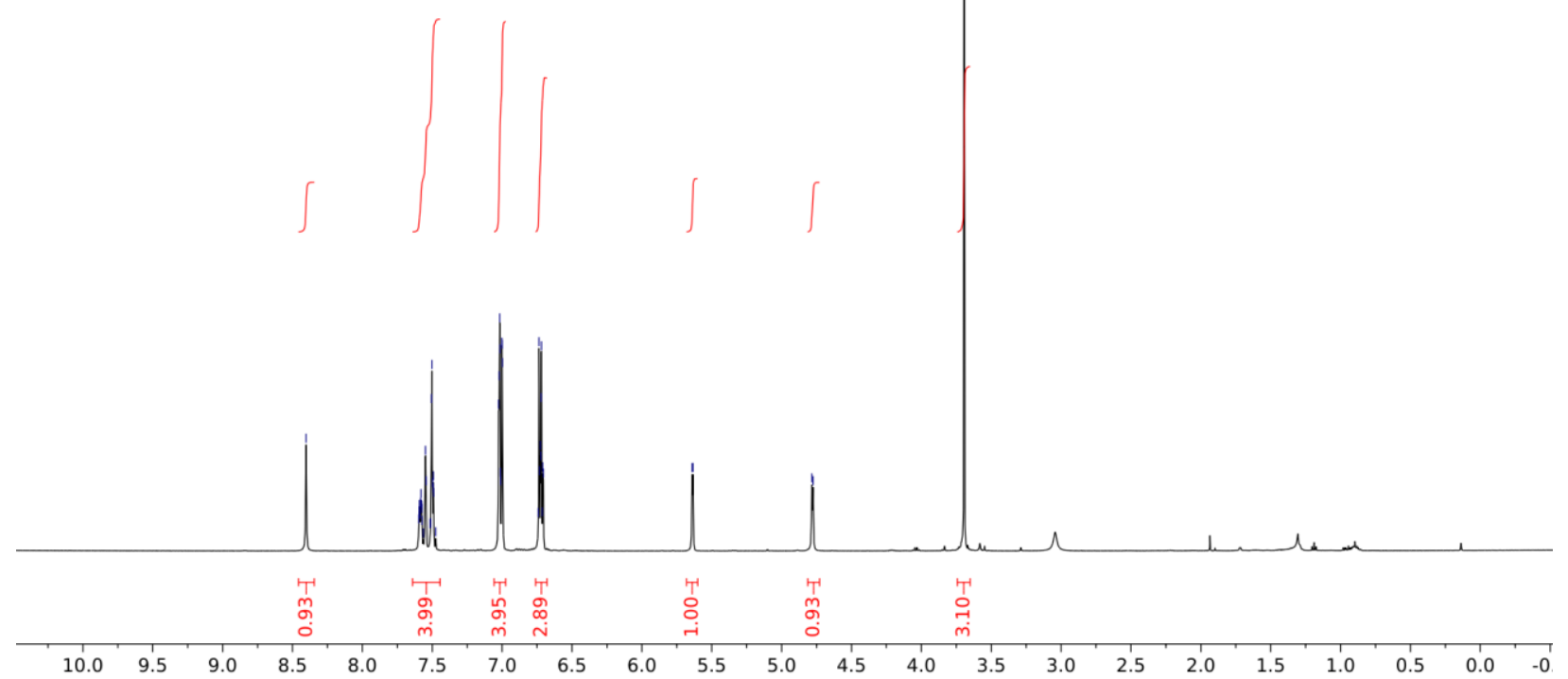

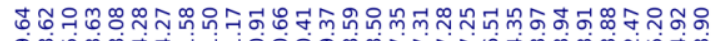
光<smiles>COc1ccc(C(O)c2cc(O)ccc2-c2cccc(C(F)(F)F)c2)cc1</smiles>

$5 \mathbf{q}$

${ }^{13} \mathrm{C}\left\{{ }^{1} \mathrm{H}\right\}$ NMR $\left(125 \mathrm{MHz}\right.$, THF- $\left.d_{8}\right)$

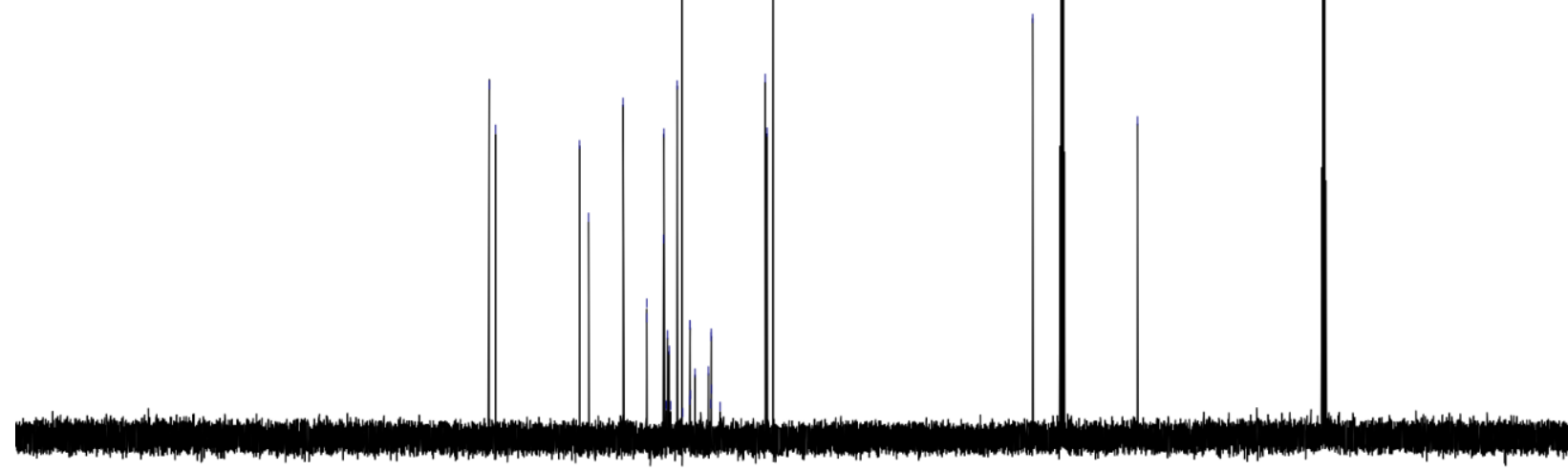

$\begin{array}{lllllllllllllllllllllllll}230 & 220 & 210 & 200 & 190 & 180 & 170 & 160 & 150 & 140 & 130 & 120 & 110 & 100 & 90 & 80 & 70 & 60 & 50 & 40 & 30 & 20 & 10 & 0 & -10\end{array}$ 


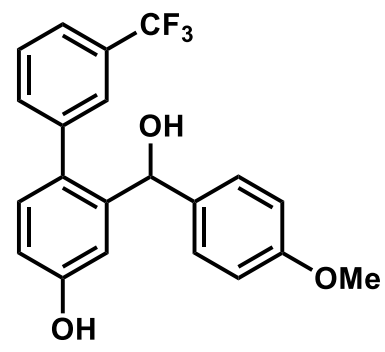

ذ్

$5 q$

${ }^{19} \mathrm{~F}$ NMR $\left(470 \mathrm{MHz}, \mathrm{THF}-\mathrm{d}_{8}\right)$

$\begin{array}{lllllllllllllllllllllll}20 & 10 & 0 & -10 & -20 & -30 & -40 & -50 & -60 & -70 & -80 & -90 & -100 & -110 & -120 & -130 & -140 & -150 & -160 & -170 & -180 & -190 & -2\end{array}$




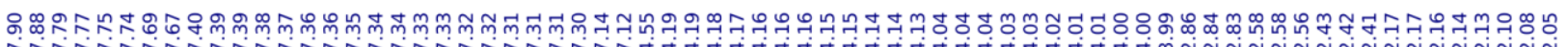

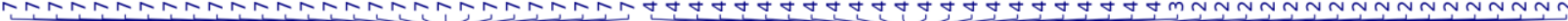<smiles>O=C1CCC2(CC1C=C1c3ccccc3-c3ccccc31)OCCO2</smiles>

10

${ }^{1} \mathrm{H}$ NMR $\left(500 \mathrm{MHz}, \mathrm{CDCl}_{3}\right)$

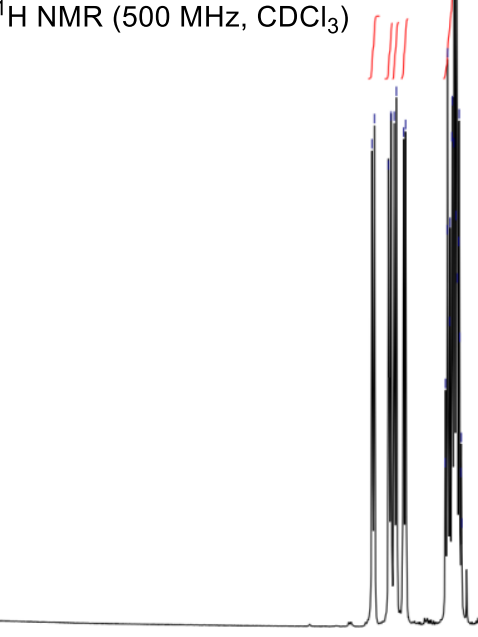

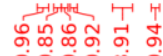

100000

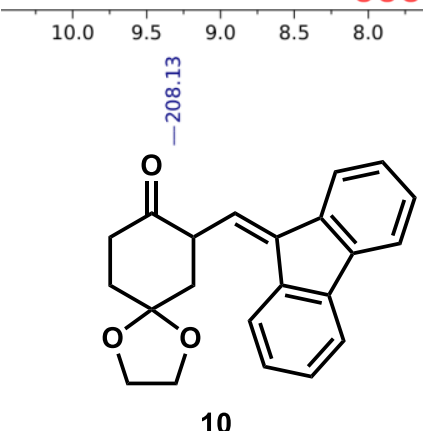

${ }^{13} \mathrm{C}\left\{{ }^{1} \mathrm{H}\right\}$ NMR $\left(125 \mathrm{MHz}, \mathrm{CDCl}{ }_{3}\right)$
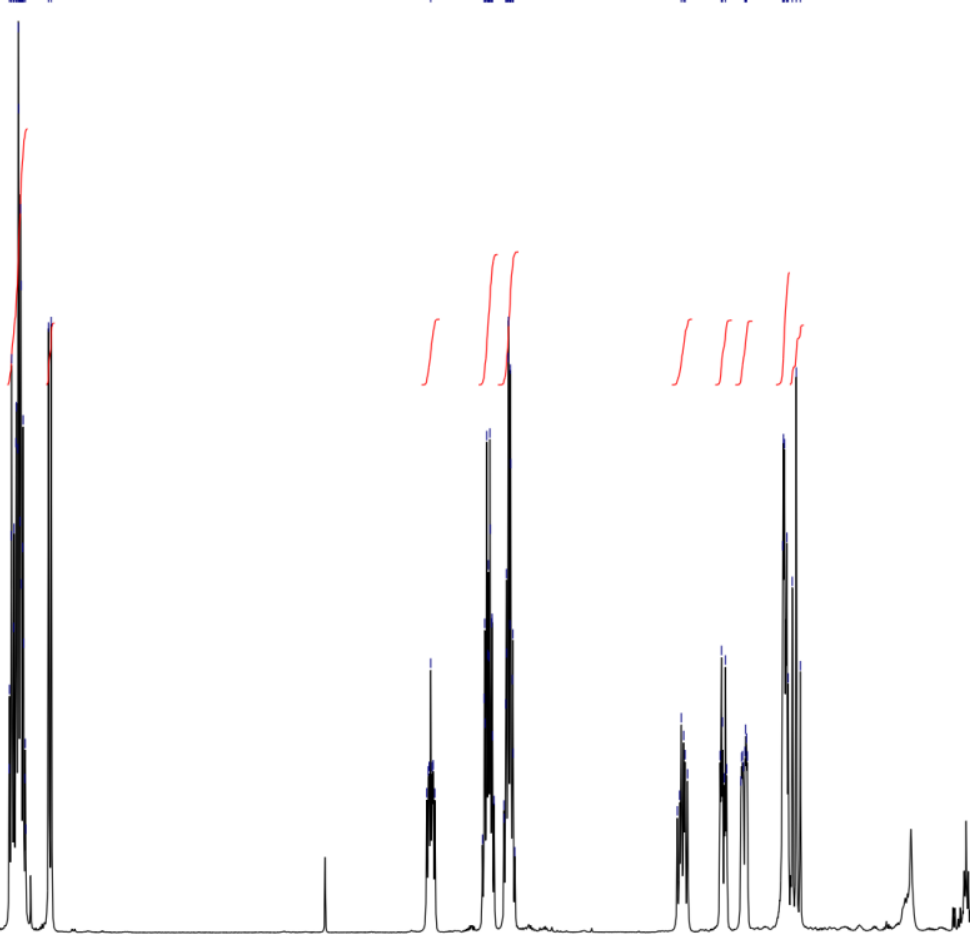

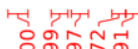
iótio

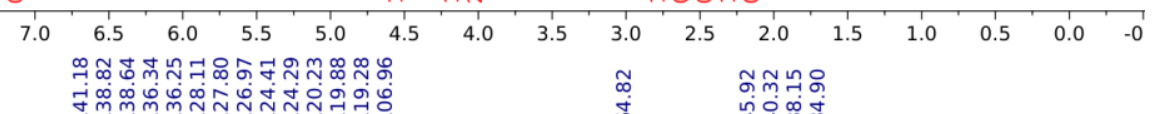

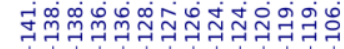

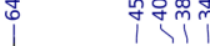

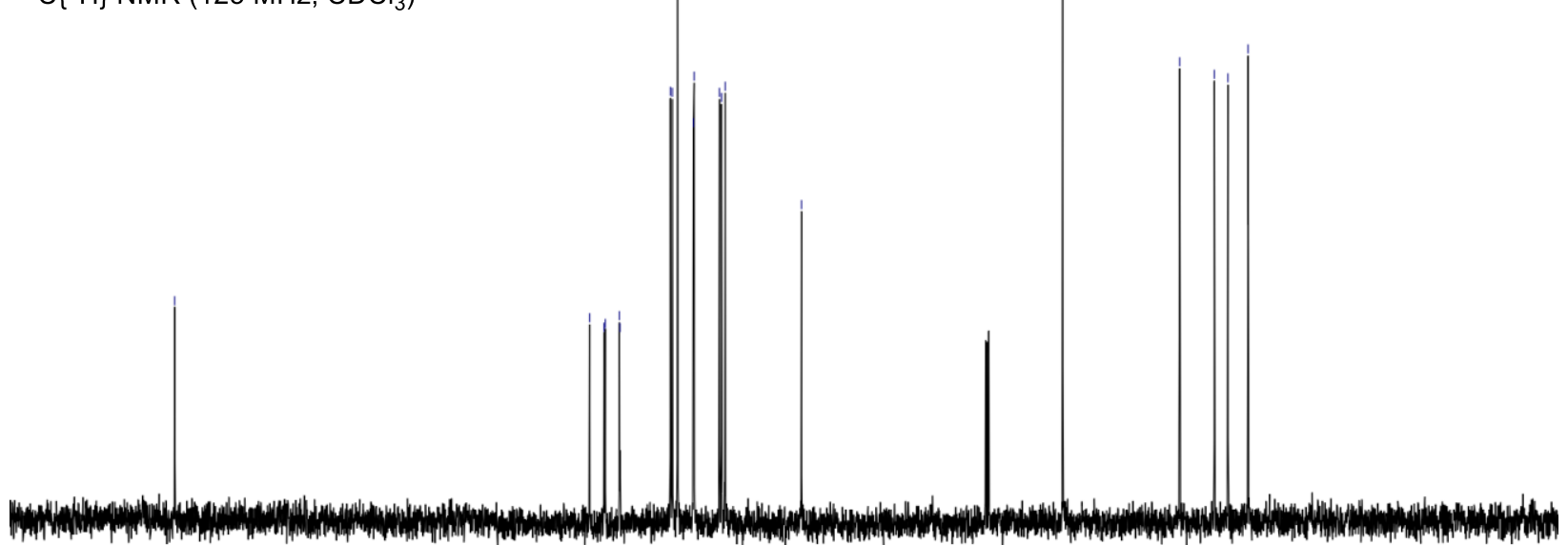

$\begin{array}{lllllllllllllllllllllllll}230 & 220 & 210 & 200 & 190 & 180 & 170 & 160 & 150 & 140 & 130 & 120 & 110 & 100 & 90 & 80 & 70 & 60 & 50 & 40 & 30 & 20 & 10 & 0 & -10\end{array}$ 
$\mathrm{MeO}$<smiles>Cc1ccc(C2(O)CCC3(CC2C=C2c4ccccc4-c4ccccc42)OCCO3)cc1</smiles>

${ }^{1} \mathrm{H}$ NMR $\left(500 \mathrm{MHz}, \mathrm{CDCl}_{3}\right)$

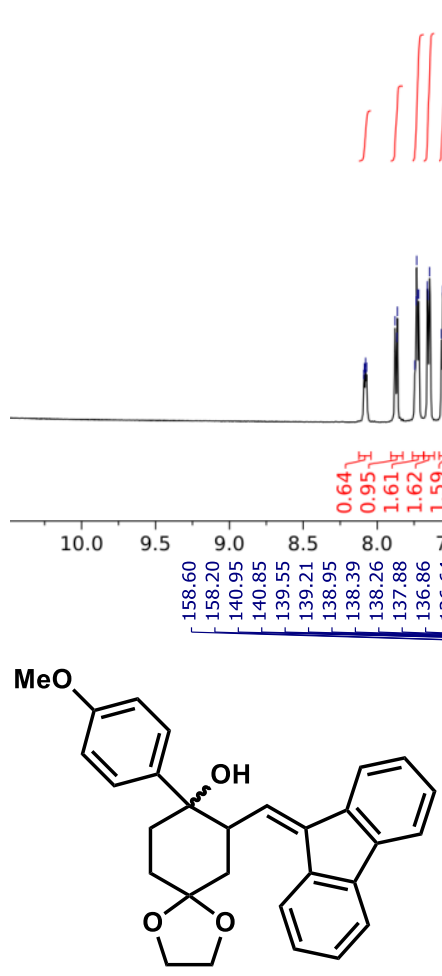

11a

${ }^{13} \mathrm{C}\left\{{ }^{1} \mathrm{H}\right\} \operatorname{NMR}\left(125 \mathrm{MHz}, \mathrm{CDCl}_{3}\right)$

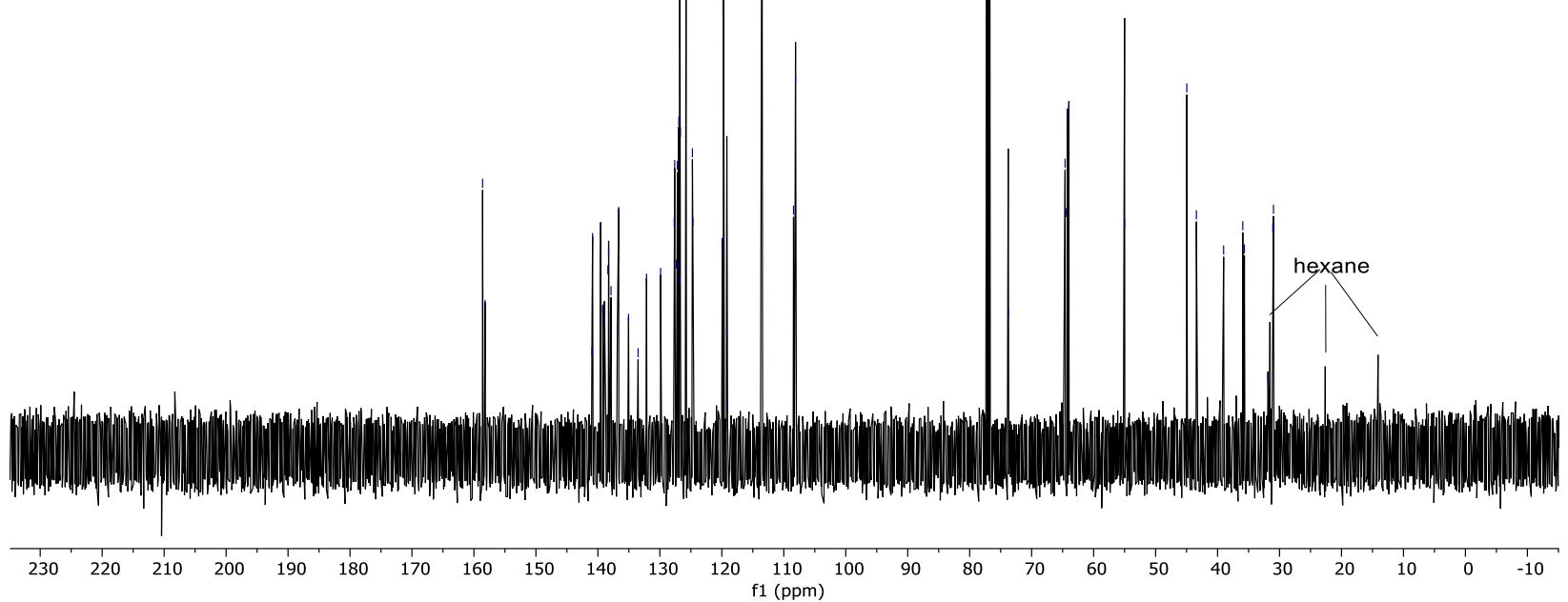




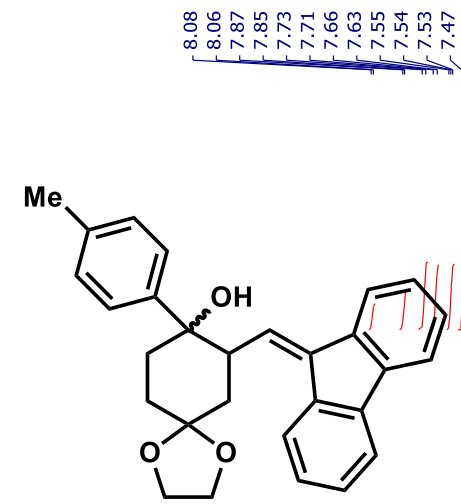

11b

${ }^{1} \mathrm{H}$ NMR $\left(600 \mathrm{MHz}, \mathrm{CDCl}_{3}\right)$

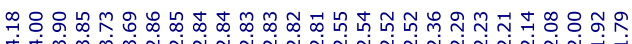

ivininiti

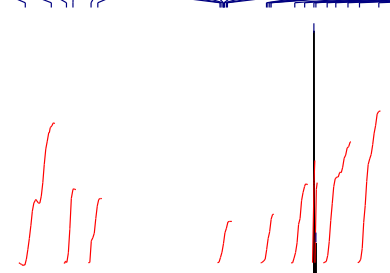

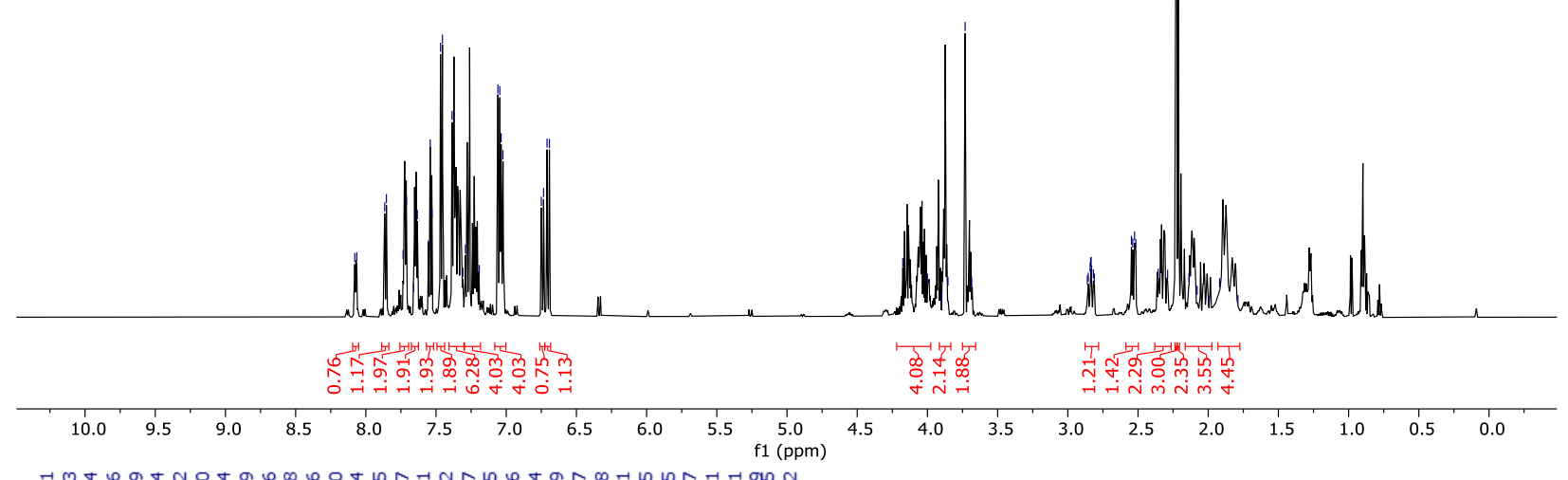

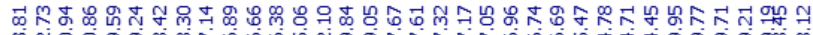

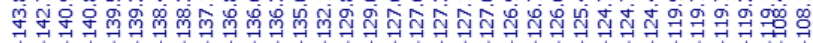

Me<smiles>O[C@]1(c2ccc(F)cc2)CCC2(CC1C=C1c3ccccc3-c3ccccc31)OCCO2</smiles>

$11 \mathrm{~b}$

${ }^{13} \mathrm{C}\left\{{ }^{1} \mathrm{H}\right\} \mathrm{NMR}\left(150 \mathrm{MHz}, \mathrm{CDCl}_{3}\right)$

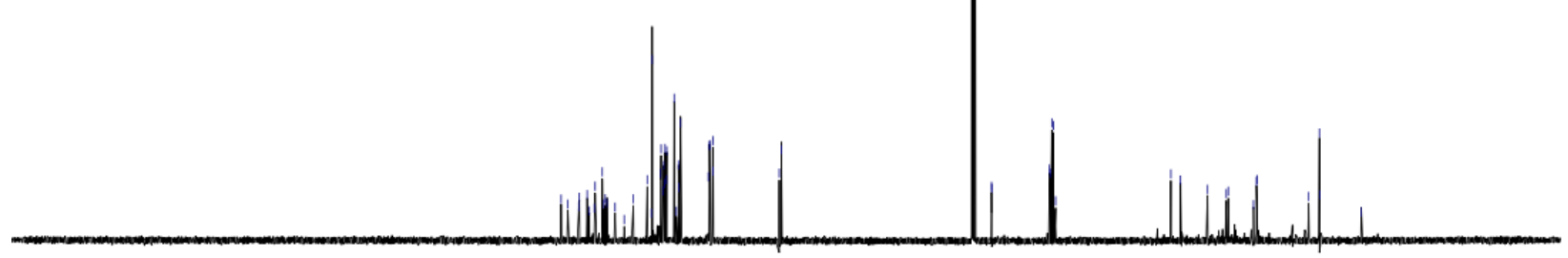

230

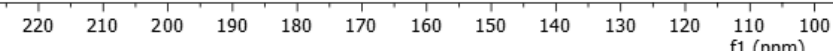

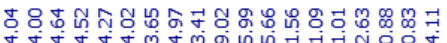

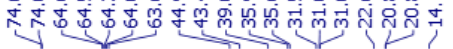




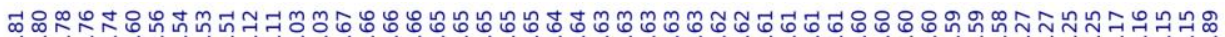

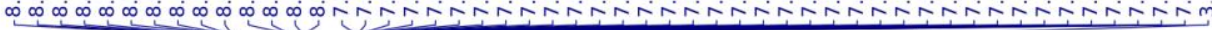<smiles>COc1ccc2c3ccc(O)cc3c3c4ccccc4c4ccccc4c3c2c1</smiles>

${ }^{1} \mathrm{H}$ NMR $\left(500 \mathrm{MHz}\right.$, THF- $\left.d_{8}\right)$
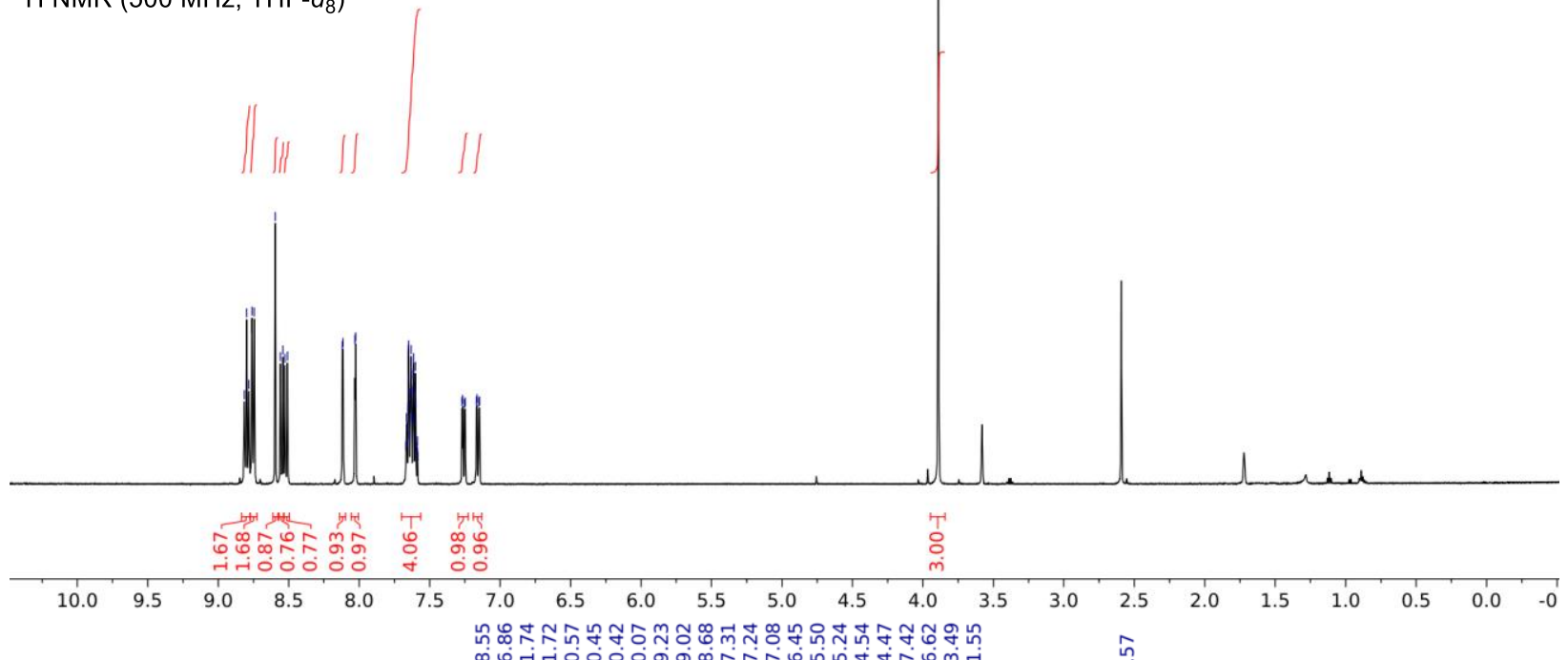<smiles>COc1ccc2c3ccc(O)cc3c3c4ccccc4c4ccccc4c3c2c1</smiles>

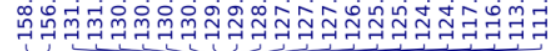

${ }^{13} \mathrm{C}\left\{{ }^{1} \mathrm{H}\right\}$ NMR $\left(125 \mathrm{MHz}\right.$, THF- $\left.d_{8}\right)$

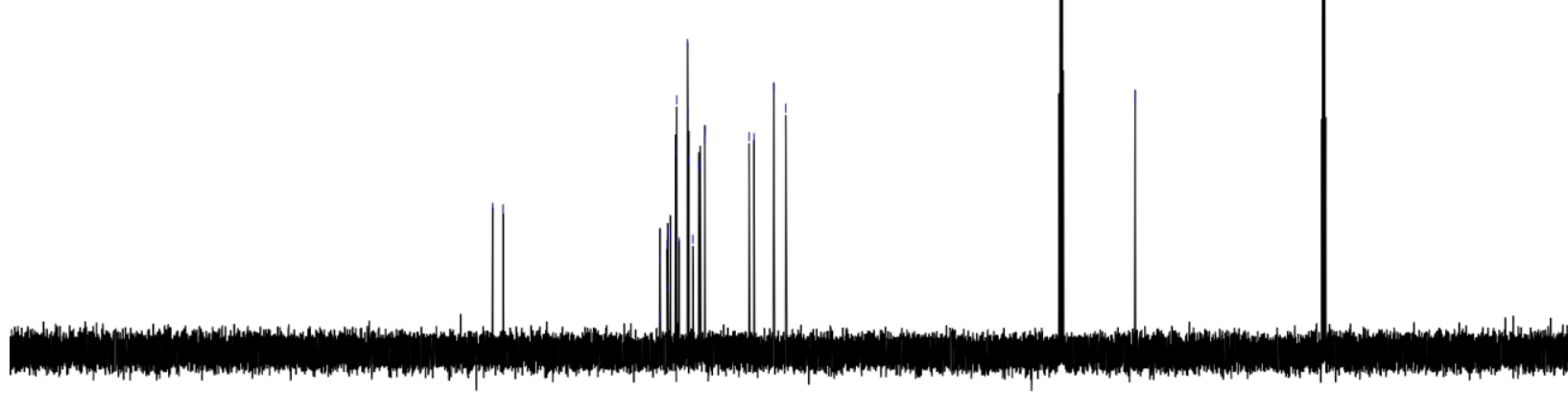

$\begin{array}{lllllllllllllllllllllllll}230 & 220 & 210 & 200 & 190 & 180 & 170 & 160 & 150 & 140 & 130 & 120 & 110 & 100 & 90 & 80 & 70 & 60 & 50 & 40 & 30 & 20 & 10 & 0 & -10\end{array}$ 


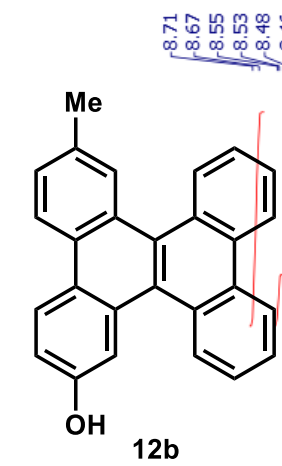

${ }^{1} \mathrm{H}$ NMR $\left(600 \mathrm{MHz}, \mathrm{CDCl}_{3}\right)$

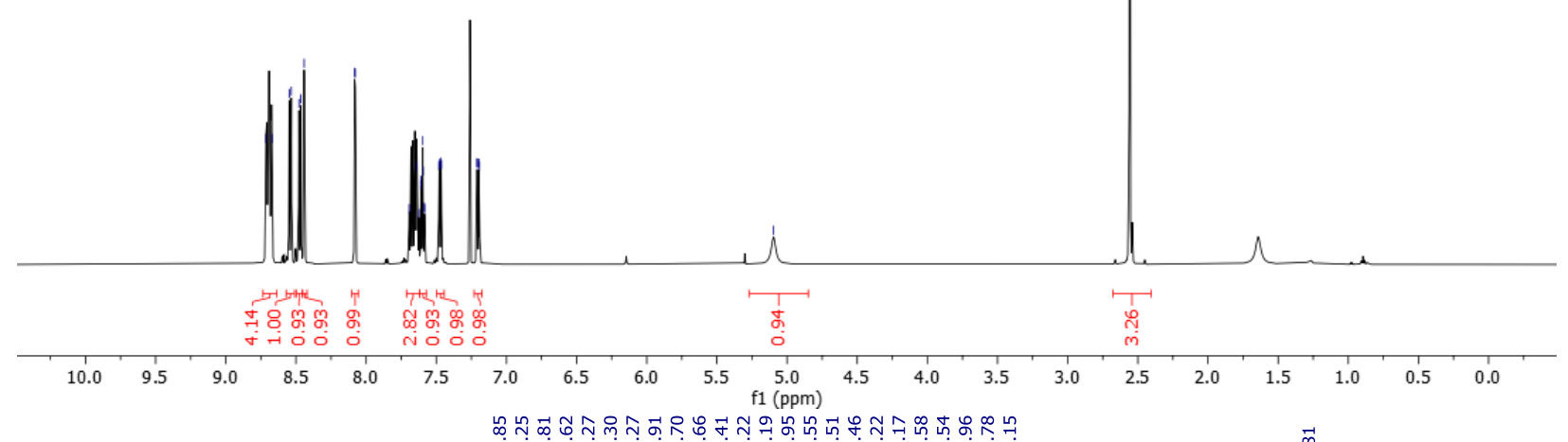<smiles>Cc1ccc2c3ccc(O)cc3c3c4ccccc4c4ccccc4c3c2c1</smiles>

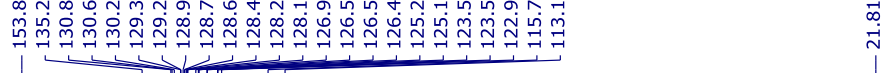

${ }^{13} \mathrm{C}\left\{{ }^{1} \mathrm{H}\right\}$ NMR $\left(150 \mathrm{MHz}, \mathrm{CDCl}_{3}\right)$

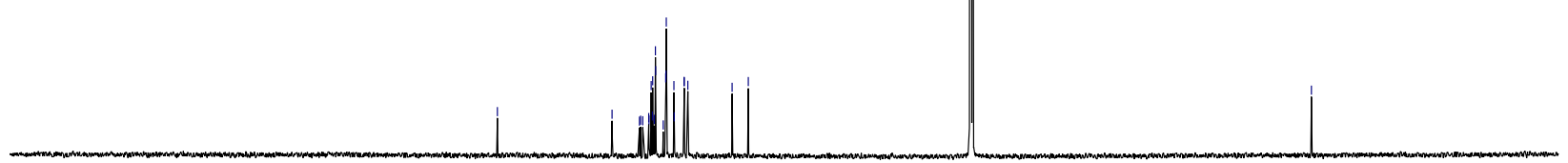




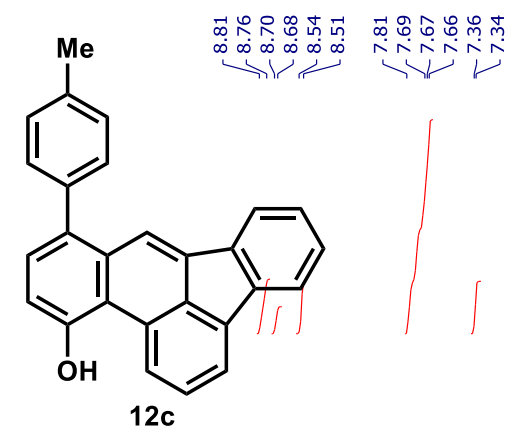

${ }^{1} \mathrm{H}$ NMR $\left(600 \mathrm{MHz}, \mathrm{CDCl}_{3}\right)$
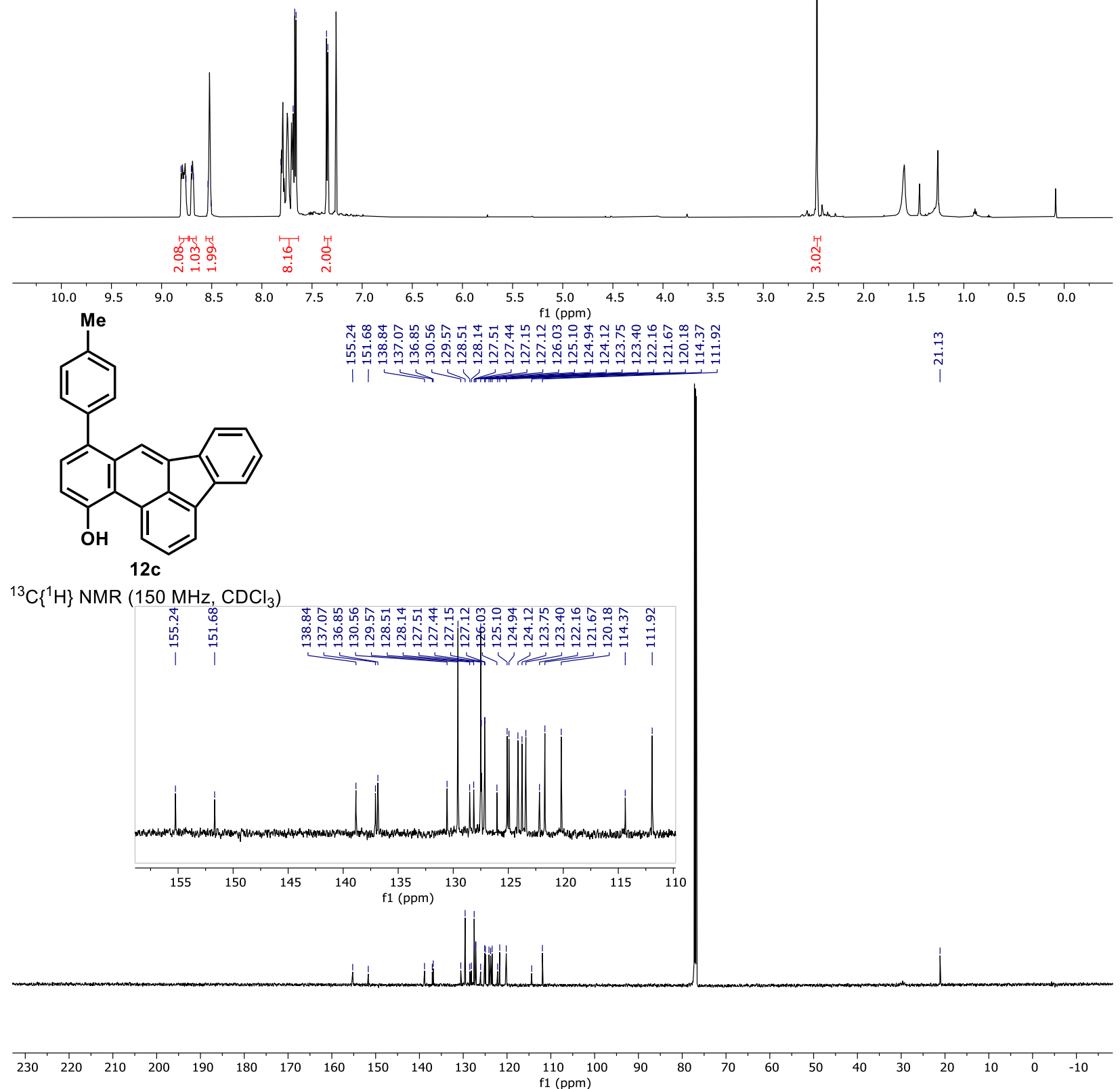
<smiles>CCc1ccc(-c2ccc(O)cc2C=C2c3ccccc3-c3ccccc32)cc1</smiles>

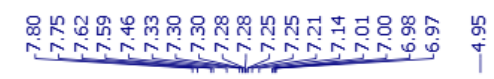

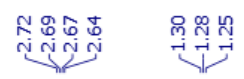

${ }^{1} \mathrm{H}$ NMR $\left(300 \mathrm{MHz}, \mathrm{CDCl}_{3}\right)$

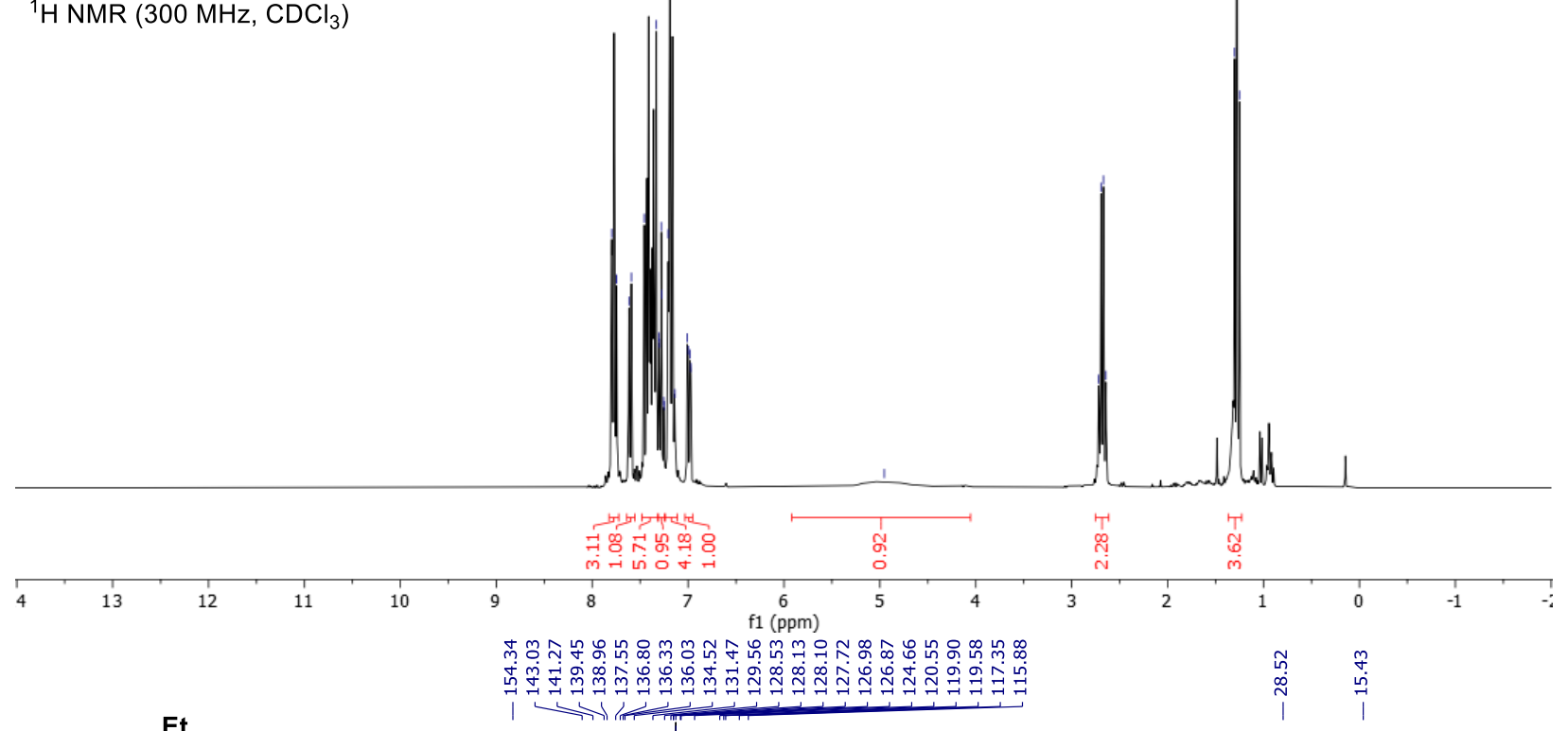<smiles>CCc1ccc(-c2ccc(O)cc2C=C2c3ccccc3-c3ccccc32)cc1</smiles>

${ }^{13} \mathrm{C}\left\{{ }^{1} \mathrm{H}\right\} \mathrm{NMR}\left(75 \mathrm{MHz}, \mathrm{CDCl}_{3}\right)$

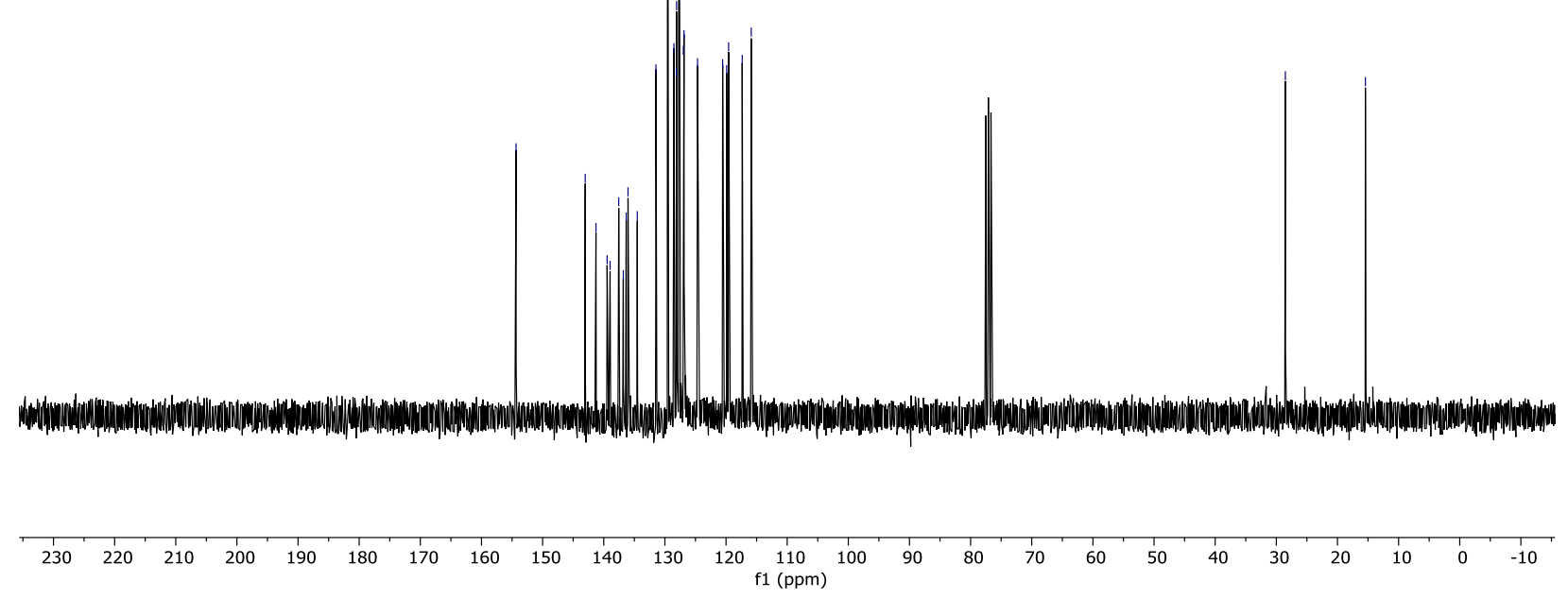



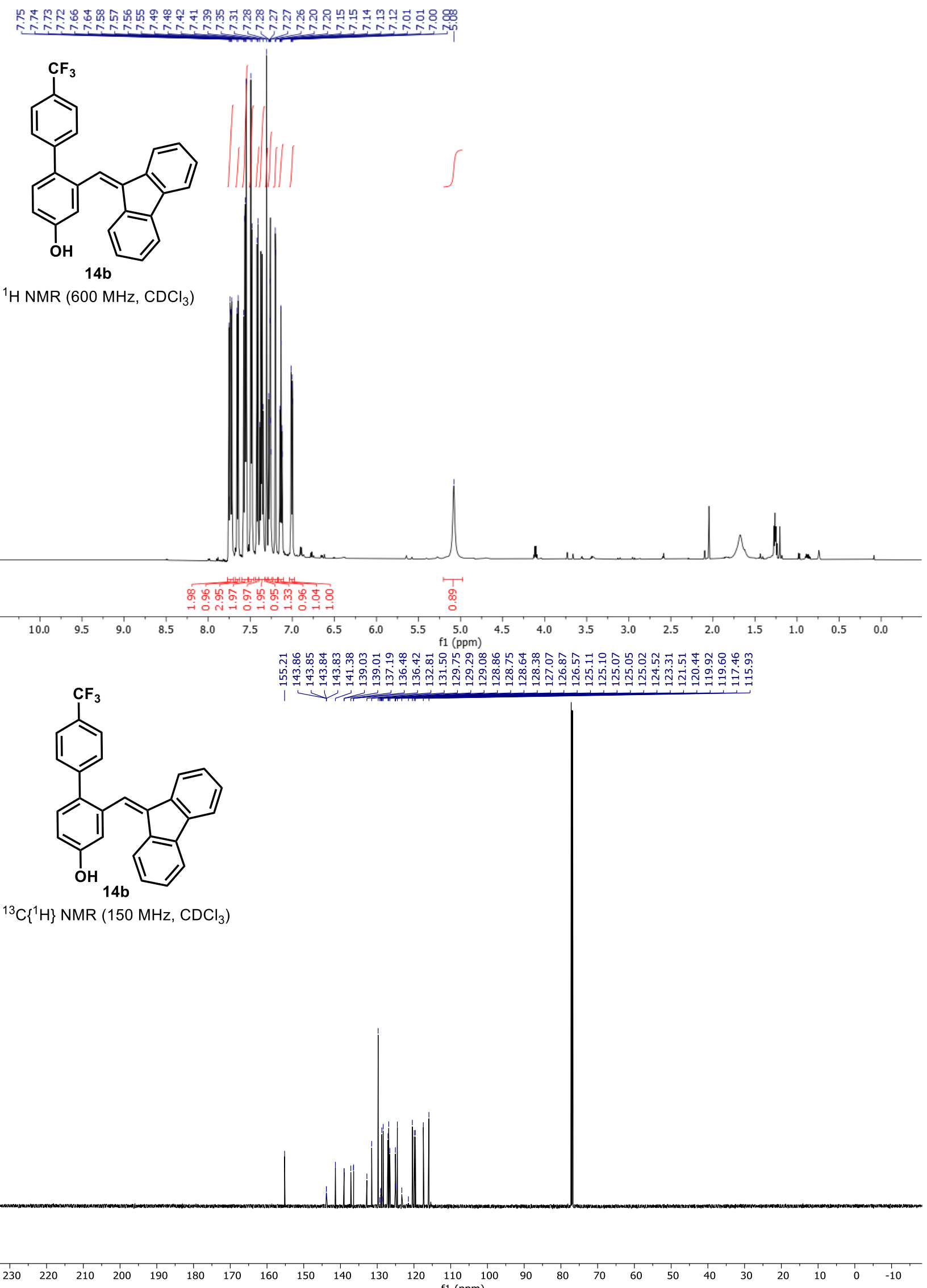

(ppm) 


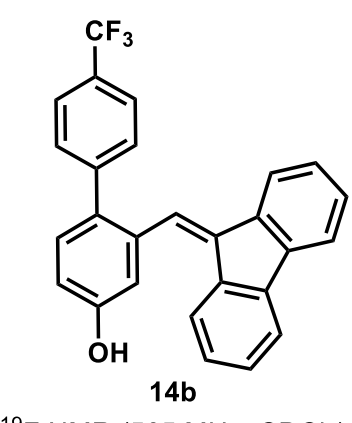

${ }^{19} \mathrm{~F} \mathrm{NMR}\left(565 \mathrm{MHz}, \mathrm{CDCl}_{3}\right)$

\begin{tabular}{lllllllllllllllllllllllll}
\hline 30 & 20 & 10 & 0 & -10 & -20 & -30 & -40 & -50 & -60 & -70 & -80 & -90 & -100 & -110 & -120 & -130 & -140 & -150 & -160 & -170 & -180 & -190 & -20
\end{tabular}




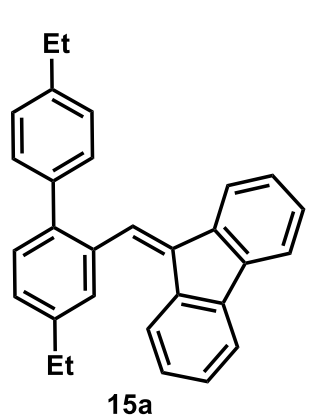

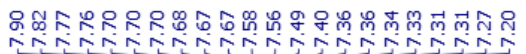

\section{מू⿻}

ayinghy

'H NMR (300 MHz, $\left.\mathrm{CDCl}_{3}\right)$
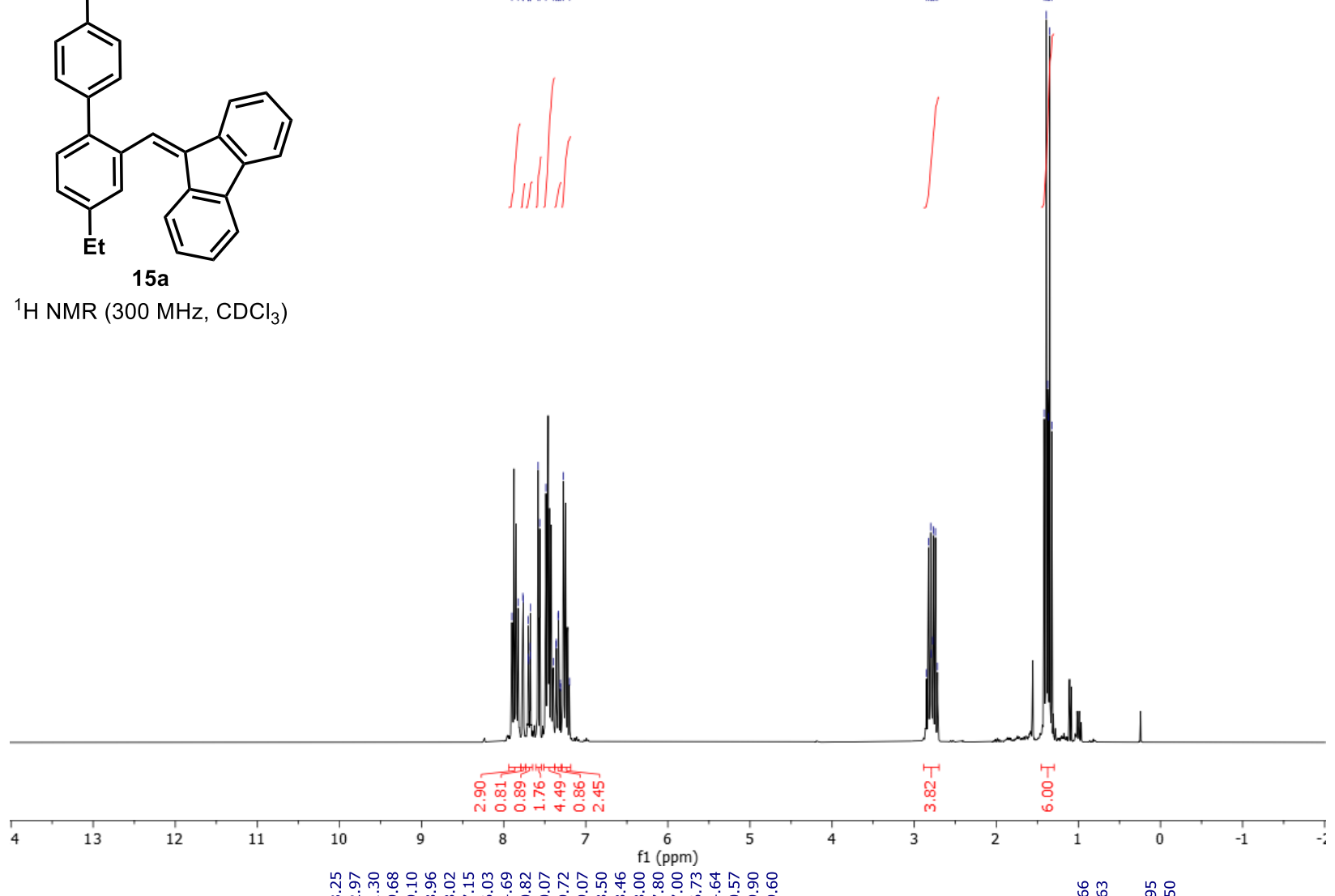<smiles>CCc1ccc(-c2ccc(O)cc2C=C2c3ccccc3-c3ccccc32)cc1</smiles>

${ }^{13} \mathrm{C}\left\{{ }^{1} \mathrm{H}\right\}$ NMR $\left(75 \mathrm{MHz}, \mathrm{CDCl}_{3}\right)$

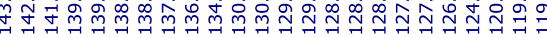

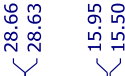

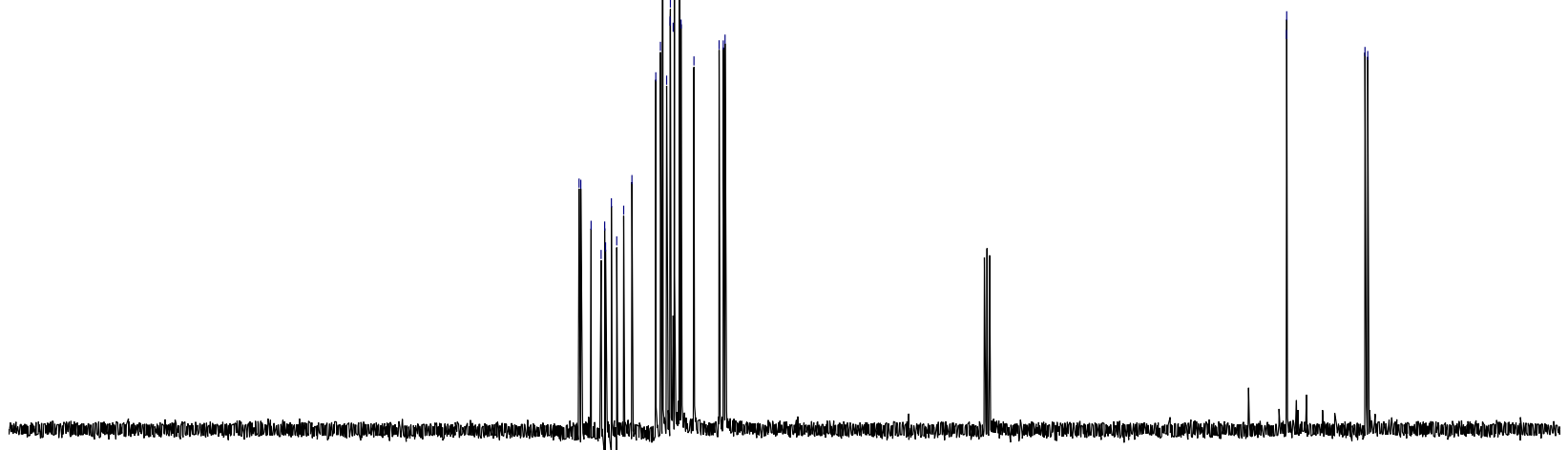

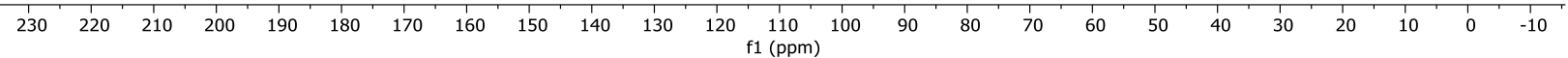


<smiles>CCc1ccc(-c2ccc(C(F)(F)F)cc2)c(C=C2c3ccccc3-c3ccccc32)c1</smiles>

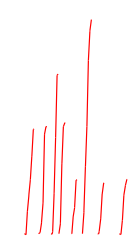

${ }^{1} \mathrm{H}$ NMR $\left(600 \mathrm{MHz}, \mathrm{CDCl}_{3}\right)$

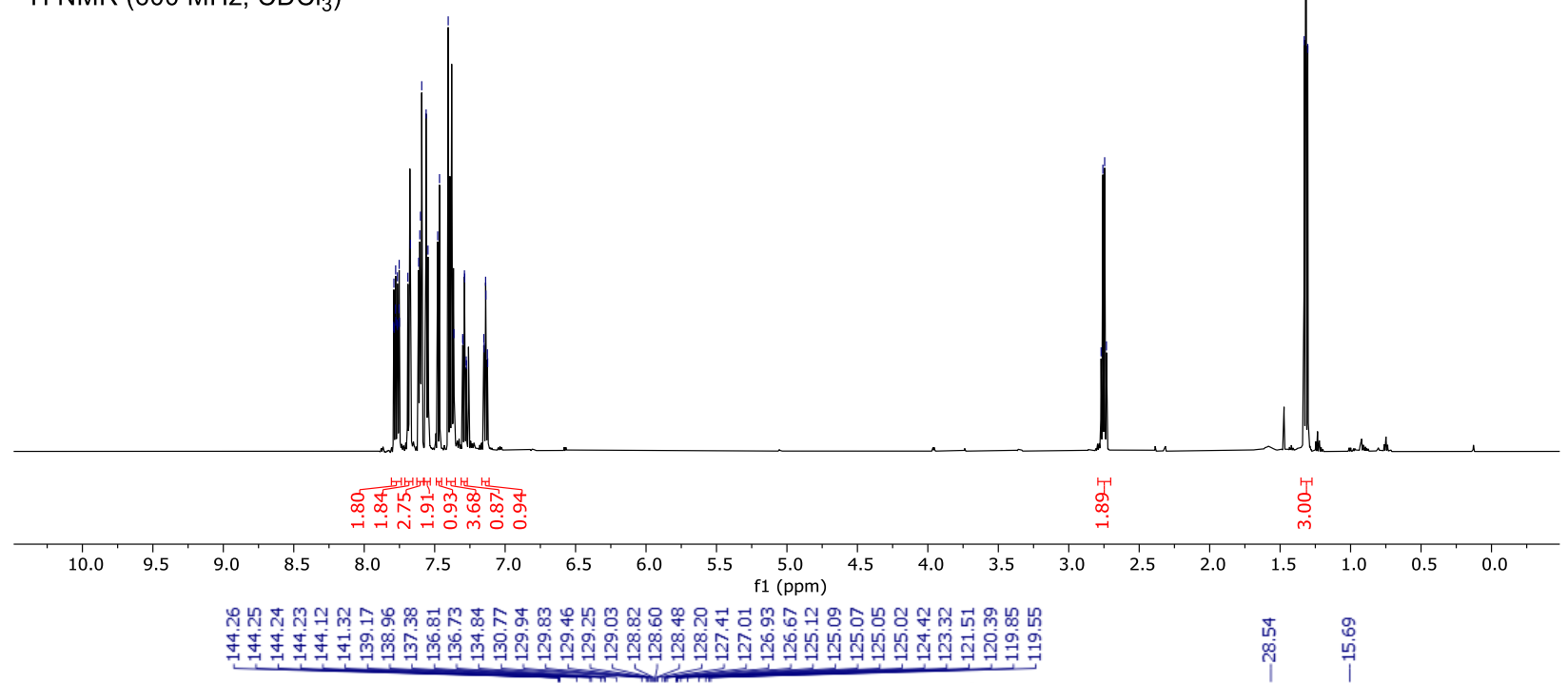<smiles>CCc1ccc(-c2ccc(C(F)(F)F)cc2)c(C=C2c3ccccc3-c3ccccc32)c1</smiles>

${ }^{13} \mathrm{C}\left\{{ }^{1} \mathrm{H}\right\}$ NMR $\left(150 \mathrm{MHz}, \mathrm{CDCl}_{3}\right)$

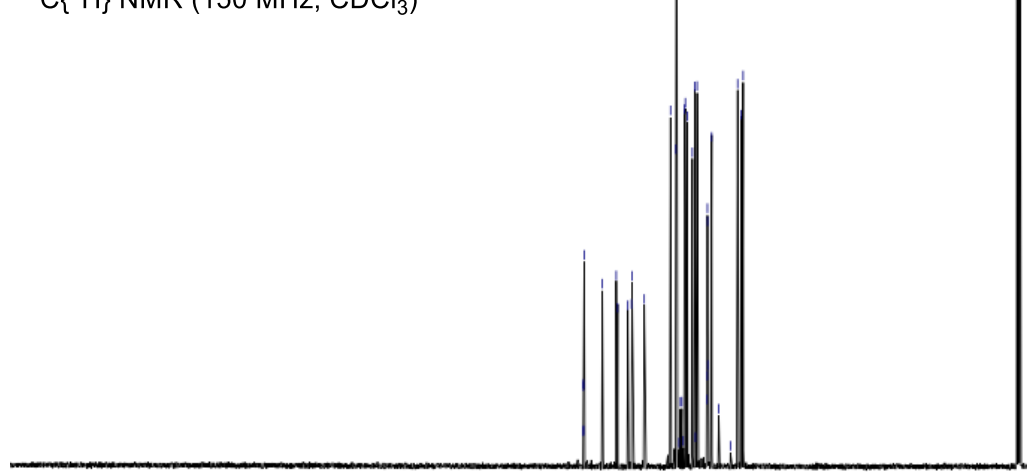

230

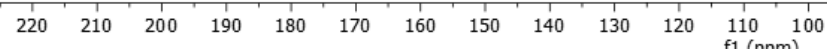
1 (ppm) 


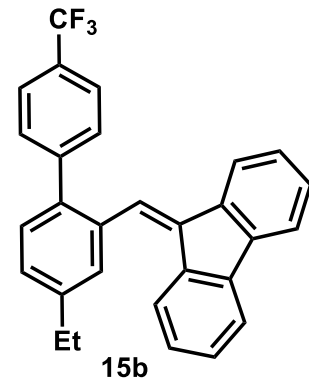

${ }^{13} \mathrm{~F} \mathrm{NMR}\left(565 \mathrm{MHz}, \mathrm{CDCl}_{3}\right)$

\begin{tabular}{llllllllllllllllllllllll}
\hline 30 & 20 & 10 & 0 & -10 & -20 & -30 & -40 & -50 & -60 & -70 & $\underset{-10}{1}$ & -90 & -100 & -110 & -120 & -130 & -140 & -150 & -160 & -170 & -180 & -190 & -20
\end{tabular}


<smiles>CCc1ccc2c3ccc(CC)cc3c3c4ccccc4c4ccccc4c3c2c1</smiles>

'H NMR ( $300 \mathrm{MHz}, \mathrm{CDCl}_{3}$ )
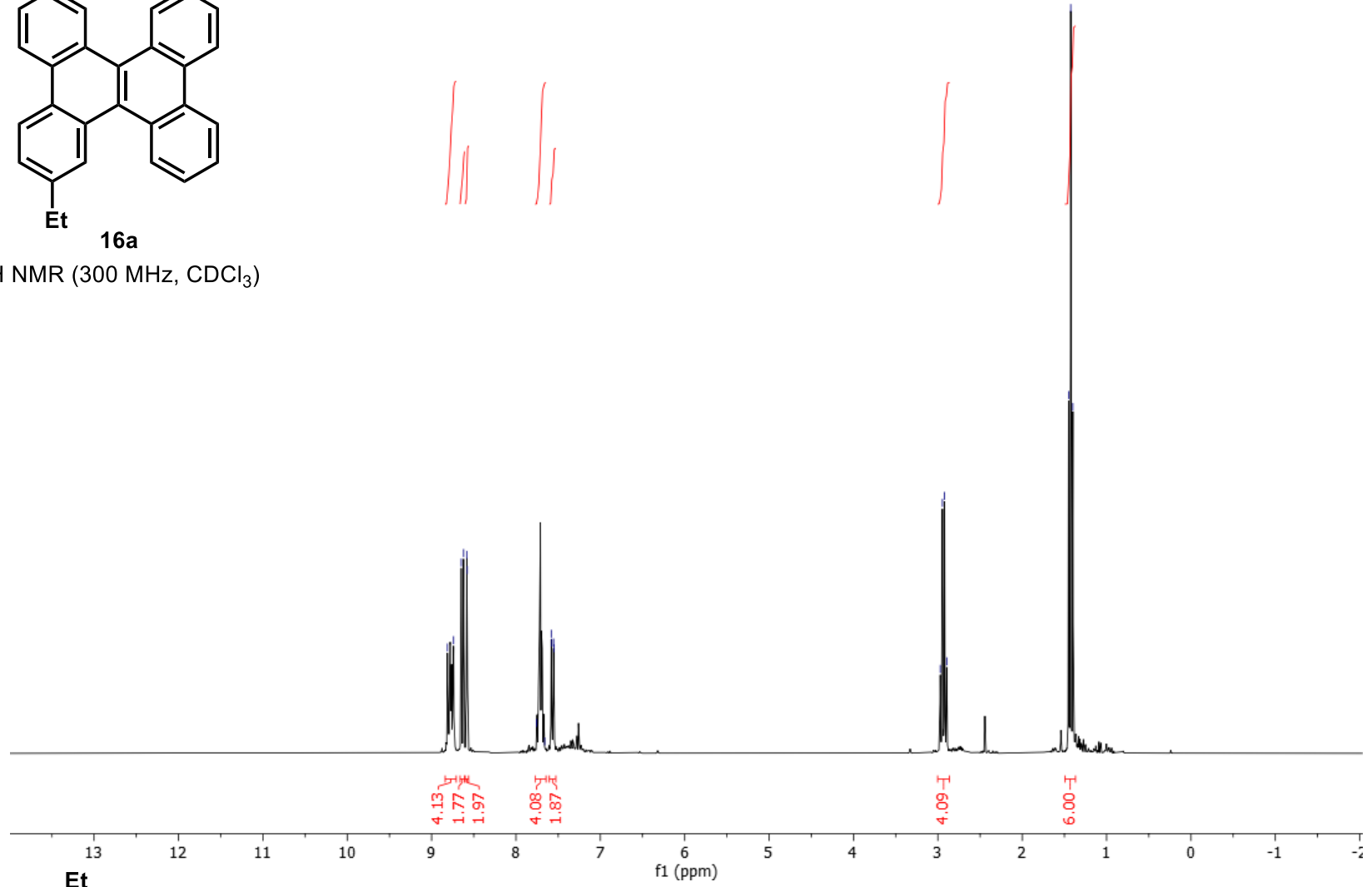<smiles>CCc1ccc2c3ccc(CC)cc3c3c4ccccc4c4ccccc4c3c2c1</smiles>

${ }^{13} \mathrm{C}\left\{{ }^{1} \mathrm{H}\right\}$ NMR $\left(75 \mathrm{MHz}, \mathrm{CDCl}_{3}\right)$
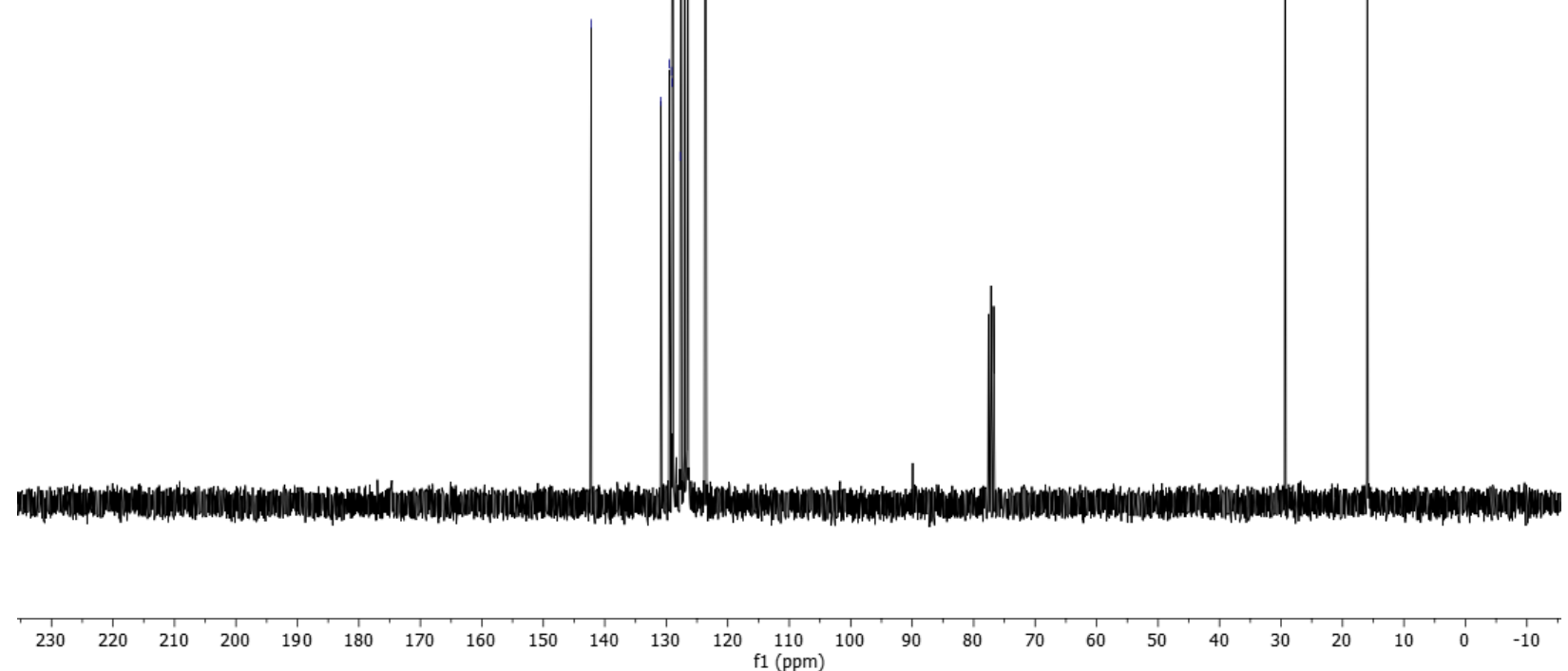

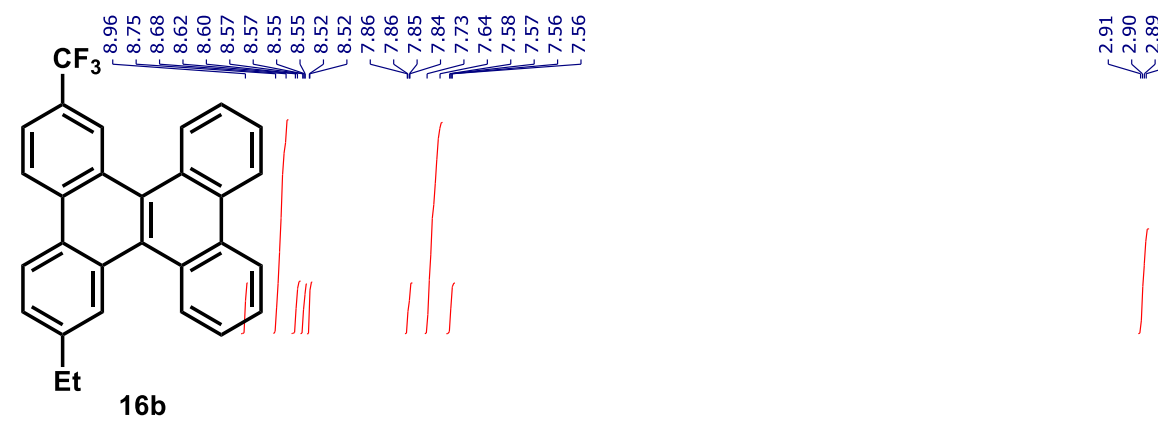

${ }^{1} \mathrm{H}$ NMR $\left(600 \mathrm{MHz}, \mathrm{CDCl}_{3}\right)$

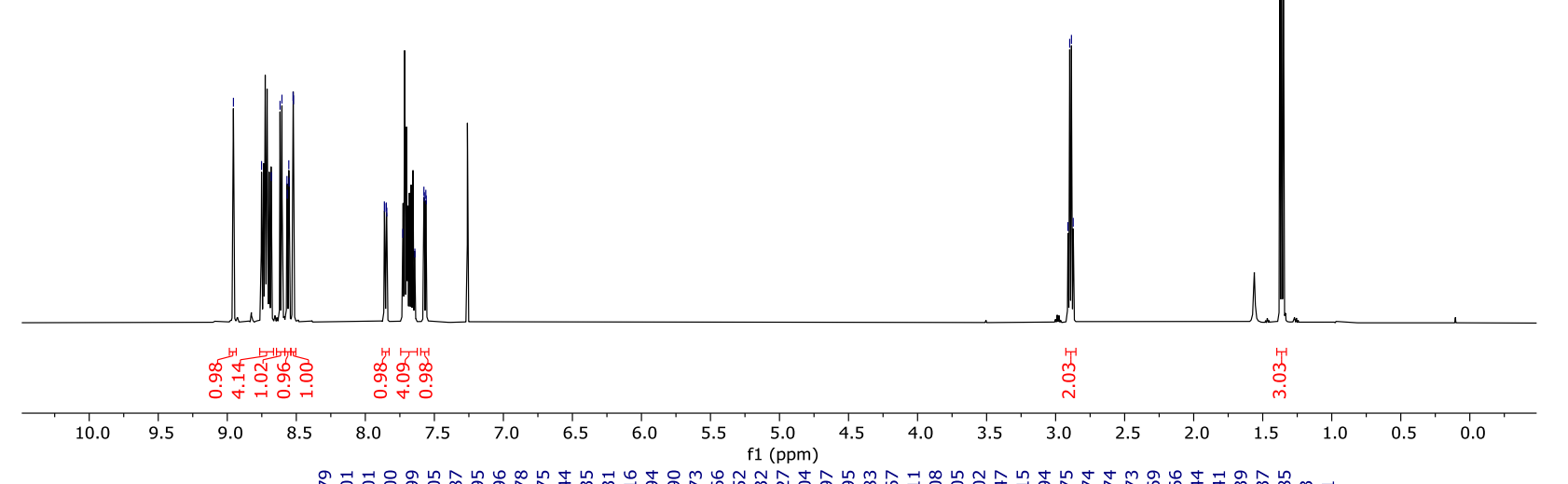

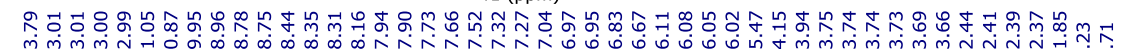

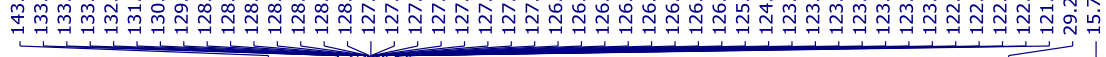<smiles>CCc1ccc2c3ccc(C(F)(F)F)cc3c3c4ccccc4c4ccccc4c3c2c1</smiles>

${ }^{13} \mathrm{C}\left\{{ }^{1} \mathrm{H}\right\}$ NMR $\left(150 \mathrm{MHz}, \mathrm{CDCl}_{3}\right)$

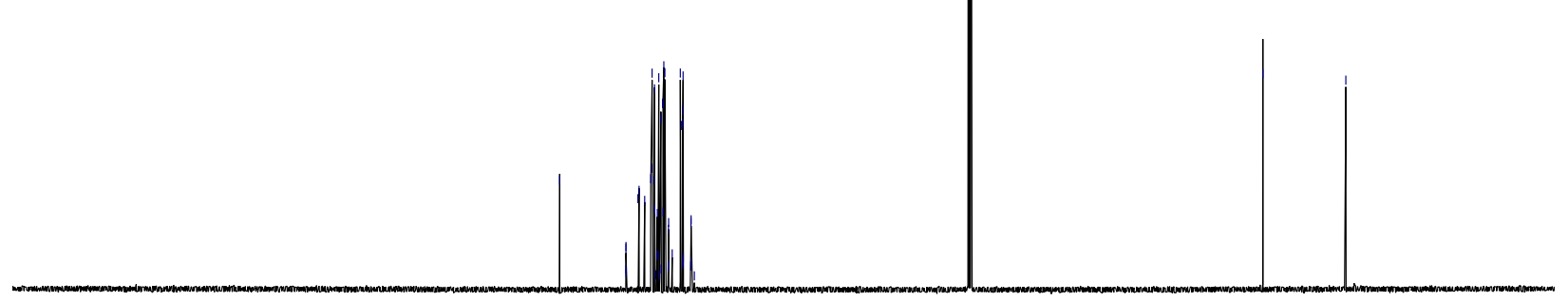

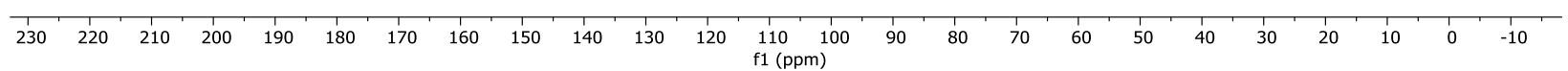




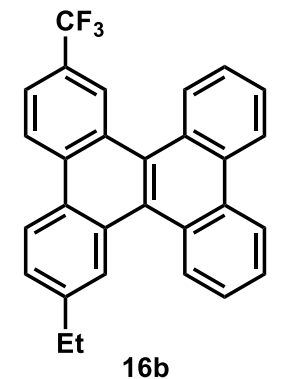

${ }^{19} \mathrm{~F}$ NMR $\left(565 \mathrm{MHz}, \mathrm{CDCl}_{3}\right)$

$\begin{array}{llllllllllllllllllllllllllllllll}1 & 20 & 10 & 0 & -10 & -20 & -30 & -40 & -50 & -60 & -70 & -80 & -90 & -100 & -110 & -120 & -130 & -140 & -150 & -160 & -170 & -180 & -190 & -201\end{array}$




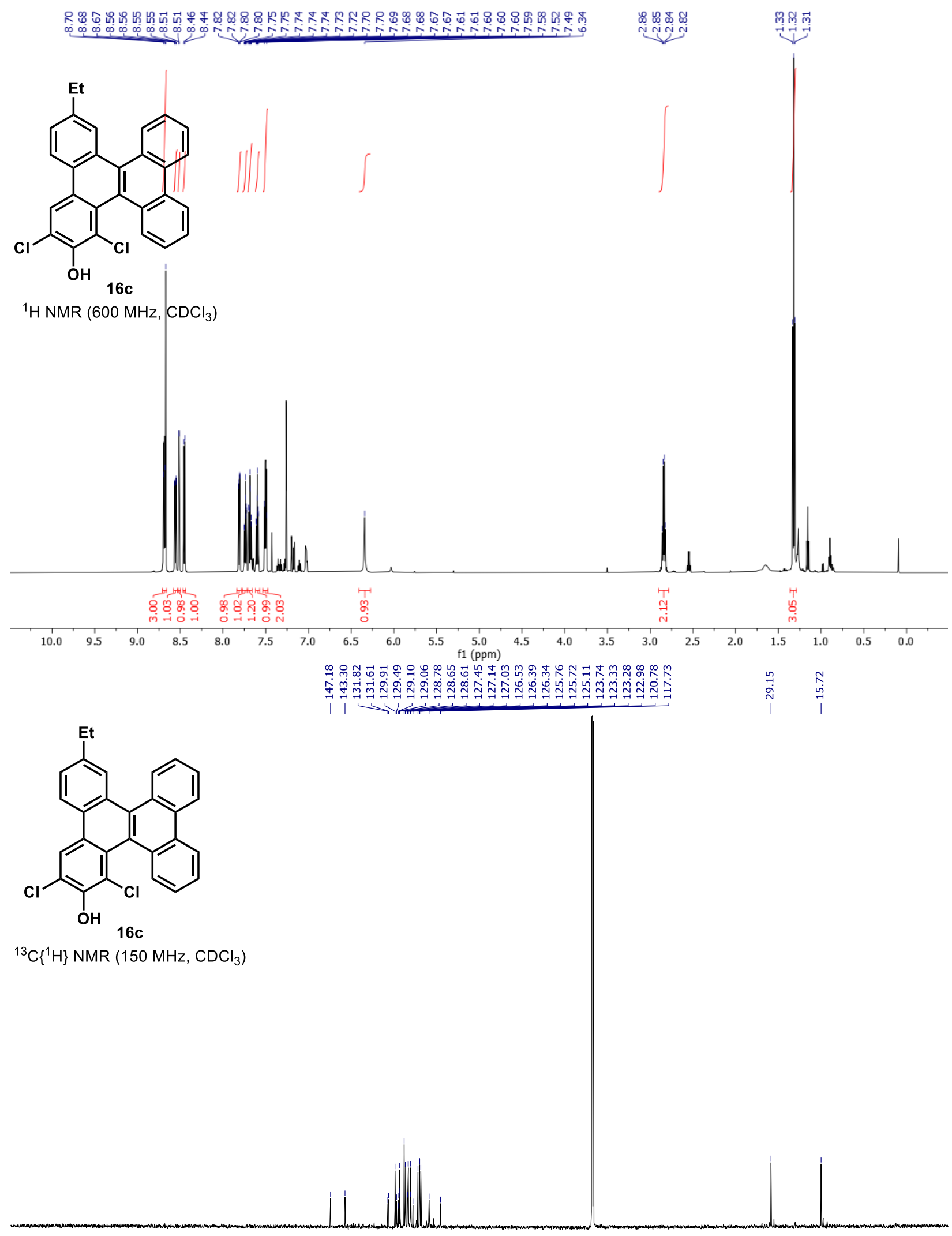

$\begin{array}{lllllllllllllllllllllllll}1 & 1 \\ 230 & 220 & 210 & 200 & 190 & 180 & 170 & 160 & 150 & 140 & 130 & 120 & 110 & 100 & 90 & 80 & 70 & 60 & 50 & 40 & 30 & 20 & 10 & 0 & -10\end{array}$ 


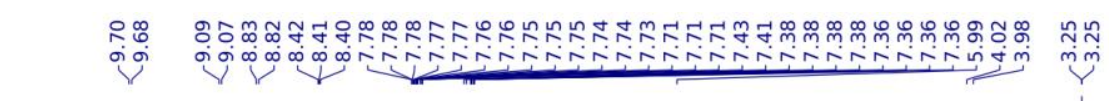<smiles>COc1ccc2c3c4c5c6c(cccc6c6ccccc6c5c2c1)O[C@]34O[14CH3]</smiles>

${ }^{1} \mathrm{H}$ NMR $\left(500 \mathrm{MHz}, \mathrm{THF}-d_{8}\right)$

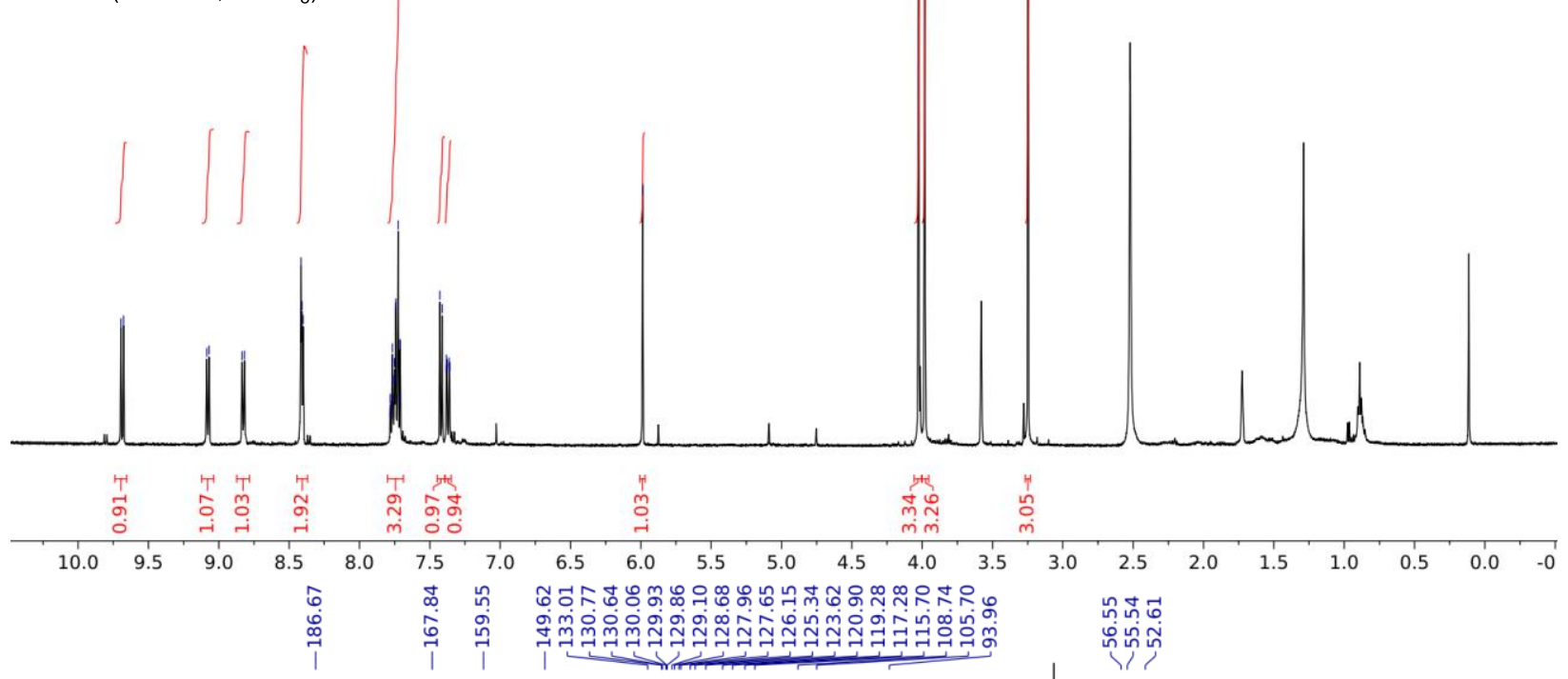<smiles></smiles>

${ }^{13} \mathrm{C}\left\{{ }^{1} \mathrm{H}\right\}$ NMR $\left(125 \mathrm{MHz}\right.$, THF- $\left.d_{8}\right)$

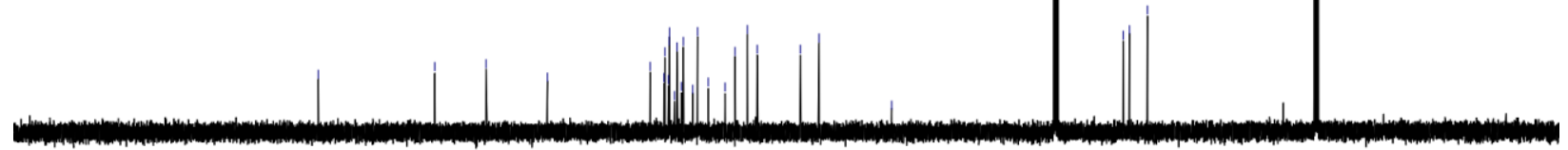

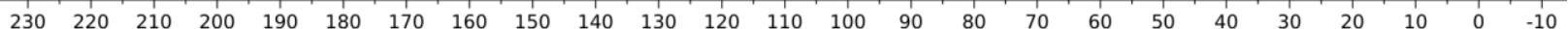



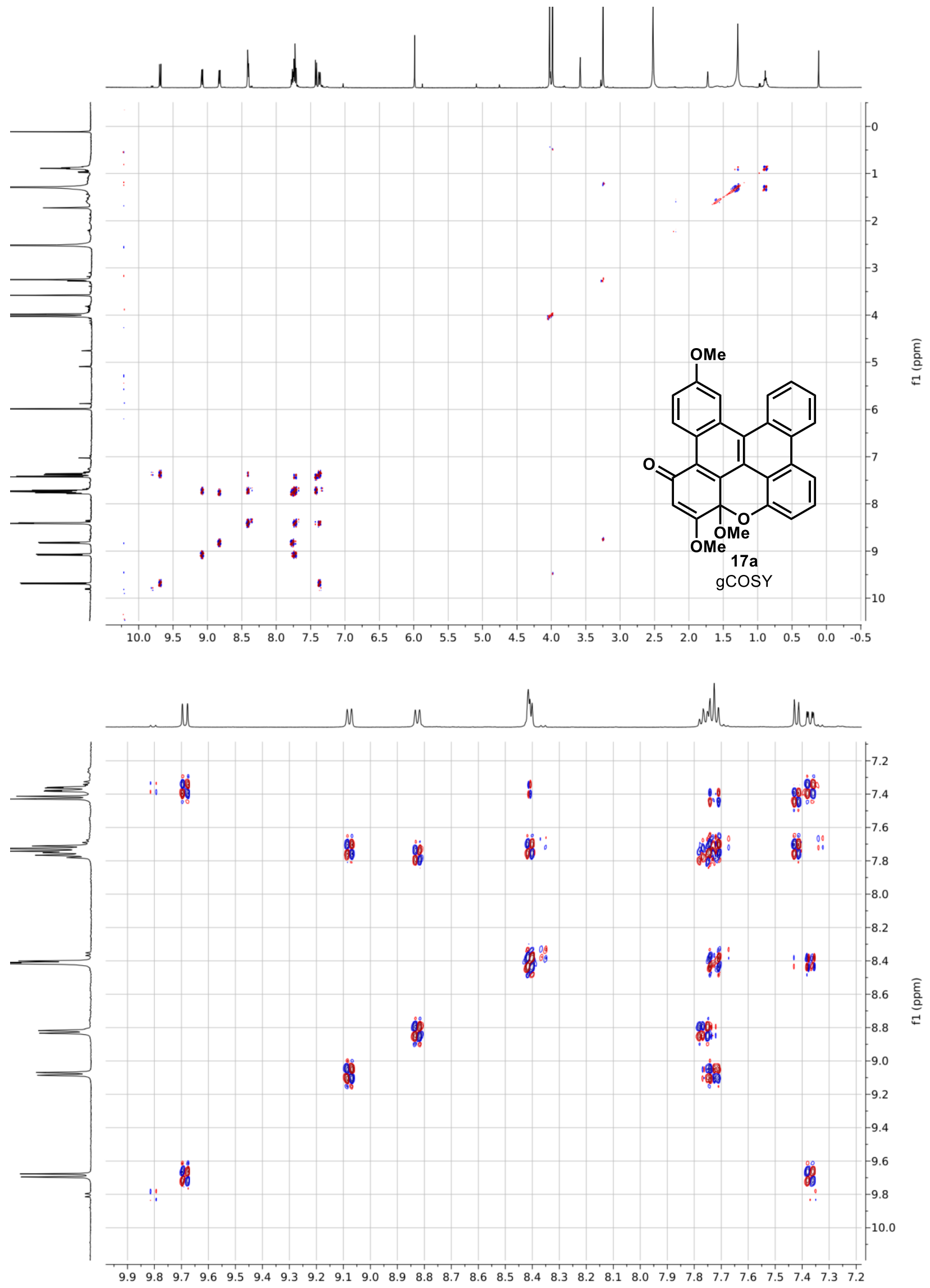


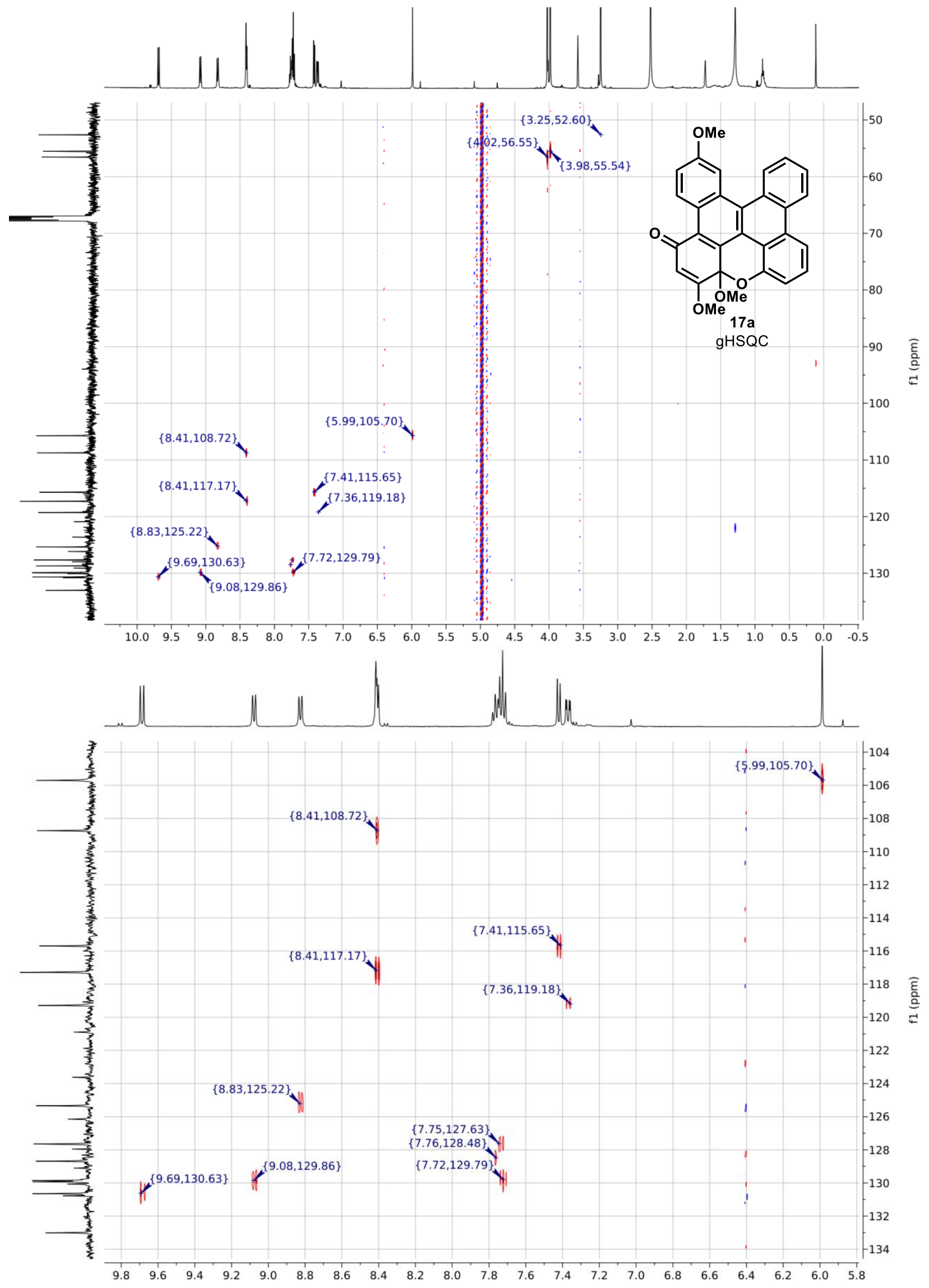




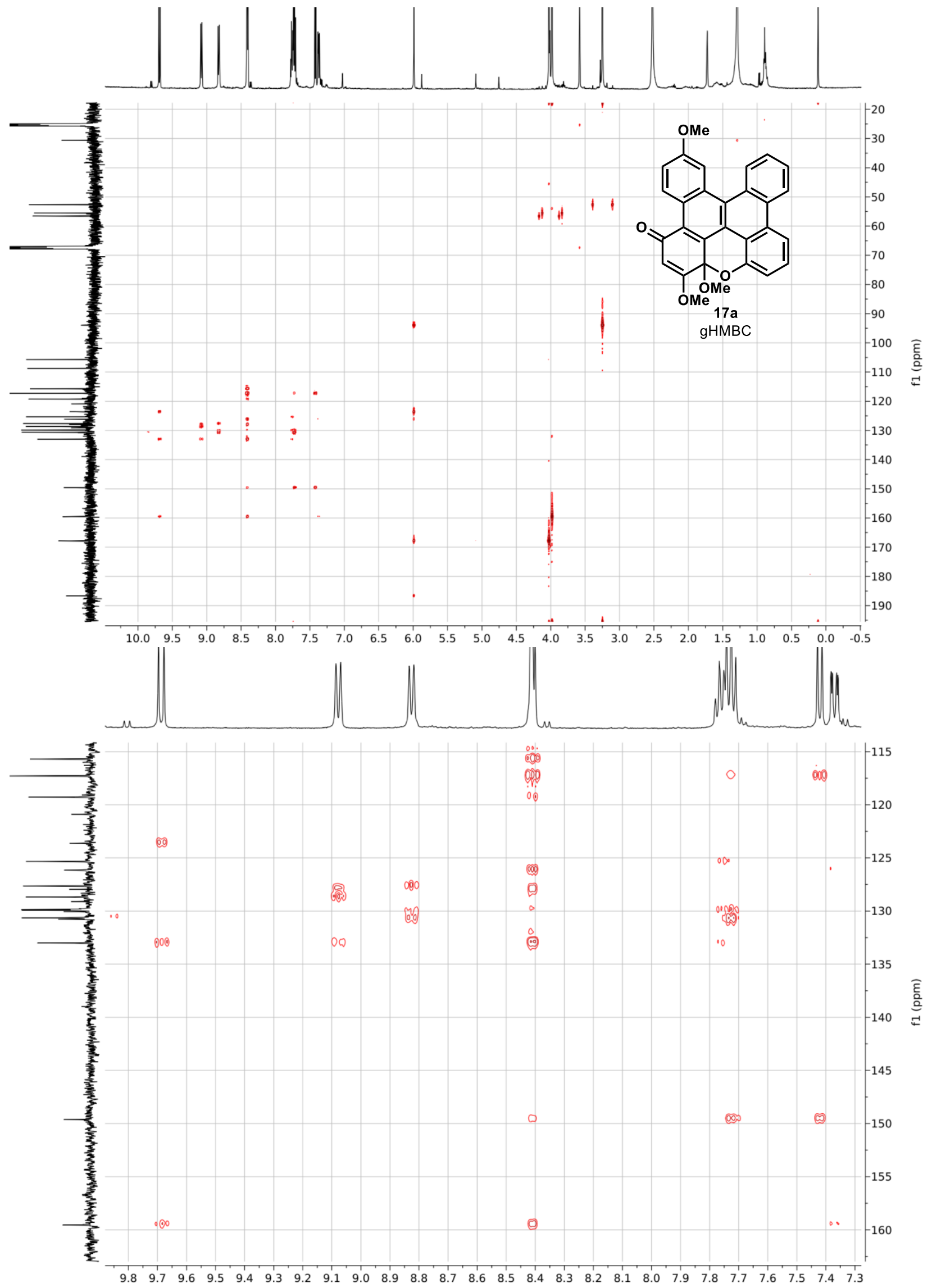

